\title{
Ecosystem metabolism in salt marsh tidal creeks and ponds: Applying triple oxygen isotopes and other gas tracers to novel environments
}

\author{
by \\ Evan M. Howard \\ B.A. in Chemistry, University of Washington 2009 \\ B.S. in Oceanography, University of Washington 2009 \\ Submitted in partial fulfillment of the requirements for the degree of \\ Doctor of Philosophy \\ at the \\ MASSACHUSETTS INSTITUTE OF TECHNOLOGY \\ and the \\ WOODS HOLE OCEANOGRAPHIC INSTITUTION
}

February 2017

(C) 2017 Evan M. Howard. All rights reserved.

The author hereby grants to MIT and WHOI permission to reproduce and to distribute publicly paper and electronic copies of this thesis document in whole or in part in any medium now known or hereafter created.

Author.

Evan M. Howard

Joint Program in Oceanography/Applied Ocean Science and Engineering Massachusetts Institute of Technology and Woods Hole Oceanographic Institution

Certified by

Rachel H.R. Stanley Assistant Professor, Wellesley College Thesis Co-Supervisor

Certified by....

Scott D. Wankel

Associate Scientist, Woods Hole Oceanographic Institution

Thesis Co-Supervisor

Accepted by.

Shuhei Ono

Associate Professor, Massachusetts Institute of Technology Chairperson, Joint Committee for Chemical Oceanography 


\title{
Ecosystem metabolism in salt marsh tidal creeks and ponds: Applying triple oxygen isotopes and other gas tracers to novel environments
}

\author{
by \\ Evan M. Howard \\ Submitted to the MIT-WHOI Joint Program in Oceanography/Applied Ocean Science and \\ Engineering in partial fulfillment of the requirements for the degree of \\ Doctor of Philosophy in Chemical Oceanography
}

\begin{abstract}
Salt marshes are physically, chemically, and biologically dynamic environments found globally at temperate latitudes. Tidal creeks and marshtop ponds may expand at the expense of productive grasscovered marsh platform. It is therefore important to understand the present magnitude and drivers of production and respiration in these submerged environments in order to evaluate the future role of salt marshes as a carbon sink. This thesis describes new methods to apply the triple oxygen isotope tracer of photosynthetic production in a salt marsh. Additionally, noble gases are applied to constrain air-water exchange processes which affect metabolism tracers. These stable, natural abundance tracers complement traditional techniques for measuring metabolism. In particular, they highlight the potential importance of daytime oxygen sinks besides aerobic respiration, such as rising bubbles. In tidal creeks, increasing nutrients may increase both production and respiration, without any apparent change in the net metabolism. In ponds, daytime production and respiration are also tightly coupled, but there is high background respiration regardless of changes in daytime production. Both tidal creeks and ponds have higher respiration rates and lower production rates than the marsh platform, suggesting that expansion of these submerged environments could limit the ability of salt marshes to sequester carbon.
\end{abstract}

Thesis supervisor: Rachel H.R. Stanley

Title: Assistant Professor, Wellesley College

Thesis supervisor: Scott D. Wankel

Title: Associate Scientist, Woods Hole Oceanographic Institution 


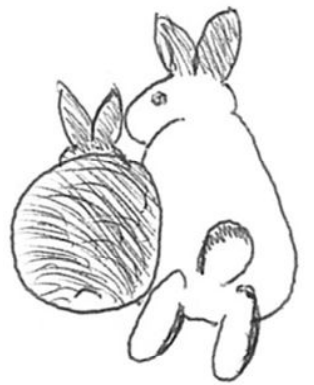

Dedicated in memory of Isabella and Walter. 


\section{Acknowledgments}

Financial support for my doctoral research was provided by the United States Department of Defense through the National Defense Science and Engineering Graduate Fellowship Program, the National Science Foundation under grant OCE-1233678, and the Woods Hole Oceanographic Institution (WHOI) under grants from the WHOI Coastal Ocean Institute, Ocean and Climate Change Institute, and Ocean Life Institute. WHOI Academic Programs Office also provided funding support for research, through the Ocean Ventures Fund, and for my stipend, as graduate research assistantships including an assistantship from the United States Geological Survey administered by WHOI. I am very grateful for this financial support which allowed me earn a living wage while focused on my doctoral research.

In addition, the great majority of this work would not have been possible without the outstanding logistical and in kind support of many scientific teams and individuals, including the Plum Island Ecosystems Long Term Ecological Research site and TIDE experiment staff and scientists (these projects funded by NSF OCE 1238212, NSF DEB 1354494, and NE Climate Science Center grant DOI G12AC00001), Nancy Pau at the Parker River National Wildlife Refuge who granted permits for the work in Chapters 4 and 5, and Liz Kujawinski and Krista Longnecker at WHOI who invited me to participate on cruise KN210-04 in the South Atlantic (funded by NSF grant OCE 1154320).

Specific acknowledgments are also found in each chapter of this thesis, but I'd like to thank a number of individuals in particular who helped me plan for, collect, and analyze the data that underlies this dissertation. First my advisor, Rachel Stanley has supported me over every step of the last five years, whether in the lab or in the field, and she has helped me develop into a better scientist, educator, and communicator. It has been a great pleasure to work with her and I am profoundly glad that she took me on as her student. Her undergraduate students at Wellesley College are very fortunate to have her as a mentor. Second, I am very thankful for the support and advice of my thesis committee, including Anne Giblin, Phil Gschwend, Amanda Sipvak, and my co-advisor Scott Wankel. All have devoted considerable time and energy to helping me succeed and I am lucky to have a team of such distinguished and thoughtful scientists guiding and improving my work. Anne in particular has been an important mentor and role-model for me outside of WHOI. I'd also like to thank Meg Tivey, who chaired my defense and provided me with detailed comments on every chapter. Kim Vaeth also gave me thoughtful feedback on three of the four data chapters in this work, which greatly improved their quality for communicating my scientific work.

Zoë Sandwith was involved in nearly every aspect of my research as the laboratory manager for my advisor Rachel Stanley, and this dissertation would not have been possible without her. Inke Forbrich and Jimmy Nelson were experienced and thoughtful sources of advice and support for every aspect of my work in the marsh, and went out of their way to make me feel like part of a larger scientific community. Anne Giblin and Linda Deegan did the same, and invited me into the Plum Island Ecosystem and TIDE Project community and helping me take advantage of the wellspring of resources and expertise associated with these projects. Justin Ossolinski also helped collect data that I use in three of the following chapters, including both open ocean and marsh work. Jen Karolewski, now a student in the MIT/WHOI Joint Program, was my partner in swampling and provided essential help collecting and analyzing data for Chapters 4 and 5 of this work. I am also grateful to Ashley Bulseco-McKim, David Behringer, Hap Garritt, Katherine Hoering, Kate Morkeski, and the "Sams" (the power team of Sam Kelsey and Sam Bond) who contributed instruments, advice, and muscle power to working in the marsh. I'd also like to thank the staff of the WHOI Isotope Geochemistry Facility for their amazing support with noble gas sampling and analysis: Bill Jenkins, Dempsey Lott, Joanne Goudreau, Josh Curtice, and Kevin Cahill. 
I also would like to thank my collaborators on the various papers associated with some of the work that went into this dissertation, some of whom were mentioned above in other contexts. These include my advisor Rachel Stanley, as well as Jen Bowen, Kevin Cahill, Colleen Durkin, Inke Forbrich, Anne Giblin, Gwenn Hennon, Pat Kearns, Dempsey Lott, Francois Ribalet, and Amanda Spivak.

I'd especially like to thank my labmate Cara Manning, who made our lab such an enjoyable place to work and without whose insights and advice I could not have completed this thesis. Cara's friendship and scientific collaboration has been the best part of my time in the Joint Program. I have many other friends at WHOI and MIT that have made my time here enjoyable and productive: Thank you all.

My family of course deserves thanks as well for supporting my scientific aspirations and being interested in even the most esoteric aspects of my research. My mother, father, and brother have cheered me one throughout my oceanography studies, over a decade at this point, and even earlier encouraged my interest in the sciences by way of trips to places like the Royal Tyrell Museum of Paleontology (a trip to the Canadian badlands was a grand family adventure). My grandfather and nana provided early inspiration for a career in science and technology, going so far as to send me vintage electronics textbooks. Thank you also to the Sawatzki "outlaws" that have embraced me as their own.

Most importantly and finally, thanks to my partner Kate Sawatzki. We've been partners in life and rabbit husbandry for a decade, and it is very much her responsibility that we moved to Boston and I embarked on doctoral research. I would not be who or where I am today without her, and this is definitely for the best (a couple of Boston winters excluded). 


\section{Contents}

\section{Chapter 1}

Introduction

p. 11

Chapter 2

Biological production, export efficiency, and phytoplankton communities across $8000 \mathrm{~km}$ of the South Atlantic: Basin scale similarity with mesoscale variability

p. 19

Chapter 3

Evaluating the effects of nutrient addition on whole ecosystem oxygen metabolism in salt marsh tidal creeks

p. 59

\section{Chapter 4}

Using noble gases in a shallow aquatic environment to compare common gas exchange parameterizations and to constrain efflux of oxygen by ebullition

p. 117

Chapter 5

Production and respiration, and unusual metabolic processes in a salt marsh pond: insights from multiple chemical tracers

p. 135

Chapter 6

Conclusion

p. 173

Bibliography

p. 179 


\section{Chapter 1-Introduction}

\section{Salt marshes-ecologically rich environments with globally significant carbon fluxes}

Salt marshes, which are tidally flooded coastal meadows, are a dominant coastal environment at temperate latitudes globally, and are important in mediating the flow of carbon and nutrients between terrestrial and freshwater environments and the coastal ocean (Cai 2011, Deegan et al. 2012). Net primary production (the balance between photosynthetic production and autotrophic respiration only) is at least an order of magnitude greater in salt marshes than in the open ocean per unit area, and on par with that in tropical forests (Valiela 1995). A small but significant fraction of the carbon dioxide taken up by high rates of primary production in salt marshes is sequestered from the atmosphere on the scale of hundreds to thousands of years because of its rapid burial as peat (Hopkinson et al. 2012); organic matter burial in salt marshes is up to two orders of magnitude faster than in tropical forests on an areal basis, making salt marshes and tropical forests similar global carbon sinks (60-90 $\mathrm{Tg} \mathrm{C} \mathrm{y}^{-1}$ each) despite the fact that salt marshes cover less than 2\% as much surface area (Duarte et al. 2005, Mcleod et al. 2011).

High primary production and subsequent peat accumulation in the grass covered salt marsh platforms provide the basis for a number of ecosystem services. These include physical protection of shoreline from storm surge and erosion, habitat and food for ecologically and economically important species (including the species that contribute $70-80 \%$ of the value of United States fisheries), and filtering of nutrients from coastal waters (Valiela and Teal 1979, Lellis-Dibble et al. 1998, Shepard et al. 2011). However, salt marshes are susceptible to human driven stressors that jeopardize the provision of these ecosystem services (Bromberg Gedan et al. 2009).

In particular, increased nitrogen loading from agricultural runoff and wastewater may alter ecosystem level metabolic fluxes such that the marsh becomes less effective at sequestering carbon (Deegan et al. 2012). Organic matter released from the marsh into the coastal ocean is expected to be respired and returned to the atmosphere, exacerbating carbon dioxide emissions and their contribution to climate change (Cai 2011; Hopkinson et al. 2012, Duarte et al. 2013a). Enhanced nitrogen loading may also lead to algal blooms in surrounding coastal waters that can cause hypoxia, fish kills, and shading and death of seagrasses (McGlathery 2001, Paerl 2006, Thronson and Quigg 2008).

Sea-level rise and infilling of mad-made drainage ditches, extensively used from colonial periods through the early $19^{\text {th }}$ century to control mosquitos and manage hay production, are also hypothesized to contribute to expansion of tidal creeks and marshtop ponds throughout the marsh (Hartig et al. 2002, Wilson et al. 2014). Nutrient loading may similarly contribute to geomorphological change and expansion of these environments at the expense of the productive, grass covered marsh platform (Deegan et al. 2012).

The metabolism of tidal creeks and ponds, as compared to the better studied grasses, is the primary focus of this thesis (Fig. 1). Because of sea-level rise and increased nutrients in estuaries, marshtop ponds and tidal creeks may be expected to play a larger role in overall salt marsh metabolism in the future. Thus, the primary producers and metabolic rates that characterize these environments will become increasingly important to the overall carbon cycling in salt marshes. It is therefore important to understand both the present magnitude and drivers of production and respiration in these environments and how these fluxes may change when evaluating the future role of salt marshes as a carbon sink. 

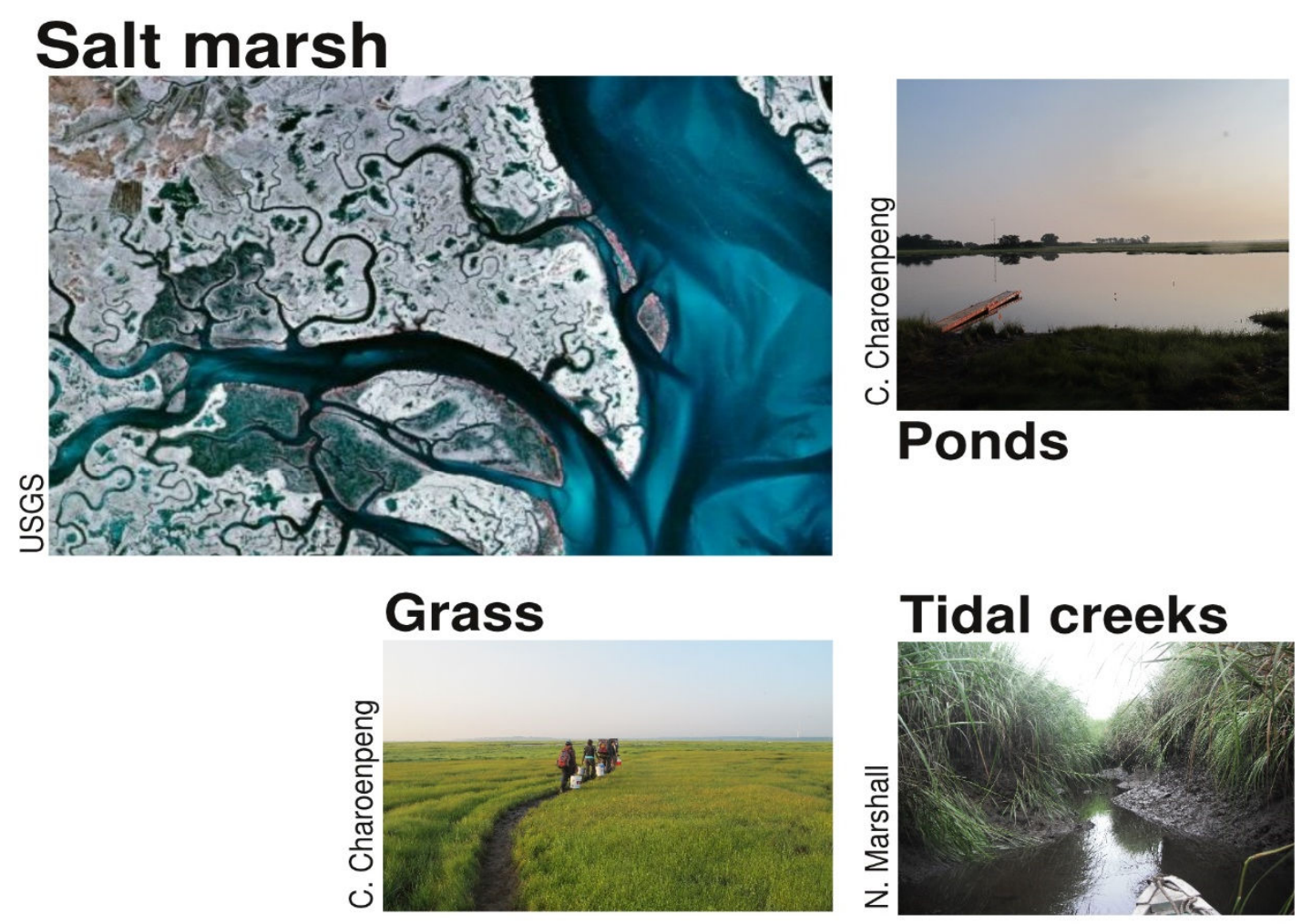

Figure 1: Different environments within the Plum Island Ecosystems Long Term Ecological Research salt marsh in northeastern Massachusetts, USA. Aerial photograph shows tidal creeks and ponds (black), grass covered high marsh platform (gray, light brown) and low marsh with grass and mudflats (green, dark brown). Ponds, tidal creeks, and the grass covered high marsh from this setting are pictured separately as well.

\section{Primary production in salt marshes—vascular plants, seaweeds, and benthic microalgae}

The importance of salt marsh primary production and resultant storage or export of organic matter to adjacent estuaries has been recognized for decades (Teal 1962). Along the United States' East coast, salt marsh macrophyte (visible) plant net primary production is dominated by marsh cordgrass, Spartina alterniflora and $S$. patens. Annual aboveground net primary production by S.patens and S. alterniflora is 50 and $90 \mathrm{~mol} \mathrm{C} \mathrm{m}^{-2} \mathrm{y}^{-1}$, respectively, in the Plum Island Estuarine system in Northeastern Massachusetts, the site of much of my thesis work (Deegan et al. 2012, Morris et al. 2013). Despite these high production rates, Spartina detritus does not appear to support much of the salt marsh food web at this location (Galván et al. 2011). Belowground production of Spartina roots and rhizomes can be greater than aboveground production, and contributes to the impressive carbon burial rates in salt marshes (Dame and Kenny 1986, Turner et al. 2009).

In areas of the marsh that are generally submerged, macrophytes including the seagrass Ruppia maritima and macroalgae such as Ulva intestinalis are important primary producers, contributing between 1 to 30 mol C m${ }^{-2} \mathrm{y}^{-1}$ or more depending on the nutrient supply (Roman et al. 1990, Peckol and Rivers 1996, Kinney and Roman 1998). Ulva in particular opportunistically bloom during nutrient enrichment.

A final important group of primary producers in the salt marsh are the benthic microalgae. These microbial photosynthesizers, including diatoms, cyanobacteria, and green algae, are known to be important food sources for consumers in salt marsh ecosystems (Galván et al. 2008, 2011), including both 
in submerged environments and on the marsh platform. Microalgae mediate nutrient and carbon fluxes in shallow environments year-round, and if light-limited by macrophyte shading in the spring and summer may actually have peak production when macrophyte grasses are least productive (Van Raalte et al. 1976, McGlathery et al. 2004, Sand-Jensen and Nielsen 2004). In salt marshes in particular, microalgae can contribute as much as $10-40 \%$ of annual net primary production on the rarely flooded marsh platform, and are the important or even dominant producers on creek banks, mudflats (Van Raalte et al. 1976, Pinckney and Zingmark 1993, Sullivan and Currin 2000), and shallow ponds on the marsh platform (Gallagher and Daiber 1974). Annual primary production by benthic microalgae in salt marshes ranges from around 4-25 mol C m $\mathrm{y}^{-1}$, although there are significant uncertainties associated with the techniques used to measure microalgae production and validate models of annual production (Williams and Lefèvre 1996, Sullivan and Currin 2000).

It is increasingly important to understand drivers and magnitudes of production by primary producers in the submerged environments as these environments expand. Yet the predicted range and measured uncertainty of previous estimates of production by microalgae, macroalgae, and other submerged producers makes it difficult to evaluate their role in salt marsh metabolism, and thus the role of submerged tidal creeks and ponds on salt marsh carbon cycling. Further, a variety of processes consume and produce metabolic tracers including oxygen and dissolved inorganic carbon that are commonly used to examine production. These processes, such as light enhanced consumption of oxygen or anaerobic metabolisms, may vary with environmental conditions in ways that are not readily captured by standard methods of measuring metabolism fluxes.

\section{Measuring metabolism in submerged environments - existing methods}

Community metabolic fluxes in aquatic ecosystems include respiration, gross primary production (total carbon fixation prior to any respiration), and net community production (synonymously, net ecosystem production or net ecosystem metabolism), the net difference between carbon fixation and respiration in in the ecosystem. Respiration rates vary between autotrophs and heterotrophs, and may be limited by both organic matter and electron acceptor availability. A variety of respiratory mechanisms exist as well, including aerobic and anaerobic respiration pathways. In sunlit systems, gross primary production is generally a measure of total photosynthesis, but chemolithoautotrophs can also fix inorganic carbon and produce organic matter, and may be important primary producers in estuarine sediments. In principle, net community production is the simplest rate to quantify, as it is the net balance of biological carbon fluxes in the ecosystem and should be readily related to the varying abundance of terminal metabolites such as dissolved oxygen and dissolved inorganic carbon. However, variable rates of chemically and physically driven sinks and sources of metabolites can complicate interpretation of the biological fluxes.

In lakes and estuaries in general, net community production and respiration are often evaluated from rates of oxygen production using techniques such as paired light-dark bottle or core incubations (Odum 1956, Cebrian et al. 2007), or diurnal differences of in situ oxygen concentrations in the water column (Vallino et al. 2005, Staehr et al. 2010). Respiration is inferred from the net community production rate in dark experiments or overnight, where photosynthesis is inferred to be zero. Barring major roles of anoxygenic photosynthesis, chemolithoautotrophy, and anaerobic respiration metabolisms (which change concentrations of dissolved inorganic carbon but not oxygen) or non-aerobic sinks of oxygen (such as unconstrained physical fluxes or unconventional biological oxygen utilization pathways), gross primary production can then be calculated as the sum of the measured net community production in the light and inferred respiration.

However, production rates calculated using this standard method may be in error for methodological or environmental reasons. Incubations of water or sediment may introduce methodological biases, and in situ oxygen concentrations are sensitive to many processes besides production. Both incubation and open 
water methods either assume that respiration in the light equals respiration in the dark or must model gross primary production using generalized relationships such as production-biomass or photosynthesisirradiance curves derived from other methods of measuring primary production. Light respiration may exceed dark respiration by factors of 1.5 to to 10 or more in water column and benthic marine systems (Bender et al. 1987, Fenchel and Glud 2000, Pringault et al. 2007) because of processes including lightenhancement of mitochondrial respiration (i.e. dark respiration) and additional biological oxygen sinks driven by high daytime concentrations of irradiance such as photoreduction, photorespiration, and chemical oxidation of reduced compounds.

Alternative methods for assessing gross primary production are varied, with different methodological limitations. In general these techniques fall into three major categories: measurements of Chlorophyll- $a$ or biomass combined with models of growth under various environmental conditions (light, nutrients), more nuanced interpretations of variability in oxygen concentrations, and isotope uptake experiments. Sediment and water column chlorophyll can be measured using fluorometry or high performance liquid chromatography, either on extracted samples or in situ (Underwood and Kromkamp 1999, Kromkamp et al. 2006, Brotas et al. 2007). Unfortunately, the relationship between chlorophyll content and gross primary production depends strongly on species dependent physiology and physical factors such as light attenuation and chlorophyll to carbon ratios, which can vary by almost an order of magnitude (Flynn 2003, Ross and Geider 2009), though theoretical advances suggest that these limitations could be overcome moving forward (Halsey et al. 2010). Additionally, spectrophotometric estimation of Chlorophyll-a in salt marshes may be complicated by interference from degradation products (MacIntyre et al. 1996).

Oxygen microelectrodes in sediments can provide highly precise in situ measurements of benthic production that are independent of the light-dark assumption (Revsbech and Jørgensen 1983). However, these measurements tend to be limited to a very small spatial scale and require correction for benthic community dynamics such as the diel vertical migration of benthic diatoms, which limits their usefulness in determining production at an ecosystem scale (Brotas et al. 2007). The eddy correlation technique uses paired microelectrodes and velocimeters to integrate benthic fluxes over a spatial scale of tens of square meters with high temporal resolution, but can reintroduce assumptions related to light-dark respiration ratios (Berg et al. 2003, Glud et al. 2010).

Gross primary production can be estimated from uptake of radiocarbon labeled bicarbonate in short-term bottle or sediment incubations, or by uptake of $\mathrm{H}_{2}{ }^{18} \mathrm{O}$ in bottle incubations (Gazeau et al. 2007, KrauseJensen et al. 2011). The radiocarbon method has long been used in estuaries and marshes, but rates vary substantially with incubation time and there is uncertainty as to whether they reflect gross primary production or something closer to net primary production (gross primary production minus autotrophic respiration only; Dring and Jewson 1982; Williams and Lefèvre 1996). Indeed, many conclusions about the magnitude and stoichiometry of production based on radiocarbon incubations have subsequently been largely dismissed as artifacts of methodological biases related to that method (Williams and Robertson 1991). Despite this limitation, radiocarbon incubations are the only technique summarized here that accounts for chemoautotrophy, which may be of comparable magnitude to oxygenic photosynthesis in poorly flushed sediments (Tobias and Neubauer 2009). In the open ocean, gross primary production determined from ${ }^{18} \mathrm{O}$ tends to be similar or greater to that obtained from light-dark bottle incubations. However, work in a lake and estuaries that are well below oxygen saturation suggest that ${ }^{18} \mathrm{O}$ derived GPP can be depressed because of intracellular recycling of labeled ${ }^{18} \mathrm{O}^{16} \mathrm{O}$, with large uncertainties related to fractionation during respiratory pathways (Yacobi et al. 2007, Gazeau et al. 2007), and the method is perhaps unsuited for work in the generally low oxygen salt marsh environments. In either type of incubation experiment, bottle effects complicate interpretation of production estimates (Quay et al. 2010).

Clearly there are many potential approaches to measuring production and in particular gross primary production in estuaries that may be applicable to submerged salt marsh environments. I introduce one new 
method as a tool for studying production in salt marshes which has strengths that compliment standard methods.

\section{Applying the triple oxygen isotope method to salt marsh environments}

In this thesis, I use the natural abundance ratios of triple oxygen isotopes $\left({ }^{16} \mathrm{O},{ }^{17} \mathrm{O}\right.$, and $\left.{ }^{18} \mathrm{O}\right)$ to estimate gross primary production. This method has been applied in marine environments, particularly the pelagic ocean, for more than a decade (Luz and Barkan 2000, Juranek and Quay 2013), but is not typically used in estuarine systems. This technique combines some of the strengths of the isotopic and oxygen based methods - it provides a direct measurement of photosynthetic rate, and, when used in situ, integrates over large spatial scales. At the same time, it does not require an artificial isotope spike but relies on natural abundance stable isotopes. Moreover, the triple oxygen isotope method is relatively insensitive to normal aerobic respiration and other mass-dependent fractionation processes, and thus does not require major assumptions regarding the relative rates of light and dark respiration.

The triple oxygen isotope method is based on the different isotopic signatures of dissolved molecular oxygen $\left(\mathrm{O}_{2}\right)$ that is added to a body of water via gas exchange versus that generated in situ during photosynthesis. Atmospheric $\mathrm{O}_{2}$ has a distinct ratio of stable oxygen isotopes ${ }^{16} \mathrm{O}:{ }^{17} \mathrm{O}:{ }^{18} \mathrm{O}$ set by mass independent fractionation during dissociation of ozone in the stratosphere (Thiemens and Heidenreich 1983, Thiemens et al. 2012). Photosynthesis fractionates seawater in a mass dependent manner and generates $\mathrm{O}_{2}$ with an isotopic composition similar to that of the water it was produced from (Luz and Barkan 2000, Barkan and Luz 2011). All measured aerobic respiration pathways have mass dependent fractionation with a nearly constant ratio of ${ }^{17} \mathrm{O}:{ }^{16} \mathrm{O}$ to ${ }^{18} \mathrm{O}:{ }^{16} \mathrm{O}$ isotope enrichment factors (Angert et al. 2003, Luz and Barkan 2005, 2009). The constancy of the ratio between the respiration enrichment factors means that ${ }^{18} \mathrm{O}:{ }^{16} \mathrm{O}$ can be related to ${ }^{17} \mathrm{O}:{ }^{16} \mathrm{O}$ in such a way that respiration effects cancel out. The combined triple oxygen isotope tracer, typically represented by the notation ${ }^{17} \Delta$, depends primarily on the source of the dissolved $\mathrm{O}_{2}$ (Fig. 2), i.e. atmospheric or photosynthetic $\mathrm{O}_{2}$. With an independent estimate of the amount of atmospheric oxygen dissolved in the water using a gas exchange parameterization, the concentration of photosynthetic $\mathrm{O}_{2}$ can be solved for, and it's time rate of change is directly related to gross primary production.

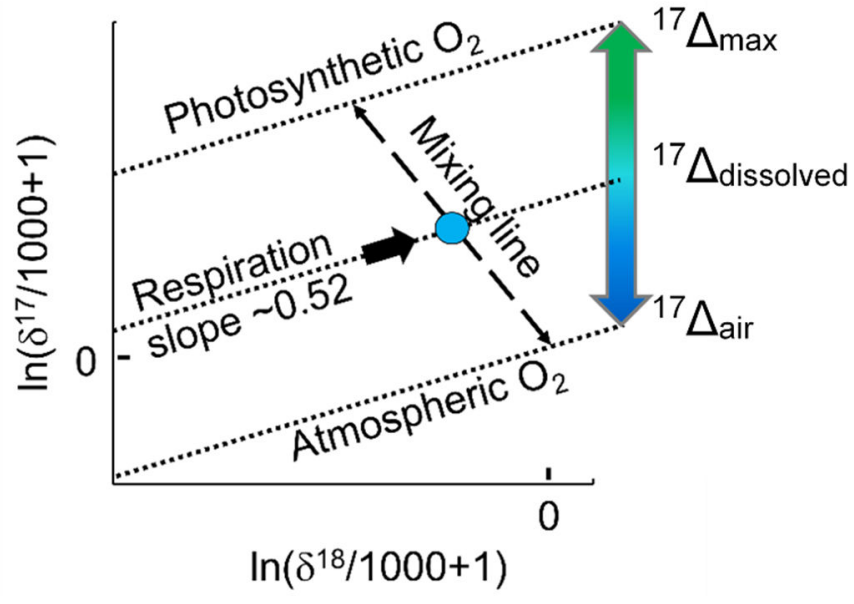

Figure 2: Triple oxygen isotope systematics for dissolved oxygen, after Juranek and Quay (2013). ${ }^{17} \Delta_{\text {dissolved }}$ is the water value, which falls along a mixing line between ${ }^{17} \Delta_{\text {air }}$, the atmospheric endmember, and ${ }^{17} \Delta_{\max }$ the expected photosynthetic endmember for a given system. Respiration along the slope $\gamma_{R}=0.5179 \pm 0.006$ does not change the relative relationship of the oxygen isotope ratios, plotted here in standard isotope notation $\left(\delta^{x}=\left({ }^{x} \mathrm{O}:{ }^{16} \mathrm{O}_{\text {sample }} /{ }^{x} \mathrm{O}:{ }^{16} \mathrm{O}_{\text {standard }}-1\right)\right)$. 
Analysis of data from salt marshes presents some new challenges for the triple oxygen isotope method. In the mixed layer of the pelagic ocean, steady state between photosynthetic production and gas exchange is generally assumed, and the ratio of $\mathrm{O}_{2}$ from each source is equal to the ratio of gross primary production to gas exchange (Hendricks et al. 2004). However, a steady state interpretation is poorly suited to tidal creeks and marshtop ponds, which experience large diel swings in chemical, physical, and biological parameters. So even with an independent estimate of gas exchange, additional constraints related to the physical transport and time evolution of $\mathrm{O}_{2}$ in the water are required. Kaiser (2011) and Prokopenko et al. (2011) derive equations that include additional corrections for equilibrium and kinetic fractionation factors related to gas exchange and photosynthesis, and that do not require an assumption of steady state in dissolved $\mathrm{O}_{2}$ concentration or isotopic ratios if both are monitored. The triple oxygen isotope method is typically also applied assuming a known seawater isotopic composition. However, in salt marshes a substantial fraction of the estuarine water may be derived from meteoric water (from precipitation, rivers, and groundwater) and local seawater may not be similar isotopically to the mean global seawater composition (Luz and Barkan 2010, Li et al. 2015). Additionally, the small photosynthetic fractionations vary between algae types (Helman et al. 2005), such as diatoms versus cyanobacteria, and the mean fractionations assumed for seawater include groups that are unrepresentative of salt marshes, such as coccolithophores. Thus when applying the triple oxygen isotope method in estuarine or coastal environments, care must be taken to account for local differences in water isotopic composition and community structure.

The triple oxygen isotope technique provides a direct, natural abundance measure of gross primary production that yields some improvements over many existing techniques, but the triple oxygen isotope method has weaknesses as well. Sample processing (described in detail in the thesis chapters) is technically challenging, and requires a minimum quantity of total dissolved $\mathrm{O}_{2}$ in the sample for precise and accurate measurement in the isotope ratio mass spectrometer. Low $\mathrm{O}_{2}$ concentrations typical of some tidal stages and periods in the salt marsh require modified sample processing that is particularly timeintensive, and at particularly low $\mathrm{O}_{2}$ large uncertainties can make the method impractical. Additionally, like any oxygen based method it will not capture anoxygenic photosynthesis or chemolithoautotrophic production. Temporal resolution of triple oxygen isotope derived rates from discrete samples is by necessity coarser than $\mathrm{O}_{2}$ concentration based methods since the latter can use rapid response sensors. Finally, production must be relatively high to minimize uncertainties relative to measured values.

Despite these limitations, the triple method provides a useful and novel tool for evaluating primary production in salt marsh tidal creeks and ponds, especially when used in tandem with other metabolism measurement techniques. The triple oxygen isotope method complements standard methods in particular because it requires few assumptions about the relative rate of daytime respiration versus nighttime respiration; indeed, unlike most existing methods, the magnitude of daytime oxygen sinks can be determined by directly comparing triple oxygen isotope derived gross primary production to net community production from measured daytime oxygen fluxes.

\section{Applying noble gases to constrain physical fluxes in salt marsh environments}

In physically dynamic salt marsh environments, physical fluxes of metabolic products such as $\mathrm{O}_{2}$ may be as large or larger than metabolic fluxes. Thus, accurate determination of metabolic fluxes requires careful accounting for physical processes. As part of this thesis, I use the dissolved noble gases in order to constrain air-water fluxes. The stable noble gases (helium, neon, argon, krypton, and xenon) are a suite of naturally occurring, biologically inert tracers that vary widely in solubility and are sensitive to a range of physical processes over the same timescales as $\mathrm{O}_{2}$ and carbon dioxide (Stanley and Jenkins 2013). These processes include diffusive gas exchange and bubbling from the sediments, or ebullition. More soluble gases including krypton and xenon are primarily sensitive to changes in temperature while less soluble neon and helium are highly sensitive to bubble processes. Argon exhibits intermediate sensitivity to these 
processes, similarly to $\mathrm{O}_{2}$. Thus the noble gases allow comparison of published gas exchange parameterizations in addition to characterization of other important, site-specific air-water fluxes that may affect oxygen concentrations and triple oxygen isotope ratios. As air-water gas exchange is frequently the largest source of error for metabolic rates derived from dissolved gas tracers (Raymond and Cole 2001, Emerson et al. 2008, Staehr et al. 2010), noble gas data plays an important role in selecting appropriate air-water exchange parameterizations and maximizing accuracy of calculated metabolism rates.

\section{Thesis overview}

This thesis applies dissolved gas tracers previously used in open ocean settings to less studied salt marsh tidal creeks and pond, in order to better understand the role of these settings to salt marsh carbon cycling and how that may change in a period of increasing human influence on coastal environments. Salt marsh tidal creeks and ponds are physically, chemically, and biologically dynamic environments that have additional complications and require additional considerations compared to surface ocean environments. The following chapters have an overarching theme of applying and modifying methods for dissolved gas tracers of integrated, in situ biological and physical effects (triple oxygen isotopes, oxygen to argon ration, noble gases) to environments where metabolic rates may be poorly constrained by existing techniques.

Chapter 2 uses the triple oxygen isotopes and oxygen to argon ratio in a more standard open ocean setting, but with some modifications to the calculation scheme that allow more accurate assessment of metabolism using these tracers in specific environments in which normal processing of the tracer data falls short. For example, in this chapter equations are introduced to use triple oxygen isotopes in environments where isotopic measurements are influenced by mixing of water masses with different isotopic composition. In Chapter 3, triple oxygen isotopes are used in salt marsh tidal creeks, requiring consideration of even more extensive mixing and physical fluxes which modify the composition of dissolved oxygen simultaneously with biological fluxes. Benthic chamber experiments are introduced as a way to simplify the physical system, but lead to new methodological considerations and biases to the tracer measurements. In Chapter 4, I use dissolved noble gases in a salt pond to evaluate the suitability of literature gas exchange parameterizations to this environment and identify additional important physical fluxes of gases that may influence metabolism tracers. This work sets the stage for Chapter 5, in which I use both the triple oxygen isotope tracer and more standard metabolic tracers (dissolved oxygen and dissolved inorganic carbon) to measure production, respiration, and net metabolism over hourly to seasonal timescales. The combination of tracers allows me to systematically evaluate light-dependent oxygen uptake compared to dark respiration and the potential role of anaerobic and anoxygenic metabolisms on carbon cycling in the salt marsh pond.

I highlight the common themes and guiding questions of the four data analysis chapters below. Taken together, the answers to these questions leads to better understanding of the role of salt marshes in the carbon cycle compared to other marine settings, and how that role may evolve over time in these changing environments. 


\section{Themes:}

Relationships between metabolism and community of primary producers (Chapters 2, 3, 5).

Application of dissolved gas tracers in marine settings where they have not previously been used (Chapters 2, 3, 4, 5).

Methodological modifications for evaluating production in a setting where standard triple oxygen isotope data processing cannot be used $(2,3,5)$.

Physical and biological processes often neglected in ocean settings that may be important for interpreting metabolism (Chapters 3, 4).

\section{Research questions:}

\section{Chapter 2-ocean setting}

How are metabolism, carbon export, and community structure related in the surface South Atlantic Ocean?

\section{Chapter 3-metabolism in salt marsh tidal creeks}

How does nutrient enrichment affect community metabolism in tidal creeks, and does this response differ between the creek-bottom sediments and the integrated creek ecosystem?

\section{Chapter 4-air-water gas exchange in a salt marsh pond}

What air-water diffusive gas exchange parameterization is most appropriate to this small, shallow aquatic system, and are there other physical gas fluxes that are large compared to this flux?

\section{Chapter 5-metabolism in a salt marsh pond}

What are the metabolic rates in a marshtop pond, and how do they vary over hourly to seasonal timescales? Compared to oxygenic photosynthesis and aerobic respiration, do unusual oxygen or carbon metabolisms play a major role in controlling carbon cycling in this environment? 


\section{Chapter 2}

\section{Biological production, export efficiency, and phytoplankton communities across $8000 \mathrm{~km}$ of the South Atlantic: Basin scale similarity with mesoscale variability}

A version of this chapter is under review for potential publication in Global Biogeochemical Cycles. The authors are: Evan M. Howard, Woods Hole Oceanographic Institution, Massachusetts Institute of Technology; Colleen A. Durkin, Moss Landing Marine Laboratories; Gwenn M.M. Hennon, LamontDoherty Earth Observatory; Francois Ribalet, University of Washington; Rachel H.R. Stanley, Wellesley College.

\section{Data availability}

Supporting data are included in the electronic supplementary information (not in this text because of space and file size constraints). The electronic supplementary information is available from the MBL/WHOI library (https://darchive.mblwhoilibrary.org/handle/1912/1172). Additional hydrographic data are available through the Biological and Chemical Oceanography Data Management Office (http://www.bco-dmo.org/project/2204). 


\begin{abstract}
In situ oxygen tracers (triple oxygen isotope and oxygen/argon ratios) were used to evaluate meridional trends in surface biological production and export efficiency across $\sim 8000 \mathrm{~km}$ of the tropical and subtropical South Atlantic in March-May 2013. We used observations of pico-, nano-, and microphytoplankton to evaluate community structure and diversity, and assessed the relationships of these characteristics with production, export efficiency, and particulate organic carbon (POC) fluxes. Rates of productivity were relatively uniform along most of the transect with net community production (NCP) between 0 and $10 \mathrm{mmol} \mathrm{O}_{2} \mathrm{~m}^{-2} \mathrm{~d}^{-1}$, gross primary production (GPP) between 40 and $100 \mathrm{mmol} \mathrm{O}_{2} \mathrm{~m}^{-2} \mathrm{~d}^{-1}$, and NCP/GPP, a measure of export efficiency ranging from 0.1-0.2 (0.05-0.1 in carbon units). However, notable exceptions to this basin scale homogeneity included two locations with highly enhanced NCP and export efficiency compared to surrounding regions. POC flux correlated with GPP rather than with NCP. No significant production differences were observed between different phytoplankton communities, but the variance of production and diversity correlated between biogeographic provinces, indicating a possible shared driver of variability of production and diversity. Overall these results highlight the similarity of biological production over large regions of the Atlantic on the scale of thousands of kilometers, overlain with high variability on the scale of hundreds of kilometers. This intriguing increase in variability on small scales supports growing evidence that mesoscale and smaller processes may be key controls on the biological carbon pump.
\end{abstract}

\title{
Keywords
}

Biological carbon pump, community structure, diversity, triple oxygen isotopes, oxygen argon, particulate organic carbon, South Atlantic, mesoscale

\section{Acknowledgments and data}

Cruise KN210-04 was supported by NSF grant OCE 1154320 to E.B. Kujawinski and K. Longnecker (WHOI). The author was supported by grants from the Woods Hole Oceanographic Institution (WHOI) Ocean and Climate Change Institute and WHOI Ocean Life Institute to R.H.R.S., and the National Defense Science and Engineering Graduate Fellowship program. I would like to thank the captain, crew, and shipboard scientific technicians of the RV Knorr for at sea support, and cruise principal investigators E.B. Kujawinski and K. Longnecker for inviting participation on KN201-04 and generous in-kind support. Thanks as well to Colleen A. Durkin, Gwenn M.M. Hennon, and Francois Ribalet for their constructive feedback on this work in addition to the version under review on which they are coauthors. I am grateful to Z. Sandwith for cruise setup and laboratory assistance with gas tracer analyses and J. Swallwell for assistance with setup and operation of the SeaFlow continuous underway flow cytometry system, and to B. Van Mooy and J. Ossolinski for support in collecting sediment trap samples. 


\section{Introduction}

Marine systems play an important role in the global carbon cycle as sinks or sources of atmospheric carbon dioxide [Takahashi et al. 2009]. The biologically mediated processes controlling fixation and export of carbon from the euphotic zone to the deep ocean, called the biological carbon pump [Volk and Hoffert 1985], are a major control on atmospheric $\mathrm{CO}_{2}$. A primary means of transporting organic matter to the deep ocean is through sinking particulate organic carbon (POC) such as cells, detrital aggregates, and zooplankton fecal pellets [Benitez-Nelson et al. 2001, Ducklow et al. 2001]. Dissolved organic carbon (DOC) fluxes are additionally an important component of carbon export to depth [Hansell et al. 2009, 2013]. Fluxes of POC and DOC out of the surface mixed layer are modulated by community structure and production of phytoplankton and zooplankton [Boyd and Newton 1995, Boyd and Trull, 2007, Lomas and Bates 2004, Guidi et al. 2009, 2016]. Plankton community structure is variable across space (tens of meters to ocean basins) and time (hours to decadal time scales), and may be a major factor determining the magnitudes of production rates and carbon export, and thus the efficiency of the biological carbon pump in sequestering carbon to the deep ocean [Karl et al. 2001, 2012, Vaillancourt et al. 2003, Richardson and Jackson 2007, Henson et al. 2012]. Investigators have described correlations of community structures with patterns of primary production and carbon export in a variety of nutrient regimes [Pollard et al. 2009, Moreno-Ostos et al. 2011, Lockwood et al. 2012, Cassar et al. 2015]. Transition regions at gyre edges between higher and lower nutrient regimes can be zones of high biomass and intensified production [Juranek et al. 2012, Ostle et al. 2015] with unique phytoplankton communities distinct from the regions to either side [Kavanaugh et al. 2014a, Palevsky et al. 2013, Ribalet et al. 2010].

Such relationships between physical ocean structure and nutrients, biological community structure, and the biological carbon pump can be combined with expanding availability of remotely sensed data [Johnson et al. 2009, McClain 2009, Xing et al. 2011] in order to model primary production and carbon export over regional and global scales [Behrenfeld and Falkowski 1997, Westberry et al. 2008, Utiz et al. 2010]. In some parts of the ocean, satellite informed model results poorly reproduce in situ observations of production and export in terms of both magnitude and variability [Friedrichs et al. 2009, Palevsky et al. 2013, Emerson 2014, Lee et al. 2015, Świrgoń and Stramska 2015], suggesting that there are still gaps in the mechanistic understanding of what drives production and export. The food-web approach of Siegel et al. [2014] demonstrates that a better understanding of how community structure affects production can improve model predictions of carbon export [Stukel et al. 2015]. A growing body of literature suggests that physical variability is an important determinant of community structure [Barton et al. 2010], production [Nicholson et al. 2008], and export [Ayers and Lozier 2012]. In particular submesoscale to mesoscale (kilometers to hundreds of kilometers) variability may play an important role in increasing phytoplankton community diversity [Clayton et al. 2013, 2014, Lévy et al. 2014, 2015] and concentrating or enhancing biological activity [McGillicuddy et al. 2007, Klein and Lapeyre 2009, Estapa et al. 2015] at small scales.

Variability in carbon export is affected by both community structure and the underlying physical variation driving changes in phytoplankton production. We evaluate biological carbon pump fluxes and phytoplankton community structure from the mesoscale to the basin scale across an $8000 \mathrm{~km}$ transect in the western subtropical and equatorial South Atlantic Ocean by combining measurements of gross primary production and net community production rates (triple oxygen isotope and oxygen argon natural abundance tracers) with concurrent in situ, high resolution measurements of cyanobacteria and picoeukaryote abundances (underway flow cytometry), nano-and microphytoplankton abundances (visual microscopy), and POC export fluxes below the surface mixed layer (drifting sub-surface sediment traps). Gross primary production (GPP) is equal to total photosynthetic production prior to any respiration or other recycling of oxygen or organic carbon [Williams 1993]. Net community production (NCP) is equal to GPP minus community respiration, and expresses the net carbon drawdown or oxygen production by the biological pump. NCP should be balanced by export of carbon over sufficient scales of time and space 
[Emerson et al. 1997, Brix et al. 2006], including dissolved organic carbon (DOC) and zooplankton mediated export in addition to POC sinking out of the mixed layer [Emerson 2014]. The NCP/GPP ratio is a measure of metabolic efficiency akin to the f-, e-, and ef-ratios [Eppley and Peterson 1979, Murray et al. 1996, Laws et al. 2000]. Thus the ratio of NCP/GPP has been called an "export ratio in $\mathrm{O}_{2}$ currency" [Juranek and Quay 2013]. Higher NCP/GPP ratios indicate that a larger fraction of production is not respired locally over the residence time of the oxygen based tracers.

In particular, we evaluate (1) how changes in the spatial variability of phytoplankton abundance, community structure, and diversity and production relate to export from the basin scale to the mesoscale, (2) whether differences in net community production and particulate organic carbon export rates are potentially balanced by dissolved organic carbon production, and (3) if export is proportional to autotroph production (GPP) in a transect where picophytoplankton dominate the phytoplankton community [Richardson and Jackson 2007, Stukel and Landry 2010, Guidi et al. 2016].

\section{Methods}

All samples were collected aboard the $R V$ Knorr (KN210-04). The cruise traversed $8480 \mathrm{~km}$ and nearly $22^{\circ}$ of latitude of the western South and Equatorial Atlantic (Figure 1) between Montevideo, Uruguay, and Bridgetown, Barbados from 25 March through 9 May of 2013, in austral fall. The cruise track encompasses a number of surface physical regimes, including the South Atlantic subtropical front and transition region, subtropical gyre, and the tropics and equatorial current system [Tsuchiya et al. 1994, Stramma and Schott 1999, Mémery et al. 2000]. Finally, the cruise passed through the pelagic and mesohaline portions of the Amazon River plume [Hu et al. 2004]. These physically defined regions overlap distinct biogeographic provinces [Longhurst 1995], including the South Subtropical transition zone (SST), the South Atlantic Gyre (SAG), the Western Tropical Atlantic (WTA) and the Amazon River Plume (ARP). Samples for gas tracer analysis were collected from surface waters while underway and at discrete stations (Table 1). At three stations (locations 2, 9, and 20) the Knorr remained stationary for one to two days. At three other stations, the ship followed drifting particle traps [Durkin et al. 2016] in a quasi-Lagrangian manner for one to three days (locations 7, 25, 31). At all of these stations with longer sampling durations (Table 1), multiple discrete gas tracer samples were collected either at arrival and departure from the station, or as a time-series over the duration of the stay. Gas tracer estimates of production across the transect were compared with concurrent underway observations of surface cyanobacterial and picoeukaryote populations [Hennon 2015, Hennon et al. in prep]. Data were also compared with concurrent observations of surface eukaryotic phytoplankton abundance and the phytoplankton and carbon fluxes captured in free-floating particle interceptor traps at $125 \mathrm{~m}$ reported by Durkin et al. [2016]. Particulate nitrogen fluxes analyzed simultaneously with particulate carbon were not previously reported, but analyzed as in Durkin et al. [2016]. 

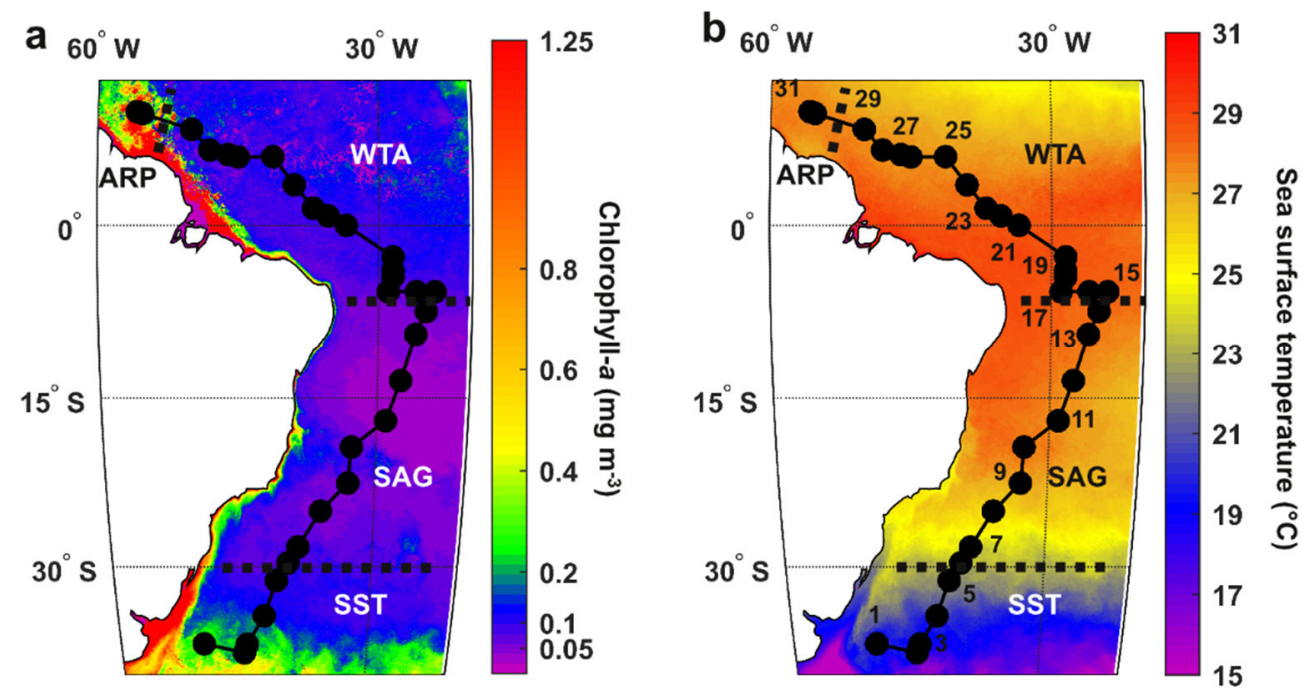

Figure 1: Sample locations in the Equatorial and South Atlantic overlaid on satellite derived (a) sea surface Chlorophyll- $a$ and (b) daytime sea surface temperature averaged from March through May 2013 (with odd station numbers labeled). Four biogeographic provinces identified as in the text (South Subtropical Transition zone, SST; South Atlantic Gyre, SAG; Western Tropical Atlantic, WTA; Amazon River Plume, ARP) are noted in each panel and separated by dashed lines. Sea surface data are from MODIS-Aqua satellite and obtained from the Giovanni online data system (Acker and Leptoukh [2007]; http:Giovanni.gsfc.nasa.gov/Giovanni/) maintained by the NASA Goddard Earth Science Data and Information Services Center. 
Table 1: Sample locations and replicates with respect to biogeographic provinces

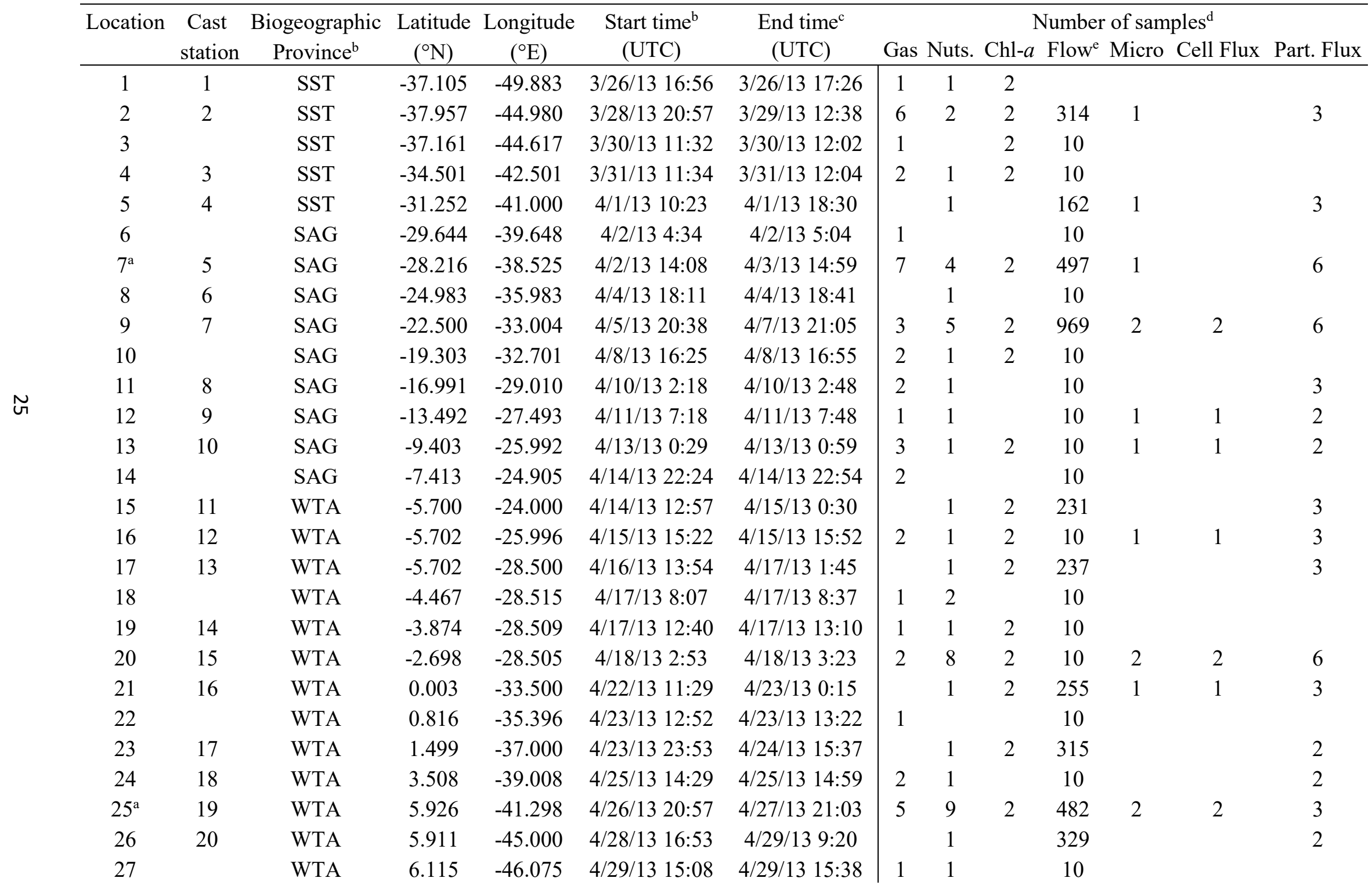


Table 1: Sample locations and replicates with respect to biogeographic provinces, continued

\begin{tabular}{|c|c|c|c|c|c|c|c|c|c|c|c|c|c|}
\hline \multirow[t]{2}{*}{ Location } & \multirow{2}{*}{$\begin{array}{c}\text { Cast } \\
\text { station }\end{array}$} & \multirow{2}{*}{$\begin{array}{c}\text { Biogeographic } \\
\text { Province }^{\mathrm{b}}\end{array}$} & \multirow{2}{*}{$\begin{array}{l}\text { Latitude } \\
\left({ }^{\circ} \mathrm{N}\right)\end{array}$} & \multirow{2}{*}{$\begin{array}{c}\text { Longitude } \\
\left({ }^{\circ} \mathrm{E}\right)\end{array}$} & \multirow{2}{*}{$\begin{array}{l}\text { Start time }^{\mathrm{b}} \\
\text { (UTC) }\end{array}$} & \multirow{2}{*}{$\begin{array}{l}\text { End time }^{c} \\
\text { (UTC) }\end{array}$} & \multicolumn{7}{|c|}{ Number of samples ${ }^{d}$} \\
\hline & & & & & & & Gas & Nuts. & Chl- $a$ & Flow $^{\mathrm{e}}$ & Micro & Cell Flux & Part. Flux \\
\hline 28 & 21 & WTA & 6.513 & -48.008 & $4 / 30 / 1311: 19$ & $4 / 30 / 1311: 49$ & 2 & 1 & 2 & 10 & 2 & 2 & 3 \\
\hline 29 & 22 & WTA & 8.279 & -49.984 & 5/1/13 14:06 & $5 / 1 / 1314: 36$ & 1 & 1 & 2 & 10 & & & 2 \\
\hline 30 & & ARP & 9.835 & -55.860 & $5 / 3 / 138: 48$ & $5 / 3 / 139: 18$ & 2 & 1 & 2 & 10 & & & \\
\hline $31 \mathrm{a}$ & 23 & ARP & 9.670 & -55.283 & $5 / 3 / 13$ 15:39 & $5 / 6 / 1314: 33$ & 10 & 4 & 2 & 1418 & 2 & 2 & 6 \\
\hline
\end{tabular}

a. quasi-Lagrangian sampling

b. South Subtropical Transition (SST), South Atlantic Gyre (SAG), Western Tropical Atlantic (WTA), Amazon River Plume (ARP)

c. corresponds to evaluated period of underway flow cytometry

d. Gas tracers (gas), nutrients (nut.), Chlorophyll- $a$ (Chl-a), Flow cytometry (Flow), microscopy (micro), cell and particle number flux (Cell Flux), particulate organic carbon and particulate nitrogen flux (Part. Flux)

e. number of 3 minute averaging periods 


\subsection{Underway sampling line}

Surface water was collected using the ship's underway flow-through system at $5 \mathrm{~m}$ depth, and passed through an inline de-bubbler and a Seabird Electronics SBE45 thermosalinograph prior to sample collection. In order to minimize biofouling, the underway line was flushed with bleach at the beginning and halfway through the cruise (April 3, 2013) by dissolving 150-200 g pelletized sodium hypochlorite in the $\sim 150$ L sea chest ( $\sim 0.1 \%$ sodium hypochlorite solution) [after Juranek et al. 2010 ]. After each cleaning, the underway line was then flushed for 12-15 hours by normal seawater flow prior to commencing sampling. $1 \mathrm{~L} \mathrm{~min}^{-1}$ of water from the underway line was diverted for sampling of gas tracers, nutrients, and Chlorophyll- $a$.

\subsection{Discrete gas tracer theory, sampling, and analysis}

\subsection{1 $\mathrm{O}_{2} / \mathrm{Ar}$ and triple oxygen isotope ratio theory and calculation of production rates}

We use the oxygen to argon ratio as a tracer of net oxygen production in the surface mixed layer. Argon acts as a biologically inert analogue of oxygen in seawater, and the difference in saturation state between the two gases is assumed to correspond primarily to the balance of photosynthesis and respiration [e.g. Craig and Hayward 1987, Spitzer and Jenkins 1989, Emerson et al. 1991]. Extending the equations by Cassar et al. [2011] such that argon is not assumed to be at saturation and neglecting horizontal and vertical mixing, the time evolution of oxygen relative to argon in the mixed layer is controlled by production and gas exchange:

$$
\mathrm{z} \frac{\partial}{\partial \mathrm{t}}\left(\mathrm{O}_{2}\left[\frac{\Delta \mathrm{O}_{2}}{\mathrm{Ar}}\right]\left[\frac{\mathrm{Ar}}{\mathrm{Ar}_{\mathrm{sat}}}\right]\right) \rho_{s w}=\mathrm{NCP}-\mathrm{k}_{\mathrm{O} 2} \mathrm{O}_{2} \text { sat }\left[\frac{\Delta \mathrm{O}_{2}}{\mathrm{Ar}}\right]\left[\frac{\mathrm{Ar}}{\mathrm{Ar}_{\mathrm{sat}}}\right] \rho_{s w}
$$

where $\mathrm{z}$ is the mixed layer depth defined as a $0.125 \mathrm{~kg} \mathrm{~m}^{-3}$ offset in potential density from the surface [Levitus 1982], $\mathrm{O}_{2}$ and $\mathrm{Ar}$ are measured concentrations (molal), and $\mathrm{O}_{2 \text { sat }}$ and $\mathrm{Ar}_{\text {sat }}$ are saturation concentrations (molal) [Garcia and Gordon 1992, 1993, Hamme and Emerson 2004]. The ratio $\Delta \mathrm{O}_{2} / \mathrm{Ar}=\left[\left(\mathrm{O}_{2} / \mathrm{Ar}\right) /\left(\mathrm{O}_{2} / \mathrm{Ar}\right)_{\text {saturation }}-1\right] \times 100 \%$ reflects the net biological oxygen production or consumption [Hendricks et al. 2004, Kaiser et al. 2005]. In particular, the "measured" Ar is actually derived from the measured $\mathrm{O}_{2}$ and $\mathrm{O}_{2} / \mathrm{Ar}$. NCP is an areal rate $\left(\mathrm{mol} \mathrm{O}_{2} \mathrm{~m}^{-2} \mathrm{~d}^{-1}\right)$ and $\rho_{\mathrm{sw}}$ is the in situ density of seawater. $\mathrm{k}_{\mathrm{O} 2}$ is the gas transfer coefficient $\left(\mathrm{m} \mathrm{d}^{-1}\right)$ calculated from $6 \mathrm{~h}$ NCEP/NCAR Reanalysis winds [Kalnay et al. 1996] for the cruise track from March to May 2013 using the gas exchange parameterization of Stanley et al. [2009] and the 60 day weighting scheme of Reuer et al. [2007]. The relationship between windspeed and gas exchange is constrained to $\sim \pm 15-20 \%$ [Stanley et al. 2009, Ho et al. 2011, Wanninkhof et al. 2014], and is in general the largest source of error in resulting NCP rates.

The triple isotope composition of dissolved oxygen is related to photosynthetic oxygen production and is largely independent of respiration. Juranek and Quay [2013] discuss the theory, advantages, and limitations of this method in detail. Measured stable oxygen isotope ratios $\left({ }^{18} \mathrm{O} /{ }^{17} \mathrm{O} /{ }^{16} \mathrm{O}\right)$ can be used to define the triple oxygen isotope tracer ${ }^{17} \Delta$ in order to conceptualize the relative contribution of photosynthetic oxygen to different water samples, with greater values indicating increased rates of production relative to gas exchange with the atmosphere. In this work we use the definition ${ }^{17} \Delta=\left(\ln \left[\left({ }^{17} \mathrm{O} /{ }^{16} \mathrm{O}\right)_{\text {measured }}\left({ }^{17} \mathrm{O} /{ }^{16} \mathrm{O}\right)_{\text {air }}\right]-\lambda \ln \left[\left({ }^{18} \mathrm{O} /{ }^{16} \mathrm{O}\right)_{\text {measured }} /\left({ }^{18} \mathrm{O} /{ }^{16} \mathrm{O}\right)_{\text {air }}\right]\right) \times 10^{6}$ per meg [Angert et al. 2003] relative to an atmospheric oxygen standard, where $\lambda=0.518$ is the ratio of the mass dependent isotope enrichment factors for ${ }^{17} \mathrm{O} /{ }^{16} \mathrm{O}$ and ${ }^{18} \mathrm{O} /{ }^{16} \mathrm{O}$. Gross primary production $\left(\mathrm{mmol} \mathrm{O}_{2} \mathrm{~m}^{-2} \mathrm{~d}^{-1}\right)$ is calculated for the surface mixed layer in terms of a balance between gas exchange and production using the oxygen isotope ratios and $\lambda$. If the measured ${ }^{17} \mathrm{O} /{ }^{16} \mathrm{O}$ and ${ }^{18} \mathrm{O} /{ }^{16} \mathrm{O}$ ratios are notated as ${ }^{17} \mathrm{X}$ and ${ }^{18} \mathrm{X}$ :

$$
\mathrm{z} \frac{\partial}{\partial \mathrm{t}}\left(\mathrm{O}_{2}{ }^{17} \Delta\right) \rho_{s w}=\operatorname{GPP}\left(\frac{{ }^{17} \mathrm{X}_{\mathrm{P}}-{ }^{17} \mathrm{X}}{{ }^{17} \mathrm{X}}-\lambda \frac{{ }^{18} \mathrm{X}_{\mathrm{P}}-{ }^{18} \mathrm{X}}{{ }^{18} \mathrm{X}}\right)-\mathrm{k}_{\mathrm{O} 2} \mathrm{O}_{2 \text { sat }}\left(\frac{{ }^{17} \mathrm{X}-{ }^{17} \mathrm{X}_{\text {sat }}}{{ }^{17} \mathrm{X}}-\lambda \frac{{ }^{18} \mathrm{X}-{ }^{18} \mathrm{X}_{\text {sat }}}{{ }^{18} \mathrm{X}}\right) \rho_{\text {sw }}
$$


where ${ }^{i} X_{P}$ is the photosynthetic production ratio and ${ }^{i} X_{\text {sat }}$ the saturated equilibrium ratio for each isotope (Table S1; this and all other supplementary tables are available electronically as described in the data availability statement). Alternate choices for ${ }^{\mathrm{i}} \mathrm{X}_{\mathrm{P}}$ and ${ }^{\mathrm{i}} \mathrm{X}_{\text {sat }}$ and $\lambda$ may be appropriate under some circumstances [Kaiser 2011, Nicholson 2011, Kaiser and Abe 2012]. The above equation is identical to Equation S8 in the Supplemental Information of Prokopenko et al. [2011], and neglects fractionation effects during gas exchange and bubble fluxes [Kaiser 2011].

Nicholson et al. [2014] suggest including corrections to Equation 2 to account for seasonal entrainment and mixing across the base of the thermocline (e.g. left side of Eq. 2 becomes $\left.\mathrm{z}(\partial / \partial \mathrm{t})\left(\mathrm{O}_{2}{ }^{17} \Delta\right) \rho_{\mathrm{sw}}-C_{G P P}\right)$. These corrections are expressed as the tracer difference from an appropriate point measured below the mixed layer multiplied by the entrainment $\partial z / \partial t$ (only when the mixed layer deepens) and mixing rate $\mathrm{K}_{\mathrm{z}} /\left(\mathrm{z}_{d}-\mathrm{z}\right)$, where $\mathrm{K}_{\mathrm{z}}$ is the vertical diffusivity coefficient $\left(\mathrm{m}^{2} \mathrm{~d}^{-1}\right)$ across the thermocline at $\mathrm{z}_{\mathrm{d}}$, the depth of the sub-mixed layer sample. We apply the same approach to calculating NCP from $\mathrm{O}_{2}$ and Ar.

Simplifying the expressions of Nicholson et al. [2014] in terms of measured variables (see supplemental text for derivation), in the case where $\partial \mathrm{z} / \partial \mathrm{t}>0$, we obtain corrections $C_{\mathrm{NCP}}$ and $C_{\mathrm{GPP}}$ for Equations 1 and 2:

$$
\begin{gathered}
C_{\mathrm{NCP}}=\left(\frac{\partial \mathrm{z}}{\partial \mathrm{t}}+\frac{\mathrm{Kz}}{\left(\mathrm{z}_{\mathrm{d}}-\mathrm{z}\right)}\right)\left(\mathrm{O}_{2 \text { sat }}\left[\frac{\mathrm{O}_{2 \mathrm{~d}}-\mathrm{O}_{2}}{\mathrm{O}_{2 \text { sat }}}-\frac{\mathrm{Ar}_{\mathrm{d}}-\mathrm{Ar}}{\mathrm{Ar}_{\mathrm{sat}}}\right]\right) \rho_{\mathrm{sw}} \\
C_{\mathrm{GPP}}=\left(\frac{\partial \mathrm{z}}{\partial \mathrm{t}}+\frac{\mathrm{Kz}}{\left(z_{d}-\mathrm{z}\right)}\right) \mathrm{O}_{2 d}\left(\left[\frac{{ }^{17} X_{d}}{{ }^{17} X}-1\right]-\lambda\left[\frac{{ }^{18} X_{d}}{{ }^{17} X}-1\right]\right) \rho_{s w}
\end{gathered}
$$

where the $d$ subscript refers to measurements below the base of the mixed layer ( $\sim 20 \mathrm{~m}$ below the bottom of the mixed layer). Entrainment is estimated from the Monthly Isopycnal and Mixed-layer Ocean Climatology [Schmidko et al. 2013] based on Argo profiler data, and $\partial \mathrm{z} / \partial \mathrm{t}$ is set to zero for a shoaling mixed layer. $K_{z}$ is estimated to have a lower bound of $1.0 \times 10^{-5} \mathrm{~m}^{2} \mathrm{~s}^{-1}\left(8.6 \times 10^{-1} \mathrm{~m}^{2} \mathrm{~d}^{-1}\right)$ across the bottom of the mixed layer based on direct measurements along a similar transect at the same time of year [Mouriño-Carballido et al. 2011]. The time rate of change terms generally cannot be evaluated using the discrete gas samples, so steady-state is assumed and Equations 1 and 2 are solved for NCP and GPP as:

$$
\begin{gathered}
\mathrm{NCP}=\mathrm{k}_{\mathrm{O} 2} \mathrm{O}_{2} \text { sat }\left[\frac{\Delta \mathrm{O}_{2}}{\mathrm{Ar}}\right]\left[\frac{\mathrm{Ar}}{\mathrm{Ar}}\right] \rho_{s w}-C_{N C P} \\
\mathrm{GPP}=\left[\mathrm{k}_{\mathrm{O} 2} \mathrm{O}_{2 \text { sat }}\left(\frac{{ }^{17} \mathrm{X}-{ }^{17} \mathrm{X}_{\text {sat }}}{{ }^{17} \mathrm{X}}-\lambda \frac{{ }^{18} \mathrm{X}-{ }^{18} \mathrm{X}_{\text {sat }}}{{ }^{18} \mathrm{X}}\right) \rho_{S w}-C_{G P P}\right] /\left(\frac{{ }^{17} \mathrm{X}_{\mathrm{P}}-{ }^{17} \mathrm{X}}{{ }^{17} \mathrm{X}}-\lambda \frac{{ }^{18} \mathrm{X}_{\mathrm{P}}-{ }^{18} \mathrm{X}}{{ }^{18} \mathrm{X}}\right)
\end{gathered}
$$

The potential role of upwelling and surface divergence is not accounted for in this formulation. To calculate NCP and GPP for sample locations without depth profiles, sub-mixed layer values of $\Delta \mathrm{O}_{2} / \mathrm{Ar}$ (and thus $\mathrm{Ar} / \mathrm{Ar}_{\mathrm{sat}}$ ), ${ }^{17} \Delta$, and ${ }^{17} \mathrm{X}$ (see section 2.2.4) were approximated using weighted-mean averaging from adjacent casts. Oxygen profiles at the sample locations were additionally used to constrain likely subsurface values of $\Delta \mathrm{O}_{2} / \mathrm{Ar}$. Five locations (noted in Figure 3) are separated from the nearest depth profiles by more than $2^{\circ}$ latitude, and interpolating sub-mixed layer $\Delta \mathrm{O}_{2} / \mathrm{Ar}$ for entrainment and mixing corrections introduces additional uncertainty in the rate of NCP; the dynamic range of the corrections immediately below the mixed layer is five times greater for $\Delta \mathrm{O}_{2} / \mathrm{Ar}$ relative to its uncertainty than for ${ }^{17} \Delta$.

We address probable systematic biases introduced in the gas mass balances for NCP and GPP in the supplemental text. These include lateral advection [Peterson and Stramma 1991, Lumpkin and Garzoli 2005, Brandt et al. 2006], upwelling [Helber et al. 2007, Grodsky et al. 2008, Rhein et al. 2010, Kadko and Johns 2011], and greater vertical diffusivity [Carr et al. 1992, Wunsch and Ferarri 2004, Haskell et al. 2015]. Most assumptions above are reasonable for this dataset. However, diel cycles of the gas tracers [Sarma et al. 2006, Ferrón et al. 2015, Nicholson et al. 2015] can lead to large errors in steady-state production rates as calculated above (as high as $\pm 50 \%$ ) if samples are taken during daily periods of peak tracer accumulation and deficit [Hamme et al. 2012]. While this is not a major bias in this dataset (see 
supplemental text and Figures S1 and S2, at the end of this chapter), it may be a larger source of error in other studies when it is not possible to sample multiple timepoints within a given day.

\subsubsection{Conversion between oxygen and carbon units}

Carbon and oxygen based rates of net community production are related by the ratio of oxygen production to carbon uptake during photosynthesis, $\mathrm{O}_{2} / \mathrm{C}=1.4$ for nitrate based production and 1.1 for growth on ammonia [Laws 1991], or similarly 1.0-1.2 for nitrogen fixation-supported growth [Kirk 1994 and references therein]. A relationship exists between carbon based net primary production (NPP, GPP minus autotrophic respiration only [Falkowski et al. 2003]) derived from 24 hour radiocarbon incubations and ${ }^{18} \mathrm{O}$ incubation based GPP [Marra et al. 2002] or in situ triple oxygen isotope based GPP [Juranek et al. 2013]; the ratio of GPP (moles of oxygen)/NPP (moles carbon) is roughly 2.7 across a number of ocean regions and experiments [Juranek and Quay 2013 and references therein]. We use this ratio to compare our rates to radiocarbon incubations in other studies, but the incubations were shorter than 24 hours and thus may have lower and less well defined conversion factors [Dring and Jewson 1982, Williams and Lefèvre 1996, Behrenfeld et al. 2008, Marra 2009, Halsey et al. 2010]. Combining the conversion of NCP to carbon units and GPP to NPP leads to a form equivalent to the e-ratio ([NCP/(1.1 to 1.4$)] /[\mathrm{GPP} / 2.7]=$ $\mathrm{NCP} / \mathrm{NPP}$ ) in carbon units.

\subsubsection{Gas tracer sampling}

Discrete seawater samples were collected for analysis of oxygen concentration, $\mathrm{O}_{2} / \mathrm{Ar}$ ratio, and triple oxygen isotope ratios from Niskin bottles on the CTD rosette at cast locations and from the underway line between cast stations (Table 1). Oxygen concentration was generally sampled in replicate following Joint Global Ocean Flux Study protocols [Knap et al. 1996] and titrated after equilibrating to room temperature by a modified amperometric Winkler titration [Carpenter 1965a, 1965b, Knapp et al. 1989]. Full water column depth profiles of oxygen were collected during stationary cast sampling throughout the cruise in order to calibrate the oxygen sensor on the SBE911+. At a subset of the stations, depth profiles below the mixed layer were sampled for $\mathrm{O}_{2} / \mathrm{Ar}$ and triple oxygen isotope ratios simultaneously with oxygen concentration. The $\mathrm{O}_{2} / \mathrm{Ar}$ and triple oxygen isotope ratios in dissolved oxygen were sampled using custom made evacuated $500 \mathrm{~mL}$ glass flasks with gas-tight valves pre-poisoned with mercuric chloride [Emerson et al. 1995]. The spatial scale associated with underway samples ranged from stationary sampling to integration over $\sim 3 \mathrm{~km}$ depending on ship's speed.

\subsection{4 $\mathrm{O}_{2} / \mathrm{Ar}$ and triple oxygen isotope ratio analytical methods}

Discrete water samples were analyzed for both dissolved $\mathrm{O}_{2} / \mathrm{Ar}$ and triple oxygen isotope ratios at Woods Hole Oceanographic Institution on an isotope ratio mass spectrometer using methods similar to those of Barkan and Luz [2003]. Sample water and gas headspace were equilibrated at room temperature and the headspace further processed on an automated line which collected $\mathrm{O}_{2}$ and Ar while separating and discarding water vapor and $\mathrm{N}_{2}$ [Stanley and Howard 2013]. The $\mathrm{O}_{2}$ and Ar were passed to a Thermo Scientific MAT 253 mass spectrometer and the $\mathrm{O}_{2} / \mathrm{Ar},{ }^{17} \mathrm{O} /{ }^{16} \mathrm{O}$, and ${ }^{18} \mathrm{O} /{ }^{16} \mathrm{O}$ ratios determined. Samples were referenced to a gas secondary standard with similar $\mathrm{O}_{2} / \mathrm{Ar}$ to seawater (Scott Specialty Gases). Additionally, equilibrated water and atmospheric air standards (air standard is Woods Hole beach air stored in a valved $2 \mathrm{~L}$ electropolished stainless steel cylinder) were analyzed every nine samples.

All samples were corrected for the effects of interactions with Ar and trace $\mathrm{N}_{2}$ at the ion source [Abe and Yoshida 2003] and sample size [Stanley et al. 2010], with corrections applied both on ${ }^{17} \mathrm{O} /{ }^{16} \mathrm{O}$ and ${ }^{18} \mathrm{O} /{ }^{16} \mathrm{O}$ directly and on ${ }^{17} \Delta$. Since the Ar corrections were more linear for ${ }^{17} \mathrm{O} /{ }^{16} \mathrm{O}$ and ${ }^{17} \Delta$, we recalculated ${ }^{18} \mathrm{O} /{ }^{16} \mathrm{O}$ from those measurements, which was especially important for low $\mathrm{O}_{2}$ (i.e. high relative $\mathrm{Ar}$ ) samples. Recalculated values of ${ }^{18} \mathrm{O} /{ }^{16} \mathrm{O}$ were on average $2 \times 10^{-3}$ per mil lower than the measured ratio in this dataset. Water standards run concurrently with our samples (13 replicates over 19 days) were precise to $0.1 \%$ in $\Delta \mathrm{O}_{2} / \mathrm{Ar}, 9 \times 10^{-3}$ per mil in ${ }^{17} \mathrm{O} /{ }^{16} \mathrm{O}, 15 \times 10^{-3}$ per mil in ${ }^{18} \mathrm{O} /{ }^{16} \mathrm{O}$, and 3 per meg in ${ }^{17} \Delta\left(3 \times 10^{-3}\right.$ 
per mil). Sample duplicate precision was $0.1 \%$ in $\Delta \mathrm{O}_{2} / \mathrm{Ar}$ and 5 per meg in ${ }^{17} \Delta$ (5 pairs from either cast or underway samples).

\subsection{Nutrient and Chlorophyll-a concentrations}

Nutrients were collected from the ship's underway line simultaneously with dissolved gas samples, and filtered through $0.2 \mu \mathrm{m}$ sterile syringe filters (surfactant-free cellulose acetate, Corning). Additional, unfiltered samples were collected from $5 \mathrm{~m}$ Niskin bottles during CTD rosette casts (data accessible through the National Science Foundation Biological and Chemical Oceanography Data Management Office, BCO-DMO, http:/www.bco-dmo.org/project/2204). Both sample sets were photometrically analyzed on a Technicon Autoanalyzer II at Oregon State University following the methods of Gordon et al. [1994]. The nitrite and total nitrate plus nitrite concentrations used in this study from both filtered and unfiltered datasets showed no evidence of systematic bias. Phosphate and ammonia concentrations did have systematic offsets between filtered and unfiltered datasets and thus are not evaluated in this study. Four Chlorophyll- $a$ samples were collected from the underway system at each sample location, and duplicate samples were filtered sequentially at $10 \mu \mathrm{m}$ and $0.2 \mu \mathrm{m}$ (polycarbonate, Millipore). Chlorophyll- $a$ was extracted with $90 \%$ acetone and then determined fluorometrically on a Triology fluorometer (Turner) by G.M.M. Hennon, according to the methods of Arar and Collins [1997].

\subsection{Phytoplankton diversity and community structure}

The concentration (cells $\left.\mathrm{L}^{-1}\right)$ of surface phytoplankton communities of picoplankton $(0.5-2 \mu \mathrm{m})$ populations identified by continuous underway flow cytometry (reported by Hennon [2015] and Hennon et al. [in prep]) and nano- and microplankton $(\sim 5 \mu \mathrm{m}$ and $20-200 \mu \mathrm{m})$ identified by light microscopy (reported in Durkin et al. [2016]) were used to assess community structure and diversity. In brief, Prochlorococcus, Synechococcus, and picoeukaroyte abundances were identified based on characteristic fluorescence and light scattering [Ribalet et al. 2010, Swallwell et al. 2011]. For this study, continuous data and associated statistics were averaged over the 30 minutes surrounding discrete underway sampling, or, if stationary, over the duration of particle trap deployment, in order to integrate over a similar time and space resolution as the underway gas sampling. Surface phytoplankton abundances reported by Durkin et al. [2016] at the same locations as trap deployments were quantified by light microscopy from $0.5 \mathrm{~L}$ samples. Microphytoplankton morphotypes were identified to the genus level where possible.

Surface community structure associations were identified using principal component analysis (PCA) on the phytoplankton abundances normalized to variance, and locations with distinct community structures were identified by k-means clustering of PCA coordinates using tools in R software (FactoMineR package, R project [Lê et al. 2008]). The number of clusters used in the analysis was determined by identifying the fewest number of clusters that explained a large fraction of total variance. To determine which principal components defined the clustering of similar communities, dominant principal component coordinates of each cluster were identified (cluster coordinate magnitudes accounting for $>50 \%$ of the absolute magnitude). The phytoplankton with the greatest variable loadings on the dominant principal coordinates of each cluster were identified to determine which phytoplankton influenced the clustering of locations. Additionally, the Shannon-Weaver diversity index $(\mathrm{H})$ was calculated from cell concentrations (separated by phytoplankton type) in R software (vegan package, R project). Community structure and diversity were only evaluated at stations with microscopy data in addition to continuous flow cytometry observations. Additional approaches to evaluating diversity, such as normalizing to biomass of each phytoplankton type, are considered in the work of Hennon et al. [in prep].

\subsection{Biogeographic provinces}

All sample types were grouped by biogeographic province using a clustering approach informed by average daily sea surface temperature, salinity, and Chlorophyll- $a$ fluorescence [in the manner of Reygondeau et al. 2013, Kavanaugh et al. 2014b] as reported in Hennon [2015]. Clustered sample 
locations were then assigned names corresponding to the regions identified by Longhurst [1995]. Biogeographic provinces in this dataset include the South Subtropical Transition region, the South Atlantic Gyre, and the Western Tropical Atlantic (Figure 1). An additional warm, low salinity, very high Chlorophyll- $a$ fluorescence cluster of samples specific to the mesohaline Amazon River Plume in this study is grouped separately from the other stations in the Western Tropical Atlantic. The identified provinces roughly correspond to physical circulation features of the surface ocean, such as the subtropical/subpolar frontal region, subtropical gyre, and equatorial current systems [e.g. Peterson and Stramma 1991].

\subsection{Other statistical tests}

We evaluated correlations between production rates, particle export, phytoplankton abundances, and hydrographic variables throughout the transect and between biogeographic provinces. Binning data by provinces allows us to compare data over sufficient scales to maximize dataset overlap and minimize spatial and temporal disagreements between tracer types. We calculated Pearson linear correlation coefficients (r) pairwise between each variable. Normality of the data could not be assumed because of limited overlap between datasets and the small number of sample locations across the transect ( $\mathrm{n}=9$ to 30 for each variable). Therefore we additionally calculated the non-parametric Spearman's rank correlation coefficient $(\rho)$, in which higher ranks correspond to variable pairs that increase monotonically with respect to each other without assuming a linear dependency. Significance was assigned with the standard value of $\alpha=0.05$ [Fisher 1956].

In order to quantify whether average properties differ significantly between biogeographic provinces, we used the non-parametric equivalent of an ANOVA, the Kruskal-Wallis test [Kruskal and Wallis 1952, Meyer and Seaman 2013] with $\alpha=0.05$ and the Dunn-Šidák [Šidák 1967] correction for multiple comparisons (more precisely, the median is compared in rank based tests). We used the non-parametric ksample Anderson-Darling test [Scholz and Stephens 1987, Trujillo-Ortiz et al. 2007] to evaluate whether the sample distribution in each region was significantly different from other regions (and by inference whether underlying processes contributed differently to variability in the observations). Finally, we explicitly tested whether the variance differed between sample sets in each region with the BrownForsythe test [Brown and Forsythe 1974] a non-parametric equivalent of the $F$-test that allows dissimilarity of sample distributions. All statistical analyses described in this section were performed using Matlab software (release 2015b, The Mathworks, Natick, MA).

\section{Results}

\subsection{Gas tracers}

\subsubsection{Surface and subsurface gas tracers}

Gas tracer results in the surface $250 \mathrm{~m}$ are shown in Figure 2 and tabulated in Table S1 (supplemental information). Oxygen concentrations and CTD data are accessible through BCO-DMO (http://www.bcodmo.org/project/2204). Oxygen concentrations were highest in the Southern Subtropical Transition zone and immediately below the mixed layer of the South Atlantic Gyre (Figure 2a). This sub-mixed layer maximum was also associated with increased $\Delta \mathrm{O}_{2} / \mathrm{Ar}$ and ${ }^{17} \Delta$ (Figure $2 \mathrm{~b}$ and $2 \mathrm{~d}$ ) likely accumulated during production within the euphotic zone but below the mixed layer [Hendricks et al. 2005, Howard et al. 2010] — such production is not included in gas-tracer based production estimates for the surface mixed layer. Surface $\Delta \mathrm{O}_{2} / \mathrm{Ar}$ and $\mathrm{Ar} / \mathrm{Ar}_{\text {sat }}$ (Figure 2c) were generally slightly in excess of atmospheric equilibrium because of biological production and bubble dissolution processes [e.g. Nicholson et al. 2011]. The shoaling pycnoclines in the equatorial region were associated with low oxygen concentration, decreased $\Delta \mathrm{O}_{2} / \mathrm{Ar}$, increased Ar saturation state (south of the equator), and high ${ }^{17} \Delta$. Ar supersaturations of up to $18 \%$ below the mixed layer were likely the result of the temperature differences between mixing 
water masses and the nonlinear response of saturation state to diapycnal mixing [Gehrie et al. 2006, Ito et al. 2007, Emerson et al. 2012].

Underway and surface cast gas tracer samples were compared to verify that processes within the underway sampling line did not bias gas tracer measurements. No consistent offset in either $\Delta \mathrm{O}_{2} / \operatorname{Ar}$ or ${ }^{17} \Delta$ was observed over the cruise, and no systematic bias was inferred. Duplicate precision from either the underway or cast samples was similar to that for paired cast and underway samples (standard deviation $0.2 \% \Delta \mathrm{O}_{2} /$ Ar and 5 per meg in ${ }^{17} \Delta, \mathrm{n}=14$ pairs). However, ${ }^{17} \Delta$ in underway samples from April $18^{\text {th }}$ and $25^{\text {th }}\left(2.7^{\circ} \mathrm{S}\right.$ and $\left.3.5^{\circ} \mathrm{N}\right)$ were more than two standard deviations greater than the paired cast samples, but no differences in $\Delta \mathrm{O}_{2} / \mathrm{Ar}$ in those samples were observed. Inline degassing was unlikely as underway line temperatures were similar to surface water, and air leaks or bubble entry during cast sampling were unlikely as both underway and cast samples were similarly and substantially elevated above atmospheric $\mathrm{O}_{2} / \mathrm{Ar}$. Thus the differences may reflect the influence of fine scale environmental heterogeneity, so we do not discard these data points from the results.

\subsubsection{Rates of net community production and gross primary production}

Net community production and gross primary production in the surface mixed layer (Figures 3 and 4c) were high in the South Subtropical Transition zone, with generally low to moderate values along the rest of the transect (NCP $\sim 5$ to $15 \mathrm{mmol} \mathrm{O}_{2} \mathrm{~m}^{-2} \mathrm{~d}^{-1}$, GPP $\sim 40$ to $100 \mathrm{mmol} \mathrm{O}_{2} \mathrm{~m}^{-2} \mathrm{~d}^{-1}$ in general). Production rates are tabulated in the supplemental information (Table S2) along with all other results from this work and the previously reported results of Durkin et al. [2016] and Hennon et al. [in prep]. All results are averaged by sample location, including over multiday periods. NCP and GPP had overall standard deviations of 3 and $22 \mathrm{mmol} \mathrm{O}_{2} \mathrm{~m}^{-1} \mathrm{~d}^{-1}(25 \%$ and $30 \%$, including location replicates separated in time) respectively (Table S2). Two separate duplicate values of GPP reported in Table S2 were excluded from analysis, as they were within uncertainties of zero, differed from their duplicates by an order of magnitude, and were smaller than their associated NCP (errors associated with the mixing correction become proportionally very large when production rates are low). Uncertainties for these duplicate pairs are still included in the replicate precisions reported above.

The NCP/GPP ratio (Figure 4d) was between 0.1 and 0.2 across most of the transect, i.e. 10-20\% of photosynthetic oxygen in the surface mixed layer is likely offset by organic matter export below the pycnocline or laterally advected over the residence time of oxygen in the mixed layer. The relative uniformity in NCP and GPP across the basin was punctuated by large variations over hundreds of kilometers, which were particularly evident in changes to NCP/GPP and peaks in apparent carbon cycling efficiency (e.g. $37.2^{\circ} \mathrm{S}$ and $2.7^{\circ} \mathrm{S}$, see section 5). 

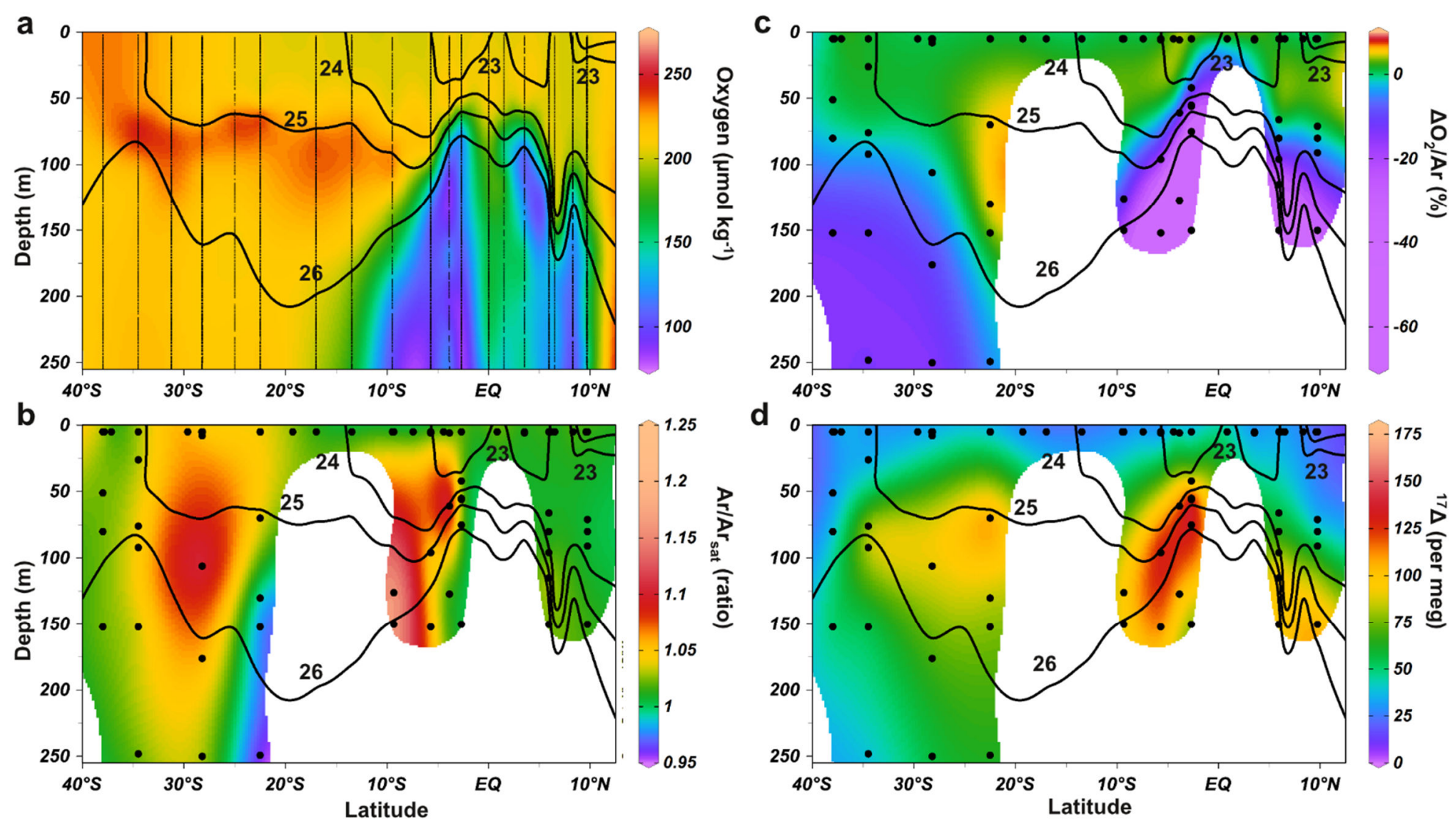

Figure 2: Meridional sections of (a) oxygen concentration, (b) biological oxygen supersaturation $\Delta \mathrm{O}_{2} / \operatorname{Ar}$ (supersaturation or undersaturation relative to atmospheric equilibrium), (c) argon saturation relative to atmospheric equilibrium $\mathrm{Ar} / \mathrm{Ar}_{\text {sat }}$, and (d) the triple oxygen isotope tracer ${ }^{17} \Delta$ (relative to the triple oxygen isotope tracer value of atmospheric oxygen). Sample locations are identified as black circles (lines for calibrated oxygen concentrations from conductivity temperature depth casts) and labeled black contours are isopycnals of neutral density. Sections are gridded with horizontal and vertical length-scales of $1^{\circ}$ Latitude and $20 \mathrm{~m}$ depth using Data-Interpolating Variational Analysis in Ocean Data View (Schlitzer [2015]; http:odv.awi.de). Tracer values from the first sample location along the transect $\left(37.1^{\circ} \mathrm{S}, 49.9^{\circ} \mathrm{W}\right)$ are not plotted so that latitude increases monotonically along the section. 

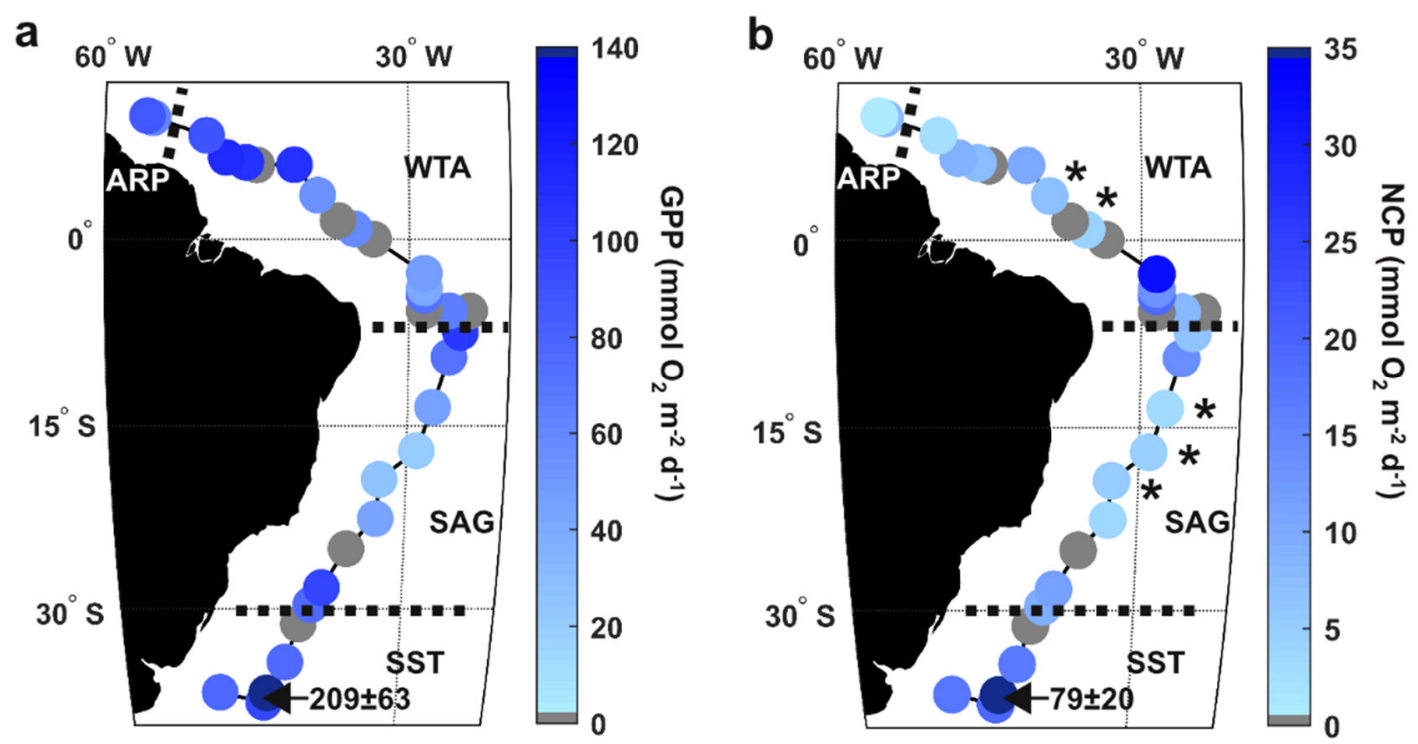

Figure 3: Surface mixed layer rates of (a) gross primary production, GPP, and (b) net community production, NCP. Stations with no production rate estimates are marked in gray, and one station with offscale rates $\left(37.2^{\circ} \mathrm{S}, 44.6^{\circ} \mathrm{W}\right)$ is marked in dark blue and noted in the panels. Sample locations marked with asterisks in panel $\mathbf{b}$ are more than $2^{\circ}$ Latitude from the nearest depth profile (see text section 2.2.1). Biogeographic provinces are noted with dashed lines and abbreviations as in Fig. 1.

\subsection{Nitrate, nitrite, and Chlorophyll-a concentrations}

The total nitrate and nitrite concentrations (Figure 4b) were higher in the South Subtropical Transition zone and the Western Tropical Atlantic $\left(0.05\right.$ to $\left.0.20 \mu \mathrm{mol} \mathrm{N} \mathrm{L}^{-1}\right)$ than in the South Atlantic Gyre or Amazon River Plume, similar to the general pattern in NCP and the NCP/GPP ratio (see section 5 and supplemental tables for statistical comparisons). Nitrite concentrations were low across the subtropics and tropics (typically less than $0.05 \mu \mathrm{mol} \mathrm{N} \mathrm{L}{ }^{-1}$ ). The nitrite and total nitrate plus nitrite duplicate precisions were 0.006 and $0.03 \mu \mathrm{mol} \mathrm{N} \mathrm{L}^{-1}$, respectively. The standard deviation of all samples at each sample location was similar. Two measurements of total nitrite plus nitrate were discarded as outliers (one each from the filtered and unfiltered sample sets) because they were more than two standard deviations higher than both their duplicates and all other samples.

Peak values of Chlorophyll- $a$ (Figure $4 \mathrm{~b}$ ) were found in the SST both for the 0.2 to $10 \mu \mathrm{m}$ and $>10 \mu \mathrm{m}$ size fractions (up to $0.20 \mathrm{mg} \mathrm{m}^{-3}$ and $0.13 \mathrm{mg} \mathrm{m}^{-3}$ respectively). Values in the $>10 \mu \mathrm{m}$ fraction were low $\left(<0.03 \mathrm{mg} \mathrm{m}^{-3}\right)$ or below detection across the rest of the transect, while the 0.2 to $10 \mu \mathrm{m}$ fraction followed a similar trend to GPP; lowest in the SAG $\left(\sim 0.05 \mathrm{mg} \mathrm{m}^{-3}\right)$ but higher in the WTA and ARP $\left(\sim 0.1 \mathrm{mg} \mathrm{m}^{-3}\right)$. Thus small phytoplankton likely contributed significantly to the standing stock of Chlorophyll- $a$ across the transect, while Chlorophyll- $a$ associated with larger cells contributed most to the total in the colder, higher nutrient SST. Replicate precision was $9 \%(0.2$ to $10 \mu \mathrm{m})$ and in the case of the $>10 \mu \mathrm{m}$ fraction generally varied between 10 and $100 \%$ (uncertainties were very large relative to mean values at the lowest concentrations). 

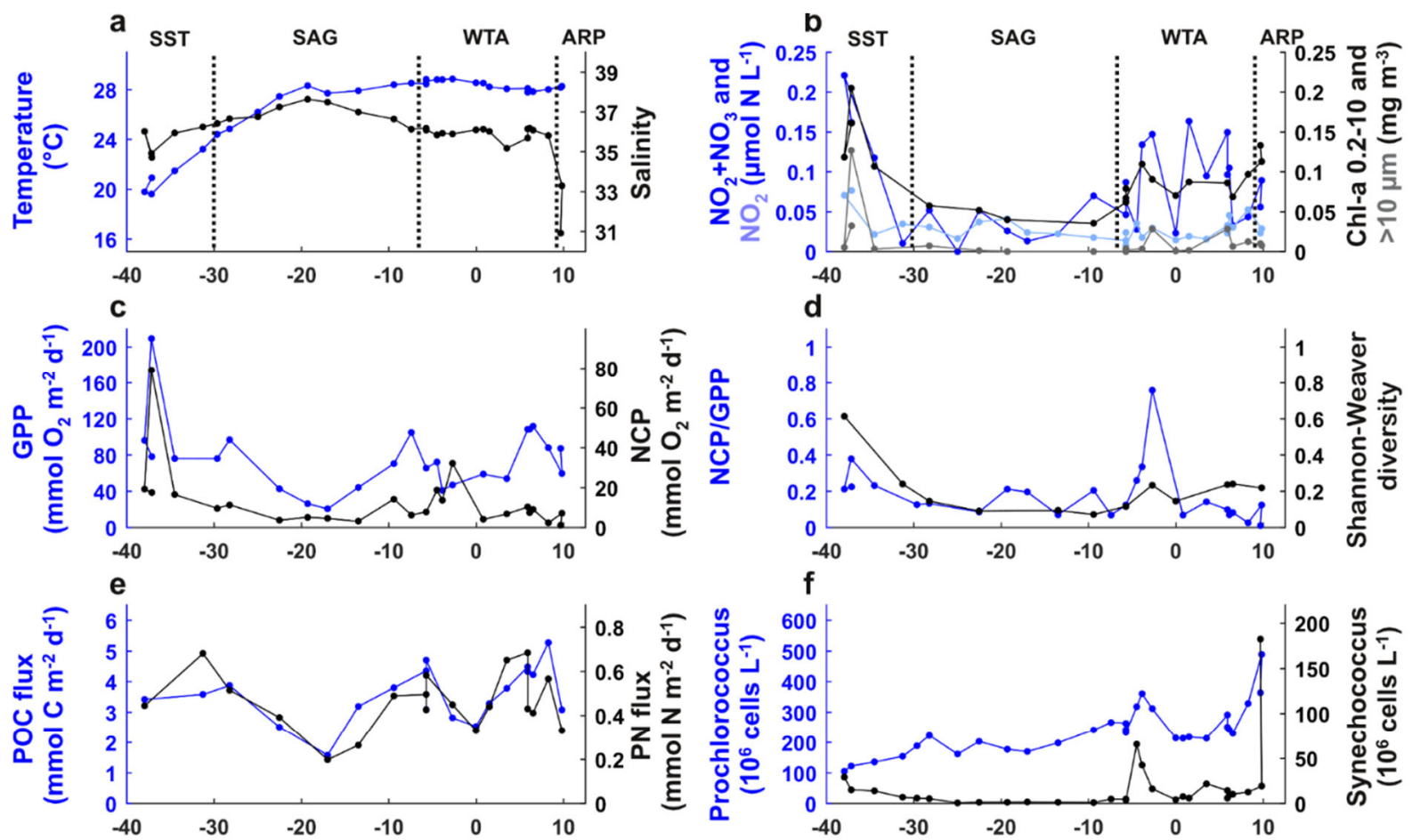

$$
\text { f }
$$
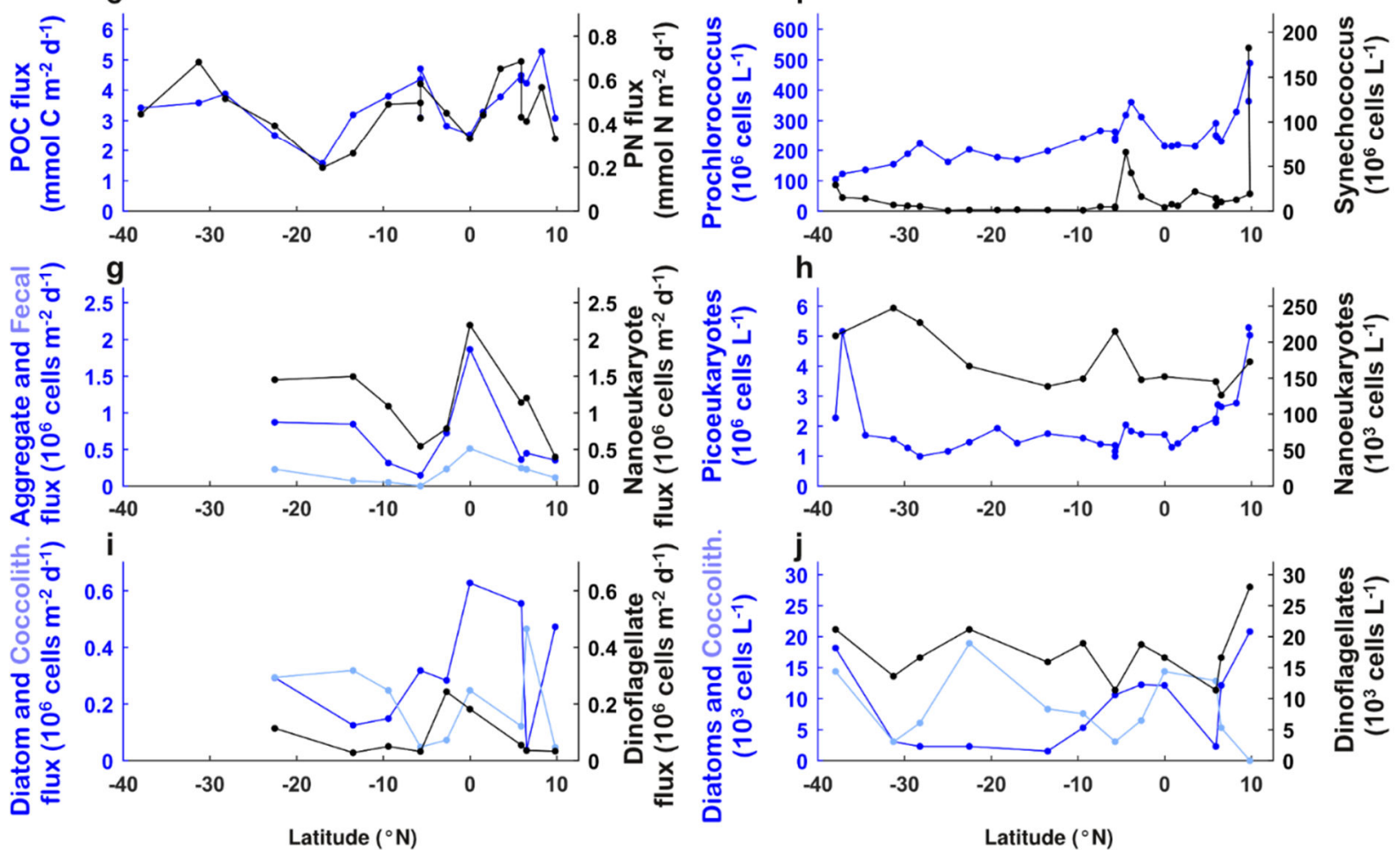

Figure 4: Measured and calculated parameters plotted against Latitude. Biogeographic provinces are noted with dashed lines and abbreviations (see text). Top row: (a) Temperature (blue) and salinity (black), and (b) total nitrite plus nitrate (blue) and nitrite only (light blue) concentrations, as well as Chlorophyll- $a$ concentrations for the $0.2 \mu \mathrm{m}-10 \mu \mathrm{m}$ (black) and $>10 \mu \mathrm{m}$ (gray) size fractions. The same color scheme is applied throughout. Second row: (c) gross primary production, GPP, and net community production, NCP, and (d) the NCP/GPP ratio and Shannon-Weaver diversity $(\mathrm{H})$ for the total pico-, nano-, and microphytoplankton community (see text). Left hand column from third row down: particle fluxes at 125 $\mathrm{m}$, including (e) particulate organic carbon (POC) and particulate nitrogen (PN) fluxes, (g) cell aggregate, fecal pellet, and nanoeukaryote number fluxes, and (i) diatom, coccolithophores, and dinoflagellate number fluxes. Right hand column from third row down: abundances fo (f) Prochlorococcus and Synechococcus, (h) picoeukaryotes and nanoeukaryotes, and (j) diatoms, coccolithophores, and dinoflagellates. 


\subsection{Phytoplankton abundance and diversity}

Picophytoplankton (Figure $4 \mathrm{f}$ and $4 \mathrm{~h}$ ) and nano- and microphytoplankton (Figure $4 \mathrm{~h}$ and $4 \mathrm{j}$ ) abundances are presented in Hennon et al. [in prep] and Durkin et al. [2016]. We focus here on how meridional trends in surface cell concentrations and resulting community diversity compare to gas tracer based production rates. Cyanobacteria (Prochlorococcus and Synechococcus) and picoeukaryotes $\left(\sim 10^{8}, 10^{7}\right.$, and $10^{6}$ cells $\left.\mathrm{L}^{-1}\right)$ were orders of magnitude more abundant than larger nanoeukaryotes and microeukaryotes such as diatoms $\left(\sim 10^{5}\right.$ and $10^{3}$ to $10^{4}$ cells $\left.\mathrm{L}^{-1}\right)$. Picoeukaryotes followed a broadly similar trend to GPP (see supplemental tables for statistical comparisons), with particularly high abundances coincident with the $\mathrm{NCP} / \mathrm{GPP}$ peak at $37.2^{\circ} \mathrm{S}$ and lower but variable abundance across the rest of the transect, with the exception of the Amazon River Plume, which had high picoeukaryote abundance but average GPP. Nanoeukaryote abundances also followed a similar trend to GPP. Abundances of cyanobacteria, nanoeukaryotes, diatoms, and coccolithophores all peaked in the equatorial region near where NCP, GPP, $\mathrm{NCP} / \mathrm{GPP}$, and Chlorophyll- $a$ peak as well, but not at identical locations. Uncertainties in cell abundance for each cell type are similar (average relative standard deviations of 3-13\% for cell types measured by flow cytometery, and 1-8\% for light microscopy; Table S2).

Shannon-Weaver diversity (Figure 4d) was low across the transect, reflecting the numerical dominance of Prochlorococcus. Diversity was highest in the SST where the lowest abundance of Prochlorococcus and highest abundances of nano- sized and microphytoplankton were observed. Community diversity was low throughout the SAG, and slightly higher in the WTA and ARP due to higher abundances of larger phytoplankton. Diversity increased at $2.7^{\circ} \mathrm{S}$ compared to adjacent stations in the equatorial region, in a similar region to peaks in abundances of cyanobacteria, diatoms and coccolithophores, and coincident with the large peaks in NCP and the NCP/GPP ratio. Rarefaction curves (Figure S3) indicate that microscopy sampling was insufficient to detect all phytoplankton morphotypes likely to be present within the mixed layer - thus diversity may be underrepresented to different degrees across the transect and reported $\mathrm{H}$ is likely a lower bound.

\subsection{Particle fluxes}

Fluxes at $125 \mathrm{~m}$ of POC, as well as number fluxes of individual nano-size and microeukaryote cell types, and number fluxes of cell aggregates and fecal pellets (Figure $4 \mathrm{e}, 4 \mathrm{~g}$, and $4 \mathrm{i}$ ) have been previously reported in Durkin et al. [2016]. Particle number fluxes were more variable in the WTA than in the SAG, in a similar manner to the observed variability of net community production, gross primary production, nutrients and Chlorophyll- $a$. Particle number fluxes were not measured in the SST. These fluxes did not clearly vary in the same manner as gas tracer based production fluxes. Cell and particle type number fluxes had average relative standard deviations of roughly $30-60 \%$.

While POC and PN fluxes were broadly similar throughout the transect (generally 2 to $5 \mathrm{mmol} \mathrm{C} \mathrm{m}^{-2} \mathrm{~d}^{-1}$ and 0.2 to $0.7 \mathrm{mmol} \mathrm{N} \mathrm{m}^{-2} \mathrm{~d}^{-1}$ ), there was high variability on the scale of hundreds of kilometers, similar to the gas tracer based production rates. In particular, POC flux followed a similar pattern as GPP rather than tracking phytoplankton abundances or particle number fluxes. POC and PN flux standard deviations are $0.8 \mathrm{mmol} \mathrm{C} \mathrm{m}^{-2} \mathrm{~d}^{-1}(24 \%)$ and $0.1 \mathrm{mmol} \mathrm{N} \mathrm{m}^{-2} \mathrm{~d}^{-1}(28 \%)$, similar to the average relative standard deviations in NCP and GPP.

\section{Comparison of production rates with literature values}

\subsection{Net community production and gross primary production}

Rates of net community production and gross primary production presented in this work are compared with other published studies in the South Atlantic (Table 2). Other methods used to calculate primary production in this region include one study in the Amazon River Plume also using the $\mathrm{O}_{2} / \mathrm{Ar}$ and triple 
oxygen isotope tracers as well as dissolved inorganic carbon drawdown [Yeung et al. 2012] and multiple years of coverage across the basin in May and October as part of the Atlantic Meridional Transect Program [Robinson et al. 2006, 2009], using radiocarbon uptake [Marañón et al. 2003, Tilstone et al. 2009] and oxygen light-dark bottle incubations [Serret et al. 2015]. All rates are converted to oxygen fluxes using the conversions described in section 2.2.2.

Table 2: Production rate comparisons to literature values

\begin{tabular}{|c|c|c|c|c|c|c|}
\hline $\begin{array}{c}\text { Biogeographic } \\
\text { province }\end{array}$ & & \multicolumn{2}{|c|}{$\left(\mathrm{mmol} \mathrm{O} \mathrm{m}^{-2} \mathrm{~d}^{-1}\right)$} & Methods & Time of year & Reference \\
\hline \multirow[t]{3}{*}{ SST } & 115 & \pm 64 & $33 \pm 31$ & $\mathrm{O}_{2} / \mathrm{Ar}, \mathrm{TOI}$ & Mar-May & this study \\
\hline & 130 & \pm 70 & $66 \pm 68$ & $\mathrm{O}_{2}$ bottle & May/Oct & Serret et al. $2015^{\mathrm{a}}$ \\
\hline & 98 & \pm 26 & & $6 \mathrm{~h}{ }^{14} \mathrm{C}-\mathrm{NPP}$ & May/Oct & Tilstone et al. $2009^{\mathrm{b}}$ \\
\hline \multirow[t]{4}{*}{ SAG } & 60 & \pm 32 & $7 \pm 4$ & $\mathrm{O}_{2} / \mathrm{Ar}, \mathrm{TOI}$ & Mar-May & this study \\
\hline & 58 & \pm 4 & $10 \pm 2$ & O2bottle & May/Oct & Serret et al. 2015 \\
\hline & 47 & \pm 4 & & $6 \mathrm{~h}{ }^{14} \mathrm{C}-\mathrm{NPP}$ & May/Oct & Tilstone et al. $2009^{\mathrm{b}}$ \\
\hline & 38 & \pm 18 & & $6 \mathrm{~h}{ }^{14} \mathrm{C}-\mathrm{NPP}$ & May/Oct & Marañón et al. $2003^{c}$ \\
\hline \multirow[t]{5}{*}{ WTA } & 75 & \pm 27 & $11 \pm 9$ & $\mathrm{O}_{2} / \mathrm{Ar}, \mathrm{TOI}$ & Mar-May & this study \\
\hline & 100 & \pm 40 & $28 \pm 38$ & O2bottle & May/Oct & Serret et al. $2015^{\mathrm{a}}$ \\
\hline & 58 & \pm 11 & & $6 \mathrm{~h}{ }^{14} \mathrm{C}-\mathrm{NPP}$ & May/Oct & Tilstone et al. $2009^{\mathrm{b}}$ \\
\hline & 152 & \pm 25 & $15 \pm 6$ & $\mathrm{O}_{2} / \mathrm{Ar}, \mathrm{TOI}$ & May-June & Yeung et al. 2012 \\
\hline & & & $9 \pm 4$ & DIC & May-June & Yeung et al. $2012^{\mathrm{d}}$ \\
\hline \multirow[t]{3}{*}{ ARP } & 73 & \pm 19 & $4 \pm 4$ & $\mathrm{O}_{2} / \mathrm{Ar}, \mathrm{TOI}$ & Mar-May & this study \\
\hline & 206 & \pm 39 & $78 \pm 6$ & $\mathrm{O}_{2} / \mathrm{Ar}, \mathrm{TOI}$ & May-June & Yeung et al. 2012 \\
\hline & & & $23 \pm 7$ & DIC & May-June & Yeung et al. $2012^{\mathrm{d}}$ \\
\hline
\end{tabular}

a. estimated from subset of reported data over similar locations as this study, May and October average b. $6 \mathrm{~h}$ radiocarbon incubation during morning modeled to $12 \mathrm{~h}$ average using daily light, converted assuming $\mathrm{GPP}(\mathrm{O}) / \mathrm{NPP}(\mathrm{C})=2.7$, May and October average

c. $6 \mathrm{~h}$ radiocarbon incubation during afternoon assumed constant throughout sunlit hours, converted assuming $\mathrm{GPP}(\mathrm{O}) / \mathrm{NPP}(\mathrm{C})=2.7$

d. converted to oxygen units following assumptions of authors that $\mathrm{O}_{2} / \mathrm{C}=1.1$ for diazotroph fueled growth

Similar results were observed using different techniques over different sampling months and several years. GPP and NCP rates in the South Subtropical Transition, South Atlantic Gyre, and Western Tropical Atlantic agreed within standard deviations between this study (late April to early May) and the May/October averages from the studies cited in Table 2. Higher NCP in the transition region compared to the subtropical gyre is consistent with results in other frontal regions [Juranek et al. 2012, Ostle et al. 2015, Palevsky et al. 2013], and may be related to enhanced nutrient supply from the subpolar gyre [Ayers and Lozier 2010, Palter et al. 2013]. Oxygen based rates in the SST and WTA (this work and Serret et al. [2015]) both had increased variability in the transition zone and equatorial region compared to the subtropical gyre; in particular, infrequent but high GPP and NCP features were separated from regionally typical values over distances of $\sim 100 \mathrm{~km}$ in both datasets. 
Notably, these other studies used incubation based methods, which have different biases and assumptions [Serret et al. 2015, Williams and Lefèvre 1996] than those in the gas tracer methods (supplemental information of this study, Juranek et al. [2013]). Despite these complicating factors, the in situ rates presented in this study agreed well with incubation based rates. The oxygen incubation results in particular had similar variability to our results. Notably, bottle incubations are not subject to potential biases in the mass balances for NCP and GPP in this study, suggesting that trends in variability identified between biogeographic provinces are not strongly influenced by uncertainties in physical fluxes. This agreement between datasets widely separated in time suggests that high variability and associated higher production rates may be persistent regional characteristics on top of basin scale homogeneity.

In contrast, mean production rates estimated from the two mesohaline Amazon River Plume stations in this study (early May 2013) were significantly smaller (3 times smaller GPP, 20 times smaller NCP) than rates at two mesohaline stations reported by Yeung et al. [2012] (May-June 2010), despite similar methods, gas exchange, and mixed layer depth. The higher production rates in Yeung et al. [2012] were likely caused by recurring, but transient, blooms of diatom-diazotroph associations that were not observed in our study. During this study, the diatom community was primarily composed of Coscinodiscus, Pseudo-nitzschia, Cylindrotheca, and Nitzschia, not the diazotroph-harboring Hemiaulus or Rhizosolenia. NCP rates from stations sampled by Yeung et al. [2012] in the Western Tropical Atlantic (rather than inside the Amazon River Plume) were in agreement with the rates measured in this study for the WTA.

\subsection{Metabolic and export efficiency}

The NCP/GPP ratio was generally between 0.1 and 0.2 , similar to ratios observed in most studies to date [Juranek et al. 2013 and references therein]. On the basis of physiological studies [Behrenfeld et al. 2008, Halsey et al. 2010], a NCP/GPP ratio of approximately 0.4 may be a theoretical maximum [Juranek and Quay 2013]. A NCP/GPP ratio of 0.4 is equivalent to a carbon based NCP/NPP of roughly 0.8 . High $\mathrm{NCP} / \mathrm{GPP}$ and e-ratios (POC flux/NPP) (0.7-0.8 in carbon units) have been observed in the subpolar North Atlantic during the spring bloom [Buesseler 1998, Quay et al. 2012] and the Bering Sea [Prokopenko et al. 2011]. Two locations in this study had ratios near or exceeding an NCP/GPP of 0.4: The first location at $37.2^{\circ} \mathrm{S}$ (SST), had NCP/GPP $=0.38$ (standard deviation 0.17 ; carbon based $\mathrm{NCP} / \mathrm{NPP}=0.73$ ), as well as the highest GPP and NCP fluxes and highest Chlorophyll- $a$ concentrations in both size fractions of the transect (see section 5).

NCP/GPP from the second location (WTA outlier in Figure 4, $2.7^{\circ} \mathrm{S}$ ) was implausibly high $(0.76$, standard deviation 0.32 ), equivalent to carbon based $\mathrm{NCP} / \mathrm{NPP}=1.45$, which implies a net carbon fixation signal in excess of local production, though the uncertainty is large. This high export ratio derives from moderate NCP combined with low GPP. The large ${ }^{17} \Delta$ below the mixed layer (Figure 2) implies that there was substantial production or accumulation of photoysynthetic oxygen at this station that was missed by the surface mixed layer production model, however this feature was associated with lower rather than higher $\Delta \mathrm{O}_{2} / \mathrm{Ar}$, (i.e. respiration) - while the integrated euphotic zone NCP/GPP at this location was likely lower than in the mixed layer alone, the anomalous mixed layer values cannot be explained by enhanced mixing (which would lead to a lower ratio). Similarly, this peak in NCP/GPP was probably not explained by an anomalous increase in particle export efficiency as the export turnover rates (defined as the number flux/[concentration $\times$ mixed layer depth]) of individual cells were low to moderate at $2.7^{\circ} \mathrm{S}$ compared to elsewhere in the SAG and WTA [Durkin et al. 2016].

This unusual NCP/GPP was likely a result of temporal and spatial variability of NCP and GPP, such as has been observed in Lagrangian experiments [Hamme et al. 2012]. Rapid changes in the balance of production and respiration fluxes add uncertainty to estimates of both NCP and GPP, potentially making the corresponding NCP/GPP less representative of average conditions. A potential driver for such changes is illustrated by the adjacent feature at $3.9^{\circ} \mathrm{S}$, characterized by anomalously high cyanobacterial abundance and Chlorophyll- $a$ concentrations in both size fractions. While surface abundance and export fluxes of larger phytoplankton were not sampled at this station, abundant Rhizosolenia were identified in 
surface water, a genus that blooms [Murray et al. 1994, Carpenter et al. 1999] or accumulates along surface frontal systems [Yoder et al. 1994, Kemp et al. 2006, Wilson et al. 2008] and may host symbiotic diazotrophs [Zehr et al. 2000, Foster et al. 2009]. Similarly high ratios (NCP/GPP=0.47, carbon based $\mathrm{NCP} / \mathrm{NPP}=1.15$ with $\mathrm{O}_{2} / \mathrm{C}=1.1$ ) were determined in such a diatom-diazotroph association bloom in the mesohaline front of the Amazon River Plume in 2010 [Yeung et al. 2012]. It is possible that at $2.7^{\circ} \mathrm{S}$ the transitory presence of conditions similar to those observed at $3.9^{\circ} \mathrm{S}$ influenced our measured production rates.

\section{Links between spatial variability of phytoplankton community structure and production}

A striking feature of the results presented here is the large-scale homogeneity of rates of biological production over thousands of kilometers in the subtropical and tropical Atlantic. However, on mesoscales there is an increase in the variability of biological production. The gas-tracer derived measure of export was also homogenous at larger spatial scales, consistent with previous estimates of particulate carbon export over the same region [Owens et al. 2015, Durkin et al. 2016], but variability at smaller scales. A similar pattern of large-scale homogeneity but small-scale variability is seen in biological community characteristics such as surface abundances of Chlorophyll- $a$ and phytoplankton taxa and community diversity. Given these similar patterns of variability and scale, how are primary production, particle export, and surface biological community variables related? In this section we discuss whether observed variability may be related to the time and space scales of sampling as opposed to true environmental variability. Then we discuss potential physical underpinnings for the observed scales of variability. We then identify relationships between measures of production, export, and community structure, paying special attention to related scales of variability.

\subsection{Interpreting data over multiple scales of space and time}

Comparisons of variability are central to the analysis in this chapter and subsequent interpretations of relationships between various aspects of the phytoplankton community and production. However, this approach must be used with caution. We compare measurements of community structure, production, and export that integrate across different scales of space and time, and thus may be more or less sensitive to different processes over various scales. It is possible that calculations in this work relating variability of properties across the transect and between biogeographic provinces do not reflect true environmental variability because of the different sensitivities and small number of samples in some regions.

In particular, picophytoplankton are evaluated primarily in the surface mixed layer, and although flow cytometry measurements are sensitive to growth processes on the order of hours as well as sample integration over several kilometers of the surface ocean through the underway line, picophytoplankton can be evaluated continuously over larger scales of space and time. Microphytoplankton are sparsely evaluated across the transect (sample spacing of hundreds of $\mathrm{km}$ ), but unlike the picophytoplankton measurements are evaluated both in the surface mixed layer and below. Carbon and cell export are also infrequently evaluated in space and time, but integrate export processes over a few days and across much of the euphotic zone, meaning that these fluxes can include effects on production and community structure that are missed by measurements of the surface mixed layer only. The dissolved gas tracers of production are used to evaluate only surface mixed layer production, and discrete samples are expected to integrate over one to two weeks and many kilometers based on the residence time of the tracers with respect to gas exchange.

The sampling resolution of these methods shows that the biological community and its related carbon fluxes are more variable than was believed a decade ago, but are consistent with recent work regarding the dynamic nature and biological and physical fluxes on small scales of space and time [e.g. McGillicuddy et al. 2007, Estapa et al. 2015, Ferrón et al. 2015]. For example, we find that the dissolved 
gas tracers vary substantially with biological activity over the timescale of hours, not weeks as would be expected from the gas exchange timescale (see section 2 and supplementary information). While we argue in this study that our sampling plan probably avoided systematic bias across the transect from diel patterns, variability based on the timing of sampling may be large enough to complicate interpretations of variability in space. Even if differences in timing may be neglected, variability on small spatial scales (tens to hundreds of $\mathrm{km}$ ) appears to be an important consideration when interpreting ocean ecology and carbon cycling. Interpreting variability and how it is related between biological parameters and underlying mechanisms is complicated by difficulty distinguishing differences caused by environmental variability versus sampling variability. Thus it is especially important moving forward to evaluate the sources of variability at these scales and to evaluate any underling drivers.

\subsection{Links between mesoscale physical and biological variability}

Identifying sources of variability at smaller scales is particularly relevant to understanding the mechanisms of the biological carbon pump. There is a growing body of literature that mixing of biomass and nutrients on the mesoscale [McGillicuddy et al. 2007, Klein and Lapeyre 2009] and submesoscale [Lévy et al. 2012, Estapa et al. 2015, Mahadevan 2016] may be key controls on the biological carbon pump by driving enhanced transient production and export pulses such as those described at the ocean time-series [Sweeney et al. 2003, Nicholson et al. 2008, Giesbrecht et al. 2012]. Similarly mixing and dispersion enhance modeled phytoplankton community diversity at the mesoscale while homogenizing diversity at the regional scale [Lévy et al. 2012, 2014, Clayton et al. 2013]. Such infrequent or spatially heterogenous features may have a disproportionate impact on seasonal and annual cycles of productivity in the surface ocean.

Two features in particular in this study highlight mesoscale variability in production and biological properties:

1) An underway sample location in the South Subtropical Transition $\left(37.2^{\circ} \mathrm{S}\right)$ was characterized by very high GPP, NCP, NCP/GPP ratio and Chlorophyll- $a$ concentrations (Figure 4). In addition, this location was slightly fresher and cooler than adjacent stations, and was associated with a $\sim 100 \mathrm{~km}$ diameter low absolute dynamic topography feature propagating westward along the northern edge of the subtropical front (data and time-series visualization available from Ssalto/Duacs, distributed by Aviso with support from Cnes, http://www.aviso.altimetry.fr/duacs/), i.e. a cyclonic eddy. Cyclonic eddies on subtropical gyre boundaries may be associated with enhanced nutrient supply, local diversity, and chlorophyll abundance [McGillicuddy et al. 2016]. The association of the cyclonic eddy with the very large production rates at this sample location are consistent with the idea that mesoscale eddies may contribute to the generally observed high net community production in subpolar/subtropical frontal regions [Juranek et al. 2012, Lockwood et al. 2012, Ostle et al. 2015]. More variable POC export fluxes in the SST than in the other regions has previously been observed, and attributed to episodic addition of nutrients by mesoscale features [Thomalla et al. 2006].

2) High variability of production rates and community characteristics were observed in the equatorial region of the Western Tropical Atlantic. On the southern flank of this region there are peaks in NCP, $\mathrm{NCP} / \mathrm{GPP}$ ratio, total nitrite and nitrate, Chlorophyll- $a$ in the greater than $10 \mu \mathrm{m}$ fraction, and community diversity $\left(2.7^{\circ} \mathrm{S}\right)$, cyanobacterial abundance and Chlorophyll- $a$ in the 0.2 to $10 \mu \mathrm{m}$ fraction $\left(3.9^{\circ} \mathrm{S}\right)$, nano- and microeukaryote abundance $\left(5.7^{\circ} \mathrm{S}\right)$, and fluxes of nano- and microeukaryotes, aggregates, and fecal pellets $\left(0^{\circ}\right)$. The scales of variability separating these peaks from each other and from nearby sample locations with properties close to the regional mean is on the order of $\sim 200 \mathrm{~km}$, the same order scale as variability in satellite observed chlorophyll associated with mesoscale eddies near the equator [Doney et al. 2003, Chelton et al. 2011, Cheng et al. 2014]. Not every parameter was measured at every station, and each measurement type is sensitive to distinct physical and biological influences and methodological biases, which may contribute to apparent variability in these properties. 


\subsection{Relationships between variability of phytoplankton, production, and export}

Many biological parameters measured in this study have qualitatively similar trends and variability to the oxygen based production fluxes and export efficiency (Figure 4). However, these features do not necessarily correlate well on a sample by sample basis. For example, while net community production, total cyanobacterial abundance, Chlorophyll- $a$ concentrations ( 0.2 to $10 \mu \mathrm{m}$ size fraction), and particle fluxes all have visually similar peaks in the equatorial region, the highest values of each property are separated by up to $5^{\circ}$ of latitude. Thus there are a limited number of significant correlations across the transect (Table S3).

The biogeographic provinces identified in section 2.5 are an ecologically meaningful way to bin the sample results in order to maximize dataset overlap and minimize small-scale spatial and temporal offsets between data types. When grouped by province (Figures 5 and S4), oxygen based productivity and particulate organic carbon fluxes generally have similar mean magnitudes, but have notable differences in relative variability. The most variable regions (for most measurements) are the South Subtropical Transition zone and the Western Tropical Atlantic, which skew towards higher values of GPP, NCP, $\mathrm{NCP} / \mathrm{GPP}$ ratio and community diversity. Thus variability at subregional scales may reflect the frequency or magnitude of sporadic biological production and carbon uptake (see section 4.2).

As there are a small number of sample locations for each property and skewed data distributions for some properties, we use non-parametric tests for evaluating differences in regional distributions of each variable. Significantly different $(\mathrm{p} \leq 0.05)$ regional medians, sample distributions, and variances are summarized in Table 3. Although regional medians sometimes differ (for example net community production, Shannon-Weaver diversity, and chlorophyll in the small size fraction are higher in the South Subtropical Transition than the South Atlantic Gyre) the variance and distribution of observed values vary more between regions than the medians do. Furthermore, the variability and distribution of many measured parameters follow similar patterns (Figure 5, Table 3), suggesting that similar processes influence distributions within specific regions. In particular, for many variables the SAG has a different sample distribution and smaller variance than the SST and WTA. Distribution patterns of NCP and community diversity are similar, as are regional patterns in variance in the NCP:GPP ratio and particulate organic carbon flux and Shannon-Weaver diversity. Regional means of POC flux as well as GPP are not significantly different, but variability of both fluxes differs significantly between regions. Thus patterns of regional distribution and variance suggest potential links between biological production rates and diversity. 
Table 3: Region differences in property median, distribution, and variance

\begin{tabular}{|c|c|c|c|}
\hline $\begin{array}{l}\text { Test } \\
\text { Variable }\end{array}$ & $\begin{array}{l}\text { Kruskal-Wallis }^{\mathrm{a}} \\
\text { (Median) }\end{array}$ & $\begin{array}{l}\text { Anderson-Darling } \\
\text { (Distribution) }\end{array}$ & $\begin{array}{l}\text { Brown-Forsythe } \\
\text { (Variance) }\end{array}$ \\
\hline Gross primary production & & & all $\neq$ except $\mathrm{SST} \sim \mathrm{ARP}$ \\
\hline Net community production & SST $>$ SAG & $\mathrm{SST} \neq \mathrm{SAG}, \mathrm{WTA}, \mathrm{ARP}$ & $\mathrm{WTA} \neq \mathrm{SST}, \mathrm{SAG}, \mathrm{ARP} ; \mathrm{SAG} \neq \mathrm{ARP}$ \\
\hline $\mathrm{NCP} / \mathrm{GPP}$ ratio & & $\mathrm{SST} \neq \mathrm{SAG}, \mathrm{ARP}$ & $\mathrm{SAG} \neq \mathrm{WTA}$ \\
\hline $\mathrm{NO}_{3}+\mathrm{NO}_{2}$ & & $\mathrm{SAG} \neq \mathrm{WTA}$ & $\mathrm{SAG} \neq \mathrm{WTA}$ \\
\hline $\mathrm{NO}_{2}$ & & & $\mathrm{SST} \neq \mathrm{SAG}, \mathrm{WTA}, \mathrm{SAG} \neq \mathrm{WTA}$ \\
\hline Chlorophyll-a 0.2 to $10 \mu \mathrm{m}$ & $\mathrm{SAG}<\mathrm{SST}, \mathrm{ARP}$ & all except $\neq \mathrm{SST} \sim \mathrm{ARP}$ & $\mathrm{SST} \neq \mathrm{SAG}, \mathrm{WTA}, \mathrm{SAG} \neq \mathrm{WTA}$ \\
\hline Chlorophyll-a $>10 \mu \mathrm{m}$ & & $\mathrm{SAG} \neq \mathrm{ARP}$ & $\mathrm{SST} \neq \mathrm{SAG}, \mathrm{WTA}, \mathrm{SAG} \neq \mathrm{WTA}$ \\
\hline Cyanobacteria & $\mathrm{SST}<\mathrm{WTA}, \mathrm{ARP} ; \mathrm{SAG}<\mathrm{ARP}$ & all regions & all $\neq$ except $\mathrm{SST} \sim \mathrm{ARP}$ \\
\hline Picoeukaryotes & $\mathrm{SAG}<\mathrm{ARP}$ & $\mathrm{SST} \neq \mathrm{SAG} ; \mathrm{ARP} \neq \mathrm{SAG}, \mathrm{WTA}$ & $\mathrm{ARP} \neq \mathrm{SST}, \mathrm{WTA}$ \\
\hline Nanoscale and larger eukaryotes & & $\mathrm{SST} \neq \mathrm{SAG}, \mathrm{WTA}$ & $\mathrm{SAG} \neq \mathrm{WTA}$ \\
\hline Shannon-Weaver diversity $(\mathrm{H})$ & SST $>$ SAG & $\mathrm{SAG} \neq \mathrm{SST}, \mathrm{WTA}$ & $\mathrm{SAG} \neq \mathrm{WTA}$ \\
\hline \multicolumn{4}{|l|}{ Nanoscale and larger flux } \\
\hline \multicolumn{4}{|l|}{ Cell aggregate flux } \\
\hline \multicolumn{4}{|l|}{ Fecal pellet flux } \\
\hline Particulate organic carbon flux & & & $\mathrm{SAG} \neq \mathrm{WTA}$ \\
\hline Particulate nitrogen flux & & & \\
\hline
\end{tabular}

a. with Dunn-Šidák correction for multiple comparisons 

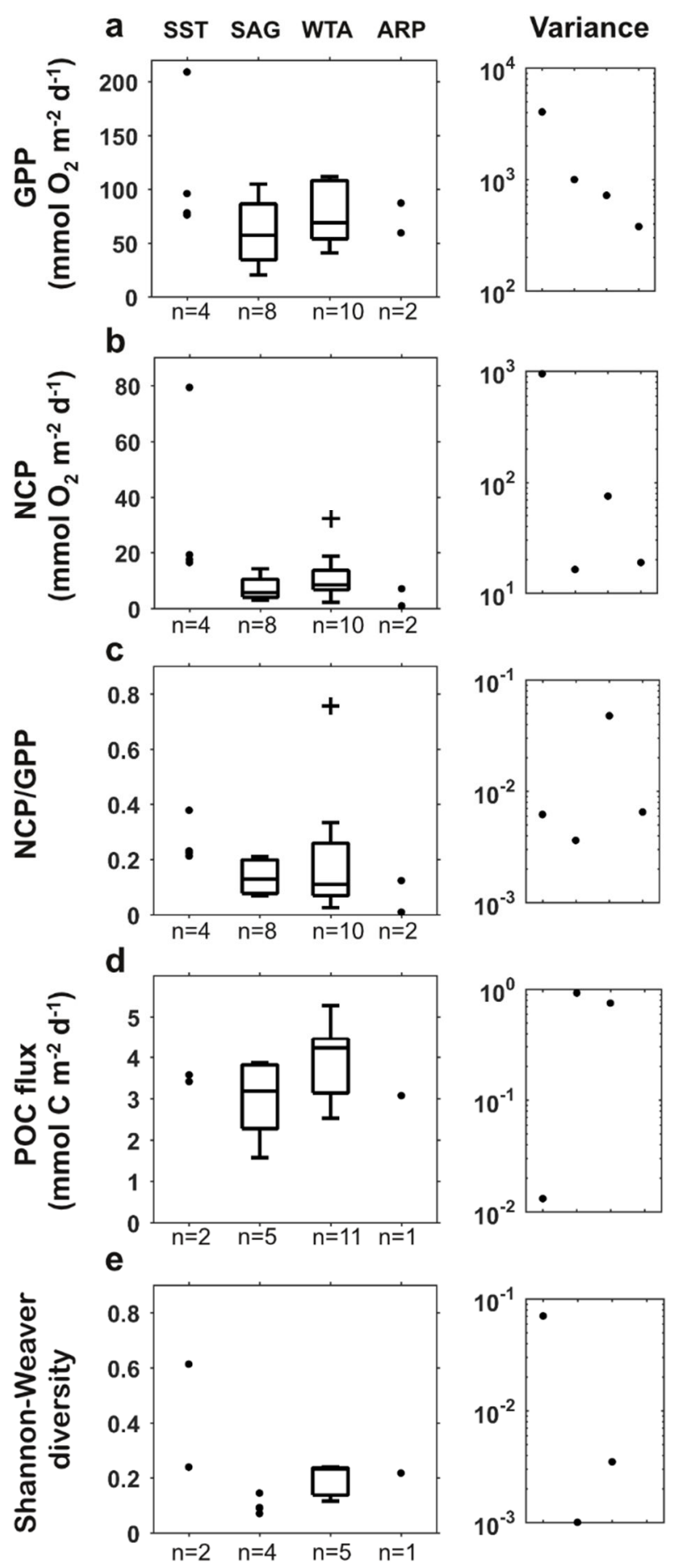

Figure 5: Selected parameters in this study binned by biogeographic provinces. Individual data values are plotted for four or fewer samples in a region, and box pots for five or more samples. Potnetial outliers (greater than 1.5 times interquartile range from middle $50 \%$ of data) are marked as crosses on box plots. Variance (standard deviation squared) for each region is plotted in the righthand column. (a) Gorss primary production, GPP, (b) net community production, $\mathrm{NCP}$, and (c) the NCP/GPP ratio in the surface mixed layer. (d) particulate organic carbon (POC) flux at $125 \mathrm{~m}$. (e) Shannon-Weaver diversity (H) for the total pico-, nano-, and microphytoplankton community (See text) at $5 \mathrm{~m}$. 
Correlations for data grouped by biogeographic province were used to test whether two variables scale by the same magnitude between different regions (mean, supplemental Table S4) and whether variability changes in a similar manner between regions (standard deviation, supplemental Table S5). Significant regional correlations ( $\mathrm{p}<0.05$; Tables S4 and S5) of means include Shannon-Weaver diversity to GPP $(\mathrm{p}=0.009)$ and Chlorophyll- $a>10 \mu \mathrm{m}(\mathrm{p}=0.02)$, as well as chlorophyll in the large size fraction to GPP, $\mathrm{NCP}$, and nitrite concentration ( $\mathrm{p}=0.008,0.04,0.02$ respectively). Interestingly, mean POC fluxes significantly correlate with GPP across the transect (Figure 6), but not with NCP, even though NCP is generally thought to be balanced by POC export on sufficiently long temporal and spatial scales.
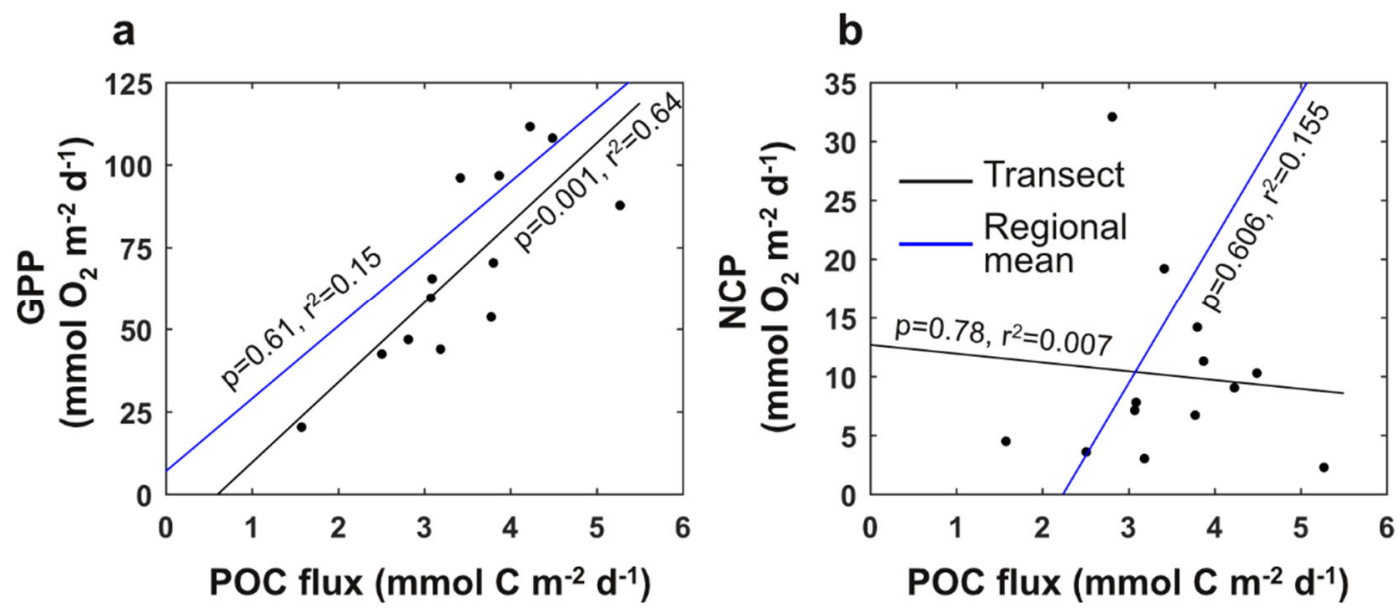

Figure 6: Correlations of particulate organic carbon (POC) flux at $125 \mathrm{~m}$ with (a) gross primary production (GPP), and (b) net community production (NCP) in the surface mixed layer. Correlations include either all stations with concurrent measurements across the transect (black line) or regional means (blue line) including locations where only one of the fluxes was measured. GPP significantly correlates with POC flux across the transect ( $\mathrm{p}$ scores are for F-test of regression significance).

Stronger correlations are observed between standard deviations than with regional means. For example, the standard deviation of gross primary production correlates with that of net community production, nitrite concentration, and the $>10 \mu \mathrm{m}$ fraction of Chlorophyll- $a$, but not with the standard deviation of POC fluxes. Standard deviation of Chlorophyll- $a$ in the large size fraction is also significantly correlated to NCP and nutrients. Standard deviation of the Shannon-Weaver diversity is correlated on the regional level with more than half of all variables, including GPP and NCP, nutrients, and Chlorophyll- $a$ in both size fractions. This broad similarity in how variance scales between regions suggests that the underlying mechanisms causing increased variability in each region, such as the scale-dependent physical processes identified above, affect each property similarly.

Our findings - that variability and locations of maxima in production and community parameters are linked and that the highest variability is observed on the mesoscale rather than basin scales - are consistent with the ideas in section 5.2 regarding the importance of transient physical and biological events. This linked variability of biological production and community characteristics distinguishes between regions that are otherwise similar in the mean sense in this study, and are evidence that diversity and production may be influenced by similar controlling factors. 


\section{Interpreting relationships of community structure, production, and export}

\subsection{Community structure, diversity, and production}

In order to evaluate the potential connection between distinct surface phytoplankton communities and production measures, community structure associations were identified. No clear link between community structure and production rates was observed in this dataset (details below). However, gross primary production and diversity may be connected, and changes in diversity are plausibly explained by variability in nutrient supply driven by mixing at multiple spatial scales. Other important controls on community structure and diversity such as top-down virus and grazer effects were not evaluated in this study.

Phytoplankton groups best describing differences in surface community composition between each location were identified by principal component analysis (section 2.4), and the results clustered into groups of locations that described five distinct community structures (Figure 7a-c). Two of these community structures were represented by only a single location $(\mathrm{D}$, the southernmost station near the subpolar edge of the South Subtropical Transition and B, a station in the Amazon River Plume). Analogously, centroid clustering of community composition by location (using a Bray-Curtis dissimilarity matrix [Bray and Curtis 1957]) also identifies the same significantly different community structures between these two locations and all other locations. Of the remaining three community clusters, Cluster E was associated with a single biogeographic province (the Western Tropical Atlantic) and Clusters $\mathrm{A}$ and $\mathrm{C}$ were spread across multiple provinces. There was no significant correlation (KruskalWallis test, section 2.6) between these community structures and GPP, NCP, NCP/GPP, or POC flux (Figure 7d-g). As in a similar Southern Ocean dataset [Cassar et al. 2015], diatom influenced community structures did not necessarily have greater production or export as might be expected.

Additionally, locations within a given cluster did not all share a common primary particle export mechanism (of the export pathways hypothesized by Durkin et al. [2016] from the same dataset, such as a primarily aggregation/disaggregation controlled pathway) and the surface communities often do not correspond with the hypothesized export pathway; clearly, the phytoplankton community in the surface does not necessarily reflect what is sinking below the mixed layer at the time of sampling. The community composition data were not collected at the same spatial resolution as the production measures - at this larger spatial scale community structure and production were generally similar across the subtropics and mesoscale variability may have been missed. Thus this lack of correlation may be because the number of locations with concurrent productivity data was too small to detect significant differences, or because the surface community structure is unrepresentative of the portion of the community responsible for production and export over the space and time scales of our observations.

The patterns of regional variability in Shannon-Weaver diversity (Figure 5) provide an additional basis for identifying and discussing the relationship between phytoplankton communities and production rates. Phytoplankton community diversity has been proposed to scale to phytoplankton biomass [Irigoien et al 2004, Vallina et al. 2014] and net primary production rates [Napoléon et al. 2014] in a manner similar to terrestrial vegetation. Regional means of gross primary production (which can be scaled to NPP, section 2.2.2) and diversity correlate significantly $(\mathrm{p}=0.009)$, and the correlations between variance of diversity, production rates, nutrients, and chlorophyll between regions (Table S4) suggests that they share a common underlying driver (e.g. mesoscale eddies). Furthermore, observed phytoplankton diversity across the South Atlantic was similar to patterns derived from self-assembling phytoplankton communities in global circulation and biogeochemistry models [Barton et al. 2010, Clayton et al. 2013, Lévy et al. 2014, 2015]. These models suggest mixing at both basin and mesoscales and resulting effects on nutrient supply can explain the diversity patterns we observe. However, on a station to station basis across the entire transect GPP and diversity are not significantly correlated across the transect for either a linear or quadratic correlation $(\mathrm{F}=0.91, \mathrm{p}=0.17$ linear; $\mathrm{F}=2.0 ; \mathrm{p}=0.45$ quadratic $)$. 

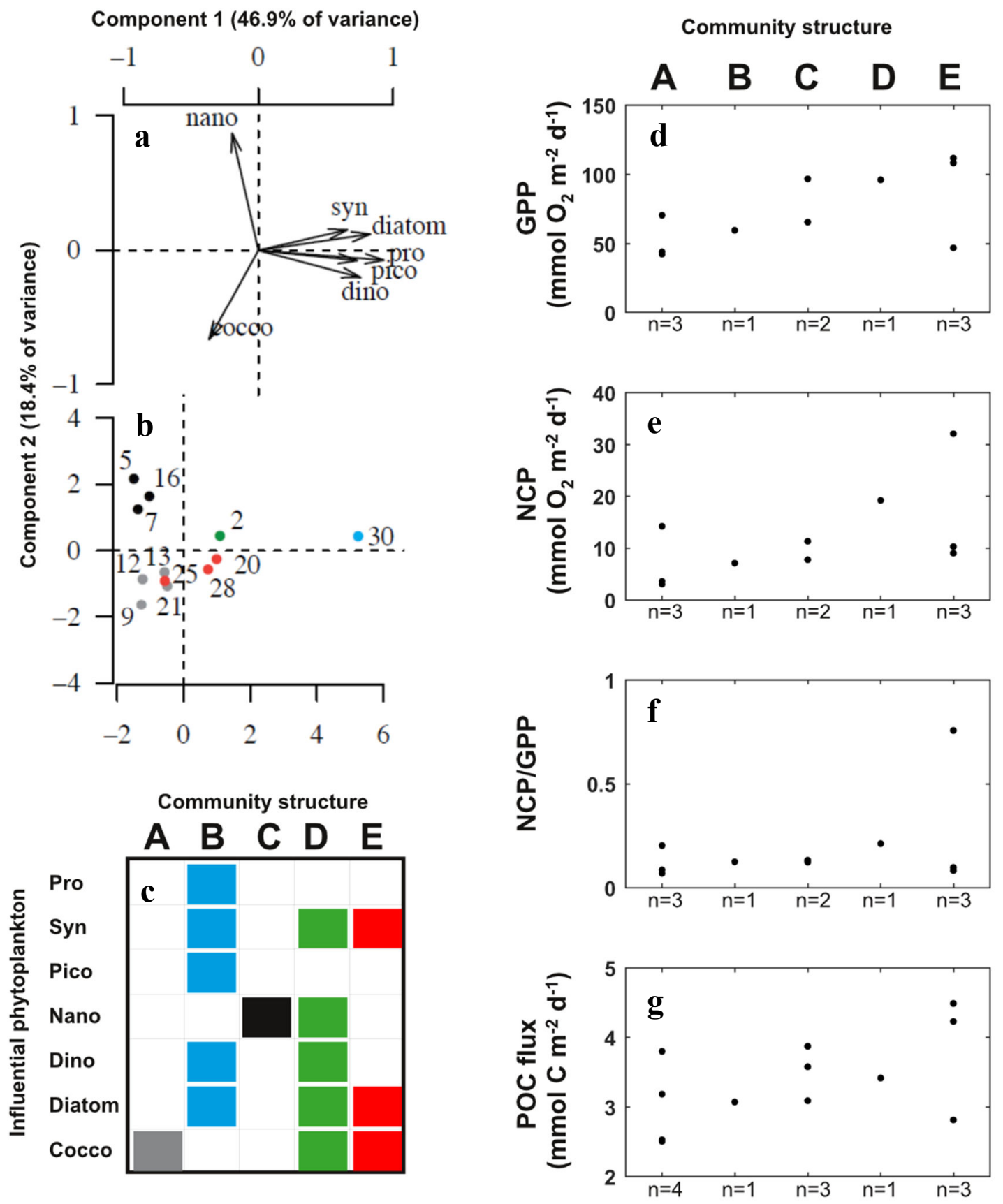

Figure 7: Results of the principal component analysis (PCA) and clustering of community structure types described in the text: (a) Component loadings of phytoplankton groups onto first and second components of PCA, (b) the component scores of each sample location in component space. Location component scores are grouped across all components of the PCA using k-means clustering to identify five community structure types (colors in panel $\mathbf{b}$, indentified in the legend, $\mathbf{c}$ ). Gross primary production (GPP), net community production (NCP), the NCP/GPP ratio, and the particulate organic carbon (POC) flux are plotted for each of the location (n) to which a community structure was assigned (panels d-g). No significant differences in these fluxes were identified between community types (see text). 
The correlation of diversity with Synechococcus abundance $\left(\mathrm{p}=7 \times 10^{-5}\right)$ and Chlorophyll- $a$ concentrations in the smaller size fraction ( $\mathrm{p}=0.01$; most of the biomass) across the transect indicates that the abundance of pico- and nanophytoplankton may be particularly relevant to explaining abundance based diversity shifts in this dataset. Significant basin scale correlations between nitrite and total nitrate plus nitrite concentrations and diversity $\left(\mathrm{p}=1 \times 10^{-4}, \mathrm{p}=0.008\right)$, as well as the regional correlations between variance of diversity and nutrients ( $\mathrm{p}=0.02$ with nitrite and $\mathrm{p}=0.03$ with nitrate plus nitrite), suggest that changes in the availability of inorganic nitrogen may play a key role in allowing other phytoplankton to better compete for this primary limiting nutrient relative to the numerically dominant Prochlorococcus; most identified isolates of Prochlorococcus are able to grow only on ammonia, though some strains have recently been discovered to assimilate nitrite and nitrate [Moore et al. 2002, Martiny et al. 2009, Berube et al. 2015]. While none of the phytoplankton types evaluated in this study have significant correlations with nitrite concentration, nutrient supply rates (not evaluated) are more likely to be environmentally relevant in an ecosystem where limiting nutrients are subject to rapid uptake.

\subsection{Production rates and particulate and dissolved organic carbon export}

The relationship between particulate organic carbon and nitrogen fluxes and gross primary production, but not net community production, was unexpected. Conceptually, NCP should be balanced by export production over sufficient scales of time and space [Emerson et al. 1997]. Particulate carbon flux is often assumed or measured to dominate carbon export [Michaels and Silver 1988, Estapa et al. 2015] and usually measurements of particle flux are considered synonymous with export flux. Disagreement between particle export and oxygen based NCP has been observed previously [Steinberg et al. 2001, Martz et al. 2008], potentially because of methodological biases in each technique [Gardner 2000, Buesseler et al. 2007, 2008, Martz et al. 2008]. In particular, depth dependent biases act differently on gas tracer and trap based methods; particle fluxes at $125 \mathrm{~m}$ are below the base of the mixed layer and may be reduced by attenuation [Martin et al. 1987], while production below the base of the mixed layer but within the euphotic zone may constitute a substantial portion of total NCP [Howard et al. 2010] that is not incorporated in the mixed-layer oxygen budget in this work.

Mismatch in the temporal [Steinberg et al. 2001] and spatial scales integrated by the two types of measurements could also play a role. Recent work in the subtropical North Atlantic [Estapa et al. 2015] found that on submesoscales particulate export and NCP are decoupled, but when integrating over several measurements throughout a mesoscale transect the two fluxes agree in magnitude. Surface production and export may be spatially decoupled at the mesoscale as well in physically dynamic regions [Plattner et al. 2005]. NCP and POC flux sampling across the entire transect ( $\mathrm{n}=13$ locations with coincident measurements) may not have sufficient resolution that the average fluxes are comparable. The lack of correlation between NCP and POC fluxes across the transect in this dataset could thus reflect both methodological issues in the comparison and inadequate spatial resolution of the comparisons. However, GPP should be affected by many of the same potential biases as NCP and to a similar degree.

This mismatch between NCP and POC fluxes may reflect a large contribution of dissolved organic carbon or zooplankton mediated fluxes. Both of these latter fluxes are reflected in gas-tracer based estimates of $\mathrm{NCP}$, but not in particle trap methods. It has previously been observed that a sizable fraction of NCP can be funneled into DOC export or storage with some community structures [Emerson et al. 2014, Ducklow et al. 2015]. Vertically migrating zooplankton may produce fecal pellets, respire, or excrete as DOC surface derived organic matter at depth, with associated carbon fluxes anywhere from 0 to $39 \%$ as large as the POC flux in equatorial and subtropical regions [Zhang and Dam 1997, Steinberg et al. 2000, AlMutairi and Landry 2001].

Here we quantitatively compare export ratios of NCP and POC flux using NCP/GPP in order to evaluate the potential role of DOC or zooplankton mediated fluxes in balancing organic matter production. NCP and GPP are converted to carbon based units as described in section 2.2.2. NCP and the gas tracer implied export ratio is more variable and generally greater than the POC flux and export ratio (Figure 8). 
Comparing only stations where both NCP and POC export fluxes were evaluated, POC flux at $125 \mathrm{~m}$ accounts for $82 \%$ of total NCP across the transect (standard deviation $81 \%, 95 \%$ confidence interval of 38 to $126 \%$ ). The mean value is not well constrained, reflecting the high variability of NCP, the potential variability of the DOC flux, and poor sample resolution [Estapa et al. 2015]. Nonetheless, the mean value is equivalent to DOC storage or export accounting for $18 \%$ of NCP, in agreement with estimates that DOC accounts for $17 \%$ of euphotic zone NCP in the Atlantic [Romera-Castillo et al. in press] and 20\% of export to below $130 \mathrm{~m}$ globally [Hansell 2013]. The POC flux to NCP relationship is equivalent but slightly better constrained in the South Atlantic Gyre, 76 (standard deviation 46\%, $n=5$ ). Observations are more sparse in the South Atlantic than in the North Atlantic, but modeled annual DOC fluxes are on the order of 1-2 mmol DOC m $\mathrm{m}^{-2} \mathrm{~d}^{-1}$ in the western subtropical and tropical Atlantic [Hansell et al. 2012], or $\sim 25 \%$ of our measured NCP. Thus the average difference between NCP and POC export can plausibly be explained by DOC fluxes in the subtropical and tropical regions.

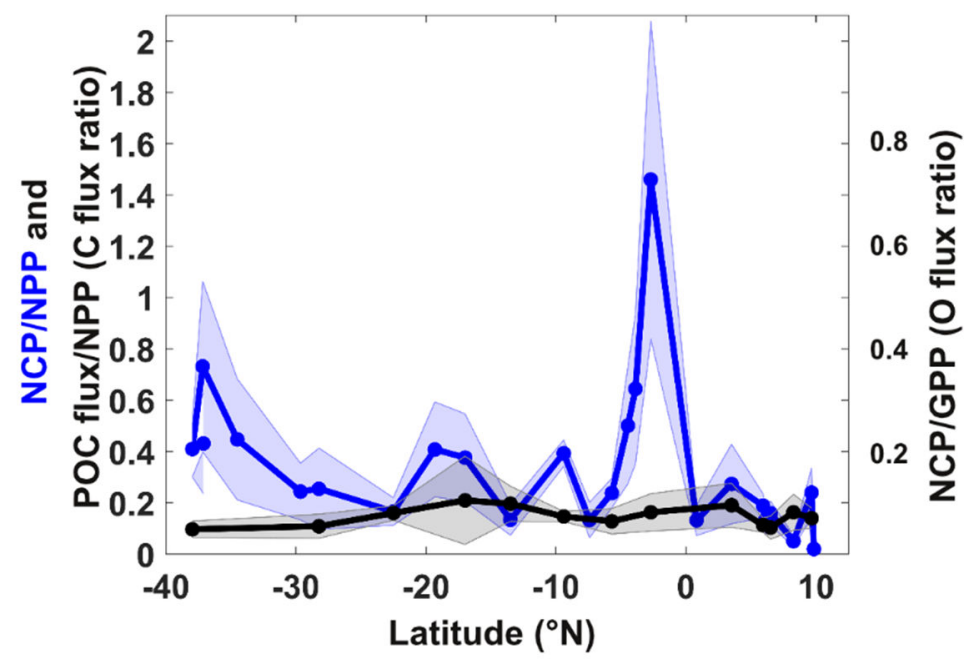

Figure 8: Export ratio estimates from $\mathrm{O}_{2} / \mathrm{Ar}$ based net community production (NCP) and particulate organic carbon (POC) flux, relative to net primary production (NPP; scaled from triple oxygen isotope based gross primary production, GPP). The NCP based export ratio and standard deviation are in blue, and the POC flux based export ratio and uncertainties are in black. The export ratios are reported in carbon units on the left hand axis, and oxygen units on the right hand axis. The POC flux based export ratio is generally lower and less variable than the NCP based export ratio. NCP/NPP peaks at $37.2^{\circ} \mathrm{S}$ and $2.9^{\circ} \mathrm{S}$ are discussed in the text.

The correlation between gross primary production and POC flux across the transect (Figure 6a) may provide a clue that particle export is directly linked to total photosynthetic production despite decoupling of net community production and particulate organic carbon fluxes as hypothesized above. This is consistent with recent findings that picophytoplankton can export carbon in proportion to their contribution to net primary production (or proportionally GPP, section 2.2.2) in tropical and subtropical regions, possibly through cell aggregation and fecal packaging by zooplankton [Richardson and Jackson 2007, Stukel and Landry 2010, and Guidi et al. 2016]. Hennon et al. [in prep] found that in situ growth rates of Prochlorococcus and Synechococcus could account for around 50\% of total GPP in the South Subtropical Transition zone, and 70\% GPP across the South Atlantic Gyre and Western Tropical Atlantic. Thus the link between POC export and photosynthesis is plausibly explained by the packaging and export of cyanobacteria derived carbon. 


\section{Conclusion}

We used a suite of in situ measurements of community structure, biological production, and particulate export to evaluate how the biological carbon pump varies meridionally across the Equatorial and South Atlantic Ocean in austral fall of 2013. This work highlights the basin scale homogeneity and mesoscale variability of rates of biological production and carbon export over the transect. While we did not observe significant differences in carbon cycling between different community structure types, regional shifts in the variance of community diversity and primary production were correlated, along with chlorophyll and nutrient concentrations, suggesting that these processes share underlying drivers of variability at regional scales. Observed spatial scales of variability were consistent with the scale of mesoscale eddies, particularly in the South Subtropical Transition zone and Western Tropical Atlantic, providing a plausible physical link between nutrient supply, community diversity, and production; our results are consistent with a growing body of literature ascribing local variability and particularly transient enhancement of community diversity, production, and export to mesoscale structure. In this work, abundance based community diversity and production rates were not significantly correlated throughout the transect, however when binned by biogeographic province, diversity correlated with gross primary production.

In addition, particulate organic carbon and nitrogen export correlated with gross primary production, suggesting that there may be a link between photosynthesis and ultimate particle export when picophytoplankton dominate production [Hennon et al. in prep]. This finding is consistent with the hypothesis of Richardson and Jackson [2007] that picoplankton contribute to export in proportion to their contribution to community autotrophic production, regardless of the expected slow sinking rates of individual small cells. At the same time, the lack of correlation between net community production and POC export may indicate the importance of dissolved organic carbon or zooplankton mediated fluxes to export, or could be indicative of methodological or sample distribution biases; quantitatively, the relative magnitude of POC to NCP fluxes implies a DOC export rate consistent with both global and regional estimates of the contribution of DOC to carbon export from the surface mixed layer.

Additional specific findings of interest include:

1. Good agreement exists between production rates based on in situ biogeochemical tracers (this study) and incubation based methods [Marañón et al. 2003, Tilstone et al. 2009, Serret et al. 2015] across the South and Equatorial Atlantic surface ocean.

2. Disagreement between production rates measured at the mesohaline edge of the Amazon River Plume in early May 2013 (this study) with those from May-June 2010 [Yeung et al. 2012] using nearly identical methods points to the dynamic nature of this environment and potentially to the importance of blooms by diatom-diazotroph associations in enhancing community production.

3. A high chlorophyll, high productivity, and potentially high export ratio feature in the South Subtropical Transition zone may have been associated with a westward propagating cyclonic eddy.

4. Observed community diversity generally agrees with predictions from global circulation and biogeochemistry models.

5. Some common assumptions in the calculation of gross primary production and net community production from oxygen based tracers may introduce additional errors to rate estimates. In particular, results may be sensitive to the time of sampling because diel cycles may be large relative to average tracer values. 


\section{Supporting information}

\section{A. Derivation of mixing corrections to gross primary production and net community production}

Nicholson et al. 2014 derive mixing corrections for gross primary production to account for entrainment in their supplementary material. Their equation S7 is reproduced here, with measured ${ }^{17} \mathrm{O} /{ }^{16} \mathrm{O}$ and ${ }^{18} \mathrm{O} /{ }^{16} \mathrm{O}$ ratios notated as ${ }^{17} \mathrm{X}$ and ${ }^{18} \mathrm{X}$ as in the main text of this work, and the subscript $d$ indicating the measured value of a property at some depth below the mixed layer:

$$
C_{G P P}=\left(\frac{\partial \mathrm{z}}{\partial \mathrm{t}}\right)\left(\left[\frac{1}{{ }^{17} X}\left({ }^{17} \mathrm{O}_{2 d}-{ }^{17} \mathrm{O}_{2}\right)-\left(\mathrm{O}_{2_{d}}-\mathrm{O}_{2}\right)\right]-\lambda\left[\frac{1}{{ }^{18} X}\left({ }^{18} \mathrm{O}_{2_{d}}-{ }^{18} \mathrm{O}_{2}\right)\left(\mathrm{O}_{2 d}-\mathrm{O}_{2}\right)\right]\right)
$$

As the measured oxygen concentrations are in molal units, Eq. S1 is multiplied by the density of seawater, $\rho_{\text {sw. }}$ If Eq. $\mathrm{S} 1$ is distributed, it becomes:

$$
C_{G P P}=\left(\frac{\partial \mathrm{z}}{\partial \mathrm{t}}\right)\left(\left[\frac{{ }^{17} \mathrm{O}_{2 d}}{{ }^{17} X}-\frac{{ }^{17} \mathrm{O}_{2}}{{ }^{17} X}-\mathrm{O}_{2 d}+\mathrm{O}_{2}\right]-\lambda\left[\frac{{ }^{18} \mathrm{O}_{2_{d}}}{{ }^{18} X}-\frac{{ }^{18} \mathrm{O}_{2}}{{ }^{18} X}-\mathrm{O}_{2_{d}}+\mathrm{O}_{2}\right]\right) \rho_{s w}
$$

Using the definitions of ${ }^{17} \mathrm{X}$ and ${ }^{18} \mathrm{X}$, the second term in each of the bracketed expressions simplifies to $\mathrm{O}_{2}$, and the surface concentration of bulk oxygen cancels out of the expression:

$$
C_{G P P}=\left(\frac{\partial \mathrm{z}}{\partial \mathrm{t}}\right)\left(\left[\frac{{ }^{17} \mathrm{O}_{2 d}}{{ }^{17} X}-\mathrm{O}_{2_{d}}\right]-\lambda\left[\frac{\mathrm{O}_{2 d}}{{ }^{18} X}-\mathrm{O}_{2_{d}}\right]\right) \rho_{s w}
$$

Finally, the first term in each of the bracketed expressions can be multiplied by $\mathrm{O}_{2 d} / \mathrm{O}_{2 d}$ in order to rewrite the concentrations of the rare isotopologues in terms of ${ }^{17} \mathrm{X}$ and ${ }^{18} \mathrm{X}$, and the common factor of $\mathrm{O}_{2 d}$ removed from all the terms within the parentheses. This leaves Eq. S4 in terms of only measured variables.

$$
C_{G P P}=\left(\frac{\partial \mathrm{z}}{\partial \mathrm{t}}\right) \mathrm{O}_{2_{d}}\left(\left[\frac{{ }^{17} X_{d}}{{ }^{17} X}-1\right]-\lambda\left[\frac{{ }^{18} X_{d}}{{ }^{17} X}-1\right]\right) \rho_{s w}
$$

The equation for correcting for vertical diffusivity is identical to that for entrainment except that the mixing rate is equal to the vertical diffusivity coefficient $\mathrm{K}_{\mathrm{z}}$ over the change in depth. Thus mixing from entrainment and vertical diffusivity can be combined into a single expression, Eq. S5, which is identical to Eq. 4 in the main text $(\partial z / \partial t$ set to 0 for shoaling):

$$
C_{\mathrm{GPP}}=\left(\frac{\partial \mathrm{z}}{\partial \mathrm{t}}+\frac{\mathrm{Kz}}{\left(z_{d}-z\right)}\right) \mathrm{O}_{2 d}\left(\left[\frac{{ }^{17} X_{d}}{{ }^{17} X}-1\right]-\lambda\left[\frac{{ }^{18} X_{d}}{{ }^{17} X}-1\right]\right) \rho_{S w}
$$

By similar reasoning net community production may be approximated as a function of the difference between the net biological oxygen production signal at depth and in the surface:

$$
C_{\mathrm{NCP}}=\left(\frac{\partial \mathrm{z}}{\partial \mathrm{t}}+\frac{\mathrm{Kz}}{\left(\mathrm{z}_{\mathrm{d}}-\mathrm{z}\right)}\right)\left(\mathrm{O}_{2 \mathrm{sat}_{\mathrm{d}}}\left[\frac{\Delta \mathrm{O}_{2}}{\mathrm{Ar}}\right]_{\mathrm{d}}\left[\frac{\mathrm{Ar}}{\mathrm{Ar} \mathrm{sat}_{\mathrm{d}}}\right]_{\mathrm{d}}-\mathrm{O}_{2 \text { sat }}\left[\frac{\Delta \mathrm{O}_{2}}{\mathrm{Ar}}\right]\left[\frac{\mathrm{Ar}}{\mathrm{Ar}_{\mathrm{sat}}}\right]\right) \rho_{\mathrm{sw}}
$$

This form is convenient because it expresses the components of the correction in the same form as the gas exchange term. However the biological oxygen supersaturation $\left(\Delta \mathrm{O}_{2} / \mathrm{Ar}\right)$ is not mixed conservatively.

The exact form can be derived by combining the separate mass balances for oxygen and argon. For the entrainment term only:

$$
\begin{aligned}
& C_{\mathrm{O}_{2}}=\left(\frac{\partial \mathrm{z}}{\partial \mathrm{t}}\right)\left(\mathrm{O}_{2 \mathrm{~d}}-\mathrm{O}_{2}\right) \rho_{\mathrm{sw}} \\
& C_{\mathrm{Ar}}=\left(\frac{\partial \mathrm{z}}{\partial \mathrm{t}}\right)\left(A r_{\mathrm{d}}-\mathrm{Ar}\right) \rho_{\mathrm{sw}}
\end{aligned}
$$


Multiplying Eq. $\mathrm{S} 8$ by $\mathrm{O}_{2}$ sat $/ \mathrm{Ar}_{\text {sat, }}$, subtracting from Eq. S7, and multiplying the resulting mass balance by $\mathrm{O}_{2 \text { sat }} / \mathrm{O}_{2}$ sat in identical manner to the derivation of the net community production expression in Howard et al. [2011] leads to the following expression (Eq. 3 in main text):

$$
C_{\mathrm{NCP}, \text { exact }}=\left(\frac{\partial \mathrm{z}}{\partial \mathrm{t}}\right)\left(\mathrm{O}_{2 \text { sat }}\left[\frac{\mathrm{O}_{2 \mathrm{~d}}-\mathrm{O}_{2}}{\mathrm{O}_{2 \text { sat }}}-\frac{\mathrm{Ar}_{\mathrm{d}}-\mathrm{Ar}}{\mathrm{Ar}_{\mathrm{sat}}}\right]\right) \rho_{\mathrm{sw}}
$$

The approximate expression in Eq. S6 can be rewritten in the same form as Eq. S9 by substituting the definition of $\Delta \mathrm{O}_{2} / \mathrm{Ar}$, multiplying by $\mathrm{O}_{2}$ sat $/ \mathrm{O}_{2}$ sat and $\mathrm{Ar}_{\text {sat }} / \mathrm{Ar}_{\text {sat }}$ where appropriate and cancelling terms:

$$
\begin{aligned}
& C_{\mathrm{NCP}}=\left(\frac{\partial \mathrm{z}}{\partial \mathrm{t}}\right)\left(\mathrm{O}_{2 \text { sat }_{\mathrm{d}}}\left[\frac{\mathrm{O}_{2 \mathrm{~d}} / \mathrm{Ar}_{\mathrm{d}}}{\mathrm{O}_{2 \text { sat d }} / \mathrm{Ar}_{\text {sat d }}}-1\right]\left[\frac{\mathrm{Ar}}{\mathrm{Ar}_{\text {sat }}}\right]_{\mathrm{d}}-\mathrm{O}_{2 \text { sat }}\left[\frac{\mathrm{O}_{2} / \mathrm{Ar}_{\mathrm{O}_{\text {sat }} / \mathrm{Ar}_{\text {sat }}}}{\mathrm{O}_{\text {sat }}}-1\right]\left[\frac{\mathrm{Ar}}{\mathrm{Ar}_{\text {sat }}}\right]\right) \rho_{\mathrm{sw}} \\
& C_{\mathrm{NCP}}=\left(\frac{\partial \mathrm{z}}{\partial \mathrm{t}}\right)\left(\mathrm{O}_{2 \mathrm{~d}}-\mathrm{O}_{2 \mathrm{sat}_{\mathrm{d}}}\left[\frac{\mathrm{Ar}}{\mathrm{Ar}_{\mathrm{sat}}}\right]_{\mathrm{d}}-\mathrm{O}_{2}+\mathrm{O}_{2 \text { sat }}\left[\frac{\mathrm{Ar}}{\mathrm{Ar}_{\mathrm{sat}}}\right]\right) \rho_{\mathrm{sw}} \\
& C_{\mathrm{NCP}}=\left(\frac{\partial \mathrm{z}}{\partial \mathrm{t}}\right)\left(\mathrm{O}_{2 \text { sat }}\left[\frac{\mathrm{O}_{2 \mathrm{~d}}-\mathrm{O}_{2}}{\mathrm{O}_{2 \text { sat }}}-\frac{\left(\frac{\mathrm{O}_{2} / \mathrm{Ar}_{\mathrm{Aat}}}{\mathrm{O}_{2} / \mathrm{Ar}_{\mathrm{sat}}}\right) \mathrm{Ar}_{\mathrm{d}}-\mathrm{Ar}}{\mathrm{Ar}}\right]\right) \rho_{\mathrm{sat}}
\end{aligned}
$$

Thus the approximate form adds a factor to the argon concentration at depth that is close to one, as the saturation ratio of oxygen to argon stays relatively constant with depth; the solubility functions of oxygen and argon are very similar. The approximate expression (Eqs. S6 or S12) is within $0.15 \mathrm{mmol} \mathrm{m}^{-2} \mathrm{~d}^{-1}$ of the exact expression in all cases in this dataset $(<1 \%$ relative standard error except when net community production is very close to zero) and is an acceptable formulation in most situations.

\section{B. Sources of systematic bias in oxygen tracer mass balances}

We present some important additional considerations related to the calculation of net community production (NCP) and gross primary production (GPP) using the $\mathrm{O}_{2} / \mathrm{Ar}$ and triple oxygen isotope tracers. First, we address the probable biases in the gas mass balances used in this paper. Next we note how diel biological cycles influence uncertainty in NCP and GPP, and finally the role of argon saturation state in the calculation of NCP. These important caveats may have implications for the use of oxygen to argon and triple oxygen isotope ratios in other work.

\section{B.1 Lateral advection, upwelling, and mixing}

Equations 5 and 6 in the main text (NCP and GPP) ignore lateral advection, as well as the potential role of upwelling and surface divergence. While this is a reasonable assumption across the South Atlantic Gyre, with surface current velocities on the order of $\mathrm{cm} \mathrm{s}^{-1}$ and low variability in the tracer values, it may not hold in the equatorial region, particularly between $\sim 5^{\circ} \mathrm{S}$ and $5^{\circ} \mathrm{N}$, which has complex surface and near surface zonal flows related to the North and South Equatorial Currents and Equatorial Counter Current, with velocities up to tens of $\mathrm{cm} \mathrm{s}^{-1}$ [Peterson and Stramma 1991, Lumpkin and Garzoli 2005, Brandt et al. 2006]. In general, surface transport of the $\mathrm{O}_{2} / \mathrm{Ar}$ and triple oxygen isotope tracers cannot be constrained because advective gradients cannot be determined at high enough resolution in this study to determine zonal flow. However, within the equatorial region the triple oxygen isotope tracer ${ }^{17} \Delta$ does not vary between adjacent sample locations by more than twice the standard deviation of a single location. Thus differences in the tracer are driven primarily by gas exchange and vertical exchange. Gradients in $\Delta \mathrm{O}_{2} / \mathrm{Ar}$ are larger relative to uncertainties than the lateral gradients in ${ }^{17} \Delta$, but generally the difference between stations is no larger than the standard deviation at either station. These relatively small changes over degree scales suggest that surface gradients can be neglected within the uncertainty bounds of the GPP and NCP rates: as a worst case scenario, with a $50 \mathrm{~cm} \mathrm{~s}^{-1}$ surface current [Lumpkin and Garzoli 2005], and the maximum observed gradients in the equatorial region of 15 per meg ${ }^{17} \Delta$ over $220 \mathrm{~km}$, and 
$1.5 \% \Delta \mathrm{O}_{2} /$ Ar over $145 \mathrm{~km}$, and $3 \mu \mathrm{mol} \mathrm{O}_{2} \mathrm{~m}^{-3}$ difference in surface oxygen saturation concentration over the same distance, then over the range of observed production rates GPP is altered by no more than $4 \%$ and NCP by no more than $2 \%$.

Vertical gradients in the tracers are large compared to horizontal gradients, and upwelled water would act similarly to entrainment or mixing in decreasing surface $\Delta \mathrm{O}_{2} / \mathrm{Ar}$ and increasing ${ }^{17} \Delta$. While upwelling was not directly evaluated in this study, it is likely minimal compared to in the Eastern Equatorial Atlantic [Rhein et al. 2010, Kadko and Johns 2011] as the cruise transect was well west of the main upwelling region and associated surface chlorophyll $a$ feature [e.g. Grodsky et al. 2008]. Measured rates close to our transect location are $\sim 0 \mathrm{~m} \mathrm{~d}^{-1}$ [Kadko and Johns 2011] (4.7 ${ }^{\circ} \mathrm{S}, 26.8^{\circ} \mathrm{W}$, May 2009, error estimated from reported results as $\left.\sim 0.05 \mathrm{~m} \mathrm{~d}^{-1}\right)$. Satellite based divergence indicates annual minimum upwelling in March and April near our cruise track with near zero divergence to net convergence [Helber et al. 2007]. Thus we believe an assumption of near zero upwelling is justified for this dataset, but we recognize that such an assumption makes our GPP estimate an upper bound and our NCP estimate a lower bound.

Finally, GPP and NCP are sensitive to the vertical diffusivity coefficient, $\mathrm{K}_{\mathrm{z}}$, which likely varies across the South Atlantic. Increased vertical diffusivity would act to lower our GPP estimates and raise apparent $\mathrm{NCP}$ and the NCP/GPP ratio, as with upwelling. We choose $\mathrm{K}_{\mathrm{z}}=1 \times 10^{-5} \mathrm{~m}^{2} \mathrm{~s}^{-1}\left(8.6 \times 10^{-1} \mathrm{~m}^{2} \mathrm{~d}^{-1}\right)$ as representative of mixing across the base of the mixed layer based on microscale measurement derived $\mathrm{K}_{\mathrm{z}}$ by Mouriño-Carballido et al. [2011] at the same time of year (they find average $\mathrm{K}_{\mathrm{z}}$ in the SAG and WTA of $0.3 \pm 0.5 \times 10^{-5} \mathrm{~m}^{2} \mathrm{~s}^{-1}$ over the entire surface $150 \mathrm{~m}$ ). This estimate is consistent with global values for below the mixed layer (order of $10^{-5} \mathrm{~m}^{2} \mathrm{~s}^{-1}$ ) [Wunsch and Ferarri 2004], diffusivity derived from thermocline argon supersaturation for decadal scale mixing in the subtropical North Pacific (no more than $\left.2 \times 10^{-5} \mathrm{~m}^{2} \mathrm{~s}^{-1}\right)$ [Emerson et al. 2012], and a tracer release experiment in the Southern Ocean $\left(1 \pm 2 \times 10^{-5} \mathrm{~m}^{2}\right.$ $\mathrm{s}^{-1}$ ) [Law et al. 2003]. In the open ocean, values up to the order of $10^{-4} \mathrm{~m}^{2} \mathrm{~s}^{-1}$ are observed primarily in actively upwelling regions such as the Eastern Equatorial Pacific [Carr et al. 1992, Haskell et al. 2015] and Eastern Equatorial Atlantic [Kadko and Johns 2011, Rhein et al. 2010]. Our choice of $\mathrm{K}_{\mathrm{z}}$ may not be high enough for the WTA or the more energetically mixed boundary regions such as the SST, but is a reasonable lower bound across the transect. Increasing $\mathrm{K}_{\mathrm{z}}$ by an order of magnitude to $1 \mathrm{x}^{-4} 0^{-4} \mathrm{~m}^{2} \mathrm{~s}^{-1}$ on average decreases GPP by $26 \%$, and increases NCP and the NCP/GPP ratio by $6 \%$ and $36 \%$ respectively. If $\mathrm{K}_{\mathrm{z}}$ is instead set to 5 or $2 \times 10^{-5} \mathrm{~m}^{2} \mathrm{~s}^{-1}$, the changes in GPP, NCP, and NCP/GPP are $-11 \%, 3 \%$, and $12 \%$ and $-3 \%, 1 \%$, and $4 \%$ respectively compared to rates at $\mathrm{K}_{\mathrm{z}}=1 \times 10^{-5} \mathrm{~m}^{2} \mathrm{~s}^{-1}$. The sensitivity of absolute rates and NCP/GPP ratio is low except when NCP and GPP are low and of similar magnitude (primarily the $\mathrm{SAG}$ ) - in this case the higher mixing rates (e.g. $\mathrm{K}_{\mathrm{z}}$ greater than $\sim 3 \times 10^{-5} \mathrm{~m}^{2} \mathrm{~s}^{-1}$ ) may increase the $\mathrm{NCP} / \mathrm{GPP}$ ratio by 0.1 or more. However, this region is also where we would expect stratification to be strongest and $\mathrm{K}_{\mathrm{z}}$ to be most similar to the minimum bound we chose. Direct evaluation of upwelling and vertical mixing rates over a similar timescale to the oxygen based tracers would be preferable to the assumptions above; the ${ }^{7} \mathrm{Be}$ approach is a promising technique for just such an approach [Haskell et al. 2015, Kadko and Johns 2011].

\section{B.2 Sample time in relation to diel cycles}

In principle, triple oxygen isotope and $\mathrm{O}_{2} / \mathrm{Ar}$ derived rates of NCP and GPP integrate over the residence time of dissolved oxygen in the mixed layer, set primarily by the gas transfer coefficient and the mixed layer depth. The residence time is generally on the order of one to two weeks, and the tracer is expected to average sufficient time and space that diel cycles and small-scale heterogeneity in production and respiration are averaged out [Juranek and Quay 2013]. In practice daily and sub-daily variations in oxygen, the $\mathrm{O}_{2} / \mathrm{Ar}$ ratio, and triple oxygen isotopes can be used to glean additional information about localized metabolic cycling [Hamme et al. 2012, Ferrón et al. 2015, Nicholson et al. 2015], and gasexchange fluxes [Sarma et al. 2006] on shorter time scales. These daily cycles dominate the time rate of change of the tracers over seasonal cycles, and steady state solutions to GPP and NCP as were calculated in this work may be in error. ${ }^{17} \Delta$ and $\Delta \mathrm{O}_{2} / \mathrm{Ar}$ at a single sample location $\left(28.2^{\circ} \mathrm{S}\right)$ over one day are 
plotted against local time in Figure S1a. The systematic increase in the tracers until sunset and then decrease until the following midday is likely related to the balance of diel wind and productivity cycles. Similar patterns of a diel cycle were observed at locations in each biogeographic province (not plotted). Treating the data with the standard steady-state assumption and ignoring short term variability in gas exchange and productivity results in the GPP and NCP rates in Figure S1b. In this case, a sample taken around 06:00 local time leads to estimates of GPP and NCP close to the mean rates, while rates derived from samples taken in the early evening would be $40-60 \%$ higher than those from midday samples - this range is similar to the difference between adjacent sample locations. Sample times in this dataset are rough evenly distributed throughout the day within each region (Figure S2) and time of sampling likely does not bias the regional analysis. For comparison across locations with and without time-series data, we report the location mean and standard deviation of all replicates, regardless of time of day sampled, and assume steady state in all calculations. While it may not be possible to evaluate the nominal sample time for a given location a priori when planning a study, if it is not possible to sample multiple timepoints within a given day it may be preferable to avoid sampling during morning and dusk-i.e. during daily periods of expected peak tracer accumulation and deficit (as suggested by Hamme et al. [2012]).

\section{B.3 Argon supersaturation and the calculation of net community production}

Biological oxygen saturation [Hendricks et al. 2004] (or equivalently supersaturation, $\Delta \mathrm{O}_{2} / \mathrm{Ar}[$ Kaiser et al. 2005]) is defined in order to calculate NCP, often with the explicit assumption that Ar is at saturation in the absence of direct measurements of argon or oxygen concentration paired with the $\mathrm{O}_{2} / \mathrm{Ar}$ ratio. Assuming argon is at $100 \%$ saturation is a reasonable approximation in most cases, and neglecting $\mathrm{Ar} / \mathrm{Ar}_{\text {sat }}$ in the gas exchange term generally leads to small systematic errors of no more than a few percent in NCP. However, when incorporating terms for mixing, entrainment, or upwelling the gradient in $\mathrm{Ar} / \mathrm{Ar}_{\text {sat }}$ may be substantial; some locations in this study have 5 to $10 \%$ supersaturation differences between the surface and below the pycnocline. With the gas exchange, entrainment and mixing in this study, setting $\mathrm{Ar} / \mathrm{Ar}_{\text {sat }}=1$ leads to systematic underestimation of NCP and the NCP/GPP ratio by $3 \%$ on average $(3,5,1$, and $1 \%$ underestimation in the SST, SAG, WTA, and ARP). The underestimation at individual sample locations may be more significant however, with NCP up to $9 \%$ too low. This is similar to the systematic uncertainty associated with the choices of gas exchange parameterization $(\sim 15 \%)$ or vertical diffusivity ( up to $10 \%$ in general). Thus when including diapycnal exchange terms in the mass balance for NCP, measured argon or oxygen concentrations are preferable to assuming that argon is saturated with respect to the atmosphere when subsurface argon supersaturations are expected to be large [q.v. Gehrie et al. 2006]. 


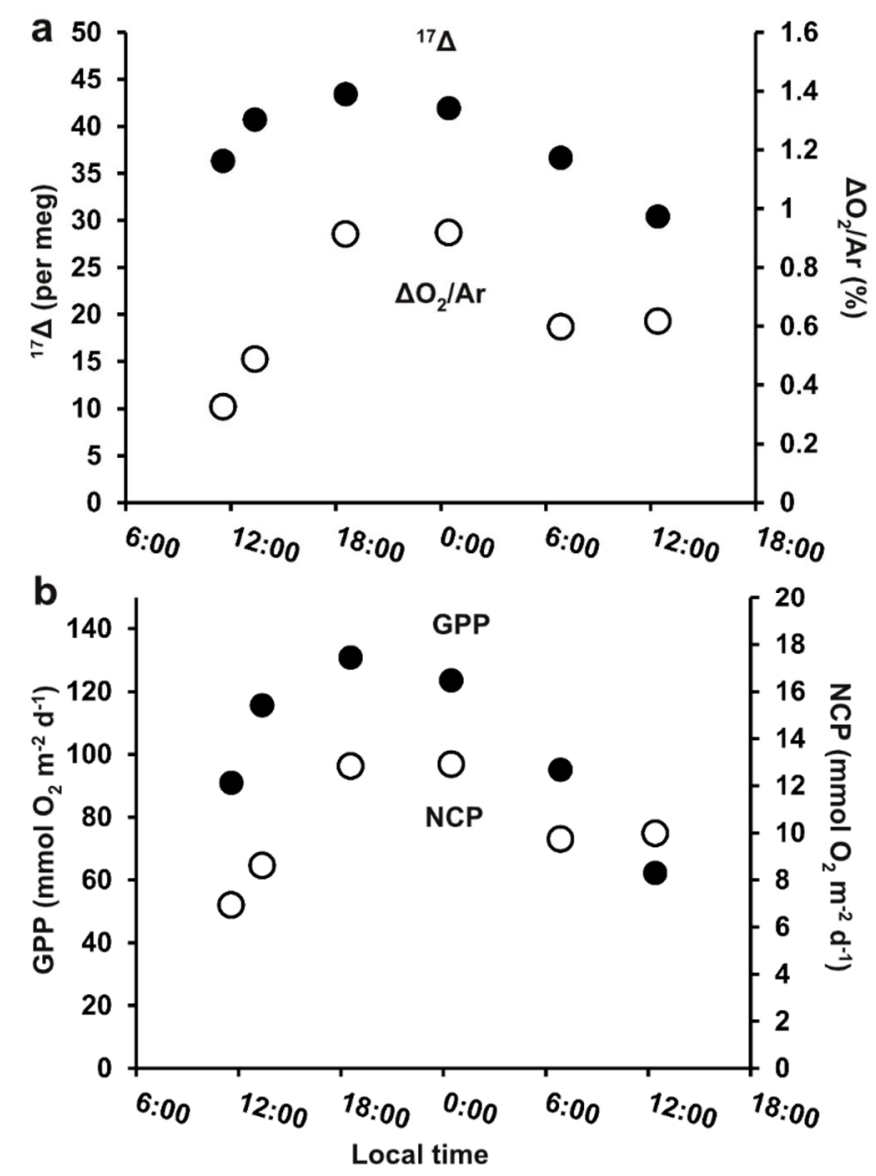

Figure S1: Underway sampling at $28.2^{\circ} \mathrm{S}$ relative to local noon; measured (a) the triple oxygen isotope tracer ${ }^{17} \Delta$ and biological oxygen supersaturation $\Delta \mathrm{O}_{2} / \mathrm{Ar}$ compared to (b) gross primary production (GPP) and net community production (NCP) calculated by assuming steady state conditions and neglecting the observed time rate of change (as may be required without time-series sampling). The three quasiLagrangian stations in the South Atlantic Gyre, Western Tropical Atlantic, and the Amazon River Plume have different timing of peak relative to minimum tracer values and day to day variability; no specific time of day is close to the mean value in all three cases and the optimal sample period for assuming steady state cannot be determined a priori in this dataset. 


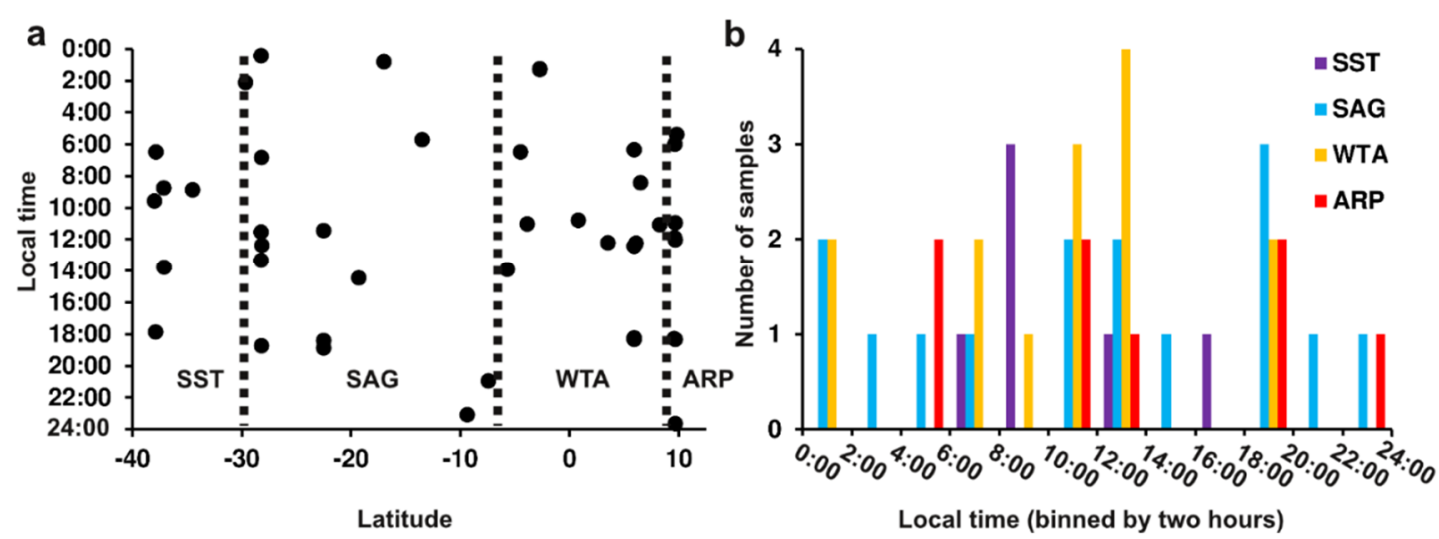

Figure S2: Gas tracer sampling time relative to local noon (a) across the cruise transect and (b) binned in two hour intervals by biogeographic provinces as defined in the text. No systematic trends in sample time are found within or bewtern biogeographic provinces for this dataset.

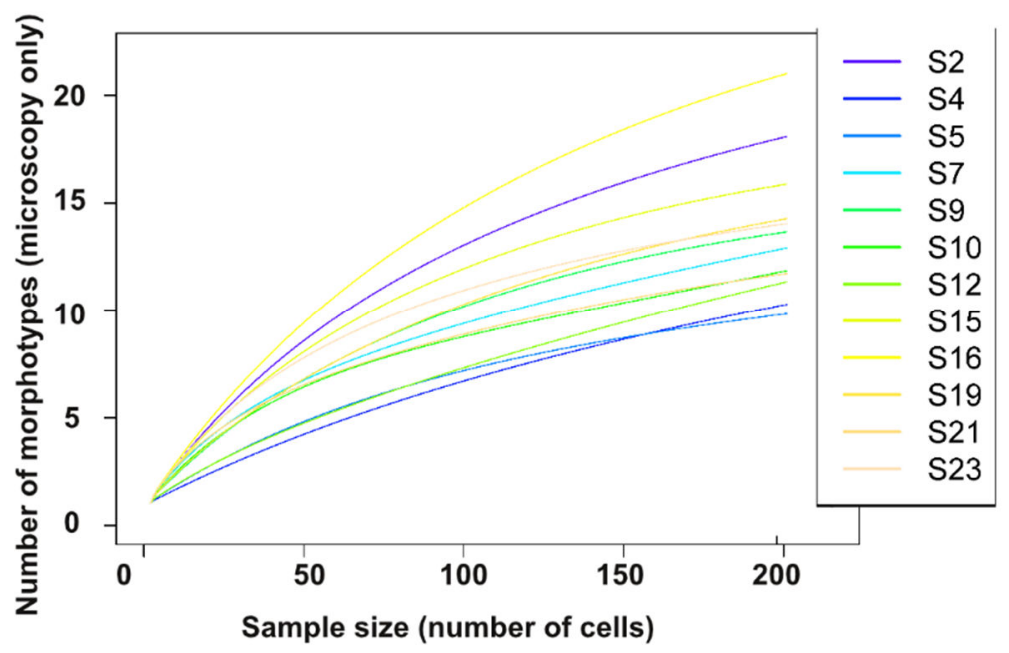

Figure S3: Rarefaction curves of number of morphotypes of microphytoplankton identified by light microscopy. Curves are identified by station number. Curves generally have not leveled within the number of observations, indicating that not all microphytoplankton morphotypes likely to be present have been identified. 

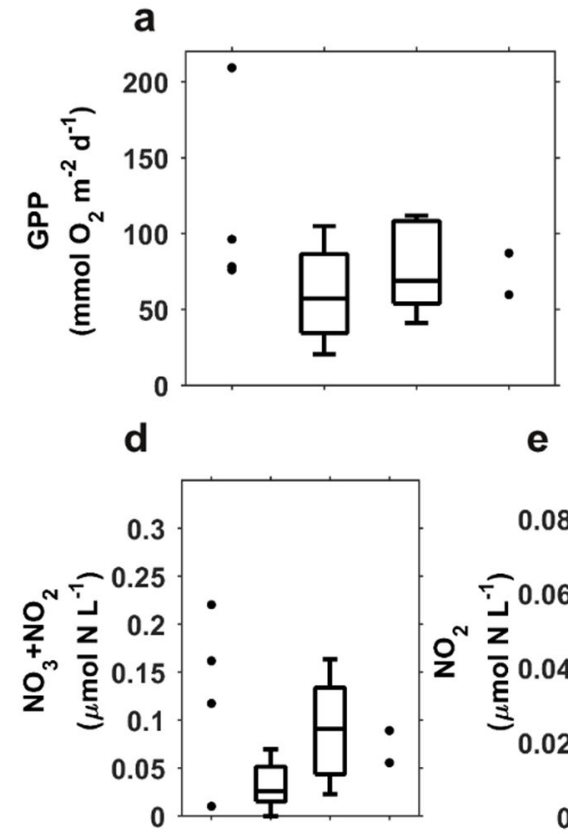

h

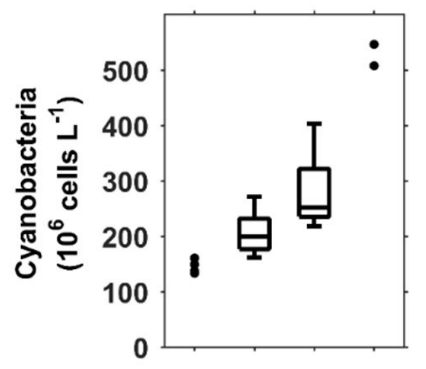

I

i

m
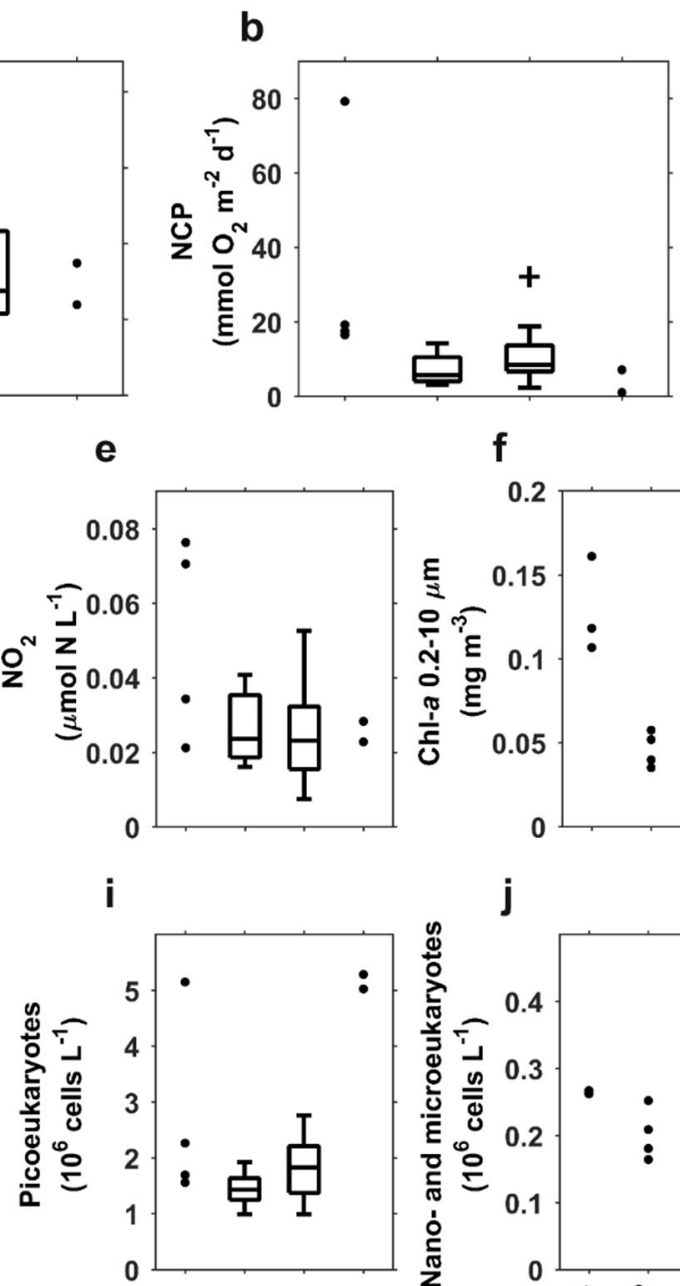
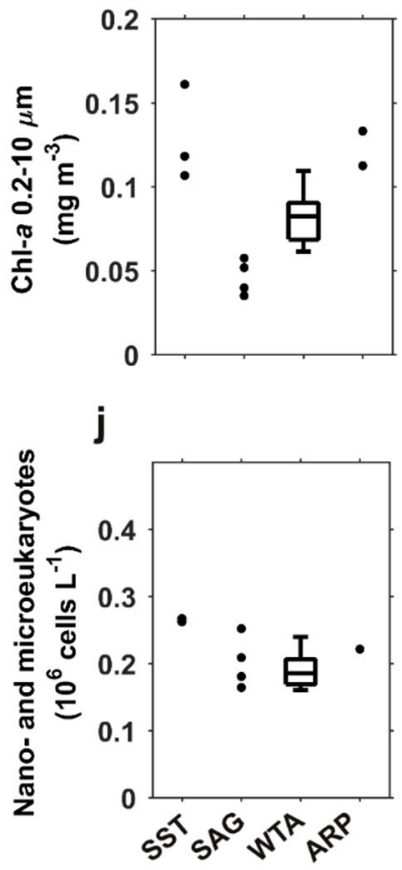

C
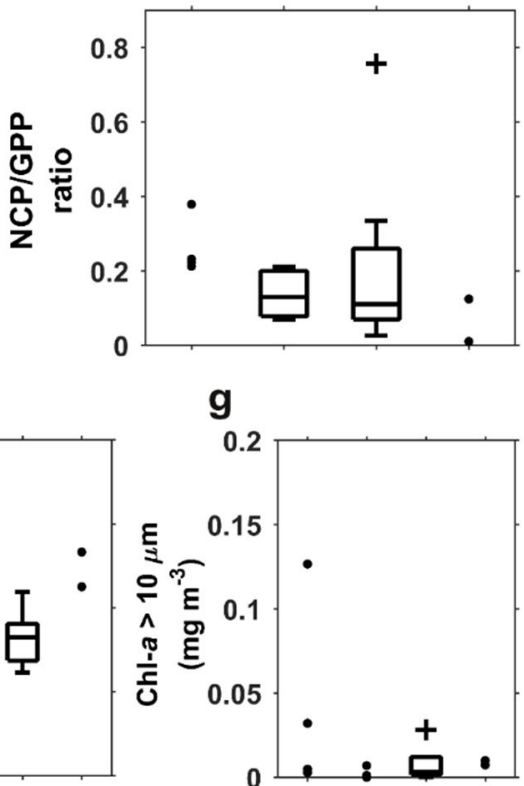

$\mathbf{k}$

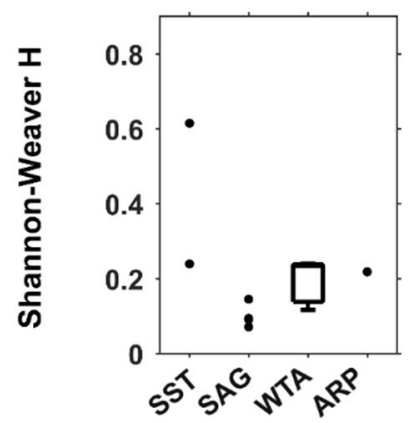

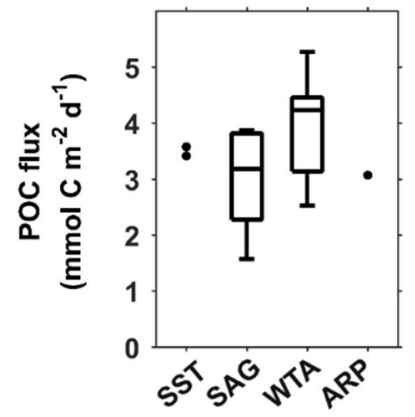

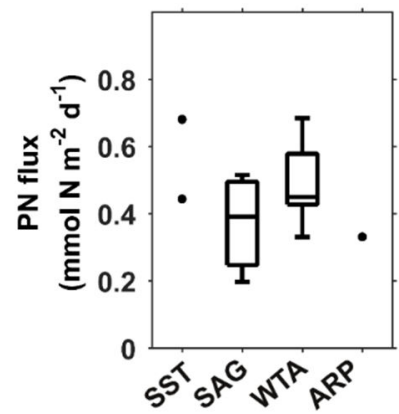

Figure S4: Biological parameters in this study binned by biogeographic provinces as identified in text, as in Fig. 5. Top row: (a) gross primary production (GPP), (b) net community production (NCP), and (c) the NCP/GPP ratio in the surface mixed layer. Second row: Surface concentrations of (d) total nitrite plus nitrate (e) nitrite, (f) Chlorophyll- $a$ in the $0.2-10 \mu \mathrm{m}$ and (g) $10 \mu \mathrm{m}$ size fractions. Third row: Abundances of (h) cyanobacteria (Prochlorococcus plus Synechococcus), (i) picoeukaryotes, and (j) nano- and microeukaryotes, as well as $(\mathbf{k})$ Shannon-Weaver diversity $(\mathrm{H})$ for phytoplankton community (see text). Final row: export fluxes of (l) particulate organic carbon (POC) and (m) particulate nitrogen (PN) fluxes at $125 \mathrm{~m}$. Panels a, b, c, k, and 1 are replotted from Fig. 5 in the main text for ease of comparison with other properties. 


\section{Supporting information tables}

Table captions are provided here, but tables are not reprinted within the printed thesis because of the large size of these documents. Instead, supporting tables can be found at the MBL/WHOI library website in conjunction with the digital version of this thesis; https://darchive.mblwhoilibrary.org/handle/1912/1172.

Table S1: Isotopic results, including definitions and ancillary properties. A. Equations and definitions used in the calculation of gross primary production from the dissolved triple oxygen isotope measurements. B. Isotopic measurement information and resulting values of gross primary production and net community production for each sample, prior to averaging by location. Isotope and gas ratio definitions are defined in part $\mathrm{A}$ and in the main text.

Table S2: Results summarized by sample location. Mean values and uncertainties of all measured variables at each sample location, as well as number of replicates for each type of sample, $n$.

Biogeographic province acronymns are South Subtropical Transition zone (SST), South Atlantic Gyre (SAG), Western Tropical Atlantic (WTA), and Amazon River Plume (ARP). Variable acronymns are gross primary production (GPP), net community production (NCP), the net community production to gross primary production ratio $(\mathrm{NCP} / \mathrm{GPP})$, Shannon-Weaver diversity $(\mathrm{H})$, particulate organic carbon (POC) flux, and particulate nitrogen (PN) flux, each as defined in the text.

Table S3: Correlation matrix of mean values across transect. Correlation matrix of mean values by location for each variable, with Pearson correlation coefficients ( $r$ ) shown for the upper half only of the symmetric matrix. Variables names and abbreviations are listed in boxed legend. Shaded values are significant values of $r$ at $\alpha=0.05(p<0.05)$. Values of $p$ for Pearson correlation coefficient only are in parentheses for significant correlations. Boxed values are significant $(\mathrm{p}<0.05)$ using the non-parametric Spearman's rank correlation coefficeint $(\rho)$.

Table S4: Correlation matrix of regional mean. Correlation matrix of mean values by region for each variable, with Pearson correlation coefficients ( $r$ ) shown for the upper half only of the symmetric matrix. Variables names and abbreviations are listed in boxed legend. Shaded values are significant values of $r$ at $\alpha=0.05(p<0.05)$. Values of $p$ are in parentheses for significant correlations. There are too few regions to test Spearman's rank based correlation.

Table S5: Correlation matrix of regional standard deviation. Correlation matrix of standard deviation by region for each variable, with Pearson correlation coefficients ( $r$ ) shown for the upper half only of the symmetric matrix. Variables names and abbreviations are listed in boxed legend. Shaded values are significant values of $r$ at $\alpha=0.05(p<0.05)$. Values of $p$ are in parentheses for significant correlations. There are too few regions to test Spearman's rank based correlation. 


\section{Chapter 3}

\section{Evaluating the effects of nutrient addition on whole ecosystem oxygen metabolism in salt marsh tidal creeks}

Some of the data reported in this chapter has been separately analyzed and published as: Kearns, P.J., H.H. Angell, E.M. Howard, L.A. Deegan, R.H.R. Stanley, and J.L. Bowen. 2016. Nutrient enrichment induces dormancy and decreases diversity of active bacteria in salt marsh sediments. Nature Comm. 7: 12881, doi:10.1038/ncomms 12881

\section{Data availability}

Data used in this work are reported in the attached appendices. Additionally, open water oxygen metabolism data and associated advective volume transport are accessible from the LTER Network Information System Data Portal (http://dx.doi.org/10.6073/pasta/fe47a9461bd332fae3ac7792af21c2b0). 


\begin{abstract}
Tidally submerged creek banks in salt marshes host large metabolic fluxes likely driven by benthic microalgae and heterotrophic microbes that mediate exchange of nutrients and terminal metabolic products between the marsh and estuarine water. I compare short-term tidal creek metabolic fluxes derived from oxygen at a tidal creek with nutrients enriched above ambient levels (nutrient amended creek) to metabolic fluxes from an unamended control creek in order to test the effect of long-term nutrient amendment of tidal creek watersheds on the balance of autotrophic and heterotrophic processes on a daily to weekly scale. I present two studies conducted one year apart. In August 2012, I used an open water oxygen mass balance over one to three weeks to estimate net community production (or overnight community respiration; NCP) at similar settings in each creek. Noble gas concentrations were additionally sampled to help constrain gas exchange. In August 2013 sediment gross primary production (GPP), NCP and community respiration fluxes were determined from three midday benthic chamber experiments in the creek bottoms at low tide. The 2013 experiment was at the same site in the nutrient amended creek as in 2012, but at a more shaded site at the unamended control creek. I did not find a significant effect of nutrient amendment on NCP in the tidal creeks over the study periods. In the 2012 open water work, mean daily NCP was $-0.13 \pm 0.04 \mathrm{mmol} \mathrm{O}_{2} \mathrm{~m}^{-2} \mathrm{~min}^{-1}\left(-190 \pm 60 \mathrm{mmol} \mathrm{O}_{2} \mathrm{~m}^{-2} \mathrm{~d}^{-1}\right)$ at both a nutrient amended and unamended control tidal creek. Based on NCP fluxes at just high tide when the creek was is contact with the marsh banks and low platform, both GPP and respiration may have been enhanced in the submerged area in or around the nutrient amended creek, even though on balance these processes did not change mean NCP. During the 2013 benthic chamber experiments, the unamended creek received less light which likely affected relative rates of production between the two creeks. At the unamended creek, NCP fluxes from ambient-light chamber treatments were indistinguishable from respiration in dark treatments, roughly $-0.01 \mathrm{mmol} \mathrm{O}_{2} \mathrm{~m}^{-2} \mathrm{~min}^{-1}$. This respiration rate is much lower than in the open water experiment; very low oxygen concentrations in these chambers may have led to measurement biases and kinetic inhibition of aerobic respiration. At the nutrient amended creek, sediment NCP was $0.06 \pm 0.02$ and $0.05 \pm 0.05 \mathrm{mmol} \mathrm{O}_{2} \mathrm{~m}^{-2} \mathrm{~min}^{-1}$ over two days with high light flux, similar to peak rates from the open water study. Chamber GPP estimated from triple oxygen isotopes was lower than oxygen concentration based NCP; this discrepancy may be explained by oxygen isotope systematics in porewater or complications arising from the interplay of low initial oxygen concentrations in the chambers with high rates of production. Despite this inconsistency, GPP can be compared between the two creeks; based on available nutrient and benthic chlorophyll data, light differences between the two creeks likely played an important role in increasing GPP at the nutrient amended creek relative to the unamended creek. Because light was likely below the saturating irradiance for the benthic community, GPP could be normalized for differences in photosynthetically active radiation over the experimental periods in order to evaluate the magnitude of the effect size for light differences, resulting in 1.7 times greater GPP at the nutrient amended creek than at the unamended creek. This significant difference in GPP between creeks may indicate that GPP increases with nutrient amendment even as the system resists changes to NCP, similar to the results of the open water timeseries. An important conclusion of this work is that triple oxygen isotope ratios and noble gas concentrations are promising tracers of biological and physical processes in salt marshes and estuarine environments, but physical fluxes such as advection play an important role in controlling their systematics. Physical and chemical processes in salt marsh sediments are poorly understood with respect to these tracers, and more work is required to fully unlock the utility of these stable, natural abundance dissolved gases.
\end{abstract}

\title{
Keywords
}

Salt marsh tidal creeks, metabolism, oxygen, triple oxygen isotopes, TIDE project

\section{Acknowledgments}

This work would not have been possible without the outstanding logistical and in kind support of the Plum Island Ecosystems Long Term Ecological Research site and TIDE experiment staff and scientists 
(these projects funded by NSF OCE 1238212, NSF DEB 1354494, and NE Climate Science Center grant DOI G12AC00001). In particular, thanks to James Nelson and Linda Deegan for loading the water quality sondes and boats used in this study. Also, I thank the WHOI Mechanical and Electrical Shops for constructing the benthic chambers used in this study. Additionally, there were numerous undergraduate students, graduate students, and technical staff that assisted in various aspects of this project, and in particular sample collection: Thank you to all my swamplers! This project would not have been possible without the laboratory assistance of Zoë Sandwith at WHOI. Additionally, thanks to Anne Giblin, Amanda Spivak, and my advisor Rachel Stanley for constructive feedback in developing this manuscript. Funding for this research was provided by NSF OCE 1233678 and the WHOI Coastal Ocean Institute. E. Howard was supported by the National Defense Science and Engineering Graduate Fellowship. 


\section{Introduction}

Salt marshes are a dominant coastal environment at temperate latitudes globally, and are important in mediating the flow of organic matter and nutrients between terrestrial and freshwater environments and the coastal ocean (Fagherazzi et al. 2013). A small but significant fraction of the carbon dioxide taken up by high rates of primary production in salt marshes (Valiela 1995) is sequestered from the atmosphere on the scale of hundreds to thousands of years because of its rapid burial as peat (Hopkinson et al. 2012); high rates of organic matter burial make salt marshes a global carbon sink of similar magnitude to tropical forests (60-90 Tg C y-1) despite covering less than 2\% as much surface area (Duarte et al. 2005; Mcleod et al. 2011). High primary production and subsequent peat accumulation in salt marshes provide the basis for a number of ecosystem services. These include physical protection of shoreline from storm surge and erosion, habitat and food for ecologically and economically important species, and filtering of nutrients from coastal waters (Lellis-Dibble et al. 1998; Valiela and Teal 1979; Shepard et al. 2011). However, salt marshes are susceptible to human driven stressors that jeopardize the provision of these ecosystem services (Bromberg Gedan et al. 2009). In particular, increased nitrogen loading from agricultural runoff and wastewater may alter ecosystem level metabolic fluxes such that the marsh becomes less effective at sequestering carbon (Deegan et al. 2012). Organic matter released from the marsh into the coastal ocean is expected to be respired and returned to the atmosphere (Cai 2011; Hopkinson et al. 2012; Duarte et al. 2013).

Anthropogenically driven nutrient enrichment is a global phenomenon that may profoundly alter patterns of salt marsh production. A key question related to how higher nutrient concentrations affect salt marshes is which marsh environments are susceptible to changes in nutrient supply. The importance of salt marsh primary production and resultant storage or export of organic matter to adjacent estuaries has been recognized for decades (Teal 1962), and much subsequent research has focused on the role of the salttolerant grasses that dominate the marsh platform, primarily Spartina alterniflora and Spartina patens in the northeastern United States (Deegan et al. 2012, Morris et al. 2013). Artificial and natural nutrient amendment may in some instances act to increase aboveground macrophyte plant production while simultaneously decreasing belowground production, which could decrease the soil stability of the marsh platform (Turner 2010; Davey et al. 2011). It has been proposed that top-heavy plants are more likely to lodge, or fall over, which can limit production and increase drag from tidal currents contributing to mechanical breakdown of marsh banks (Deegan et al. 2012). Additionally, increased nitrate may stimulate denitrification driven organic matter breakdown in the marsh platform (Koop-Jakobsen and Giblin 2010). The combination of these processes may act to decrease organic matter burial and accelerate mechanical breakdown of marsh sediments, which may contribute to geomorphological change such as marsh platform subsidence and conversion to mudflats (Deegan 2002).

Tidal submergence connects the salt marsh platform and the estuarine source of nutrients, but only on the highest spring tides does this connection extend onto the grass-covered high marsh platform. Instead, it is the tidally filled and drained tidal creeks, the submerged environments, which act as the primary interface between the estuary and the salt marsh. Thus any interpretation of the impacts of increased nutrients on salt marsh metabolism and carbon fluxes is incomplete without addressing the effects on processes at this important interface. In the tidally filled and drained tidal creeks that channel water through the marsh platform, and in mudflats adjacent to the marsh platform, benthic microalgae appear to be the primary producers most intimately associated with the transfer of organic matter and nutrients at the interfaces between the grass-dominated marsh platform and the open water estuarine environments (Tobias et al. 2003, McGlathery et al. 2004, Sand-Jensen and Nielsen 2004). These microbial photosynthesizers, including diatoms, cyanobacteria, and green algae, are known to be important food sources for consumers in salt marsh ecosystems (Galván et al. 2008, 2011, Bowen et al. 2009). Benthic microalgae are known to be nitrogen limited in more porous sediments and a dominant factor controlling estuarine nutrients, so increasing nutrient supply could stimulate their production (Tyler et al. 2003, Engelsen et al. 2008, Sundbäck et al. 2011). However the preferential recycling of sediment ammonia by some benthic 
microalgae communities (particularly in muddy sediments like those found in salt marsh tidal creeks) and the interplay between water column uptake and sediment release can decouple benthic production and water column nutrients (Tobias et al. 2003, Spivak and Ossolinski 2016). Artificial nutrient enrichment has been shown to increase benthic microalgal biomass in some estuarine environments (Cook et al. 2007). In salt marshes and surrounding mudflats, long term nutrient enrichment appears to increase benthic Chlorophyll-a content and may stimulate release of labile organic matter (Bowen et al. 2009, Pascal et al. 2013). While benthic microalgal production is assumed to increase simultaneously with these indirect indicators of growth, very few estimates of production have been made in nutrient enrichment studies in salt marshes (e.g. Van Raalte et al. 1976, Bowen et al. 2009). Further, it is unclear what if any effect altered primary production might have on the net balance of autotrophic and heterotrophic processes. For example, does enhanced production exceed or stimulate community consumption by releasing more labile organic matter? Additionally, other primary producers like macroalgae may have more direct responses to water column nutrients (Peckol and Rivers 1996) than benthic microalgae. Thus community level metabolism fluxes may or may not clearly and directly respond to nutrient enrichment. Given the important role of benthic microalgae as primary producers and limited and sometimes contradictory data regarding the response of microalgal production to fertilization, direct observations of the magnitude and drivers of tidal creek production fluxes are necessary to evaluate how salt marshes metabolism may change with increased nutrient supply.

In this work, I test the hypothesis that nutrient addition to salt marsh tidal creeks at the watershed level enhances creek primary production and alters the net metabolic balance between autotrophic and heterotrophic processes. I present two studies, each comparing ecosystem metabolic fluxes in two salt marsh tidal creeks adjacent to the Plum Island Sound estuary, Massachussets, USA. One creek is a control and the other the experimental treatment in part of a decade long nutrient amendment experiment meant to simulate moderately to highly nutrient enriched conditions at the watershed level (see 3 Study location; Deegan et al. 2007). The first study presented here, conducted in 2012, employs an open-water mass balance approach (Vallino et al. 2005; Nidzieko et al. 2014 ), hereafter referred to as the 'open water (2012)' study, while the second study, conducted in 2013, employs a benthic chamber flux technique (Hopkinson 1999; Jahnke et al. 2000; Cebrian et al. 2007), hereafter referred to as the 'benthic chamber experiments (2013)'. The open water (2012) experiment integrates all physical and biological fluxes in the system; the more complex fluxes are not always well defined. The benthic chamber experiments (2013) isolate sediment fluxes from most water transport and gas exchange processes. This simplifies the system at the expense of altering the physical and chemical environment experienced by the primary producers, and potentially altering the metabolic fluxes being measured. In addition, the benthic chambers integrate over a small and not necessarily representative patch of the heterogeneous environment.

In each experiment, I evaluate the net community production (NCP), the oxygen evolved (or carbon fixed) in excess of biological oxygen consumption. NCP is a measure of the metabolic status of the system, where positive NCP indicates net autotrophy and organic matter production, and negative NCP indicates that oxygen consumption exceeds local photosynthesis. The oxygen demand of the system depends on oxygen consumption both by aerobic respiration and oxidation of other terminal metabolic products in the sediments. These sinks ultimately reflect biological fluxes, and I refer to the combined oxygen consumption as community respiration. For the benthic chamber experiments (2013), I also present estimates of gross primary production (GPP), the total photosynthetic production prior to any respiration, using the novel triple oxygen isotope tracer. This paper is organized as follows: a background section (section 2 ) examines in detail the tracers used to evaluate NCP, community respiration, and GPP. The study site and watershed scale experimental treatment are then described (3). The experimental design and methods, results, and discussion are then treated separately for each of the two studies (sections 4-6 and 7-9, respectively for the 2012 and 2013 studies). I conclude (10) with a synthesis of the two studies and discussion of how they inform our understanding of nutrient effects on tidal creek metabolic fluxes. 


\section{Background}

A number of in situ and in vitro techniques are used to estimate net community production, gross primary production, and community respiration. These techniques generally fall into three major categories: measurements of Chlorophyll-a combined with model of production dependence on environmental factors like irradiance (MacIntyre et al. 1996, Kromkamp et al. 2006, Brotas et al. 2007), measurements of oxygen $\left(\mathrm{O}_{2}\right)$ concentration (Odum 1956; Revsbech and Jørgensen 1983; Berg et al. 2003; Vallino et al. 2005), and isotope uptake experiments (Dring and Jewson 1982; Williams and Lefèvre 1996; Gazeau et al. 2007; Yacobi et al. 2007). Each approach has its strengths and weaknesses, but in the interests of brevity I only describe in detail the in situ dissolved gas tracers and techniques used in this work. In addition, the biologically inert noble gases may provide in situ tracers of water transport and diffusive gas exchange in this system, and I describe the theory and use of these tracers.

2.1 Oxygen concentration based net community production and community respiration: In both the open water (2012) and benthic chamber experiment (2013) studies, I evaluate changes in oxygen concentration with time to derive rates of NCP in the light when photosynthesis is occurring, and community respiration in the dark. Dark, or mitochondrial, respiration may be lower than respiration in sunlit sediments because of light enhanced mitochondrial respiration, photorespiration, and photochemical oxidation (eg Fenchel and Glud 2000). Because of the lack of a direct tracer for respiration separate from production, most authors assume that respiration in the light equals respiration in the dark, but careful experimentation reveals that light respiration may exceed dark respiration by factors of 1.5 to 6.5 in water column and benthic marine systems (Bender et al. 1987; Fenchel and Glud 2000; Pringault et al. 2007). If the NCP and community respiration rates from these techniques are well constrained, GPP can be estimated by addition (GPP $=\mathrm{NCP}+$ community respiration). In the open water (2012) study, in situ oxygen concentrations and a tidally varying water budget are used to build a gas exchange dependent, non-steady state mass balance from which metabolic fluxes are isolated (sensu Nidzieko et al. 2014). In the benthic chamber experiments (2013) the oxygen concentration rates of change with time in each chamber are interpreted directly as metabolic rates, with some adjustments for entrainment of ambient creek water when withdrawing samples.

2.2 Triple oxygen isotope ratios and gross primary production: Natural abundance ratios of dissolved triple oxygen isotopes $\left({ }^{16} \mathrm{O},{ }^{17} \mathrm{O}\right.$, and $\left.{ }^{18} \mathrm{O}\right)$ were sampled in both studies. These ratios are sensitive to the source of dissolved oxygen, and can be used to determine GPP directly. This method has been applied in marine environments for more than a decade (Luz and Barkan 2000; Juranek and Quay 2013 and references therein), but is not typically used in estuarine systems. This technique combines some of the strengths of the isotopic and oxygen concentration based methods - it provides a direct measurement of photosynthetic rate, and, when used in situ, integrates over large spatial scales. At the same time, it does not require an artificial isotope spike but relies on natural abundance stable isotopes, and requires no assumptions regarding the relative rates of light and dark respiration so long as the isotopic fractionation associated with those processes is identical. The triple oxygen isotope method is based on the different isotopic signatures of oxygen that is added to a body of water via gas exchange versus that generated in situ during photosynthesis. Atmospheric oxygen has a distinct ratio of stable oxygen isotopes ${ }^{16} \mathrm{O}:{ }^{17} \mathrm{O}:{ }^{18} \mathrm{O}$ set by mass independent fractionation during dissociation of ozone in the stratosphere (Thiemens and Heidenreich 1983; Thiemens et al. 2012). Photosynthesis fractionates seawater in a mass dependent manner and generates oxygen with an isotopic composition similar to that of the water it was produced from (Luz and Barkan 2000; Barkan and Luz 2011).

All measured aerobic respiration pathways have mass dependent fractionation with a nearly constant ratio of ${ }^{17} \mathrm{O}:{ }^{16} \mathrm{O}$ to ${ }^{18} \mathrm{O}:{ }^{16} \mathrm{O}$ isotope enrichment factors (Angert et al. 2003; Luz and Barkan 2005, 2009). The constancy of the ratio between the respiration enrichment factors (notated as $\gamma_{R}$ and equal to $0.5179 \pm 0.006)$ means that the oxygen isotopes can be related in such a way that respiration effects cancel 
out. The combined isotope signal for any given sample of dissolved oxygen can be defined after Angert et al. (2003) as:

$$
{ }^{17} \Delta=\ln \left(\frac{{ }^{17} O /{ }^{16} O_{\text {sample }}}{{ }^{17} O /{ }^{16} O_{\text {air }}}\right)-\gamma_{R} \ln \left(\frac{{ }^{18} O /{ }^{16} O_{\text {sample }}}{{ }^{18} O /{ }^{16} O_{\text {air }}}\right)
$$

${ }^{17} \Delta$ depends primarily on the source of the dissolved oxygen (Fig. 1), i.e. atmospheric or photosynthetic oxygen, and is a useful way of visualizing the relationship between the dissolved isotope ratios and endmembers. GPP can be solved for using an independent estimate of the gas exchange flux into the water. Or, in the absence of gas exchange, GPP can be evaluated directly from the time evolution of the isotope ratios.

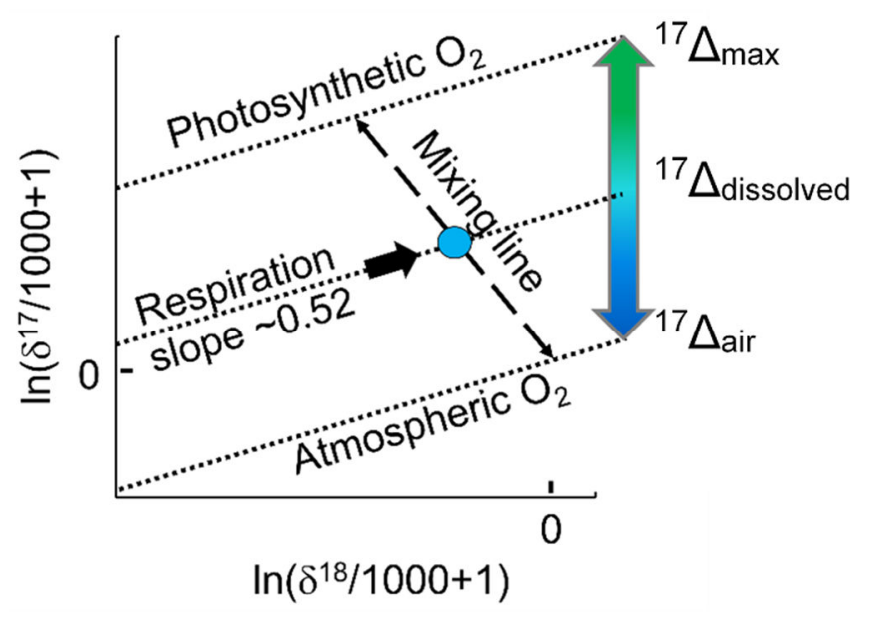

Figure 1: Triple oxygen isotope systematics for dissolved oxygen, after Juranek and Quay (2013). ${ }^{17} \Delta_{\text {dissolved }}$ is the water value, which falls along a mixing line between ${ }^{17} \Delta_{\text {air }}$, the atmospheric endmember, and ${ }^{17} \Delta_{\max }$ the expected photosynthetic endmember for a given system. Respiration along the slope $\gamma_{R}=0.5179 \pm 0.006$ does not change the relative relationship of the oxygen isotope ratios, plotted here in standard isotope notation $\left(\delta^{x}=\left({ }^{x} \mathrm{O}:{ }^{16} \mathrm{O}_{\text {sample }} /{ }^{x} \mathrm{O}:{ }^{16} \mathrm{O}_{\text {standard }}-1\right)\right)$.

A direct, natural abundance measure of GPP provides improvements over existing techniques, but the triple oxygen isotope method has weaknesses as well. Sample processing is technically challenging, and requires a minimum quantity of total dissolved oxygen in the sample for precise and accurate measurement in the isotope ratio mass spectrometer. Like all oxygen based methods, it cannot constrain chemoautotrophy and is sensitive to physical processes like advection. Temporal resolution is limited by discrete sampling. Finally, production must be moderately high to minimize uncertainties, yet not so high that nearly all oxygen in a study system is photosynthetic in origin and the tracer becomes insensitive to subsequent production (see 8 Discussion: Part 2). Despite these limitations, the triple oxygen isotope method provides a useful and novel tool for evaluating primary production in salt marsh tidal creeks.

2.3 Noble gas concentrations as tracers of gas exchange properties: The stable noble gases (He, Ne, Ar, $\mathrm{Kr}, \mathrm{Xe}$ ) are in situ, natural abundance tracers of physical transport of water and gas exchange. Because they have varying temperature dependencies of solubility and diffusion rates (Wood and Caputi 1966; Weiss 1971, Weiss and Kyser 1978; Hamme and Emerson 2004), it is possible to simultaneously solve mass balances for several gases at once and evaluate diffusive gas exchange and bubble processes (Spitzer and Jenkins 1989; Hamme and Emerson 2006; Stanley et al. 2009a). He and Ne are low solubility and particularly sensitive to bubble processes, $\mathrm{Kr}$ and $\mathrm{Xe}$ are more soluble and have the largest temperature dependencies, while Ar has intermediate solubility and very similar solubility and molecular 
diffusion rates to oxygen. The noble gas approach has advantages over traditional gas exchange estimates and could be particularly useful in salt marsh tidal creeks: Unlike wind speed parameterizations (e.g. Wanninkhof 1992), noble gas based estimates are sensitive to site specific conditions, including the effects of precipitation, surfactant films, and bottom-generated turbulence (Raymond and Cole 2001; Harrison et al. 2012). Sampling does not alter turbulence at the air-water interface, a drawback of measurements from floating dome studies, and tracer dispersal does not limit sampling period or location as with deliberate tracer releases (Abril et al 2009; Ho et al 2011). The noble gas method has drawbacks as well, including uncertainty in the solubility of $\mathrm{Kr}$ and $\mathrm{Xe}$ and potentially large sampling errors caused by small bubbles or air leaks in the samples. Because the noble gases are sensitive to water transport and mixing, accuracy is limited by how well the water fluxes and concentration gradients are constrained. Finally, the processes controlling noble gas systematics in sediment porewaters and their exchange with creek water are not well understood in salt marsh tidal creeks. Nonetheless, the noble gases provide additional constraints on gas exchange that are useful in evaluating site specific gas exchange for calculating NCP and GPP in the open water (2012) study.

\section{Study location}

The Plum Island Sound estuary, located in Northeastern Massachusetts, USA, is roughly $60 \mathrm{~km}^{2}$, with two-thirds of that area covered by tidal salt marsh (Wilson et al. 2014). The Parker River bounds the northern end of the estuary, while the Ipswich River enters the estuary to the south where the estuary empties into the Gulf of Maine (Fig. 2). The salt marshes surrounding the tidally dominated Rowley River, located midway up the estuary, are the focus of a number of studies of salt marsh biogeochemistry as part of the Plum Island Ecosystems Long Term Ecological Reserve site (pie-lter.ecosystems.mbl.edu). In particular, the Trophic cascades and Interacting control processes in a Detritus-based aquatic Ecosystem (TIDE; mbl.edu/tide) experiment at the site focuses on the effects of chronic nutrient addition on biogeochemical fluxes and community structure in tidal creek drainages (e.g. Koop-Jacobsen and Giblin 2009; Viellard et al 2011; Deegan et al. 2012).

The experiments in this work were located in Sweeney and West Creeks, primary tidal creeks $\sim 2 \mathrm{~km}$ apart that eventually merge to tertiary creeks that branch from the Rowley River (Fig. 2). These creeks are paired as part of the TIDE experiment (Deegan et al. 2007, 2009), with Sweeney amended to receive $\sim 70$ $\mu \mathrm{M}$ nitrate throughout the flooding tide over the last ten years, and West acting as an unamended control with dissolved nitrate concentrations of $\sim 5 \mu \mathrm{M}$. The tidal creeks (hereafter referred to as nutrient amended and unamended) have no permanent freshwater source, and salinity is expected to be controlled primarily by tidally driven flooding of brackish estuary water from the Rowley River. At the outset of the nutrient amendment study, paired creeks and surrounding marsh were selected for similarity in geomorphology and water column chemistry - additional details of the site comparison and TIDE methods can be found in Deegan et al. (2007). At the sample locations in the open water (2012) study, the creeks are 2-4 m across, with muddy bottoms incised by a few centimeter deep, permanently flooded central channel. Steeply sloping banks $\sim 2.5$ tall are capped by 2-3 m wide bands of tall-form Spartina alterniflora in the top third of the tidal range. Beyond is a high marsh platform dominated by Spartina patens which floods to several $\mathrm{cm}$ depth near Spring tides. Artificial ditches incise the marsh platform and drain into both tidal creeks every 10-20 m. The benthic chamber experiments (2013) in the unamended creek were undertaken in a different primary creek branch from the 2012 study (Fig. 2). At this location, the creek width varied from 1-3 m across along the length used in the experiments and with more steeply sloping banks than the 2012 site. 


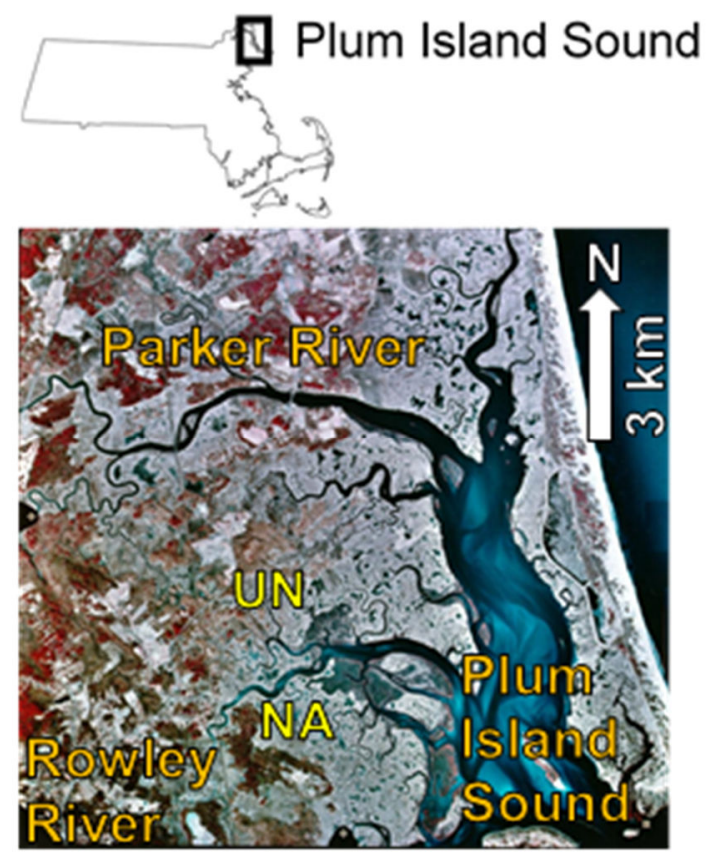

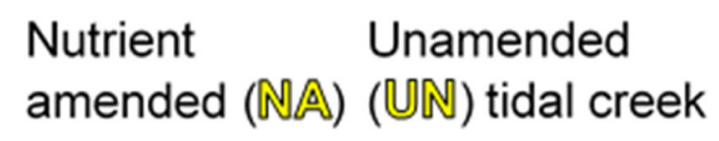
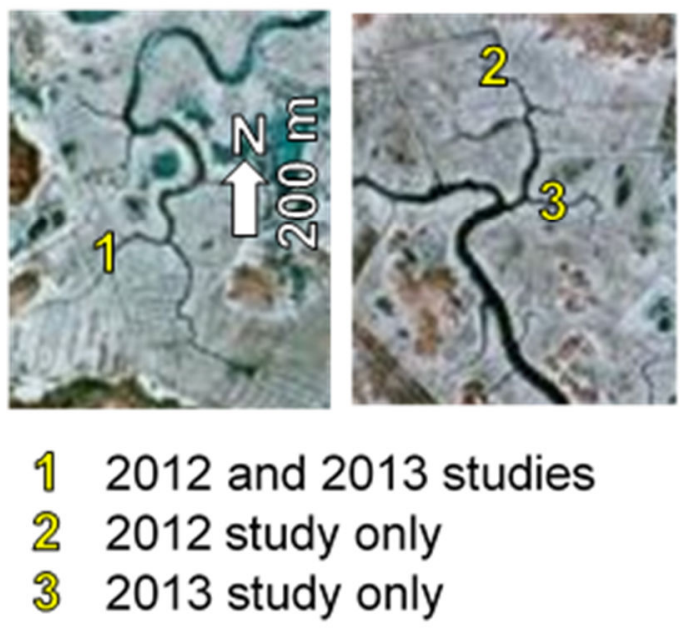

Figure 2: Plum Island Sound estuary and tidal creek study sites (nutrient amended, NA, and unamended control, UN). The two tidal creeks are roughly $2 \mathrm{~km}$ apart. The 2013 benthic chamber study at the unamended creek was located on a different primary creek branch of the unamended creek than the 2012 open water study. The open water study and benthic chamber experiments were collocated at the nutrient amended tidal creek. Images from USGS National Aerial Photographic Program courtesy of Plum Island Ecosystems Long Term Ecological Reserve site (pie-lter.ecosystems.mbl.edu).

\section{Experimental methods: Part 1, water column (2012)}

The 2012 experiment was focused on determining in situ metabolic fluxes in the tidal creek water columns, without experimental manipulations beyond the TIDE experiment's whole watershed nutrient amendment. The nutrient amended and unamended creeks were instrumented with hydrographic sensors in July and August 2012, and over the deployment period discrete water samples were also obtained. Descriptions of the experimental methods and sample analyses follow.

4. 1 Deployed equipment: Sensors deployed at each creek included YSI TM 6 series water property sondes, upward facing acoustic Doppler velocimeters (ADVs), and Onset ${ }^{\circledR}$ HOBO pressure loggers. Relative locations of deployed equipment are diagrammed in Appendix I: Fig. A1. The sondes measured and recorded water temperature, conductivity, and dissolved oxygen saturation state using a Clark type polarographic electrode with a nominal accuracy of $2 \%$. However, such sensors may systematically underestimate oxygen saturation state up to $4 \%$ near saturation, perhaps because of $\mathrm{O}_{2}$ consumption by the electrode (Glazer et al. 2004). The sondes at the nutrient amended and unamended creeks were respectively secured on the creek bottom 115 and $129 \mathrm{~m}$ upstream from the nearest confluence with another primary creek. An ADV was placed $10 \mathrm{~m}$ downstream of the water property sonde at the amended creek, but $100 \mathrm{~m}$ downstream at the unamended creek for use with another experiment. Several acoustic Doppler current profiler sections were made with a Sontek ${ }^{\circledR}$ RiverSurveyor in October 2012 in order to calibrate the ADV data to water flux through each creek at the sample sites. The water pressure loggers were mounted $2 \mathrm{~cm}$ above the bottom adjacent to the sondes. 
4.2 Water sampling: Discrete water samples of dissolved oxygen isotopes were collected in replicate simultaneously at both creeks over 3-5 days in both July and August. Samples were collected at 110 and $124 \mathrm{~m}$ upstream from the nearest confluence with another creek, $5 \mathrm{~m}$ downstream from the sonde locations. Additional samples were collected over 3 days in late October at the unamended creek only, after seasonal fertilization had ended in the nutrient amended creek. Samples were collected from $10 \mathrm{~cm}$ below the creek surface over five timepoints spanning the ebbing tides only. Oxygen concentrations, temperature, and salinity were measured at the same depth for comparison with the sondes. Noble gas concentration were collected during two days at each creek in July and August 2012. An additional transect along each creek was sampled for noble gases in late October, 2013, in order to evaluate alongcreek gradients. Porewater samples from the tall-form Spartina alterniflora platform adjacent to the unamended creek were collected at high tide in late October, 2014. Sample collection and analysis for the measured properties are described next.

4.3 Photosynthetically active radiation (PAR) and windspeed: 15 minute averages of PAR flux at 400$700 \mathrm{~nm}$ and windspeed at $3 \mathrm{~m}$ height were obtained from a weather station located approximately $5 \mathrm{~km}$ northwest of the creeks and maintained by the Long Term Ecological Reserve (http://www.pielter.org/Weather/). PAR and windspeed were not evaluated separately for each sample site in 2012, but inter-creek variability was observed during sampling.

4.4 Temperature, salinity, and dissolved oxygen concentration: Water temperature, conductivity, and dissolved oxygen saturation state were measured every 10 minutes by the water property sondes. Each sonde was calibrated by Long Term Ecological Reserve personnel prior to deployment. Temperature and derived salinity are used with the solubility function of oxygen (Garcia and Gordon 1992, 1993) to derive oxygen concentration. At each discrete sample absolute salinity $\left(\mathrm{S}_{\mathrm{A}}\right.$; Millero et al. 2008) of sampled water at each timepoint was measured on handheld salinity refractometers with accuracy and precision of $2 \pm 1 \mathrm{~g}$ salt $/ \mathrm{kg}$ seawater, and temperature was checked using a handheld thermometer with accuracy and precision of $0.2+/-0.4{ }^{\circ} \mathrm{C}$, similar to that of the sondes. For one creek each sampling day, in situ oxygen saturation state at $10 \mathrm{~cm}$ below the surface was measured with an optical dissolved oxygen sensor (YSI TM ProODO, accuracy and precision $1 \pm 0.1 \%$ of air saturation). These measurements were used to correct the sonde oxygen time-series for a small, linear drift in sensor response with time. No significant offsets were found in the weighted residuals between the sondes and handheld sensor following the drift correction.

4.5 Acoustic Doppler velocimeters $(A D V S)$ and pressure loggers: Nortek ${ }^{\circledR}$ and Sontek ${ }^{\circledR}$ ADVs were deployed at the nutrient amended and unamended creeks, respectively. ADV principles of operation in stream and marsh settings are described in the literature (eg. Morlock et al. 2002; Ganju et al. 2005; Stevens et al. 2007); in brief these instruments exploit the Doppler shift of acoustic pulses which reflect off particles in the water to determine velocity at a single point within the channel. Care was not taken prior to deployment to synchronize the settings of the two instruments or optimize the number of velocity cells, sampling bounds, and signal to noise thresholds for the local creek environments, limiting the utility of these data (see Results: Part 1). Data were processed into average along-creek velocity vectors for every 10 minutes of deployment and quality assessed. Pressure logger data were corrected to account for sensor height above creek bottom, and converted to water depth using sonde temperature and salinity and functions from the Gibbs SeaWater toolbox of the Thermodynamic Equation of Seawater 2010 (www.teos-10.org), in MATLAB (Mathworks, release 2014a).

4.6 Triple oxygen isotope ratios and ${ }^{17} 4$ : The triple oxygen isotope ratios of ${ }^{16} \mathrm{O},{ }^{17} \mathrm{O}$, and ${ }^{18} \mathrm{O}$ in dissolved oxygen were sampled in pre-poisoned, evacuated glass flasks. These flasks were filled with $200-400 \mathrm{~mL}$ of water while submerged, with care taken to exclude bubble and debris. The temperature equilibrated headspace from these samples was run through an automated processing line which separates and removes most of the $\mathrm{N}_{2}$ (Stanley and Howard 2013). The processed headspace was then inlet to a Finnigan TM MAT 253 isotope ratio mass spectrometer where the ${ }^{17} \mathrm{O}:{ }^{16} \mathrm{O}$ and ${ }^{18} \mathrm{O}:{ }^{16} \mathrm{O}$ ratios were measured (Barkan and Luz 2003). Corrections were applied for pressure and matrix effects from Ar and 
any remaining $\mathrm{N}_{2}$ (Abe and Yoshida 2003) and for size differences between sample and standard (Stanley et al., 2010), and the resulting ratios reported as $\delta^{17}$ and $\delta^{18}$, where the $\delta$ value of the heavy isotope $x$ relative to the more abundant ${ }^{16} \mathrm{O}$ is defined in standard isotope notation as $\delta^{x}=\left({ }^{x} \mathrm{O}:{ }^{16} \mathrm{O}\right.$ sample $/{ }^{x} \mathrm{O}:{ }^{16} \mathrm{O}$ standard -1). All samples were referenced to oxygen in sea-level air.

The precise relationship between the triple oxygen isotope ratios is critically important to the accurate calculation of gross oxygen production, more so than the exact value of either $\delta^{17}$ and $\delta^{18} \cdot{ }^{17} \Delta$ can be measured more precisely than the absolute values of $\delta^{17}$ or $\delta^{18}$ by isotope ratio mass spectrometry. For example, the duplicate precision of the samples in the benthic chamber experiments (2013) was ${ }^{17} \Delta \pm 6 \mathrm{x}$ $10^{-6}$, or 6 per meg, and $\delta^{18} \pm 2 \times 10^{-4}$, or 0.2 per mil $\left(\delta^{17}\right.$ has similar precision). Therefore ${ }^{17} \Delta$ can be combined with the isotope ratio of one of the two rare isotopes to precisely determine the remaining isotope ratio with greater precision than it can be measured, preserving the specific mass independent signature of the triple oxygen isotopes if $\delta^{17}$ and $\delta^{18}$ are used to calculate oxygen production. ${ }^{17} \Delta$ was measured directly, and was corrected for pressure and matrix affects in the same manner as the individual isotope ratios. However, multiple definitions of ${ }^{17} \Delta$ have been presented (Kaiser 2011); care must be taken to use consistent definitions and constants as the results of this approach are necessarily dependent on the definition of ${ }^{17} \Delta$. In both the 2012 and 2013 experiments $\delta^{18}$ was measured with slightly greater precision than $\delta^{17}$; thus the measured $\delta^{18}$ and ${ }^{17} \Delta$ were used to calculate an inferred value of $\delta^{17}$.

4.7 Noble gas concentrations: Dissolved He, $\mathrm{Ne}, \mathrm{Ar}, \mathrm{Kr}$, and $\mathrm{Xe}$ were sampled on a subset of days and timepoints by hand pumping water through a short length of Tygon ${ }^{\circledR}$ tubing connected to a $\sim 25 \mathrm{~cm}$ length of copper tube. The tubing was knocked during pumping to dislodge any bubbles, and clamped shut at each end to seal the sample. The October 2014 porewater samples were obtained using the porewater extraction apparatus and peristaltic pump system described in Koop-Jakobsen and Giblin (2009). Noble gas concentrations are measured on the extraction line and quadrupole mass spectrometer described in Stanley et al. (2009b), and saturation concentration were calculated using the sonde temperatures and derived salinities based on published solubility coefficients (Xe, Wood and Caputi 1966; He, Weiss, 1971; Kr, Weiss and Kyser 1978; Ne, Ar, Hamme and Emerson 2004).

5 Results: Part 1, water column (2012)

Water depth, salinity, and dissolved oxygen concentration at each creek over the instrument deployment period in 2012 are presented in Fig. 3. PAR flux from the offsite meteorological tower is also plotted identically for both creeks. At the nutrient amended creek, the sonde was buried by a collapsing bank roughly a week into the deployment, and only the truncated record for which all properties are available is plotted. Water depth varied with tidal forcing, and had slightly different timing and magnitude at each site because the channel morphology and distance from the Rowley River were not identical. Oxygen and water mass balance results were assigned a digital object identifier (doi:10.6073/pasta/fe47a9461bd332fae3ac7792af21c2b0) and are available through the Long Term Ecological Reserve data repository (https://pasta.Iternet.edu/package/eml/knb-lter-pie/415/1)

5.1 Salinity: Salinity generally tracked the tide in both creeks, with peak salinity associated with the inflow of estuarine water ( $\mathrm{S} \sim 31$ in Gulf of Maine near estuary mouth) at high tide and lowest salinity at low tide when the flux of porewater from the adjacent marsh platform is likely to be highest (eg. Wilson and Morris, 2012). Interestingly, the nutrient amended creek had a larger range of salinity over the tidal cycle ( $\Delta \mathrm{S} \sim 6$, vs. 3 at the unamended creek) and a more rapid freshening over the low tide period. This suggests increased freshwater input or accumulation relative to bulk creek water compared to the unamended creek, at least near the instrumented locations. In the longer unamended creek record, the high tide salinity was relatively stable throughout the spring-neap cycle (peak tidal range near August 1, and minimum tidal range near August 11,2012). $\Delta \mathrm{S}$ over the tidal cycle was generally smaller when there was less variation in tidal height. The large decrease in creek salinity on Aug 10 was associated with $\sim 2$ 
$\mathrm{cm}$ of precipitation (data not shown). No appreciable precipitation fell over the rest of the instrument record; the slow rebound of salinity towards values prior to the rain event indicated that the residence time of porewater draining into the creek from the marsh surface was on the order of several days. Based on the October 2013 sampling transects, temperature and salinity were relatively homogenous along the length of the creeks.

5.2 Dissolved oxygen concentration: Oxygen $\left(\mathrm{O}_{2}\right)$ concentration varied between $\sim 25-275 \mu \mathrm{mol} \mathrm{kg}^{-1}(\sim 10-$ $120 \%$ of saturation with respect to air), and did not closely track PAR as would be expected if biological production and respiration was the primary control on tidal creek oxygen abundance (Fig. 3). Oxygen concentrations in each creek are plotted against water depth, PAR flux, and time of day in Fig. 4. A single lunar day ( 25 hours) with an initial low tide at midnight and two complete tidal cycles is overlaid in color on each panel in order to highlight how oxygen concentrations do or don't vary with tidal stage, light, and time of day; the purpose of this approach is to identify which processes (tides, daily light flux) $\mathrm{O}_{2}$ concentration may be linked to and whether concentration changes follow tidal or light periodicity. Daytime and nighttime high tides were associated with increased oxygen concentration, as were some (primarily daytime) low tides. Creek $\mathrm{O}_{2}$ is not correlated with instantaneous PAR flux; the relationship between the two properties at any given tidal stage was mediated by tidal movement of water parcels between the estuary and the tidal creeks. The combined influence of tidal transport and light is illustrated in the panels plotting dissolved oxygen against time of day; tidal advection causes large increases in $\mathrm{O}_{2}$ regardless of light, but there are some increases of oxygen at midday that do not follow the tidal cycle. While following similar trends with respect to tidal stage, oxygen concentrations were variable between the amended and unamended creeks. In particular, the lowest oxygen concentrations in the nutrient amended creek were $\sim 25-50 \mu \mathrm{mol} \mathrm{kg}{ }^{-1}$ lower than in the unamended creek (lowest concentrations in plotted gray dots in any pair of panels, also refer to simultaneous $\mathrm{O}_{2}$ timeseries in Fig. 3); this difference was likely caused by real differences in the biological or physical cycling of oxygen between the creeks rather than a calibration error between the instruments.

5.3 Acoustic Doppler velocimeters (ADVs): ADV results from these particular deployments were unsatisfactory for quantitative evaluation of advective fluxes in both creeks. The signal to noise ratio was very low $(\sim 1)$ at water depths less than half the tidal depth, because of the constrained volume away from the creek bed from which to draw observations; increased friction and turbulence near the edges of creeks complicated interpretation of ADV data, and reflections from banks or other hard objects are known to cause very large biases in acoustic data (Morlock et al. 2002; Stevens et al. 2007). These complications were also present at higher tidal stages, but in addition the vegetated banks dramatically altered the channel hydrodynamics (Nepf 2012). ADVs are typically deployed in unvegetated stretches of channel $>10 \mathrm{~m}$ across and well away from banks in marsh settings (eg. Voulgaris and Meyers 2004; Ganju et al. 2005). When the signal was significant, point velocity data at the center of the channel was on average 45 times greater than expected values based on the modeled volume change as well as acoustic Doppler current profiler (ADCP) measurements $100 \mathrm{~m}$ farther downstream in 2006 (Luciano 2007), which indicates that increased friction and reduced flow near creek edges must play a large role compared to flow through the center of the channel. In short, the choice of deployment location at the amended creek precluded quality data collection during this experiment using the available equipment.

The instrument at the unamended creek was expected to have fewer issues with these complications, but again data quality was poor through much of the tidal cycle. Because this instrument was deployed $100 \mathrm{~m}$ from the experiment site at the unamended creek, the data needed to be calibrated to flow at that site. However, ADCP calibration transects at the sample site did not achieve acceptable results with the four repeat transects performed at each of three tidal stages. The very low signal to noise ratio and high reflectance spikes in the data indicate that the ADCP experienced the same problems as the ADV in the narrower portion of the creek. Therefore, the best remaining option for calculating water fluxes with the data at hand was a tidal stage to creek volume relationship (volume change method, Ganju et al. 2012) that is not calibrated to any direct estimates of water flux (see 6 Discussion: Part 1). 

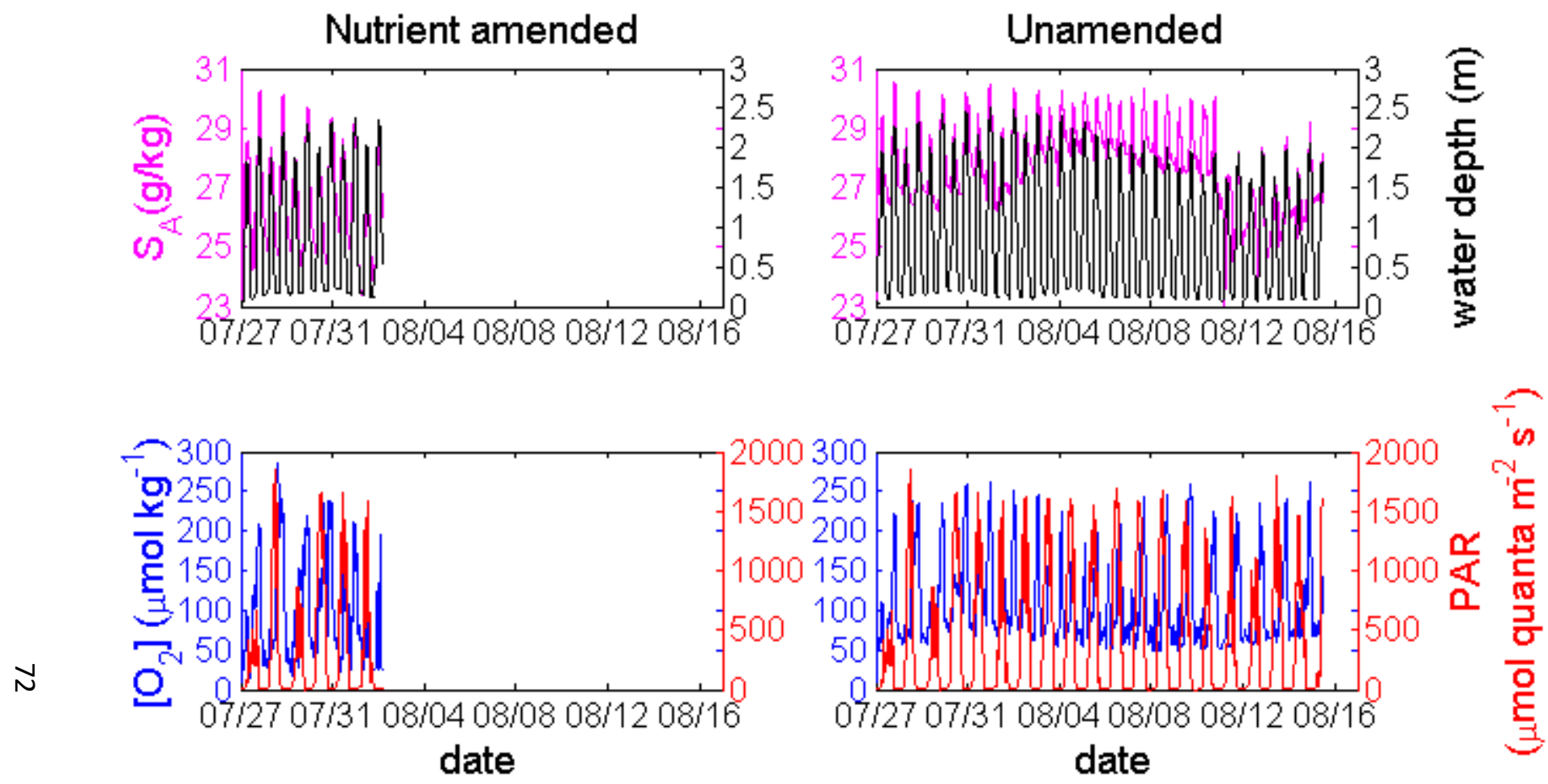

Figure 3: Absolute salinity $\left(\mathrm{S}_{\mathrm{A}}\right)$ and tidal creek water depth over time series (top panels), in nutrient amended and unamended control tidal creeks. Oxygen concentration and photosynthetically active radiation (PAR) flux over time series (bottom panels). PAR record is from weather station $\sim 5$ $\mathrm{km}$ northwest of study sites, and is not evaluated separately at each site. 

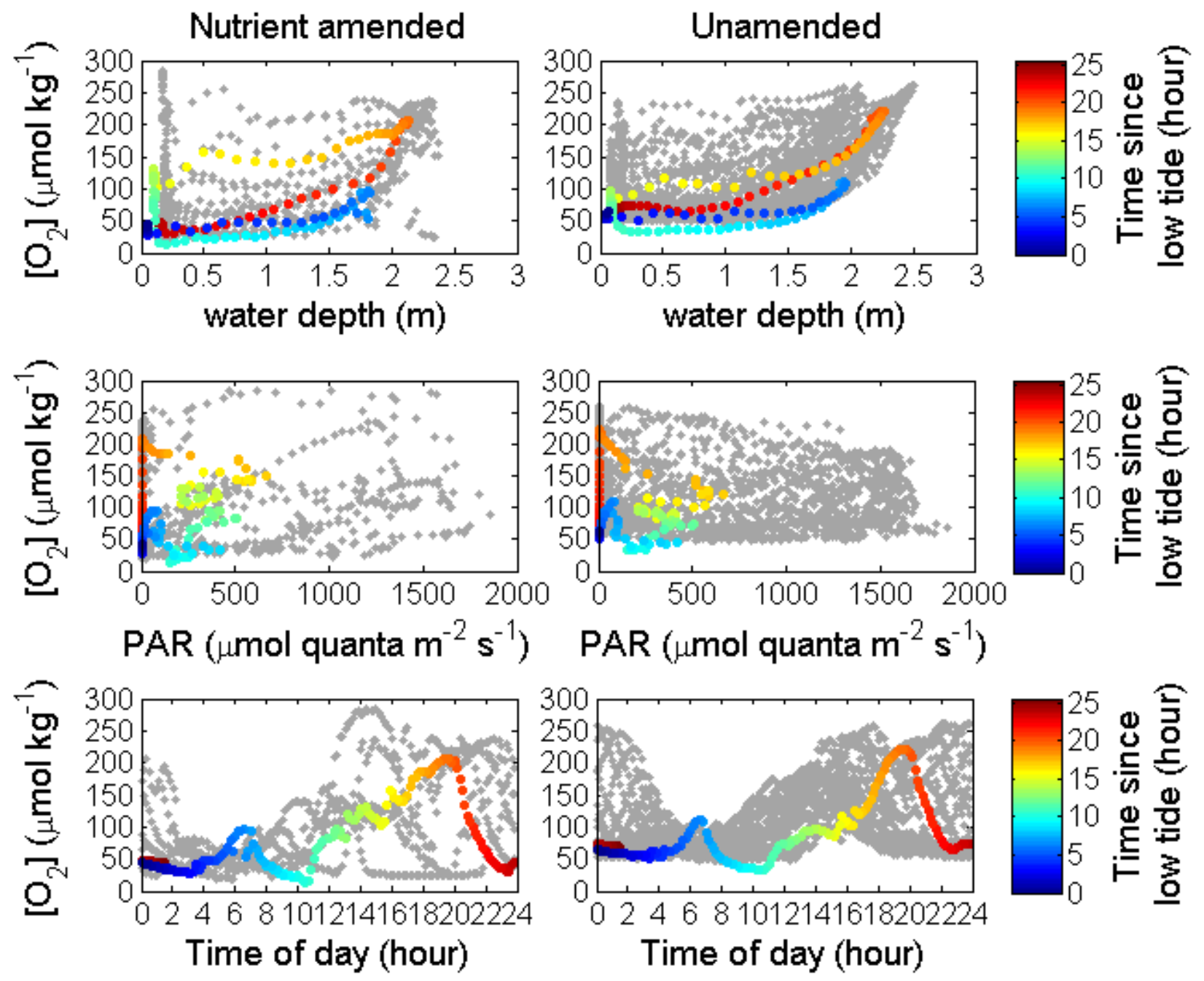

Figure 4: Oxygen concentration for the nutrient amended and unamended control tidal creeks plotted against tidal creek water depth (top panels), photosynthetically active radiation (PAR) flux, (middle panels), and time of day (bottom panels). Time series includes three times more data points at unamended creek. The first lunar day ( 25 hours, 2 complete tidal cycles) is overlaid in color, and begins at midnight local time. 
5.4 Triple oxygen isotopes: Dissolved triple oxygen isotopic ratios from the 2012 experiments were analyzed prior to the development of the low-oxygen methods described in section 7 Experimental methods: Part 2 for the 2013 experiment. After applying to these samples the same filtering criteria which was applied to samples from the 2013 experiment, oxygen isotope samples from only four, noncontiguous timepoints from four separate days had likely acceptable data quality for analysis (Fig. 5). Therefore, no further analysis of triple oxygen isotopic ratios or derived production rates is presented in this work.

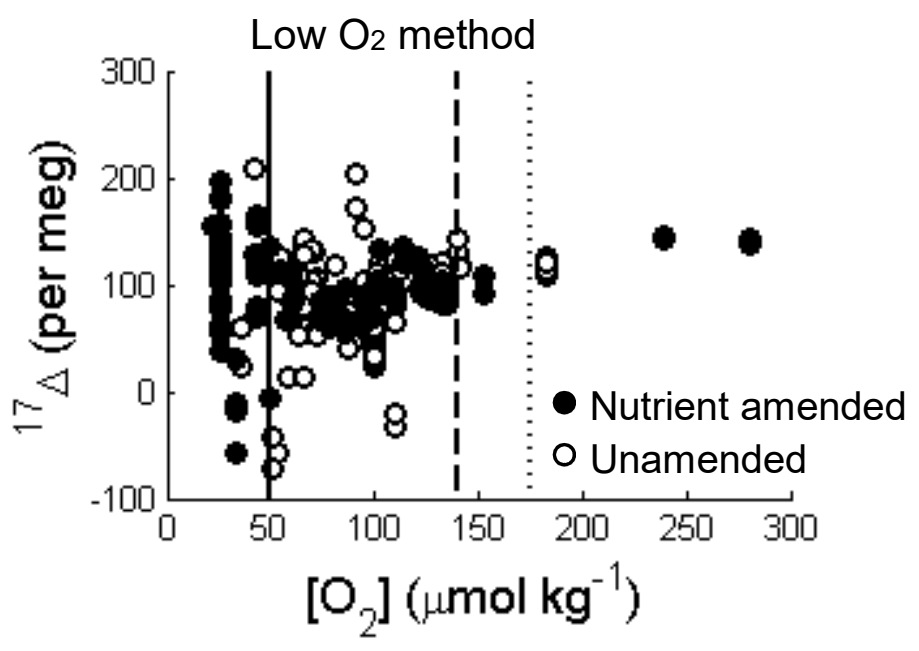

Figure 5: Triple oxygen isotope $\operatorname{tracer}{ }^{17} \Delta$ for all samples from the open water (2012) study. Samples with oxygen concentration greater than the dashed line can be run with accuracy greater than the change in ${ }^{17} \Delta$ between timepoints using the standard methods described in Experimental methods: Part 1 for the 2012 study. Samples with oxygen concentrations between the solid and dotted lines can be run with high accuracy and precision using the low-oxygen method described in Experimental methods: Part 2 for the 2013 study. All samples from the 2012 study were run using standard methods, and only samples from four timepoints on separate days have acceptable accuracy.

5.5 Noble gas concentrations: Noble gas results are presented in Table A1. Concentrations are reported

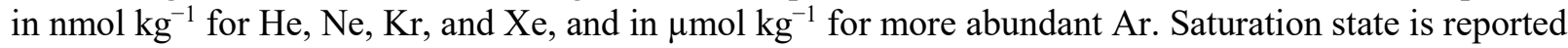
as percent of the expected saturation concentration in equilibrium with 1 atmosphere pressure at the in situ temperature and salinity. The reported uncertainties are likely lower bounds because of the smaller sample size and less robust seal used with these samples compared to the dataset from which the uncertainties were derived (13 g water and hand-clamped seals versus $45 \mathrm{~g}$ and cold-welded seal). The nutrient amended and unamended creeks had broadly similar changes of noble gas concentrations and saturation states with time, both throughout a single tidal cycle and between days and seasons. This is consistent with the similar magnitudes of transport and diffusive exchange processes at the two creeks. All five gases generally increased in concentration and saturation state from high to mid tide or low tide during the ebbing tide. Mid to low tide changes were variable; for the single day with data at both mid and low tide, $\mathrm{Ar}, \mathrm{Kr}$, and $\mathrm{Xe}$ increased in saturation state while concentrations changed slightly, while the lighter gases decrease in both concentration and saturation state towards atmospheric equilibrium.

Along-creek gradients in noble gas concentrations and saturation states were weak to insignificant during high tide. Greater along-creek variability was observed at mid tide, however it is unclear whether this was 
caused by differences in processes along the creek or by the time-delay of sampling between locations during the period when advective transport was most rapid. For example, the mid tide sample at $90 \mathrm{~m}$ in the unamended creek was more similar to samples taken closer in time rather than in space (eg more like $150 \mathrm{~m}$ sample than $115 \mathrm{~m}$ sample). It may be that there were no along-creek gradients in noble gases at mid-tide, and any trend was an artifact of sampling limitations in the non-steady state environment. At high tide, the October 2013 creek transect samples were highly supersaturated with He and Ne but near saturation for $\mathrm{Ar}, \mathrm{Kr}$, and $\mathrm{Xe}$. In contrast, the October 2014 porewater samples (sampled at high tide as well) had a nearly constant saturation state across all five gases. This trend may suggest that enrichments in the lighter (less soluble and more bubble sensitive) gases were not caused by creek exchange with porewater of different concentration, but instead by a bubble injection process (see section 6.3).

6 Discussion: Part 1, water column (2012)

The major fluxes of oxygen in the nutrient amended and unamended creek must be determined in order to derive and compare biological production between the two sites. The correlation of oxygen and tidal stage indicates that water fluxes related to tidal cycling are a major control on oxygen in the system, so constraining the water budgets and associated oxygen transport fluxes of the creeks is of paramount importance. In addition, oxygen was rarely near saturation in either creek, and diffusive air-water gas exchange should therefore be an important flux. The noble gases provide additional tracers of gas transport and air-water exchange, but interpreting patterns of noble gas concentrations and saturation states is not straightforward in this salt marsh setting. The following discussion focuses on constraining the aspects of the water budget most important to controlling oxygen concentration, evaluating the oxygen budget with respect to the water budget and gas exchange, and determining the relative net community production at each creek. The noble gas results are then discussed with regard to how they could inform the evaluation of oxygen fluxes.

6.1 Water budget: The water volume in the tidal creeks at any given time is a function of the water depth (tidal stage) and creek geometry. The change in water volume in the creek must be balanced by the inflow and outflow of water throughout the tidal cycle. In the simplest case where freshwater inputs from rain or groundwater, as well as evaporation, are negligible compared to the creek volume, and the surrounding marsh platform doesn't store significant water, the water budget for a tidal creek can be expressed as:

$$
\frac{\partial V}{\partial t}=u \cdot A_{c}+\cdots
$$

where $\partial V / \partial t$ is the change in water volume in the creek with time, $u$ is the advective velocity, and $A_{c}$ is the cross sectional area perpendicular to the creek axis at the sample location. Both $\partial V / \partial t$ and $u$ are defined so that positive values indicate water coming into to creek, a flood tide. The ellipses in equation 2 represent other processes that may be important to the water budget. $A_{c}$ is estimated from a creek transect indexed to the pressure logger depth. With estimates of cross sectional areas along the entire creek (Will Kearney, unpublished data) paired with water depth, the change in water volume can be calculated throughout the time-series (cross sectional area with tidal height and volume flux data accessible from LTER database along with associated sonde data as indicated in the data availably statement). Two limitations of this method are that the creek transect locations may miss discontinuous features that alter the volume, such as cracks and slumps in the creek bank, and that the transects do not always extend far enough onto the creek platform to fully define the tidal prism during spring tide events that overtop the marsh platform.

Therefore the calculated creek volume is most in error (too low) near high spring tides.

Other processes that might be included in the water budget were the freshwater budget (Ganju et al. 2012), storage in marshtop ponds (Wilson et al. 2014), and porewater absorption to sediments (swelling), infiltration, and drainage over daily to biweekly tidal cycles (Nuttle and Hemond 1988; Gardner and Gaines 2008). I distinguish low salinity groundwater from brackish porewater in this discussion, in that 
groundwater refers specifically to the freshwater component entering the system, while porewater refers to water in the sediments derived from the mixture of creekwater, rain, and groundwater inputs. Ideally this or similar studies should have measurements or modeled estimates for each of these terms. It is unclear that these fluxes are important to the water budgets in the open creek compared to tidal advection, however. In a much larger salt marsh watershed drained by a tidal creek (West Falmouth Harbor, MA), Ganju et al. (2012) found that the net freshwater flux was less than $0.5 \%$ of the total volume flux over any time period. The large salinity swings at the tidal creeks in this study could be explained by freshwater fluxes on the order of only $1-2 \%$ of the total volume flux. Pond and marsh porewater infiltration and drainage over the spring-neap tidal cycle could be important fluxes relative to the water cycling in the marsh platform, but drainage tends to be protracted such that instantaneous fluxes are low compared to creek advection except during overtopping spring tides. For example, marsh porewater drainage at a similar site on the Parker River ( $\sim 5 \mathrm{~km}$ north along Plum Island Sound) was estimated to be $\sim 0.1 \mathrm{~m}^{3}$ tide ${ }^{-1}$ per meter of creek (Gardner and Gaines 2008). This would be equivalent to $10 \mathrm{~m}^{3}$ per tide at each creek, or less than $1 \%$ of the tidal volume (roughly $1000 \mathrm{~m}^{3}$, with roughly $50 \mathrm{~m}^{3}$ remaining at low tide), though long-term drainage is likely to be more heterogeneous at our site because of the artificial ditches draining the marsh platform. Tidally driven porewater advection into and out of creek banks is thought to involve 2-3 times more water on a daily basis than the long term spring-neap infiltration and drainage cycle (Harvey et al. 1987; Nuttle and Hemond 1988). Using the 1D model of Nuttle (1988), I find that this flux is again on the order of $1 \%$ or less of the total tidal volume. More nuanced 2D models result in similar fluxes for modeled systems (Wilson and Gardner 2006; Wilson and Morris 2012). Based on radium activity (following Charette 2007), tidally drive porewater advection is similarly estimated to be 0.8 to $1.5 \%$ of the tidal volume at nearby tidal creek on the Rowley River (E. Chung, H. Dulaiova, and M. Charette, unpublished) which has a similar tidal range and width but a smaller tidal prism than the creeks in my study.

Advection could not be directly evaluated from the ADV data because of poor data quality. If the other fluxes can be discounted as major sources of water, then the advective water flux should be approximately equal to the left side of Eq. 2. Because of the direction and timing of porewater fluxes relative to tidal stage (Wilson and Morris 2012), in ignoring porewater fluxes I introduce small systematic positive biases near high tides and negative biases on low tides. The largest likely source of error from this estimate is underestimation of the submerged volume near high tides (particularly spring tides that flood the marsh) because of limited transect data, which could lead to 5-7\% underestimation of the magnitude of $u \cdot A_{c}$. The net result of these combined errors would be a systematic offset in advective fluxes such that the 'true' value of $u \cdot A_{c}$ may be more positive by $3 \%$ through most of the tidal cycle except for high ebbing tides, which may be as much as $8-13 \%$ more negative with the highest errors when water depth is greater than $\sim 2 \mathrm{~m}$. Water transport over the marsh platform on spring tides could be better constrained with well pressure data or more extensive topography in the calculation of the tidal prism. For now I assume that advection dominated the mass balance of water in the tidal systems. The resultant advection from Eq. 2 was poorly constrained ( $>3 \%$ systematic error) for every other ebb tide (once a day) prior to August 6, but is otherwise $\sim 3 \%$ in error.

Oxygen budget: The oxygen mass balance can be similarly defined to the water budget, but with additional terms related to gas exchange and the balance of photosynthesis and respiration, or net community production:

$$
\frac{\partial V\left[\mathrm{O}_{2}\right]}{\partial t}=u \cdot A_{c}\left[\mathrm{O}_{2}\right]+k_{O_{2}}\left(\left[\mathrm{O}_{2}\right]_{s a t}-\left[\mathrm{O}_{2}\right]\right)+\mathrm{NCP}+\cdots
$$

where $\left[\mathrm{O}_{2}\right]$ is the oxygen concentration measured by the sonde, $k_{O 2}$ is the oxygen air-water transfer coefficient assuming diffusive gas exchange only, and $\left[\mathrm{O}_{2}\right]_{\text {sat }}$ is the expected saturation concentration at equilibrium with the atmosphere given the in situ temperature and salinity. Most of these quantities are only estimated at the sampling sites near the instrument deployments and the creeks are not homogenous 
along their entire lengths with respect to oxygen. Thus Eq. 3 is solved only for a control volume at the sampling location, though the entire creek volume is needed to accurately estimate $u$ from Eq. 2 .

While the additional water fluxes may be small compared to advection, the role of these fluxes in the oxygen mass balance cannot necessarily be discounted. In particular, while porewater moving from the creek into the sediments should remove oxygen from the control volume in the same manner as tidal advection, that oxygen may be rapidly consumed in the near-surface sediments by microbial respiration, and porewater reentering the sediments will be oxygen depleted. The situation is further complicated by the prevalence of anaerobic metabolisms in salt marsh systems which release reduced terminal metabolic products to the porewater that may subsequently be reoxidized and consume dissolved oxygen in the creek water. Sulfur reduction and oxidation is likely the dominant such sediment metabolism cycle in New England tidal creeks (Howarth 1984). Bioturbation and particularly bioirrigation in the creeks could increase the exposure of reduced compounds to creek water and enhance sediment oxygen uptake. Rather than try to account for these oxygen sinks separately from aerobic respiration, I extend the (arbitrary) control volume far enough into the creek sediments such that any consumption of oxygen from whatever source that effects measured water column oxygen concentrations is included in the resulting estimate of $\mathrm{NCP}$. In this case the transport of oxygen by porewater fluxes may be safely neglected with no additional error beyond that associated with the uncertainty in determining $u$ from $\partial V / \partial t$.

Finally, the choice of gas exchange parameterization is especially important since our method of solving for advection forces the advective flux and time rate of change of oxygen to be very nearly equal; thus the gas exchange flux must be the dominant control on the magnitude and sign of NCP in order to maintain the above mass balance. Determining the correct air-water transfer of oxygen is not trivial.

Parameterizations of gas exchange in terms of wind, currents, or turbulence in one estuarine system often show poor agreement with direct measurements when applied to other locations (Raymond and Cole 2001; Borges et al. 2004a; Ho et al. 2011), and gas exchange can vary widely between similar sites separated by only short distances because of the interplay of screening by vegetation, fetch, and current direction relative to winds (Kremer et al. 2003; Borges et al. 2004b; Zappa et al. 2007). Additionally, most such studies evaluate diffusive gas fluxes only, however, bubble processes have been shown to play a small but significant role in the open ocean (Hamme and Emerson 2006; Stanley et al. 2009a; Nicholson et al. 2011a). Enhanced turbulence in estuarine systems relative to the open ocean may greatly increase bubble injection and transport (Baschek et al. 2006; Crosswell 2015). Gas stripping during ebullition (bubbling from the sediments of methane or nitrogen; King and Wiebe 1978; Chanton and Whiting 1995) is possible; this processes has a tidal dependence based on hydrostatic pressure (Chanton et al. 1989). Ebullition in these creeks is more likely to come from photosynthetic oxygen production (Chapter 4), which may remove oxygen production in a way that is missed by diffusive gas exchange models. Consistent with this idea, numerous bubbles were observed in the water column of both study creeks.

Despite the potentially important role of bubble processes in air-water gas exchange in the study creeks, I apply only published, diffusion based models of air-water gas exchange in estuaries. In particular, given the very limited fetch possible in the tidal creeks and sheltering tall-from Spartina alterniflora along the banks, it is likely that current velocity plays a significant role in driving turbulence and gas exchange across the creek surface. I use the parameterization of Borges et al. (2004b) from the Scheldt River estuary as the default for this study because it incorporates short term variability in both windspeed and current velocity in a shallow, limited fetch channel. The normalized gas transfer coefficient $k_{600}$ in this parameterization is evaluated as:

$$
k_{600}=1+1.719\left(100 u^{0.5}\right)\left(z^{-0.5}\right)+2.58\left(w_{10}\right) \quad \mathrm{cm} \mathrm{h}^{-1}
$$

where $u$ and $w_{10}$ are water current and windspeed at $10 \mathrm{~m}$ above ground in $\mathrm{cm} \mathrm{h}^{-1}$, and $z$ is water depth in m. $w_{10}$ is calculated from the windspeed data at $3 \mathrm{~m}$ using the wind profile power approximation with an exponent of 0.13 . The normalized gas transfer coefficient is converted to $k_{O 2}$ using the Schmidt number based on in situ temperature and salinity using the coefficients of Wanninkhof (1992). 
6.2 Net community production (NCP): With assumptions regarding the water and oxygen budgets as above, the net community production can be estimated by difference, using Eq. 3. Advection through the creek cross-section at the sample location is unchanged by the size of the control volume, while gas exchange and NCP are both scale dependent. In order to compare the relative size of gas exchange and NCP to the magnitude and uncertainty of advection, these fluxes can be scaled to the entire length of the creek (because each creek is a tidal system with one outlet, the advective flux through any control volume or length of creek is identical to that through the whole creek; NCP and gas exchange can only be compared to advection on an equivalent scale over the whole creek length). These proportional oxygen fluxes through the study creeks are plotted in Fig. $\mathbf{6}$ in order to demonstrate the dominant role of physical advection of $\mathrm{O}_{2}$ compared to $\mathrm{NCP}$. As expected based on the approximate solution of the water budget, gas exchange and NCP were tightly coupled. Both fluxes were on the order of $3-5 \%$ of peak advective oxygen transport at mid-tides, similar to the error in the advective flux. However, in general only every other tide had peak advective oxygen transport greater than $1 \mathrm{~mol} \mathrm{O}_{2} \mathrm{~min}^{-1}$; at lower rates of advective oxygen transport NCP was larger than the uncertainties in advective transport. For example, NCP was equal to or greater than $10 \%$ of the advective oxygen flux for $80 \%$ of the time series, including all high and low tides. Thus even with the large uncertainties in the water and oxygen budgets, it is probable that the net metabolic flux estimated over the time-series is significant relative to the error bounds associated with the water balance.
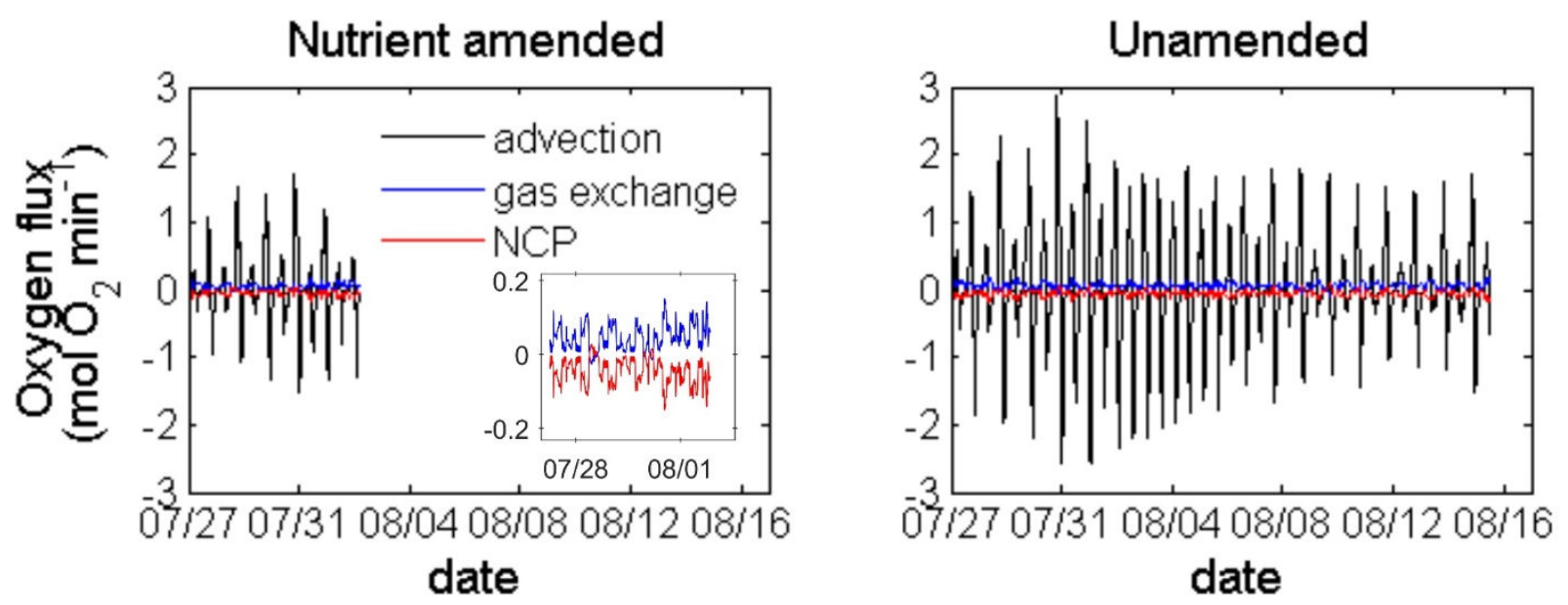

Figure 6: Oxygen fluxes at the nutrient amended and unamended creeks associated with advection, gas exchange, and net community production (NCP). Advection is scale independent, while gas exchange and NCP are scaled to the length of the creeks in order to compare the relative magnitudes and uncertainties of these processes with advection on the same scale.

Areal NCP for the evaluated control volumes at each creek is plotted against water depth (from the unamended creek only) in Fig. 7. The running average of NCP over the 25 hour lunar day is plotted for the unamended creek only as well. NCP in both creeks had large negative excursions near the beginning and end of each low tide. These rates were abrupt relative to the rest of the signal, occurring over 30-50 minutes. Excursions in the data may be the result of model errors or represent important, short-term biogeochemical fluxes. In favor of the former argument, these transitions occurred when creek surface area changed most rapidly, meaning that apparent NCP changes may be attributable to rapid increase or decrease of area over which the mass balance is divided. However, if this was the case NCP rates should become more negative during the ebbing tide and more positive during the rising tide; this does not match 
the observations of negative excursions on both ends of the low tide period. Similar excursions occur if the rates are normalized to length of creek rather than area (not shown), so it is unlikely that the rapid change in area relative to metabolic rates is entirely responsible. One plausible explanation is that these negative excursions are controlled by horizontal porewater fluxes driven in and out of the creek banks by changing water pressure with the tides. Porewater moving into the sediments removes oxygen from the creek, while returning porewater carries an oxygen deficit and reduced terminal metabolic products such as sulfide that would rapidly oxidize in the creek water. Applying the 1D-porewater advection model of Nuttle (1988) to the creek study sites does indicate maximum, albeit small, porewater fluxes concurrent with the negative NCP excursions.

Comparing the nutrient amended and unamended creeks, there were some differences in NCP with time but no consistent trends apparent in the time series. Unlike the results of Nidzieko et al. (2014) using similar methods in a similar salt marsh tidal creek system, I find no relationship between the spring-neap tidal cycle and average NCP over the fortnightly tide cycle. In our experiment, high tides occurred in both day and night, so a net respiratory contribution by the marsh platform was not expected as in the other study. Fig. 8 compares creek NCP against time of day to highlight biologically driven cycling. Rates are normalized to both length and area of the control volume in each creek, and a single lunar day is overlaid in color as with the oxygen results. Again there were no obvious differences in NCP between creeks over the six days with data at both creeks. NCP was generally heterotrophic. There was still a distinct tidal component, with higher areal NCP associated with high tides ( $\sim 6$ and $\sim 19$ hrs into tidal day from low tide, though this pattern is not obvious in the results normalized to creek length instead of submerged area). Within the creek watersheds, this may be explained by partial or complete submergence of tall-form Spartina alterniflora on the creek banks, which could contribute to the subaqueous production signal. Nighttime high tides may similarly have had less heterotrophic NCP because of the up creek advection of signal from estuarine productivity earlier in the day (viz. the residence time of dissolved oxygen in the estuary was greater than in the tidal creeks, which have high respiration and rapid tidal flushing). There were three overnight high tides at the unamended creek in which estimated NCP was greater than 0 , which should not be biologically possible. The most likely explanation for this is an error in the high tide creek volume and underestimation of the amount of oxygen that can accounted for by tidal advection. In any case, the values were $\sim 0.02 \mathrm{mmol} \mathrm{O}_{2} \mathrm{~m}^{-2} \mathrm{~min}^{-1}$, or roughly equal to the uncertainty propagated from the calculation of the advected oxygen flux $\left(\sim 0.03 \mathrm{mmol} \mathrm{O}_{2} \mathrm{~m}^{-2} \mathrm{~min}^{-1}\right)$.

The environmental variability can be assessed over the whole dataset in terms of the data spread and trend with time of day. The average NCP (Fig. 8) was identical at each creek, $-0.13 \pm 0.04 \mathrm{mmol} \mathrm{O}_{2} \mathrm{~m}^{-2} \mathrm{~min}^{-1}$, with a daily range between approximately -0.4 and $0 \mathrm{mmol} \mathrm{O}_{2} \mathrm{~m}^{-2} \mathrm{~min}^{-1}$. The average daily cycle (e.g. Fig. 9) had a minimum in NCP (peak heterotrophy) over the morning and increased to near balance between oxygen production and consumption in the afternoon, followed by a slow decrease over the rest of the day. The reason for a NCP minimum that lasts well into the period of peak PAR is unclear. Creek geometry and shading by the banks may play an important role in limiting available light; NCP did not start to increase until 9-10 am, three hours after dawn. Respiration may also have accelerated with increased temperature (Thamdrup et al. 1998; Duarte et al. 2014). 


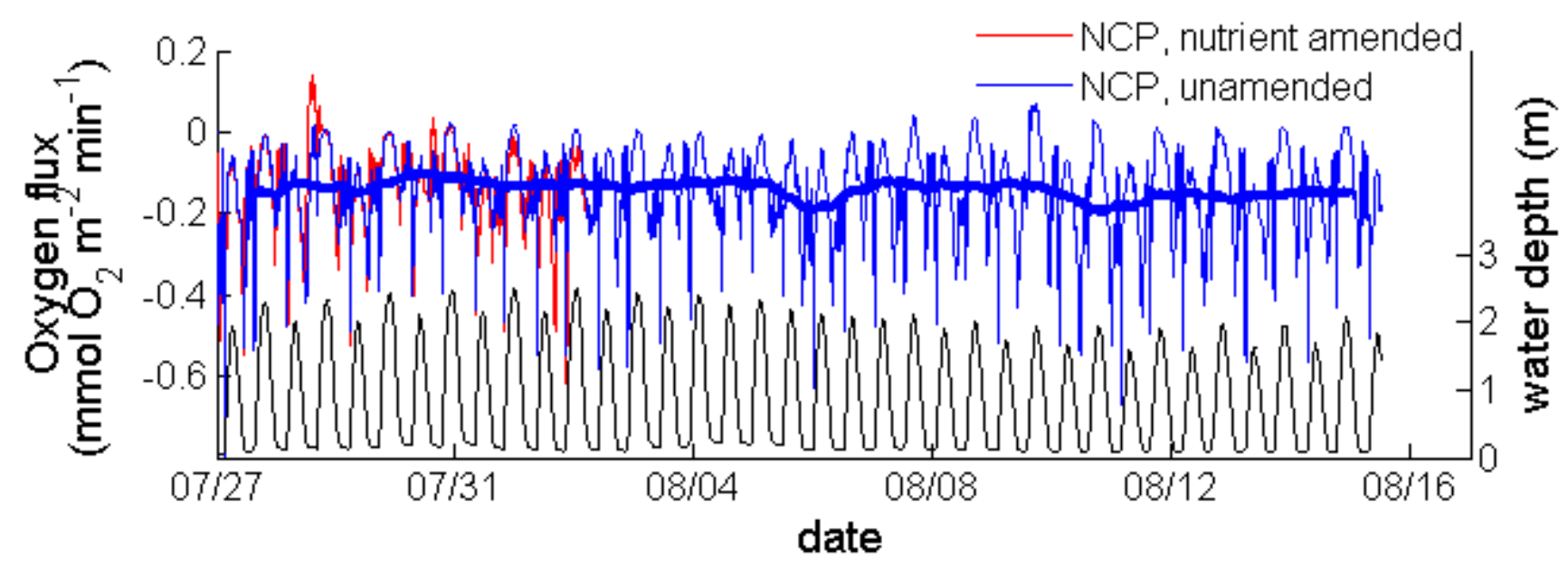

Figure 7: Net community production (NCP) in areal units at the nutrient amended (red), and unamended control (blue) tidal creeks. Thick blue line is moving average of NCP over the 25 hour lunar day at unamended creek only. Water depth (black) in the unamended tidal creek is plotted on the right axis. Large negative excursions in NCP are associated with the beginning and end of low tide. 

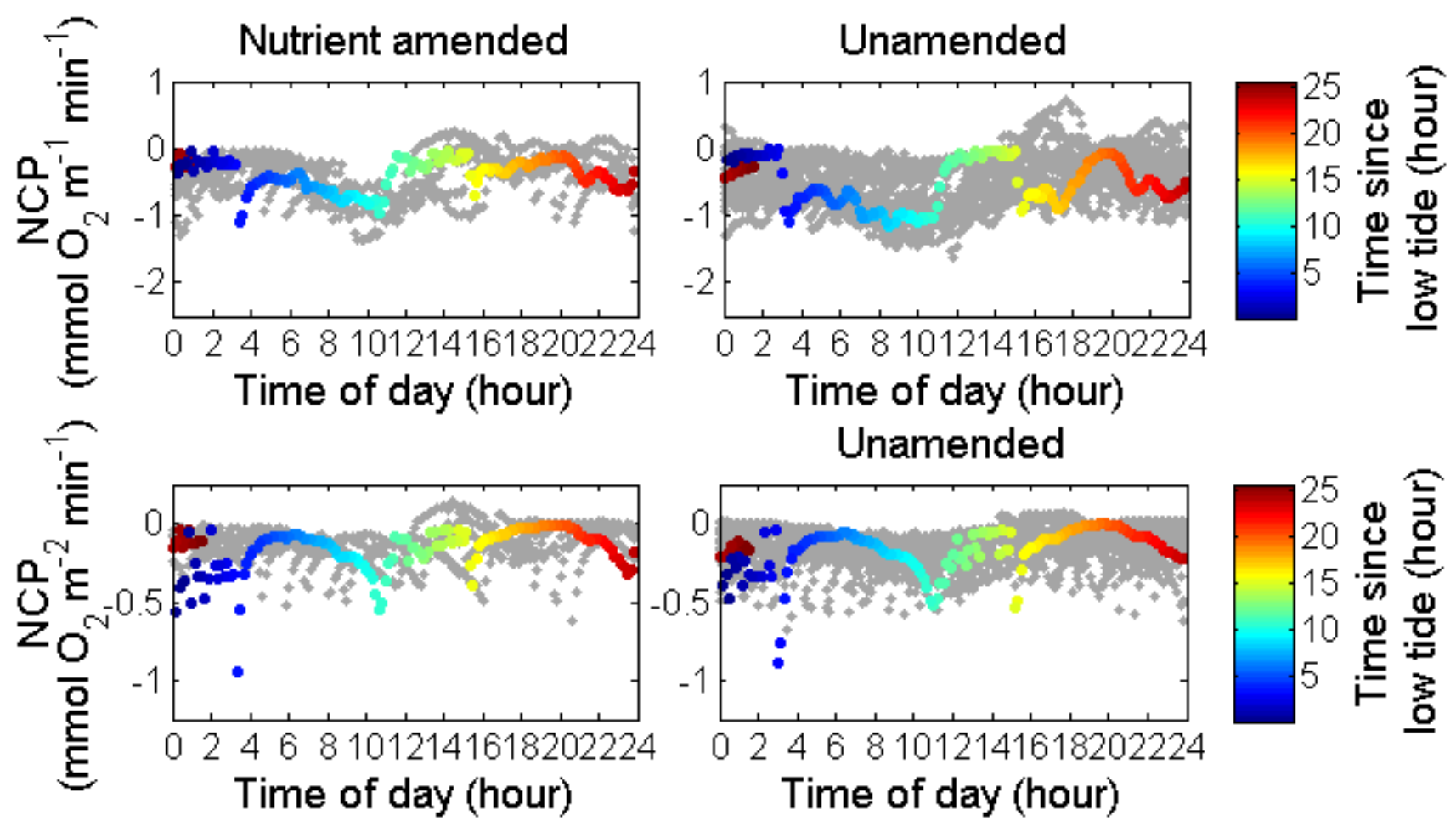

Figure 8: Net community production (NCP) at the nutrient amended and unamended control tidal creeks, per length of creek (top panels) and normalized to creek surface area (bottom panels). As in Fig. 4, the time series includes three times more data points at unamended creek. The first lunar day ( $\sim 25$ hours, 2 complete tidal cycles) is overlaid in color, and begins at midnight local time. 

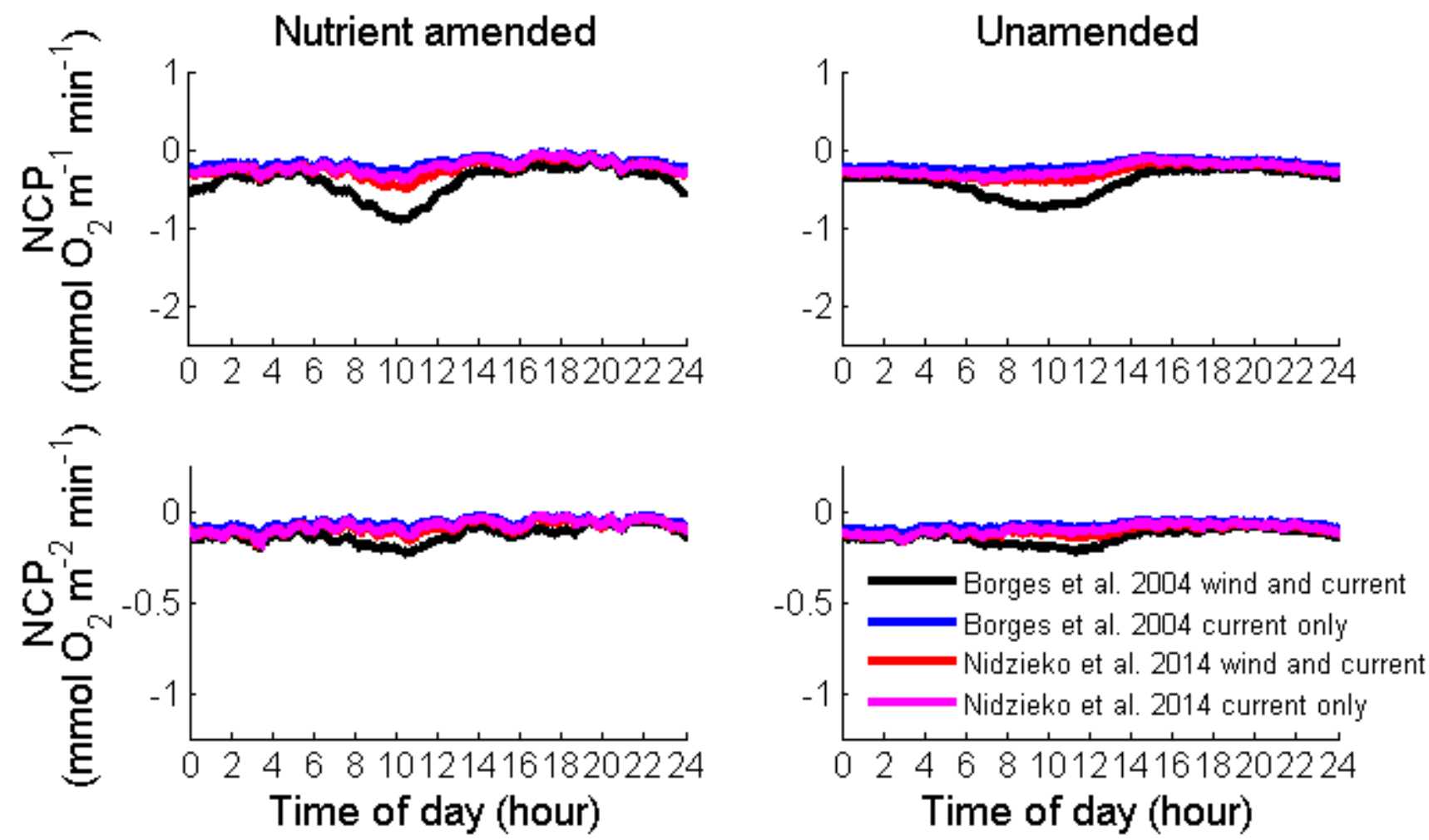

Figure 9: Length and area normalized net community production (NCP; top and bottom panels respectively) determined from the open water oxygen mass balance using four different gas exchange parameterizations (Eqs. 4-7 in text). The Nidzieko et al. (2014) gas exchange parameterization is based in part on the quadratic windspeed dependence of Wanninkhof (1992) which has zero gas transfer at zero windspeed. Thus at low windspeeds this parameterization is indistinguishable from a current only parameterization. 
After accounting for advection, the choice of gas exchange parameterization is the largest source of uncertainty in calculating NCP. In addition to the parameterization assumed in Eq. 4, I compare NCP estimates from three other parameterizations, including the current only component of the Borges et al. (2004b), the windspeed and current parameterization of Nidzieko et al. (2014), and the current only parameterization of Nidzieko et al. (2014) alone (Eqs. 5-7):

$$
\begin{array}{clrl}
k_{600}= & 1+1.719\left(100 u^{0.5}\right)\left(z^{-0.5}\right)+2.58\left(w_{10}\right) & \mathrm{cm} \mathrm{h}^{-1} & \text { (Borges et al. 2004b) } \\
& k_{600}=1.719\left(100 u^{0.5}\right)\left(z^{-0.5}\right) & \mathrm{cm} \mathrm{h}^{-1} & \text { (Borges et al. 2004b) } \\
k_{600}= & 0.31 w_{10}{ }^{2}\left(\frac{600}{660}\right)^{-0.5}+3600\left(\frac{u D}{z}\right)^{0.5} & \mathrm{~cm} \mathrm{~h}^{-1} & \text { (Nidzieko et al. 2014) } \\
k_{600}=3600\left(\frac{u D}{z}\right)^{0.5} & \mathrm{~cm} \mathrm{~h}^{-1} & \text { (Nidzieko et al. 2014) }
\end{array}
$$

where $u, z$, and $w_{10}$ are defined as before in Eq. 4. $D$ is the molecular diffusivity $\left(\mathrm{cm} \mathrm{s}^{-1}\right)$ of the gas of interest (carbon dioxide as written). The numerator of the first term in Eq. 6 (600) is the Schmidt number and is chosen in order to make the equations directly comparable. Schmidt number scaling is employed to solve for in situ $k_{O 2}$ as before. Note that while Eqs. 4 and $\mathbf{5}$ were empirically determined using direct measurement of fluxes, Eq. $\mathbf{6}$ is an amalgam of the open-ocean windspeed parameterization of Wanninkhof (1992) and a theoretical relationship developed for tracer transport in streams and rivers. These parameterizations are chosen because they have been previously applied to shallow channels with tidal flow and rely only on measurements collected with this study. Treating current-driven gas transfer independently from windspeed parameterizations may be preferable for this study because the tidal creeks are below the height at which surface windspeed is reduced to zero by sheltering vegetation (Raupach 1994) except on the highest of tides and conditions ideal for maximum fetch (Kremer et al. 2003).

Half-hour moving averages of NCP calculated from each of the parameterizations in Eqs. 5-7 are plotted in Fig. 9. The four parameterizations showed good agreement overall, though the Borges et al. (2004b) parameterization including windspeed, used as the base case in this study, led to the greatest magnitude and variability of fluxes. The other three parameterizations averaged $-0.11 \mathrm{mmol} \mathrm{O}_{2} \mathrm{~m}^{-2} \mathrm{~min}^{-1}$ at both creeks, $\sim 0.02$ more positive than the base case. It is unsurprising that the windspeed component of the Borges et al. (2004b) paper leads to greater gas exchange and NCP, as it was developed using estuarine data while the Wanninkhof (1992) parameterization was developed for the open ocean and has a near zero intercept at low windspeed (implicitly assuming that other sources of turbulence are negligible). While ocean parameterizations based on windspeed perform well in some very large estuaries (Ho et al. 2011), most investigators have found that shallow environments have enhanced turbulence that leads to some minimum gas exchange even at very low windspeeds $\left(<2 \mathrm{~m} \mathrm{~s}^{-1}\right)$ and with slow or non-uniform currents (Caffrey 2003, 2004; Kremer et al. 2003; Borges et al. 2004a; Zappa et al. 2007; Cole et al. 2010). In any case, it is not clear that any one of the above treatments is superior to the others in this setting, so I use the range between estimates as a measure of the uncertainty in the gas exchange parameterization. In the morning when NCP was most negative the parameterizations agreed to \pm 0.06 (range 0.12 ) $\mathrm{mmol} \mathrm{O}_{2} \mathrm{~m}^{-2}$ $\min ^{-1}$, or $\pm 45 \%$ relative standard deviation (RSD). The maximum agreement in the early evening was \pm 0.02 (range 0.04 ) $\mathrm{mmol} \mathrm{O}_{2} \mathrm{~m}^{-2} \mathrm{~min}^{-1}$, or $\pm 25 \%$ RSD. In summary, during the experimental period mean net community production in the tidal creeks was similar between the nutrient amended and unamended treatments throughout the day $\left( \pm 0.02 \mathrm{mmol} \mathrm{O}_{2} \mathrm{~m}^{-2} \mathrm{~min}^{-1}\right)$ and within uncertainties ( \pm 0.02 to $0.06 \mathrm{mmol}$ $\mathrm{O}_{2} \mathrm{~m}^{-2} \mathrm{~min}^{-1}$ ). Average NCP was always heterotrophic and significantly different than zero.

It is important to note that while the mean NCP was not observed to be significantly different between the nutrient amended creek and unamended control creek over the whole period of comparison, comparisons over specific tidal periods point to more nuanced conclusions. As part of work presented in Kearns et al. (2016), I reanalyzed the same data filtered by just the periods surrounding high tide, when the creek metabolism is influenced by sediment processes in the creek banks and Spartina alterniflora habitat. For 
high- tides only, I found that nighttime NCP was significantly more negative in the nutrient amended creek than the unamended control creek. At the same time, NCP was significantly more positive in the nutrient amended creek than the unamended creek during daytime high tides. Thus there is evidence that, at least during high tide, daytime NCP is more autotrophic (and by inference GPP is larger) and nighttime respiration is more heterotrophic in the nutrient enriched system. At this tidal stage the creek water integrates metabolism not only from creek bottom sediments, but also from grasses on the upper creek banks and low marsh platform. Assuming similar light conditions and primary producer biomass between creeks (see section 9), this effect could be plausibly related to differences in nutrient status; previous work has shown differences in grass and benthic microalgal production on the low marsh platform around the fertilized creeks (Bowen et al. 2009, Deegan et al. 2012). Kearns et al. (2016) combined genomic and RNA transcriptomic analysis in the sediment microbial community with metabolic rates to argue that in the creek bank and low marsh sediments surrounding the nutrient enricheded creek, community activity and metabolism was likely altered, leading to increased nitrate uptake, primary production, and respiration of the resulting organic matter.

If enhanced production is balanced by enhanced respiration in these habitats over daily timescales in this manner, then mean NCP over the day would be expected to be unchanged; the result reported in this chapter simply averages over any differences in a way that hides the effects on production and respiration. Results of sediment incubations using cores from the same creeks (Spivak and Ossolinski 2016) indicated that heterotrophic carbon uptake by sediment bacterial community is tightly coupled to benthic microalgal production. That study did not support a direct fertilization effect on microalgal production, but the bacterial community was found to rapidly utilize organic carbon from a variety of sources including Spartina debris; nitrate addition could enhance production by other primary producers such as grasses or macroalgae (e.g. the filamentous green algae observed along the upper creek banks) and still ultimately stimulate respiration by the bacterial community. In the context of the question asked by this project (Is the net balance of ecosystem metabolism more or less autotrophic in a nutrient enriched tidal creek than in an unamended control creek?), the approach described in sections $\mathbf{6 . 1}$ and $\mathbf{6 . 2}$ for comparing across a whole day or longer periods is appropriate and the answer is likely no, over weekly timescales at least. But if the question is instead, Does ecosystem photosynthesis and respiration change under nutrient enrichment? then the answer is likely yes, at least over sub-daily periods in specific environments in the area flooded by the tidal creek.

6.3 Noble gases: The noble gases provide natural abundance in situ tracers of physical processes including water transport and diffusive and bubble gas exchange processes. With sufficient sampling resolution and understanding of key fluxes, they may provide a valuable tool to better understand gas fluxes in salt marshes and constrain biological oxygen fluxes. Unfortunately the simplifying assumptions used in the water and oxygen budgets for this study are not well justified with respect to the noble gases. In particular, the porewater fluxes can't necessarily be neglected as the porewater and creek water concentrations appear to be controlled by separate processes.

The October 2014 noble gas samples were uniformly supersaturated on the order of 10\%; the most likely process affecting more and less soluble gases similarly is an increase in pressure relative to the reference case of one atmosphere. Tidal inundation of the marsh platform could increase pressure in the pore space in this manner. The phenomenon of 'excess air,' or supersaturation caused by the trapping and subsequent partial or complete dissolution of air bubbles is sometimes invoked to explain groundwater supersaturations of noble gases (Heaton and Vogel 1981; Klump et al. 2007); if air in the pore space was completely dissolved, the expected ratio of $\mathrm{Ne} / \mathrm{He}$ in the porewater would be 3.47 , and that of Xe/ $\mathrm{Ne}$ 0.005 . The measured ratios in the porewater are instead $4.25(\mathrm{Ne} / \mathrm{He})$ and $0.060(\mathrm{Xe} / \mathrm{Ne})$, with more soluble gases more enriched relative to the less soluble gases than expected for complete dissolution of trapped air, consistent with an equilibrium process (and thus the uniform saturation state of the noble gases). This implies that at the time the porewater noble gas concentrations were set, air remained trapped in the pore space at equilibrium with the porewater. 
Meanwhile the less soluble gases ( $\mathrm{He}, \mathrm{Ne}$ ) were preferentially enriched in the creek water, while the other noble gases were near atmospheric equilibrium. Given the uniform enrichment of the porewater, these enrichments cannot be explained by creek water mixing with porewater, but are instead most likely related to air injection by bubble processes. One obvious source of bubbles to the creeks is air flushed from the porespace (inferred to be present above) during tidal flooding. Just as increasing water pressure causes dissolution of air explaining porewater enrichments, some air is likely expelled from the pore space in the creek banks. The pore air expelled into the creeks in this manner would preferentially enrich $\mathrm{He}$ and $\mathrm{Ne}$ in the creek water (just like the better understood process of air injection from the water surface). Bubbles of atmospheric air could also be entrained into the creek by turbulent processes along the creek banks during flooding, and would similarly enrich $\mathrm{He}$ and Ne relative to the more soluble gases.

The noble gas dynamics in the creeks depend not only on processes related to tidal flushing and interactions between air and water in the pore space, but also on air-water gas exchange processes. Surfactant films that may weaken diffusive exchange are observed at both study sites, particularly near high tides, and turbulence along the creek banks introduces bubbles as the tide drops. The relative standard deviation of the noble gas concentrations in the creeks is twice as large as the RSD of the saturation state, indicating that in general air-water diffusion and bubbles act rapidly to maintain saturation state relative to atmospheric equilibrium throughout the day, rather than maintaining stable concentrations. Ignoring the potentially large role of pore space and bubble processes for noble gases, and assuming that most of the change in noble gas concentrations in the creeks was driven by gas exchange rather than advection of water based on the small along-creek gradients in the noble gases, noble gas based $k_{O 2}$ was approximately $10^{-6}$ to $10^{-5} \mathrm{~m} \mathrm{~s}^{-1}$, the same as the range in $k_{O 2}$ estimated with the four gas exchange parameterizations. With a better mechanistic understanding of the noble gas dynamics in the tidal creeks, noble gas data could allow us to distinguish between published parameterizations as applied to salt marshes and determine system specific gas exchange.

7 Experimental methods: Part 2, benthic chamber experiments (2013)

The 2013 experiment used light and dark benthic chambers at the nutrient amended and unamended creeks to isolate sediment dissolved oxygen concentration and isotope ratio fluxes from the water transport and gas exchange fluxes. Three daily experiments were conducted, August 27-29, 2013, with sampling of the chamber volumes and surrounding creeks water. Descriptions of the experimental methods and sample analyses follow.

7.1 Deployed equipment: Stirred benthic chambers made of clear acrylic plastic similar to those of Rao and Charette (2011) were built to enclose $\sim 8.4 \mathrm{~L}$ of water and a similar volume of sediment (chamber radius $14.6 \mathrm{~cm}$, height $30.5 \mathrm{~cm}$ ). All chamber materials were cleaned, soaked in salt water for one week, and dried for two days prior to use. The morning of each daily experiment, six chambers were pushed 12$13 \mathrm{~cm}$ into the creek bottom sediment at $1 \mathrm{~m}$ intervals along each creek, with alternate chambers darkened with light-blocking fabric shrouds (dark treatment). A $30 \mathrm{~m}$ stretch of each creek was used for the three daily experiments, with chambers placed in different $10 \mathrm{~m}$ sections on subsequent days. The chamber tops were left open during chamber insertion, and closed with gas tight plugs roughly two hours before lowtide and immediately before the initial timepoint of each experiment. Each chamber was then stirred with an acrylic bar at $30 \mathrm{rpm}$ both to homogenize the chamber volumes for sampling and to minimize changes in pressure driven porewater exchange relative to the open creek sediments; the speed was chosen based on pressure gradients created by chamber stirring in in other muddy systems (Tengberg et al. 2005, Rao and Charette 2011) and the estimated mean pressure field from creek advection over the experimental periods. Additionally, closed-bottom containers containing $8.2 \mathrm{~L}$ of water in isolation from the sediments were used evaluate the relative rates of tracer fluxes in creek water alone compared to the sediment plus water chambers (light and dark water treatments). 
7.2 Water sampling: Each chamber was sampled for salinity, dissolved oxygen concentration, and dissolved oxygen isotopic ratios at four timepoints spanning 4-5 hours centered on a midday low tide. The initial sampling order was randomized for each day's experiment but consistent between timepoints. 125 $\mathrm{mL}$ of water was withdrawn from each chamber using a syringe attached to $6 \mathrm{~m}$ of pre-soaked Tygon ${ }^{\circledR}$ e-series phthalate free tubing attached to a port in the chamber lid. The initial $60 \mathrm{~mL}$ was discarded to flush the volume sitting in the sample tubing, and the rest used for sample collection. Up to an additional $575 \mathrm{~mL}$ water was withdrawn directly through the tubing into evacuated flasks after Stanley and Howard (2013) to sample dissolved oxygen isotopic ratios. The mean and one standard deviation volume of water withdrawn was $265 \pm 170 \mathrm{~mL}$, or $3 \pm 2 \%$ of the chamber volume. A second port with a $10 \mathrm{~cm}$ length of tubing allowed replacement volume to be pulled in from the surrounding creek water near the sediment water interface. Ambient creek water was sampled at two locations along each $10 \mathrm{~m}$ length of creek using an identical length of tubing anchored near the creek bottom. Sample collection and analysis for the measured properties are described next.

7.3 Photosynthetically active radiation (PAR) and light intensity: PAR flux at 400-700 $\mathrm{nm}$ was measured at chamber height in the tidal creeks and on the adjacent creek platform using a LI-COR ${ }^{\circledR}$ underwater spherical sensor and upward facing quantum sensor, respectively. Onset ${ }^{\circledR}$ HOBO pendant luminous flux (weighted according to visible light as opposed to full light spectrum) and temperature loggers placed inside two light and two dark chambers at each creek qualitatively confirmed that light did or did not penetrate the chambers. The light record from the loggers also gave a qualitative measure of the light history of each chamber, including the light decrease caused by sediment settling on the chamber lids between cleanings (with a cloth on a pole) after each sample timepoint.

7.4 Temperature, salinity, and dissolved oxygen concentration: Water temperature was recorded using the HOBO pendants inside two light and two dark chambers at each creek. A small volume of sampled water was immediately transferred by syringe into a thermally insulated vial with an optical dissolved oxygen sensor (YSI TM Pro-ODO). The instrument reading was allowed to stabilize (generally $<1$ minute), and the oxygen saturation state at the temperature of the water sample recorded. The instruments were calibrated to or better than the manufacturer's reported instrument accuracy and precision of $1 \pm 0.1 \%$ of air saturation, or $1-3 \%$ of measured values. Absolute salinity $\left(\mathrm{S}_{\mathrm{A}}\right.$; Millero et al. 2008) of sampled water at each timepoint was measured on handheld salinity refractometers with accuracy and precision of $2 \pm 1 \mathrm{~g}$ salt $/ \mathrm{kg}$ seawater from the water sample used to measure oxygen. Salinity and chamber temperature were used to calculate in situ concentration and saturation state of dissolved oxygen (Garcia and Gordon 1992).

7.5 Triple oxygen isotope ratios and ${ }^{17} \Delta$ : The triple oxygen isotope ratios of ${ }^{16} \mathrm{O},{ }^{17} \mathrm{O}$, and ${ }^{18} \mathrm{O}$ in dissolved oxygen were sampled in identical flasks to the 2012 experiments. These flasks were attached to the chamber sample tubing with care taken to avoid bubbles and limit contamination by atmospheric oxygen, and then slowly opened to draw a $\sim 200 \mathrm{~mL}$ water sample into the vacuum (Stanley and Howard 2013). The headspace from the samples was processed similarly to that from the 2012 experiments, with modifications as follows to improve data quality.

The ratio of oxygen to argon in a sample relative to air can be independently useful as a tracer of net oxygen production (Craig and Hayward 1987). However, the $\mathrm{O}_{2}$ :Ar ratio of samples at our study site was generally much lower than in typical seawater because of low dissolved oxygen concentrations. The resulting Ar matrix effects of samples containing ambient Ar led to larger corrections than raw values of $\delta^{17}$ and $\delta^{18}$ when the concentration of oxygen dropped below $\sim 70 \%$ of saturation and Ar was near saturation. Based on instrument response when samples from a trial run of this experiment were analyzed, Ar was manually separated by running the sample through a gas chromatography column held in an isopropanol and dry ice bath $\left(-80^{\circ} \mathrm{C}\right)$. Once the Ar was eluted (observed using a quadrupole mass spectrometer attached to the carrier gas outflow), the bath was removed and the remaining oxygen processed normally. An additional liquid nitrogen trap was added to the processing line in order to remove methane, which was occasionally present in the samples and interfered with normal analysis on 
the isotope ratio mass spectrometer; methane concentrations were not quantitatively determined. Ar was discarded with $\mathrm{N}_{2}$ during sample processing for samples with $\left[\mathrm{O}_{2}\right]<140-175 \mu \mathrm{mol} \mathrm{kg}-1$ ( $70 \%$ of saturation). ${ }^{17} \Delta, \delta^{17}$, and $\delta^{18}$ were derived as before.

High precision measurement of ${ }^{17} \Delta$ becomes more difficult as the amount of oxygen available for analysis in a sample decreases, and as oxygen isotopic ratios become less homogenous in the sampled environment. Analysis of these samples using the modified method above is impractical at $\left[\mathrm{O}_{2}\right]<50$ $\mu \mathrm{mol} \mathrm{kg}{ }^{-1}$, and these samples were not run or the results discarded (eg all dark chamber experiments, all day three samples). 17 additional samples were discarded because they were analyzed at $50<\left[\mathrm{O}_{2}\right]<140$ $\mu \mathrm{mol} \mathrm{kg} \mathrm{kg}^{-1}$ prior to development of the low- $\mathrm{O}_{2}$ method - a low $\mathrm{O}_{2}$ :Ar ratio, and potentially the presence of methane, led to analytical uncertainty in ${ }^{17} \Delta$ similar or greater than the change in value between adjacent timesteps for these samples.

8 Results: Part 2, benthic chamber experiments (2013)

Results from each of the three daily chamber experiments in August 2013 are plotted in Figs. 10 (August 27, 2013 experiment), 11 (August 28), and 12 (August 29). For each site, PAR, chamber oxygen concentrations, and chamber ${ }^{17} \Delta$ are plotted as measured. When calculating production fluxes, oxygen concentration and isotopic ratios were corrected for entrainment of ambient creek water to replace sampled volumes by using the tracer values in the surrounding creek, also presented in the figures. Chamber temperature and salinity are not plotted, but are tabulated in Appendix I: Table A2 with the associated values of oxygen concentration and oxygen isotope ratios.

8.1 Temperature and salinity: Temperature in the chambers generally tracked ambient water temperature in both the light and dark chambers, on August 27 and 28 warming $\sim 2-5^{\circ} \mathrm{C}$ over the experimental period at the nutrient amended creek, warming by $1-3^{\circ} \mathrm{C}$ at the unamended creek, and remaining unchanged at both creeks on August 29. Salinity was not observed to systematically change within measurement errors. Therefore, all chamber salinity measurements at each site on a given day were binned to determine average salinity for the calculation of oxygen saturation state.

8.2 Photosynthetically active radiation (PAR): PAR is plotted both in terms the incident flux on the marsh platform, as well as the PAR flux penetrating the creek water to the level of the chambers (panels a and $\mathbf{d}$ of Figs. 10-12). The two locations received similar instantaneous (Figs. 10-12) and integrated incident PAR on the marsh platform over the experimental period on August 27 and 28 (integrated PAR $31.0 \pm 1.1 \times 10^{-3}$ and $31.5 \pm 0.5 \times 10^{-3}$ mol quanta $\mathrm{m}^{-2}$ ); it is unclear why water column PAR at the unamended creek was initially higher than marsh platform PAR, but this was prior to the chamber deployments and the sensor may not have been submerged. PAR flux between the two sites was more variable on August 29, which was overcast, and the integrated fluxes were less similar. The platform sensor at the amended site was covered for 75 minutes to protect it from rain; interpolating the missing values, the integrated PAR was $4.3 \times 10^{-3}$ versus $6.4 \times 10^{-3}$ mol quanta $\mathrm{m}^{-2}$ at the nutrient amended and unamended creeks, respectively. However, the difference in integrated rate is mostly a consequence of the much longer experiment at the unamended creek that day (nutrient amended experiment from 10:15-15:15, unamended from 11:00-18:00); the integrated flux was within $0.4 \times 10^{-3}$ mol quanta $\mathrm{m}^{-2}$ over identical time periods at each site. 


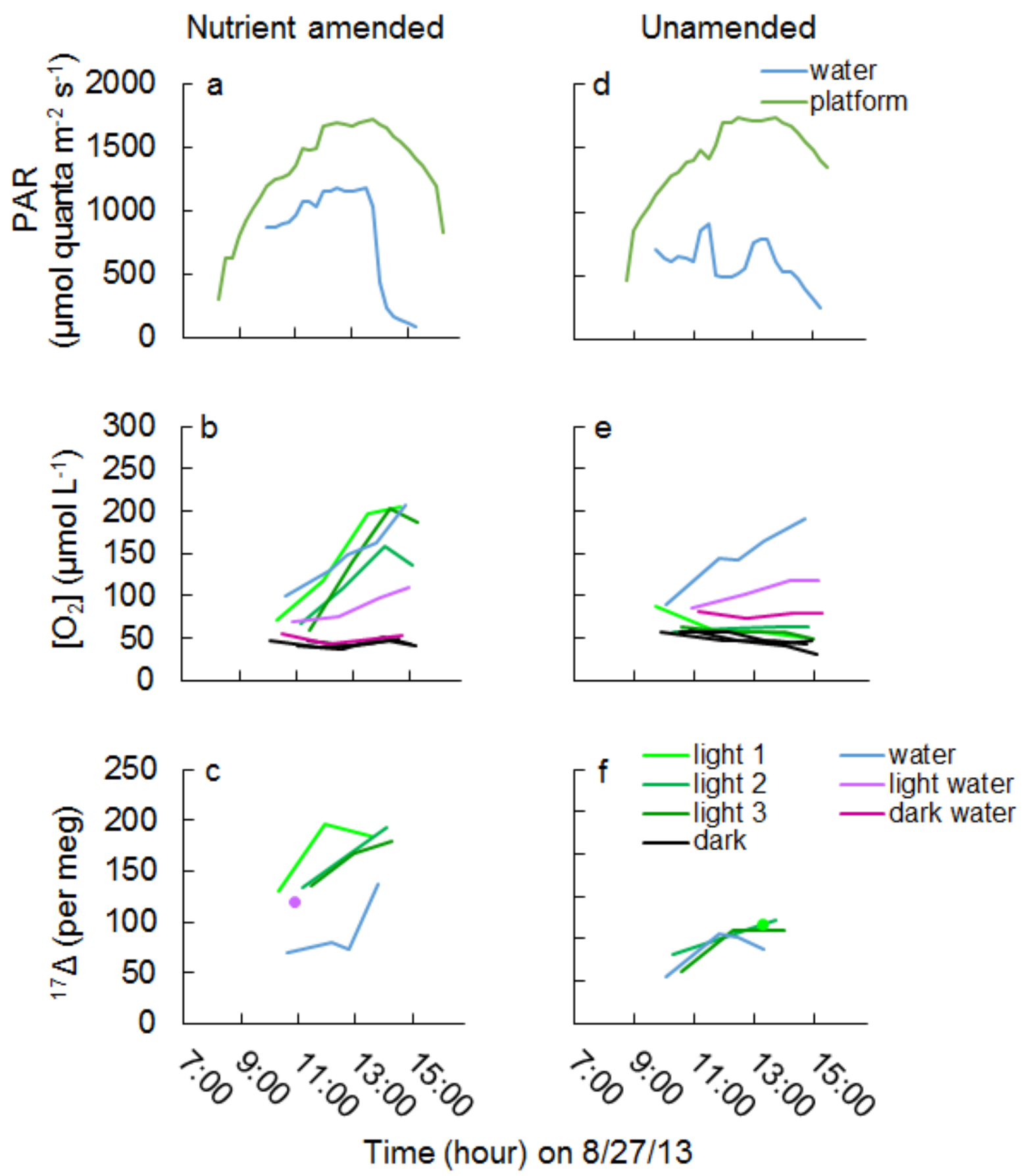

Figure 10: Results from August 27, 2013 benthic chamber experiments at the nutrient amended and unamended control creeks. Panels a and $\mathbf{c}$ plot photosynthetically active radiation (PAR) flux arriving at the marsh platform adjacent to the benthic chambers (green), and at the water level of the submerged chambers (blue). Dissolved oxygen concentrations (b and e) and triple oxygen isotope tracer ${ }^{17} \Delta$ values (c and f) are plotted for each chamber for which there is data at each creek, including three clear, ambient light sediment treatments ('light'), three shrouded, dark treatments ('dark'), and one light and dark chamber at each creek containing water isolated from the sediments ('light water' and 'dark water'). Tidal creek water column oxygen concentrations and isotope ratios are used to correct chamber values for entrainment of ambient water during sampling when calculating production rates. 

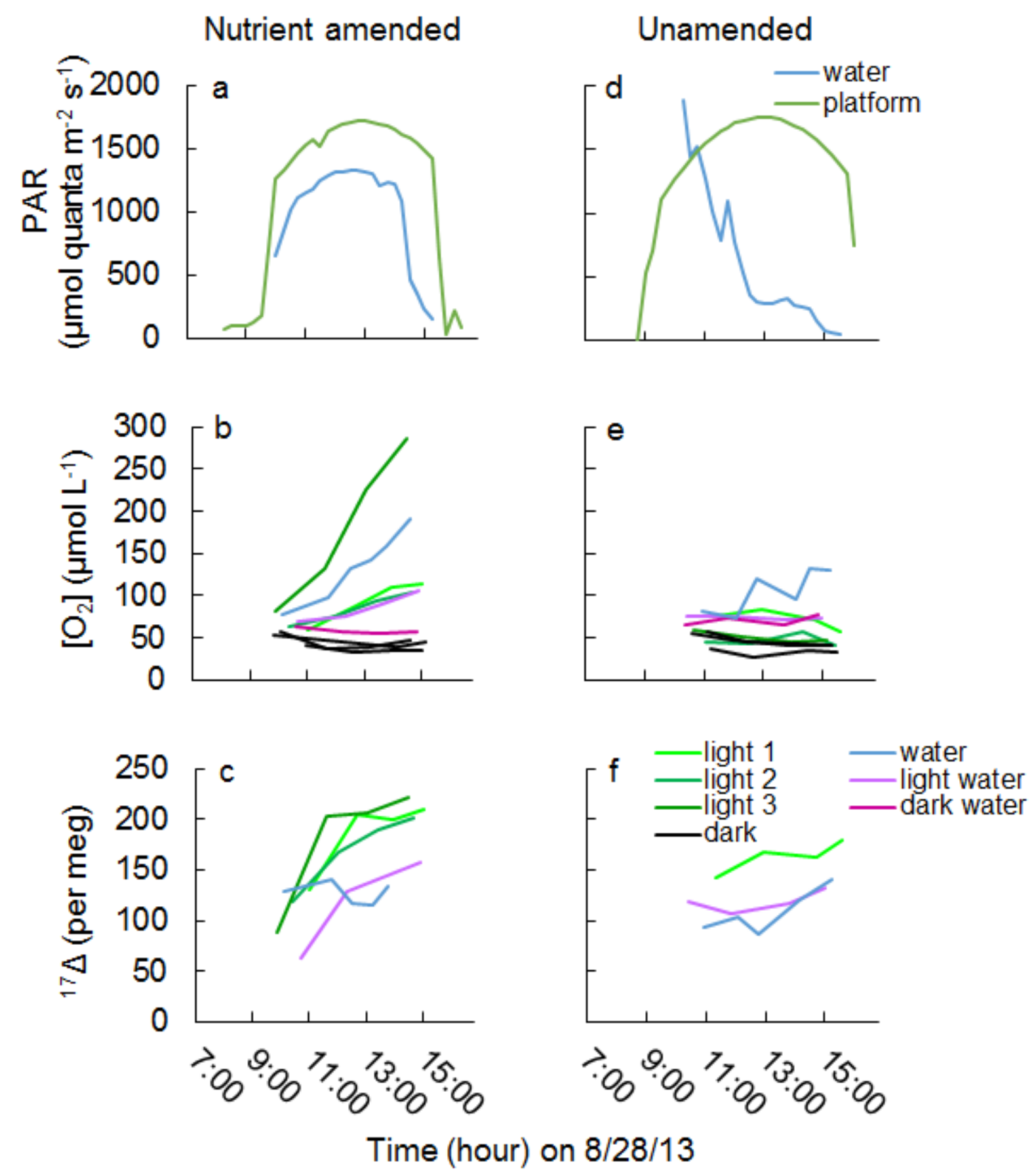

Figure 11: Results from August 28, 2013 benthic chamber experiments at the nutrient amended and unamended control creeks. Panels and chamber notation are as in Fig. 10, including photosynthetically active radiation (PAR) on the marsh platform and in the creek and dissolved oxygen concentrations and triple oxygen isotope $\operatorname{tracer}{ }^{17} \Delta$ values in each chamber. 


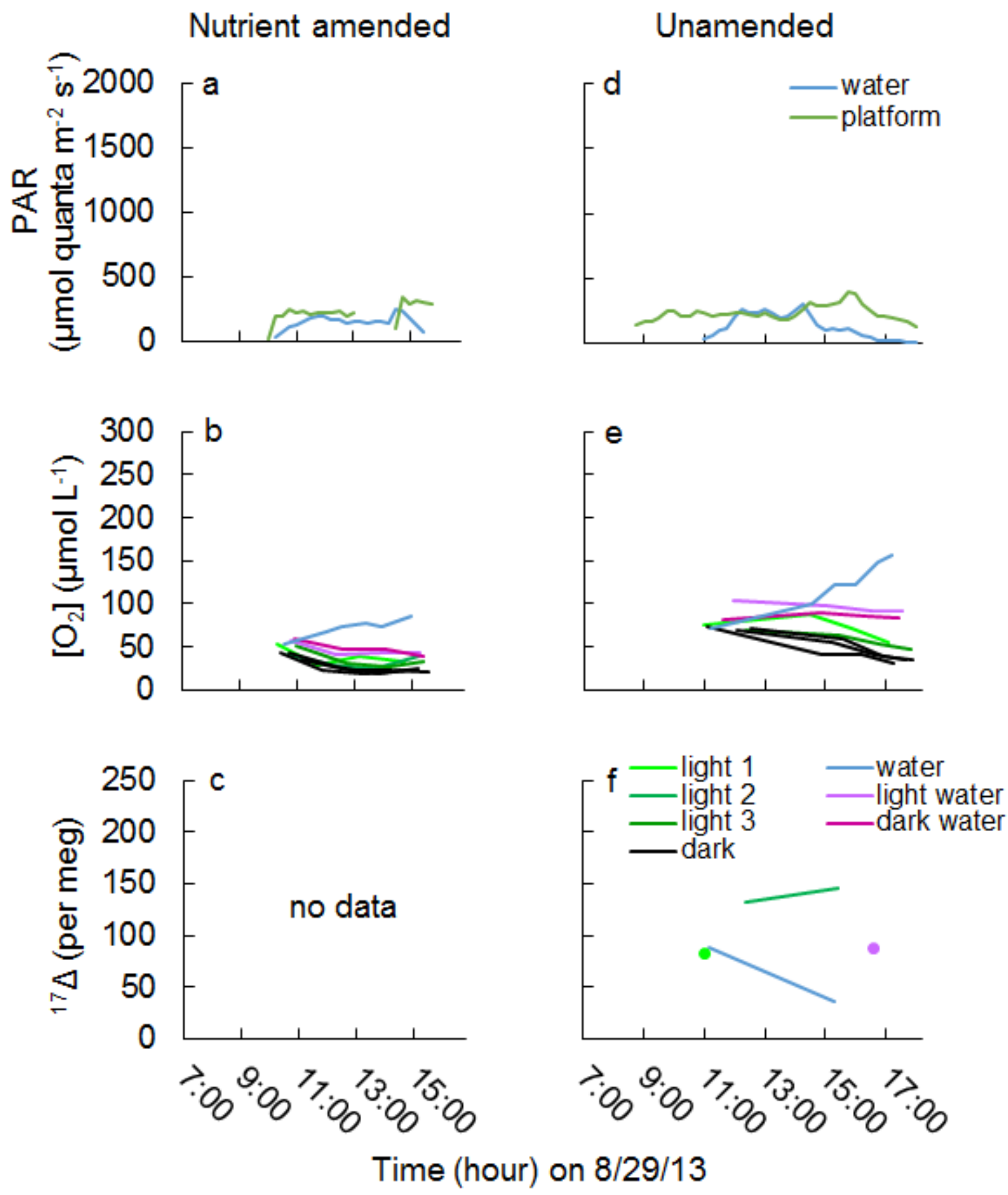

Figure 12: Results from August 29, 2013 benthic chamber experiments at the nutrient amended and unamended control creeks. Panels and chamber notation are as in Fig. 10, including photosynthetically active radiation (PAR) on the marsh platform and in the creek and dissolved oxygen concentrations and triple oxygen isotope tracer ${ }^{17} \Delta$ values in each chamber. Production during the August 29 experiment was light limited at both tidal creeks. 
The PAR flux at chamber depth was markedly different between creeks, however. PAR flux at the experiment site at the nutrient amended creek closely followed incident PAR on the marsh platform all days. The flux at the unamended creek was irregular and flattened compared to marsh platform PAR, suggesting increased shading related to creek geometry compared to the amended creek. On August 28, the reason for the apparently higher PAR flux in the water than on the platform at the beginning of the experiment in not clear. In all cases, light attenuation to the submerged sediments was greater at the unamended site; the amended creek received 31\% and 67\% more light that the unamended site on August 27 and 28. The amended creek had 18\% lower integrated PAR than the unamended creek on August 29, with about a third of that deficit accounted for by the difference in experimental periods.

8.3 Dissolved oxygen concentration: Oxygen concentrations for each chamber experiment are plotted in panels b and e of Figs. 10-12. Clear sediment chambers (light treatment) are individually labeled as 'light' 1-3. Dark treatments ('dark,' $n=3$ ), open water measurements ('water,' $n=2$, pooled) and light and dark water treatments in the chambers with bottoms and isolated from the sediments ('light water' and 'dark water,' $\mathrm{n}=1$ each) are also identified. Chamber oxygen concentration varies between $\sim 18-280 \mu \mathrm{mol}$ $\mathrm{kg}^{-1}$ (8-137\% of saturation), similar to the results of the 2012 water column experiment. Only one chamber at the nutrient amended creek (light treatment chamber 3 on August 28, 2013) became supersaturated; all other chambers at both creeks remained below saturation. Dark treatment chamber timepoints were calculated as being near or below $50 \mu \mathrm{mol} \mathrm{kg}{ }^{-1}$ in general, and in most cases showed little or no systematic variation with time - this may be indicative of limited measurement accuracy at low saturation state based on the sampling method used as well as the nonlinearity of aerobic respiration at low oxygen concentrations (see Discussion: Part 2).

Oxygen concentrations in light treatment chambers at the nutrient amended creek were greater than in the dark treatment chambers and increased with time as would be expected with net photosynthesis, except on the third, cloudy day. Oxygen concentrations in these light sediment chambers were similar to those in the surrounding creek water; the creek concentrations were influenced by advective and diffusive solute transport along the creeks as well as sediment processes. Light treatments in the unamended creek, however, generally had lower oxygen concentrations with small rates of change and were more similar to dark treatments even as the overall creek water oxygen concentration rose. During the August 29 experiment, light and dark treatments had similar trends in oxygen with time in both creeks indicating that the processes controlling oxygen concentration were similar between chambers and creeks on the day with the smallest PAR flux to the chambers. The light and dark water treatments tended to have concentrations intermediate between the light and dark sediment treatments and were less variable with time than chambers with sediments or the open water column.

8.4 Triple oxygen isotope ratios and ${ }^{17} \Delta$ : The derived ${ }^{17} \Delta$ from the oxygen isotope ratios of the subset of samples that could be analyzed (see Experimental methods: Part 2) is plotted in panels $\mathbf{c}$ and $\mathbf{f}$ of Figs. 10-12. In several cases, insufficient data points were available for subsequent production rate analysis. Nonetheless, the triple oxygen isotopes reveal three important trends. First, the ${ }^{17} \Delta$ tracer generally increased in each sampled chamber over the experimental period, even when oxygen concentration was declining, indicating accumulation of photosynthetic oxygen in the remaining dissolved oxygen pool. Second, there was on average a leveling or small decrease of ${ }^{17} \Delta$ near low tide (begins $\sim 12: 00$ to 13:00 over the three experiments) despite peak PAR flux to the creek bottom over the same period. Decreases in the ${ }^{17} \mathrm{O}$-excess could be partially explained by nonlinear isotope mixing caused by the addition of ambient creek water with low dissolved oxygen and ${ }^{17} \Delta$ during sampling; mixing of porewater with even lower values of these tracers into the chambers potentially plays some role as well (see Discussion: Part 2). Finally, ${ }^{17} \Delta$ was on average higher in the light treatments at the nutrient amended creek than at the unamended creek, indicating a greater accumulation of the photosynthetic tracer relative to other sources of oxygen either preceding or during the experiments. 
9 Discussion: Part 2, benthic chamber experiments (2013)

Unlike the 2012 experiment, advective water transport and gas exchange should not play a role in interpreting biological oxygen fluxes from the sediments within the experimental chambers. Ideally, changes in oxygen concentration and isotope ratios should be directly proportional to metabolic fluxes. However, the resulting rates of gross primary production and net community production are not consistent between tracers. The following discussion covers the determination and comparison of net community production and gross primary production between the tidal creeks and potential drivers of discrepancies between rates.

9.1 Net community production and sediment oxygen demand fluxes: Dissolved oxygen fluxes were derived using a linear fit of the oxygen concentration in each chamber versus time. The rates' fits to the data were improved by correcting oxygen concentrations at earlier timepoints for the inflow of water from the surrounding creek to replace the volume removed during sampling and sequentially adding these effects to make a new slope. For example the corrected oxygen concentration at the preceding timestep $\left[\mathrm{O}_{2}\right]_{t-1}$ relative to that at the current timestep, $\left[\mathrm{O}_{2}\right]_{t}$, was determined as:

$$
\left[O_{2}\right]_{t-1}=\left[O_{2}\right]_{t}\left(1-f_{e x}\right)+\left[O_{2}\right]_{w, t-1}\left(f_{e x}\right)
$$

where $f_{e x}$ is the fraction of the chamber volume exchanged and $\left[\mathrm{O}_{2}\right]_{w, t-1}$ is the oxygen concentration of the entrained water at $\mathrm{t}-1$. Rates derived in this manner agreed well with piecewise integration of oxygen production or consumption in each chamber, which does not assume linear oxygen evolution (relative standard deviation 11\%). All further discussion of oxygen fluxes refers to linearly fit data. Sediment oxygen demand was determined from the oxygen fluxes in the dark chambers. Net community production was calculated from the oxygen flux in the clear, sunlit chambers.

NCP based on an entrainment corrected linear fit to the chamber oxygen data is summarized in Fig. 13. Piecewise integrated rates are listed in Table 1 for comparison with GPP values. As expected from the low oxygen concentrations and small changes with time, NCP was close to zero in most dark treatments (mean and standard deviation across all three days of $-0.007 \pm 0.005 \mathrm{mmol} \mathrm{O}_{2} \mathrm{~m}^{-2} \mathrm{~min}^{-1}$ ); NCP was negative and close to zero in light treatments at the unamended creek as well $\left(-0.009 \pm 0.005 \mathrm{mmol} \mathrm{O}_{2} \mathrm{~m}^{-2}\right.$ $\left.\mathrm{min}^{-1}\right)$. NCP in the light treatments at the unamended creek was always indistinguishable from sediment oxygen demand in the dark chambers within one standard deviation. On August 29, when PAR was low at both creeks compared to the other experiments, light and dark sediment and water treatments were all similar at both tidal creeks (eg, mean and standard deviation for light treatments at nutrient amended and unamended creeks were $-0.008 \pm 0.005$ and $-0.009 \pm 0.001 \mathrm{mmol} \mathrm{O}_{2} \mathrm{~m}^{-2} \mathrm{~min}^{-1}$, and dark treatments at each creek were $-0.008 \pm 0.003$ and $-0.015 \pm 0.001 \mathrm{mmol} \mathrm{O}_{2} \mathrm{~m}^{-2} \mathrm{~min}^{-1}$ ).

The higher NCP in the nutrient amended creek compared to the unamended creek could support the hypothesis that nutrient addition may enhance productivity. However, both nutrient and light supply to the creeks covary, and the literature for benthic microalgae suggests that in muddy, low-porosity sediments such as those at the creek bottom these primary producers should be more dependent on sediment nutrient fluxes than water column supply (Tobias et al. 2003). Indeed, sediment cores taken from the same creeks and incubated under similar light conditions showed no nutrient effect on NCP (Spivak et al. 2016), suggesting that light limitation in the unamended creek may drive the observations in the benthic chambers. Water collected from the amended creek in August (during an incoming tide; Spivak and Ossolonski 2016) had nitrate concentrations of roughly $120 \mu \mathrm{M}$, while similar incoming tide water from the unamended creek had $5 \mu \mathrm{M}$ nitrate, but NCP was not significantly different between cores submerged beneath high or low nutrient water. In sediment cores from the creek mudflats in august, Chlorophyll- $a$ concentrations were around $15 \mathrm{mg} \mathrm{m}^{-2}$ in the nutrient amended creek and $35 \mathrm{mg} \mathrm{m}^{-2}$ in the unamended creek (Spivak and Ossolinski 2016; not significantly different in that study), so differences in primary producer biomass are probably secondary to light effects as well. In the natural creek environment during the chamber experiments, nutrients and benthic chlorophyll were likely near the 
expected levels as well; nitrate concentrations in two of the light chambers on August $28^{\text {th }}, 2013$ in the nutrient amended creek were between 80 and $170 \mu \mathrm{M}$, in excess of the expected nutrient amendment to $\sim 70 \mu \mathrm{M}$ (but similar to peak values previously observed near low tide because of difficulty metering the nutrient addition at very low creek volumes; Linda Deegan, 2014 TIDE Meeting). In 2008 and 2009, the last years for which data were reported by the TIDE project

(doi:10.6073/pasta/90cb9d605614ac3e6249de7ae2eb55cf), benthic chlorophyll was very similar in the mudflats of the two creeks; areal concentrations were within $15 \%$, with sediment Chlorophyll- $a$ concentrations around 20 to $30 \mathrm{mg} \mathrm{m}^{-2}$.

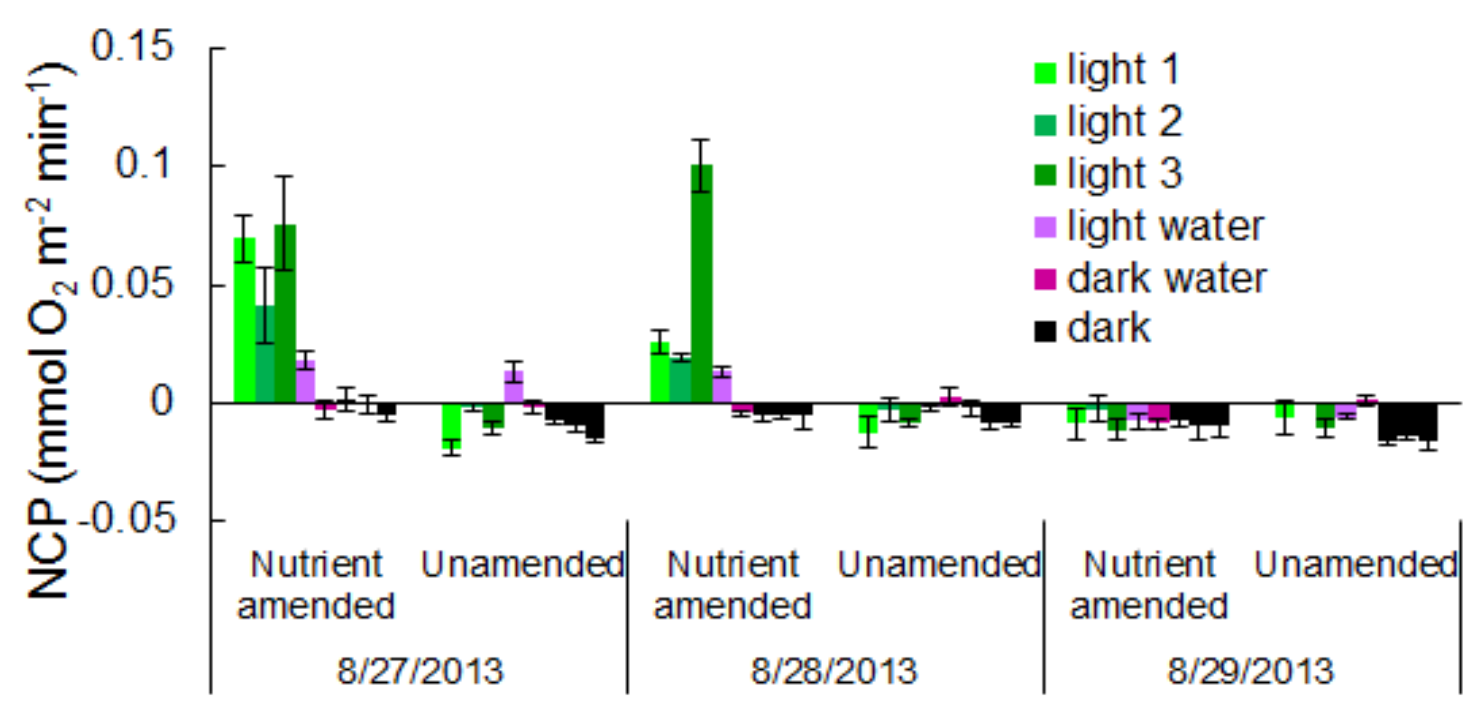

Figure 13: Net community production (NCP) rates at the nutrient amended and unamended control tidal creeks, for each day of the benthic chamber experiments (2013) based on a linear fit to the entrainment corrected concentration data (not the piecewise integration method for determining the rates). One standard error bars are based on the uncertainty of the slope fit. Chamber notation is identical to Fig. 12, including clear, ambient light sediment treatments ('light'), shrouded 'dark' sediment treatments, and light and dark treatments of chambers enclosing water only ('light water' and 'dark water').

Taken together, the similar dark and light chamber rates at the unamended control creek, similar NCP at both creeks on August $29^{\text {th }}$ (the cloudy day), and similar benthic chlorophyll at both creeks even with high nitrate concentrations at the nutrient amended creek, indicate that chamber production in the unamended creek was likely light limited compared to the nutrient amended creek and differences in NCP cannot be ascribed to nutrient treatment. The chamber locations in the creek bottoms were clear of any visible macroalgae, so primary production was most likely supported by benthic microalgae (eg. diatoms, cyanobacteria). While benthic microalgae in some coastal environments can adapt to maintain growth with PAR of only a few $\mu$ mol quanta $\mathrm{m}^{-2} \mathrm{~s}^{-1}$ (Cahoon 1999; Glud et al. 2002), intact sediment communities of benthic microalgae in unvegetated, low-porosity salt marsh sediments (tidal creeks, submerged mud flats) have saturation irradiances (below which a system is light limited) between 600 to $1200 \mu \mathrm{mol}$ quanta $\mathrm{m}^{-2} \mathrm{~s}^{-1}$, with some reported values in excess of $2000 \mu \mathrm{mol}$ quanta $\mathrm{m}^{-2} \mathrm{~s}^{-1}$ (Whitney and Darley 1983; Pinckney and Zingmark 1993, MacIntyre et al. 2006). These very high saturation irradiances in intact communities are thought to occur because of community structure and light penetration differences with depth in the sediment (MacIntyre et al. 1996). Based on the PAR record and measured NCP rates, it is plausible that the algal community at the unamended control site had 
consistently lower potential production than at the nutrient amended site, where midday PAR was near the likely saturating irradiance for the community. Given the equivalent rates of NCP (light chamber) and respiration (dark chambers) at unamended creek and in both creeks on the cloudy day, it is apparent that oxygen demand by the sediment community was also high compared to photosynthesis at these low light levels. The compensation irradiance (the PAR flux below which NCP is heterotrophic) for intact sediment microbial communities can be as high as $250 \mu \mathrm{mol}$ quanta $\mathrm{m}^{-2} \mathrm{~s}^{-1}$ (Gattuso et al. 2006); if the chamber enclosure or suspended sediments from stirring decreased light inside the chambers compared to open water then the enclosed sediments could have been pushed below this threshold.

NCP was positive in the light treatments at the nutrient amended creek on August 27 and $28(0.062 \pm 0.018$ and $0.049 \pm 0.045 \mathrm{mmol} \mathrm{O}_{2} \mathrm{~m}^{-2} \mathrm{~min}^{-1}$ ), when PAR during the experimental period was greater than 600 $\mu \mathrm{mol}$ quanta $\mathrm{m}^{-2} \mathrm{~s}^{-1}$ for most of the experimental period. The high coefficient of variation for the light treatments on August 27 and 28 ( 0.57 combined) indicates the patchiness of sediment production on the chamber scale. While more exaggerated because of the larger fluxes, this is no larger than the variability of oxygen fluxes in the dark treatments or light-limited light treatments ( 0.65 and 0.59 , respectively). The small NCP in the light and, particularly, dark water treatments compared to the sediment chambers indicates that sediment metabolism played a driving role in overall creek fluxes.

The dark treatment rates were at the low end for sediment oxygen demand in salt marsh sediments in general (eg. -0.3 to $-0.4 \mathrm{mmol} \mathrm{O}_{2} \mathrm{~m}^{-2} \mathrm{~min}^{-1}$ annual average; Gattuso et al. 1998, Middelburg et al. 2004). Making a more direct comparison to tidal creeks, in 2006 the rates of sediment oxygen demand from cores removed from the same tidal creek mud flats and incubated in a laboratory setting were -0.25 and $0.42 \mathrm{mmol} \mathrm{O}_{2} \mathrm{~m}^{-2} \mathrm{~min}^{-1}$ from the unamended control and nutrient amended creeks, respectively (not significantly different in that study; Koop-Jakobsen and Giblin 2010). The rates reported in this benthic chamber study are much lower, similar to rates in offshore coastal sediments (e.g Jahnke et al. 2000; $-0.02 \mathrm{mmol} \mathrm{O}_{2} \mathrm{~m}^{-2} \mathrm{~min}^{-1}$ ). Thus the dark treatment rates may be too low. Indeed, minimum gross primary production derived from adding NCP and sediment oxygen demand was not significantly different from light treatment NCP within errors, implying an unusually low fraction of respiration compared to production. NCP is usually significantly less than GPP, with NCP/GPP rarely observed to exceed 0.1 to 0.4 in benthic systems (Gattuso et al. 1998; Jahnke et al. 2000), similar to the theoretical ratio for phytoplankton of 0.3 to 0.4 (Halsey et al. 2010, 2014), though occasionally higher values are observed, eg. 0.7 in a shallow Arctic fjord (Attard et al. 2014). Oxygen consumption may have been too low for methodological reasons related to how I measured oxygen or to the chamber deployment, described below.

After inserting the sediment chambers into the sediments, the first measured timepoint in the dark treatments had oxygen concentrations near or below $50 \mu \mathrm{mol} \mathrm{kg}{ }^{-1}$. The measurement technique for oxygen relied on withdrawing water from the chamber and adding a small $(\sim 20 \mathrm{~mL})$ subsample to an open-topped insulated vial through a narrow tube for measurement. The dissolved oxygen was well below saturation, and oxygen would be added to the sample throughout the transfer and measurement. In practice, probe accuracy suffers at very low concentrations and sufficient oxygen could have been added to the sample to maintain the observed minimum concentration of $25-30 \mu \mathrm{mol} \mathrm{kg}{ }^{-1}$. The low concentrations in the dark treatments also complicated the interpretation of sediment oxygen demand because aerobic respiration nonlinearly decreases at low oxygen concentrations because of both respiratory kinetics (Longmuir 1954; Dromgoole 1978) and increasing thermodynamic favorability of other electron acceptors present in relatively high concentrations, such as nitrate (Brewer et al. 2014). Pointing to the possibility of this latter mechanism, higher sediment oxygen demand was observed when chambers maintained higher concentrations, as in the unamended creek on August 29. Additionally, large volume sediment cores removed from each site and incubated with creek water near saturation initially had sediment oxygen demand an order of magnitude higher than the chamber experiments, but these rates dropped off exponentially below $\sim 100 \mu \mathrm{mol} \mathrm{kg}{ }^{-1}$ (E. Howard and A. Spivak, unpublished data). Both lines of evidence suggest that the oxygen concentrations measured were not an adequate tracer of total 
respiration in the experiment dark treatments. The same arguments apply to the light chambers at similarly low concentrations. Thus NCP in the light limited chambers may have been even more heterotrophic than estimated here.

9.2 Gross primary production fluxes: In the absence of gas exchange and other physical fluxes, photosynthetic production can be solved for as a function of the oxygen concentration and the time evolution of $\delta^{17}$ and $\delta^{18}$ (Kaiser 2011; Prokopenko et al. 2011). Equation 42 of Kaiser (2011) can be rewritten in terms of the average isotopic composition between two sample timepoints as:

$$
G P P=\left[O_{2}\right]_{\text {avg }} \frac{\left(\frac{1}{1+\delta_{\text {avg }}^{17}}\right)\left(\frac{\partial \delta^{17}}{\partial t}\right)-\gamma_{R}\left(\frac{1}{1+\delta_{\text {avg }}^{18}}\right)\left(\frac{\partial \delta^{18}}{\partial t}\right)}{\left(\frac{\partial \delta_{P}^{17}-\delta_{a v g}^{17}}{1+\delta_{\text {avg }}^{17}}\right)-\gamma_{R}\left(\frac{\partial \delta_{P}^{18}-\delta_{\text {avg }}^{18}}{1+\delta_{\text {avg }}^{18}}\right)}
$$

where $\delta^{17}{ }_{P}$ and $\delta^{18}{ }_{P}$ define the endmember composition of purely photosynthetic oxygen. The values of the isotopic endmembers vary with the initial composition of the water split during Photosystem II (Luz and Barkan, 2000), as well as small fractionations during photosynthesis that are specific to algal clades photosynthesizing, eg. diatoms, green algae, and cyanobacteria (Helman et al. 2005; Eisenstadt et al. 2010; Luz and Barkan 2011). The initial oxygen isotope composition of the water in the tidal creek was not measured, however it can be estimated based on the isotopic composition of New England meteoric and river waters (Dutton et al. 2005; Li et al. 2015) and an estimate of the fraction of meteoric waters versus the seawater isotopic standard (Vienna Standard Mean Ocean Water, VSMOW) from salinity. The isotopic composition of local seawater is similar to VSMOW (water $\delta^{18} \cong-1, \delta^{17} \cong-0.5$ per mil versus VSMOW; Luz and Barkan 2010), though ongoing photosynthesis and respiration in the surrounding estuary has likely modified the water from this isotopic composition. ${ }^{17} \Delta_{\max }$ is calculated from $\delta^{17}{ }_{P}$ and $\delta^{18}{ }_{P}$ with $\gamma=0.5154$ (not $\gamma_{\mathrm{R}}$ ) and is the maximum observable ${ }^{17} \Delta$ in a system with both photosynthesis and respiration (Luz and Barkan 2000; Nicholson 2011b); ${ }^{17} \Delta_{\max }$ is plotted versus salinity in Fig. 14 for three clades of primary producers to illustrate the effect of initial water composition and photosynthetic fractionations on the production calculation (assuming $\delta^{18}{ }_{\text {meteoric }}=-8 \pm 5$ per mil versus VSMOW and ${ }^{17} \Delta_{\text {meteoric }}(\gamma=0.528)=37 \pm 4$ per meg). In calculating GPP, the community composition is assumed to be half diatoms and half cyanobacteria, which is consistent with the observed dominance of cyanobacteria and diatoms cultured from the creek bottoms at each site (glass plate and mesh screen substrates deposited on the creek bottom for roughly two weeks to collect organisms, which were then cultured and identified; K. Mackey and R. Stanley unpublished data).

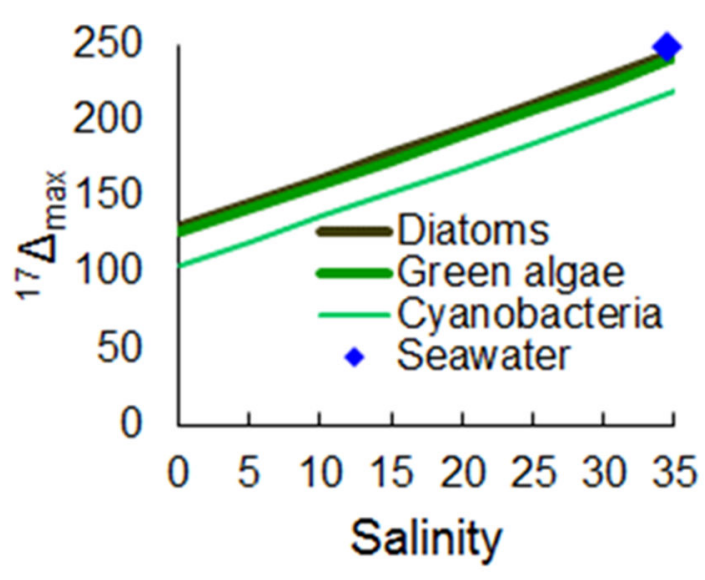

Figure 14: The maximum observable triple oxygen isotope tracer value in a system with concurrent photosynthesis and respiration, ${ }^{17} \Delta_{\max }$, a function of primary producer type and salinity of system. ${ }^{17} \Delta_{\max }$ determined for ocean phytoplankton communities is marked as blue point, and is close to the result of a pure diatom culture. Photosynthetic fractionations from Eisenstadt et al. (2010), Helman et al. (2005), and Luz and Barkan (2011), and initial composition of meteoric water assumed to be $\mathrm{S}_{\mathrm{A}}=0 \mathrm{~g} / \mathrm{kg}, \delta^{18}{ }_{\text {meteoric }}$ $=-8 \pm 5$ per mil versus Vienna Standard Mean Ocean Water $($ VSMOW $)$ and ${ }^{17} \Delta_{\text {meteoric }}(\gamma=0.528)=37 \pm 4$ per meg (Dutton et al. (2005), Li et al. (2015), Luz and Barkan (2011). $\mathrm{S}_{\mathrm{A}}, \delta^{18}{ }_{\text {meteoric }}$, and ${ }^{17} \Delta_{\text {meteric }}$ defined in text. VSMOW defined as $\mathrm{S}_{\mathrm{A}}=34.5 \mathrm{~g} / \mathrm{kg}, \delta^{18}=-23.324$ per mil and $\delta^{17}=-11.883$ per mil versus air. 
Eq. 9 is discretized as in Stanley and Howard (2013), with the exception that the average isotopic composition is used for rate calculations as above. I make this change in order to minimize the systematic error caused by pairing the instantaneous oxygen concentration and isotopic composition with the central difference approximation of the $\delta^{17}$ and $\delta^{18}$ time rate of change terms. When calculating the isotope derivatives with time, the isotopic composition at the preceding timestep $\delta_{t-1}^{x}$ is corrected for entrainment of ambient creek water in the same manner as oxygen according to the mass balance:

$$
\delta_{t-1}^{x}\left[O_{2}\right]_{t-1}=\delta_{t}^{x}\left[O_{2}\right]_{t}\left(1-f_{e x}\right)+\delta_{w, t-1}^{x}\left[O_{2}\right]_{w, t-1}\left(f_{e x}\right)
$$

The notation is identical to the oxygen mass balance in Eq. 8. The piecewise GPP rates are integrated over each timestep and summed to determine an average rate over the experimental period.

Gross primary production from the triple oxygen isotopes is listed in Table 1 with NCP evaluated in identical fashion over the shorter time period for which oxygen isotope ratios were sampled. A linear fit as previously used with oxygen to determine NCP was not appropriate for determining GPP because the isotope ratios and resultant production varied nonlinearly with time. August 27 and 28 GPP at the nutrient amended creek was 2.6 times higher than at the unamended creek (mean and standard deviation for both days at amended [ $\mathrm{n}=6]$ and unamended [ $\mathrm{n}=3$ ] creeks $0.059 \pm 0.012$ and $0.023 \pm 0.010 \mathrm{mmol} \mathrm{O}_{2} \mathrm{~m}^{-2} \mathrm{~min}^{-1}$ respectively). GPP scales with absorbed light in laboratory cultures of planktonic diatoms and green algae (Halsey et al. 2013), which in turn depends on the incident PAR flux (in addition to the chlorophyll content and adsorption characteristics of the cells). Over hourly periods, GPP by benthic microalgae in another New England estuary closely tracks incident PAR flux in situ as well (Stanley and Howard 2013), however the sandy site in that study may have different production dynamics than the very different environment in tidal creeks.

Based on the above observations, it may be that GPP can be adjusted for differences in light availability in order to provide an ex post facto estimate of the differences in productivity between the creeks that are not explained by light differences between the creeks. This approach was not possible using oxygen concentration based metabolism because NCP could not be distinguished from respiration in the unamended tidal creek, but the triple oxygen isotopes allow GPP measurement even in the light limited system. In the analysis for NCP above, I suggested that the sediment community in the unamended creek is likely below saturating irradiances, while the nutrient amended creek is plausibly near the community saturating irradiance. GPP over the range of irradiances between 0 and saturating irradiance can be approximated as linearly related to PAR, and scaled to the expected GPP across varying light up to the saturating irradiance. This technique has significant uncertainties as the saturating irradiance for the tidal creek community is unknown and light is not constant, and likely overestimates extrapolated GPP on the order of 10 to $20 \%$ as the saturating irradiance is approached depending on the specific parameters of the photosynthesis irradiance curve. Testing this approach on the Stanley and Howard (2013) dataset (in a very different, sandy system) leads to scaled results similar to observations (within one standard error) over the range of PAR below $1200 \mu \mathrm{mol}$ quanta $\mathrm{m}^{-2} \mathrm{~s}^{-1}$. Despite the difficulties mentioned above, this approach may be a practical stand in absent more detailed information on the relationship between light and production during the experimental periods and assuming that the photosynthetic slope $(\alpha)$ does not change to a great degree over the experimental period. However, this may be a poor assumption for benthic microalgae in a tidally varying system (Blanchard and Montagna 1992). 
Table 1: Piecewise integrated rates of gross primary production (GPP) derived from triple oxygen isotopes and of net community production (NCP) from oxygen concentrations over the time range for which GPP can be estimated in each chamber. Apparent GPP is lower than NCP at four of the 6 light treatments at the nutrient amended creek. GPP rates are normalized to photosynthetically active radiation (PAR) flux over the entire chamber deployment period in order to estimate the amount of creek to creek GPP difference that may be accounted for by light differences.

\begin{tabular}{|c|c|c|c|c|}
\hline \multirow{6}{*}{ 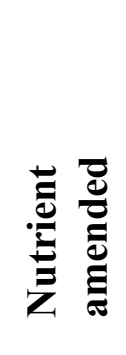 } & Chamber & $\begin{array}{c}\text { Time } \\
8 / 27 / 2013\end{array}$ & \multicolumn{2}{|c|}{$\left(\mathrm{mmol} \mathrm{O} \mathrm{m}^{-2} \min ^{-1}\right)$} \\
\hline & Light 1 & $10: 19$ to $13: 32$ & $0.049 \pm 0.006$ & $0.080 \pm 0.008$ \\
\hline & Light 2 & $11: 10$ to $14: 06$ & $0.062 \pm 0.007$ & $0.064 \pm 0.008$ \\
\hline & Light 3 & $11: 27$ to $14: 18$ & $0.055 \pm 0.006$ & $0.104 \pm 0.008$ \\
\hline & Mean (Light 1-3) & & $0.056 \pm 0.007$ & $0.083 \pm 0.020$ \\
\hline & PAR normalized & & $0.069 \pm 0.008$ & \\
\hline & Light 2 & $10: 16$ to $13: 43$ & $0.016 \pm 0.002$ & $0.000 \pm 0.008$ \\
\hline & Light 3 & $10: 35$ to $13: 59$ & $0.018 \pm 0.002$ & $-0.006 \pm 0.009$ \\
\hline & Mean (Light 2-3) & & $0.017 \pm 0.001$ & $-0.003 \pm 0.005$ \\
\hline & PAR normalized & & $0.028 \pm 0.002$ & \\
\hline
\end{tabular}

\begin{tabular}{|c|c|c|c|c|}
\hline \multirow{6}{*}{ 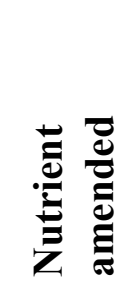 } & \multicolumn{3}{|c|}{$8 / 28 / 2013$} & \\
\hline & Light water & $10: 41$ to $14: 53$ & $0.053 \pm 0.005$ & $0.014 \pm 0.008$ \\
\hline & Light 1 & $11: 00$ to $15: 02$ & $0.058 \pm 0.008$ & $0.024 \pm 0.008$ \\
\hline & Light 2 & $10: 23$ to $14: 40$ & $0.047 \pm 0.006$ & $0.018 \pm 0.008$ \\
\hline & Light 3 & $11: 38$ to $14: 28$ & $0.081 \pm 0.012$ & $0.118 \pm 0.008$ \\
\hline & Mean (Light 1-3) & & $0.062 \pm 0.017$ & $0.054 \pm 0.056$ \\
\hline & PAR normalized & & $0.062 \pm 0.017$ & \\
\hline ְِ & Light water & $10: 26$ to $14: 59$ & $0.007 \pm 0.001$ & $-0.002 \pm 0.008$ \\
\hline$=$ & Light 1 & $11: 21$ to $13: 59$ & $0.034 \pm 0.006$ & $-0.013 \pm 0.008$ \\
\hline $\bar{F}$ & PAR normalized & & $0.057 \pm 0.010$ & \\
\hline g & & $8 / 29 / 2013$ & & \\
\hline 8 & Light 3 & $12: 21$ to $15: 27$ & $0.015 \pm 0.004$ & $-0.003 \pm 0.008$ \\
\hline$\underset{\Xi}{E}$ & PAR normalized & & $0.084 \pm 0.020$ & \\
\hline
\end{tabular}

Applying this light scaling approach thus likely provides an upper bound on the effect of light differences on production. Normalizing GPP by the PAR flux over each experimental period relative to that at the nutrient amended creek on August 28 (the peak PAR flux of the 2013 experiments, $\sim 1200 \mu$ mol quanta $\mathrm{m}^{-2} \mathrm{~s}^{-1}$ ) results in GPP on August 27 and 28 of $0.066 \pm 0.013$ and $0.038 \pm 0.017 \mathrm{mmol} \mathrm{O}_{2} \mathrm{~m}^{-2} \mathrm{~min}^{-1}$ at the amended and unamended creeks. In other words, with the largest possible effect ascribed to differences in light between the creeks, GPP at the nutrient amended creek was on average 1.7 times greater than at the unamended creek, a significant difference (Student's T-test, $\mathrm{p}=0.04$ ). The rate from the unamended creek on August 29 was only between the initial two timepoints, rather than over 3-4 timepoints as with the 
other rates - nonetheless it is interesting to note that the PAR normalized rate for this chamber was actually the highest of any chamber at either creek, perhaps indicating that the community generates oxygen more efficiently relative to PAR during lower light conditions (as observed by Fisher and Halsey 2016).

Differences in available light may have played a major role in the GPP differences between the two tidal creeks, but even after adjusting for light differences, production in the creek with added nutrients was nearly twice as large as in the creek with ambient nutrient concentrations. While the PAR normalized GPP rates at each creek were significantly different, the mean values had overlapping one standard deviation error bars, and the difference on August 27 and 28 should be interpreted with caution. Also, spatial heterogeneity in production could explain the differences in the mean rates. But given the otherwise similar hydrographic and characteristics and microbial community composition of the tidal creeks (Deegan et al. 2007, Bowen et al. 2011) and assumptions related to nutrients and benthic chlorophyll above, it is possible that the increased photosynthetic production of oxygen was the result of the nutrient enrichment experiment.

In the work of Spivak and Ossolinski (2016), NCP from ${ }^{13} \mathrm{C}$ labeled bicarbonate uptake by the sediment community was $0.004 \mathrm{mmol} \mathrm{C} \mathrm{m}^{-2} \mathrm{~min}^{-1}$ (mean of sediment cores from both creeks, which were not significantly different in that study), an order of magnitude lower than the oxygen-derived rates in the nutrient amended creek in this study; at that lower rate, concentrations of nitrate were sufficiently high to support expected nitrogen demand to produce organic matter at the Redfield ratio in the nutrient amended creek (in August), but not in the unamended creek. While it may be expected that most of the nutrient supply in the chambers is met by sediment recycling of ammonia, it is possible that nitrate directly or indirectly (through nitrogen cycle reprocessing; Koop-Jakobsen and Giblin 2009, 2010) contributes to nitrogen availability to support GPP in the sediment chambers. Further, the lack of clear nutrient driven differences in NCP (section 9.1) does not preclude a nutrient effect on GPP. For example, GPP normalized to absorbed light (dependent on incident PAR flux) is independent of cellular growth rate for some planktonic algae (Halsey et al. 2013, 2014) in nitrogen limited culture, while NCP varies with both growth rate and oxidation of terminal metabolic products from anaerobic respiration by the rest of the microbial community. This is consistent with the results of the 2012 study in this work that suggested while on average there was no difference in NCP between creeks, there was enhanced production and respiration over specific tidal stages in the nutrient fertilized creek despite no overall difference in NCP (though again in the case of the 2012 data grass may have contributed production and has previously been shown to be nutrient limited in this setting).

9.3 Comparison of fluxes and complicating processes: While gross primary production (integrated piecewise between timepoints) should be consistent for comparison between chambers, GPP does not appear to be consistent with NCP calculated in the same manner over the same time intervals. For example, while the net flux can only become more negative than the gross flux with increasing respiration or decreasing photosynthesis, the calculated NCP was greater than GPP at the nutrient amended creek for all light treatment chambers on August 27 and one of the three on August 28. The measurement uncertainties and replicate standard deviations for both rates were relatively well constrained, so a systematic error is the most likely cause for this discrepancy. Two processes that could potentially help explain the observations are a) tidally forced porewater advection through the creek bottom sediments, and b) reaching ${ }^{17} \Delta_{\max }$ within the chambers during the experiment. Below I discuss each in detail, but the latter may be more likely - indeed, the triple oxygen isotope measurements suggest a new method of determining photosynthetic fractionations of the triple oxygen isotopes in a natural setting.

Models of porewater flow driven by changes in water depth (Nuttle 1988; Wilson and Morris 2012) predict peak porewater flow out of the creek bottom sediments near low tide. A simple 1D porewater model of the study creeks (after Nuttle 1988) predicts peak low-tide porewater discharge into the creek on the order of 5-15 L per meter of creek. While only a small fraction of that would be transported through 
the creek bottom in the center of the channel (Gardner 2005), this could have been sufficient to replace up to $1 \%$ of the chamber volume over the experimental time period, with overlying water pushed out through the sample replacement volume intake port. Chamber stirring might also enhance porewater exchange. Replacing chamber volume with porewater would not necessarily invalidate the NCP estimates, as any oxygen deficit introduced with the porewater represents respiration in the sediments.

The relationship between the three stable oxygen isotopes is not conserved during mixing, and mixing water parcels with different oxygen concentration can lead to deviations in ${ }^{17} \Delta$ and GPP (Nicholson et al. 2014). $5-20 \%$ of the chamber volume would have to be replaced to cause changes in ${ }^{17} \Delta$ larger than the uncertainty in the measurement, depending on the porewater $\mathrm{O}_{2}$. However, the uncertainty and methodological errors associated with the measurement of the triple oxygen isotopes become quite large at low $\mathrm{O}_{2}$, so changes in porewater exchange could still bias the triple oxygen isotope method. Sediment isotopic fractionations could also modify the measured triple oxygen isotope ratios. If all the oxygen entering the sediments is completely consumed, no additional fractionation processes should effect the oxygen isotopic ratios in the water column. However, even though direct effects of enhanced oxygen uptake by bioturbation and bioirrigation are integrated into any net oxygen fluxes, these processes could help extend oxygen gradients deeper into the sediments (Koretsky et al. 2002) and allow partial consumption of oxygen in the sediments. Infauna abundance is not significantly different in the two tidal creeks (Pascal et al. 2013), but in either creek water flushed through irrigated sediments and returned to the overlying creek could both enhance porewater exchange and allow for incomplete consumption of $\mathrm{O}_{2}$ by sediment processes (e.g. oxidation of reduced compounds). The triple oxygen isotope systematics of such processes have not been evaluated and could modify the interpretation of GPP.

Apparent GPP could also be reduced if the chamber ${ }^{17} \Delta$ approached ${ }^{17} \Delta_{\text {max }}$. This occurs when nearly all of the dissolved oxygen in a chamber is produced from photosynthesis, and once this condition is reached the dissolved oxygen isotope ratios remain proportionally constant and are no longer effective tracers of photosynthesis. Oxygen concentrations in the light treatments that were not light limited doubled or tripled over the course of the experiment, so this was a distinct possibility. Appendix I: Table A2 lists estimated ${ }^{17} \Delta_{\max }$ for each date and creek. I find that in general the chambers in which oxygen based NCP exceeded triple oxygen isotope derived GPP (suggesting a possible bias to GPP) had ${ }^{17} \Delta$ that approached the estimated ${ }^{17} \Delta_{\max }$ early in the experiment (within one to two hours of the initial timepoint). Given the spatial heterogeneity of rates, it is plausible that the benthic community structure and thus ${ }^{17} \Delta_{\max }$ varied between chambers (e.g. by up to $\sim 25$ per meg if comparing a patch of primarily diatoms with one of primarily cyanobacteria). Most light treatments at the nutrient amended creek appear to reach an asymptotic maximum within one to two standard deviations of predicted ${ }^{17} \Delta_{\max }$. Therefore I may underestimate GPP in the nutrient amended creek via this mechanism even when triple oxygen isotopederived GPP exceeds oxygen concentration-derived NCP. Excluding later timepoints in order to calculate GPP only when the triple oxygen isotope tracer was most sensitive does not consistently lead to higher GPP. For example, the higher oxygen concentrations later in the experiment meant that calculated GPP was higher between timepoints near the end of the experiment than near the middle of the experiment in four of the six light treatments (at either creek) for which I have multiple piecewise rates to compare.

This asymptotic behavior of ${ }^{17} \Delta$ during high production makes it difficult to use the triple oxygen isotope ratios for measuring rates in such circumstances. However, this exact effect could be very useful to the triple oxygen isotope community. If chamber incubations in natural settings are manipulated so as to maximize the proportion of $\mathrm{O}_{2}$ from photosynthesis, the observed ${ }^{17} \Delta_{\max }$ could be combined with measurements of water oxygen isotope ratios to measure the photosynthetic fractionation of oxygen by the in situ sediment community. This could be an improvement over existing techniques, which measure $\mathrm{O}_{2}$ evolved from phytoplankton cultures in near anoxic culture (Helman et al. 2005, Luz and Barkan et al. 2011); such anoxic environments may alter production and the resulting measurements with very small amounts of $\mathrm{O}_{2}$ for analysis may suffer from additional uncertainties as discussed above. 
Both porewater advection into the chambers from the underlying sediments and loss of sensitivity of the triple oxygen isotope tracer as the system approached ${ }^{17} \Delta_{\max }$ could explain negative errors that contributed to decreased GPP and the discrepancy with oxygen concentration-based NCP rates. However, neither is entirely consistent with the observations. A remaining mechanism that could drive changes in the relationship of the oxygen isotope ratios is if the fractionation slope of ${ }^{17} \mathrm{O}$ relative to ${ }^{18} \mathrm{O}$ is larger during oxygen consuming porewater processes than that measured for respiration-in this case the ${ }^{17} \Delta$ calculated from the triple oxygen isotope system would be too large and would not correctly remove the massdependent fractionation associated with various respiration processes. The respiration fractionation slope appears to be remarkably stable across the range of organisms and aerobic respiration pathways measured (Angert et al 2003; Luz and Barkan 2005, 2009). However, there are no published measurements of triple oxygen isotope fractionations of dissolved oxygen in sediment porewaters, or in the oxygen pool during common sedimentary processes such as sulfide oxidation. It is unclear how large an error this might introduce; study of oxygen isotope systematics in porewaters may be a particularly relevant problem moving forward as the triple oxygen isotope tracer is used to study productivity in shallow and nearshore environments (Sarma et al. 2008, Yeung et al. 2012, Munro et al. 2013, Stanley and Howard 2013) where sediment-water interactions may play a significant role.

\section{Conclusion}

Both the 2012 and 2013 experiments illustrate the role of the dynamic physical environment in controlling oxygen fluxes and biological productivity in salt marsh tidal creeks. In particular, tidal cycling plays a major role in controlling fluxes of oxygen through the system, and net community production is a small but important flux overlaying the physically driven cycling. In the 2013 benthic chamber experiments, the light differences between the study sites were an important control on dissolved oxygen concentrations and triple isotope ratios. Sediment production fluxes were heterogeneous within the tidal creeks, but there were also broad similarities at the whole system level between creeks. I hypothesized that nutrient additions to salt marsh tidal creeks at the watershed level would enhance creek primary production and alter the net metabolic balance between autotrophic and heterotrophic processes. Neither experiment provided unequivocal evidence for a nutrient amendment effect on net community production or gross primary production in the submerged creek environments. However, the hypothesis cannot be dismissed entirely either; even though mean NCP was similar between the creeks over the evaluated periods, both the open water (2012) and benthic chamber (2013) studies pointed to potential enhancement of GPP in the nutrient amended creek, which may have been balanced by enhanced respiration in order to lead to no net change in NCP. In the following paragraphs, I compare the results of the two experiments (using the base case for gas exchange in the open water work), compare NCP estimates to the literature, and evaluate the results with respect to the TIDE nutrient enrichment experiment. I conclude with key findings and recommendations for improvement in similar studies and directions for future research.

10.1 Tidal creek study comparison: The open water (2012) and benthic chamber (2013) studies are complimentary for studying the net community production in the integrated creek systems and creek sediments. While creek bank and bottom sediments are an important interface in the tidal creek system because of the large and tidally varying surface area to volume ratio, the open water fluxes are not necessarily directly comparable to the benthic chamber sediment fluxes. The benthic chambers integrate over a small area in a heterogeneous sediment setting, and average fluxes can be biased by limited replication. The open water NCP has large fluxes and uncertainties associated with physically driven oxygen fluxes, and integrates additional sources and sinks of oxygen on the marsh platform and creek banks, such as production by filamentous green algae and Spartina alterniflora. Both studies measure only subaqueous production and respiration. This is a particularly important limitation as production on the banks may peak when the sediments are not submerged. For example, benthic diatoms are known to migrate vertically in sediments in order to optimize light exposure, and may move to the surface during 
daytime low tides (Round and Palmer 1966; Happey-Wood and Jones 1988; Consalvey et al. 2004 and references therein). Finally, while the same nutrient amended site was used for both studies, the 2013 unamended site was in a different, much narrower branch of the unamended creek. Despite these limitations, the two studies have important similarities and differences in estimated NCP.

In the 2012 open water study over the days with data at both creeks, using the base case for gas exchange, average NCP was $-0.13 \pm 0.04 \mathrm{mmol} \mathrm{O}_{2} \mathrm{~m}^{-2} \mathrm{~min}^{-1}\left(-190 \pm 60 \mathrm{mmol} \mathrm{O}_{2} \mathrm{~m}^{-2} \mathrm{~d}^{-1}\right)$ at both creeks but with distinct daily variations. Over $10 \mathrm{am}-3 \mathrm{pm}$, roughly the experimental period for the three August 2013 experiments, average NCP was $-0.15 \pm 0.05$ and $-0.16 \pm 0.03 \mathrm{mmol} \mathrm{O}_{2} \mathrm{~m}^{-2} \mathrm{~min}^{-1}$ at the nutrient amended and unamended creeks, respectively over the experimental period. This was significantly more heterorophic than the autotrophic rates in the 2013 light treatments that were less likely to be light limited $\left(0.062 \pm 0.018\right.$ and $0.049 \pm 0.045 \mathrm{mmol} \mathrm{O}_{2} \mathrm{~m}^{-2} \mathrm{~min}^{-1}$ on August 27 and 28 at the nutrient amended creek only). The 2013 dark treatment rates and NCP from the light limited experiments were an order of magnitude smaller than open water NCP, at roughly $-0.01 \mathrm{mmol} \mathrm{O}_{2} \mathrm{~m}^{-2} \mathrm{~min}^{-1}$, but it is likely that NCP was biased towards zero in those treatments (see Discussion: Part 2). Creek bank porewater drainage and incorporation of more of the heterotrophic biological community (eg. grazers) may explain a more heterotrophic open water (2012) NCP compared to the chamber experiments (2013) that are not light limited. The rates may be more comparable than the averages suggest as well. Daily variability was quite large in the 2012 rates, ranging between approximately -0.4 and $0 \mathrm{mmol} \mathrm{O}_{2} \mathrm{~m}^{-2} \mathrm{~min}^{-1}$, with some early afternoon peak production rates of $0.1 \mathrm{mmol} \mathrm{O}_{2} \mathrm{~m}^{-2} \mathrm{~min}^{-1}$ or greater, encompassing the range of the benthic chamber experiments. In addition, the experiments were a year apart, and temperature, salinity, nutrient concentrations, and PAR were not identical. While incident PAR on the creek platform was similar between years $\left(\sim 1600 \mu \mathrm{mol}\right.$ quanta $\mathrm{m}^{-2} \mathrm{~s}^{-1}$, with some cloudier days), I do not have site-specific data for the average PAR reaching the creek bottom, which is particularly relevant for comparing the unamended creek data. Thus some disagreement is to be expected based on environmental variability, and it is encouraging that the data ranges overlap for the two studies.

10.2 Comparison to literature values: In the interest of clarity, I primarily compare net community production in the two studies presented here to oxygen-based literature rates for salt marsh and estuarine environments. Converting to carbon units requires some assumptions regarding the carbon to oxygen stoichiometry of various aerobic and anaerobic metabolic processes (Hopkinson et al. 1999), and ratios may range from 0.8-1.4 for various processes (Laws 1991; Jahnke et al. 2000; Ní Longphuirt et al. 2007). An oxygen to carbon ratio of $1: 1$ for NCP is commonly assumed in the estuarine literature. Daily average NCP from the open water (2012) study $\left(-0.13 \pm 0.04 \mathrm{mmol} \mathrm{O}_{2} \mathrm{~m}^{-2} \mathrm{~min}^{-1}\right.$ for both creeks) falls within the range of literature values for salt marsh tidal creeks and channels $\left(-0.14\right.$ to $+0.4 \mathrm{mmol} \mathrm{O}_{2} \mathrm{~m}^{-2} \mathrm{~min}^{-1}$; Vallino et al. 2005; Cebrian et al. 2007; Nidzieko et al. 2014), and salt marsh dominated estuaries ( -0.16 to $-0.08 \mathrm{mmol} \mathrm{O}_{2} \mathrm{~m}^{-2} \mathrm{~min}^{-1}$; Caffrey 2004; Gazeau et al. 2007; Cai 2011). The most methodologically comparable studies to the open water work, by Vallino et al. (2005) in the nearby Parker River and by Nidziko et al. (2014) in Elkhorn Slough, California, had average daily rates of roughly $-0.05 \mathrm{mmol} \mathrm{O}_{2}$ $\mathrm{m}^{-2} \mathrm{~min}^{-1}$ each, and peak daily autotrophy of 0.2 to $0.3 \mathrm{mmol} \mathrm{O}_{2} \mathrm{~m}^{-2} \mathrm{~min}^{-1}$ (versus roughly 0.1 in this study). Midday benthic metabolism from the benthic chambers that were not light limited (0.05 to 0.06 $\mathrm{mmol} \mathrm{O}_{2} \mathrm{~m}^{-2} \mathrm{~min}^{-1}$ ) are an order of higher than $\mathrm{NCP}$ calculated from ${ }^{13} \mathrm{C}$ bicarbonate uptake by primary producers in sediment cores taken from the bottom of the same creeks in August (Spivak and Ossolinski 2016), but are consistent with sediment chamber fluxes in Gulf Coast salt marsh tidal creeks $(\sim 0.03$ mmol O $\mathrm{O}_{2}^{-2} \mathrm{~min}^{-1}$; Cebrian et al. 2007) and a coastal site off Georgia (0.02 to $0.09 \mathrm{mmol} \mathrm{O}_{2} \mathrm{~m}^{-2} \mathrm{~min}^{-1}$; Jahnke et al. 2000). The generally good agreement between both studies in this work and literature NCP supports the conclusion that tidally flushed channels in salt marshes are often net heterotrophic, with brief daytime autotrophy and large diel variability.

One additional interesting feature of the 2012 open water data is that nightime dark respiration, estimated from average $\mathrm{NCP}$ between $6 \mathrm{pm}$ and 6am, was less heterotrophic than the average daytime rates $(-0.11$ to $-0.12 \mathrm{mmol} \mathrm{O}_{2} \mathrm{~m}^{-2} \mathrm{~min}^{-1}$ from $6 \mathrm{pm}$ to 6 am versus -0.15 to $-0.16 \mathrm{mmol} \mathrm{O}_{2} \mathrm{~m}^{-2} \mathrm{~min}^{-1}$ from $10 \mathrm{am}$ to 
$3 \mathrm{pm})$, while the most negative NCP rates were between dawn and 9-10am $\left(<-0.2 \mathrm{mmol} \mathrm{O}_{2} \mathrm{~m}^{-2} \mathrm{~min}^{-1}\right)$. Respiration cannot be distinguished from NCP in the open water study, and the discrepancy between GPP and NCP in the 2013 chamber data and possibly biased dark treatment rates (an order of magnitude lower than the 2012 overnight rates) preclude direct comparison of respiration in the light versus dark. Nonetheless, the results of the open water study could support the interpretation that light respiration exceeded dark respiration by a factor of two or more in this system, similar to literature observations in other systems. Night versus daytime dark treatment benthic chamber experiments by Jahnke et al. (2000) in a shallow shelf setting similarly found nighttime fluxes two times smaller than daytime fluxes. It is unclear how the expected light enhancement of mitochondrial respiration, photorespiration, and photochemical oxidation (Fenchel and Glud 2000) could explain the differences in fluxes in the dark treatments of the Jahnke et al. (2000) study, perhaps indicating the important role of endogenous circadian rhythms in benthic metabolic fluxes (Cosper 1982; Ashworth et al. 2013). On the other hand, higher $\mathrm{O}_{2}$ consumption over that period may reflect built up oxygen demand from reduced compounds released into the water column from the sediments.

10.3 Relevance to TIDE study: One important result from the first decade of the TIDE experiment is that nutrient amendment alters production and respiration on a large scale on the marsh platform. At the nutrient amended creek, the combination of altered distribution of Spartina production and biomass, increased aerobic respiration, and increased denitrification all on the marsh platform may act to decrease organic matter burial and accelerate mechanical breakdown of marsh sediments. Such a mechanism is proposed to explain observed geomorphological changes including creek bank fracturing and collapse at the nutrient amended creek (Deegan et al 2012). An important and related question is whether production and respiration are similarly altered in the submerged tidal creek environments. This submerged environment is the interface between the introduced nutrients and the creek banks and marsh platform for much of the tidal cycle. Thus a nutrient amendment effect on creek water column and sediment metabolic fluxes would be particularly compelling.

However, the two studies presented in this work provide mixed evidence about whether nutrient amendment altered metabolic fluxes in the tidal creek. Whole system net community production was not different between the nutrient amended and unamended creek sites in the open water study (2012). But, there were indications that at least over short periods and specific tidal stages, both production and respiration were enhanced, and that these fluxes could have balanced out to no net change in NCP over the daily timescales evaluated. At different sites in the same creek watersheds, the benthic chamber experiments (2013) were likely influenced to a large degree by light differences between the creek sites. Tantalizingly, the comparison of triple oxygen isotope derived gross primary production normalized to incident PAR indicated 1.7 times greater GPP in the nutrient amended creek than the unamended control creek. This ratio is identical to the increase in potential denitrification in creek bank sediments (Deegan et al. 2012) and similar to the increase in marsh platform and creek bottom sediment denitrification (Koop-Jakobsen and Giblin 2009, 2010), which may imply links between the magnitude of production and nitrogen cycle changes within the creeks. It is also similar to the estimated doubling in seasonal GPP in the nutrient amended creek sediments relative to the unamended control over the first two years of the TIDE experiment based on a chlorophyll and irradiance based model of benthic microalgal production (Bowen et al. 2009). However, the average, PAR normalized GPP rates overlapped within the one standard deviation bounds for the rates at each creek, and the oxygen isotope tracer results must be interpreted cautiously because of the unexpected bias relative to rates derived from oxygen concentrations.

Thus the results of this work could be interpreted both in support of and against the mechanism posited by Deegan et al. (2012). While marsh platform processes can drive the observations of the TIDE experiment, it is perhaps surprising that NCP in the nutrient amended tidal creek is not clearly altered by the exposure and oxidation of buried organic matter from collapsing creek banks. Results of investigations on the creeks' microbial communities may help explain this discrepancy: Bowen et al. (2011) found that 
microbial community composition resisted change during long-term nutrient amendment (though the active community may be changing; Kearns et al. 2016), and in addition to the estimated change in seasonal GPP, bacterial production increased by a similar proportion in the same creek environments and was most likely supported by labile carbon from the benthic microalgae (Bowen et al. 2009). Close coupling of carbon fluxes between benthic microalgae and the bacterial community was similarly reported by Spivak and Ossolinski (2016). Thus respiration might be expected to increase proportionally with production without changing NCP. As noted above, chamber GPP and open water high tide only oxygen fluxes are consistent with this sort of dynamic metabolic balance. It is also likely that rapid tidal flushing and exchange with the broader estuary obscures any small, long-term changes in creek metabolism relative to the large daily NCP fluxes. In the short time series for which data are available at both creeks, cumulative NCP is not distinguishable between the nutrient amended and unamended tidal creeks. In this case a seasonal time series may be essential in order to determine whether NCP diverges between creek treatments.

\subsection{Key findings and recommendations:}

Findings:

- There is no clear nutrient amendment effect on mean daily subaqueous net community production (NCP), but at high tides both production and respiration may be enhanced at the nutrient amended creek in a manner that leads to little net change in NCP. Triple oxygen isotope derived gross primary production (GPP) rates may indicate increased GPP with fertilization as well.

- Both tidal creeks in this study are on average heterotrophic over daily to weekly timescales, but with large daily variability. Light limitation is an important control on sediment NCP and GPP. Tidal creek respiration in the light is likely at least double that in the dark.

- Noble gases are promising tracers of bubble and gas exchange fluxes in salt marshes, but reflect the complex history of exchange between creek water, porewater, and the atmosphere over multiple scales of time and space. Gas exchange parameterizations dependent primarily on current speed are consistent with noble gas dynamics in the tidal creeks.

Recommendations:

- Each study type (open water mass balance, benthic chamber fluxes) has different sensitivities, uncertainties, and particularly complications in complex estuarine environments. Ideally, multiple approaches should be employed in order to investigate production, including subaerial fluxes which both these approaches miss.

- For open water mass balances, accurate water fluxes are necessary to distinguish biological fluxes from potentially larger physical fluxes of oxygen. Sufficiently sensitive sensors or tracers of advection, porewater exchange, and gas exchange as applicable should be deployed or measured during salt marsh studies. Seasonal time-series may be necessary to distinguish the net effect of ecosystem scale manipulations.

- When studying metabolic rates in benthic chambers, a goal should be to avoid so much oxygen turnover through photosynthesis that the triple oxygen isotope tracer is no longer an effective tracer of GPP. However, at the extreme where nearly all oxygen in a chamber is derived from photosynthesis, the triple oxygen isotope systematics could be exploited to study the photosynthetic oxygen isotope fractionations in intact sediment communities.

- The porewater systematics of triple oxygen isotope ratios and noble gas concentrations are virtually unknown, and must be studied to use these tracers carefully and effectively in the salt marsh. Triple oxygen isotopes in particular are sensitive to even small changes in mass-dependent fractionation. Dissolved oxygen isotope fractionations associated with chemical oxidation of reduced compounds like sulfide have not been studied, and may be different than typical aerobic respiration. 
Appendix I

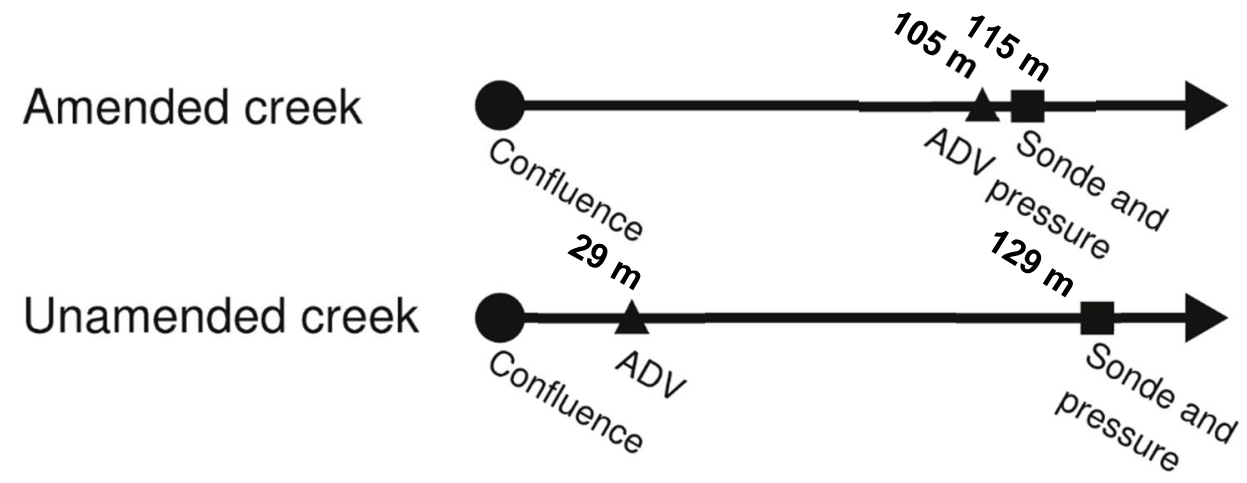

Figure A1: Locations of instruments in each tidal creek relative to confluence with next primary tidal creek. Arrows point upstream. 
Table A1: Noble gas concentrations of nutrient amended tidal creek(nmol kg ${ }^{-1}$ for $\mathrm{He}, \mathrm{Ne}, \mathrm{Kr}, \mathrm{Xe}$, and $\mu \mathrm{mol} \mathrm{kg}^{-1}$ for Ar) and saturation states (\%) from the water column (2012) experiments and follow-up sampling in Oct. 2013 and Oct. 2014. Minimum error bounds for accuracy and precision of this dataset are $0.2 \pm 0.2 \%$ of saturation ( $\mathrm{He}, \mathrm{Ne}, \mathrm{Kr}$ ), and $0.2 \pm 0.1 \%$ of saturation ( $\mathrm{Ar}, \mathrm{Xe}$ ) (W. Jenkins, personal communication). Salinity and temperature are reported to unit of precision for each sample. Values for unamended tidal creek follow on next page.

\begin{tabular}{|c|c|c|c|c|c|c|c|c|c|c|c|}
\hline & Tide stage & Time & & Location & $\begin{array}{l}\text { Temperature } \\
\left({ }^{\circ} \mathrm{C}\right)\end{array}$ & $\begin{array}{l}\text { Absolute salinity } \\
(\mathrm{g} / \mathrm{kg})\end{array}$ & $\begin{array}{l}{[\mathrm{He}]} \\
\left(\mathrm{nmol} \mathrm{kg}^{-1}\right)\end{array}$ & $\begin{array}{l}{[\mathrm{Ne}]} \\
\left.(\mathrm{nmol} \mathrm{kg})^{-1}\right)\end{array}$ & $\begin{array}{l}{[\mathrm{Ar}]} \\
\left(\mu \mathrm{mol} \mathrm{kg}{ }^{-1}\right)\end{array}$ & $\begin{array}{l}{[\mathrm{Kr}]} \\
\left(\mathrm{nmol} \mathrm{kg}{ }^{-1}\right)\end{array}$ & $\begin{array}{l}{[\mathrm{Xe}]} \\
\left(\mathrm{nmol} \mathrm{kg}{ }^{-1}\right)\end{array}$ \\
\hline & & & & & & & (\% of saturat & at in situ tem & erature and sal & nity in parenth & eses) \\
\hline & High tide & $7 / 27 / 12$ & $6: 43$ & $110 \mathrm{~m}$ & 22.77 & 28.55 & $1.87(108.6)$ & $7.45(107.4)$ & $11.00(100.0)$ & $2.30(96.1)$ & $0.32(101.0)$ \\
\hline & Low tide & - & $12: 45$ & - & 21.11 & 24.32 & $1.89(106.7)$ & $7.60(105.5)$ & $11.86(101.9)$ & $2.54(99.2)$ & $0.36(105.9)$ \\
\hline & High tide & $7 / 28 / 12$ & $7: 20$ & - & 21.27 & 28.16 & $1.91(110.2)$ & $7.66(108.8)$ & 11.15 (98.5) & $2.36(95.0)$ & $0.33(99.1)$ \\
\hline & Mid tide & - & $10: 10$ & - & 20.56 & 27.45 & $1.92(110.5)$ & $7.79(109.7)$ & $11.87(103.0)$ & $2.50(98.5)$ & $0.35(102.1)$ \\
\hline & Low tide & - & $13: 42$ & - & 26.61 & 24.89 & $1.80(102.5)$ & $7.18(103.2)$ & 11.34 (107.5) & $2.46(108.9)$ & $0.34(117.8)$ \\
\hline & High tide & $10 / 29 / 13$ & $38: 58$ & $150 \mathrm{~m}$ & 6.7 & 29 & $2.14(120.8)$ & $8.96(114.4)$ & $14.76(97.6)$ & $3.36(96.1)$ & $0.50(94.9)$ \\
\hline & - & - & $8: 45$ & $125 \mathrm{~m}$ & 7.2 & 28.5 & $2.11(119.1)$ & 8.89 (113.6) & $14.58(97.2)$ & $3.42(96.1)$ & 0.49 (93.2) \\
\hline & - & - & $8: 31$ & $100 \mathrm{~m}$ & 7.3 & 29 & $2.11(119.3)$ & $8.86(113.6)$ & $14.46(96.9)$ & $3.38(95.2)$ & $0.48(92.7)$ \\
\hline & - & - & $8: 12$ & $75 \mathrm{~m}$ & 7.4 & 30 & $2.10(119.3)$ & $8.79(113.5)$ & $14.43(97.7)$ & 3.35 (95.7) & $0.48(93.7)$ \\
\hline & Mid tide & - & $11: 59$ & $140 \mathrm{~m}$ & 7.0 & 29.5 & $2.18(123.5)$ & $9.22(118.4)$ & 15.44 (103.2) & $3.60(101.3)$ & $0.52(99.0)$ \\
\hline & - & - & $11: 47$ & $125 \mathrm{~m}$ & 6.8 & 30 & $2.16(122.7)$ & $9.16(117.8)$ & 15.27 (101.9) & $3.57(100.1)$ & $0.51(97.4)$ \\
\hline & - & - & $11: 36$ & $100 \mathrm{~m}$ & 6.8 & 31 & $2.17(123.9)$ & $9.15(118.4)$ & $15.09(101.5)$ & $3.54(100.2)$ & $0.49(93.8)$ \\
\hline & - & - & $11: 25$ & $75 \mathrm{~m}$ & 7.1 & 31 & $2.15(123.2)$ & 9.07 (117.6) & $14.91(101.0)$ & 3.49 (99.6) & 0.49 (95.6) \\
\hline
\end{tabular}


Table A1, continued.

\begin{tabular}{|c|c|c|c|c|c|c|c|c|c|c|}
\hline & Tide stage & Time & Location & $\begin{array}{l}\text { Temperature } \\
\left({ }^{\circ} \mathrm{C}\right)\end{array}$ & $\begin{array}{l}\text { Absolute salinity } \\
(\mathrm{g} / \mathrm{kg})\end{array}$ & $\begin{array}{l}{[\mathrm{He}]} \\
\left(\mathrm{nmol} \mathrm{kg}{ }^{-1}\right)\end{array}$ & $\begin{array}{l}{[\mathrm{Ne}]} \\
\left(\mathrm{nmol} \mathrm{kg}{ }^{-1}\right)\end{array}$ & $\begin{array}{l}{[\mathrm{Ar}]} \\
\left(\mu \mathrm{mol} \mathrm{kg}{ }^{-1}\right)\end{array}$ & $\begin{array}{l}{[\mathrm{Kr}]} \\
\left(\mathrm{nmol} \mathrm{kg}{ }^{-1}\right)\end{array}$ & $\begin{array}{l}{[\mathrm{Xe}]} \\
\left.(\mathrm{nmol} \mathrm{kg})^{-1}\right)\end{array}$ \\
\hline \multirow{15}{*}{ 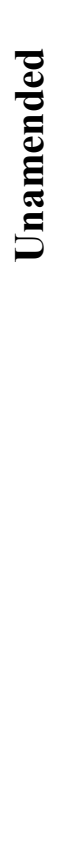 } & High tide & $7 / 27 / 12 \quad 6: 39$ & $124 \mathrm{~m}$ & 22.21 & 29.42 & $1.86(108.5)$ & $7.45(107.2)$ & $11.07(100.2)$ & $2.33(96.6)$ & $0.33(102.0)$ \\
\hline & Low tide & $13: 00$ & - & 21.12 & 26.51 & $1.89(108.2)$ & $7.63(107.2)$ & $11.57(100.8)$ & $2.45(97.0)$ & 0.34 (101.9) \\
\hline & High tide & $7 / 28 / 12 \quad 7: 22$ & - & 20.83 & 28.73 & $1.87(108.5)$ & $7.73(109.9)$ & $11.33(98.5)$ & $2.41(96.3)$ & $0.33(99.5)$ \\
\hline & Mid tide & $10: 30$ & - & 20.49 & 26.88 & $1.93(110.3)$ & $7.76(109.0)$ & $11.72(103.0)$ & $2.46(96.5)$ & $0.34(99.0)$ \\
\hline & Low tide & $13: 42$ & - & 22.83 & 26.43 & $1.86(106.8)$ & $7.53(107.0)$ & $11.70(107.5)$ & $2.49(102.7)$ & $0.35(108.8)$ \\
\hline & High tide & $10 / 30 / 13 \quad 9: 45$ & $150 \mathrm{~m}$ & 7.1 & 31 & $2.12(121.3)$ & $9.00(116.7)$ & $14.78(100.1)$ & $3.45(98.4)$ & $0.49(96.2)$ \\
\hline & - & $9: 34$ & $130 \mathrm{~m}$ & 7.3 & 31.5 & $2.11(121.1)$ & $8.88(115.7)$ & $14.66(100.1)$ & $3.45(98.8)$ & $0.48(95.5)$ \\
\hline & - & $9: 23$ & $115 \mathrm{~m}$ & 7.4 & 31 & $2.08(119.5)$ & $8.84(115.0)$ & $14.53(99.1)$ & $3.40(97.9)$ & $0.48(94.3)$ \\
\hline & - & 9:07 & $90 \mathrm{~m}$ & 7.4 & 31 & $2.10(120.3)$ & $8.84(115.0)$ & $14.56(99.3)$ & $3.41(98.1)$ & $0.48(94.3)$ \\
\hline & Mid tide & $10 / 30 / 1312: 45$ & $150 \mathrm{~m}$ & 7 & 30 & $2.18(124.1)$ & $9.25(119.1)$ & $15.47(103.8)$ & $3.62(102.3)$ & $0.52(100.0)$ \\
\hline & & $12: 36$ & $130 \mathrm{~m}$ & 6.9 & 30 & $2.16(123.0)$ & $9.20(118.3)$ & $15.38(102.9)$ & $3.61(101.7)$ & $0.52(98.2)$ \\
\hline & - & $12: 26$ & $115 \mathrm{~m}$ & 6.8 & 30 & $2.16(122.7)$ & $9.17(117.7)$ & $15.28(102.0)$ & $3.59(100.9)$ & $0.53(100.9)$ \\
\hline & - & $13: 08^{*}$ & $90 \mathrm{~m}$ & 7.3 & 30.5 & $2.15(123.0)$ & $9.21(119.2)$ & $15.39(104.3)$ & $3.62(103.4)$ & $0.51(99.7)$ \\
\hline & High tide & $10 / 29 / 14 \quad 17: 18$ & $\mathrm{PW} 1^{* *}$ & 12.6 & 31.5 & $1.87(108.5)$ & $8.01(109.2)$ & $14.78(113.1)$ & $3.51(116.5)$ & $0.48(112.9)$ \\
\hline & 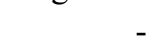 & $17: 22$ & $\mathrm{PW} 2^{* *}$ & 12.6 & 30 & $1.95(112.2)$ & $8.24(111.3)$ & $14.25(108.7)$ & $3.37(110.7)$ & $0.46(106.9)$ \\
\hline
\end{tabular}

* Farthest downstream location sampled last instead of first.

** Porewater (PW) samples taken from $130 \mathrm{~m}$ upstream from nearest confluence and $1 \mathrm{~m}$ back from bank Sample water withdrawn from $10 \mathrm{~cm}$ below bank platform surface, roughly $1 \mathrm{~m}$ apart, with different in situ temperatures and salinities. 
Table A2: Data from the benthic chamber experiments (2013). Tabulated values are defined as follows with assigned or experimental one standard errors: temperature $(\mathrm{T}) \pm 0.1^{\circ} \mathrm{C}$; mean absolute salinity $\left(\mathrm{S}_{\mathrm{A}}\right)$ across all chambers $\pm 0.2 \mathrm{~g} / \mathrm{kg}$; height of water column within chamber (h, for determining areal rates) $\pm 0.7 \mathrm{~cm}$; the chamber volume $(\mathrm{V}) \pm \sim 0.25 \mathrm{~L}$; the fraction of the chamber volume removed during sampling (f) and replaced by entrained creek water \pm 0.002 ; oxygen concentration $\left(\left[\mathrm{O}_{2}\right]\right.$ reported here in volumetric units) $1-3 \%$ of the value but with potential positive systematic bias on the order of $10 \mu \mathrm{mol} \mathrm{L}^{-1}$ below $50 \mu \mathrm{mol} \mathrm{L}-1$ (see text), and the entrainment corrected oxygen concentration for the preceding time step $\left(\left[\mathrm{O}_{2}\right]_{\mathrm{t}-1, \mathrm{c}}\right.$, the initial point for each new piecewise rate) similar uncertainties; the ${ }^{18} \mathrm{O}:{ }^{16} \mathrm{O}$ ratio, reported in standard isotope notation as ${ }^{18} \delta \pm 0.2$ per mil $\left(2 \times 10^{-4}\right)$, and the entrainment corrected value for the preceeding timestep ${ }^{18} \delta_{\mathrm{t}-1, \mathrm{c}} \pm 0.3$ per mil; expected maximum isotopic ratio possible with both photosynthesis and respiration occurring in a closed system $\left({ }^{18} \delta_{\max }\right) \pm 6$ per mil depending on assumptions about the isotopic composition of water and dominant primary producers in the tidal creek system (see text for derivation); the triple oxygen isotope tracer ${ }^{17} \Delta$ has a replicate standard error of \pm 6 per meg $\left(6 \times 10^{-6}\right)$ per meg, and the entrainment corrected and maximum values $\left({ }^{17} \Delta_{\mathrm{t}-1, \mathrm{c}}\right.$ and $\left.{ }^{17} \Delta_{\mathrm{max}}\right)$ have uncertainties of 6 and 12 per meg respectively. ${ }^{18} \delta$ and ${ }^{17} \Delta$ can be used to determine ${ }^{17} \delta$ with greater relative accuracy than direct measurement of each isotope ratio separately. On August $29^{\text {th }}$, the second light chamber at the unamended creek was not fully sealed; the values were similar to ambient creek water and are not reported here.

\begin{tabular}{|c|c|c|c|c|c|c|c|c|c|c|c|c|c|c|c|}
\hline \multicolumn{2}{|c|}{ Chamber } & Time & $\begin{array}{c}\mathrm{T} \\
\left({ }^{\circ} \mathrm{C}\right) \\
\end{array}$ & $\begin{array}{c}\mathrm{S}_{\mathrm{A}} \\
(\mathrm{g} / \mathrm{kg})\end{array}$ & $\begin{array}{c}\mathrm{h} \\
(\mathrm{cm})\end{array}$ & $\begin{array}{l}\mathrm{V} \\
(\mathrm{L}) \\
\end{array}$ & $\mathrm{f}$ & $\begin{array}{c}{\left[\mathrm{O}_{2}\right]} \\
\left(\mu \mathrm{mol} \mathrm{L}^{-1}\right) \\
\end{array}$ & $\begin{array}{c}{\left[\mathrm{O}_{2}\right]_{\mathrm{t}-1, \mathrm{c}}} \\
\left.(\mu \mathrm{mol} \mathrm{L})^{-1}\right)\end{array}$ & $\begin{array}{c}{ }^{18} \delta \\
10^{-3} \\
\end{array}$ & $\begin{array}{c}{ }^{18} \delta_{\mathrm{t}-1, \mathrm{c}} \\
10^{-3} \\
\end{array}$ & $\begin{array}{c}{ }^{18} \delta_{\max } \\
10^{-3} \\
\end{array}$ & $\begin{array}{l}{ }^{17} \Delta \\
10^{-6} \\
\end{array}$ & $\begin{array}{c}{ }^{17} \Delta_{\mathrm{t}-1, \mathrm{c}} \\
10^{-6}\end{array}$ & $\begin{array}{c}{ }^{17} \Delta_{\max } \\
10^{-6} \\
\end{array}$ \\
\hline \multirow{13}{*}{ 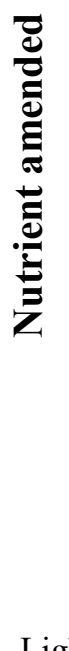 } & Light 1 & $8 / 27 / 13 \quad 10: 19$ & 23.0 & 26.5 & 12.7 & 8.4 & 0.081 & 72 & & -5.2 & & -22.7 & 131 & & 204 \\
\hline & - & $8 / 27 / 1311: 56$ & 24.6 & 26.5 & - & - & 0.060 & 118 & 75 & -13.5 & -4.7 & -22.7 & 196 & 125 & 204 \\
\hline & - & $8 / 27 / 1313: 32$ & 26.1 & 26.5 & - & - & 0.048 & 197 & 118 & -15.3 & -12.9 & -22.7 & 184 & 188 & 204 \\
\hline & - & $8 / 27 / 13 \quad 14: 39$ & 26.4 & 26.5 & - & - & 0.015 & 205 & 195 & & & & & & \\
\hline & Light 2 & $8 / 27 / 1311: 10$ & 23.7 & 26.5 & 12.7 & 8.4 & 0.048 & 67 & & -4.8 & & -22.7 & 135 & & 204 \\
\hline & - & $8 / 27 / 13 \quad 12: 39$ & 24.6 & 26.5 & - & - & 0.048 & 109 & 69 & & -4.5 & & & 130 & \\
\hline & - & $8 / 27 / 1314: 06$ & 26.1 & 26.5 & - & - & 0.045 & 159 & 111 & -14.6 & $*$ & -22.7 & 193 & $*$ & 204 \\
\hline & - & 8/27/13 15:04 & 26.4 & 26.5 & - & - & 0.015 & 136 & 159 & & & & & & \\
\hline & Light 3 & $8 / 27 / 1311: 27$ & 23.9 & 26.5 & 12.7 & 8.4 & 0.047 & 59 & & -2.1 & & -22.7 & 136 & & 204 \\
\hline & - & $8 / 27 / 13 \quad 12: 57$ & 24.6 & 26.5 & - & - & 0.049 & 140 & 63 & -14.5 & -2.3 & -22.7 & 168 & 131 & 204 \\
\hline & - & $8 / 27 / 13 \quad 14: 18$ & 26.1 & 26.5 & - & - & 0.050 & 203 & 140 & -15.3 & -14.1 & -22.7 & 179 & 163 & 204 \\
\hline & - & $8 / 27 / 13$ 15:14 & 26.4 & 26.5 & - & - & 0.015 & 187 & 201 & & & & & & \\
\hline \multirow{4}{*}{\multicolumn{2}{|c|}{$\begin{array}{r}\text { Light water } \\
- \\
-\end{array}$}} & $8 / 27 / 1310: 53$ & 23.5 & 26.5 & 12.4 & 8.2 & 0.047 & 69 & & -1.1 & & -22.7 & 120 & & 204 \\
\hline & & $8 / 27 / 13 \quad 12: 28$ & 24.6 & 26.5 & - & - & 0.054 & 75 & 70 & & & & & & \\
\hline & & $8 / 27 / 13$ 13:54 & 26.1 & 26.5 & - & - & 0.048 & 97 & 78 & & & & & & \\
\hline & & $8 / 27 / 1314: 55$ & 26.4 & 26.5 & - & - & 0.016 & 110 & 100 & & & & & & \\
\hline
\end{tabular}




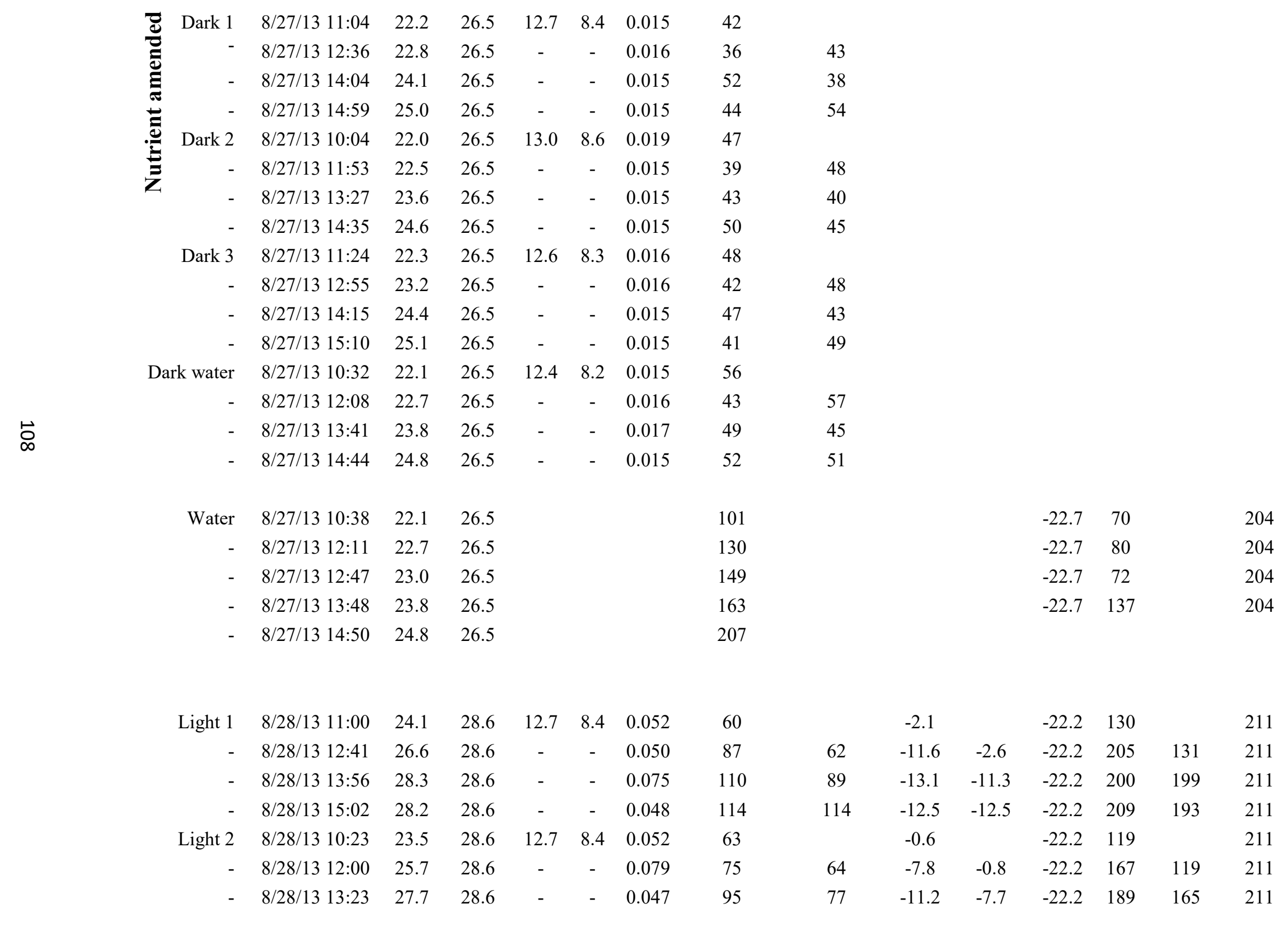




\begin{tabular}{|c|c|c|c|c|c|c|c|c|c|c|c|c|c|c|}
\hline - & 8/28/13 14:40 & 28.3 & 28.6 & - & - & 0.048 & 105 & 97 & -11.0 & -11.0 & -22.2 & 201 & 184 & 211 \\
\hline Light 3 & 8/28/13 9:54 & 22.8 & 28.6 & 12.7 & 8.4 & 0.048 & 82 & & & & & & & \\
\hline - & 8/28/13 11:38 & 25.3 & 28.6 & - & - & 0.048 & 132 & 82 & -14.2 & & -22.2 & 203 & & 211 \\
\hline- & 8/28/13 13:03 & 27.0 & 28.6 & - & - & 0.043 & 226 & 131 & -15.7 & -13.9 & -22.2 & 206 & 201 & 211 \\
\hline- & $8 / 28 / 1314: 28$ & 28.3 & 28.6 & - & - & 0.045 & 285 & 222 & -15.6 & -15.5 & -22.2 & 222 & 203 & 211 \\
\hline sht water & 8/28/13 10:41 & 23.7 & 28.6 & 12.4 & 8.2 & 0.048 & 70 & & 1.6 & & -22.2 & 64 & & 211 \\
\hline- & 8/28/13 12:19 & 26.0 & 28.6 & - & - & 0.090 & 76 & 70 & -4.6 & 1.4 & -22.2 & 129 & 67 & 211 \\
\hline - & $8 / 28 / 1313: 36$ & 27.7 & 28.6 & - & - & 0.049 & 90 & 81 & & -4.9 & & & 127 & \\
\hline- & $8 / 28 / 1314: 53$ & 28.2 & 28.6 & - & - & 0.057 & 106 & 93 & -9.1 & $*$ & -22.2 & 157 & $*$ & 211 \\
\hline Dark 1 & $8 / 28 / 1310: 56$ & 22.2 & 28.6 & 12.7 & 8.4 & 0.017 & 42 & & & & & & & \\
\hline- & $8 / 28 / 1312: 38$ & 23.2 & 28.6 & - & - & 0.015 & 32 & 43 & & & & & & \\
\hline- & 8/28/13 13:52 & 24.3 & 28.6 & - & - & 0.022 & 34 & 34 & & & & & & \\
\hline- & 8/28/13 15:00 & 25.5 & 28.6 & - & - & 0.015 & 36 & 37 & & & & & & \\
\hline Dark 2 & 8/28/13 9:49 & 22.1 & 28.6 & 13.0 & 8.6 & 0.015 & 52 & & & & & & & \\
\hline$* *$ & $8 / 28 / 13$ 14:09 & 24.9 & 28.6 & - & - & 0.015 & 39 & 53 & & & & & & \\
\hline- & $8 / 28 / 1315: 10$ & 25.7 & 28.6 & - & - & 0.015 & 44 & 41 & & & & & & \\
\hline Dark 3 & $8 / 28 / 1310: 03$ & 22.1 & 28.6 & 12.6 & 8.3 & 0.015 & 57 & & & & & & & \\
\hline- & 8/28/13 11:41 & 22.5 & 28.6 & - & - & 0.015 & 38 & 57 & & & & & & \\
\hline- & 8/28/13 13:11 & 23.7 & 28.6 & - & - & 0.015 & 40 & 38 & & & & & & \\
\hline - & 8/28/13 14:35 & 25.1 & 28.6 & - & - & 0.015 & 47 & 41 & & & & & & \\
\hline Dark water & $8 / 28 / 13 \quad 10: 37$ & 22.1 & 28.6 & 12.4 & 8.2 & 0.015 & 63 & & & & & & & \\
\hline- & 8/28/13 12:14 & 22.9 & 28.6 & - & - & 0.015 & 57 & 63 & & & & & & \\
\hline- & $8 / 28 / 1313: 31$ & 24.1 & 28.6 & - & - & 0.015 & 55 & 58 & & & & & & \\
\hline- & 8/28/13 14:50 & 25.4 & 28.6 & - & - & 0.015 & 57 & 57 & & & & & & \\
\hline Water & 8/28/13 10:06 & 22.1 & 28.6 & & & & 78 & & & & -22.2 & 128 & & 211 \\
\hline- & 8/28/13 11:46 & 22.5 & 28.6 & & & & 97 & & & & -22.2 & 140 & & 211 \\
\hline- & 8/28/13 12:31 & 23.0 & 28.6 & & & & 133 & & & & -22.2 & 117 & & 211 \\
\hline- & $8 / 28 / 1313: 15$ & 23.7 & 28.6 & & & & 143 & & & & -22.2 & 116 & & 211 \\
\hline - & 8/28/13 13:45 & 24.3 & 28.6 & & & & 159 & & & & -22.2 & 134 & & 211 \\
\hline
\end{tabular}




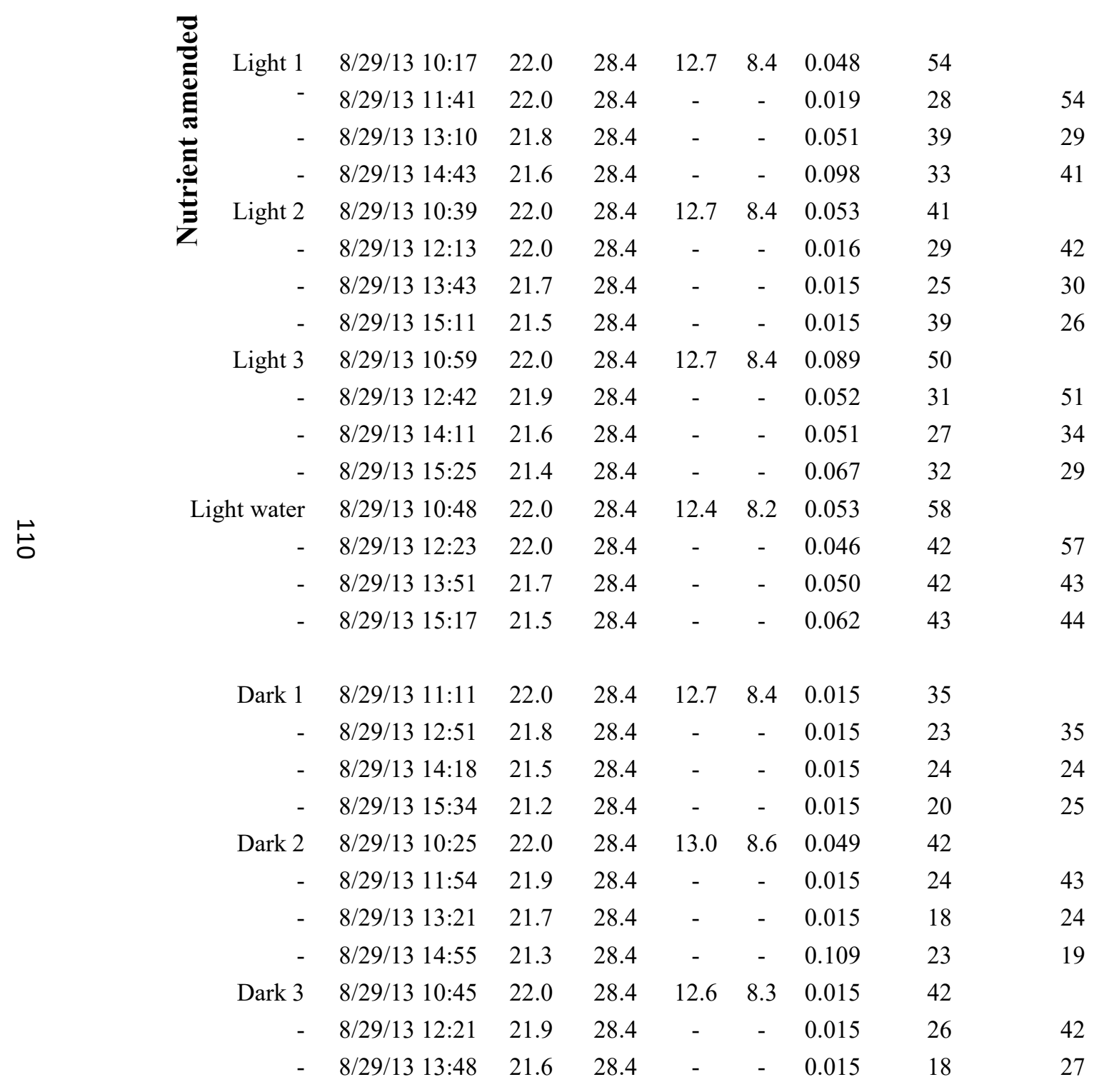




\begin{tabular}{|c|c|c|c|c|c|c|c|c|}
\hline \multirow{3}{*}{ - } & $8 / 29 / 1315: 13$ & 21.2 & 28.4 & - & - & 0.015 & 25 & 19 \\
\hline & $8 / 29 / 1310: 56$ & 22.0 & 28.4 & 12.4 & 8.2 & 0.015 & 60 & \\
\hline & $8 / 29 / 13 \quad 12: 38$ & 21.8 & 28.4 & - & - & 0.015 & 47 & 59 \\
\hline$\ddot{E}$ & 8/29/13 14:08 & 21.5 & 28.4 & - & - & 0.015 & 47 & 47 \\
\hline 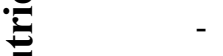 & $8 / 29 / 1315: 23$ & 21.2 & 28.4 & - & - & 0.015 & 40 & 48 \\
\hline Water & $8 / 29 / 13 \quad 10: 32$ & 22.0 & 28.4 & & & & 53 & \\
\hline- & 8/29/13 11:59 & 21.9 & 28.4 & & & & 68 & \\
\hline- & $8 / 29 / 1312: 32$ & 21.8 & 28.4 & & & & 73 & \\
\hline- & $8 / 29 / 1313: 24$ & 21.6 & 28.4 & & & & 78 & \\
\hline- & $8 / 29 / 1313: 58$ & 21.5 & 28.4 & & & & 73 & \\
\hline- & $8 / 29 / 1314: 57$ & 21.3 & 28.4 & & & & 86 & \\
\hline
\end{tabular}

占

\begin{tabular}{|c|c|c|c|c|c|c|c|c|c|c|c|c|c|c|}
\hline Chamber & Time & $\begin{array}{c}\mathrm{T} \\
\left({ }^{\circ} \mathrm{C}\right) \\
\end{array}$ & $\begin{array}{c}\mathrm{S}_{\mathrm{A}} \\
(\mathrm{g} / \mathrm{kg})\end{array}$ & $\begin{array}{c}\mathrm{h} \\
(\mathrm{cm})\end{array}$ & $\begin{array}{l}\mathrm{V} \\
(\mathrm{L}) \\
\end{array}$ & $\mathrm{f}$ & $\begin{array}{c}{\left[\mathrm{O}_{2}\right]} \\
\left(\mu \mathrm{mol} \mathrm{L}{ }^{-1}\right)\end{array}$ & $\begin{array}{c}{\left[\mathrm{O}_{2}\right]_{\mathrm{t}-1, \mathrm{c}}} \\
(\mu \mathrm{mol} \mathrm{L}-1)\end{array}$ & $\begin{array}{c}{ }^{18} \delta \\
10^{-3} \\
\end{array}$ & $\begin{array}{c}{ }^{18} \delta_{\mathrm{t}-1, \mathrm{c}} \\
10^{-3}\end{array}$ & $\begin{array}{c}{ }^{18} \delta_{\max } \\
10^{-3} \\
\end{array}$ & $\begin{array}{l}{ }^{17} \Delta \\
10^{-6} \\
\end{array}$ & $\begin{array}{c}{ }^{17} \Delta_{\mathrm{t}-1, \mathrm{c}} \\
10^{-6} \\
\end{array}$ & $\begin{array}{c}{ }^{17} \Delta_{\max } \\
10^{-6} \\
\end{array}$ \\
\hline Light 1 & $8 / 27 / 139: 43$ & 22.0 & 25.4 & 12.7 & 8.4 & 0.046 & 88 & & & & & & & \\
\hline ס्य & $8 / 27 / 1311: 39$ & 22.5 & 25.4 & - & - & 0.046 & 60 & 89 & & & & & & \\
\hline $\bar{E}$ & 8/27/13 13:17 & 23.3 & 25.4 & - & - & 0.049 & 58 & 64 & -0.9 & & -22.9 & 119 & & 200 \\
\hline Е & $8 / 27 / 1314: 35$ & 24.3 & 25.4 & - & - & 0.015 & 52 & 63 & & & & & & \\
\hline $\mathbf{S} \quad$ Light 2 & $8 / 27 / 1310: 16$ & 22.1 & 25.4 & 12.6 & 8.3 & 0.046 & 58 & & 5.1 & & -22.9 & 81 & & 200 \\
\hline - & $8 / 27 / 13 \quad 12: 01$ & 22.6 & 25.4 & - & - & 0.040 & 62 & 60 & & 4.3 & & & 82 & \\
\hline- & $8 / 27 / 13$ 13:43 & 23.5 & 25.4 & - & - & 0.048 & 64 & 65 & -3.1 & $*$ & -22.9 & 121 & $*$ & 200 \\
\hline- & $8 / 27 / 13 \quad 14: 48$ & 24.4 & 25.4 & - & - & 0.015 & 63 & 68 & & & & & & \\
\hline Light 3 & $8 / 27 / 13 \quad 10: 35$ & 22.1 & 25.4 & 12.9 & 8.5 & 0.046 & 62 & & 3.7 & & -22.9 & 60 & & 200 \\
\hline- & $8 / 27 / 1312: 16$ & 22.8 & 25.4 & - & - & 0.046 & 57 & 64 & -1.6 & 3.1 & -22.9 & 110 & 63 & 200 \\
\hline- & $8 / 27 / 1313: 59$ & 23.8 & 25.4 & - & - & 0.048 & 57 & 61 & -2.4 & -2.0 & -22.9 & 111 & 109 & 200 \\
\hline- & $8 / 27 / 1314: 58$ & 24.5 & 25.4 & - & - & 0.015 & 50 & 63 & & & & & & \\
\hline Light water & $8 / 27 / 1310: 54$ & 22.3 & 25.4 & & & 0.081 & 86 & & & & & & & \\
\hline
\end{tabular}




\begin{tabular}{|c|c|c|c|c|c|c|c|c|c|c|c|c|c|c|c|}
\hline \multirow{4}{*}{ 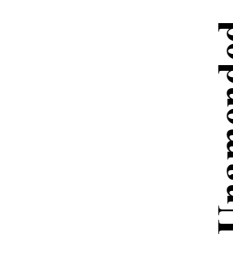 } & - & 8/27/13 12:41 & 23.0 & 25.4 & & & 0.048 & 102 & 87 & & & & & & \\
\hline & - & 8/27/13 14:10 & 24.1 & 25.4 & & & 0.079 & 119 & 104 & & & & & & \\
\hline & - & 8/27/13 15:09 & 24.5 & 25.4 & & & 0.015 & 118 & 124 & & & & & & \\
\hline & Dark 1 & $8 / 27 / 139: 55$ & 21.8 & 25.4 & 12.7 & 8.4 & 0.019 & 57 & & & & & & & \\
\hline & - & $8 / 27 / 1311: 59$ & 22.0 & 25.4 & - & - & 0.015 & 46 & 58 & & & & & & \\
\hline & - & $8 / 27 / 1313: 35$ & 22.6 & 25.4 & - & - & 0.015 & 47 & 48 & & & & & & \\
\hline & - & $8 / 27 / 1314: 44$ & 23.5 & 25.4 & - & - & 0.015 & 44 & 49 & & & & & & \\
\hline & Dark 2 & $8 / 27 / 1310: 31$ & 21.9 & 25.4 & 12.7 & 8.4 & 0.019 & 58 & & & & & & & \\
\hline & - & 8/27/13 12:12 & 22.1 & 25.4 & - & - & 0.019 & 57 & 59 & & & & & & \\
\hline & - & $8 / 27 / 1313: 48$ & 22.9 & 25.4 & - & - & 0.019 & 43 & 59 & & & & & & \\
\hline & - & $8 / 27 / 1314: 55$ & 23.7 & 25.4 & - & - & 0.015 & 47 & 45 & & & & & & \\
\hline & Dark 3 & $8 / 27 / 1310: 51$ & 21.9 & 25.4 & 12.6 & 8.3 & 0.015 & 60 & & & & & & & \\
\hline & - & $8 / 27 / 13 \quad 12: 27$ & 22.2 & 25.4 & - & - & 0.015 & 48 & 61 & & & & & & \\
\hline & - & $8 / 27 / 1314: 04$ & 23.1 & 25.4 & - & - & 0.015 & 41 & 49 & & & & & & \\
\hline & - & $8 / 27 / 13$ 15:04 & 23.9 & 25.4 & - & - & 0.015 & 32 & 43 & & & & & & \\
\hline & Dark water & $8 / 27 / 1311: 08$ & 21.9 & 25.4 & 12.4 & 8.2 & 0.015 & 82 & & & & & & & \\
\hline & - & $8 / 27 / 13 \quad 12: 44$ & 22.4 & 25.4 & - & - & 0.015 & 74 & 83 & & & & & & \\
\hline & - & 8/27/13 14:17 & 23.3 & 25.4 & - & - & 0.015 & 79 & 75 & & & & & & \\
\hline & - & $8 / 27 / 13 \quad 15: 15$ & 24.0 & 25.4 & - & - & 0.015 & 80 & 81 & & & & & & \\
\hline & Water & 8/27/13 10:05 & 21.8 & 25.4 & & & & 90 & & & & -22.9 & 55 & & 200 \\
\hline & - & 8/27/13 11:49 & 22.0 & 25.4 & & & & 145 & & & & -22.9 & 105 & & 200 \\
\hline & - & $8 / 27 / 13$ 12:29 & 22.3 & 25.4 & & & & 142 & & & & -22.9 & 103 & & 200 \\
\hline & - & 8/27/13 13:20 & 22.6 & 25.4 & & & & 165 & & & & -22.9 & 87 & & 200 \\
\hline & - & $8 / 27 / 13 \quad 14: 41$ & 23.7 & 25.4 & & & & 190 & & & & & & & \\
\hline & Light 1 & $8 / 28 / 1311: 21$ & 22.4 & 24.9 & 12.7 & 8.4 & 0.048 & 76 & & -5.0 & & -23.1 & 142 & & 199 \\
\hline & - & $8 / 28 / 1312: 57$ & 23.0 & 24.9 & - & - & 0.083 & 83 & 76 & -8.3 & -4.6 & -23.1 & 167 & 140 & 199 \\
\hline & - & $8 / 28 / 1314: 45$ & 23.6 & 24.9 & - & - & 0.047 & 71 & 86 & -6.2 & -7.6 & -23.1 & 162 & 157 & 199 \\
\hline
\end{tabular}




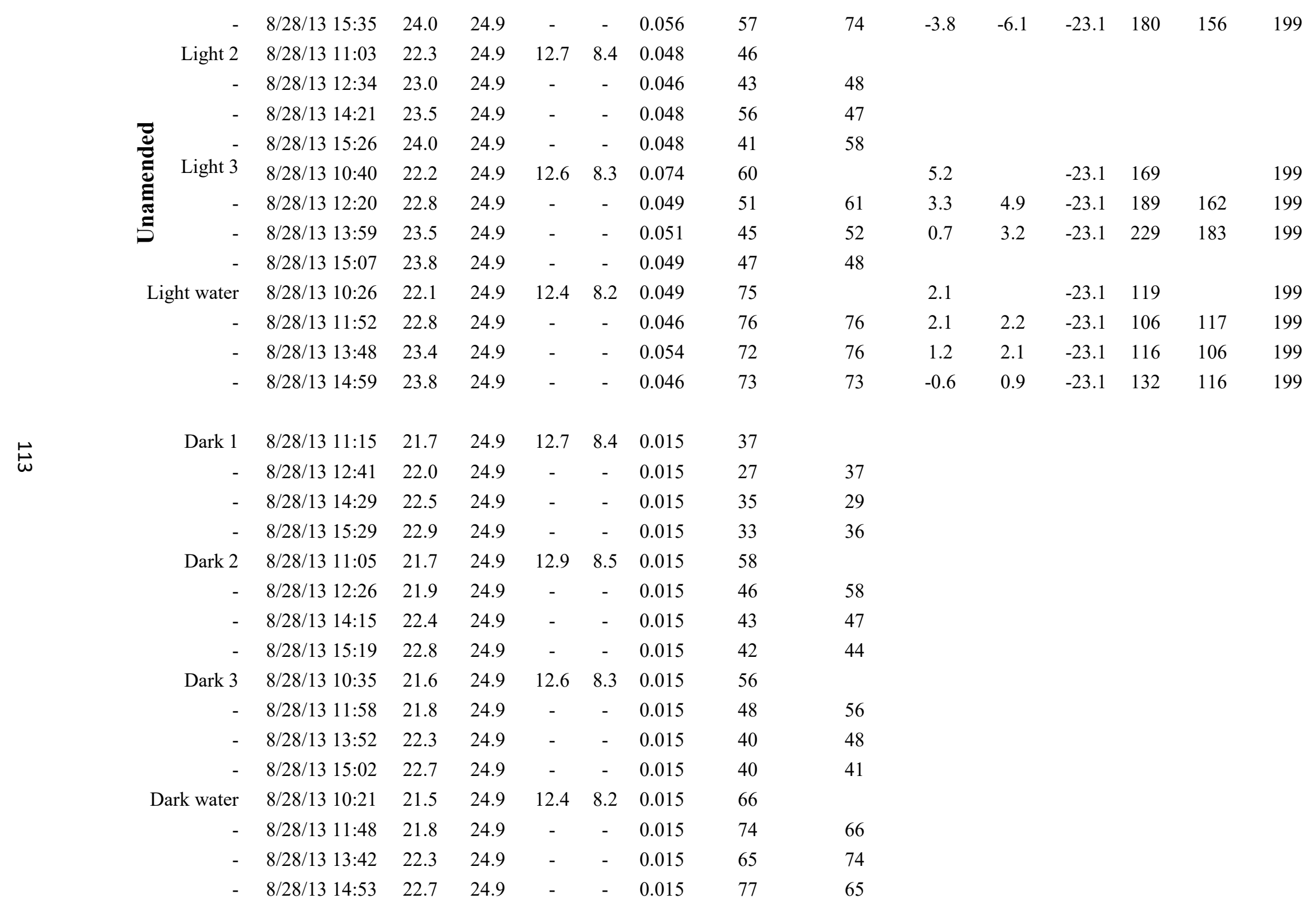




\begin{tabular}{|c|c|c|c|c|c|c|c|c|c|c|c|c|c|c|}
\hline \multirow{6}{*}{$\begin{array}{r}\text { Water } \\
- \\
- \\
- \\
-\end{array}$} & $8 / 28 / 13 \quad 10: 56$ & 21.7 & 24.9 & & & & 82 & & & & -23.1 & 94 & & 199 \\
\hline & 8/28/13 12:05 & 21.8 & 24.9 & & & & 73 & & & & -23.1 & 103 & & 199 \\
\hline & $8 / 28 / 13 \quad 12: 47$ & 22.0 & 24.9 & & & & 120 & & & & -23.1 & 87 & & 199 \\
\hline & 8/28/13 14:08 & 22.4 & 24.9 & & & & 97 & & & & -23.1 & 120 & & 199 \\
\hline & $8 / 28 / 13 \quad 14: 35$ & 22.5 & 24.9 & & & & 131 & & & & -23.1 & 89 & & 199 \\
\hline & $8 / 28 / 1315: 14$ & 22.8 & 24.9 & & & & 130 & & & & -23.1 & 140 & & 199 \\
\hline Light 1 & 8/29/13 10:58 & 22.2 & 26.3 & 12.7 & 8.4 & 0.053 & 75 & & 4.6 & & -22.7 & 82 & & 203 \\
\hline- & $8 / 29 / 1314: 29$ & 21.9 & 26.3 & - & - & 0.048 & 88 & 75 & & & & & & \\
\hline- & $8 / 29 / 1315: 53$ & 22.0 & 26.3 & - & - & 0.053 & 72 & 89 & & & & & & \\
\hline- & $8 / 29 / 13$ 17:05 & 22.2 & 26.3 & - & - & 0.015 & 56 & 75 & & & & & & \\
\hline Light 3 & 8/29/13 12:21 & 22.3 & 26.3 & 12.6 & 8.3 & 0.049 & 69 & & 4.9 & & -22.7 & 133 & & 203 \\
\hline- & $8 / 29 / 13 \quad 15: 27$ & 21.7 & 26.3 & - & - & 0.048 & 64 & 69 & -1.0 & 4.7 & -22.7 & 146 & 130 & 203 \\
\hline - & $8 / 29 / 13 \quad 16: 49$ & 22.1 & 26.3 & - & - & 0.052 & 54 & 67 & & & & & & \\
\hline- & $8 / 29 / 1317: 51$ & 22.2 & 26.3 & - & - & 0.015 & 47 & 59 & & & & & & \\
\hline \multirow{4}{*}{$\begin{array}{r}\text { Light water } \\
- \\
- \\
-\end{array}$} & 8/29/13 11:57 & 22.3 & 26.3 & 12.4 & 8.2 & 0.049 & 105 & & 4.2 & & & & & \\
\hline & $8 / 29 / 1315: 03$ & 21.7 & 26.3 & - & - & 0.050 & 98 & 103 & 1.9 & & & & & \\
\hline & $8 / 29 / 13 \quad 16: 33$ & 22.1 & 26.3 & - & - & 0.051 & 91 & 99 & 1.8 & & -22.7 & 88 & & 203 \\
\hline & $8 / 29 / 1317: 32$ & 22.2 & 26.3 & - & - & 0.015 & 92 & 94 & & & & & & \\
\hline Dark 1 & 8/29/13 11:06 & 22.1 & 26.3 & 12.7 & 8.4 & 0.043 & 74 & & 6.9 & & -22.7 & 131 & & 203 \\
\hline- & $8 / 29 / 1314: 55$ & 21.4 & 26.3 & - & - & 0.046 & 41 & 74 & & & & & & \\
\hline- & $8 / 29 / 1316: 12$ & 21.6 & 26.3 & - & - & 0.043 & 42 & 44 & & & & & & \\
\hline - & $8 / 29 / 13 \quad 17: 16$ & 21.9 & 26.3 & - & - & 0.015 & 32 & 45 & & & & & & \\
\hline Dark 2 & 8/29/13 12:05 & 22.0 & 26.3 & 12.9 & 8.5 & 0.015 & 69 & & & & & & & \\
\hline- & $8 / 29 / 13$ 15:14 & 21.3 & 26.3 & - & - & 0.015 & 55 & 69 & & & & & & \\
\hline- & $8 / 29 / 1316: 37$ & 21.7 & 26.3 & - & - & 0.015 & 42 & 56 & & & & & & \\
\hline- & $8 / 29 / 13 \quad 17: 41$ & 22.0 & 26.3 & - & - & 0.015 & 34 & 43 & & & & & & \\
\hline \multirow{2}{*}{$\begin{array}{r}\text { Dark } 3 \\
-\end{array}$} & $8 / 29 / 13 \quad 12: 31$ & 21.9 & 26.3 & 12.6 & 8.3 & 0.015 & 72 & & & & & & & \\
\hline & $8 / 29 / 13 \quad 15: 34$ & 21.4 & 26.3 & - & - & 0.015 & 59 & 72 & & & & & & \\
\hline
\end{tabular}




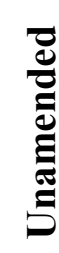

\begin{tabular}{|c|c|c|c|c|c|c|}
\hline $8 / 29 / 13$ 16:54 & 21.8 & 26.3 & - & - & 0.015 & 38 \\
\hline $8 / 29 / 13$ 17:55 & 22.0 & 26.3 & - & - & 0.015 & 34 \\
\hline $8 / 29 / 1311: 38$ & 22.0 & 26.3 & 12.4 & 8.2 & 0.015 & 81 \\
\hline $8 / 29 / 1314: 50$ & 21.4 & 26.3 & - & - & 0.015 & 89 \\
\hline 8/29/13 16:25 & 21.7 & 26.3 & - & - & 0.015 & 86 \\
\hline $29 / 13$ 17:27 & 21.9 & 26.3 & - & - & 0.015 & 83 \\
\hline
\end{tabular}

\begin{tabular}{|c|c|c|}
\hline Water & $8 / 29 / 1311: 11$ & 22.1 \\
\hline - & $8 / 29 / 1314: 32$ & 21.4 \\
\hline - & $8 / 29 / 13$ 15:19 & 21.3 \\
\hline - & $8 / 29 / 13$ 15:59 & 21.5 \\
\hline - & $8 / 29 / 13 \quad 16: 45$ & 21.8 \\
\hline - & $8 / 29 / 1317: 11$ & 21.9 \\
\hline
\end{tabular}

*Water withdrawn, but triple oxygen isotope sample could not be analyzed. For piecewise rate calculation, used t-1 values for preceding timestep (eg $\mathrm{t}-2$ for $\left[\mathrm{O}_{2}\right]$ ) as starting point and correct as able for entrained water.

**Water level was too low to sample without risking drawing air through replacement volume port, long delay between timepoints. 


\section{Chapter 4}

\section{Using noble gases in a shallow aquatic environment to compare common gas exchange parameterizations and to constrain efflux of oxygen by ebullition}

This chapter is under review for potential publication in Limnology and Oceanography: Letters. The authors are: Evan M. Howard, Woods Hole Oceanographic Institution, Massachusetts Institute of Technology; Rachel H.R. Stanley, Wellesley College; Inke Forbrich, Marine Biological Laboratory; Anne E. Giblin, Marine Biological Laboratory; Dempsey E. Lott III, Woods Hole Oceanographic Institution; Kevin L. Cahill, Woods Hole Oceanographic Institution

\section{Data availability}

Data used in this work are accessible through the Biological and Chemical Oceanography Data Management Office (http://www.bco-dmo.org/project/529583). Ancillary meteorological data are accessible from the LTER Network Information System Data Portal (Giblin and Forbrich 2016; http://dx.doi.org/10.6073/pasta/14eb405f583ae2384b2c6c5714776214). 


\begin{abstract}
Accurate determination of air-water gas exchange fluxes is critically important for calculating ecosystem metabolism rates from dissolved oxygen in shallow aquatic environments. We present a unique dataset of the noble gases neon, argon, krypton, and xenon in a salt marsh pond to evaluate three commonly-used windspeed based gas exchange parameterizations. These noble gases are sensitive to a variety of physical processes, including bubbles in particular. We thus additionally use this dataset to evaluate the contribution of bubbling from the sediments (ebullition) to gas fluxes. We find that while the literature gas exchange parameterizations do well in modeling more soluble gases, up to $24 \%$ of net oxygen production may be lost from the pond via ebullition during periods of biologically and physically produced supersaturation. In this environment, neglecting ebullition results in greater biases to metabolism rates than any differences introduced by the choice between the three gas exchange parameterizations.
\end{abstract}

\title{
Keywords
}

Bubbles, salt marsh pond metabolism, neon, argon, krypton, xenon

\section{Acknowledgments}

This work would not have been possible without the outstanding logistical and in kind support of the Plum Island Ecosystems Long Term Ecological Research site and TIDE experiment staff and scientists (these projects funded by NSF OCE 1238212, NSF DEB 1354494, and NE Climate Science Center grant DOI G12AC00001). Thanks to Nancy Pau at the Parker River National Wildlife Refuge for permitting this work. All samples were collected with the assistance of Jennifer Karolewski, who was supported by the Woods Hole Oceanographic Institution (WHOI) Summer Student Fellowship program. I also wish to thank Amanda C. Spivak for the use of the water quality sonde in this study, Joshua M. Curtice, Joanne Goudreau, and William J. Jenkins at the WHOI Isotope Geochemistry Facility (http://www.whoi.edu/IGF) for feedback on sampling methods and interpretation of noble gas data, and Kim Vaeth and Philip M. Gschwend for constructive feedback in developing this manuscript. Funding for this research was provided by NSF OCE 1233678 and the WHOI Coastal Ocean Institute. E. Howard was supported by the National Defense Science and Engineering Graduate Fellowship. 


\section{Introduction}

Shallow aquatic environments such as estuaries and lakes vary widely in biological productivity and may be both important sinks and sources of carbon dioxide $\left(\mathrm{CO}_{2}\right)$ to the atmosphere. Estuaries and lakes have dynamic diel cycles of oxygen $\left(\mathrm{O}_{2}\right)$ influx and efflux; measurements of $\mathrm{O}_{2}$ are often paired with parameterizations of diffusive gas exchange based on windspeed to calculate net ecosystem metabolic fluxes (Caffrey 2004, Staehr et al. 2010). While an accurate estimate of air-water gas exchange fluxes is critical to determining these metabolic fluxes, gas exchange is not well constrained in shallow environments.

Existing gas exchange parameterizations for coastal and inland waters often lack fundamental mechanisms of gas transfer, such as bubble processes, turbulence generated from bottom stress or buoyancy fluxes, and fetch limitation of wind-driven turbulence (MacIntyre et al. 2010, Vachon and Prairie 2013, Crosswell 2015). A further limitation of many existing gas exchange parameterizations in these settings is that they are derived using the efflux of a single gas such as sulfur hexafluoride, $\mathrm{CO}_{2}$, or methane $\left(\mathrm{CH}_{4}\right)$ (Cole et al. 2010). Relationships between influx processes and gas exchange may not be measurable using the efflux only of these gases. Most importantly, these gases have very different sensitivities to air injection (bubbles added by breaking waves) and ebullition (the formation and rise of bubbles from the sediments) than $\mathrm{O}_{2}$ and other intermediate solubility gases - making gas exchange parameterizations from any single tracer difficult to scale to other gases of interest. In shallow aquatic environments, bubble processes in particular may contribute to fluxes of dissolved $\mathrm{O}_{2}$ because of windtide interactions (Crosswell 2015) and because photosynthesis or methanogenesis may lead to ebullition (Cheng et al. 2014). The result of these limitations is that gas exchange parameterizations may be site specific, and fluxes predicted using different parameterizations can range over an order of magnitude (Kremer et al. 2003, Borges et al. 2004). Determination of gas exchange fluxes is thus often the largest source of error in estimates of metabolic rates.

The noble gases neon (Ne), argon (Ar), krypton (Kr), and xenon (Xe) are a suite of naturally occurring, biologically inert tracers that vary widely in solubility and are sensitive to a range of physical processes over the same timescales as $\mathrm{O}_{2}$ and $\mathrm{CO}_{2}$, including diffusive gas exchange and bubbling. More soluble gases including $\mathrm{Kr}$ and $\mathrm{Xe}$ are primarily sensitive to changes in temperature while less soluble $\mathrm{Ne}$ is highly sensitive to bubble processes. Ar exhibits intermediate sensitivity to these processes, similarly to $\mathrm{O}_{2}$. The processes expected to affect noble gas concentrations in a shallow, tidally-isolated aquatic setting are plotted in Fig. 1, along with the resulting changes to $\mathrm{Ne}$ and $\mathrm{Kr}$ saturation state relative to their solubility equilibrium concentrations, i.e. $\Delta \mathrm{Ne}=\left([\mathrm{Ne}] /[\mathrm{Ne}]_{\mathrm{sat}}-1\right) * 100 \%$. Diffusive gas exchange, temperature changes and bubble processes are expected to largely control changing saturation states of noble gases in surface waters [10]. 
Figure 1: Processes expected to change noble gas saturation state relative to atmospheric equilibrium in a tidally isolated salt marsh pond. A conceptual diagram of the pond (a): dissolved gas fluxes are identified with arrows, including diffusive gas exchange, ebullition from biologically generated bubbles, air (bubble) injection from breaking waves, and exchange with adjacent porewater. Other processes that change the saturation state of dissolved gases and may affect fluxes (boxed to the right of the diagram) include changes in pressure, temperature, and salinity $(\Delta \mathrm{P}, \mathrm{T}$, and $\mathrm{S})$ and evaporation of pond water. The effects of these processes on the saturation state of neon (Ne, a less soluble gas) and krypton ( $\mathrm{Kr}$, a more soluble gas) (b) are plotted relative to initial conditions at saturation with respect to the atmosphere $\left(\mathrm{T}=27^{\circ} \mathrm{C}, \mathrm{S}=30, \mathrm{P}=1 \mathrm{~atm}\right)$. Gas exchange adds and removes the gases at $0 \%$ (at saturation). Processes that are well constrained by measured environmental variables are identified with solid lines. Processes marked with dashed lines are derived from literature estimates in other settings, and the actual magnitude of effects in the ponds will depend on in situ conditions (see main text and supplementary information for more details). Porewater exchange may affect dissolved gases differently depending on the porewater composition (modeled as $\mathrm{T}=12^{\circ} \mathrm{C}, \mathrm{S}=25$, between $30 \%$ undersaturated and $30 \%$ supersaturated). 


\section{a Diffusive gas}
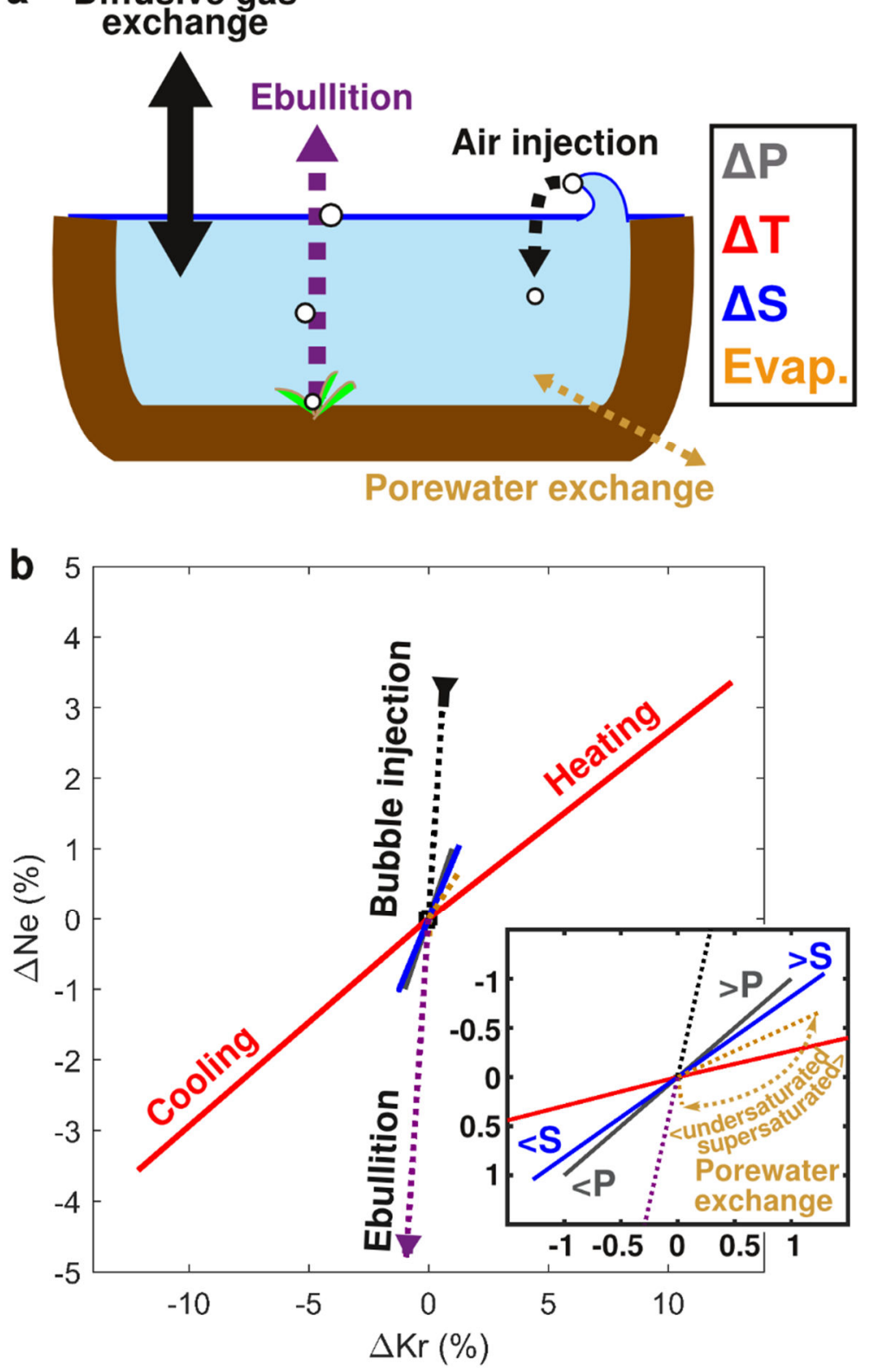

Figure 1: Processes expected to change noble gas saturation state relative to atmospheric equilibrium in a tidally isolated salt marsh pond. Caption on preceding page. 
In this study, we analyze a unique dataset of $\mathrm{Ne}, \mathrm{Ar}, \mathrm{Kr}$, and $\mathrm{Xe}$ in order to address three goals: First, we evaluate how three commonly used gas exchange parameterizations derived from studies in lakes (Cole and Caraco 1998, Crusius and Wanninkhof 2003, Vachon and Prairie 2013) perform in predicting gas exchange in an analogous marine environment, a salt marsh pond. Shallow $(<0.5 \mathrm{~m})$ brackish ponds are abundant and dynamic features of temperate salt marshes (Wilson et al. 2014). Gas exchange parameterizations specific to such environments have not been experimentally determined, and picking an appropriate parameterization is critical for the determination of metabolic fluxes in the pond. Next, we evaluate the contribution of other potential drivers of air-water fluxes of dissolved gases that are not included in the three models, in particular ebullition. We conclude by estimating how much photosynthetic $\mathrm{O}_{2}$ might be removed from the pond by ebullition and the resulting effect on metabolic rates calculated from an oxygen mass balance.

\section{Methods}

\section{Setting and sampling methods}

The study location is a salt marsh pond in the Plum Island Ecosystems Long Term Ecological Research site in Massachusetts, USA $\left(42.7411^{\circ} \mathrm{N}, 70.8309^{\circ} \mathrm{W}\right)$. The pond is circular, covers $7000 \mathrm{~m}^{2}$, and has 25 $\mathrm{cm}$ mean depth. Five to ten $\mathrm{m}$ of grass-covered high marsh separates the pond from an adjacent tidal creek. Water from the creek floods the pond during spring-tides every two weeks. In summer 2014, three sub-daily sampling periods when the pond was tidally isolated were sampled, including two daytime periods and one nighttime period. Environmental conditions and the expected maximum change in noble gas saturations state over these periods are presented in Table 1 (mean saturation states of the noble gases over each experimental period are also summarized in the supplementary information).

Temperature, salinity, and $\mathrm{O}_{2}$ saturation state data were collected every 15 minutes using a YSI ${ }^{\mathrm{TM}}$ Exo2 water quality sonde located near the center of the pond that was calibrated before and after each sampling period. Atmospheric pressure and meteorological measurements used to calculate the windspeed at $10 \mathrm{~m}$ height $\left(\mathrm{U}_{10}\right)$ were determined using a meteorological tower located roughly $200 \mathrm{~m}$ from the study pond. $\mathrm{U}_{10}$ was calculated using the stability corrected log wind profile (Walmsley 1988) corrected for displacement of the zero-wind level by ground obstructions $(\sim 5 \mathrm{~cm})$ and the roughness length scale of grasses on the high marsh $(\sim 1 \mathrm{~cm})$ (e.g. Tsai and Tsuang 2005).

Dissolved noble gases were sampled using crimped copper tube sampling (Jenkins et al. 2010), with the modification that water samples were hand-pumped through the copper tube using pre-soaked plastic tubing extended $\sim 1 \mathrm{~m}$ from the pond bank (10 $\mathrm{m}$ from the sonde). Gases were extracted and concentrations of noble gases determined on a quadrupole mass spectrometer Stanley et al. 2009b; Ne and Ar were determined using peak height manometry and $\mathrm{Kr}$ and $\mathrm{Xe}$ using ratiometric isotope dilution. 
Table 1. Environmental variability during sample period and expected effects on noble gas saturation states.

\begin{tabular}{|c|c|c|c|c|c|}
\hline Study period & Jul. $29^{\text {th }}$ & $(6: 00-19: 00)$ & & & \\
\hline Parameter & Mean(std. dev.) & [Range] & Maximum change: & $\Delta \mathrm{Ne}$ & $\Delta \mathrm{Kr}$ \\
\hline Temperature $\left({ }^{\circ} \mathrm{C}\right)$ & $24.5(2.8)$ & [20.4 to 27.7$]$ & & $4.6 \%$ & $17.1 \%$ \\
\hline Salinity & $27.9(0.2)$ & [27.7 to 28.3 ] & & $0.3 \%$ & $0.4 \%$ \\
\hline$\Delta \mathrm{O}_{2}(\%)^{\mathbf{b}}$ & $1(33)$ & {$[-56$ to 41$]$} & & & \\
\hline $\mathrm{U}_{10}\left(\mathrm{~m} \mathrm{~s}^{-1}\right)^{\mathbf{c}}$ & $4.4(1.5)$ & [1.6 to 6.5$]$ & via air injection ${ }^{\mathrm{e}}$ & $<1.7 \%$ & $<0.3 \%$ \\
\hline $\operatorname{PAR}(\mathrm{mmol} \mathrm{m}-2 \mathrm{~s}-1)^{\mathbf{d}}$ & $0.96(0.60)$ & [0.04 to 1.75$]$ & & & \\
\hline Atmospheric pressure $(\mathrm{kPa})$ & $100.769(0.184)$ & {$[100.410$ to 101} & 105] & $0.7 \%$ & $0.7 \%$ \\
\hline Study period & Aug. $25^{\text {th }}$ & $(20: 30-00: 45)$ & & & \\
\hline Parameter & Mean(std. dev.) & [Range] & Maximum change: & $\Delta \mathrm{Ne}$ & $\Delta \mathrm{Kr}$ \\
\hline Temperature $\left({ }^{\circ} \mathrm{C}\right)$ & $26.3(0.8)$ & [25.2 to 27.7$]$ & & $1.4 \%$ & $5.3 \%$ \\
\hline Salinity & $32.0(0.1)$ & [31.8 to 32.2$]$ & & $0.2 \%$ & $0.3 \%$ \\
\hline$\Delta \mathrm{O}_{2}(\%)$ & $-42(16)$ & {$[-63$ to -10$]$} & & & \\
\hline $\mathrm{U}_{10}(\mathrm{~m} \mathrm{~s}-1)$ & $1.6(0.6)$ & {$[0.9$ to 2.7$]$} & via air injection & $<0.1 \%$ & $<0.1 \%$ \\
\hline $\operatorname{PAR}(\mathrm{mmol} \mathrm{m}-2 \mathrm{~s}-1)$ & $0.00(0.00)$ & {$[0.00$ to 0.00$]$} & & & \\
\hline Atmospheric pressure $(\mathrm{kPa})$ & $101.910(0.015)$ & [101.892 to 101 & 934] & $<0.1 \%$ & $<0.1 \%$ \\
\hline
\end{tabular}

\begin{tabular}{l|llrrr} 
Study period & Aug. $26^{\text {th }}$ & $(9: 15-15: 45)$ & & \\
\cline { 1 - 2 } Parameter & Mean(std. dev.) & {$[$ Range $]$} & Maximum change: & $\Delta \mathrm{Ne}$ & $\Delta \mathrm{Kr}$ \\
\hline Temperature $\left({ }^{\circ} \mathrm{C}\right)$ & $28.8(2.4)$ & {$[24.0$ to 31.2$]$} & & $4.1 \%$ & $15.8 \%$ \\
Salinity & $32.1(0.2)$ & {$[31.8$ to 32.4$]$} & & $0.3 \%$ & $0.4 \%$ \\
$\Delta \mathrm{O}_{2}(\%)$ & $15(32)$ & {$[-51$ to 50$]$} & & & \\
$\mathrm{U}_{10}(\mathrm{~m} \mathrm{~s}-1)$ & $3.9(1.4)$ & {$[1.5$ to 5.7$]$} & via air injection & $<0.4 \%$ & $<0.1 \%$ \\
PAR $(\mathrm{mmol} \mathrm{m-2} \mathrm{s-1)}$ & $1.30(0.31)$ & {$[0.54$ to 1.61$]$} & & & \\
Atmospheric pressure $(\mathrm{kPa})$ & $101.838(0.114)$ & {$[101.634$ to 101.997$]$} & $0.4 \%$ & $0.4 \%$
\end{tabular}

Other poorly constrained processes: Maximum change: $\quad \Delta \mathrm{Ne} \quad \Delta \mathrm{Kr}$

Ebullition ${ }^{\mathrm{f}}$

Porewater exchangeg

$\sim 5 \% \quad \sim 1 \%$

$<0.7 \% \quad<1.2 \%$

a Practical Salinity Scale of 1978, unitless

b Saturation state relative to solubility equilibrium of gas, as defined in text

c Windspeed at $10 \mathrm{~m}$, stability corrected using log wind profile, as defined in text

d Photosynthetically active radiation at $400-700 \mathrm{~nm}$ wavelengths

${ }^{\mathbf{e}}$ Overestimate for pond, calculated from parameterization from Stanley et al. 2009a for open ocean environment

${ }^{\mathbf{f}}$ Estimated from expected effect of rates in Cheng et al. 2014

${ }^{\mathbf{g}}$ Estimated for upper bound of tidally variant porewater exchange rates using the model of Nuttle and Hemond 1988 


\section{Model evaluations}

The three gas exchange parameterizaitons evaluated in this study were chosen because they are representative of a pond environment with no tidal currents, are functions of readily measureable environmental parameters such as windspeed, and have been applied in other studies as well as modeling toolboxes (Winslow et al. 2016). Each parameterization was originally derived following the form:

$$
\frac{\partial \mathrm{C}}{\partial \mathrm{t}} * \mathrm{z} * \rho=\mathrm{k}_{C} *\left(\mathrm{C}_{\mathrm{sat}}-\mathrm{C}\right) * \rho
$$

where $\mathrm{z}$ is water depth $(\mathrm{m}), \mathrm{C}$ the concentration of a given gas $\left(\mu \mathrm{mol} \mathrm{kg} \mathrm{kg}^{-1}\right), \mathrm{C}_{\text {sat }}$ the temperature, salinity, and pressure dependent saturation concentration of that gas, and $\rho$ is density $\left(\mathrm{kg} \mathrm{m}^{-3}\right)$ to convert concentrations to volumetric units. $\mathrm{k}_{C}$ is the gas transfer velocity $\left(\mathrm{m} \mathrm{h}^{-1}\right)$, and is scaled between gases using the Schmidt number ( $\mathrm{Sc}$, the unitless ratio of kinematic viscosity of water to molecular diffusivity of the gas):

$$
\frac{\mathrm{k}_{a}}{\mathrm{k}_{b}}=\left(\frac{\mathrm{Sc} \mathrm{c}_{\mathrm{a}}}{\mathrm{Sc}_{\mathrm{b}}}\right)^{-0.5}
$$

where the subscripts represent two gases, and the exponent of -0.5 is appropriate for a rough surface (ripples or waves). $\mathrm{k}_{C}$ is often scaled to that of $\mathrm{CO}_{2}$ in fresh water at $20^{\circ} \mathrm{C}(\mathrm{Sc}=600)$, reported as $\mathrm{k}_{600}$.

The parameterizations for $\mathrm{k}_{600}\left(\mathrm{~cm} \mathrm{~h}^{-1}\right)$ evaluated in this study are reproduced here:

$$
\begin{array}{clr}
\text { Cole and Caraco (1998), Eq. 5: } & \mathrm{k}_{600}=2.07+2.15 * \mathrm{U}_{10} 1.7 \\
\begin{array}{clr}
\text { Crusius and Wanninkhof (2003), } & \mathrm{k}_{600}=0.72(0.36) * \mathrm{U}_{10} & {\left[\mathrm{U}_{10}<3.7 \mathrm{~m} \mathrm{~s}^{-1}\right]} \\
\text { Eqs. 5 and 6: } & \mathrm{k}_{600}=-13.3(27.1)+4.33(5.31) * \mathrm{U}_{10} & {\left[\mathrm{U}_{10}>3.7 \mathrm{~m} \mathrm{~s}^{-1}\right]}
\end{array} \\
\begin{array}{rrr}
\text { Vachon and Prairie (2013), } & \mathrm{k}_{600}=2.51(0.99)+1.48(0.34) * \mathrm{U}_{10} \\
\text { Table 2, Eq. B: } & & +0.39(0.08) * \mathrm{U}_{10} * S A
\end{array}
\end{array}
$$

Standard deviations for parameterization terms are in parentheses (Eq. 3 uncertainties could not be assessed using the data reported in Cole and Caraco 1998). In Eq. 5, $S A$ is the surface area $\left(\mathrm{km}^{2}\right)$ of the water body; this acts to integrate the effects of fetch. Non-zero intercepts (gas exchange in the absence of wind) in Eqs. 3 and 5 are empirical fits for non-wind sources of turbulence, such as buoyancy fluxes.

Each parameterization was evaluated by initializing a bulk water model with the measured concentrations of $\mathrm{Ne}, \mathrm{Ar}, \mathrm{Kr}$, and $\mathrm{Xe}$ and running the discretized form of Eq. 1 forward at 15 minute timesteps using $\mathrm{k}_{C}$ predicted for each gas (Eqs. 2-5). The saturation state of each gas $\left(\Delta C=\left[C / C_{\text {sat }}-1\right]^{*} 100 \%\right)$ was determined using recent solubility functions (Lott and Jenkins, personal communication) measured on the same instrument used to analyze the samples in this study. The choice of solubility functions is discussed in the supplementary information (Wood and Caputi 1966, Weiss and Kyser 1978, Hamme and Emerson 2004, Manning et al. 2016a). The modeled gas saturation states were compared to those of the samples by calculating the root mean square error (RMSE) over each sampling period and over the entire study period. Systematic model misfits that vary between the three gases may indicate that fluxes other than diffusive gas exchange occurred (e.g. processes identified in Fig. 1, Table 1). 


\section{Results and Discussion}

\section{Model results}

Measured and modeled noble gas saturation states are plotted in Fig. 2 (Xe not shown for figure clarity but Xe data are included in database). Uncertainties in sample saturation state are primarily caused by heterogeneity of pond temperature and salinity. Modeled values of $\Delta \mathrm{Kr}$ and $\Delta \mathrm{Xe}$, the most soluble of the four gases, generally agree well with sampled saturation state, though the initial rise of Xe was underestimated by the model evaluations on the morning of Aug $26^{\text {th }} . \Delta \mathrm{Ar}$ and especially $\Delta \mathrm{Ne}$ tended to be lower than model predictions during the day. As the pond cooled at night, $\Delta \mathrm{Ne}$ and $\Delta \mathrm{Ar}$ were in better agreement with modeled values, excluding a single sample which has a pattern of supersaturation for $\mathrm{Ne}$ and $\mathrm{Ar}$ characteristic of sample contamination by a small air bubble (the effect is minimal on $\mathrm{Kr}$ and $\mathrm{Xe}$ ).

The model evaluation with the lowest RMSE compared to measurements of each gas varied by sampling period, but in general the parameterization from Vachon and Prairie (2013) resulted in good fits to the data (Table 2). Over the entire study, this parameterization led to the best agreement to the pooled data for all gases, and for $\mathrm{Ne}, \mathrm{Ar}$, and $\mathrm{Kr}$ separately. If the first Xe sample on Aug $26^{\text {th }}$ was assumed to be unrepresentative of mean pond conditions and increased by one standard deviation, or if the models were initialized at the second sample point, all three models gave equivalently good fits for Xe.

\section{Interpreting noble gas signals}

A notable feature of the dataset is the low saturation state of Ne and Ar compared to that expected from the diffusive gas exchange; this offset was larger than the difference between the parameterizations. In addition to gas exchange, differences in temperature, salinity and atmospheric pressure effect both measured and modeled values similarly. Many of the other processes identified as drivers of changing saturation state (Fig. 1) are expected to have small effects on the observations. Potential effects of evaporation (Linacre 1993), porewater exchange (Nuttle and Hemond 1988), and air injection (Stanley et al. 2009a) are evaluated in the supplementary information, but are expected to cause $\sim 1 \%$ or less difference in saturation state for all gases. There was a small increase in nighttime $\Delta \mathrm{Ne}$ compared to the model evaluations not readily explained by the evaluated processes; as measured $\Delta \mathrm{Ne} \approx 0 \%$, there was likely another source of diffusive exchange (e.g. enhanced nighttime buoyancy flux; MacIntyre et al. 2010) that was not well represented by the non-zero intercepts in the parameterizations.

Ebullition is the remaining and most plausible explanation for observed differences between measured and modeled saturation states. Indeed, pronounced decreases in $\Delta \mathrm{Ne}$ and $\Delta \mathrm{Ar}$, particularly on Jul. $29^{\text {th }}$, are a sign of ebullition since gas exchange alone is sufficient to explain $\Delta \mathrm{Kr}$ and $\Delta \mathrm{Xe}$ and ebullition removes less soluble gases preferentially. Supporting this idea, we observed abundant bubbles rising from the pond bottom in the vicinity of benthic macroalgae. Direct measurements of $\mathrm{O}_{2}$ and $\mathrm{CH}_{4}$ ebullition from shallow marine sediments (Cheng et al. 2014) are sufficiently large to explain such decreases in $\Delta \mathrm{Ne}$ and $\Delta \mathrm{Ar}$ (Fig. 1b), with high spatial and temporal variability; similar variability in ebullition may explain the larger differences between modeled and measured $\Delta \mathrm{Ne}$ and $\Delta \mathrm{Ar}$ on Jul. $29^{\text {th }}$ than on Aug. $26^{\text {th }}$. 

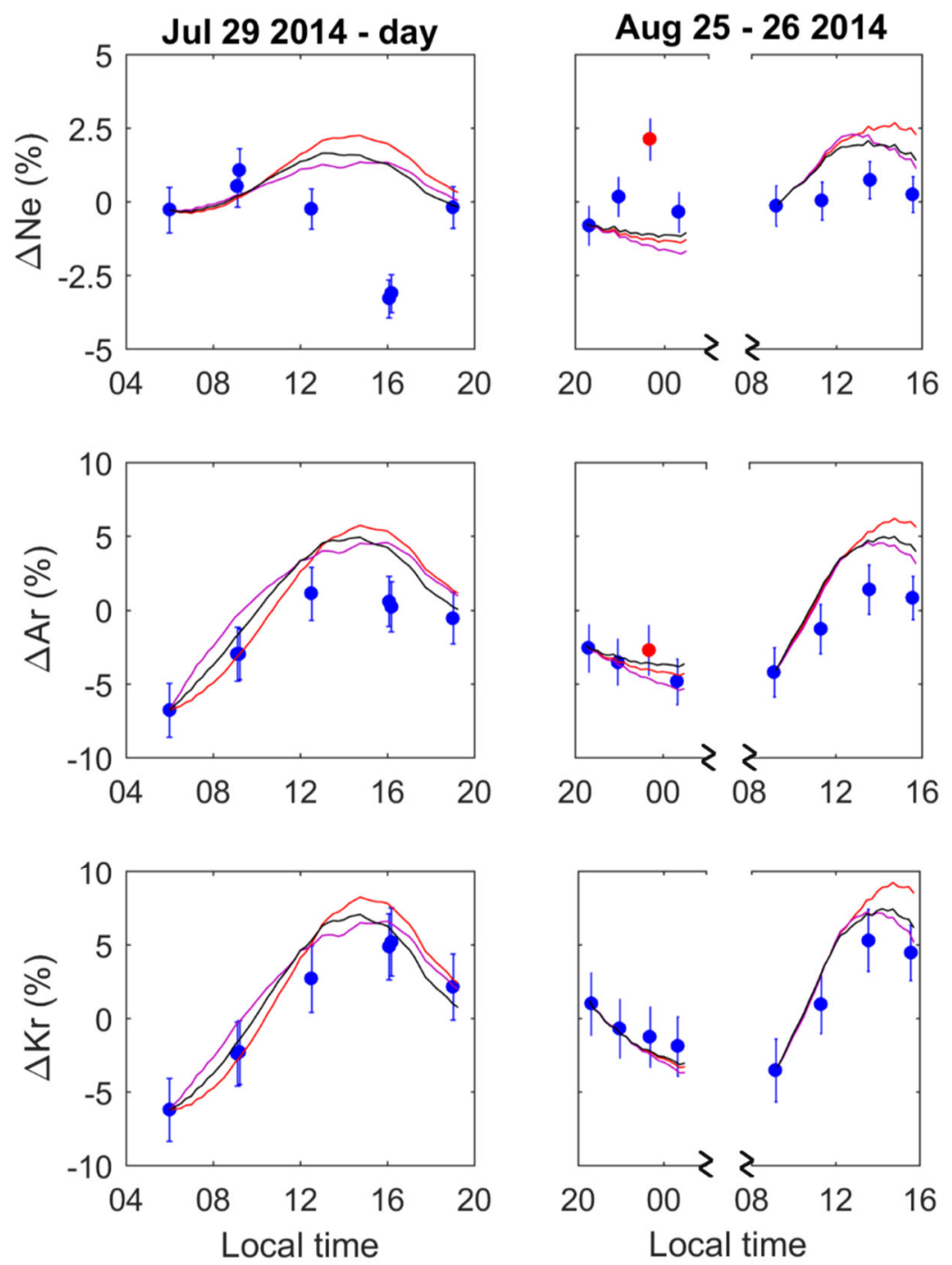

Cole and Caraco (1998)

Vachon and Prairie (2013)

Crusius and Wanninkhof (2003)

Figure 2: Saturation state of neon (Ne), argon (Ar), and krypton $(\mathrm{Kr})$ in blue circles (with one standard deviation error bars) for the three study periods, relative to in situ solubility corrected for atmospheric pressure. Modeled saturation states are plotted as lines based on initial conditions and the gas exchange parameterizations from Cole and Caraco (1998; red), Crusius and Wanninkhof (2003; purple), and Vachon and Prairie (2013; black). On night of Aug. 25 $5^{\text {th }}$, measurements of Ne and Ar from one sample (red circle) were contaminated by an air bubble during sampling (see text). 
Table 2. Root mean square error (RMSE) for model evaluations with each gas exchange parameterization.

Study period Jul. $29^{\text {th }}(6: 00-19: 00)$

\begin{tabular}{l|ccc}
\hline \multirow{2}{*}{ RMSE } & $\begin{array}{l}\text { Cole and } \\
\text { Caraco (1998) }\end{array}$ & $\begin{array}{l}\text { Crusius and } \\
\text { Wanninkhof (2003) }\end{array}$ & $\begin{array}{l}\text { Vachon and } \\
\text { Prairie (2013) }\end{array}$ \\
\cline { 2 - 4 } $\mathrm{Ne}$ & 2.5 & 2.1 & 2.1 \\
$\mathrm{Ar}$ & 2.5 & 2.5 & 2.1 \\
$\mathrm{Kr}$ & 1.6 & 1.5 & 1.4 \\
$\mathrm{Xe}$ & 1.9 & 2.0 & 1.8
\end{tabular}

Study period Aug. $25^{\text {th }}$ (20:30 - 00:45)

\begin{tabular}{l|ccc}
\hline \multirow{2}{*}{ RMSE } & $\begin{array}{l}\text { Cole and } \\
\text { Caraco (1998) }\end{array}$ & $\begin{array}{l}\text { Crusius and } \\
\text { Wanninkhof (2003) }\end{array}$ & $\begin{array}{l}\text { Vachon and } \\
\text { Prairie (2013) }\end{array}$ \\
\cline { 2 - 4 } $\mathrm{Ne}$ & 2.0 & 2.2 & 1.9 \\
$\mathrm{Ar}$ & 0.8 & 1.2 & 0.8 \\
$\mathrm{Kr}$ & 0.9 & 1.0 & 0.8 \\
$\mathrm{Xe}$ & 0.7 & 0.9 & 0.7
\end{tabular}

Study period Aug. $26^{\text {th }}(9: 15-15: 45)$

\begin{tabular}{l|ccc}
\hline \multirow{2}{*}{ RMSE } & $\begin{array}{l}\text { Cole and } \\
\text { Caraco (1998) }\end{array}$ & $\begin{array}{l}\text { Crusius and } \\
\text { Wanninkhof (2003) }\end{array}$ & $\begin{array}{l}\text { Vachon and } \\
\text { Prairie (2013) }\end{array}$ \\
\cline { 2 - 3 } $\mathrm{Ne}$ & 1.6 & 1.2 & 1.1 \\
$\mathrm{Ar}$ & 3.4 & 2.4 & 2.7 \\
$\mathrm{Kr}$ & 2.7 & 1.5 & 1.7 \\
$\mathrm{Xe}^{\mathrm{a}}$ & 1.8 & 3.4 & 2.9
\end{tabular}

Pooled data from all three periods

\begin{tabular}{l|ccc}
\hline \multirow{2}{*}{ RMSE } & $\begin{array}{l}\text { Cole and } \\
\text { Caraco (1998) }\end{array}$ & $\begin{array}{l}\text { Crusius and } \\
\text { Wanninkhof (2003) }\end{array}$ & $\begin{array}{l}\text { Vachon and } \\
\text { Prairie (2013) }\end{array}$ \\
\cline { 2 - 4 } $\mathrm{Ne}$ & 2.1 & 1.9 & 1.8 \\
$\mathrm{Ar}$ & 2.6 & 2.2 & 2.1 \\
$\mathrm{Kr}$ & 1.9 & 1.4 & 1.3 \\
$\mathrm{Xe}^{\mathrm{a}}$ & 1.6 & 2.3 & 2.0 \\
$\mathrm{All} \mathrm{gases}^{\mathrm{a}}$ & 2.1 & 2.0 & 1.7 \\
\multicolumn{2}{l}{${ }^{\text {a See Model results text regarding Xe on Aug. 26 }} 6^{\text {th }}$} &
\end{tabular}




\section{Calculating ebullition from neon saturation state}

The gas exchange parameterizations did well in explaining observed changes in $\mathrm{Kr}$ and $\mathrm{Xe}$, and ebullition could explain most of the difference between modeled and observed values of $\mathrm{Ne}$ and Ar. Therefore we combine our observations of $\mathrm{Ne}$ saturation state with the gas exchange models to constrain ebullition. If bubbles forming at the sediment-water interface equilibrate with respect to noble gases in the surrounding water before rising (timescale of minutes; Holocher et al. 2003), then the concentrations in water can be related to those in the bubble, $\mathrm{C}_{\mathrm{b}}\left(\mu \mathrm{mol} \mathrm{m} \mathrm{m}^{-3}\right.$ bubble) by Henry's law solubility equilibrium $\left(H_{b w}\right)$ such that:

$$
\mathrm{C}_{\mathrm{b}}=\mathrm{C} * H_{b w, C}
$$

where $H_{b w, C}\left(\mu \mathrm{mol} \mathrm{m}{ }^{-3}\right.$ gas / $\mu$ mol kg-1 water) is calculated from Henry's law for exchange between the pond and atmosphere, adjusted for the depth of bubble formation; the bubble equilibrium concentration is fixed by the water equilibrium concentration, which is in turn controlled by atmospheric composition. In other words, $H_{b w, C}$ between water and both gas phases must be identical other than a pressure-proportional increase with depth. Disequilibrium develops as bubbles escape to the air. The mass balance of a bubble sensitive gas can be used to calculate an ebullition rate, $E\left(\mathrm{~mL}\right.$ bubbles $\left.\mathrm{m}^{-2} \mathrm{~h}^{-1}\right)$ :

$$
\begin{aligned}
& \frac{\partial \mathrm{C}}{\partial \mathrm{t}} * \mathrm{z} * \rho=\mathrm{k}_{C} *\left(\mathrm{C}_{\mathrm{sat}}-\mathrm{C}\right) * \rho-E * \mathrm{C}_{\mathrm{b}} * 10^{-6} \\
& E=-\rho *\left[\frac{\partial \mathrm{C}}{\partial \mathrm{t}} * \mathrm{z}-\mathrm{k}_{C} *\left(\mathrm{C}_{\mathrm{sat}}-\mathrm{C}\right)\right] /\left[\mathrm{C}_{\mathrm{b}} * 10^{-6}\right]
\end{aligned}
$$

Eq. 8 rearranges the mass balance in Eq. 7, and the factor of $10^{-6}$ converts from $\mathrm{m}^{3}$ to $\mathrm{mL}$ of bubble volume per unit area.

The model framework used to evaluate the gas exchange parameterizations can be used to calculate the ebullition rate from $\mathrm{Ne}$ (with gas exchange calculated from Eq. 5). Ne concentration is interpolated between sample points in order to calculate $E$ over each timestep (Eq. 8), with the restriction that negative values of $E$ are set to zero (ebullition is not allowed to add noble gases to the water). Eq. 7 is solved for $\mathrm{Ne}$ and the other noble gases, and modeled results including ebullition compared to those from gas exchange only (Fig. 3a).

The improved fit of Ar using Ne-derived ebullition indicates that assuming bubble equilibrium with respect to the noble gases is reasonable. This is consistent with visual observations of bubbles stuck to macroalgae and plant leaves. However if the noble gas distributions in bubbles are diffusion-rate limited, then greater ebullition is required to explain decreased $\Delta \mathrm{Ne}$ relative to gas exchange. Forcing positive solutions to $E$ biases ebullition high; there is no restoring force besides gas exchange if $E$ is overestimated because of interpolation or sample uncertainties. Iteratively adjusting the magnitude of the ebullition flux over each sampling period to minimize RMSE suggests that the best fit ebullition rate may be up to $20 \%$ lower than estimated above with $E \geq 0$. 

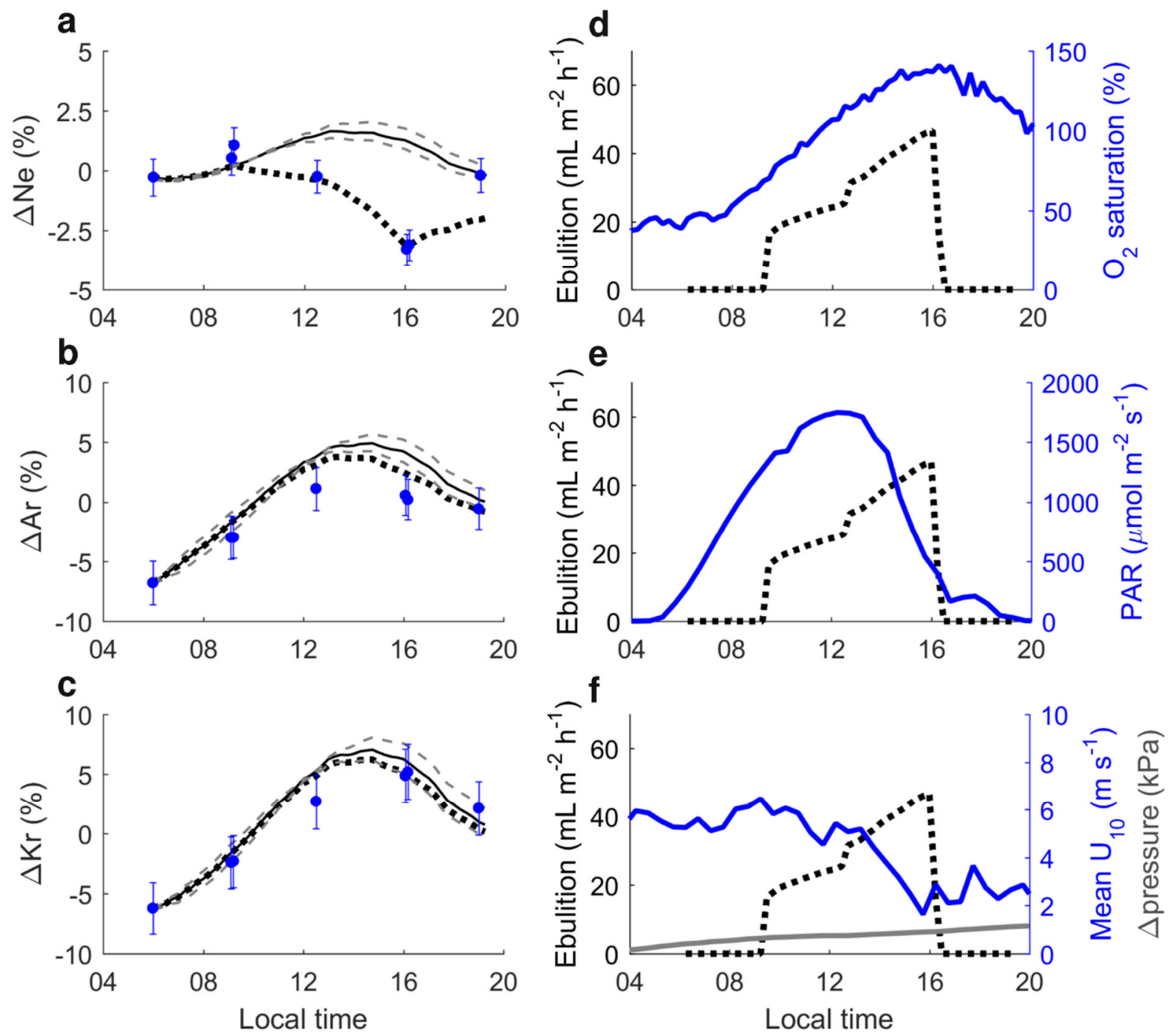

Figure 3: The neon (Ne; a), argon (Ar; b), and krypton (Kr; c) saturation states on Jul. $29^{\text {th }}$ relative to in situ solubility adjusted for atmospheric pressure (blue circles and one standard deviation error bars) compared to modeled saturation states from the gas exchange parameterization of Vachon and Prairie (2013) only (black line and dashed gray uncertainty bounds based on reported standard deviation of parameterization terms), and to gas exchange including the effects of ebullition (black dashed line) derived from $\mathrm{Ne}$ mass balance. Ebullition rates (black dashed line again) are plotted against environmental parameters (blue lines), including oxygen $\left(\mathrm{O}_{2}\right)$ saturation state $(\mathbf{d})$, photosynthetically active radiation flux (PAR, 400-700 nm; e), and (f) mean windspeed at $10 \mathrm{~m}\left(\mathrm{U}_{10}\right)$ as well as the pressure difference from 1 atm $(101.325 \mathrm{kPa})$. 


\section{Relationship between ebullition and environmental processes}

Photosynthetic $\mathrm{O}_{2}$ production in sunlit sediments is a likely source of ebullition. Production and respiration cause large diel changes in $\mathrm{O}_{2}(10 \%$ to $150 \%$ of saturation at the sonde location, over bare sediment). $\mathrm{O}_{2}$ is distributed heterogeneously within the pond, reflecting the spatial variability of production; a survey of $\mathrm{O}_{2}$ around the perimeter of the study pond revealed peak $\mathrm{O}_{2}$ of $200 \%$ to $300 \%$ of saturation in areas with visible benthic algae or vascular plants. Photosynthetic production should track photosynthetically active radiation (PAR, 400-700 nm; Stanley and Howard 2013). Local $\mathrm{O}_{2}$ supersaturations may form bubbles at nucleation points (such as photosynthesizing algae), and these bubbles break free and rise to the water surface because of buoyancy increases, physical disturbances, or transient decreases in pressure, e.g. from wave troughs (Maeck et al. 2014).

On Jul. $2^{\text {th }}$, Ne-derived ebullition tracks $\mathrm{O}_{2}$ saturation state in the water column (Fig. 3b). Ebullition occurs during a period of decreasing windspeed and rising atmospheric pressure, and does not track but follows peak PAR. On Aug $26^{\text {th }}$ (results not plotted) the rate of ebullition was half that of Jul. $29^{\text {th }}$. In this case, peak PAR was slightly later in the day and $\mathrm{O}_{2}$ did not become supersaturated until later in the day. At the same time, windspeed was stable and pressure was $1 \mathrm{kPa}$ greater than over the Jul. $29^{\text {th }}$ study period. While sonde $\mathrm{O}_{2}$ was not supersaturated over the entire period of ebullition on either day, local supersaturation could still generate bubbles. Thus we surmise that $\mathrm{O}_{2}$ saturation state, affected by both biological production and temperature, was related to the magnitude of ebullition, and lower pressure may have stimulated bubble release as expected from other work (Maeck et al. 2014).

\section{The effect of ebullition on production rates}

Photosynthetic $\mathrm{O}_{2}$ escaping submerged environments via ebullition is not included in diffusive gas exchange fluxes, meaning metabolism estimates from gas exchange models underestimate $\mathrm{O}_{2}$ efflux and thus biological production. If ebullition is also present at night (e.g. bubbles of $\mathrm{CH}_{4}$ or molecular nitrogen, $\mathrm{N}_{2}$ ), $\mathrm{O}_{2}$ could instead be stripped from the water during ebullition and aerobic respiration overestimated. In either case, the net effect is a negative (heterotrophic) bias in net metabolism derived from diel $\mathrm{O}_{2}$ cycling. We use the Ne-derived bubble flux to estimate the amount of photosynthetic $\mathrm{O}_{2}$ lost through ebullition in this study. To do so, we assume bubbles contain primarily $\mathrm{O}_{2}, \mathrm{~N}_{2}$, and $\operatorname{Ar}$ (and water vapor). We further assume that $\mathrm{N}_{2}$ is homogenously distributed throughout the pond with a similar saturation state to $\mathrm{Ne}$ or $\operatorname{Ar}$ (which have similar solubility to $\mathrm{N}_{2}$ ) because $\Delta \mathrm{N}_{2}$ caused by denitrification should be small compared to gas exchange during daytime periods of high $\mathrm{O}_{2}$. The fraction of the ebullition flux that is $\mathrm{O}_{2}$ is then solved by difference from $\mathrm{C}_{\mathrm{b}}$ of each gas and the total gas in the bubble (from the ideal gas law).

Applying this approach, we find that ebullition accounts for losses of $-6.9(1.4) \mathrm{mmol} \mathrm{O}_{2} \mathrm{~m}^{-2}$ on Jul. 29 and $-2.7(0.5) \mathrm{mmol} \mathrm{O}_{2} \mathrm{~m}^{-2}$ on Aug. $26^{\text {th }}$ (standard deviation of propagated error in parentheses), and thus ebullition accounted for $113 \%$ and $12 \%$ as much $\mathrm{O}_{2}$ as net diffusive gas exchange (supplementary table). This results in $24 \%$ and $5 \%$ greater net metabolism when ebullition is included compared to using a standard gas exchange parameterization without ebullition $\left(28.8\right.$ and $57.6 \mathrm{mmol} \mathrm{O}_{2} \mathrm{~m}^{-2}$ not including ebullition; see supplementary information). The underestimates of productivity are slightly smaller on an hourly basis during periods of peak productivity - mean ebullition rates are $-1.0(0.3) \mathrm{mmol} \mathrm{O}_{2} \mathrm{~m}^{-2} \mathrm{~h}^{-1}$ and $-0.4(0.1) \mathrm{mmol} \mathrm{O}_{2} \mathrm{~m}^{-2} \mathrm{~h}^{-1}$ on Jul. $29^{\text {th }}$ and Aug. $26^{\text {th }}$ during the $\sim 9: 00$ to 17:00 periods when ebullition was observable, leading to $20 \%$ and $5 \%$ greater net metabolism, respectively. Each ebullition estimate becomes a more important fraction of the net metabolism over longer periods because integrated net metabolism is smaller in magnitude as periods of net heterotrophy are included (e.g. night). Neglecting ebullition when calculating metabolism then introduces a systematic bias similar or greater in size than the differences between gas exchange parameterizations. Thus ebullition of photosynthetic oxygen may 
be a significant flux compared to diffusive gas exchange in this setting, and an important process to consider when using dissolved gases to study ecosystem metabolism and biogeochemical cycling in other shallow aquatic environments such as sea grass beds.

\section{Conclusion}

We found that three commonly used gas exchange parameterizations all did well predicting noble gas saturation states over sub-daily periods in a salt marsh pond, particularly for more soluble gases. The parameterization of Vachon and Prairie (2013) had the lowest RMSE overall. Because this parameterization is fetch dependent (unlike the other parameterizations), smaller ponds such as those abundant in salt marshes would have much lower predicted gas exchange rates using the Vachon and Prairie (2013) parameterization than using the other parameterizations. We also demonstrated the utility of using noble gases to evaluate ebullition. Mass balances including ebullition better explained the observations than diffusive gas exchange alone. Ebullition fluxes in this setting could release $5-24 \%$ of $\mathrm{O}_{2}$ production. The limited number of samples in this study were not sufficient to develop predictive models of ebullition, but the recent development of field portable, low-cost mass spectrometers designed for measuring dissolved noble gases (Manning et al. 2016b) promises to expand the utility of this approach. 


\section{Supplementary information}

\section{Choice of solubility functions}

The new solubility functions chosen (Lott and Jenkins, personal communication) lead to slightly lower saturation states for $\mathrm{Xe}, \mathrm{Kr}$, and $\mathrm{Ne}$ for our samples than previously published solubilities (Wood and Caputi 1966, Weiss and Kyser 1978, Hamme and Emerson 2004). We chose the recent solubility functions because all noble gas solubilities were measured from the same samples on the same instrument, which was also the instrument used to analyze the samples in this study. Thus using these solubility functions as a set is more internally consistent than using a set of solubilities measured at different laboratories (Manning et al. 2016a). In practice the effect is small because both modeled and measured saturation state scale proportionally. We do not report the recent solubility functions by Lott and Jenkins in this work, as they are being prepared for submission, and the choosing these functions over previously published works does not substantially change the results discussed in this work or the applicability of the method. Mean saturation concentrations of each gas from the Lott and Jenkins solubility functions are summarized in Table S1 (refer to Table 1 in main text for temperature and salinity), and saturation concentrations are listed with each measurement with the original data, accessible as described in the data availability statement.

Table S1. Mean saturation concentration of the noble gases. Standard deviation (in parentheses) calculated from Monte Carlo simulation (3000 iterations) of saturation state assuming random environmental variability within one standard deviation of observed variability around the pond at a single timepoint (temperature $1^{\circ} \mathrm{C}$ and salinity 0.5 on PSS-78).

$$
[\mathrm{C}]_{\mathrm{sat}}\left(\mu \mathrm{mol} \mathrm{kg} \mathrm{kg}^{-1}\right)
$$

\begin{tabular}{cccc} 
& July 29 & August 25 & August 26 \\
\hline $\mathrm{Ne}$ & $6.95(0.13) \times 10^{-3}$ & $6.80(0.04) \times 10^{-3}$ & $6.74(0.14) \times 10^{-3}$ \\
$\mathrm{Ar}$ & $10.8(0.6)$ & $10.2(0.2)$ & $10.0(0.6)$ \\
$\mathrm{Kr}$ & $2.36(0.16) \times 10^{-3}$ & $2.22(0.05) \times 10^{-3}$ & $2.16(0.15) \times 10^{-3}$ \\
$\mathrm{Xe}$ & $3.13(0.03) \times 10^{-4}$ & $2.90(0.01) \times 10^{-4}$ & $2.80(0.02) \times 10^{-4}$
\end{tabular}

\section{Other physical processes that affect noble gas saturation states}

Compared to modeled values, plausible rates of evaporation $\left(<2000 \mathrm{~kg} \mathrm{~h}^{-1}\right.$; Linacre 1993) could account for $<1.3 \%$ increase in saturation state over the sampling period. The effect of porewater exchange depends both on the exchange rate (Nuttle and Hemond 1988) and on the concentration of the porewater, which are poorly constrained. Nonetheless, $\Delta \mathrm{Kr}$ and $\Delta \mathrm{Xe}$ from porewater exchange should be $<1.5 \%(\mathrm{Kr}$ and $\mathrm{Xe}$ are the noble gases most sensitive to mixing and porewater exchange); this value assumes porewater concentrations up to $30 \%$ undersaturated or supersaturated, and porewater $\mathrm{C}_{\text {sat }}$ with temperature $\left(12^{\circ} \mathrm{C}\right)$ and salinity $(25)$ similar to the annual mean for the surrounding estuary combined with a very high exchange rates $\left(4 \mathrm{~m}^{3} \mathrm{~h}^{-1}\right.$, the peak rate through a $2 \mathrm{~m}$ tidal creek bank (Nuttle and Hemond 1988), sustained over a $\sim 6 \mathrm{hr}$ half tidal period). With only slightly warmer porewater temperatures $\left(\right.$ e.g. $\left.15^{\circ} \mathrm{C}\right)$ or more plausible exchange rates the expected effect becomes negligible. Air injection could be identified from increases in $\Delta \mathrm{Ne}$ when windspeed is sufficiently high to generate breaking waves. $\Delta \mathrm{Ne}$ was higher than modeled values only on the night of Aug. $25^{\text {th }}$; during this period, air injection should not be observable (Stanley et al. 2009a) as windspeed was too low to generate breaking waves $\left(\mathrm{U}_{10}=1.4\right.$ to $\left.1.6 \mathrm{~m} \mathrm{~s}^{-1}\right)$. Some other process which enhances turbulence and diffusive exchange other than windspeed (see main text) may lead to these increased saturation states. 
The noble gas concentrations for the more soluble gases can be used to estimate a standardized gas transfer velocity, $\mathrm{k}_{600}$, for comparison with the gas exchange parameterizations. $\mathrm{k}_{600}$ for $\mathrm{Kr}$ and $\mathrm{Xe}$, as well as modeled $\mathrm{k}_{600}$ from the Vachon and Prairie (2013) parameterization are reported in Table S2, based on the time rate of change over the two timepoints and the air-water gradient at the second timepoint (instead of the average gradient, see below). The noble gas based gas transfer velocities should be interpreted with caution as they are evaluated between only two consecutive measurements and short term variation in gas exchange and measurement uncertainty may lead to average air-water gradients and changes in concentration with time that are apparently opposing (negative values of $\mathrm{k}_{600}$ ) over the period between samples. More frequent sampling would be expected to avoid such biases.

Table S2. $\mathrm{k}_{600}$ from measured noble gases and the Vachon and Prairie (2013) windspeed parameterization (standard deviation in parentheses).

\begin{tabular}{ccccccc} 
Date & \multicolumn{2}{c}{ Period (h:mm) } & mean u 10 & \multicolumn{2}{c}{$\mathrm{k}_{600}\left(\mathrm{~cm} \mathrm{~h}^{-1}\right)$} & Vachon \\
& Initial time & Final time & $\left(\mathrm{m} \mathrm{s}^{-1}\right)$ & $\mathrm{Kr}^{\mathrm{a}}$ & $\mathrm{Xe}^{\mathrm{a}}$ & $\begin{array}{c}\text { and } \\
\text { Prairie }\end{array}$ \\
\hline 29-Jul & $5: 59$ & $9: 09$ & 5.6 & 8.5 & 6.1 & 6.1 \\
29-Jul & $9: 09$ & $12: 30$ & 5.7 & 8.5 & 7.4 & 6.2 \\
29-Jul & $12: 30$ & $16: 07$ & 3.7 & 4.4 & 4.1 & 4.9 \\
29-Jul & $16: 07$ & $19: 00$ & 3.1 & -0.8 & -1.3 & 4.5 \\
\hline & Time-weighted mean & $4.4(1.4)$ & $5.3(4.4)$ & $4.2(3.8)$ & $5.4(0.8)$ \\
& Excluding negative k & $4.9(1.1)$ & $7.0(2.4)$ & $5.8(1.7)$ & $5.7(0.7)$
\end{tabular}

\begin{tabular}{|c|c|c|c|c|c|c|}
\hline 25-Aug & $20: 36$ & $21: 56$ & 2.1 & 6.3 & -2.7 & 3.9 \\
\hline 25-Aug & $21: 56$ & $23: 21$ & 1.1 & 12.3 & 3.9 & 3.2 \\
\hline 25-Aug & $23: 21$ & $0: 39$ & 1.2 & 6.4 & 6.8 & 3.3 \\
\hline & Time & 1 & $1.5(0.6)$ & $8.4(3.4)$ & $2.7(4.9)$ & $3.5(0.4)$ \\
\hline & Excl & tive $\mathrm{k}$ & $1.1(0.1)$ & $9.5(4.1)$ & $5.3(2.1)$ & $3.2(0.1)$ \\
\hline
\end{tabular}

\begin{tabular}{ccccccc} 
26-Aug & $9: 10$ & $11: 19$ & 1.9 & 19.9 & -6.6 & 3.7 \\
26-Aug & $11: 19$ & $13: 34$ & 3.8 & 4.4 & 3.3 & 5.0 \\
26-Aug & $13: 34$ & $15: 35$ & 4.8 & 9.6 & 1.6 & 5.6 \\
\hline & Time-weighted mean & $3.5(1.5)$ & $11.2(7.9)$ & $-0.6(5.3)$ & $4.7(1.0)$ \\
& Excluding negative $\mathrm{k}$ & $4.3(0.7)$ & $6.8(3.7)$ & $2.5(1.1)$ & $5.3(0.4)$
\end{tabular}

${ }^{a}$ See supplemental text, sampling frequency can lead to apparently negative mean $\mathrm{k}_{600}$ over specific periods when concentration and/or saturation state are rapidly changing. When excluding negative values of $\mathrm{k}$ from time-weighted mean for either gas, all terms recalculated over identical time periods. 
Sonde and meteorological tower data were used to solve for an $\mathrm{O}_{2}$ mass balance similar to that of the noble gases, but neglecting ebullition and including net ecosystem metabolism $\left(N, \mathrm{mmol} \mathrm{O}_{2} \mathrm{~m}^{-2} \mathrm{~h}^{-1}\right)$, the balance of oxygen production and respiration:

$$
\frac{\partial \mathrm{O}_{2}}{\partial \mathrm{t}} * \mathrm{z} * \rho=\mathrm{k}_{\mathrm{O}_{2}} *\left(\mathrm{O}_{2_{\text {sat }}}-\mathrm{O}_{2}\right) * \rho+N
$$

$\mathrm{k}_{\mathrm{O} 2}$ was determined from Eq. 5 (main text), and sonde data noise was smoothed using a 30 minute moving-average. The diffusive gas exchange of oxygen in or out of the pond (first term on right side of Eq. S1 and $N$ were integrated over the sample periods for noble gases on Jul. $29^{\text {th }}$ and Aug. $26^{\text {th }}$ and compared to the integrated Ne-derived ebullition flux. Assuming ebullition represents loss of photosynthetic oxygen that would otherwise be accounted for in $N$, the difference in net metabolism with or without including ebullition can be compared (Table S3).

Table S3. Integrated oxygen fluxes over noble gas sampling periods.

\begin{tabular}{|c|c|c|c|}
\hline & Gas exchange ${ }^{a}$ & $\begin{array}{c}\text { Ebullition } \\
\left(\mathrm{mmol} \mathrm{O}_{2} \mathrm{~m}^{-2}\right)\end{array}$ & Net ecosystem metabolism \\
\hline $\begin{array}{l}\text { Jul. } 29^{\text {th }} \\
(6: 00-19: 00)\end{array}$ & 6.1 & -6.9 & 28.8 \\
\hline $\begin{array}{l}\text { Aug. } 26^{\text {th }} \\
(9: 15-15: 45)\end{array}$ & -22.3 & -2.7 & 57.6 \\
\hline
\end{tabular}




\section{Chapter 5-metabolism in a salt marsh pond}

\section{Production and respiration, and unusual metabolic processes in a salt marsh pond: insights from multiple chemical tracers}

Data availability

Data used in this work that is not reported in tables is accessible through the Biological and Chemical Oceanography Data Management Office (http://www.bco-dmo.org/project/529583). 


\begin{abstract}
Dissolved triple oxygen isotope ratios, oxygen concentration, and dissolved inorganic carbon concentrations were used to study rates of production and respiration in a salt marsh pond over hourly to seasonal timescales. Results highlight the heterogeneous nature of production within the pond, as well as the potential importance of light-enhanced oxygen and DIC consumption in shallow, sunlit marine systems. Plant abundance may be an important driver of pond metabolism, consistent with previous results. High rates of unusual metabolic sinks can explain the differences between various tracers of production and respiration, such as light-enhanced biological oxygen utilization and anoxygenic production in sediments: the data presented here points to the potential importance of chemosynthesis and anoxygenic photosynthesis for ecosystem carbon cycling in marsh ponds. Daytime production in the study pond was entirely consumed by dusk, and the net heterotrophic community metabolism must be subsidized by organic matter from other sources (such as buried peat). If generalizable to other marshtop ponds, high ratios of dissolved inorganic carbon to oxygen fluxes during both respiration and production may mean that ponds are more important to overall salt marsh carbon cycling than is currently thought.
\end{abstract}

\title{
Keywords
}

Salt marsh pond metabolism, production, respiration, stoichiometry

\section{Acknowledgments}

This work would not have been possible without the outstanding logistical and in kind support of the Plum Island Ecosystems Long Term Ecological Research site and TIDE experiment staff and scientists (these projects funded by NSF OCE 1238212, NSF DEB 1354494, and NE Climate Science Center grant DOI G12AC00001). Thanks to Nancy Pau at the Parker River National Wildlife Refuge for permitting this work. Many samples were collected with the assistance of Jennifer Karolewski, who was supported by the Woods Hole Oceanographic Institution (WHOI) Summer Student Fellowship program. A large number of individuals helped with the chamber deployment and sampling, including summer research students and WHOI staff; I particularly wish to thank my advisor Rachel Stanley for her many days in the field on this project, and her lab manager Zoë Sandwith for assistance in sampling and sample analysis. Joanne Goudreau and Cara C. Manning evacuated many, many bottles in support of sampling. I also wish to thank Amanda Spivak, Anne Giblin and Hap Garritt, and Linda Deegan and James Nelson for the use of the several water quality sondes in this study, and Rachel Stanley and Kim Vaeth for constructive feedback in developing this manuscript. Funding for this research was provided by NSF OCE 1233678. I was supported in part by the National Defense Science and Engineering Graduate Fellowship and by the Woods Hole Oceanographic Institution Academic Programs Office. 


\section{Introduction}

Salt marshes are highly productive ecosystems that link terrestrial and marine environments. The grass covered salt marsh platform forms peat which sequesters carbon dioxide from the atmosphere on the scale of hundreds to thousands of years (Hopkinson et al. 2012). Ponds are submerged salt marsh environments that are very different from the marsh platform; ponds are $10 \mathrm{~s}$ of $\mathrm{cm}$ deep, vary widely in size, cover up to $10 \%$ of the marsh surface and generally do not have emergent grasses (Wilson et al. 2014). Pond flora include benthic microalgae, macroalgae such as mat forming Ulva, and the seagrass Ruppia maritima. In general, ponds form on the high marsh platform ( $\sim 1.4 \mathrm{~m}$ elevation above mean sea level) and are flooded by estuarine water from nearby tidal creeks only near the spring tides (Millete et al. 2010; a quarter of high tides). Pond formation and expansion may be related to physical processes such as ice-rafting of sediments (Agrow et al. 2007), exclusion of emergent grasses and attendant belowground production by sea-level rise or infill of ditches or creeks (Hartig et al. 2002, Bromberg Gedan et al. 2011, Wilson et al. 2014), and by removal of underlying peat organic matter by respiration (Johnston et al. 2003). Ponds may also be filled or drained by geomorphological processes, meaning that these are dynamic and transient salt marsh features over years or decades.

Because of their isolation from the surrounding marine environment and infrequent tidal exchange, salt marsh ponds act as naturally contained experimental systems for studying community scale metabolism. Yet there are only a handful of studies of ecosystem metabolism in high marsh pond environments; so far, these studies indicate that these ponds are far less productive than the grass covered marsh platform (Johnston et al. 2003, Deegan et al. 2012, Spivak et al. submitted). Of the limited number of salt marsh ponds studied, roughly half have net heterotrophic community metabolisms and all have high rates of respiration compared to marsh platform grasses. In both of the above studies, metabolism was evaluated from the diel oxygen technique, using a mass-balance of dissolved oxygen with terms for production and gas exchange. This method is widely adopted in shallow aquatic systems (e.g. Staehr et al. 2010). While this technique is broadly applicable and informative, it may be biased by methodological or environmental factors (like any technique for measuring primary production, see Chapter 1); an important drawback is that oxygen concentrations may not adequately reflect the net effect of diverse metabolic processes in an environment, such as additional biological sinks of $\mathrm{O}_{2}$ that are decoupled from organic matter production or respiration, or anoxygenic primary production in sediments. Such processes occur in many shallow aquatic environments (Birmingham et al. 1982, Pinckney and Paerl 1997, Hopkinson et al. 1999, Larsen et al. 2015).

In this work, I focus on evaluating the metabolic rates within a single pond, including respiration (R), gross primary production (GPP; total photosynthetic production), and net community production (NCP; GPP-R for the whole community including both autotrophs and heterotrophs). In particular, I use a variety of tracers, including (i) the triple isotopic signature of dissolved oxygen (hereafter triple oxygen isotopes), which provides an estimate of gross primary production $\left(\mathrm{GPP}_{\mathrm{TOI}}\right)$ that is largely independent of respiration, (ii) dissolved oxygen concentrations $\left(\mathrm{O}_{2}\right)$, a standard tracer of metabolism in aquatic systems, and (iii) dissolved inorganic carbon (DIC), which is less sensitive to gas exchange than $\mathrm{O}_{2}$ but also affected by sediment uptake and release processes that may be unrelated to oxygenic photosynthesis. The variety of tracers used are sensitive to varying degrees to different mechanisms of $\mathrm{O}_{2}$ and DIC production and uptake; the combination of tracers allows evaluation of the relative roles of different, non-canonical respiration and photosynthetic pathways including light-enhanced oxygen uptake pathways and anaerobic or anoxygenic microbial metabolisms in the sediments.

The triple oxygen isotope and $\mathrm{O}_{2}$ tracers were measured in the open pond water from May through October, 2014, with resulting estimates of pond metabolism rates at hourly to weekly resolution.

Additionally, during the summer, targeted benthic sediment chamber experiments sampling all three tracers allowed comparison of metabolic rates in bare, presumably microalgae dominated, sediments to sediments dominated by macroalgal mats. I use the chamber and open pond datasets to evaluate a number 
of questions related to metabolic cycling in the marshtop pond, including: How do respiration rates and processes vary between nighttime and daytime in a shallow aquatic environment? How do the metabolism rates from each of the tracers change over various timescales? Are oxygen based metabolism rates good proxies for biological carbon fluxes in this environment? What DIC fluxes in addition to photosynthesis and respiration might be occurring?

\section{Methods}

\subsection{Setting}

The salt marsh pond study location is in the Plum Island Ecosystems Long Term Ecological Research site in Massachusetts, USA $\left(42.7411^{\circ} \mathrm{N}, 70.8309^{\circ} \mathrm{W}\right)$. The pond is approximately circular in shape, covering $7000 \mathrm{~m}^{2}$, and has a mean depth of $25 \mathrm{~cm}$ with steep banks. A swath of grass-covered high marsh, 5 to 10 $\mathrm{m}$ in width, separates the pond from an adjacent tidal creek. This creek floods the pond during alternating high tides for a few days surrounding the spring-tide, roughly every two weeks. Additional details of the study site are described in the work of Spivak et al. (submitted), including plant and algal coverage of the pond bottom.

\subsection{Sample collection and processing}

\subsubsection{Continuous instrumented data collection and processing}

Temperature, salinity (calculated from conductivity), and $\mathrm{O}_{2}$ saturation state data were collected every 15 minutes between May $7^{\text {th }}$ and October $28^{\text {th }}, 2014$ using a YSITM 6 -series or YSITMExo2 water quality sonde located near the center of the pond, $10-15 \mathrm{~m}$ from all edges and over bare sediment (Fig. 1). The sonde location was generally upwind of the discrete sample locations (section 2.2.2), and downwind of the eastern rim of the pond where seagrass and macroalgae were most abundant. The sonde location was chosen in order to integrate pond conditions along the wind fetch and be representative of the bare sediment environments characterizing roughly $80 \%$ of the pond area (section 3.2 ). A comparison of data from the sondes and discrete measurements indicates that temperature, salinity, and oxygen concentrations were similar between the sonde and sample locations. These instruments were calibrated and redeployed every two weeks, and cross calibrated in the study pond with overlapping deployment periods when multiple instruments were available. Temperature and $\mathrm{O}_{2}$ saturation state data were found to have negligible drift over each deployment period compared to instrument accuracy and be in good agreement during cross-calibration of instruments (accuracy and precision of $0.1+/-0.1{ }^{\circ} \mathrm{C}$ and $2 \pm 0.1 \%$ of oxygen saturation).

One of the 6-series sondes used for two periods in June and early July had systematic salinity offsets compared to the other instruments to which it was cross-calibrated at either end of its deployment periods - salinity data from this sonde was post-calibrated using constant offset corrections for each period $(\Delta S=-2.63$ and +2.84$)$. From mid-July through October, only the Exo2 sonde was used. Salinity derived from this sonde was also post-corrected to correct for occasional erroneously low readings (in one case likely caused by bubbles in the sensor cavity at deployment, and later potentially because of a loose sensor connection to the sonde housing) generally identified by an abrupt drop in salinity (e.g. $\Delta \mathrm{S}=-6$ over a single 15 minute timepoint); in this case the sensor data were linearly interpolated over periods of poor data quality.

Salinity is only used in this study to calculate $\left[\mathrm{O}_{2}\right]$ from $\mathrm{O}_{2}$ saturation state. Because the solubility of oxygen (Garcia and Gordon 1992, 1993) is primarily dependent on temperature, these post-corrections 
have very small effects on the calculated $\left[\mathrm{O}_{2}\right]$ compared to environmental signals. Resulting $\left[\mathrm{O}_{2}\right]$ and $\left[\mathrm{O}_{2}\right]_{\text {sat }}$ were reprocessed as a 30 minute $(\mathrm{n}=3)$ running average in order to smooth the data and remove instrument noise from subsequent metabolic rate calculations.

Photosynthetically active radiation (PAR, 400-700 nm wavelengths) and meteorological measurements used in the calculation of air-water oxygen fluxes were averaged every 30 minutes over the same period using a meteorological tower at $4 \mathrm{~m}$ height located roughly $200 \mathrm{~m}$ from the study pond. Details of the calculation of oxygen gas exchange rates using this data are described in Chapter 4.

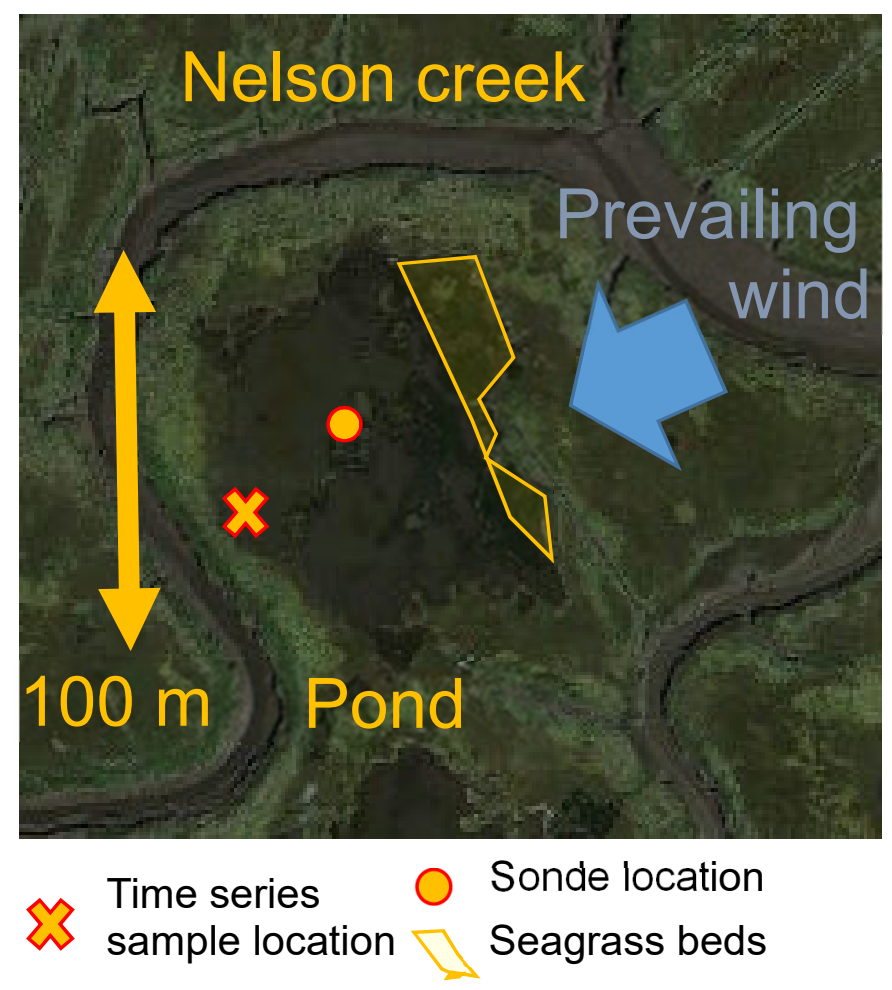

Figure 1: Pond study site, with sonde (circle) and sample locations (cross) marked, as well as areas in which seagrass beds (shaded yellow) were observed throughout the timeseries.

\subsubsection{Weekly discrete sampling and processing}

Discrete samples of the triple oxygen isotope $\left({ }^{16} \mathrm{O},{ }^{17} \mathrm{O}\right.$, and $\left.{ }^{18} \mathrm{O}\right)$ ratios in dissolved oxygen were collected roughly once a week over the instrument deployment period, resulting in 20 daytime and 2 nighttime periods (data repository TBD). Water was collected in pre-poisoned, $500 \mathrm{~mL}$ evacuated glass flasks, which were filled with roughly $300 \mathrm{~mL}$ of water while submerged at $10 \mathrm{~cm}$ depth. Care was taken to exclude bubbles and debris, and samples were collected $2 \mathrm{~m}$ from the pond edge using an inner-tube in order to avoid stirring sediments from the bottom during sampling. Samples were collected over bare sediments at three or more timepoints between 10:00 and 14:00, with a few days including morning and evening periods as well in order to confirm that results from midday sampling could be extrapolated over the entire daylight period. Additionally, concurrent timeseries over sediments with macoralgae or rooted vascular plants (e.g. Ruppia maritima) were collected on several days. In general one or more samples were taken in duplicate each day.

Triple oxygen isotope samples were processed and analyzed using a cryogenic processing line and isotope ratio mass spectrometer as described in Chapter 3; standard methods were applied when $\mathrm{O}_{2} / \mathrm{Ar}$ ratios were 
sufficiently high that Ar matrix corrections were not large compared to measured isotope ratios (i.e. when corrections were less than $10 \%$ or so of the measured values, which was generally true at $>70 \%$ of $\mathrm{O}_{2}$ saturation). At lower $\mathrm{O}_{2} / \mathrm{Ar}$ ratios (nighttime or early morning samples), Ar was stripped from the gas sample prior to measurement.

Local $\mathrm{O}_{2}$ saturation state, temperature, and salinity (in contrast to conditions at the sonde location) were measured simultaneously with each bottle sample using a handheld YSI ${ }^{\mathrm{TM}}$ Pro-ODO sensor (accuracy and precision $0.1+/-0.2{ }^{\circ} \mathrm{C}$ and $2 \pm 0.2 \%$ of oxygen saturation) and handheld refractometer (2+/-1 on PSS-78). Both instruments were calibrated daily. At the usual sampling locations along the western edge of the pond, these measurements were similar to conditions at the sonde location. However, these measurements were more varied around the pond perimeter, particularly on the eastern side of the pond where seagrasses and macroalgae were most abundant (and temperature and $\mathrm{O}_{2}$ saturation state were often higher).

On a single day concurrent with triple oxygen isotope sampling (July $29^{\text {th }}$ ), DIC samples were collected roughly hourly between dawn and dusk. Pond water was filtered (to remove particulate inorganic carbon) into clean $60 \mathrm{~mL}$ glass vials (acid washed, rinsed with deionized water, and dried at $60^{\circ} \mathrm{C}$ ). Filled vials were capped with butyl rubber septa and crimp-top seals. Samples were individually acidified and analyzed the same day on an infrared $\mathrm{CO}_{2}$ analyzer (Apollo AS-C3) and referenced to a secondary standard and thus to a certified reference standard (Dickson et al. 2007). All DIC analyses were performed by J. Karolewski (currently MIT-WHOI Joint Program).

\subsubsection{Chamber experiments}

On three days in summer (June 30, July 8, and July 22), the stirred benthic chambers described in Chapter 3 were used to conduct sediment-water flux experiments. 24 hours prior to each experiment, two temporary piers (fiberglass ladders resting on milk crates) were extended $6 \mathrm{~m}$ from the creek bank to separate areas of pond bottom with no visible macroflora (bare sediments) and with moderate to high benthic macroalgal sediment cover. The piers were deployed in different areas of the pond for each experiment. From each pier, eight chambers were deployed without tops in order to minimize sediment compression, including three light (clear) and dark (exterior covered in gaffer tape) sediment chambers each, as well as one light and dark control chamber each with closed bottoms that contained water but no sediment (water chambers).

All 16 chambers were allowed to soak and exchange freely with surrounding pond water overnight. On the day of the experiment, oxygen concentrations in each chamber were measured using the handheld YSI sensor to confirm that initial concentrations were not prohibitively low for triple oxygen isotope sample analysis Chapter 3. Beginning 40 to 60 minutes before the first sample point, each chamber was carefully lidded with the lid plugs open to avoid sediment compression and to exclude standing air bubbles on any interior surfaces. After all lids were secured, plugs were inserted in each chamber lid, and external shrouds of light-blocking fabric were secured around dark chambers. Water chamber volumes were $8.9 \mathrm{~L}$, while the water volume in sediment chambers averaged 11.9(0.1) L, 11.5(0.6) L, and 10.6(0.9) L (standard deviation in parentheses) respectively on June 30, July 8, and July 22. Each chamber was stirred at $\sim 15 \mathrm{rpm}$, slower than during the creek study described in Chapter 3 in order to avoid suspending sediments.

Chamber sample ports were connected to $7 \mathrm{~m}$ of pre-soaked Tygon ${ }^{\circledR}$ e-series phthalate free tubing. The tubing was extended underwater along the piers to shore terminated with a valve that was kept closed and submerged between use for sampling. The chamber intake ports (to replace sampled volume) were connected to $20 \mathrm{~cm}$ of tubing with the external end held fixed in ambient pond water $\sim 5 \mathrm{~cm}$ from the sediment-water interface. After all chambers were prepared, chambers were sampled at four timepoints 
between roughly $10 \mathrm{am}$ to $2 \mathrm{pm}$. The chamber sampling order was randomized at the beginning of each experiment but consistent between timepoints, and conducted in parallel between both piers. Each chamber took five to ten minutes to sample depending on the types of sampling and number of duplicates.

Salinity, temperature, oxygen saturation state, and DIC concentrations were sampled at each timepoint in all chambers using the same instruments as the weekly sampling described above. Oxygen was sampled by dipping the handheld probe into a $20 \mathrm{~mL}$ vial filled with chamber water via syringe-when concentrations of $\mathrm{O}_{2}$ were below atmospheric saturation (e.g. all dark chambers) sample transfer and airwater exchange was expected to bias the measured saturation state too high by up to $2 \%$. Triple oxygen isotope samples were generally collected from light chambers only, as the oxygen concentrations in the dark chambers were often below the threshold for sample analysis by the second sampling point. Additionally, each sample type was collected from the open pond adjacent to the chambers using an identical length of sample tubing anchored on the pond bottom next to each pier. Flushing and sample volumes were similar to those described in the creek study (Chapter 3), with 200 to $500 \mathrm{~mL}$ sampled depending on the triple oxygen isotope sample volume (on average $4 \%$ of chamber volume was sampled when oxygen isotopes were collected, and $2 \%$ of chamber volume otherwise). All samples were analyzed as described above for the weekly sampling.

\subsection{Metabolic rate calculations}

\subsubsection{Triple oxygen isotope derived gross primary production}

$\mathrm{GPP}_{\mathrm{TOI}}\left(\mathrm{mmol} \mathrm{O}_{2} \mathrm{~m}^{-2} \mathrm{~h}^{-1}\right)$ is determined between any two timepoints using the measured triple oxygen isotope composition of the dissolved oxygen, and is calculated in terms of a mass balance between gas exchange and production. Following Prokopenko et al. (2011), if the measured ${ }^{17} \mathrm{O} /{ }^{16} \mathrm{O}$ and ${ }^{18} \mathrm{O} /{ }^{16} \mathrm{O}$ ratios are notated as ${ }^{17} \mathrm{X}$ and ${ }^{18} \mathrm{X}$ :

$\mathrm{z}\left[\mathrm{O}_{2}\right] \frac{\partial}{\partial \mathrm{t}}\left({ }^{17} \Delta\right) \rho_{w}=\mathrm{GPP}_{\mathrm{TOI}}\left(\frac{{ }^{17} \mathrm{X}_{\mathrm{P}}-{ }^{17} \mathrm{X}}{{ }^{17} \mathrm{X}}-\lambda \frac{{ }^{18} \mathrm{X}_{\mathrm{P}}-{ }^{18} \mathrm{X}}{{ }^{18} \mathrm{X}}\right)-\mathrm{k}_{\mathrm{O} 2}\left[\mathrm{O}_{2}\right.$ sat $]\left(\frac{{ }^{17} \mathrm{X}-{ }^{17} \mathrm{X}_{\text {sat }}}{{ }^{17} \mathrm{X}}-\lambda \frac{{ }^{18} \mathrm{X}-{ }^{18} \mathrm{X}_{\text {sat }}}{{ }^{18} \mathrm{X}} \rho_{w}(1)\right.$

where $\mathrm{z}$ is the mean pond depth $(25 \mathrm{~cm}),\left[\mathrm{O}_{2}\right]$ is the oxygen concentration and $\left[\mathrm{O}_{2}\right]_{\text {sat }}$ is the saturation equilibrium concentration with respect to air (both molal), and

${ }^{17} \Delta=\left(\ln \left[\left({ }^{17} \mathrm{O} /{ }^{16} \mathrm{O}\right)_{\text {measured }}\left({ }^{17} \mathrm{O} /{ }^{16} \mathrm{O}\right)_{\text {air }}\right]-\lambda \ln \left[\left({ }^{18} \mathrm{O} /{ }^{16} \mathrm{O}\right)_{\text {measured }} /\left({ }^{18} \mathrm{O} /{ }^{16} \mathrm{O}\right)_{\text {air }}\right]\right)$ relative to an atmospheric oxygen standard (Angert et al. 2003), where $\lambda=0.518$ is the ratio of the mass dependent isotope enrichment factors for ${ }^{17} \mathrm{O} /{ }^{16} \mathrm{O}$ and ${ }^{18} \mathrm{O} /{ }^{16} \mathrm{O} . \rho_{W}$ is the density of the pond water. $\mathrm{k}_{\mathrm{O} 2}$ is the air water gas transfer velocity, determined using the windspeed and surface area dependent formulation from Vachon and Prarie (2013; Model B, see also Chapter 4).

${ }^{i} X_{P}$ is the photosynthetic production ratio and ${ }^{i} X_{\text {sat }}$ the saturated equilibrium ratio for each isotope. As in the creek work (Chapter 3), ${ }^{\mathrm{X}} \mathrm{X}_{\mathrm{P}}$ is calculated by assuming an initial water triple oxygen isotope composition based on a mixture of seawater (Luz and Barkan 2011) and meteoric water (Luz and Barkan 2010; Li et al. 2015). I applied the originally published photosynthetic fractionations (Helman et al. 20051 Eisenstadt et al. 2010) with the universal photosynthetic fractionation slope $\lambda_{\mathrm{P}}=0.5237$ proposed in Luz and Barkan (2011) assuming an equal mixture of production from diatoms, cyanobacteria, and green algae (but not coccolithophores or other organisms included in the "mean seawater" reinterpretation of previously published data by Luz and Barkan 2011). All parameters on the right hand side of Eq. 1 represent the mean values over the time interval $\partial \mathrm{t}$. $\left({ }^{18} \mathrm{O} /{ }^{16} \mathrm{O}\right)_{\text {measured }}$ is recalculated from $\left({ }^{17} \mathrm{O} /{ }^{16} \mathrm{O}\right)_{\text {measured }}$ and ${ }^{17} \Delta_{\text {measured }}$ to minimize uncertainty in the GPP calculation (Chapter 3). 
$\mathrm{GPP}_{\mathrm{TOI}}$ in the chamber experiments is solved in the same manner with two modifications: First, the term related to gas exchange on the right side of Eq. 1 is neglected as the chambers are not exposed to the atmosphere. Second, the change in oxygen concentration and triple oxygen isotope ratios between any two sampled timepoints are dependent not only upon production within the chamber, but are also affected by entrainment of ambient pond water during the initial sampling point. Thus the initial timepoint in each pair of samples is corrected to account for mixing with entrained pond water when determining the production rate over each interval using the mass balance:

$$
\left(\left[\mathrm{O}_{2, C}\right]{ }^{\mathrm{i}} \mathrm{X}_{C}\right) \mathrm{V}=\left(\left[\mathrm{O}_{2, w}\right]{ }^{\mathrm{i}} \mathrm{X}_{w}\right) \mathrm{V}_{w}+\left(\left[\mathrm{O}_{2}\right]{ }^{\mathrm{i}} \mathrm{X}\right)\left(\mathrm{V}-\mathrm{V}_{w}\right)
$$

where $\mathrm{V}$ is the chamber volume, and the subscripts $w$ and $C$ indicate the entrained pond water and corrected initial values, respectively.

\subsubsection{Oxygen concentration based net ecosystem metabolism, respiration, and gross primary production}

An oxygen mass balance can similarly be used to determine community metabolic rates related to oxygen production and consumption:

$$
\mathrm{z} \frac{\partial}{\partial \mathrm{t}}\left(\left[\mathrm{O}_{2}\right]\right) \rho_{w}=\mathrm{GPP}_{\mathrm{O} 2}-\mathrm{R}_{\mathrm{O} 2}-\mathrm{k}_{\mathrm{O} 2}\left(\left[\mathrm{O}_{2}\right]-\left[\mathrm{O}_{2}\right]_{\text {sat }}\right) \rho_{w}
$$

In practice $\mathrm{NCP}_{\mathrm{O} 2}\left(\mathrm{GPP}_{\mathrm{O} 2}-\mathrm{R}_{\mathrm{O} 2} ; \mathrm{mmol} \mathrm{O}_{2} \mathrm{~m}^{-2} \mathrm{~h}^{-1}\right)$ is determined during periods when photosynthesis occurs, while $\mathrm{R}_{\mathrm{O} 2}$ is evaluated from periods during which production is inferred to be zero, overnight or in dark chamber experiments. $\mathrm{R}_{\mathrm{O} 2}$ is likely greater during the daytime than at nighttime (see section 4.2), and therefore $\mathrm{GPP}_{\mathrm{O} 2}$ solved from $\mathrm{NCP}_{\mathrm{O} 2}$ and $\mathrm{R}_{\mathrm{O} 2}$ is likely a lower bound.

This mass balance can be solved at the same resolution as the continuous sonde data (15 minute intervals). However, Eq. 3 neglects other physical fluxes that could modify the interpretation of the pond data to determine metabolic rates. First, tidal exchange during spring tides is neglected in the mass balance. While in general tidal exchange does not significantly affect oxygen concentrations compared to periods of tidal isolation (Spivak et al., submitted), $\mathrm{O}_{2}$ addition or removal by tides over the intervals of active exchange and mixing may be similar or larger in magnitude than the biological fluxes being evaluated. Thus metabolic rates evaluated during this period should be interpreted with caution relative to those measured during tidal isolation.

In addition, ebullition (rising bubbles) of photosynthetic oxygen is expected to remove oxygen from the pond during daytime periods of $\mathrm{O}_{2}$ local supersaturation. $\mathrm{NCP}_{\mathrm{O} 2}$ (and $\mathrm{GPP}_{\mathrm{O} 2}$ ) from the above mass balance are then systematically biased low (too heterotrophic). Using noble gases, I found that ebulliton could remove $\sim 5-25 \%$ of daytime $\mathrm{NCP}_{\mathrm{O} 2}$ on two days during the study period (Chapter 4 ). This effect is more pronounced over a full 24 hours, as $\mathrm{NCP}_{\mathrm{O} 2}$ is close to zero over a full diel metabolism cycle. Unfortunately, ebullition cannot be evaluated throughout the time-series as cost constraints prevented high temporal resolution noble gas sampling over the majority of the experiment.

$\mathrm{NCP}_{\mathrm{O} 2}$ and $\mathrm{R}_{\mathrm{O} 2}$ in the chamber experiments can be solved using a modified mass balance that neglects gas exchange, as with the triple oxygen isotope derived rates. While the effects of entrainment into the chamber on bulk $\mathrm{O}_{2}$ are smaller than on the triple oxygen isotope composition, I calculate $\mathrm{O}_{2}$ based rates using pairwise integration with volume exchange corrections in identical manner to the calculation $\mathrm{GPP}_{\text {TOI. }}$ This allows for comparison of consistently calculated rates between the two methods. In the case where oxygen concentrations increase or decrease approximately linearly, it is standard practice to fit a straight line through multiple data to determine the mean rate over the measurement period. This approach is less accurate for the chamber experiments in this work for two reasons: First, it neglects entrainment of water with a different composition. Second, oxygen concentration frequently does not increase or decreases linearly (logarithmic increases, decreases, and midday peaks are all observed in the chamber data, consistent with $\mathrm{O}_{2}$ concentration changes in the open pond). Volume exchange corrections 
can be applied consecutively to each data point in order to apply a linear fit approach using corrected data and improve the accuracy of the estimate, however this introduces additional uncertainty as each subsequent datum must be interpolated relative to the last and still does not account for nonlinearities that reflect real differences in biological rates. The various calculation methods and associated uncertainties are demonstrated in Fig. 2.

\subsubsection{Dissolved inorganic carbon concentration based net ecosystem metabolism, respiration, and gross primary production}

A dissolved inorganic carbon mass balance can be solved for $\mathrm{NCP}_{\mathrm{DIC}}, \mathrm{R}_{\mathrm{DIC}}$, and $\mathrm{GPP}_{\mathrm{DIC}}$ in the same manner as Eq. 3 for $\mathrm{O}_{2}$. Unlike $\mathrm{O}_{2}$ based metabolism rates, the DIC based rates are sensitive to anaerobic processes as well as aerobic respiration, as well as any DIC uptake by chemoautotrophs and photoautotrophs that do not produce $\mathrm{O}_{2}$. Thus comparing the rates determined from DIC and $\mathrm{O}_{2}$ can reveal additional metabolic processes or the sensitivity of the pond ecosystem to processes that affect the two terminal metabolic products differently.

In calculating biological rates from DIC I neglect the gas exchange terms for the open water as well as the chamber work - other carbonate system parameters were not evaluated, meaning that the dissolved $\mathrm{CO}_{2}$ fraction of DIC that can exchange with the atmosphere must be estimated. If it is assumed that $\mathrm{pH}$ was near 8.0 or higher (similar to adjacent tidal creek water and estuarine conditions; Inke Forbrich, personal communication; Hopkinson 2000) then during the open water DIC timeseries (July $29^{\text {th }}$ ) $\mathrm{CO}_{2}$ was always supersaturated with respect to the atmosphere and gas exchange could have accounted for at most $4 \%$ of the decrease in DIC in the early morning. By 07:30 onwards gas exchange could account for no more than $1 \%$ of the observed DIC decrease over the time-series. Given the small expected effect and poorly constrained input estimate, I do not attempt to incorporate gas exchange when calculating the DIC based rates.

Chamber DIC data are analyzed in identical manner to $\mathrm{O}_{2}$ data so that all rates can be consistently compared. 


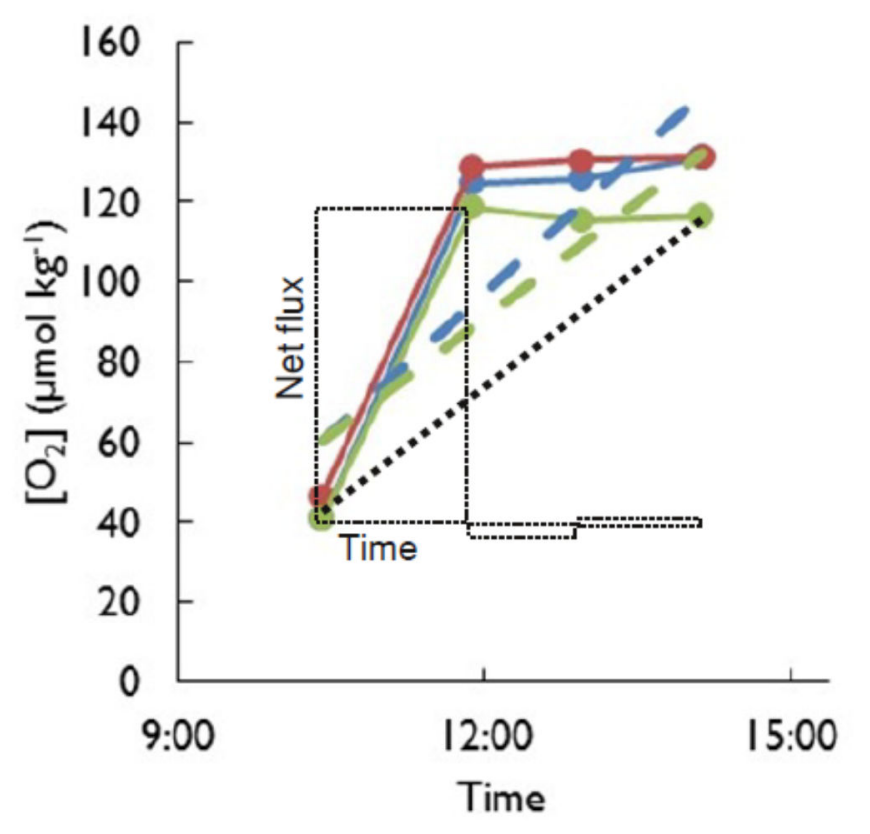

$\rightarrow-$ Measured $\quad 4.2(1.9) \mathrm{mmol} \mathrm{O}_{2} \mathrm{~m}^{-2} \mathrm{~h}^{-1}$

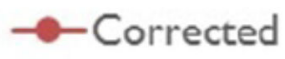

$\rightarrow-$ Interpolated

.... Integrated
$3.5(2.0) \mathrm{mmol} \mathrm{O}_{2} \mathrm{~m}^{-2} \mathrm{~h}^{-1}$

3.7 (0.3) $\mathrm{mmol} \mathrm{O}_{2} \mathrm{~m}^{-2} \mathrm{~h}^{-1}$
Figure 2: Comparison of chamber calculation methods, including linear fit through the measured concentrations (blue), linear fit through the concentrations corrected for entrainment of pond water during sampling and sequentially interpolated (green), and pairwise integration between entrainment corrected concentration and measured concentration of the following timepoint (dashed black line). Integration method is visually represented by series of boxes: the change of tracer over the period between each pair of samples is calculated, and the reported rate is the weighted average of pairwise rates. Associated rates and uncertainties for each method are in a single chamber are compared (standard deviation in parentheses).

\section{Results}

Oxygen isotopic ratios, DIC concentration data, and sonde data are available in the database described in the data availability statement. The results of metabolic rates derived from open water time-series and chamber sampling are presented separately below.

\subsection{Continuous and time-series metabolic rates}

Mean GPP ${ }_{\text {то }}$ between 10:00 and 14:00 (calculated piecewise) as well as hourly and daily mean $\mathrm{NCP}_{\mathrm{O} 2}$ over the entire open water timeseries are plotted in Fig. 3. NCP and GPP error bars (one standard deviation) are calculated using a Monte Carlo approach with 3000 iterations in tracer values are allowed to vary randomly within the one standard deviation error bounds for each iteration, and the combined uncertainty is reported as the standard deviation of all iterations. The bottom panels highlight different aspects of the data, including one of the two nighttime sampling periods and the single dawn to dusk period over which $\mathrm{NCP}_{\mathrm{DIC}}$ was additionally evaluated. Note that $\mathrm{GPP}_{\mathrm{TO}}$ depends in large part on the time rate of change of the triple oxygen isotope $\operatorname{tracer}^{17} \Delta$ (left side of Eq. 1); thus rate uncertainties may be very large when ${ }^{17} \Delta$ is similar within uncertainties between consecutive timepoints, regardless of whether the calculated rate is large or small relative to the rest of the time-series.

In Fig. 3, nighttime GPP ${ }_{\text {TOI }}$ rates are calculated as being larger than zero. I discuss this unexpected result in section 4.2, but in brief there are plausible methodological and environmental reasons that the 
nighttime GPP $\mathrm{TOI}_{\mathrm{T}}$ rate might be biased to produce a non-zero result, yet these mechanisms would not necessarily affect the calculation of daytime GPP $_{\text {тог. Daytime }}$ GPP beginning and ending the daylight period at zero production with a midday peak in photosynthesis, and is consistent with the general daytime trends of $\mathrm{NCP}_{\mathrm{O} 2}$ and $\mathrm{NCP}_{\mathrm{DIC}}$ (Fig. 3). $\mathrm{GPP}_{\mathrm{TO}}$ and $\mathrm{NCP}_{\mathrm{O} 2}$ closely follow the variability in PAR flux within a given day (Fig. 4). In general $\mathrm{NCP}_{\mathrm{O} 2}$ begins to rise when PAR flux is detectable beginning at dawn, and drops from a midday peak to below zero (net heterotrophy, $\left.\mathrm{NCP}_{\mathrm{O} 2} \sim \mathrm{R}_{\mathrm{O} 2}\right)$ as PAR decreases into dusk. $\mathrm{R}_{\mathrm{O} 2}\left(\mathrm{NCP}_{\mathrm{O} 2}\right.$ between dusk and dawn) varies around a roughly constant overnight value.

Determining mean daytime $\mathrm{GPP}_{\mathrm{TO}}$ from piecewise data primarily collected over the 10:00 to 14:00 peak production period requires scaling production to light flux during the low-light morning and evening periods when triple oxygen isotope samples were not collected (generally less than one quarter of integrated PAR for the entire day). The overall dataset of GPP ${ }_{\text {TOI }}$ to PAR (Fig. 5) is not well fit by a photosynthesis-irradiance curve, as is typically used to calculate photosynthetic production from light (e.g. MacIntyre et al. 2002). Differences in community composition, nutrient supply, and other variables over the six month time-series may contribute to this scatter. In general, the midday periods with the

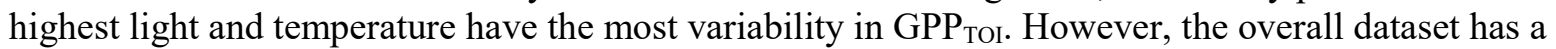
moderately robust linear relationship between production and irradiance for PAR flux less than 4 mol quanta $\mathrm{m}^{-2} \mathrm{~h}^{-1}$, with a slope of $3.8 \mathrm{mmol} \mathrm{O}_{2}$ per mol quanta $\left(95 \%\right.$ confidence interval 2.8 to $4.9, \mathrm{R}^{2}=0.45$; this relationship is identical for PAR $<5$ mol quanta $\mathrm{m}^{-2} \mathrm{~h}^{-1}$ ). I use this relationship to estimate GPP TOI $_{\text {Over }}$ the morning and evening periods during which there are no observations so that mean daytime $\mathrm{GPP}_{\mathrm{TOI}}$ can be compared to $\mathrm{GPP}_{\mathrm{O} 2}$. Using $\mathrm{NCP}_{\mathrm{O} 2}$ and nighttime $\mathrm{R}_{\mathrm{O} 2}, \mathrm{GPP}_{\mathrm{O} 2}$ must be evaluated over at least a full diel cycle because $\mathrm{R}_{\mathrm{O} 2}$ cannot be evaluated from daytime data. Mean daytime $\mathrm{NCP}_{\mathrm{O} 2}$ (dawn to dusk) and mean nighttime $\mathrm{R}_{\mathrm{O} 2}$ (dusk to following dawn) are used to calculate mean daily $\mathrm{GPP}_{\mathrm{O} 2}$ over the timeseries.

Integrated daytime rates of $\mathrm{GPP}_{\mathrm{TO}}$ and $\mathrm{GPP}_{\mathrm{O} 2}$ are plotted in Fig. 6, along with integrated daily (24 h) rates of $\mathrm{NCP}_{\mathrm{O} 2}$ and $\mathrm{R}_{\mathrm{O} 2}$. The shaded error bounds in Fig. 6 represent one standard deviation of the integrated daily rates, with a Monte Carlo approach in which individual timepoints through each day were allowed to vary within one standard deviation over 5000 iterations, and the uncertainty in the integrated daily rate was calculated from the standard deviation of the pooled iterations. In general GPP $\mathrm{TOI}_{\mathrm{T}}$ is higher than $\mathrm{GPP}_{\mathrm{O} 2}$. The highest rates of $\mathrm{GPP}_{\mathrm{TOI}}$ (on days for which multiple rates are plotted) were from areas of the pond with rooted vascular plants (e.g. Rupia). Both $\mathrm{GPP}_{\mathrm{TO}}$ and $\mathrm{GPP}_{\mathrm{O} 2}$ decrease in the fall compared to the late spring and summer. Daily mean $\mathrm{NCP}_{\mathrm{O} 2}$ is heterotrophic and relatively level across the timeseries, while rates of $\mathrm{R}_{\mathrm{O} 2}$ and $\mathrm{GPP}_{\mathrm{O} 2}$ are anti-correlated, reflecting in part the calculation method for $\mathrm{GPP}_{\mathrm{O} 2}$. 
Figure 3: Calculated GPP (red) and NCP (blue) rates in open pond. Upper panel: 172 day timeseries of

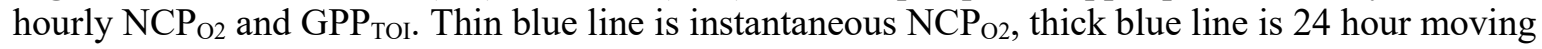
average. GPP sediment, but when two rates are presented for the same day the higher rate is from an area with extensive macroalgae or Ruppia. Bottom left panel: Nighttime $\mathrm{NCP}_{\mathrm{O} 2}$ with one standard deviation shaded error bars, and apparently positive $\mathrm{GPP}_{\mathrm{TOI}}$ (see text). Lower right panel: Daytime $\mathrm{NCP}_{\mathrm{O} 2}$ and $\mathrm{GPP}_{\mathrm{TOI}}$, as well as $\mathrm{NCP}_{\text {DIC }}$ (black, error bars smaller than symbol size). NCP and GPP error bars are calculated using a Monte Carlo approach (see text). 


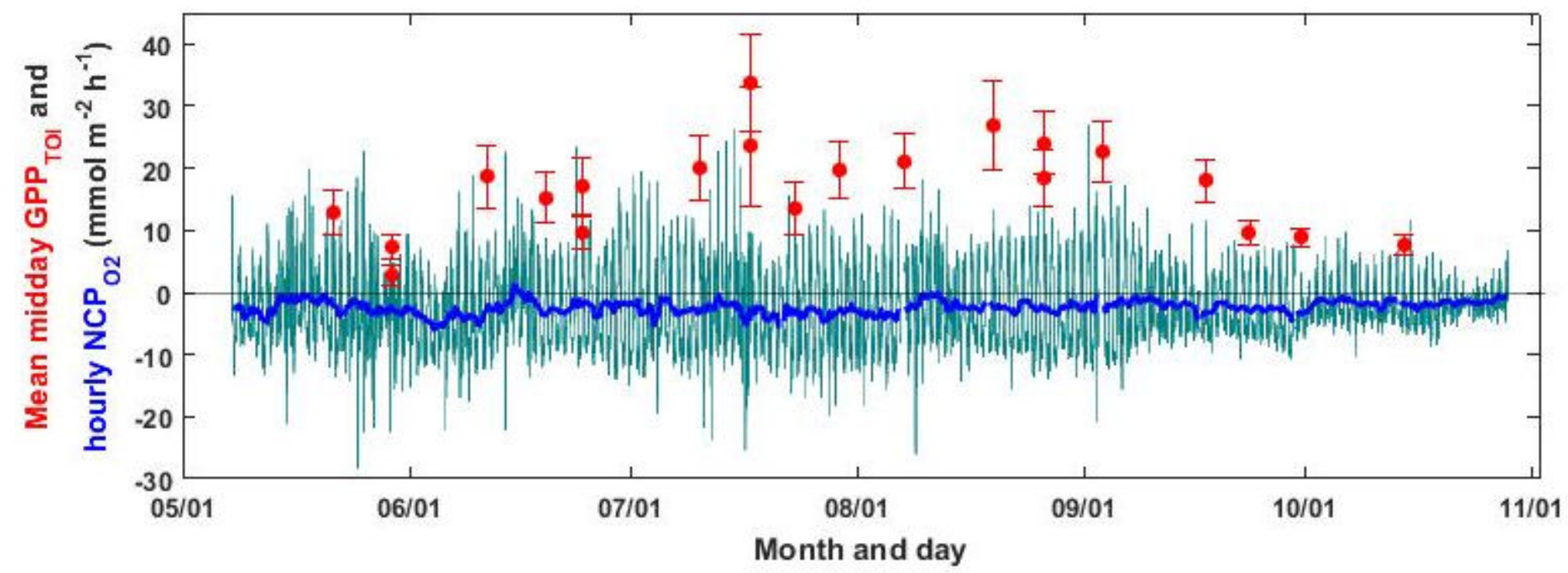

点
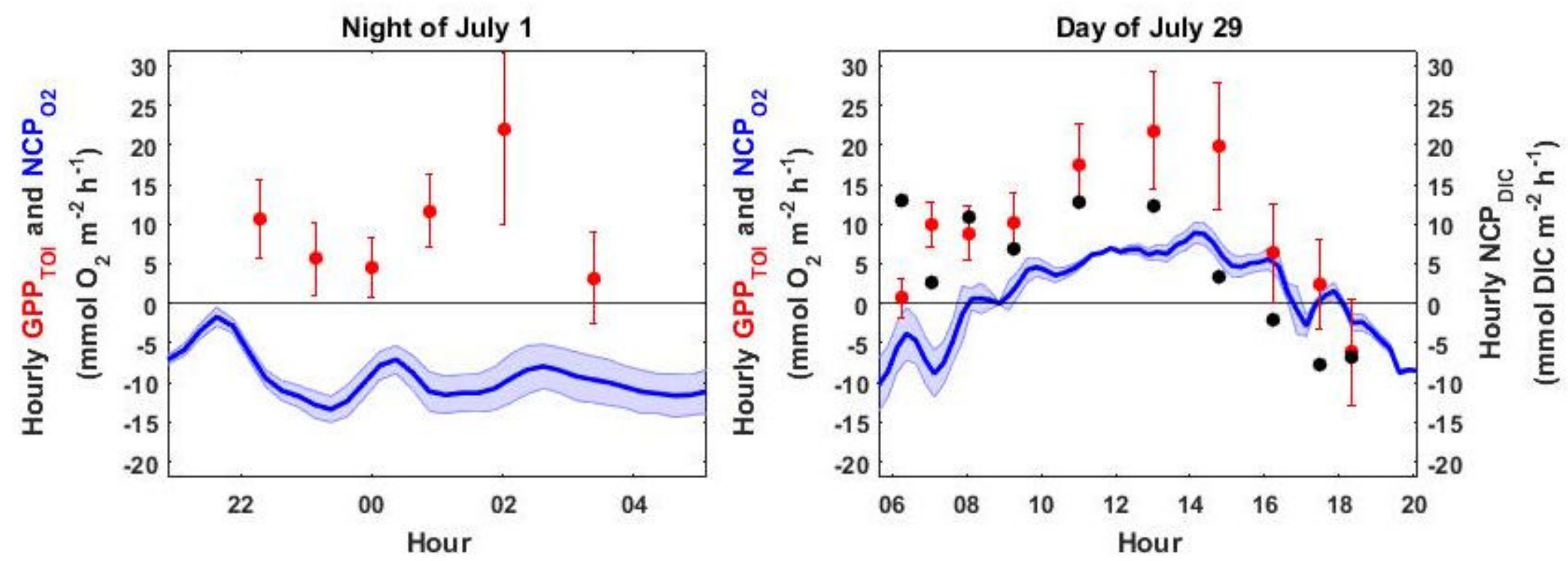

Figure 3: Calculated GPP (red) and NCP (blue) hourly rates in open pond. Figure caption on preceding page. 


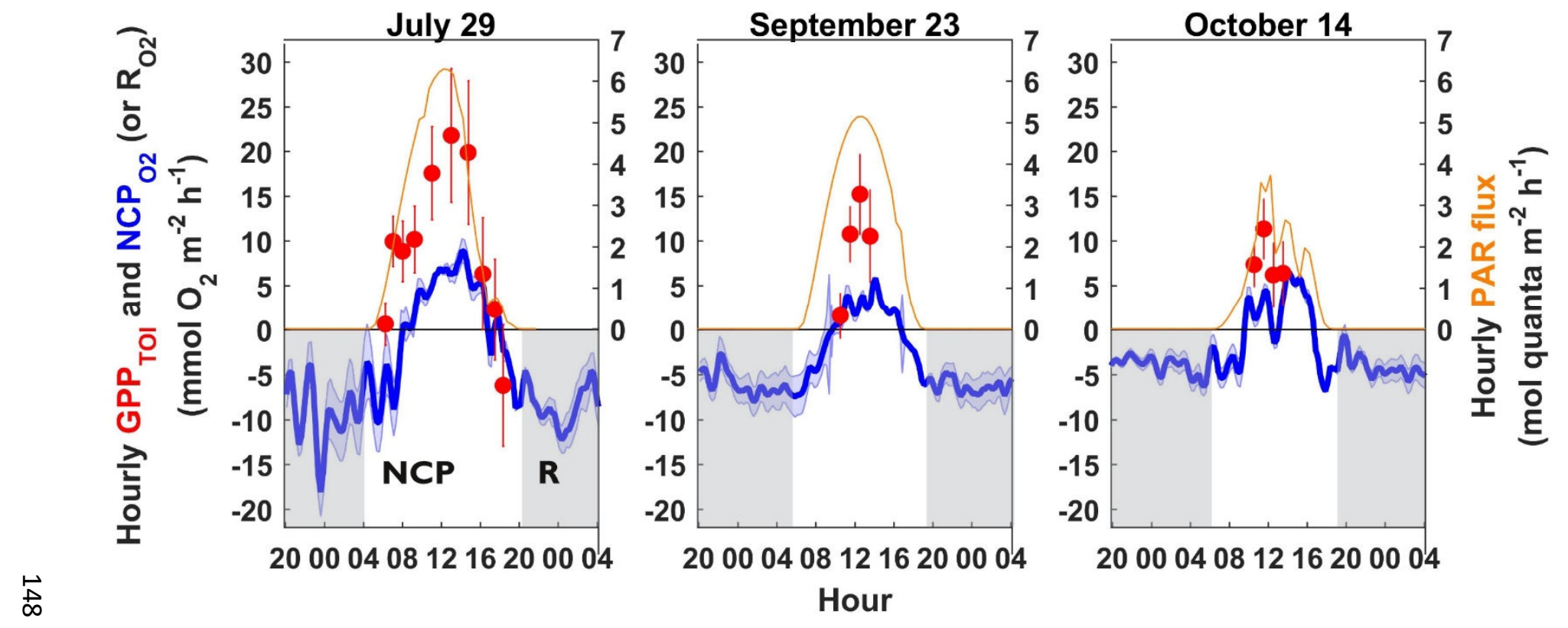

Figure 4: Examples of individual diel variability of $\mathrm{NCP}_{\mathrm{O} 2}$ (blue, with shaded one standard deviation error bars) and GPP $\mathrm{TOI}_{\mathrm{TO}}$ (red, one standard deviation error bars) with PAR flux above the pond (orange). $\mathrm{NCP}_{02}$ is equivalent to $\mathrm{R}_{\mathrm{O} 2}$ when there is no oxygen production (gray shaded periods). Error bars are calculated using a Monte Carlo approach as in Fig. 3. 


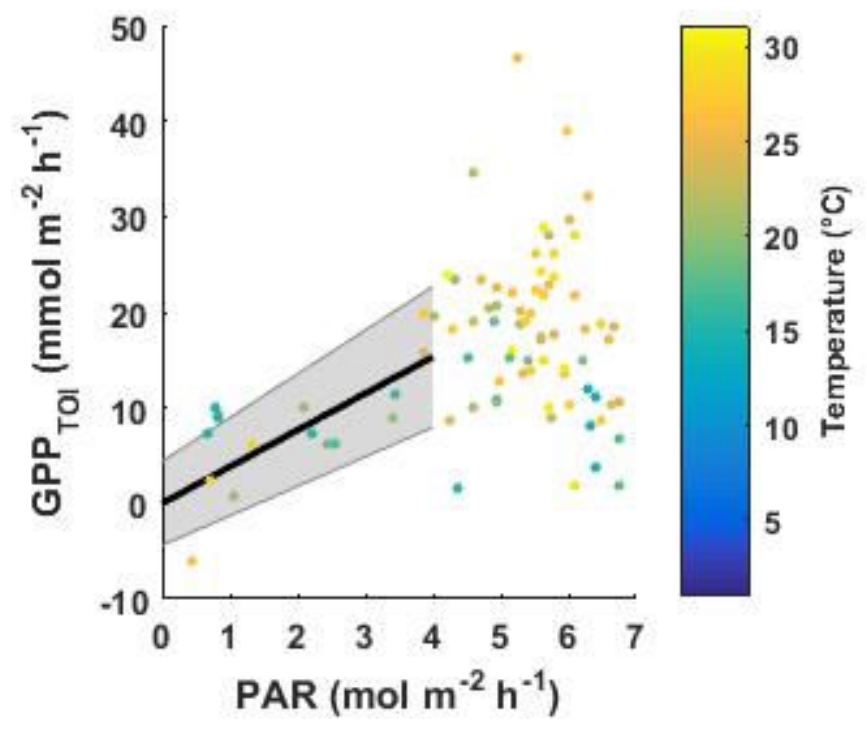

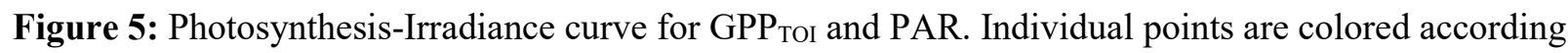
to mean pond temperature over the rate determination. The black line (and one standard deviation error bar of slope uncertainty) represents a linear regression of GPP $\mathrm{TOI}_{\mathrm{T}}$ on PAR for lower irradiances, used to calculate $\mathrm{GPP}_{\mathrm{TO}}$ over the whole day from rate measurements made primarily between 10:00 and 14:00.

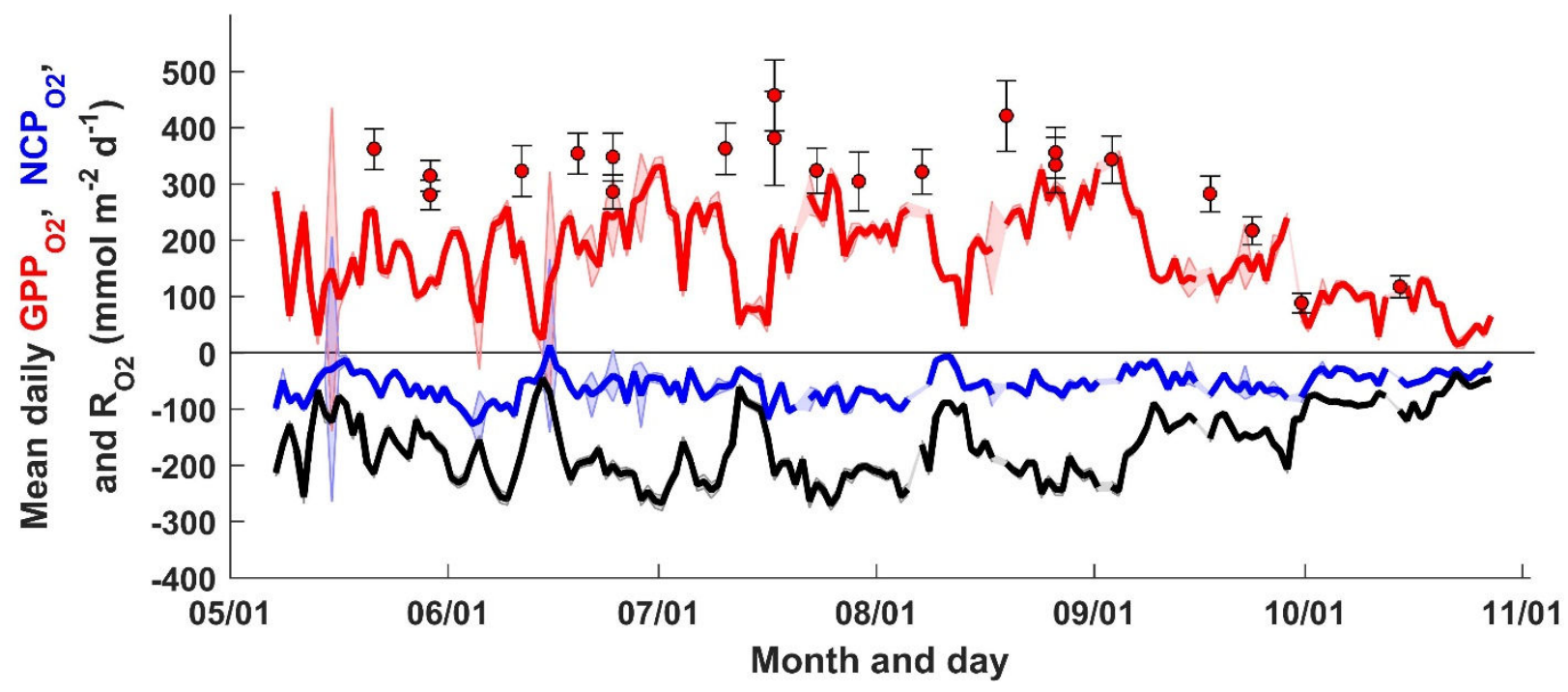

Figure 6: Daily mean dawn to dusk GPP (red line and shaded error bars), dawn to following dawn $\mathrm{NCP}_{\mathrm{O} 2}$ (blue line and shaded error bars), and nighttime $\mathrm{R}_{\mathrm{O} 2}$ (black line and shaded error bars) following each dawn to dusk period (164 days total, excluding 8 days with partial sonde data). Standard deviations represent the uncertainty of the integrated rates for each day (rather than daily variability around the mean rates), and are calculated using a Monte Carlo approach (see text). The large peaks in uncertainty in mid-May and mid-June primarily reflect uncertainty in daytime oxygen concentrations and gas exchange, which were highly variable at various timepoints on these days. 


\subsection{Chamber metabolic rates}

Metabolism rates from the chamber experiments are summarized in Table 1 (standard deviation of chamber replicates in parentheses). These include mean rates of $\mathrm{NCP}_{\mathrm{O} 2}$ and $\mathrm{NCP}_{\mathrm{DIC}}, \mathrm{R}_{\mathrm{O} 2}$ and $\mathrm{R}_{\mathrm{DIC}}$, and $\mathrm{GPP}_{\mathrm{O} 2}, \mathrm{GPP}_{\mathrm{DIC}}$, and $\mathrm{GPP}_{\mathrm{TO}}$ from macroalgal, microalgal, and water chambers over each experimental period. A positive bias of up to $0.5 \mathrm{mmol} \mathrm{O}_{2} \mathrm{~m}^{-2} \mathrm{~h}^{-1}$ was expected for undersaturated chambers based on the sampling methods, see section 2.2.3). This potential bias was corrected for in all reported rates of $\mathrm{R}_{\mathrm{O} 2}$. Additionally, open pond $\mathrm{GPP}_{\mathrm{TO}}, \mathrm{NCP}_{\mathrm{O} 2}$, and $\mathrm{NCP}_{\mathrm{DIC}}$ rates over identical periods are summarized in Table 2 for comparison; pond $\mathrm{GPP}_{\mathrm{TO}}$ and $\mathrm{NCP}_{\mathrm{DIC}}$ were derived from the water samples used to correct the rates for entrainment during sampling. The expected whole pond NCP and GPP based on the mean chamber metabolism rates (Table 2) was also calculated by assuming that macroalgae and microalgae cover $10 \%$ and $80 \%$ of the sediments, respectively (Spivak et al. submitted). In order to accurately include water column production over the whole pond area, I reduced the sediment chamber rates by the water chamber rate, and normalized the water chamber rate to the average pond depth $(25 \mathrm{~cm}$ compared to the $13 \mathrm{~cm}$ water chamber height).

In general, open water $\mathrm{GPP}_{\mathrm{TO}}$ rates were much higher than $\mathrm{GPP}_{\mathrm{TO}}$ and $\mathrm{GPP}_{\mathrm{O} 2}$ rates from any of the chambers. GPP ${ }_{\mathrm{DIC}}$ was similar to GPP from the other tracers in the water chambers, but in the sediment chambers GPP $\mathrm{DIC}_{\mathrm{C}}$ was generally much higher than $\mathrm{GPP}_{\mathrm{TOI}}$ or $\mathrm{GPP}_{\mathrm{O} 2}$. $\mathrm{NCP}_{\mathrm{DIC}}$ in the pond was double $\mathrm{NCP}_{\text {DIC }}$ in the macroalgae chambers, and GPP $\mathrm{DIC}_{\text {in }}$ the open pond (which was not evaluated) may have been much larger than in the chambers as well. $\mathrm{NCP}_{\mathrm{DIC}}$ and $\mathrm{R}_{\mathrm{DIC}}$ in the macroalgae chambers tended to be greater than $\mathrm{O}_{2}$ based rates as well. In the microalgae and water chambers $\mathrm{NCP}_{\mathrm{O} 2}, \mathrm{NCP}_{\mathrm{DIC}}, \mathrm{R}_{\mathrm{O} 2}$, and $\mathrm{R}_{\mathrm{DIC}}$ were all on average low (around 0 to $5 \mathrm{mmol} \mathrm{O}_{2}$ or DIC $\mathrm{m}^{-2} \mathrm{~h}^{-1}$ ) and similar, but with high variability between chambers and experiment days. Water chambers tended to have very low rates of respiration compared to sediment chambers.

Rates from four particular chambers were likely in error and not included in Table 1 or subsequent analysis: Of the two macroalgal light chambers sampled on June 30, one had high $\mathrm{O}_{2}$ uptake and DIC release indicating that it was behaving more like a dark chamber, and all rates from this chamber were excluded - visually this chamber was overly full of macroalgal biomass which may have limited light availability in the chamber. The second macroalgal light chamber on June 30 also had high amounts of macroalgae compared to subsequent experiments, and had very low $\mathrm{O}_{2}$ resulting in suspect rates of $\mathrm{GPP}_{\mathrm{TOI}}$ (but $\mathrm{NCP}_{\mathrm{O} 2}$ and $\mathrm{NCP}_{\mathrm{DIC}}$ are reported). One of the two dark water chambers on June 30 had a very low initial $\left[\mathrm{O}_{2}\right]\left(<20 \mu \mathrm{mol} \mathrm{kg}{ }^{-1}\right)$, but rose abruptly at the second timepoint $\left(\left[\mathrm{O}_{2}\right] \sim 130 \mu \mathrm{mol} \mathrm{kg}^{-1}\right)$. [DIC] simultaneously dropped. Along with concentrations from the surrounding pond water, these data suggests that this water chamber had a large leak during this deployment and no rates are reported from it. Finally, on the July 22 experiment, triple oxygen isotopes were sampled from a macroalgal dark chamber, and $\mathrm{GPP}_{\text {TOI }}$ from this chamber is not reported- $\left[\mathrm{O}_{2}\right]$ was $<75 \mu \mathrm{mol} \mathrm{kg}^{-1}$ at all timepoints in this chamber and apparent ${ }^{17} \Delta$ was greater than the theoretical maximum for photosynthetically produced $\mathrm{O}_{2}$ given the expected isotopic composition of the substrate water (forcing Eq. 1 to be negative despite large increases in ${ }^{17} \Delta$ ). Both mass spectrometer and volume exchange corrections were very large compared to the measured isotopic ratios, criteria that I have previously used to exclude triple oxygen isotope data (Chapter 3). 
Table 1: Mean metabolism rates (in $\mathrm{mmol} \mathrm{O}_{2} \mathrm{~m}^{-2} \mathrm{~h}^{-1}$ ) from three midday chamber experiments. Sediment chamber rates include sediment and water system, not adjusted for water only rates from water chambers.

\begin{tabular}{|c|c|c|c|c|c|c|c|c|c|c|c|c|}
\hline & \multicolumn{4}{|c|}{ Macroalgae } & \multicolumn{3}{|c|}{ Microalgae } & \multicolumn{5}{|c|}{ Water chambers ${ }^{\mathrm{c}}$} \\
\hline & Tracer & GPP & $\mathrm{NCP}$ & $\mathrm{R}^{\mathrm{b}}$ & GPP & $\mathrm{NCP}$ & $\mathrm{R}^{\mathrm{b}}$ & GPP & & $\mathrm{NCP}$ & & $\mathrm{R}^{\mathrm{b}}$ \\
\hline \multirow[t]{3}{*}{ June 30} & "TOI & ב-- & - -- & $\begin{array}{l}- \\
\end{array}$ & $\begin{array}{ll}5.1 \quad(1.1) \\
\end{array}$ & $\begin{array}{l}- \\
\end{array}$ & $\begin{array}{l}- \\
\end{array}$ & 1.5 & (1.2) & "-- & & $\begin{array}{l}- \\
\end{array}$ \\
\hline & $\mathrm{O}_{2}^{\mathrm{a}}$ & 0.9 & 0.4 & $-0.5 \quad(0.1)$ & $3.3 \quad(2.1)$ & 2.1 & $-1.7(0.9)$ & -- & -- & 1.1 & $(0.8)$ & -- \\
\hline & $\mathrm{DIC}^{\mathrm{a}}$ & 44.4 & 8.7 & $-35.8(36.0)$ & $2.2(2.4)$ & $0.7 \quad(1.7)$ & $-1.5(1.7)$ & -- & -- & 1.4 & $(0.0)$ & -- \\
\hline \multirow[t]{3}{*}{ July 8} & TOI & $5.0 \quad(2.6)$ & -- & -- & $7.2 \quad(2.8)$ & -- & -- & 2.4 & $(0.0)$ & -- & & -- \\
\hline & $\mathrm{O}_{2}$ & $5.9 \quad(2.0)$ & $3.5 \quad(1.7)$ & $-2.3(1.1)$ & $3.0 \quad(1.2)$ & $-0.6(1.2)$ & $-3.6(0.3)$ & 2.0 & $(0.4)$ & 1.8 & $(0.4)$ & $-0.2(0.1)$ \\
\hline & $\mathrm{DIC}$ & $21.6(6.4)$ & $10.6(6.4)$ & $-11.0(0.7)$ & $9.4 \quad(2.1)$ & $2.4 \quad(2.1)$ & $-7.0(0.2)$ & 2.8 & $(0.1)$ & 2.2 & $(0.1)$ & $-0.6(0.1)$ \\
\hline \multirow[t]{4}{*}{ July 22} & TOI & $7.1 \quad(3.7)$ & -- & -- & $5.3 \quad(9.0)$ & -- & -- & 0.7 & $(0.2)$ & -- & & -- \\
\hline & $\mathrm{O}_{2}$ & $7.3 \quad(1.2)$ & $5.2 \quad(0.7)$ & $-2.0 \quad(1.0)$ & $8.2(0.8)$ & $6.4 \quad(0.7)$ & $-1.7(0.5)$ & 1.7 & $(0.8)$ & 1.6 & $(0.8)$ & $-0.1 \quad(0.2)$ \\
\hline & $\mathrm{DIC}$ & $14.9(2.7)$ & $11.1(2.3)$ & $-3.7 \quad(1.4)$ & $17.0(1.6)$ & $12.4(1.6)$ & $-4.6(0.4)$ & 1.8 & $(0.5)$ & 1.7 & $(0.4)$ & $-0.1 \quad(0.3)$ \\
\hline & & GPP & $\mathrm{NCP}$ & $\mathrm{R}$ & GPP & $\mathrm{NCP}$ & $\mathrm{R}$ & GPP & & $\mathrm{NCP}$ & & $\mathrm{R}$ \\
\hline \multirow[t]{3}{*}{ Mean $^{d}$} & TOI & $\begin{array}{ll}6.1 & (1.5)\end{array}$ & - -- & "-- & 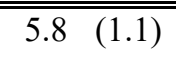 & - -- & - -- & 1.5 & (0.9) & - -- & & "-- \\
\hline & $\mathrm{O}_{2}$ & $6.6 \quad(1.0)$ & $4.4 \quad(1.2)$ & $-2.2(0.2)$ & $5.0 \quad(2.8)$ & $2.7 \quad(3.6)$ & $-2.3(1.1)$ & 1.9 & $(0.2)$ & 1.5 & $(0.3)$ & $-0.2(0.1)$ \\
\hline & $\mathrm{DIC}^{\mathrm{a}}$ & $19.3(3.2)$ & 11.8 & $-7.5 \quad(5.0)$ & $9.5 \quad(7.4)$ & $5.1 \quad(6.3)$ & $-4.4(2.7)$ & 2.3 & $(0.7)$ & 1.8 & $(0.4)$ & $-0.3 \quad(0.3)$ \\
\hline
\end{tabular}

${ }^{a}$ A single macroalgae light chamber was evaluated on June 30. A much higher volume was filled by macroalgal biomass than in subsequent experiments, and had unusual $\mathrm{O}_{2}$ and DIC based rates compared to subsequent experiments.

${ }^{\mathrm{b}} \mathrm{O}_{2}$ respiration rates were corrected for expected concentration dependent biases during sampling of low $\mathrm{O}_{2}$ chambers (see text).

${ }^{\mathrm{c}}$ Except where otherwise noted, for each daily experiment $\mathrm{n}=3$ for both light and dark sediment chambers (NCP and R rates) while $\mathrm{n}=2$ for water chambers.

${ }^{\mathrm{d}}$ Only July 8 and July 22 are averaged for mean macroalgae rates because of the low number of replicates and lack of GPP $\mathrm{TOI}_{\text {for }}$ comparison on June 30. 
Table 2: Mean metabolism rates rates (in $\mathrm{mmol} \mathrm{O}_{2} \mathrm{~m}^{-2} \mathrm{~h}^{-1}$ ) from open pond over the same periods as the chamber experiments. Additionally, expected pond rates based on chamber results adjusted for sediment and water rates in each chamber type, as well as the portion of the total pond area with each type of sediment cover (not including potential Ruppia production; see text).

\begin{tabular}{|c|c|c|c|c|c|c|c|c|c|}
\hline \multirow{5}{*}{ June $30^{\mathrm{a}}$} & \multicolumn{5}{|c|}{ Measured pond rates } & \multicolumn{4}{|c|}{ Expected pond rates } \\
\hline & \multirow{2}{*}{$\begin{array}{c}\text { Tracer } \\
\text { TOI }\end{array}$} & \multirow{2}{*}{$\begin{array}{l}\text { GPP } \\
19.9\end{array}$} & \multicolumn{3}{|c|}{ NCP } & \multicolumn{2}{|l|}{ GPP } & \multicolumn{2}{|c|}{$\mathrm{NCP}$} \\
\hline & & & $(2.0)$ & -- & & -- & & & \\
\hline & $\mathrm{O}_{2}{ }^{\mathrm{b}}$ & 22.2 & (1.7) & 11.2 & $(1.0)$ & -- & & 2.0 & \\
\hline & DIC & -- & & 15.3 & (2.4) & -- & & 1.6 & \\
\hline \multirow[t]{4}{*}{ July 8} & TOI & 20.0 & $(4.7)$ & -- & & 6.5 & $(0.4)$ & -- & \\
\hline & $\mathrm{O}_{2}{ }^{\mathrm{b}}$ & 19.6 & $(3.2)$ & 9.4 & $(2.5)$ & 3.2 & $(0.8)$ & 1.7 & $(0.8)$ \\
\hline & DIC & -- & & 21.8 & (3.6) & 10.0 & $(0.7)$ & 5.2 & $(0.7)$ \\
\hline & Pond & & & & & & & & \\
\hline \multirow[t]{4}{*}{ July 22} & TOI & 19.1 & $(0.9)$ & -- & & 5.0 & $(1.0)$ & -- & \\
\hline & $\mathrm{O}_{2}{ }^{\mathrm{b}}$ & 16.1 & $(2.3)$ & 5.1 & (1.6) & 7.5 & (1.5) & 7.3 & $(1.5)$ \\
\hline & DIC & -- & & 17.1 & $(4.7)$ & 15.3 & $(1.0)$ & 12.8 & $(0.8)$ \\
\hline & & GPP & & $\mathrm{NCP}$ & & GPP & & NCP & \\
\hline \multirow[t]{3}{*}{ Mean $^{a}$} & TOI & 19.5 & $(0.6)$ & - & & 5.4 & (1.7) & -- & \\
\hline & $\mathrm{O}_{2}{ }^{\mathrm{b}}$ & 19.3 & $(3.1)$ & 8.6 & $(2.5)$ & 4.9 & (1.8) & 4.1 & (2.9) \\
\hline & DIC & -- & & 18.1 & (3.4) & 9.8 & (1.6) & 5.4 & $(1.0)$ \\
\hline
\end{tabular}

${ }^{a}$ Only one macroalgae chamber was evaluated on June 30, and was unusual compared to all later macroalgae chambers. No GPP rates could be calculated given the missing data (Table 1). As when evaluating the mean rates for each chamber type (Table 1), June 30 macroalgae chambers are excluded when evaluating mean expected pond metabolism rates from the chamber rates. Additionally, uncertainties for expected whole pond rates for June 30 could not be evaluated with rates derived from only a single macroalgae chamber.

${ }^{\mathrm{b}} \mathrm{O}_{2}$ based GPP and NCP are derived from concentrations at the sonde location and nighttime $\mathrm{R}_{\mathrm{O} 2}$. 


\section{Discussion}

The reported metabolic rates in this work vary in experiment and tracer type, and on hourly to seasonal timescales. In this section I discuss several aspects of the metabolic rates that clarify the relationships between rates and environmental drivers of metabolic cycling in this salt marsh pond. First, (section 4.1) I compare production rates between experiment types (open water timeseries and chamber experiments) in order to distinguish differences between the whole pond environment versus the sediment water interface in macroalgal or microalgal dominated sediments. Second (section 4.2), I compare triple oxygen isotope and $\mathrm{O}_{2}$ derived GPP rates and propose specific processes that may explain observed differences between the different methods. As part of this analysis I discuss apparently positive nighttime rates calculated for $\mathrm{GPP}_{\mathrm{TOI}}$ and whether that affects the interpretation of daytime GPP ${ }_{\mathrm{TOI}}$. Third (section 4.3), I compare the $\mathrm{DIC}$ and $\mathrm{O}_{2}$ based rates and use the $\mathrm{GPP}_{\mathrm{DIC}} / \mathrm{GPP}_{\mathrm{O} 2}$ ratio in order to inform my interpretation of the processes invoked earlier in the discussion. In sections 4.2.2 and 4.3.2, a number of processes are evaluated in order to explain the results of this study. The expected effect of these processes on metabolism ratios is summarized in Table 3, and within subsections these processes are organized in descending order of likely relevance. One of several possible mechanism for driving oxidation and reduction changes in the sediments that can help explain the observed trends is metabolic fluxes driven by microbial consortia associated with cable bacteria; although I focus on this possible mechanism in sections 4.2 and 4.3 in order to illustrate in greater detail how changes in the storage and production of reduced and oxidized compounds in the sediments can influence observations of DIC and $\mathrm{O}_{2}$ in the water column (concept diagram in supplement), the data presented in this chapter could also be explained by other sediment processes which are more briefly discussed. Finally (section 4.4), I discuss the variability of production and community metabolic efficiency over a range of timescales.

\subsection{Comparison of rates from open water and chamber experiments}

GPP and NCP rates from the chamber experiments (Table 1) can be compared to rates from the open pond over the same periods (Table 2). Water chamber rates are very low compared to both sediment chambers and to overall pond metabolism; the benthic community is likely more important than plankton in explaining whole pond metabolism. Yet sediment chamber GPP from the oxygen tracers was only 20$30 \%$ as large as $\mathrm{GPP}_{\mathrm{TOI}}$ in the open pond over the same period, and chamber $\mathrm{NCP}_{\mathrm{O} 2}$ was similarly roughly $25 \%$ of pond $\mathrm{NCP}_{\mathrm{O} 2}$. Macroalgal $\mathrm{NCP}_{\mathrm{DIC}}$ was on average only $65 \%$ as large as in the open pond, and the microalgal rate was only $30 \%$ of that in the pond.

Alteration of the physical and chemical environment at the sediment-water interface during chamber deployment (chamber effects) may reduce light and nutrient supply or otherwise inhibit benthic algae production. However, chamber production rates are within the range of daytime production rates reported in other chamber studies of metabolism in shallow marine environments dominated by micro- and macroalgae (1 to $5 \mathrm{mmol} \mathrm{O}_{2} \mathrm{~m}^{-2} \mathrm{~h}^{-1}$; Jahnke et al. 2000, Cebrian et al. 2007). Studies in tidal creeks and salt marsh dominated channels using open water methods have mean daytime production rates in a similar range, with peak reported rates no greater than 12 to $18 \mathrm{mmol} \mathrm{O}_{2} \mathrm{~m}^{-2} \mathrm{~h}^{-1}$ (Vallino et al. 2005, Nidzeiko et al. 2014). Additionally, benthic algae in intact sediment communities have been reported to be well adapted to low to moderate light salt marsh environments (saturation irradiances of 1 to 4 mol quanta PAR m$~^{-2} \mathrm{~h}^{-1}$; Whitney and Darley 1983, Pinckney and Zingmark 1993, MacIntyre et al. 1996); I would expect algae in both the chambers and open pond to experience photoinhibition rather than light limitation. The higher rates in the open pond over the same time period are similar to the highest rates reported in open marsh environments (above) and in highly productive seagrass beds ( 6 to $>34 \mathrm{mmol} \mathrm{O}_{2}$ $\mathrm{m}^{-2} \mathrm{~h}^{-1}$; Murray and Wetzel 1987, Kinney and Roman 1998). 
Table 3: Some of the processes that could potentially affect $\mathrm{GPP}_{\mathrm{TO}} / \mathrm{GPP}_{\mathrm{O} 2}$ and $\mathrm{GPP}_{\mathrm{DIC}} / \mathrm{GPP}_{\mathrm{O} 2}$ ratios, and expected ratios for highest possible effect on metabolism ratios given known constraints. Darker shades indicate processes that are more likely to occur at rates sufficiently high to affect the measured ratios, and underlined ratios are potentially lower bounds.

\begin{tabular}{|c|c|c|c|}
\hline Process & $\mathrm{GPP}_{\mathrm{TOI}} / \mathrm{GPP}_{\mathrm{O} 2}$ & $\mathrm{GPP}_{\mathrm{DIC}} / \mathrm{GPP}_{\mathrm{O} 2}{ }^{\mathrm{a}}$ & Effect bound ${ }^{b}$ \\
\hline Light-enhanced mitochondrial respiration & $\overline{1.6}$ & $\overline{1}$ & Maximum possible given observed ratio ${ }^{1}$ \\
\hline Photoreduction & 1.2 & 1.2 & Maximum possible given observed ratio ${ }^{2}$ \\
\hline Ebullition & $\underline{1.1}$ & $\underline{1.1}$ & Observed with noble gases ${ }^{3}$ \\
\hline $\begin{array}{l}\text { Cable bacteria microbial consortia } \\
\text { (including chemoautotrophs) }\end{array}$ & $\underline{1.3}$ & $\underline{1.4}^{\mathrm{c}}$ & Literature rates for laboratory studies ${ }^{4}$ \\
\hline Photorespiration & 1.4 & 1 & Literature rates for laboratory study ${ }^{5}$ \\
\hline Anoxygenic photosynthesis & 1 & $\underline{1.1}$ & Literature rate for laboratory study ${ }^{6}$ \\
\hline Compounds with unusual $\mathrm{C} / \mathrm{O}$ & 1 & 2.4 & Maximum possible given observed ratio ${ }^{7}$ \\
\hline Carbonate precipitation & 1 & 1 & Literature rates for natural systems ${ }^{8}$ \\
\hline Sediment chamber mean & $1.3(1.1)$ & $2.6(0.9)$ & \\
\hline Pond during chamber experiments & $1.0(0.2)$ & $2.4(1.0)^{\mathrm{d}}$ & \\
\hline Pond over full day & $1.6(0.4)$ & -2 to $5^{d}$ & \\
\hline
\end{tabular}

${ }^{a}$ Based on assumed ratio of $\mathrm{GPP}_{\mathrm{DIC}} / \mathrm{GPP}_{\mathrm{O} 2}=1$, using rates from open water. Ratios using chamber rates are within 0.1 .

${ }^{\mathrm{b}}$ Maximum effects are within reported literature rates, which exceed the ratios reported in this work. Laboratory studies may result in lower or higher rates than natural environments, and consequently change the predicted ratios. References reported in numbered footnotes.

${ }^{c}$ Assuming both $\mathrm{O}_{2}$ drawdown by cable bacteria and DIC uptake by associated microbial consortia.

${ }^{\mathrm{d}}$ Pond $\mathrm{GPP}_{\mathrm{DIC}} / \mathrm{GPP}_{\mathrm{O} 2}$ not evaluated, but assumed to be similar to $\mathrm{NCP}_{\mathrm{DIC}} / \mathrm{NCP}_{\mathrm{O} 2}$.

${ }^{1}$ Bender et al. 1987, Fenchel and Glud 2000, Pringault et al. 2007

2 Helman et al. 2005, Halsey and Jones 2015

${ }^{3}$ Howard et al. submitted

${ }^{4}$ Vasquez-Cardenas 2015, Rao et al. 2016a, 2016b

${ }^{5}$ Birmingham et al. 1982

${ }^{6}$ Massé et al. 2002

${ }^{7}$ Rosenberg et al. 1995

${ }^{8}$ Coleman and Raiswell 1993, Ludwig et al. 2005 
Thus all three metabolic tracers point to a substantial enhancement in the rates of NCP and GPP in the open pond compared to in the macroalgal and microalgal dominated sediments. Based on the low rates of production in the water chambers, it seems likely that this difference is related to processes in parts of the pond that are characterized by different types of sediment cover. One such environment is a field of Rupia and associated epiphytes extending over roughly $10 \%$ of the sediments, primarily along the eastern edge of the pond. A comparison of production rates based on $\mathrm{O}_{2}$ in this and two other ponds (Spivak et al. submitted) reported substantially higher production in nearby smaller ponds with very high abundance of Ruppia (50 to $80 \%$ cover) and found that plant cover was a significant predictor of primary production in the ponds.

Ruppia maritima have peak daytime NCP rates of 6 to $>34 \mathrm{mmol} \mathrm{O}_{2} \mathrm{~m}^{-2} \mathrm{~h}^{-1}$ (Murray and Wetzel 1987, Kinney and Roman 1998), with the lower value representing bottle incubations of cut Rupia leaves and the higher value from chamber experiments enclosing intact plants and associated epiphytes. I calculate a predicted whole pond $\mathrm{NCP}_{\mathrm{O} 2}$ by assuming the higher rate is applicable for the $10 \%$ of total pond area with Ruppia, and using the mean chamber rates as production rates for macroalgae and microalgae covered sediments (10\% and $80 \%$ of sediments) as in section 3.2. If including Ruppia production, the resulting predicted $\mathrm{NCP}_{\mathrm{O} 2}$ is $7.6(2.9) \mathrm{mmol} \mathrm{O}_{2} \mathrm{~m}^{-2} \mathrm{~h}^{-1}$, similar to measured pond $\mathrm{NCP}_{\mathrm{O} 2}$ over the chamber experiment periods $\left(8.6(2.5) \mathrm{mmol} \mathrm{O}_{2} \mathrm{~m}^{-2} \mathrm{~h}^{-1}\right)$. Thus it is plausible that a relatively large portion of pond metabolism is driven by production of vascular plants over a relatively small area.

\subsection{Comparison of triple oxygen isotope and $\mathrm{O}_{2}$ derived GPP rates}

Triple oxygen isotope and $\mathrm{O}_{2}$ derived rates of GPP from the chamber experiments were equivalent $\left(\mathrm{p}=0.0017\right.$ and 0.0096 for a two one-sided t-test, $\theta=0.65$; Limentani et al. 2005). GPP ${ }_{\text {TOI }}$ and GPP $\mathrm{O}_{\mathrm{O} 2}$ from the open pond for just the chamber experiment days and periods are also equivalent $(\mathrm{p}=0.0364$ and 0.0237 for a two one-sided t-test, $\theta=0.24$ ). Because these data did not cover the entire period of peak production, they were not included in the daily $\mathrm{GPP}_{\mathrm{TOI}}$ rate in Fig. 6. However, mean daily $\mathrm{GPP}_{\mathrm{TO}}$ was significantly higher than $\mathrm{GPP}_{\mathrm{O} 2}$ in the open water timeseries (one-way ANOVA $\mathrm{F}=21.3, \mathrm{p}=4.8 \times 10^{-5}$ ). On average the ratio of daily $\mathrm{GPP}_{\mathrm{TO}} / \mathrm{GPP}_{\mathrm{O} 2}$ was 1.6(0.4). Based on the good overall agreement between the two tracers in the chambers and open water over the short midday chamber experiment periods, the discrepancy between the daily rates in the open pond is likely related to methodological or environmental factors that vary over the mornings and afternoon periods. Before discussing environmental processes that may lead to the difference in the daytime rates in the open pond, I examine the nighttime triple oxygen isotope data to determine whether there is any evidence for positive biases in the GPP ${ }_{\mathrm{TO}}$ results that could affect the interpretation of the daytime rates.

\subsubsection{Potential sources of methodological or environmental bias to triple oxygen isotopes as determined from nighttime results}

Nighttime GPP $_{\mathrm{TOI}}$ rates were calculated as being larger than zero (Fig. 3), which could indicate that the $\mathrm{GPP}_{\text {TOI }}$ rates were biased positive. The apparently positive rates during a period that should have had no production were calculated because the measured ${ }^{17} \Delta$ change with time was close to 0 per meg $\mathrm{h}^{-1}$ on average, while the gas exchange parameterization predicts a decrease on the order of -20 per meg $\mathrm{h}^{-1}$. Eq.

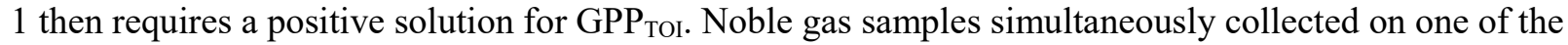
two nighttime periods indicate that the chosen gas exchange parameterization may be actually underestimating air-water exchange (Chapter 4). Therefore some other factor or process may be increasing measured ${ }^{17} \Delta$ at the nighttime sample location. It is important to clarify whether this effect is limited to the nighttime sample periods only, or whether it could affect the daytime rates and interpretation of the $\mathrm{GPP}_{\mathrm{TO} /} / \mathrm{GPP}_{\mathrm{O} 2}$ ratio. 


\section{Methodological issues:}

The method used to analyze low- $\mathrm{O}_{2}$ samples has identical precision to the standard method in this study. The two methods also agree well at moderate to high sample $\mathrm{O}_{2}$ concentrations. However, the two rates cannot be compared at the low $\mathrm{O}_{2}$ concentrations typical of the overnight samples, and it is possible that the method has greater bias as $\mathrm{O}_{2}$ decreases to the method threshold of $\sim 50 \mu \mathrm{mol} \mathrm{kg}^{-1}$ (Chapter 3).

Nighttime triple oxygen isotope samples ranged in $\mathrm{O}_{2}$ concentration from 146 to $54 \mu \mathrm{mol} \mathrm{kg} \mathrm{kg}^{-1}$. Large positive biases in ${ }^{17} \Delta$ ( 20 per meg or more) have been observed at concentrations below $80 \mu \mathrm{mol} \mathrm{kg}^{-1}$. Biases to nighttime ${ }^{17} \Delta$ at the lower concentrations could account for a positive bias to GPP ${ }_{\text {TOI }}$ rates that was one to two times as large as the standard deviation from known uncertainties, particularly if the size of the error was proportional to the decrease in $\mathrm{O}_{2}$. This estimate is in good agreement with the observed increase in $\mathrm{GPP}_{\mathrm{TOI}}$ compared to the expected rate of zero at night.

\section{Environmental conditions:}

Observation of positive GPP at night could also indicate that certain environmental conditions may bias the triple oxygen isotope method. Nighttime differences in environmental conditions could increase ${ }^{17} \Delta$ in opposition of gas exchange. For example, dissolution of bubbles on the sediments or flora that had formed in the daytime (Chapter 4) would act to release $\mathrm{O}_{2}$ with high, photosynthetically modified ${ }^{17} \Delta .{ }^{17} \Delta$ could be enhanced in a similar manner by mixing of water from Ruppia or macroalgae dominated areas during nighttime convection - during the daytime, wind-dominated mixing tended to homogenize pond water along the wind fetch, but allowed large gradients in oxygen concentration (differences in $\mathrm{O}_{2}$ saturation state of $>100$ to $200 \%$ between adjacent bare sediments and macroalgae or Ruppia dominated patches) and ${ }^{17} \Delta$ (differences of 20 to 50 per meg between patches). While it seems unlikely that such heterogeneity would persist for long after $\mathrm{O}_{2}$ became undersaturated (the $\mathrm{O}_{2}$ residence time with respect to gas exchange is 6 hours at night), in the absence of strong lateral mixing, areas with extensive Ruppia and macroalgal mats likely had much slower air-water exchange than in water over open sediments because of turbulence reduction by the vegetation (Koch and Gust 1999, Romano et al. 2003, Nepf and Ghisalberti 2008). Either dissolution of bubbles or enhanced convective mixing would likely be heterogeneous in space and time and could slow the expected overall rate of ${ }^{17} \Delta$ decrease.

The potential methodological bias to the lowest $\mathrm{O}_{2}$ samples and plausible environmental differences

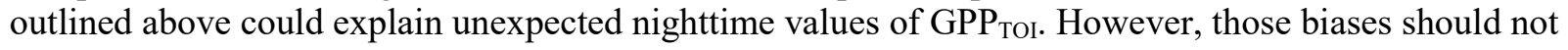
affect daytime $\mathrm{GPP}_{\mathrm{TO}}$ from the chambers or open water. Thus the observed differences in $\mathrm{GPP}_{\mathrm{TO}}$ and $\mathrm{GPP}_{\mathrm{O} 2}$ likely reflect real differences in the sensitivities of the two tracers to environmental processes. Reinforcing this interpretation, daytime $\mathrm{GPP}_{\mathrm{TO}}$ followed expected patterns similar to NCP rates, and when sampled dawn and dusk rates were zero within uncertainties. The question then becomes what $\mathrm{O}_{2}$ fluxes might explain the difference between the triple oxygen isotope derived production, which is relatively insensitive to the fate of produced oxygen, and the $\mathrm{O}_{2}$ derived flux, which depends on accurate accounting of respiration and other oxygen sinks.

\subsubsection{Environmental processes that could enhance daytime biological $\mathrm{O}_{2}$ uptake}

$\mathrm{GPP}_{\mathrm{O} 2}$ is derived from daytime $\mathrm{NCP}_{\mathrm{O} 2}$ and nighttime dark respiration $\left(\mathrm{R}_{\mathrm{O} 2}\right)$. The elevated open pond $\mathrm{GPP}_{\mathrm{TO}}$ compared to $\mathrm{GPP}_{\mathrm{O} 2}$ over the day (as opposed to peak hours in the chambers) suggests that $\mathrm{O}_{2}$ is being removed more rapidly than expected from $\mathrm{R}_{\mathrm{O} 2}$ alone. I identify five primary sinks that could remove $\mathrm{O}_{2}$ at greater rates than predicted from dark respiration: Light-enhanced mitochondrial respiration, photoreduction, ebullition, oxygen transport by the benthic community into the sediments, and photorespiration. They are discussed below in order of decreasing probable importance.

\section{Evaluation of specific processes:}

Light-enhanced mitochondrial respiration is known to increase the oxygen uptake of phytoplankton and benthic communities by 1.5 to 6 times the rate during extended periods of darkness (Bender et al. 1987; 
Fenchel and Glud 2000; Pringault et al. 2007). GPP ${ }_{\mathrm{TO}}$ is unaffected by any changes in mitochondrial respiration; an increase of only $45 \%$ for light versus dark respiration would be sufficient to bring $\mathrm{GPP}_{\mathrm{O} 2}$ in line with GPP

Similarly GPP $\mathrm{TOI}_{\text {is }}$ known to include $\mathrm{O}_{2}$ utilized by photoreduction (the Mehler reaction; Helman et al. 2005, Nicholson et al. 2012), while $\mathrm{R}_{\mathrm{O} 2}$ (and thus $\mathrm{GPP}_{\mathrm{O} 2}$ ) does not. The Mehler reaction is a small component part of oxygen cycling in the surface ocean (Marra and Barber 2004), but for algae exposed to high irradiance and DIC it can consume $15-75 \%$ of GPP in order to protect against light stress, produce ATP for carbon concentrating mechanisms, and help nitrogen fixers maintain locally anaerobic environments (Berman-Frank et al. 2001, Halsey and Jones 2015). The triple oxygen isotope fractionations for photoreduction have been determined only for a single freshwater cyanobacteria (Synechocystis sp. strain PCC 6803; Helman et al. 2005), but the relative effect of this process can be estimated by modifying Eq. 3 to apply this fractionation for all production calculations:

$$
\begin{aligned}
\mathrm{z}\left[\mathrm{O}_{2}\right] \frac{\partial}{\partial \mathrm{t}}\left({ }^{17} \Delta\right) \rho_{w}=\mathrm{GPP}_{\mathrm{TOI}, \mathrm{M}}\left(\frac{{ }^{17} \mathrm{X}_{\mathrm{P}}-{ }^{17} \mathrm{X}}{{ }^{17} \mathrm{X}}-\lambda \frac{{ }^{18} \mathrm{X}_{\mathrm{P}}-{ }^{18} \mathrm{X}}{{ }^{18} \mathrm{X}}\right) & -\mathrm{k}_{\mathrm{O} 2}\left[\mathrm{O}_{2 \text { sat }}\right]\left(\frac{{ }^{17} \mathrm{X}-{ }^{17} \mathrm{X}_{\text {sat }}}{{ }^{17} \mathrm{X}}-\lambda \frac{{ }^{18} \mathrm{X}-{ }^{18} \mathrm{X}_{\text {sat }}}{{ }^{18} \mathrm{X}}\right) \rho_{w} \\
& +\mathrm{M}\left(\frac{{ }^{17} \mathrm{X}_{\mathrm{M}}-{ }^{17} \mathrm{X}}{{ }^{17} \mathrm{X}}-\lambda \frac{{ }^{18} \mathrm{X}_{\mathrm{M}}-{ }^{18} \mathrm{X}}{{ }^{18} \mathrm{X}}\right)
\end{aligned}
$$

where $\mathrm{M}$ is the assumed rate of the Mehler reaction, and ${ }^{i} \mathrm{X}_{\mathrm{M}}$ are the isotopic endmembers for $\mathrm{O}_{2}$ derived from the measured dissolved $\mathrm{O}_{2}$ using ${ }^{18} \varepsilon=-9.6$ and ${ }^{18} \varepsilon=-4.8 \%$ relative to the substrate water (Helman et al. 2005). $\mathrm{GPP}_{\mathrm{TO} I} / \mathrm{GPP}_{\mathrm{O} 2}=1$ (all difference between the two rates is explained by photoreduction) when the Mehler reaction consumes $18 \%$ as much $\mathrm{O}_{2}$ as the "true" value of GPP. In this case calculated GPP is decreased by $23 \%$, and $\mathrm{GPP}_{\mathrm{O} 2}$ is increased by $22 \%$.

Ebullition of photosynthetic oxygen from the benthic community was identified as a potentially large oxygen sink in Chapter 4; mean daytime oxygen loss from ebullition could account for $-0.4(0.1)$ to $-1.0(0.3) \mathrm{mmol} \mathrm{O}_{2} \mathrm{~m}^{-2} \mathrm{~h}^{-1}$ lost to the atmosphere on the days for which noble gas data were available. Ebullition is expected to vary in space and time, and may be greatest in areas with the greatest local supersaturations of oxygen, such as Ruppia beds. The effect of ebullition can be approximated by modifying Eq. 1 to include equilibrium isotope fractionation with bubbles on the sediments or benthic flora:

$$
\begin{aligned}
\mathrm{z}\left[\mathrm{O}_{2}\right] \frac{\partial}{\partial \mathrm{t}}\left({ }^{17} \Delta\right) \rho_{w}=\mathrm{GPP}_{\mathrm{TOI}, \mathrm{E}}\left(\frac{{ }^{17} \mathrm{X}_{\mathrm{P}}-{ }^{17} \mathrm{X}}{{ }^{17} \mathrm{X}}-\lambda \frac{{ }^{18} \mathrm{X}_{\mathrm{P}}-{ }^{18} \mathrm{X}}{{ }^{18} \mathrm{X}}\right) & -\mathrm{k}_{\mathrm{O} 2}\left[\mathrm{O}_{2 \text { sat }}\right]\left(\frac{{ }^{17} \mathrm{X}-{ }^{17} \mathrm{X}_{\text {sat }}}{{ }^{17} \mathrm{X}}-\lambda \frac{{ }^{18} \mathrm{X}-{ }^{18} \mathrm{X}_{\text {sat }}}{{ }^{18} \mathrm{X}}\right) \rho_{w} \\
& +\mathrm{E}\left(\frac{\left({ }^{17} \mathrm{X}-{ }^{17} \mathrm{X}_{\text {sat }}\right.}{{ }^{17} \mathrm{X}}-\lambda \frac{{ }^{18} \mathrm{X}-{ }^{18} \mathrm{X}_{\text {sat }}}{{ }^{18} \mathrm{X}}\right)
\end{aligned}
$$

$\mathrm{GPP}_{\mathrm{TOI}, \mathrm{E}}$ is the $\mathrm{GPP}_{\mathrm{TOI}}$ rate corrected for ebullition, and $\mathrm{E}$ is the ebullition rate. I neglect small differences in pressure as well as minor corrections for the difference between bubble invasion and evasion (Kaiser 2011). Ebullition has a very small effect on calculated rates of $\mathrm{GPP}_{\mathrm{TO}}$, while $\mathrm{GPP}_{\mathrm{O} 2}$ is reduced by $\mathrm{E}$ because Eq. 3 does not include ebullition as a loss term. Thus if the entire difference between $\mathrm{GPP}_{\mathrm{TOI}}$ and $\mathrm{GPP}_{\mathrm{O} 2}$ is caused by ebullition, mean $\mathrm{GPP}_{\mathrm{O} 2}$ equals $\mathrm{GPP}_{\mathrm{TOI}}\left(\mathrm{GPP}_{\mathrm{TOI}} / \mathrm{GPP}_{\mathrm{O} 2}=1\right)$ over the timeseries when mean $\mathrm{E}$ is $38 \%$ of GPP $-4.8 \mathrm{mmol} \mathrm{O}_{2} \mathrm{~m}^{-2} \mathrm{~h}^{-1}$. Observed mean daytime rates of ebullition are only $10-20 \%$ of the predicted $\mathrm{E}$ value required to explain the rate difference.

Enhanced oxygen transport into the sediments during daytime could remove $\mathrm{O}_{2}$ from the water column in excess of rates predicted by diffusion alone (order of $0.1 \mathrm{mmol} \mathrm{O}_{2} \mathrm{~m}^{-2} \mathrm{~h}^{-1}$ ), decreasing $\mathrm{GPP}_{\mathrm{O} 2}$. Production and storage of reduced chemicals in the sediments overnight can increase sediment oxygen demand in a time-varying manner and enhance oxygen consumption in the daytime when oxygen gradients into the sediments are strongest, leading to stronger diffusive gradients over short periods. In Fig. 3, NCP 
appears to be autotrophic earlier in the day than $\mathrm{NCP}_{02}$, which could occur if oxygen produced by photosynthesis was rapidly consumed by microbial or chemical oxidation of iron and manganese sulfides in the sediments. In particular sulfide and pyrite $\left(\mathrm{FeS}_{2}\right)$ can be rapidly oxidized with large gross sulfur oxidation fluxes compared to net accumulation or loss of these compounds (Luther et al. 1982, Giblin and Howarth 1984, Swider and Mackin 1989), and the consumption of such reduced compounds is ultimately (indirectly) linked to oxidation driven by $\mathrm{O}_{2}$ from the overlying water column.

Additionally, microbial metabolisms often mediate the production and storage of reduced chemicals and enhance oxygen uptake by sediments. As one example, sulfur oxidizing cable bacteria consume $\mathrm{O}_{2}$ in excess of diffusion limited sediment uptake by transporting electrons along cell chains from sulfide that is centimeters deep to $\mathrm{O}_{2}$ at the sediment water interface, and are apparently ubiquitous in marine sediments globally, including northern temperate salt marshes and salt marsh ponds (Larsen et al. 2015, Rao et al. 2016b). These bacteria can consume 1 to $3 \mathrm{mmol} \mathrm{O}_{2} \mathrm{~m}^{-2} \mathrm{~h}^{-1}$ in laboratory studies (Vasquez-Cardenas 2015, Rao et al. 2016a), though minimum cable bacteria $\mathrm{O}_{2}$ consumption rates from cores taken from a salt marsh pond in the St. Laurence Estuary, Canada were on the order of only $0.1 \mathrm{mmol} \mathrm{O}_{2} \mathrm{~m}^{-2} \mathrm{~h}^{-1}$ (Rao et al. 2016b). In most studies to date cable bacteria account for $>50 \%$ of sediment oxygen uptake for water at $\mathrm{O}_{2}$ concentration near equilibrium with the atmosphere; these rates should be thermodynamically favored to increase with greater $\mathrm{O}_{2}$ concentration, while this mechanism would be relatively ineffective at night with very low $\mathrm{O}_{2}$. Based on transcriptomic data, sulfide oxidizing cable bacteria are present and metabolically very active compared to most of the microbial community in the study pond (Patrick Kearns, personal communication).

Other biologically mediated processes in the sediments could enhance daytime oxygen uptake as well. First, diffusive fluxes could be larger than expected in parts of the pond because of bioturbation or bioirrigation by benthic infuana (eg. Koretsky et al. 2002), which allows deeper $\mathrm{O}_{2}$ penetration into the sediments and more interaction with reduced compounds. While there is no data on infauna abundance in the ponds, I did not observe burrows or visible evidence of bioturbation (e.g. snail tracks) in the ponds despite observing many such features in the adjacent tidal creek-low nighttime $\mathrm{O}_{2}$ in the pond and warm daytime temperatures may be less favorable for infauna then the creeks. In addition, the metabolic activity of cable bacteria in the pond (Patrick Kearns, personal communication) may provide evidence that there is not regular bioturbation by fauna, as bioturbation breaks the cables and halts cable bacteria metabolism (Rao et al. 2016b). An additional possibility for transport of $\mathrm{O}_{2}$ into the sediments is that seagrasses are known to diffuse $\mathrm{O}_{2}$ from their roots into the surrounding sediments in order to limit sulfide and metal toxicity (Pedersen et al. 2004, Jovanovic et al. 2015). Reported rates of oxygen transport in Ruppia are far too small to affect the pond $\mathrm{O}_{2}$ fluxes (nanomoles to micromoles of $\mathrm{O}_{2} \mathrm{~m}^{-2} \mathrm{~h}^{-1}$ depending on plant density). However, there could still be substantial root respiration (as opposed to efflux to sediments) that decreases the net release of produced oxygen to the surrounding water.

Finally, photorespiration (in which RuBisCO binds $\mathrm{O}_{2}$ instead of $\mathrm{CO}_{2}$ ) is more common at high temperature and high concentrations of $\mathrm{O}_{2}$ relative to $\mathrm{CO}_{2}$. Photorespiration can be a significant sink of $\mathrm{O}_{2}$ for some algal and seagrass species (Birmingham et al. 1982, Buapet et al. 2013). However, the $\mathrm{CO}_{2}$ in the pond is probably well in excess of atmospheric abundance $\left(\mathrm{CO}_{2}\right.$ estimated from DIC and assumed $\mathrm{pH}$ near 8.0(NBS) in CO2Sys v2.1), so it seems unlikely that photorespiration is a major sink in this environment compared to some of the other likely $\mathrm{O}_{2}$ consuming processes. It would also decrease DIC uptake in direct proportion to $\mathrm{O}_{2}$ consumption, which is inconsistent with observations of elevated $\mathrm{DIC} / \mathrm{O}_{2}$ flux ratios (see section 4.3).

\section{Summary:}

Of the processes identified above, light-enhanced mitochondrial respiration, and photoreduction consume $\mathrm{O}_{2}$ in excess of nighttime respiration and are the right order of magnitude to explain the differences between $\mathrm{GPP}_{\mathrm{TO}}$ and $\mathrm{GPP}_{\mathrm{O} 2}$ in the open pond, separately or in combination. Ebullition is not large enough to explain the difference on its own, but still could explain $10-20 \%$ of the difference. Enhanced daytime 
sediment $\mathrm{O}_{2}$ uptake mediated by the biological community, for example as mediated by cable bacteria, is also potentially of the right magnitude to influence production rates. If these processes are cumulatively large enough to explain the differences between the GPP rates, it suggests that in this environment $\mathrm{NCP}_{\mathrm{O} 2}-\mathrm{R}_{\mathrm{O} 2}$ is not equal to $\mathrm{GPP}_{\mathrm{O} 2}$, but is instead closer to $\mathrm{NPP}_{\mathrm{O} 2}$ (i.e. photoreduction is a very large portion of autotrophic respiration).

Any mechanisms active in the pond should also influence production in the chamber experiments, in which $\mathrm{GPP}_{\mathrm{TO}}$ and $\mathrm{GPP}_{\mathrm{O} 2}$ were found to be equivalent. The chamber experiments were much shorter than a day however (2-3 h), and the chambers could have reduced incident light by enough to make the light dependent mechanisms less favorable, and if ebulliton in the pond is concentrated near Ruppia beds then ebullition could have a very small effect in the chambers compared to the open pond. Furthermore, even with relatively large rates of other oxygen uptake or loss processes, divergence of the rates from each tracer would not be apparent over the experiment period relative to the variability between the sediment chambers. For example, based on maximum observed gas bubble volumes in macroalgae chambers on the order of $5 \mathrm{~mL}$ at the end of the experiments, chamber $\mathrm{GPP}_{\mathrm{O} 2}$ should have been reduced by at most around $0.2 \mathrm{mmol} \mathrm{O}_{2} \mathrm{~m}^{-2} \mathrm{~h}^{-1}$, less than or similar to the uncertainty in most chamber rates. If the difference between GPP rates from each tracer is related to these environmental processes, it is the cumulative difference in their effect over the entire day that explains the diverging observations.

\subsection{Comparison of $\mathrm{O}_{2}$ and DIC derived metabolism rates}

\subsubsection{Unusual DIC to $\mathrm{O}_{2}$ flux ratios during respiration and production}

The DIC derived NCP, R, and GPP can be compared to $\mathrm{O}_{2}$ rates to further evaluate whether the processes proposed above could explain differences in the rates of the oxygen-based tracers, and their relationship to carbon cycling in the pond. In the chamber experiments, mean $\mathrm{NCP}_{\mathrm{DIC}}$ and $\mathrm{R}_{\mathrm{DIC}}$ were in good agreement with $\mathrm{O}_{2}$ rates in the light water chambers, but DIC rates were generally two to three times greater than those of $\mathrm{O}_{2}$ in the sediment chambers; rates in the dark water chamber were too small to allow a valid comparison. Fig. 7 plots the distribution of ratios of $\mathrm{NCP}_{\mathrm{DIC}} / \mathrm{NCP}_{\mathrm{O} 2}\left(\right.$ or $\left.\mathrm{R}_{\mathrm{DIC}} / \mathrm{R}_{\mathrm{O} 2}\right)$ from each experimental chamber. This plot excludes ratios from chambers where either $\mathrm{NCP}_{\mathrm{DIC}}$ or $\mathrm{NCP}_{\mathrm{O} 2}$ was close to zero compared to uncertainties to avoid biasing the results (the uncertainty in the resulting very high or low ratios is much larger than the ratio itself). Ratios from the pond water over the chamber experiment period are also plotted. The maximum expected $\mathrm{R}_{\mathrm{DIC}} / \mathrm{R}_{\mathrm{O} 2}$ for aerobic respiration is 1 -ratios with less DIC uptake than $\mathrm{O}_{2}$ release are expected for oxygenic photosynthesis by algae $\left(\mathrm{GPP}_{\mathrm{DIC}} / \mathrm{GPP}_{\mathrm{O} 2}\right.$ is 0.9 for growth on ammonia; Laws 1991) and Ruppia (0.8; Murray and Wetzel 1987). The water chambers were not significantly different from the expected ratios (at $\alpha=0.05, \mathrm{p}$-values for the plotted subset of chambers reported in Table 4). The dark water chambers (not plotted but summarized in Table 4) had very low respiration rates and only two chambers fit the ratio criteria above (leading to a high ratio that was nonetheless not statistically significantly different from 1); mean $\mathrm{R}_{\mathrm{O} 2}$ and $\mathrm{R}_{\mathrm{DIC}}$ fluxes across dark water chambers were generally in good agreement. In contrast to the agreement between measured ratios and the expected ratio in water chambers, the sediment chambers had ratios of $\mathrm{GPP}_{\mathrm{DIC}} / \mathrm{GPP}_{\mathrm{O} 2}$ and $\mathrm{R}_{\mathrm{DIC}} / \mathrm{R}_{\mathrm{O} 2}$ significantly greater than 1 . Additionally, the $\mathrm{NCP}_{\mathrm{DIC}} / \mathrm{NCP}_{\mathrm{O} 2}$ flux ratio in the pond water column over the same periods $(2.4(1.0))$ is similarly significantly greater than 1 and similar to the mean ratio from the

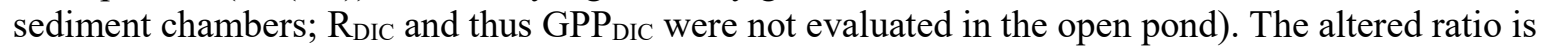
therefore likely related to sediment processes, including benthic biology. 
Table 4: Mean $\mathrm{DIC} / \mathrm{O}_{2}$ flux ratios from chamber experiments

\begin{tabular}{|c|c|c|c|c|c|c|c|c|c|c|}
\hline Chamber type & $\mathrm{GPP}_{\mathrm{DIC}} / \mathrm{GPP}_{\mathrm{O} 2}{ }^{\mathrm{a}}$ & $\mathrm{n}^{\mathrm{b}}$ & $\mathrm{p}$ for $>1: 1$ & $\mathrm{NCP}_{\mathrm{D}}$ & $/ \mathrm{NCP}_{\mathrm{O} 2}$ & $\mathrm{n}^{\mathrm{b}}$ & $\mathrm{p}$ for $>1: 1$ & $\mathrm{R}_{\mathrm{DIC}} / \mathrm{R}_{\mathrm{O} 2}$ & $\mathrm{n}^{\mathrm{b}}$ & $\mathrm{p}$ for $>1: 1$ \\
\hline Macroalgae light & $(0.8)$ & 6 & 0.0007 & 2.7 & $(0.6)$ & 6 & 0.0005 & - & & \\
\hline Macroalgae dark & -- & & & -- & & & & $4.3 \quad(2.3)$ & 5 & 0.0160 \\
\hline Microalgae light & $(1.0)$ & 5 & 0.0294 & 1.2 & $(1.1)$ & 5 & 0.3433 & -- & & \\
\hline Microalgae dark & -- & & & -- & & & & $1.6 \quad(0.6)$ & 9 & 0.0129 \\
\hline Water light & $(0.3)$ & 2 & 0.1364 & 1.1 & $(0.3)$ & 6 & 0.1712 & -- & & \\
\hline Water dark & -- & & & -- & & & & $2.3 \quad(0.4)$ & 2 & 0.0587 \\
\hline Sediment mean & $(0.9)$ & 11 & $9 \times 10^{-5}$ & 2.0 & $(1.1)$ & 11 & 0.0067 & $2.5 \quad(1.9)$ & 14 & 0.0048 \\
\hline Pond water column ${ }^{c}$ & -- & & & 2.4 & $(1.0)$ & 6 & 0.0107 & -- & & \\
\hline
\end{tabular}

a GPP calculated using NCP from individual chambers and mean R from all dark chambers.

${ }^{b}$ Chambers with DIC or $\mathrm{O}_{2}$ flux within uncertainties of zero excluded (see text).

ค $\quad{ }^{\mathrm{s}}$ Water column $\mathrm{R}_{\mathrm{DIC}}$ was not evaluated, this GPP ${ }_{\text {DIC }}$ could not be calculated. 


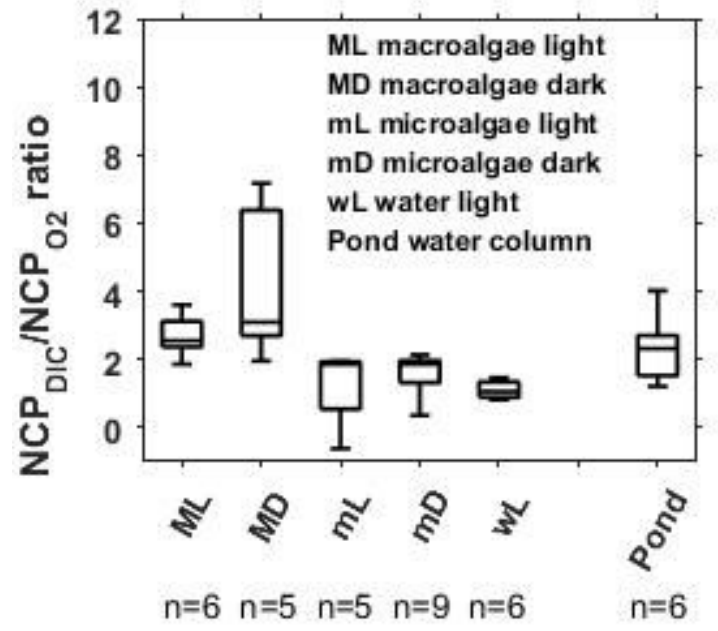

Chamber type

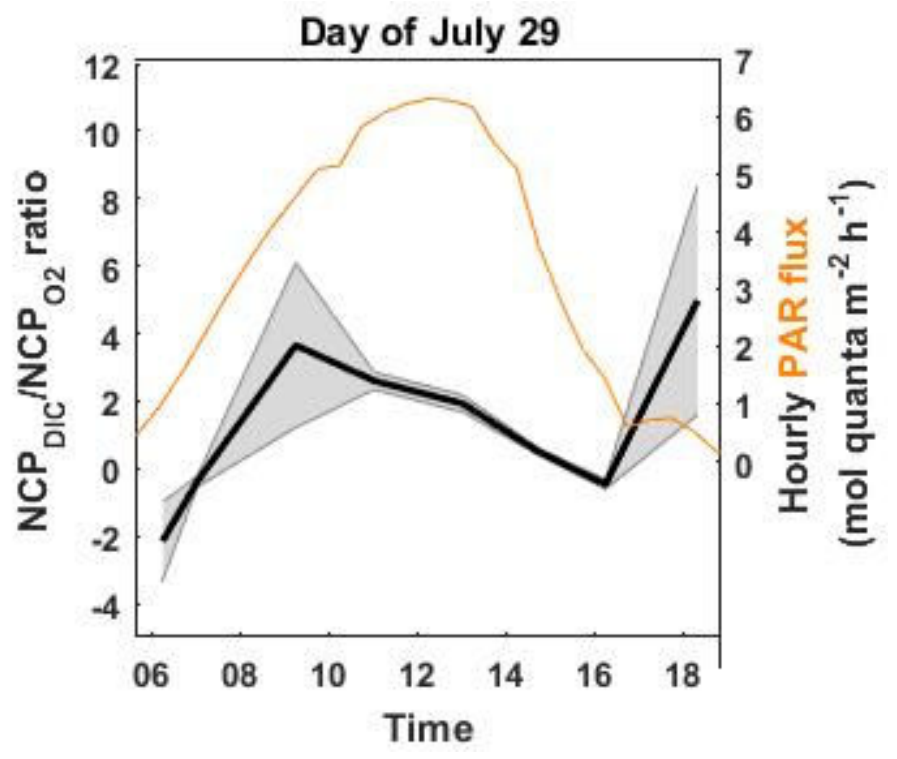

Figure 7: Measured $\mathrm{NCP}_{\mathrm{DIC}} / \mathrm{NCP}_{\mathrm{O} 2}$ ratios in the chamber experiments and open pond timeseries. Left panel: Box plots of $\mathrm{NCP}_{\mathrm{DIC}} / \mathrm{NCP}_{\mathrm{O} 2}$ ratios measured in the light and dark chamber, as well as in the pond over the chamber experiment periods. Right panel: mean flux ratio in the open pond (shaded one standard deviation error bars from a Monte Carlo approach as in Fig. 3). In both panels, ratios derived from rates within uncertainties of zero were excluded (see text). Water dark chambers not plotted because insufficient data for box plot $(\mathrm{n}=2)$ and not significantly different than zero.

Ratios of $\mathrm{R}_{\mathrm{DIC}} / \mathrm{R}_{\mathrm{O} 2}>2$ in benthic environments of the Plum Island estuary system may be readily explained. Similarly high ratios have previously been observed in the nearby Rowley River, and were attributed to release of DIC from anaerobic metabolisms in the sediments (Hopkinson et al. 1999). In that study, the increased efflux of DIC relative to $\mathrm{O}_{2}$ uptake was for the most part balanced by total alkalinity fluxes over annual timescales and there was high variability on monthly timescales of the $\mathrm{R}_{\mathrm{DIC}} / \mathrm{R}_{\mathrm{O} 2}$ ratio and alkalinity fluxes. Reduction-oxidation cycling in the sediments can create a time varying oxygen sink as well, as reduced compounds like iron sulfides build up overnight and are consumed in the day as the sediments become more oxidized. One such anaerobic metabolism that could produce DIC is sulfide oxidation coupled to sulfate reduction. The estimated diffusive sulfate flux to the sediments estimated

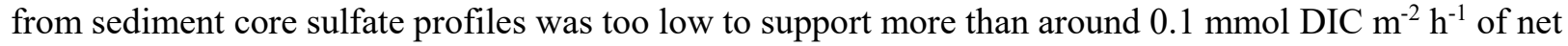
respiration (Amanda Spivak, personal communication); although open water or chamber fluxes may be larger than this calculated diffusive rate because of enhanced transport caused by bioirrigation or other processes, this flux is still an order of magnitude lower than what is needed to fuel enough DIC production to cause the observed $\mathrm{R}_{\mathrm{DIC}} / \mathrm{R}_{\mathrm{O} 2}$.

The microbial consortia associated with sulfur oxidizing cable bacteria provide one possible mechanism for driving sediment redox cycling in a time-dependent manner and enhancing DIC production in the sediments beyond what is expected from diffusive processes alone: Cable bacteria were previously identified as being a potential $\mathrm{O}_{2}$ sink, and they can also stimulate anaerobic metabolism in a manner that leads to high apparent $\mathrm{R}_{\mathrm{DIC}}$ and the coupling of DIC and alkalinity fluxes over longer timescales (Fig. S1). These bacteria engage in what is essentially cryptic sulfur cycling; oxygen is consumed from the sediment-water interface while sulfur is recycled at depth, at substantially higher rates than would be indicated by net sulfate diffusion alone. The sulfate generated is used by the microbial community to 
oxidize organic matter in the sediments and generate additional DIC. Short-term imbalances between the respiration rates of $\mathrm{O}_{2}$ and DIC associated with this process can help explain day versus night differences in oxygen consumption (section 4.2). In addition, respiratory DIC efflux from the cable bacteria microbial consortia may be enhanced by calcium carbonate dissolution over longer timescales in the same manner observed in the Rowley River (Hopkinson et al. 1999) - in a laboratory study of sediment cores with intact cable bacteria associated microbial communities (Rao et al. 2016a) this process led to $\mathrm{R}_{\mathrm{DIC}} / \mathrm{R}_{\mathrm{O} 2}$ ratios greater than 2 . In other words, the apparent $\mathrm{R}_{\mathrm{DIC}} / \mathrm{R}_{\mathrm{O} 2}$ ratio could be inflated by the activity of the cable bacteria microbial consortia by enough to explain the dark sediment chamber results and similar findings in the surrounding system. However, the calcium carbonate content of the sediments at the pond study site were not measured, and may be substantially lower than in the Rao et al. (2016a) study.

The respiratory $\mathrm{DIC} / \mathrm{O}_{2}$ flux ratios are consistent with those reported for the surrounding estuary, and can be explained by the mismatch in timing between release of DIC from anaerobic respiration in the sediments and re-oxidation of reduced metabolic byproducts such as iron and manganese sulfides in the sediments over weekly to seasonal timescales (Hopkinson et al. 1999). However, the photosynthetic flux ratios cannot be explained in the same way; the respiratory mechanisms that enhance DIC release from the sediments would counteract photosynthetic DIC uptake, leading to lower than expected $\mathrm{NCP}_{\mathrm{DIC}} / \mathrm{NCP}_{\mathrm{O} 2}$ and $\mathrm{GPP}_{\mathrm{DIC}} / \mathrm{GPP}_{\mathrm{O} 2}$ ratios. In contrast, this study finds mean $\mathrm{NCP}_{\mathrm{DIC}} / \mathrm{NCP}_{\mathrm{O} 2}$ ratios of 1.2(1.1) in microalgae dominated sediments, 2.7(0.6) in macroalgae dominated sediments, and 2.4(1.0) in the open pond. The NCP and GPP ratios presented here are much higher than the theoretical range of $\mathrm{GPP}_{\mathrm{DIC}} / \mathrm{GPP}_{\mathrm{O} 2}$ of 0.7 to 0.9 (1:1.1 to 1:1.4) for algae (Williams and Robertson 1991, Laws 1991). However, the ratios are more consistent with observations of macroalgae. For example, ratios as high as 2.4 based on similar concurrent measurements of $\mathrm{O}_{2}$ and DIC have been observed in the macroalgae Ulva (Rosenberg et al. 1995), with higher ratios corresponding to conditions during which oxygen is highly supersaturated, as is typical of the study pond at midday within the sunlit experimental chambers. Similarly high GPP ratios in a variety of algae have been previously reported based on radiocarbon incubations, but have subsequently been largely dismissed as artifacts of methodological biases related to that method (Williams and Robertson 1991).

Based on the single day with dawn to dusk DIC data, it is apparent that the $\mathrm{NCP}_{\mathrm{DIC}} / \mathrm{NCP}_{\mathrm{O} 2}$ ratio, while generally elevated over the daytime, is widely variable because of the different diel patterns of DIC and $\mathrm{O}_{2}$ production (Fig. 3, Fig. 7). DIC uptake appears to be phase-shifted earlier in the day compared to $\mathrm{O}_{2}$ production, and $\mathrm{NCP}_{\mathrm{DIC}}$ is actually lower than $\mathrm{NCP}_{\mathrm{O} 2}$ throughout the afternoon. It may be that over a 24 hour cycle $\mathrm{NCP}_{\mathrm{DIC}} / \mathrm{NCP}_{\mathrm{O} 2}$ does not significantly differ from 1 in this pond, though no nighttime DIC data are available in this work to test this hypothesis. The specific timing and timescales of different physiological and chemical carbon uptake and release processes compared to those for oxygen may be important factors explaining the observed ratios. However transient, unusual DIC/O $\mathrm{O}_{2}$ flux ratios for both respiration and production must be invoked to explain the observations.

\subsubsection{Why are the photosynthetic DIC to $\mathrm{O}_{2}$ flux ratios elevated?}

The high observed production ratios require either lower than expected $\mathrm{O}_{2}$ evolution or enhanced DIC uptake during photosynthesis that exceeds any respiratory effect on the ratios over hourly to daily timescales. Some of the processes that I proposed in section 4.1 and 4.2 to explain the difference in chamber and water column GPP (Ruppia production) and GPP $\mathrm{TOI}_{\mathrm{T}}$ versus $\mathrm{GPP}_{\mathrm{O} 2}$ in the open water (lightenhanced mitochondrial respiration, photoreduction, ebullition, and cable bacteria mediated $\mathrm{O}_{2}$ reduction) would also be expected to alter daytime $\mathrm{GPP}_{\mathrm{DIC}} / \mathrm{GPP}_{\mathrm{O} 2}$ and thus $\mathrm{NCP}_{\mathrm{DIC}} / \mathrm{NCP}_{\mathrm{O} 2}$ ratios as well. Expected effects on the measured ratios are summarized in Table 3, and discussed below in order of decreasing probable importance. 
Factors changing the $\mathrm{O}_{2}$ fluxes:

In particular, ebullition and photoreduction should both increase $\mathrm{GPP}_{\mathrm{DIC}} / \mathrm{GPP}_{\mathrm{O} 2}$ in direct proportion to the removal of $\mathrm{O}_{2}$ (which is not captured in $\mathrm{R}_{\mathrm{O} 2}$, and thus not in $\mathrm{GPP}_{\mathrm{O} 2}$. The spatial and temporal decoupling of $\mathrm{O}_{2}$ removal from the water column and DIC efflux from the sediments by sulfate reducing cable bacteria could also affect apparent daytime $\mathrm{GPP}_{\mathrm{DIC}} / \mathrm{GPP}_{\mathrm{O} 2}$ ratios. Over daylight periods with the largest $\mathrm{O}_{2}$ concentrations and thermodynamic drive for this reaction, $\mathrm{O}_{2}$ should be removed from the water column more rapidly than otherwise indicated from $\mathrm{R}_{\mathrm{O} 2}$, which was derived from dark, low $\mathrm{O}_{2}$ chambers; oxygen consuming cable bacteria have growth rates that are an order of magnitude slower under anoxic conditions than near $\mathrm{O}_{2}$ saturation (Vasquez-Cardenas et al. 2015), suggesting a strong dependence on relative $\mathrm{O}_{2}$ availability. Meanwhile DIC release from the sediments is mediated by additional metabolisms and distance beneath the sediment-water interface, including extensive chemolithoautotrophy and DIC uptake in the microbial consortia associated with the cable bacteria activity (Vasquez-Cardenas et al. 2015). Thus over short timescales oxygen may be consumed at higher than expected ratios compared to dark respiration, leading to an apparent decrease in $\mathrm{GPP}_{\mathrm{O} 2}$, and an increase in $\mathrm{GPP}_{\mathrm{DIC}} / \mathrm{GPP}_{\mathrm{O} 2}$. The analysis in section 4.2.2 suggests that $\mathrm{GPP}_{\mathrm{O} 2}$ in the open pond could indeed be missing on the order of 10 to $40 \%$ of total $\mathrm{O}_{2}$ production over a whole day depending on the combination of processes involved - removing $\mathrm{O}_{2}$ from the pond at this rate could reduce the midday $\mathrm{DIC} / \mathrm{O}_{2}$ flux ratio in the pond from 2.4(1.0) to between 1.5 and 2.2 without requiring any additional sinks of DIC.

However, the good agreement between $\mathrm{GPP}_{\mathrm{TOI}}$ and $\mathrm{GPP}_{\mathrm{O} 2}$ both in the chambers and in the pond over the midday experiment periods suggests that these effects cannot explain the full magnitude of the elevated $\mathrm{GPP}_{\mathrm{DIC}} / \mathrm{GPP}_{\mathrm{O} 2}$ compared to theory. $\mathrm{GPP}_{\mathrm{TO}}$ represents total photosynthetic oxygen production, and is largely independent of subsequent oxygen sinks (other than the Mehler reaction), while $\mathrm{GPP}_{\mathrm{O} 2}$ should be lowered by any oxygen sinks not accounted for in $\mathrm{R}_{\mathrm{O} 2}$. The agreement between $\mathrm{GPP}_{\mathrm{TOI}}$ and $\mathrm{GPP}_{\mathrm{O} 2}$ over the periods from which the $\mathrm{GPP}_{\mathrm{DIC}} / \mathrm{GPP}_{\mathrm{O} 2}$ ratio was evaluated indicate that consumption of $\mathrm{O}_{2}$ by alternative respiration processes did not lead to a measurable decrease in $\mathrm{GPP}_{\mathrm{O} 2}$ compared to GPP $\mathrm{TOI}_{\mathrm{T}}$ over the 2-3 $\mathrm{h}$ chamber experiments (as expected given the chamber variability and duration). Yet over this same period GPP $\mathrm{DIC}_{\text {was }} 2$ to 3 times greater than $\mathrm{GPP}_{\mathrm{O} 2}$ or $\mathrm{GPP}_{\mathrm{TO}}$; whatever process was responsible was observable over a short time period and was independent of the oxygen tracer used. This implies that the unusual $\mathrm{GPP}_{\mathrm{DIC}} / \mathrm{GPP}_{\mathrm{O} 2}$ ratios were in large part driven by increased DIC uptake (or slower DIC release), in addition to any extra oxygen sinks.

\section{Factors changing the DIC fluxes:}

I highlight here four processes that could increase DIC uptake in the sunlight relative to dark chambers: Photoautotrophic production by anoxygenic producers, cable bacteria activity enhanced chemolithoautotrophy, a biological stoichiometry of carbon uptake to oxygen evolution that is fundamentally greater than 1 , and differences in calcium carbonate dissolution or precipitation.

The high DIC uptake in the light may be evidence of substantial anoxygenic photosynthesis in the near surface sediments. Both green sulfur bacteria and purple sulfur bacteria were present and active in nearby creek sediments in prior years, and likely in parts of the pond environment as well (Patrick Kearns, personal communication). Such bacteria have been found to inhabit microbial mats that form on surface or shallow sediments in New England salt marshes, growing 5-15 mm below the uppermost oxygenic microalgae such as cyanobacteria and diatoms (Nicholson et al. 1987). These bacteria can oxidize sulfide to various less reduced sulfur species such as thiosulfate, and ultimately to sulfate (Van Niel 1932, Trüper 1964). During photosynthesis, these organisms take up DIC without concurrent changes in $\mathrm{O}_{2}$, effectively increasing $\mathrm{GPP}_{\mathrm{DIC}} / \mathrm{GPP}_{\mathrm{O} 2}$ for the microbial community. The corresponding reduction of sulfate by other sulfur cycling microbes generates $\mathrm{H}_{2} \mathrm{~S}$ which may build up in the dark and reduce overlying iron and manganese compounds; the stoichiometry of DIC, sulfide, and $\mathrm{O}_{2}$ leads to a net $\mathrm{R}_{\mathrm{DIC}} / \mathrm{R}_{\mathrm{O} 2}$ of roughly 2 (Van Niel 1932, Massé et al. 2002). Thus anoxygenic photosynthesis can push the $\mathrm{DIC} / \mathrm{O}_{2}$ flux ratio in 
the right direction in both the light and dark, though one caveat is that rates of photosynthesis by benthic microbes should be most light limited beneath the macroalgal canopy, yet the $\mathrm{DIC} / \mathrm{O}_{2}$ flux ratio is greatest in the macroalgae chambers. In laboratory experiments mimicking conditions in shallow marine sediments (though coarser and thus less light and less diffusion limited than the pond sediments), sulfide oxidation by co-cultures of green and purple sulfur bacteria can exceed $0.8 \mathrm{mmol} \mathrm{m}^{-2} \mathrm{~h}^{-1}$ per hour, or uptake of $1.5 \mathrm{mmol} \mathrm{DIC} \mathrm{m}^{-2} \mathrm{~h}^{-1}$ (Massé et al. 2002). Dark respiration rates in the same experiments were $0.8 \mathrm{mmol} \mathrm{O}_{2} \mathrm{~m}^{-2} \mathrm{~h}^{-1}$. These rates are still not large enough to explain the much greater GPP DIC and $\mathrm{R}_{\text {DIC }}$ than oxygen rates, but DIC uptake associated with anoxygenic photosynthesis may be higher in this system than in the laboratory experiments.

Chemolithoautotrophy in the sediments (perhaps stimulated by cable bacteria activity, which may enhance $\mathrm{O}_{2}$ drawdown in the daytime, as described above) could also enhance daytime DIC uptake and

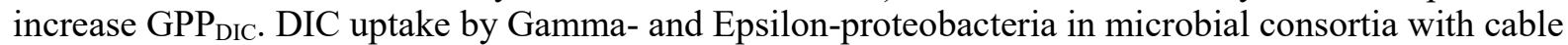
bacteria (supplementary Fig. S1) have a very strong dependence on water column oxygen concentration (and its effects on cable bacteria activity), with DIC uptake by these proteobacteria 20 times slower under anoxic conditions than at $80 \% \mathrm{O}_{2}$ saturation $\left(0.02\right.$ versus $0.4 \mathrm{mmol} \mathrm{DIC} \mathrm{m}^{-2} \mathrm{~h}^{-1}$; Vasquez-Cardenas et al. 2015). Rates are likely stimulated even more as the water column $\mathrm{O}_{2}$ becomes highly supersaturated as in the pond at midday. Even at the relatively low literature rate, these chemolithoautotrophs could increase the $\mathrm{GPP}_{\mathrm{DIC}} / \mathrm{GPP}_{\mathrm{O} 2}$ ratio by $15 \%$ to $50 \%$ compared to the oxygen drawdown by the cable bacteria alone.

Next, a substantially different $\mathrm{DIC} / \mathrm{O}_{2}$ flux stoichiometry for production would explain the ratios. For example the same pond conditions that are ideal for promoting the Mehler reaction (high irradiance, high DIC, and high $\mathrm{O}_{2}$ ) are associated with unusually high $\mathrm{GPP}_{\mathrm{DIC}} / \mathrm{GPP}_{\mathrm{O} 2}$ in macroalgae (Rosenberg et al. 1995). However, elevated ratios were observed in microalgae chambers too; there is no clear evidence of such high ratios in phytoplankton or benthic algae. To the contrary, abundant theoretical and observational evidence suggests that $\mathrm{GPP}_{\mathrm{DIC}} / \mathrm{GPP}_{\mathrm{O} 2}$ is generally 1 or less in these organisms. Transient biosynthesis of molecules with particularly high oxygen requirements (and thus lower net evolution of $\mathrm{O}_{2}$ ) might be expected to peak near midday and change the $\mathrm{DIC} / \mathrm{O}_{2}$ flux ratio over the experiment period. For example micro- and macroalgae produce mycosporine-like amino acids for UV and oxidative stress protection in high-light environments (Karsten et al. 1998, Llewellyn and Airs 2010). This explanation is pleasing because it could explain the similar $\mathrm{DIC} / \mathrm{O}_{2}$ flux ratios during both production and respiration as fundamentally related to the biochemical composition of organic matter being produced and respired in response to environmental changes. However, the resulting $\mathrm{DIC} / \mathrm{O}_{2}$ ratios for production or respiration of most such compounds are smaller than 1 (Hedges et al. 2002).

Finally, calcium carbonate precipitation is known to occur in microbial mats and is particularly influenced by the activity of sulfate reducing bacteria (Dupraz et al. 2008), and it has been proposed that this process may occur in salt marshes as well (Stephens et al. 2008). While there are many studies focused on the factors driving carbonate precipitation in microbial mats, the few published rates suggest this process can account for a DIC removal flux on the order of $0.01 \mathrm{mmol} \mathrm{DIC} \mathrm{m}^{-2} \mathrm{~h}^{-1}$ (Coleman and Raiswell 1993, Ludwig et al. 2005). While carbonate saturation state is substantially higher in lit microbial mats than in the dark, there is no apparent change in calcification rate between the light and dark (Ludwig et al. 2005). Thus, I do not expect DIC uptake by carbonate precipitation to substantially influence the observed $\mathrm{GPP}_{\mathrm{DIC}} / \mathrm{GPP}_{\mathrm{O} 2}$.

\section{Summary:}

A number of processes can alter $\mathrm{DIC} / \mathrm{O}_{2}$ production fluxes, but separately most could not explain differences over the midday, peak-production periods in the chamber experiments for both the chambers and open water given the good agreement of $\mathrm{GPP}_{\mathrm{TOI}}$ (aerobic respiration independent) and $\mathrm{GPP}_{\mathrm{O} 2}$ (potentially biased if respiration processes were missed). Oxygen consumption by light-enhanced mitochondrial respiration, photoreduction, and an active community of cable bacteria are consistent with the higher $\mathrm{GPP}_{\mathrm{TO}}$ than $\mathrm{GPP}_{\mathrm{O} 2}$ rates in the open water, but cannot explain the $\mathrm{DIC} / \mathrm{O}_{2}$ production ratios 
without the help of additional processes that affect the DIC fluxes. Three additional processes could help explain the constellation of rates: Extensive anoxygenic photosynthesis, chemolithoautotrophic DIC uptake associated with cable bacteria activity, and/or biochemically altered production ratios over this period. Macroalgae have previously been observed to have elevated $\mathrm{GPP}_{\mathrm{DIC}} / \mathrm{GPP}_{\mathrm{O} 2}$ during high light periods, and the largest discrepancies observed in this study were in the chambers over macroalgae dominated sediments. However, high ratios were also observed in the microalgae chambers and there is no observational or biochemical basis for this behavior in the literature. Anoxygenic photosynthesis or chemolithoautotrophy could both lead to $\mathrm{GPP}_{\mathrm{DIC}} / \mathrm{GPP}_{\mathrm{O} 2}$ flux ratios similar to those observed in this study, and complement the oxygen removal effect of cable bacteria with which they are often found in microbial consortia. These processes are not as well supported by the literature as the mechanisms identified to explain the $\mathrm{R}_{\mathrm{DIC}} / \mathrm{R}_{\mathrm{O} 2}$ ratios, and none of the above processes in isolation are sufficient to explain all of the observations. However, some combination of both enhanced oxygen consumption in the light or oxygen enhanced photosynthetic DIC uptake could explain both the observed $\mathrm{GPP}_{\mathrm{DIC}} / \mathrm{GPP}_{\mathrm{O} 2}$ and $\mathrm{GPP} \mathrm{TOI}_{\mathrm{T}} / \mathrm{GPP}_{\mathrm{O} 2}$ ratios. Indeed, the relatively good agreement of the triple oxygen isotope and $\mathrm{O}_{2}$ concentration based methods provides confidence that additional DIC drawdown must be occurring in the light, which points to the strong possibility of chemoautotrophy or anoxygenic photosynthesis being large and important metabolisms in this environment.

\subsection{Metabolism over daily to seasonal timescales}

Production and respiration were evaluated over a range of timescales in this study, from sub-daily to seasonal periods. Environmental factors including light, temperature, macrophyte abundance, tidal stage, and likely nutrient supply changed over the same timescales. In this section I examine the variation of production with changing environmental conditions. I additionally compare the ratios of NCP/GPP, an indicator of community metabolic efficiency, using all three tracers and examine how they change over different timescales. Finally I discuss my results with regards to ecosystem carbon cycling and the broader salt marsh context.

\subsubsection{Linking environmental variability and changes in metabolism}

Previous analysis of $\mathrm{O}_{2}$ and primary producer dynamics in this and two other ponds (Spivak et al. submitted) found that macroalgae and Ruppia abundance were important drivers of GPP differences between the ponds, but that within the study pond that is the site of the work presented in this manuscript changes in macroalgae and plant abundance were not strong predictors of changes in metabolism. In the same study, metabolic rates in this pond were lower during tidal exchange with the surrounding tidal creeks, but not other ponds. These periods are identifiable in the $\mathrm{O}_{2}$ based metabolic rates (Fig. 5) as relatively abrupt drops in the magnitude of $\mathrm{GPP}_{\mathrm{O} 2}$ and $\mathrm{R}_{\mathrm{O} 2}$ over periods with more connecting tides; while these differences may signify important changes in community metabolism, they may also reflect differences in the water mass history of the inflowing creek water compared to the previously isolated pond water that are not accounted for in the simple, isolated-system $\mathrm{O}_{2}$ mass balance. Respiration is known to be temperature dependent (e.g. Hancke and Glud 2004) and thus may be a primary driver of $\mathrm{R}_{\mathrm{O} 2}$ variability. Primary production is limited by light and nutrients in most marine settings, and nitrate in the pond is drawn down below detection limits suggesting that the system is nutrient limited.

Fig. 8 visualizes the correlations of open pond $\mathrm{GPP}_{\mathrm{TO}}, \mathrm{GPP}_{\mathrm{O} 2}$, and $\mathrm{R}_{\mathrm{O} 2}$ with respect to mean daily temperature and daytime PAR in terms of the changes $(\Delta)$ of each parameter compared to the mean value across the time-series (i.e. $\Delta$ is the centered rate or variable). Periods of tidal flooding of the marsh platform (water level $>1.46 \mathrm{~m}$ above NAVD88 baseline) are indicated as gray shaded patches - these periods are usually no more than a few hours in duration, and are clustered around the biweekly spring tides. Aside from reductions in $\mathrm{GPP}_{\mathrm{O} 2}$ and $\mathrm{R}_{\mathrm{O} 2}$ associated with some, but not all, of the flooding periods, both rates have similar high frequency variation that appears to track changes in PAR, with longer period variation that tracks changes in temperature. Significant pairwise correlations are found between $\triangle \mathrm{GPP}_{\mathrm{O} 2}$ and $\Delta \mathrm{R}_{\mathrm{O} 2}, \Delta$ temperature, and $\triangle \mathrm{PAR}\left(\mathrm{p}=4 \times 10^{-59}, 6 \times 10^{-7}\right.$, and $\left.2 \times 10^{-20}\right)$ and between $\Delta \mathrm{R}_{\mathrm{O} 2}$ and $\Delta \mathrm{PAR}$ 
$\left(\mathrm{p}=4 \times 10^{-12}\right.$ ), but not between $\Delta \mathrm{R}_{\mathrm{O} 2}$ and $\Delta$ temperature (at $\left.\alpha=0.05 ; \mathrm{p}=0.0054\right)$. Identical results are obtained if the measured rates and values are compared.

$\mathrm{GPP}_{\mathrm{O} 2}$ and $\mathrm{R}_{\mathrm{O} 2}$ are tightly coupled because the latter is used in the calculation of the former (Eq. 3), but the additional significant correlation of $\mathrm{R}_{\mathrm{O} 2}$ and $\mathrm{PAR}$, which was not used in the calculation of $\mathrm{GPP}_{\mathrm{O} 2}$, indicates that respiration is likely strongly coupled to the GPP rate in the environment, and not simply for methodological reasons. In other words, nighttime respiration seems to be tightly coupled to the production of organic matter over the preceding day, with little secondary influence of temperature on oxygen consumption rates. While $\mathrm{GPP}_{\mathrm{O} 2}$ and $\mathrm{R}_{\mathrm{O} 2}$ do seem to drop with tidal flooding after periods of tidal isolation, there is no significant pairwise correlation between tidal stage and increasing or decreasing metabolic rates. Many of these periods are associated with decreases in incident PAR that are unrelated to the tidal connectivity, and the apparent differences in community metabolism with tidal state may be coincidental. This stands in contrast to the important role of tidal flooding of the marsh platform to metabolism signals within tidal creeks (Nidzieko et al. 2014, Chapter 3).

A multiple linear regression of $\mathrm{GPP}_{\mathrm{O} 2}$ on $\mathrm{PAR}$ flux , temperature, and a proxy for tidal state (flooding does or does not occur on a given day) explains $62 \%$ of the total variance in $\mathrm{GPP}_{\mathrm{O} 2}\left(\mathrm{~F}=89, \mathrm{p}=7 \times 10^{-34}\right.$ compared to a constant model); PAR and temperature without tidal state explains $50 \%$ of the total variance in $\mathrm{GPP}_{\mathrm{O} 2}\left(\mathrm{~F}=82, \mathrm{p}=3 \times 10^{-25}\right)$ compared to a constant model), and $\mathrm{PAR}$ alone explains $41 \%$ of the variance $\left(\mathrm{F}=114, \mathrm{p}=2 \times 10^{-20}\right)$. Thus PAR seems to be the dominant measured driver of productivity in this system, with temperature and tidal connectivity playing secondary roles. These strong correlations may in part be caused by common underlying seasonal changes in production, light, and temperature across the timeseries. I do not correct for autocorrelation in this analysis, which could lead to quantitatively different percentages of variance explained by each factor, but qualitatively PAR flux would still be the dominant driver compared to temperature and tidal state.

While I cannot evaluate nutrient supply from the dataset presented here, the tidal creeks in this system usually deliver a pulse of nutrients to the marsh platform during the spring and early summer, but this excess is generally gone by mid- to late-June (Anne Giblin, personal communication). Later in the summer the creeks have low (micromolar) concentrations of nitrate (Spivak et al. submitted). Applying the above multiple linear regression model to only data after June 30 does not substantially change the percent of variance explained $\left(64 \%, \mathrm{~F}=65.3, \mathrm{p}=5 \times 10^{-24}\right)$. Thus it seems reasonable to assume that pond productivity is not benefiting from greatly enhanced nutrient supply during tidal flooding even during the early portion of the time series.

Seasonal change in pond metabolism is small compared to daily variability in production and respiration. Both $\mathrm{GPP}_{\mathrm{O} 2}$ (and $\mathrm{GPP}_{\mathrm{TOI}}$ ) and $\mathrm{R}_{\mathrm{O} 2}$ decrease in magnitude in October, but the tight coupling between the two rates leads to little change in the daily $\mathrm{NCP}_{\mathrm{O} 2}$ (Fig. 6). $\mathrm{GPP}_{\mathrm{TO}}$ evidences similar relationships with environmental parameters as $\mathrm{GPP}_{\mathrm{O} 2}$ (Fig. 8), but decreases relatively more in the fall, leading the two GPP rates to be in closer agreement. This is consistent with the expected decrease in photoreduction and other light dependent oxygen removal fluxes as PAR drops off in the fall-in the summer photoreduction could be an important sink of $\mathrm{O}_{2}$ not accounted for in $\mathrm{GPP}_{\mathrm{O} 2}$, leading that rate to be biased low relative to $\mathrm{GPP}_{\mathrm{TOI}}$, but as photoreduction decreases $\mathrm{GPP}_{\mathrm{O} 2}$ would be less depressed compared to $\mathrm{GPP}_{\mathrm{TOI}}$. 


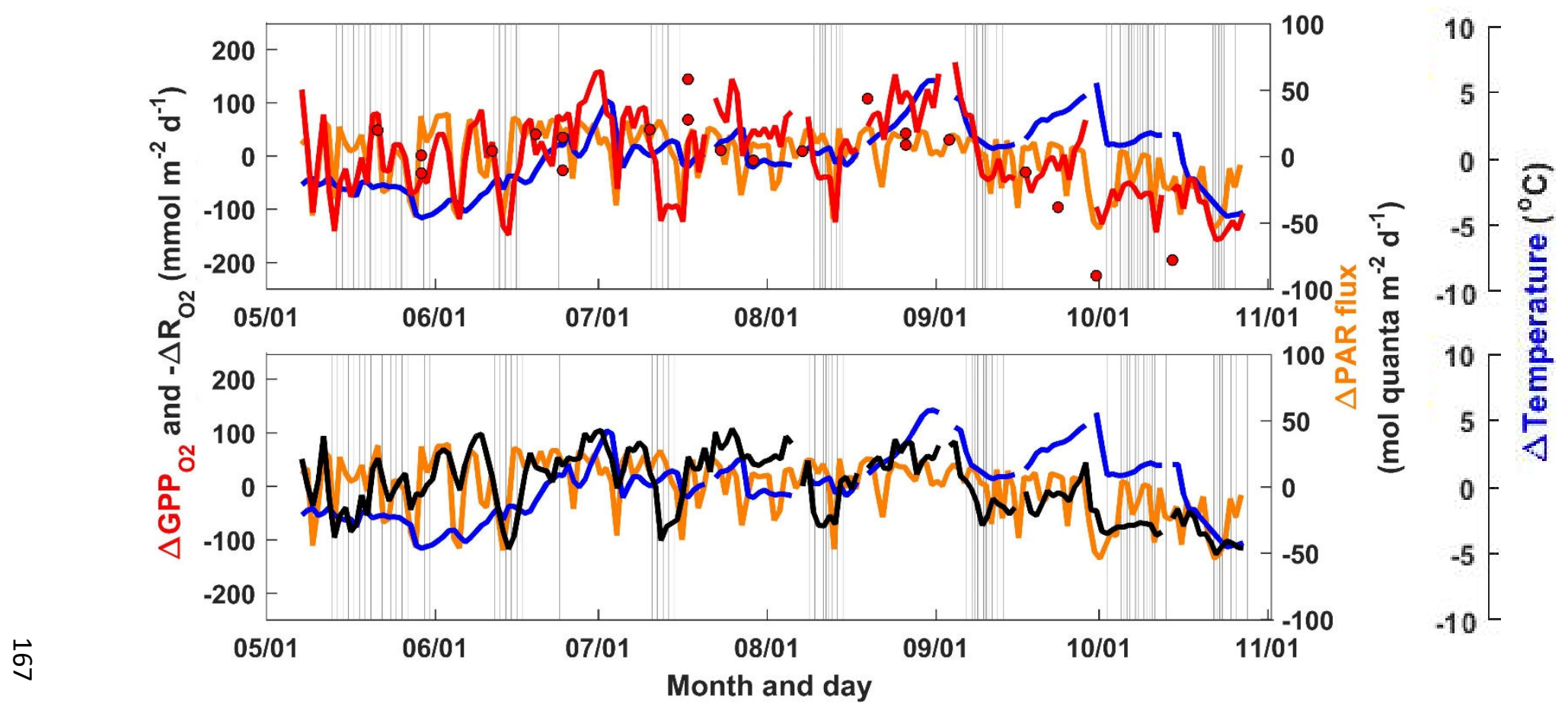

Figure 8: Change $(\Delta)$ relative to the mean values over the timeseries of $\mathrm{GPP}_{\mathrm{O} 2}$ (red line), $\mathrm{GPP}_{\mathrm{TOI}}$ (red circles), and $\mathrm{R}_{\mathrm{O} 2}$ (black), PAR flux (orange), and temperature (blue). Periods of tidal connectivity when the pond and adjacent marsh platform are flooded by tidal creeks are shaded in gray. 


\subsubsection{The net to gross production ratio and community metabolic efficiency}

The ratio of NCP/GPP can be interpreted as an index of how efficient the community is at building biomass or exporting (to sediments or outside the ecosystem boundary) organic matter produced by photosynthesis compared to respiratory requirements. A ratio near one indicates that over a given timespan virtually all production is conserved and used for growth or available for export. A ratio of zero indicates that all the production has been consumed, with no net accumulation or loss of carbon. Thus a ratio between 0 and 1 means that the ecosystem is accumulating carbon, while a ratio below zero indicates that the system is consuming more organic matter than has been produced in situ, for example by respiring buried organic matter in the pond sediments. Ratios higher than one mean that organic matter accumulation is in excess of that explained by local photosynthesis. Ratios greater than one can also be transient results of a dynamic system where photosynthesis and respiration processes are decoupled in time or space (i.e. the rates are not integrated over appropriate time or space scales) or organic matter is imported from a neighboring environment.

The NCP/GPP ratios for $\mathrm{O}_{2}$ and DIC in the chamber experiments are plotted in Fig. 9. Midday NCP/GPP ratios are generally between 0 and 1 . In pelagic ocean systems, a ratio of 0.15 is typical, so the ratios here are actually quite high and indicate that a large fraction of the organic matter produced over the chamber experiments is not respired over the midday peak production period. In general the chamber $\mathrm{NCP}_{\mathrm{O} 2} / \mathrm{GPP}_{\mathrm{TO}}$ ratios have a larger spread and positive skew than the other methods; the highest $\mathrm{NCP}_{\mathrm{O} 2} / \mathrm{GPP}_{\mathrm{TOI}}$ ratios likely do not have ecological significance, but are instead related to large uncertainties paired with small rates.

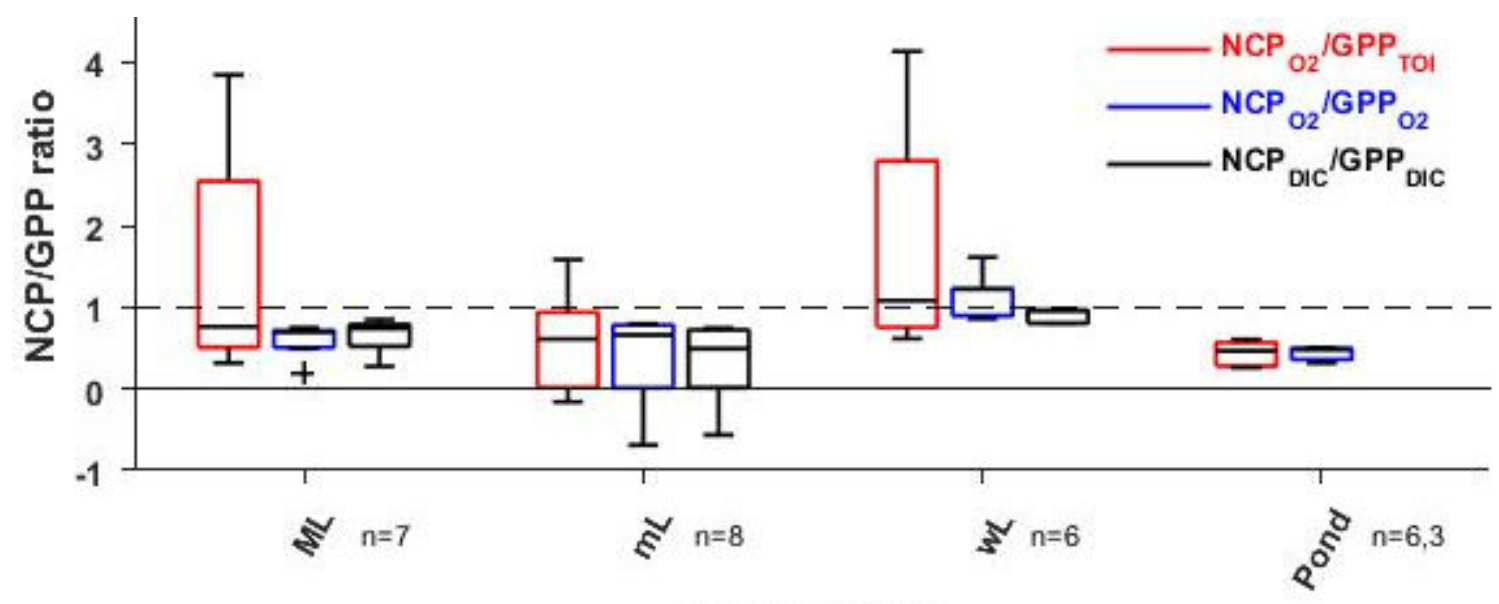

Chamber type

Figure 9: Box plots of NCP/GPP ratios from the chamber experiments during the daytime peak production period, as well as the pond over the experiment periods. Bars are 1.5 times the interquartile range, and outliers are marked as crosses. Chamber types are denoted as in Figure 7: ML indicates macroalgae light, $\mathrm{mL}$ microalgae light, and $\mathrm{wL}$ water light chambers.

Light water chamber NCP/GPP ratios are generally near one, consistent with the very small respiration rates in the water compared to sediments. Macroalgae chambers have some respiratory losses, but are still storing most of the produced organic matter over the midday period. Microalgae chambers cover a wider range of values; while these sediment systems were in general storing organic matter, respiration in two chambers exceeded photosynthetic production and in net consumed sediment organic matter even over the midday period of peak production. Pond NCP/GPP over the same periods was relatively well constrained with NCP/GPP equal to $0.4(0.1)$, similar to the mean for microalgae dominated sediments, 0.4(0.5). Overall, the NCP/GPP ratios point to photosynthesis outpacing respiration during peak production hours, leading to the accumulation of organic carbon. 
This inferred storage of organic matter can be compared to metabolic cycling over the rest of the daylight period (Fig. 10, upper panel), and over a full 24 hours (lower panel). Uncertainties are calculated through propagation of errors in quadrature for each rate. $\mathrm{NCP}_{\mathrm{DIC}} / \mathrm{GPP}_{\mathrm{DIC}}$ was not evaluated over the open pond timeseries, but the NCP/GPP ratios from the triple oxygen isotopes and $\mathrm{O}_{2}$ rates are in good agreement: Over the daytime, production and respiration are balanced, with any excess production from midday consumed over the rest of the daylight hours; over a full 24 hour day, NCP/GPP is negative, meaning that respiration exceeds daytime production. A fall trend towards even more negative NCP/GPP ratios may begin in September, which is consistent with the relatively constant NCP even as daytime production decreases (Fig. 4). The triple oxygen isotope and $\mathrm{O}_{2}$ based NCP/GPP ratios indicate that over the timeseries, daily respiration on average consumed $24(17) \%$ and $45(41) \%$ more $\mathrm{O}_{2}$ and organic matter than in situ photosynthesis produced. Therefore the pond biological community must be consuming previously stored organic matter in the sediments, such as peat underlying the pond or exposed at the pond banks, or is subsidized by allochthonous organic matter from the surrounding marsh. Daytime production appears to be largely superfluous to baseline respiration, as the integrated photosynthesis is tightly coupled to daytime consumption.
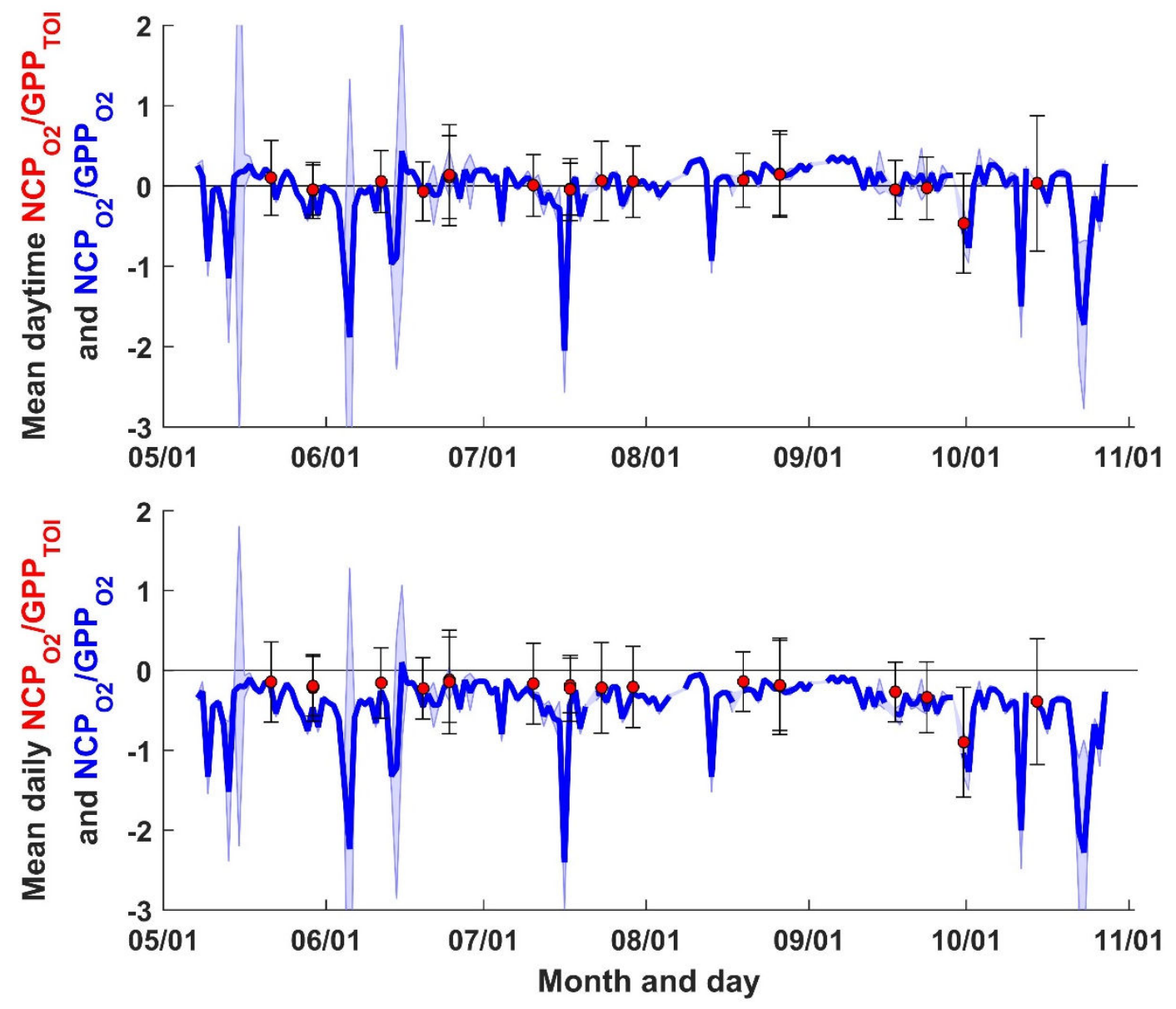

Figure 10: $\mathrm{NCP}_{\mathrm{O} 2} / \mathrm{GPP}_{\mathrm{TOI}}$ (red circles and one standard deviation error bars) and $\mathrm{NCP}_{\mathrm{O} 2} / \mathrm{GPP}_{\mathrm{O} 2}$ (blue line with shaded error bars) over the open pond timeseries. Top panel: Daytime NCP/GPP, with mean $\mathrm{NCP}_{\mathrm{O} 2}$ between dawn and dusk. Bottom panel: Daily NCP/GPP with mean $\mathrm{NCP}_{\mathrm{O} 2}$ from dawn to following dawn, including day and night. Uncertainties in the ratios are estimated using propagation of errors for each rate in quadrature. 


\subsubsection{Metabolism of the study pond in the context of other salt marsh ponds}

The net organic matter consumption inferred from the NCP/GPP ratios over the entire time-series is consistent with other findings in this and other salt marsh ponds that at least some ponds are sites of net organic matter consumption (Johnston et al. 2003, Spivak et al. submitted), as opposed to large sinks of atmospheric $\mathrm{CO}_{2}$ like the grass covered marsh platform (Forbrich and Giblin 2015). It has been hypothesized that one mechanism for pond expansion or collapse of marsh flanks into mudflats is the decomposition of the underlying or surrounding peat layers (Johnston et al. 2003, Deegan et al. 2012); in this system, net heterotrophy greatly exceeds photosynthesis over the study period and pond expansion via this mechanism seems likely.

Rates of production for this pond using a different processing approach to the $\mathrm{O}_{2}$ data were reported in Spivak et al. (submitted); in that study, daytime $\mathrm{GPP}_{\mathrm{O} 2}$ was estimated in a model framework by regressing $\mathrm{O}_{2}$ against gas exchange parameters and $\mathrm{PAR}$ in order to predict metabolism rates that minimized misfits using ordinary least squares (Winslow et al. 2016). Other than relatively small differences due to choices in data processing and gas exchange parameterizations, this approach results in similar $\mathrm{NCP}_{\mathrm{O} 2}$ (within one standard error) to the rates presented in this manuscript because identical mass balances and data are used. The modeled results for GPP are in good agreement with GPP ${ }_{\mathrm{TO}}$ in this study and the Spivak et al. modeled $\mathrm{R}$ is in good agreement with the inferred respiration rate from this study based on including light-dependent oxygen sinks from the difference of $\mathrm{GPP}_{\mathrm{TOI}}-\mathrm{NCP}_{\mathrm{O} 2}$. In other words, the model approach used by Spivak et al. (described in Winslow et al. 2016) correctly infers the magnitude of day versus night differences in oxygen sinks. $\mathrm{NCP}_{\mathrm{O} 2}$ and $\mathrm{R}_{\mathrm{O} 2}$ rates from both the work presented here and the Spivak et al. (submitted) study are of similar magnitude but generally larger in magnitude than those reported from a prior study in three smaller ponds in the same salt marsh using in situ $\mathrm{O}_{2}$ fluxes and benthic core incubations (Johnston et al. 2003; in that study mean daily $\mathrm{NCP}_{\mathrm{O} 2}-1.5$ to $0.5 \mathrm{mmol} \mathrm{O}_{2} \mathrm{~m}^{-2} \mathrm{~h}^{-1}, \mathrm{R}_{\mathrm{O} 2}-4$ to -7 mmol O $\left.\mathrm{m}^{-2} \mathrm{~h}^{-1}\right)$.

The work by Spivak et al. (submitted) additionally used data from two smaller marshtop ponds to highlight the importance of macrophyte producers in determining pond metabolic balance; the pond with the highest Ruppia coverage ( $80 \%$ ) had autotrophic $\mathrm{NCP}_{\mathrm{O} 2}$ suggesting net organic matter accumulation. The comparison of chamber experiments and the open pond rate in this work also pointed to the potential outsize importance of Ruppia compared to other producers in explaining the study pond's production rates. Plant community dynamics are likely an important driver of salt marsh pond community metabolism and pond development. While the large pond in this study is more exposed to wind driven waves and shear than the smaller nearby ponds with greater macrophyte coverage, Ruppia is relatively resilient against drag (Veiderpass and Anders 2012) and nighttime $\mathrm{O}_{2}$ concentrations over the timeseries did not approach anoxia as in the other ponds; it may be that Ruppia will spread in this pond and change the metabolic trajectory of the study pond, if deepening of the pond does not exclude these macrophytes (Hartig et al. 2002).

Even the most productive rates reported in Spivak et al. (submitted) were far less than carbon accumulation by the grass covered marsh platform (mean daily NCP rates of tens of mmol $\mathrm{C} \mathrm{m}^{-2} \mathrm{~h}^{-1}$ ) and were coupled with high rates of respiration. Using a $\mathrm{DIC} / \mathrm{O}_{2}$ flux ratio of 1 to compare to carbon respiration rates by salt marsh grasses, that study concluded that pond respiration was an important component of overall marsh respiration, and neglecting to account for ponds could underestimate marsh respiration by roughly 5 to $20 \%$. Based on the $\mathrm{DIC} / \mathrm{O}_{2}$ flux ratios reported in this work, it seems likely that the $\mathrm{DIC} / \mathrm{O}_{2}$ ratio of respiration in the pond is greater than 1 , and potentially the ratio during production as well. If so, respiration in the ponds may be even more important to total marsh respiration. 


\section{Conclusions}

Dissolved oxygen isotope ratios, oxygen concentration, and dissolved inorganic carbon concentrations were used to study metabolic rates and relationships in a salt marsh pond over hourly to seasonal timescales. Experimental approaches included benthic chamber studies and open water monitoring. The results presented in this work highlight the heterogeneous nature of production within the pond, as well as the potential importance in shallow, sunlit marine systems of light-enhanced oxygen consumption (e.g. respiration) and greater DIC uptake based primary production than expected from oxygen alone. In particular, the combination of simultaneous measurements of multiple tracers of metabolism point to the point to the potential importance of anoxygenic photosynthesis and chemolithoautotrophy as major metabolic pathways in this salt marsh pond.

I found that differences in NCP between the chamber experiments over microalgae and macroalgae dominated sediments and NCP observed in the open water over identical periods could be explained by enhanced production in areas with the seagrass Ruppia maritima, reinforcing the idea that plant abundance is an important driver of pond metabolism.

Further, I found that the smaller value of $\mathrm{GPP}_{\mathrm{O} 2}$, derived from $\mathrm{O}_{2}$ fluxes and the assumption that daytime and nighttime respiration rates were equal, compared to $\mathrm{GPP}_{\mathrm{TOI}}$ derived from oxygen isotope ratios points to the potentially high rates and important role of light-dependent oxygen sinks such as light-enhanced mitochondrial respiration and photoreduction. Unusual $\mathrm{DIC} / \mathrm{O}_{2}$ flux ratios were observed during both respiration and production; the ratio of respiration fluxes was similar to literature values and could be explained by sediment metabolisms, but the ratio during production was unexpected and could not be explained solely by the likely rates of light-enhanced $\mathrm{O}_{2}$ consumption; an additional sink of DIC in the light must occur. Anoxygenic photosynthesis and the enhanced chemolithoautotrophic DIC uptake could both be significant DIC sinks that lead to greater gross primary production than is indicated by the oxygen tracers, and need to be further evaluated in marsh pond settings.

While production outpaced respiration near midday, overall daytime production and respiration were closely coupled, indicating that nearly all daytime production was consumed or balanced by consumption of equivalent amounts of organic matter between dawn and dusk. Over a 24 hour cycle net heterotrophy is explained by a near-constant background respiration rate that must be subsidized by organic matter that was previously buried (e.g. in peat) or from the marsh platform. This steady background respiration leads to relatively constant pond NCP over daily to seasonal timescales even when GPP decreases in the fall.

Finally, the results of this study are in generally good agreement with other studies of salt marsh ponds. The good agreement of triple oxygen isotope derived GPP rates in this study with GPP rates calculated using $\mathrm{O}_{2}$ concentrations in a statistically informed model suggests that such model approaches adequately compensate for additional daytime sinks of $\mathrm{O}_{2}$ compared to nighttime respiration; however, the triple oxygen isotope system provided additional information to discriminate between the various light- or concentration-enhanced oxygen uptake mechanisms. Furthermore, the unusual ratios of carbon to oxygen during respiration and production indicate that pond metabolism may be a more important component of whole marsh metabolism than expected, particularly with regard to respiration. 


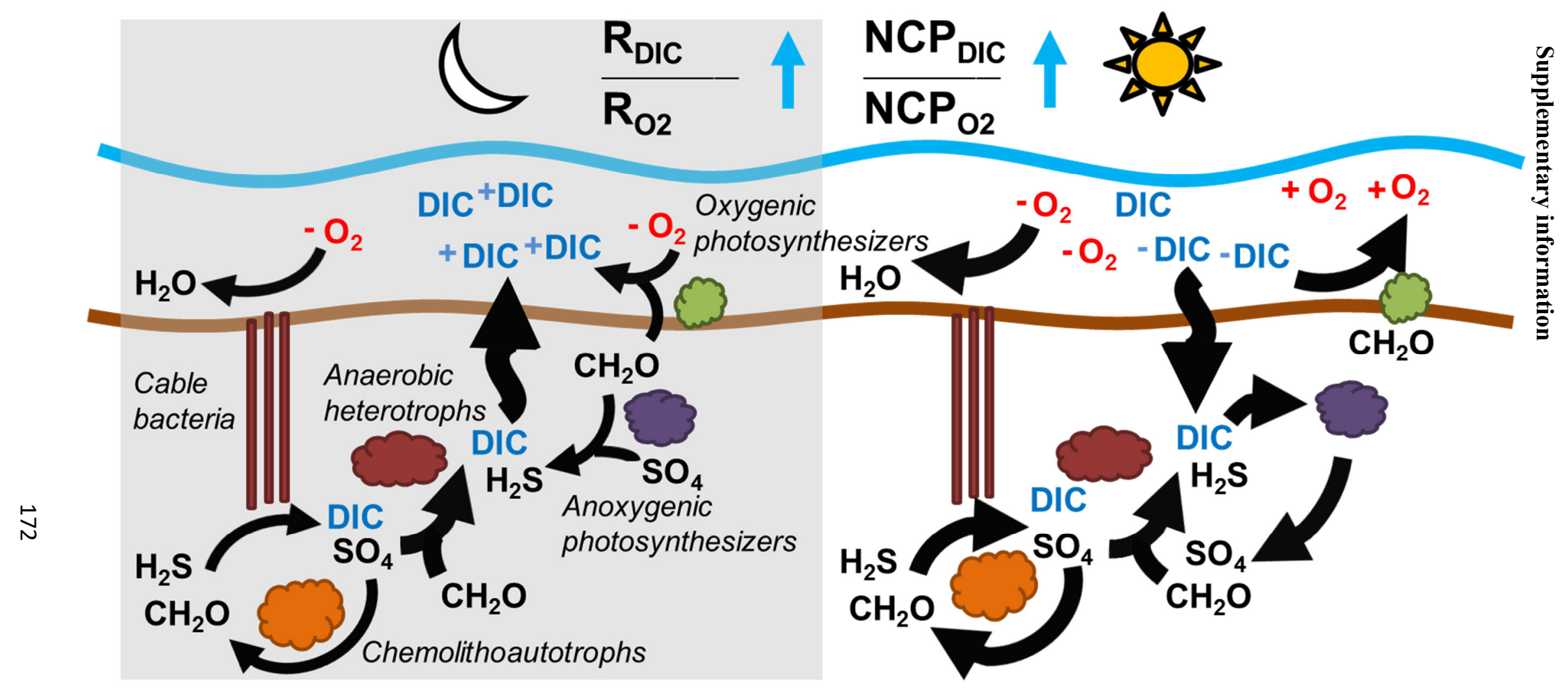

Figure S1: One possible mechanism by which microbial community structure can be affected by differences in oxygen concentrations and light in a manner that leads to day versus night differences in $\mathrm{DIC} / \mathrm{O}_{2}$ flux ratios. In this diagram, a microbial community structure including normal photosynthetic algae and heterotrophic microbes also includes a sulfide oxidizing cable bacteria and chemolithoautotrophic bacteria consortia, as well as anoxygenic photosynthesizers. Such a community could contribute to reduction and oxidation of sediment compounds in a way that decouples water column $\mathrm{O}_{2}$ and DIC from the expected stoichiometry of oxygenic photosynthesis and aerobic respiration. At night, more DIC is released than expected from aerobic respiration alone. In the day, both $\mathrm{O}_{2}$ and DIC are consumed more rapidly than expected based on normal oxygenic photosynthesis and aerobic respiration alone, increasing the DIC uptake to $\mathrm{O}_{2}$ release flux ratio. Intermediate chemical oxidants and reductants and associated processes (e.g. iron oxides, pyrite) linking sulfide and oxygen consumption are not shown. 


\section{Chapter 6--Conclusion}

\section{Summary of thesis chapters}

In the four data analysis chapters of this thesis, I applied oceanographic methods based on dissolved gas tracers of physical and biological processes to novel marine environments in which these methods previously had been rarely if ever used before (e.g. the equatorial ocean, salt marshes), and in which they can provide additional information compared to traditional metabolic tracers. I developed new ways of analyzing and processing the tracer data in order to explore physically, chemically, and biologically complicated environments, and explored addition physical and biological processes that both complicate and inform the interpretation of metabolism fluxes in these environments. I used these tools and approaches to evaluate relationships between biological communities and ecosystem scale metabolism and biogeochemical cycling. Specific scientific contributions and findings of my thesis are summarized below:

\section{Chapter 2-ocean setting}

Research question:

How are metabolism, carbon export, and community structure related in the surface South Atlantic Ocean?

Chapter 2 (under revision for Global Biogeochemical Cycles) evaluated surface ocean mixed layer productivity across 55 degrees of Latitude of the South and Equatorial Atlantic Ocean. I used triple oxygen isotopes and concurrently measured oxygen to argon ratios (a measure of net community production) in order to measure community metabolism across an ocean basin in which production and respiration have previously been evaluated solely by bottle incubation techniques. I further developed modified calculation schemes for both tracers that account for entrainment and diapycnal mixing so that surface metabolism could be evaluated in regions with strong vertical tracer gradients such as equatorial convergences, frontal systems, and the mesohaline Amazon River Plume: oxygen isotope data from some such regions previously could not be readily interpreted using standard production calculations (particularly equatorial regions of the global ocean). With my co-authors for this work, I compared surface metabolism and carbon export to depth across the ocean transect. We also used observations of pico-, nano-, and microphytoplankton to evaluate community structure and diversity, and assessed the relationships of these characteristics with production, particle export, and export efficiency; in general, there were no strong relationships between community structure and metabolism or export at the spatial scales sampled. In this chapter, I found that biological production was similar over thousands of kilometers, but variable on the mesoscale (50 to $100 \mathrm{~km}$ ), and that production, nutrient and chlorophyll concentrations, and community diversity may share underlying drivers of variability. I also found that particulate organic carbon export flux was correlated with gross primary production, but not net community production, which is unexpected compared to results from subpolar gyres but may indicate the importance of carbon export by small cells in a subtropical gyre dominated by cyanobacteria.

\section{Chapter 3-metabolism in salt marsh tidal creeks}

Research question:

How does nutrient enrichment affect community metabolism in tidal creeks, and does this response differ between the creek-bottom sediments and the integrated creek ecosystem? 
Chapter 3 examined the role of enhanced nutrient supply on tidal creek metabolism. Anthropogenically driven eutrophication is a global phenomenon that may profoundly alter patterns of salt marsh production, and could act through a variety of processes to decrease organic matter burial and convert marsh platform to mudflats, which further limits primary productivity by grasses. In an open water oxygen study as well as in a benthic chamber study including triple oxygen isotopes one year later I compared short term (hourly to weekly) creek metabolic fluxes at a nutrient amended creek and unamended control creek. In the open water study, I also sampled noble gases in order to help constrain air-water exchange fluxes; I have not found any previously presented research that evaluates a suite of noble gases in a salt marsh setting, though individual gases such as radon have been used as tracers of porewater processes. In the open water study, I found that there was no net difference in net community metabolism between nutrient treatments over an average diel cycle. Yet over specific tidal stages when the creek includes metabolism on the marsh banks and platform, there was evidence that both production and respiration were enhanced in the nutrient amended tidal creek. Although nutrient addition did not clearly result in net changes in metabolism and expected net organic matter fluxes over the experiment, specific metabolism fluxes were different between the nutrient enriched and ambient nutrient concentration creeks, with potential ecological consequences. For the chamber study, I developed a new method of processing triple oxygen isotope samples in order to evaluate gross primary production at low oxygen concentrations. The chamber study was complicated by additional methodological and environmental issues, but net community production rates were similar to the peak rates from the creek study; the comparison of triple oxygen isotope derived rates supported the idea that increased nutrients could have enhanced gross primary production. Together, these rates point to the same conclusion; production and respiration may have been enhanced by increased nutrient supply, even as the system resisted changes to net metabolism. Given the small daily differences, sampling over a seasonal timescale would be required to evaluate whether there were long term differences in organic matter storage in the creeks.

\section{Chapter 4-air-water gas exchange in a salt marsh pond}

\section{Research question:}

What air-water diffusive gas exchange parameterization is most appropriate to this small, shallow aquatic system, and are there other physical gas fluxes that are large compared to this flux?

In Chapter 4 (under review for publication in Limnology and Oceanography: Letters), I again used the noble gases in a salt marsh setting in order to help constrain physical fluxes. In the pond in particular, air-water gas exchange was the major expected flux of oxygen similar to or larger than production and respiration. Therefore the determination of air-water gas exchange fluxes was critically important for calculating ecosystem metabolism rates. Along with my coauthors, I found that there were important differences in the performance of previously published gas exchange parameterizations in explaining changes in the noble gas concentrations. However accounting for rising bubbles (ebullition) of photosynthetic oxygen could be more important than the choice of gas exchange parameterization in correctly calculating metabolic fluxes from oxygen.

\section{Chapter 5-metabolism in a salt marsh pond}

\section{Research question:}

What are the metabolic rates in a marshtop pond, and how do they vary over hourly to seasonal timescales? Compared to oxygenic photosynthesis and aerobic respiration, do unusual oxygen or carbon metabolisms play a major role in controlling carbon cycling in this environment? 
Finally, in Chapter 5 (in preparation for submission), I used the triple oxygen isotope methods developed in Chapter 3 paired with the best choice of gas exchange parameterization determined from Chapter 4 in order to evaluate gross primary production and net community production over a six month time series in a salt marsh pond. I paired this work with three chamber experiments over midday peak production periods in sediments dominated by either micro- or macroalgae; I used these datasets in combination in order to evaluate the relationships between primary producers and metabolism over hourly to seasonal timescales. Like similar studies, I found that production by seagrasses may be an important driver of system metabolism. Comparisons of metabolism rates derived from triple oxygen isotopes, oxygen, and dissolved inorganic carbon pointed to the potential importance of light-enhanced oxygen and DIC consumption in shallow, sunlit marine systems. These processes may have led to unusual production and respiration stoichiometry; this data may point to the importance of anoxygenic photosynthesis or chemolithoautotrophy as production processes in this system that are not captured by oxygen based metabolism rates. The unusual carbon to oxygen stoichiometry of metabolism means that the ponds could be more important to overall salt marsh carbon cycling than is inferred assuming typical ratios of carbon to oxygen. Finally, as with the tight coupling of changes in production and respiration observed in the tidal creeks over short periods, daytime production was found to be balanced by respiration across the timeseries; the relatively stable (across late spring, summer, and early fall) net heterotrophic community metabolism in the study pond had to be subsidized by organic matter from buried peat or the surrounding marsh platform.

\section{Broader outlook and future directions}

Coastal environments are among the most biogeochemically active and ecologically important on Earth by many measures. They are also the crucial interface between human society and the global ocean: More than half the world's population now lives within $200 \mathrm{~km}$ or so of the ocean and it is at the coasts that communities reap the benefits of marine resources and are most exposed to the risks posed by storms, sealevel changes, and algal blooms. From an environmental perspective, the coasts are both barrier and amplifier to anthropogenic impacts on the ocean. Because of the outsize import of coastal environments compared to the open ocean in many biogeochemical cycles, the trajectory of coastal environments (eutrophication, acidification, deoxygenation and hypoxia) has global consequences.

Salt marshes are one such critical coastal environment. These systems are being lost rapidly along some coastlines and estuaries, but in others large-scale environmental engineering is building salt marshes in order to provide benefits to local communities such as storm-surge protection and filtering of industrial pollutants. The construction or management of marsh environments for carbon cycling characteristics may even be a potential tool for managing greenhouse gases. Yet surprisingly little is known about the tidally submerged components of salt marshes outside the grass covered, peat storing marsh platform. Tidal creeks are the interface between the marsh and the estuary, mediating the transfer of nutrients and organic and inorganic carbon between land and sea. Ponds are abundant features on the marsh surface that can provide important ecological functions such as supporting migratory bird populations, but may expand at the expense of the grass covered platform.

Ultimately the goal of this thesis is not to place an economic or management value on these submerged environments compared to the other components of the marsh. Instead, the goal is to answer more basic questions that are the necessary antecedents to any such valuation: What are the metabolic rates in these features and how do they differ from the grassy areas? (Chapters 3, 5: In general more heterotrophic than marsh grasses). Do any differences in ponds and creeks from the grass covered marsh platform matter to the carbon cycling of salt marshes? (Chapter 5: Yes, and unusual carbon to oxygen stoichiometry may lead to surprisingly large pond fluxes of carbon dioxide). And how might these rates change in the future, as different environments with the salt marsh expand or shrink relative to each other or as nutrient supply 
to primary producers increases or decreases? (Chapters 3, 5: As ponds and creeks expand, I expect the marsh as a whole to become less efficient at sequestering carbon, but rising nutrient concentrations may have complicated effects on carbon fluxes that do not necessarily result in a change in net metabolism over daily to weekly timescales). While I do not suggest that the answers I present in this work should provide a rationale for resource managers to drain ponds (a widespread practice in the last century) or enforce thresholds for nutrient influx from surrounding properties, these are the questions that must be answered in order to inform such more complex questions about possible future changes and management options.

More generally, this thesis tries to provide new and alternate means to measure and evaluate longstanding problems in aquatic ecosystem science. Are properties that have long been used to evaluate metabolism such as oxygen ultimately a good measure of carbon cycling? (Chapter 2: In situ biogeochemical tracers of biological organic carbon and oxygen fluxes are not necessarily sensitive to the same features of metabolism and carbon cycling; Chapter 3: In the tidal creeks, oxygen has significant methodological limitations as a metabolism tracer; Chapter 5: In the marsh pond, oxygen and inorganic carbon fluxes are mediated by a set of processes that makes interpreting fluxes of oxygen as equivalent to fluxes of carbon more complicated than expected). What role do light and concentration sensitive fluxes of metabolites outside of photosynthesis play in altering metabolic fluxes in the pond, and ultimately carbon cycling? (Chapter 5: Unusual sinks of both oxygen and carbon in the daylight must be invoked to explain observed fluxes). Triple oxygen isotope ratios and dissolved noble gases were introduced in this thesis as tools in salt marsh settings; this is potentially the first time these tools have been used to study gas exchange and metabolism within a salt marsh (Chapters 3, 4, 5): The methodological hurdles associated with the techniques are surmountable, and the tracers have been proven useful in answering the above questions in a relatively complicated environment.

Indeed, some of the specific difficulties in applying these techniques (the unknown isotopic composition of water in the environment, the high cost of noble gas samples) are now far more tractable than when I performed the research reported in this thesis. Field-portable commercial cavity ring-down spectroscopy systems to measure the dissolved triple oxygen isotope composition of water are being adopted by laboratories around the world; such systems are relatively low-cost and have high sample throughput, with sufficient accuracy to improve over the mixing models used in this work (Steig et al. 2014).

Similarly, a portable noble gas quadrupole mass spectrometer made entirely from commercially available parts was recently developed that could dramatically cut the cost and improve the utility of the noble gases as tracers of physical processes in complicated estuarine environments (Manning et al. 2016). The use of natural abundance tracers with a wide range of solubility characteristics would represent a major step forward for many studies of gas exchange in shallow systems. Similarly, field-portable cavity ringdown systems to measure the triple oxygen isotope ratio of dissolved oxygen at high frequency are under development and may soon be commercially available.

In short, the methods and approaches presented in this thesis add value to traditional approaches of measuring metabolism and gas fluxes, and are ready for more widespread adoption in the estuarine and limnological community.

Specific directions of future research that would improve the utility of these methods and broaden the scope of this work include:

- In open ocean settings, evaluate community structure, nutrient supply, metabolism, and carbon export, along with tracers of physical processes such as eddy kinetic energy and turbulence dissipation, at sufficient spatial resolution to test the hypothesis that mesoscale physical processes drive shared variations in these properties. 
- In preexisting datasets of triple oxygen isotopes, use the newly introduced approach to correcting for diapycnal mixing processes in order to evaluate metabolism in environments where this was previously infeasible because of biases from such processes, including the Equatorial Pacific.

- In salt marsh tidal creeks (or ponds) evaluate nutrient enrichment effects on water column metabolism over seasonal timescales so that long term trends can be discerned from strong daily variability.

- Conduct laboratory experiments to determine whether mass dependent fractionation of the triple oxygen isotopes may be different in any porewater processes than in standard aerobic respiration, and more broadly examine the triple oxygen isotope systematics of light-dependent reactions across a range of benthic algae present in estuarine environments.

- Similarly, conduct laboratory or field studies with the specific goal of evaluating the oxygen isotope photosynthetic fractionations in estuarine species, and more broadly in natural sediment communities. The results of Chapter 3 point to a plausible new method for determining the composition of photosynthetic oxygen in intact benthic algae communities, specifically by manipulating chamber experiments to maximize production of photosynthetic oxygen relative to oxygen from the atmosphere over a short period and measuring the resulting composition. All current photosynthetic fractionation measurements on which the triple oxygen isotope method relies are determined by a single method in which algae are maintained at near-zero oxygen. This is problematic both from the perspective of phytoplankton physiology and the observed biases in the triple oxygen isotope analytical method at low oxygen concentrations (Chapter 3,5 ). Thus a new method that reflects environmental conditions would be beneficial for the field.

- When possible, incorporate natural abundance tracers of gas exchange and metabolism into studies of shallow aquatic environments (noble gases, triple oxygen isotopes, oxygen and nitrogen to argon ratios) that reduce some of the biases associated with artificial tracer additions and provide additional information about processes that are generally neglected.

- If the goal of a study is to evaluate carbon fluxes and cycling, evaluate what combination of carbon and non-carbon tracers can help best constrain direct biological fluxes, biologically mediated fluxes, and abiological fluxes. Anoxygenic production in shallow environments may be more significant compared to oxygenic photosynthesis than generally assumed, and standard techniques of measuring metabolism may yield an incomplete picture of the system. 


\section{Closing thoughts}

On the Eastern seaboard of the United States, salt marshes are both a dominant coastal environment and yet an invisible one to many of those living around them, or indeed in the middle of former marshes. As an educational volunteer in marshes and in harbors, I am consistently struck by how many individuals are unaware that such exceptional environments are within the borders of their communities, much less how they may benefit from them. This is not an indictment of public awareness - I had never stepped into a marsh before beginning this thesis. Rather, it emphasizes to me how lucky I have been over the last five years to float across a pond, slog through tidal creeks, and generally wallow in the mud during my field swampling. Yes, there have been costs in sweat and blood, but those payments have earned moments of sublime and even transcendental beauty. Work in the marsh has at times tested me, but my time in the marsh has been personally and scientifically rewarding, and so I pause here for a moment to reflect on these sediments. I leave you with this quote from a Guy de Maupassant short story that feels appropriate:

"J'aime l'eau d'une passion désordonnée: la mer, bien que trop grande, trop remuante, impossible à posséder, les rivières si jolies, mais qui passent, qui fuient, qui s'en vont, et les marais surtout où palpite toute l'existence inconnue des bêtes aquatiques. Le marais, c'est un monde entier sur la terre, monde différent, qui a sa vie propre, ses habitants sédentaires, et ses voyageurs de passage, ses voix, ses bruits et son mystère surtout."

- Guy de Maupassant, Amour, Trois Pages du Livre d'un Chasseur

\section{Or, approximately:}

"I love the water with an inordinate passion: the sea, even though it is too large, too restless, impossible to grasp; the rivers which are so pretty, but which pass by and flee away; and above all, the marshes, pulsing everywhere with all the unknowable lives of aquatic creatures. The marsh is an entire world of its own here on earth; a different world which has its own life, its own settled inhabitants and passing travelers, its own voices, sounds, and especially mystery." 


\section{Bibliography}

\section{Chapter 1}

Angert, A., S. Rachmilevitch, E. Barkan, and B. Luz. 2003. Effects of photorespiration, the cytochrome pathway, and the alternative pathway on the triple isotopic composition of atmospheric O 2. Global Biogeochem. Cycles 17, doi:10.1029/2002GB001933

Barkan, E., and B. Luz. 2011. The relationships among the three stable isotopes of oxygen in air, seawater and marine photosynthesis. Rapid Commun. Mass Spectrom. 25: 2367-9.

Berg, P., H. Røy, F. Janssen, V. Meyer, B. Jørgensen, M. Huettel, and D. de Beer. 2003. Oxygen uptake by aquatic sediments measured with a novel non-invasive eddy-correlation technique. Mar. Ecol. Prog. Ser. 261: $75-83$.

Bromberg Gedan, K., B.R. Silliman, and M. D. Bertness. 2009. Centuries of Human-Driven Change in Salt Marsh Ecosystems. Ann. Rev. Mar. Sci. 1: 117-141.

Brotas, V., C R. Mendes, and P. Cartaxana. 2007. Microphytobenthic biomass assessment by pigment analysis: comparison of spectrophotometry and High Performance Liquid Chromatography methods. Hydrobiologia 587: 19-24.

Cai, W.J. 2011. Estuarine and coastal ocean carbon paradox: CO2 sinks or sites of terrestrial carbon incineration? Ann. Rev. Mar. Sci. 3: 123-145.

Cebrian, J., C. D. Foster, R. Plutchak, K. L. Sheehan, M.-E. C. Miller, A. Anton, K. Major, K. L. Heck, and S. P. Powers. 2007. The impact of Hurricane Ivan on the primary productivity and metabolism of marsh tidal creeks in the NorthCentral Gulf of Mexico. Aquat. Ecol. 42: 391-404.

Dame, R. F., and P. D. Kenny. 1986. Variability of Spartina alterniflora primary production in the euhaline North Inlet estuary. Mar. Ecol. Prog. Ser. 32: 71-80.

Deegan, L. A., D. S. Johnson, R. S. Warren, B. J. Peterson, J. W. Fleeger, S. Fagherazzi, and W. M. Wollheim. 2012. Coastal eutrophication as a driver of salt marsh loss. Nature 490: 388-92.

Dring, M. J., and D. H. Jewson. 1982. What Does 14C Uptake by Phytoplankton Really Measure? A Theoretical Modelling Approach, Proc. R. Soc. B Biol. Sci., 214(1196), 351-368, doi:10.1098/rspb.1982.0016.

Duarte, C. M., I. J. Losada, I. E. Hendriks, I. Mazarrasa, and N. Marbà. 2013. The role of coastal plant communities for climate change mitigation and adaptation. Nat. Clim. Chang. 3: 961-968.

Duarte, C. M., J. J. Middelburg, and N. Caraco. 2005. Major role of marine vegetation on the oceanic carbon cycle. Biogeosciences 2, doi:10.5194/bg-2-1-2005

Emerson, S., C. Stump, and D. Nicholson. 2008. Net biological oxygen production in the ocean: Remote in situ measurements of O 2 and N 2 in surface waters. Global Biogeochem. Cycles 22. doi:10.1029/2007GB003095

Flynn, K. J. 2003. Do we need complex mechanistic photoacclimation models for phytoplankton? Limnol. Oceanogr. 48: 2243-2249. 
Gallagher, J., and F. Daiber. 1974. Primary production of edaphic algal communities in a Delaware salt marsh. Limnol. Ocean. 19: 390-395.

Galván, K., J. W. Fleeger, and B. Fry. 2008. Stable isotope addition reveals dietary importance of phytoplankton and microphytobenthos to saltmarsh infauna. Marine 359: 37-49.

Galván, K., J. W. Fleeger, B. Peterson, D. Drake, L. A. Deegan, and D. S. Johnson. 2011. Natural abundance stable isotopes and dual isotope tracer additions help to resolve resources supporting a saltmarsh food web. J. Exp. Mar. Bio. Ecol. 410: 1-11.

Gazeau, F. P. H., J. J. Middelburg, M. Loijens, J. P. Vanderborght, M.-D. Pizay, and J. P. Gattuso. 2007. Planktonic primary production in estuaries: a comparison of the $14 \mathrm{C}, \mathrm{O} 2$ and $18 \mathrm{O}$ methods. Aquat. Microb. Ecol. 46: 96-106.

Glud, R., P. Berg, A. Hume, P. Batty, M. Blicher, K. Lennert, and S. Rysgaard. 2010. Benthic O2 exchange across hard-bottom substrates quantified by eddy correlation in a sub-Arctic fjord. Mar. Ecol. Prog. Ser. 417: 1-12.

Halsey, K. H., A. J. Milligan, and M. J. Behrenfeld (2010), Physiological optimization underlies growth rate-independent chlorophyll-specific gross and net primary production., Photosynth. Res., 103(2), 125-37, doi:10.1007/s11120-009-9526-Z.

Hartig, E. K., V. Gornitz, A. Kolker, F. Mushacke, and D. Fallon. 2002. Anthropogenic and climatechange impacts on salt marshes of Jamaica Bay, New York City. Wetlands 22: 71-89. doi:10.1672/0277-5212(2002)022[0071:AACCIO]2.0.CO;2

Hendricks, M. B., M. L. Bender, and B. A. Barnett (2004), Net and gross O2 production in the southern ocean from measurements of biological O2 saturation and its triple isotope composition, Deep Sea Res. Part I Oceanogr. Res. Pap., 51(11), 1541-1561, doi:10.1016/j.dsr.2004.06.006.

Helman, Y., E. Barkan, D. Eisenstadt, and A. Kaplan. 2005. Fractionation of the three stable oxygen isotopes by oxygen-producing and oxygen-consuming reactions in photosynthetic organisms. Plant Physiology 138(4): 2292-2298.

Hopkinson, C. S., W.-J. Cai, and X. Hu. 2012. Carbon sequestration in wetland dominated coastal systems - a global sink of rapidly diminishing magnitude. Curr. Opin. Environ. Sustain. 4: 186-194.

Juranek, L. W., and P. D. Quay (2013), Using triple isotopes of dissolved oxygen to evaluate global marine productivity., Ann. Rev. Mar. Sci., 5, 503-24, doi:10.1146/annurev-marine-121211-172430.

Kaiser, J. (2011), Technical note: Consistent calculation of aquatic gross production from oxygen triple isotope measurements, Biogeosciences, 8(7), 1793-1811, doi:10.5194/bg-8-1793-2011.

Kinney, E., and C. Roman. 1998. Response of primary producers to nutrient enrichment in a shallow estuary. Mar. Ecol. Prog. Ser. 163: 89-98. doi:10.3354/meps163089

Krause-Jensen, D., S. Markager, and T. Dalsgaard. 2011. Benthic and Pelagic Primary Production in Different Nutrient Regimes. Estuaries and Coasts 35: 527-545.

Kromkamp, J. C., E. P. Morris, R. M. Forster, C. Honeywill, S. Hagerthey, and D. M. Paterson. 2006. Relationship of intertidal surface sediment chlorophyll concentration to hyperspectral reflectance and chlorophyll fluorescence. Estuaries and Coasts 29: 183-196. 
Lellis-Dibble, K. A., K. E. McGlynn, and T. E. Bigford. 1998. Estuarine Fish and Shellfish Species in U.S. Commercial and Recreational Fisheries: Economic Value as an Incentive to Protect and Restore Estuarine Habitat. USDC NOAA Tech. Mem. NMFS-F/SPO-90

Li, S., N.E. Levin, and L.A. Chesson. 2015. Continental scale variation in 17O-excess of meteoric waters in the United States. Geochim. Cosmochim. Acta 164: 110-126.

Luz, B., and E. Barkan. 2000. Assessment of Oceanic Productivity with the Triple-Isotope Composition of Dissolved Oxygen. Science (80-. ). 288: 2028-2031.

Luz, B., and E. Barkan. 2005. The isotopic ratios 17O/16O and 18O/16O in molecular oxygen and their significance in biogeochemistry. Geochim. Cosmochim. Acta 69: 1099-1110.

Luz, B., and E. Barkan. 2009. Net and gross oxygen production from O2/Ar, 17O/16O and 18O/16O ratios. Aquat. Microb. Ecol. 56: 133-145.

Luz, B., and E. Barkan. 2010. Variations of $17 \mathrm{O} / 16 \mathrm{O}$ and $18 \mathrm{O} / 16 \mathrm{O}$ in meteoric waters. Geochim. Cosmochim. Acta 74(22): 6276-6286.

MacIntyre, H. L., R. J. Geider, and D. C. Miller. 1996. Microphytobenthos: The Ecological Role of the "Secret Garden" of Unvegetated, Shallow-Water Marine Habitats. I. Distribution, Abundance and Primary Production. Estuaries 19: 186.

McGlathery, K J. 2001. Macroalgal blooms contribute to the decline of seagrass in nutrient-enriched coastal waters. J. Phycol. 37: 1-4.

McGlathery, K., K. Sundbäck, and I. Anderson. 2004. The importance of primary producers for the benthic nitrogen and phosphorus cycling., p. 231-261. In S.L. Nielsen, G.T. Banta, and M.F. Pedersen [eds.], The influence of Primary Producers on Estuarine Nutrient Cycling. Aquatic Ecological Progress Series.

Mcleod, E., G. L. Chmura, S. Bouillon, R. Salm, M. Björk, C. M. Duarte, C. E. Lovelock, W. H. Schlesinger, and B. R. Silliman. 2011. A blueprint for blue carbon: toward an improved understanding of the role of vegetated coastal habitats in sequestering $\mathrm{CO} 2$. Front. Ecol. Environ. 9: $552-560$.

Morris, J. T., K. Sundberg, and C. S. Hopkinson. 2013. Salt marsh primary production and its responses to relative sea level and nutrients in estuaries at Plum Island, Massachusetts, and North Inlet, South Carolina, USA. Oceanography 26: 78-84.

Odum, H. T. 1956. Primary production in flowing waters. Limnol. Oceanogr. 1: 102-11.

Paerl, H. 2006. Assessing and managing nutrient-enhanced eutrophication in estuarine and coastal waters: Interactive effects of human and climatic perturbations. Ecol. Eng. 26: doi:10.1016/j.ecoleng.2005.09.006

Peckol, P., and J.S. Rivers. 1996. Contribution by macroalgal mats to primary production of a shallow embayment under high and low nitrogen-loading rates. Estuar. Coastal Shelf Sci. 43: 311-325.

Pinckney, J., and R. Zingmark. 1993. Biomass and production of benthic microalgal communities in estuarine habitats. Estuaries 16: 887-897. doi:10.2307/1352447

Prokopenko, M. G., O. M. Pauluis, J. Granger, and L. Y. Yeung (2011), Exact evaluation of gross photosynthetic production from the oxygen triple-isotope composition of $\mathrm{O}_{2}$ : Implications for the net-to-gross primary production ratios, Geophys. Res. Lett., 38(14), doi:10.1029/2011GL047652. 
Quay, P. D., C. Peacock, K. Björkman, and D. M. Karl. 2010. Measuring primary production rates in the ocean: Enigmatic results between incubation and non-incubation methods at Station ALOHA. Global Biogeochem. Cycles 24, doi:10.1029/2009GB003665

Raymond, P.A., and J. J. Cole. 2001. Gas exchange in rivers and estuaries: Choosing a gas transfer velocity. Estuaries 24: doi:10.2307/1352954

Revsbech, N. P., and B. B. Jørgensen. 1983. Photosynthesis of benthic microflora measured with high spatial resolution by the oxygen microprofile method: Capabilities and limitations of the method. Limnol. Oceanogr. 28: 749-756.

Roman, C. T., K. W. Able, M. A. Lazzari, and K. L. Heck. 1990. Primary productivity of angiosperm and macroalgae dominated habitats in a New England Salt Marsh: a Comparative analysis. Estuar. Coast. Shelf Sci. 30: 35-45. doi:10.1016/0272-7714(90)90075-3

Ross, O. N., and R. J. Geider. 2009. New cell-based model of photosynthesis and photo-acclimation: accumulation and mobilisation of energy reserves in phytoplankton. Mar. Ecol. Prog. Ser. 383: 5371.

Sand-Jensen, K., and S. Nielsen. 2004. Estuarine primary producers, p. 17-57. In S.L. Nielsen, G.T. Banta, and M.F. Pedersen [eds.], Estuarine nutrient cycling: the influence of primary producers: the fate of nutrients and biomass. Aquatic Ecological Progress Series.

Shepard, C. C., C. M. Crain, and M. W. Beck. 2011. The protective role of coastal marshes: a systematic review and meta-analysis. PLoS One 6: e27374.

Staehr, P. A., D. Bade, M. C. Van de Bogert, G. R. Koch, C. Williamson, P. Hanson, J. J. Cole, and T. Kratz. 2010. Lake metabolism and the diel oxygen technique: State of the science. Limnol. Oceanogr. Methods 8: 628-644. doi:10.4319/lom.2010.8.0628

Stanley, R. H. R., and W. J. Jenkins. 2013. Noble Gases in Seawater as Tracers for Physical and Biogeochemical Ocean Processes, p. 55-79. In The Noble Gases as Geochemical Tracers. Springer Berlin Heidelberg.

Sullivan, M. J., and C. A. Currin. 2000. Community Structure and Functional Dynamics of Benthic Microalgae in Salt Marshes, p. 81-106. In M.P. Weinstein and D.A. Kreeger [eds.], Concepts and Controversies in Tidal Marsh Ecology. Kluwer Academic Publishers.

Teal, J. M. 1962. Energy Flow in the Salt Marsh Ecosystem of Georgia. Ecology 43: 614.

Thiemens, M. H., S. Chakraborty, and G. Dominguez. 2012. The physical chemistry of mass-independent isotope effects and their observation in nature. Annu. Rev. Phys. Chem. 63: 155-77.

Thiemens, M. H., and J. E. Heidenreich. 1983. The mass-independent fractionation of oxygen: a novel isotope effect and its possible cosmochemical implications. Science 219: 1073-5.

Thronson, A, and A Quigg. 2008. Fifty-five years of fish kills in coastal Texas. Estuars. Coasts 31: doi:10.1007/s12237-008-9056-5Valiela, I. 1995. Marine Ecological Processes, Springer.

Underwood, G. J. C., and J. Kromkamp. 1999. Primary Production by Phytoplankton and Microphytobenthos in Estuaries, p. 93-153. In D.B. Nedwell and D.G. Raffaelli [eds.], Advances in Ecological Research Estuaries. Academic Press.

Valiela, I., and J. M. Teal. 1979. The nitrogen budget of a salt marsh ecosystem. Nature 280: 652-656. 
Vallino, J. J., C. S. Hopkinson, and R. H. Garritt. 2005. Estimating estuarine gross production, community respiration and net ecosystem production: a nonlinear inverse technique. Ecol. Modell. 187: 281-296.

Van Raalte, C., I. Valiela, J. M. Teal, and D. Van Raalte. 1976. Production of epibenthic salt marsh algae : Light and nutrient limitation. Limnol. Oceanogr. 21: 862-872.

Williams, P. J. 1. B., and D. Lefèvre. 1996. Algal 14 C and total carbon metabolisms. 1. Models to account for the physiological processes of respiration and recycling, J. Plankton Res., 18(10), 19411959, doi:10.1093/plankt/18.10.1941.

Williams, P. J. I., and J. E. Robertson. 1991. Overall planktonic oxygen and carbon dioxide metabolisms: the problem of reconciling observations and calculations of photosynthetic quotients. J. Plankton Res. 13: $153-169$.

Wilson, C.A., Z.J. Hughes, D.M. Fitzgerald, C.S. Hopkinson, V. Valentine, A.S. Kolker. 2014. Saltmarsh and tidal creek morphodynamics: Dynamic equilibroum of northern latitude salt marshes? Geomorphology 213: 99-115.

Yacobi, Y. Z., N. Perel, E. Barkan, and B. Lus. Unexpected underestimation of primary productivity by $18 \mathrm{O}$ and $14 \mathrm{C}$ methods in a lake: Implications for slow diffusion of isotope tracers in and out of cells. Limnol. Oceanogr. 52: 329-337.

\section{Chapter 2}

Abe, O., and N. Yoshida. 2003. Partial pressure dependency of 17O/16O and 18O/16O of molecular oxygen in the mass spectrometer., Rapid Commun. mass Spectrom., 17(5), 395-400, doi:10.1002/rcm.923.

Acker, J. G., and G. Leptoukh. 2007. Online Analysis Enhances Use of NASA Earth Science Data, Eos, Trans. Am. Geophys. Union, 88(2), 14, doi:10.1029/2007EO020003.

Al-Mutairi, H., and M. R. Landry. 2001. Active export of carbon and nitrogen at Station ALOHA by diel migrant zooplankton, Deep Sea Res. Part II Top. Stud. Oceanogr., 48(8), 2083-2103, doi:10.1016/S0967-0645(00)00174-0.

Angert, A., S. Rachmilevitch, E. Barkan, and B. Luz. 2003. Effects of photorespiration, the cytochrome pathway, and the alternative pathway on the triple isotopic composition of atmospheric $\mathrm{O}_{2}$, Global Biogeochem. Cycles, 17(1), doi:10.1029/2002GB001933.

Arar, E. J., and G. B. Collins. 1997. Method 445.0 In Vitro Determination of Chlorophyll a and Pheophytin in Marine and Freshwater Algae by Fluorescence, Washington, DC.

Ayers, J. M., and M. S. Lozier. 2012. Unraveling dynamical controls on the North Pacific carbon sink, $J$. Geophys. Res. Ocean., 117(C1), n/a-n/a, doi:10.1029/2011JC007368. 
Ayers, J. M., and M. S. Lozier. 2010. Physical controls on the seasonal migration of the North Pacific transition zone chlorophyll front, J. Geophys. Res., 115(C5), doi:10.1029/2009JC005596.

Barkan, E., and B. Luz. 2003. High-precision measurements of $17 \mathrm{O} / 16 \mathrm{O}$ and $18 \mathrm{O} / 16 \mathrm{O}$ of O2 and O2/Ar ratio in air., Rapid Commun. mass Spectrom., 17(24), 2809-14, doi:10.1002/rcm.1267.

Barton, A. D., S. Dutkiewicz, G. Flierl, J. Bragg, and M. J. Follows. 2010. Patterns of diversity in marine phytoplankton., Science (80-. )., 327(5972), 1509-11, doi:10.1126/science.1184961.

Barton, A. D., S. Dutkiewicz, G. Flierl, J. Bragg, and M. J. Follows. 2010. Patterns of diversity in marine phytoplankton., Science (80-. )., 327(5972), 1509-1511, doi:10.1126/science.1184961.

Behrenfeld, M. J., and P. G. Falkowski. 1997. Photosynthetic rates derived from satellite-based chlorophyll concentration, Limnol. Oceanogr., 42(1), 1-20, doi:10.4319/1o.1997.42.1.0001.

Behrenfeld, M. J., K. H. Halsey, and A. J. Milligan. 2008. Evolved physiological responses of phytoplankton to their integrated growth environment., Philos. Trans. R. Soc. Lond. B. Biol. Sci., 363(1504), 2687-703, doi:10.1098/rstb.2008.0019.

Benitez-Nelson, C., K. O. Buesseler, D. M. Karl, and J. Andrews (2001), A time-series study of particulate matter export in the North Pacific Subtropical Gyre based on 234Th:238U disequilibrium, Deep Sea Res. Part I Oceanogr. Res. Pap., 48(12), 2595-2611, doi:10.1016/S0967-0637(01)00032-2.

Berube, P. M. et al. (2015), Physiology and evolution of nitrate acquisition in Prochlorococcus, ISME J., 9(5), 1195-1207, doi:10.1038/ismej.2014.211.

Boyd, P. W., and T. W. Trull (2007), Understanding the export of biogenic particles in oceanic waters: Is there consensus?, Prog. Oceanogr., 72(4), 276-312, doi:10.1016/j.pocean.2006.10.007.

Boyd, P., and P. Newton (1995), Evidence of the potential influence of planktonic community structure on the interannual variability of particulate organic carbon flux, Deep Sea Res. Part I Oceanogr. Res. Pap., 42(5), 619-639, doi:10.1016/0967-0637(95)00017-Z.

Brandt, P., F. A. Schott, C. Provost, A. Kartavtseff, V. Hormann, B. Bourlès, and J. Fischer (2006), Circulation in the central equatorial Atlantic: Mean and intraseasonal to seasonal variability, Geophys. Res. Lett., 33(7), doi:10.1029/2005GL025498.

Bray, J. R., and J. T. Curtis (1957), An Ordination of the Upland Forest Communities of Southern Wisconsin, Ecol. Monogr., 27(4), 325-349, doi:10.2307/1942268.

Brix, H., N. Gruber, D. M. Karl, and N. R. Bates (2006), On the relationships between primary, net community, and export production in subtropical gyres, Deep Sea Res. Part II Top. Stud. Oceanogr., 53(5), 698-717, doi:10.1016/j.dsr2.2006.01.024.

Brown, M. B., and A. B. Forsythe (1974), Robust Tests for the Equality of Variances, J. Am. Stat. Assoc., 69(346), 364-367, doi:10.1080/01621459.1974.10482955. 
Buesseler, K. O., C. Lamborg, P. Cai, R. Escoube, R. Johnson, S. Pike, P. Masque, D. McGillicuddy, and E. Verdeny (2008), Particle fluxes associated with mesoscale eddies in the Sargasso Sea, Deep Sea Res. Part II Top. Stud. Oceanogr., 55(10), 1426-1444, doi:10.1016/j.dsr2.2008.02.007.

Buesseler, K. O. (1998), The decoupling of production and particulate export in the surface ocean, Global Biogeochem. Cycles, 12(2), 297-310, doi:10.1029/97GB03366.

Buesseler, K. O. et al. (2007), An assessment of the use of sediment traps for estimating upper ocean particle fluxes, J. Mar. Res., 65(3), 345-416.

Carpenter, E., J. Montoya, J. Burns, M. Mulholland, A. Subramaniam, and D. Capone (1999), Extensive bloom of a N2-fixing diatom/cyanobacterial association in the tropical Atlantic Ocean, Mar. Ecol. Prog. Ser., 185, 273-283, doi:10.3354/meps 185273.

Carpenter, J. H. (1965), THE CHESAPEAKE BAY INSTITUTE TECHNIQUE FOR THE WINKLER DISSOLVED OXYGEN METHOD, Limnol. Oceanogr., 10(1), 141-143, doi:10.4319/1o.1965.10.1.0141.

Carpenter, J. H. (1965), THE ACCURACY OF THE WINKLER METHOD FOR DISSOLVED OXYGEN ANALYSIS1, Limnol. Oceanogr., 10(1), 135-140, doi:10.4319/1o.1965.10.1.0135.

Carr, M.-E., N. S. Oakey, B. Jones, and M. R. Lewis (1992), Hydrographic patterns and vertical mixing in the equatorial Pacific along $150^{\circ} \mathrm{W}, J$. Geophys. Res., 97(C1), doi:10.1029/91JC02479.

Cassar, N., P. J. DiFiore, B. A. Barnett, M. L. Bender, A. R. Bowie, B. Tilbrook, K. Petrou, K. J. Westwood, S. W. Wright, and D. Lefevre (2011), The influence of iron and light on net community production in the Subantarctic and Polar Frontal Zones, Biogeosciences, 8(2), 227-237, doi:10.5194/bg-8-227-2011.

Cassar, N., S. W. Wright, P. G. Thomson, T. W. Trull, K. J. Westwood, M. de Salas, A. Davidson, I. Pearce, D. M. Davies, and R. J. Matear (2015), The relation of mixed-layer net community production to phytoplankton community composition in the Southern Ocean, Global Biogeochem. Cycles, 29(4), 446-462, doi:10.1002/2014GB004936.

Chelton, D. B., M. G. Schlax, and R. M. Samelson (2011), Global observations of nonlinear mesoscale eddies, Prog. Oceanogr., 91(2), 167-216, doi:10.1016/j.pocean.2011.01.002.

Cheng, Y.-H., C.-R. Ho, Q. Zheng, and N.-J. Kuo (2014), Statistical Characteristics of Mesoscale Eddies in the North Pacific Derived from Satellite Altimetry, Remote Sens., 6(6), 5164-5183, doi:10.3390/rs6065164.

Clayton, S., S. Dutkiewicz, O. Jahn, and M. J. Follows. 2013. Dispersal, eddies, and the diversity of marine phytoplankton, Limnol. Oceanogr. Fluids Environ., 3(0), 182-197, doi:10.1215/215736892373515.

Clayton, S., T. Nagai, and M. J. Follows. 2014. Fine scale phytoplankton community structure across the Kuroshio Front, J. Plankton Res., 36(4), 1017-1030, doi:10.1093/plankt/fbu020. 
Craig, H., and T. Hayward. 1987. Oxygen Supersaturation in the Ocean: Biological Versus Physical Contributions, Science (80-. )., 235(4785), 199-202, doi:10.1126/science.235.4785.199.

Doney, S. C., D. M. Glover, S. J. McCue, and M. Fuentes. 2003. Mesoscale variability of Sea-viewing Wide Field-of-view Sensor (SeaWiFS) satellite ocean color: Global patterns and spatial scales, $J$. Geophys. Res. Ocean., 108(C2), doi:10.1029/2001JC000843.

Dring, M. J., and D. H. Jewson. 1982. What Does 14C Uptake by Phytoplankton Really Measure? A Theoretical Modelling Approach, Proc. R. Soc. B Biol. Sci., 214(1196), 351-368, doi:10.1098/rspb.1982.0016.

Ducklow, H. W., S. C. Doney, and S. F. Sailley. 2015. Ecological controls on biogeochemical fluxes in the western Antarctic Peninsula studied with an inverse foodweb model, Adv. Polar Sci., 122-139, doi:10.13679/J.ADVPS.2015.2.00122.

Ducklow, H., D. Steinberg, and K. Buesseler. 2001. Upper Ocean Carbon Export and the Biological Pump, Oceanography, 14(4), 50-58, doi:10.5670/oceanog.2001.06.

Durkin, C. A., B. A. S. Van Mooy, S. T. Dyhrman, and K. O. Buesseler. 2016. Sinking phytoplankton associated with carbon flux in the Atlantic Ocean, Limnol. Oceanogr., 61(4), 1172-1187, doi:10.1002/lno.10253.

Emerson, S., P. D. Quay, C. Stump, D. Wilbur, and R. Schudlich. 1995. Chemical tracers of productivity and respiration in the subtropical Pacific Ocean, J. Geophys. Res., 100(C8), 15873, doi:10.1029/95JC01333.

Emerson, S., P. Quay, D. Karl, C. Winn, L. Tupas, and M. Landry. 1997. Experimental determination of the organic carbon flux from open-ocean surface waters, Nature, 389(6654), 951-954, doi:10.1038/40111.

Emerson, S. 2014. Annual net community production and the biological carbon flux in the ocean, Global Biogeochem. Cycles, 28(1), 14-28, doi:10.1002/2013GB004680.

Emerson, S., T. Ito, and R. C. Hamme. 2012. Argon supersaturation indicates low decadal-scale vertical mixing in the ocean thermocline, Geophys. Res. Lett., 39(18), doi:10.1029/2012GL053054.

Emerson, S., C. Stump, D. Wilbur, and P. Quay. 1999. Accurate measurement of O2, N2, and Ar gases in water and the solubility of N2, Mar. Chem., 64(4), 337-347, doi:10.1016/S0304-4203(98)00090-5.

Eppley, R. W., and B. J. Peterson (1979), Particulate organic matter flux and planktonic new production in the deep ocean, Nature, 282(5740), 677-680, doi:10.1038/282677a0.

Estapa, M. L., D. A. Siegel, K. O. Buesseler, R. H. R. Stanley, M. W. Lomas, and N. B. Nelson (2015), Decoupling of net community and export production on submesoscales in the Sargasso Sea, Global Biogeochem. Cycles, 29(8), 1266-1282, doi:10.1002/2014GB004913. 
Falkowski, P. G., E. A. Laws, R. T. Barber, and J. W. Murray. 2003. Phytoplankton and Their Role in Primary, New, and Export Production, in Ocean Biogeochemistry, pp. 99-121, Springer Berlin Heidelberg, Berlin, Heidelberg.

Ferrón, S., S. T. Wilson, S. Martínez-García, P. D. Quay, and D. M. Karl. 2015. Metabolic balance in the mixed layer of the oligotrophic North Pacific Ocean from diel changes in O2/Ar saturation ratios, Geophys. Res. Lett., 42(9), 3421-3430, doi:10.1002/2015GL063555.

Fisher, R. A. 1956. Statistical methods and scientific inference., Hafner Publishing Co., Oxford.

Foster, R. A., A. Subramaniam, and J. P. Zehr. 2009. Distribution and activity of diazotrophs in the Eastern Equatorial Atlantic, Environ. Microbiol., 11(4), 741-750, doi:10.1111/j.14622920.2008.01796.x.

Friedrichs, M. A. M. et al. 2009. Assessing the uncertainties of model estimates of primary productivity in the tropical Pacific Ocean, J. Mar. Syst., 76(1), 113-133, doi:10.1016/j.jmarsys.2008.05.010.

Garcia, H. E., and L. I. Gordon. 1993. Erratum: Oxygen solubility in seawater: better fitting equations, Limnol. Oceanogr., 38(3), 656.

Garcia, H. E., and L. I. Gordon. 1992. Oxygen solubility in seawater: Better fitting equations, Limnol. Oceanogr., 37(6), 1307-1312, doi:10.4319/10.1992.37.6.1307.

Gardner, W. D. 2000. Sediment trap sampling in surface waters, in The Changing Ocean Carbon Cycle: A Midterm Synthesis of the Joint Global OCean Flux Study, edited by R. B. Hanson, H. W. Ducklow, and J. G. Field, pp. 240-284, Cambridge University Press, Cambridge.

Gehrie, E., D. Archer, S. Emerson, C. Stump, and C. Henning (2006), Subsurface ocean argon disequilibrium reveals the equatorial Pacific shadow zone, Geophys. Res. Lett., 33(18), doi:10.1029/2006GL026935.

Giesbrecht, K. E., R. C. Hamme, and S. R. Emerson (2012), Biological productivity along Line P in the subarctic northeast Pacific: In situ versus incubation-based methods, Global Biogeochem. Cycles, 26(3), doi:10.1029/2012GB004349.

Gordon, L. I., J. C. Jennings, A. A. Ross, and J. M. Krest (1993), A Suggested Protocol for Continuous Flow Automated Analysis of Seawater Nutrients (Phosphate, Nitrate, Nitrite and Silicic Acid) in the WOCE Hydrographic Program and the Joint Global Ocean Fluxes Study, WOCE Hydrographic Program Office Methods Manual WHPO 91-1, Corvallis, OR.

Grodsky, S. A., J. A. Carton, and C. R. McClain (2008), Variability of upwelling and chlorophyll in the equatorial Atlantic, Geophys. Res. Lett., 35(3), doi:10.1029/2007GL032466.

Guidi, L. et al. (2016), Plankton networks driving carbon export in the oligotrophic ocean, Nature, 532(7600), 465-470, doi:10.1038/nature16942. 
Guidi, L., L. Stemmann, G. A. Jackson, F. Ibanez, H. Claustre, L. Legendre, M. Picheral, and G. Gorsky (2009), Effects of phytoplankton community on production, size, and export of large aggregates: A world-ocean analysis, Limnol. Oceanogr., 54(6), 1951-1963, doi:10.4319/1o.2009.54.6.1951.

Halsey, K. H., A. J. Milligan, and M. J. Behrenfeld (2010), Physiological optimization underlies growth rate-independent chlorophyll-specific gross and net primary production., Photosynth. Res., 103(2), 125-37, doi:10.1007/s11120-009-9526-z.

Hamme, R. C. et al. (2012), Dissolved $\mathrm{O}_{2} / \mathrm{Ar}$ and other methods reveal rapid changes in productivity during a Lagrangian experiment in the Southern Ocean, J. Geophys. Res. Ocean., 117(C4), doi:10.1029/2011JC007046.

Hamme, R. C., and S. R. Emerson (2004), The solubility of neon, nitrogen and argon in distilled water and seawater, Deep Sea Res. Part I Oceanogr. Res. Pap., 51(11), 1517-1528, doi:10.1016/j.dsr.2004.06.009.

Hansell, D. A. (2013), Recalcitrant Dissolved Organic Carbon Fractions, Annu. Rev. Mar. Sci, 5, 421-45, doi:10.1146/annurev-marine-120710-100757.

Hansell, D. A., C. A. Carlson, and R. Schlitzer (2012), Net removal of major marine dissolved organic carbon fractions in the subsurface ocean, Global Biogeochem. Cycles, 26(1), doi:10.1029/2011GB004069.

Hansell, D., C. Carlson, D. Repeta, and R. Schlitzer (2009), Dissolved Organic Matter in the Ocean: A Controversy Stimulates New Insights, Oceanography, 22(4), 202-211, doi:10.5670/oceanog.2009.109.

Haskell, W. Z., D. Kadko, D. E. Hammond, A. N. Knapp, M. G. Prokopenko, W. M. Berelson, and D. G. Capone (2015), Upwelling velocity and eddy diffusivity from 7Be measurements used to compare vertical nutrient flux to export POC flux in the Eastern Tropical South Pacific, Mar. Chem., 168, 140150, doi:10.1016/j.marchem.2014.10.004.

Helber, R. W., R. H. Weisberg, F. Bonjean, E. S. Johnson, and G. S. E. Lagerloef (2007), SatelliteDerived Surface Current Divergence in Relation to Tropical Atlantic SST and Wind, J. Phys. Oceanogr., 37(5), 1357-1375, doi:10.1175/JPO3052.1.

Helman, Y., E. Barkan, D. Eisenstadt, and A. Kaplan. 2005. Fractionation of the three stable oxygen isotopes by oxygen-producing and oxygen-consuming reactions in photosynthetic organisms. Plant Physiology 138(4): 2292-2298.

Hendricks, M. B., M. L. Bender, and B. A. Barnett (2004), Net and gross O2 production in the southern ocean from measurements of biological O2 saturation and its triple isotope composition, Deep Sea Res. Part I Oceanogr. Res. Pap., 51(11), 1541-1561, doi:10.1016/j.dsr.2004.06.006.

Hendricks, M. B., M. L. Bender, B. A. Barnett, P. Strutton, and F. P. Chavez (2005), Triple oxygen isotope composition of dissolved $\mathrm{O}_{2}$ in the equatorial Pacific: A tracer of mixing, production, and respiration, J. Geophys. Res., 110(C12), doi:10.1029/2004JC002735. 
Hennon, G. M. M. 2015. Uncovering Mechanisms of Phytoplankton Response to Climate Change. PhD Thesis, University of Washington.

Henson, S. A., R. Sanders, and E. Madsen (2012), Global patterns in efficiency of particulate organic carbon export and transfer to the deep ocean, Global Biogeochem. Cycles, 26(1), n/a-n/a, doi:10.1029/2011GB004099.

Ho, D. T., R. Wanninkhof, P. Schlosser, D. S. Ullman, D. Hebert, and K. F. Sullivan (2011), Toward a universal relationship between wind speed and gas exchange: Gas transfer velocities measured with ${ }^{3}$ $\mathrm{He} / \mathrm{SF}{ }_{6}$ during the Southern Ocean Gas Exchange Experiment, J. Geophys. Res., 116(C4), doi:10.1029/2010JC006854.

Howard, E., S. Emerson, S. Bushinsky, and C. Stump (2010), The role of net community production in air-sea carbon fluxes at the North Pacific subarctic-subtropical boundary region, Limnol. Oceanogr., 55(6), 2585-2596.

Hu, C., E. T. Montgomery, R. W. Schmitt, and F. E. Muller-Karger (2004), The dispersal of the Amazon and Orinoco River water in the tropical Atlantic and Caribbean Sea: Observation from space and SPALACE floats, Deep Sea Res. Part II Top. Stud. Oceanogr., 51(10), 1151-1171, doi:10.1016/j.dsr2.2004.04.001.

Irigoien, X., J. Huisman, and R. P. Harris (2004), Global biodiversity patterns of marine phytoplankton and zooplankton, Nature, 429(6994), 863-867, doi:10.1038/nature02593.

Ito, T., C. Deutsch, S. Emerson, and R. C. Hamme (2007), Impact of diapycnal mixing on the saturation state of argon in the subtropical North Pacific, Geophys. Res. Lett., 34(9), doi:10.1029/2006GL029209.

Juranek, L. W., and P. D. Quay (2013), Using triple isotopes of dissolved oxygen to evaluate global marine productivity., Ann. Rev. Mar. Sci., 5, 503-24, doi:10.1146/annurev-marine-121211-172430.

Juranek, L. W., P. D. Quay, R. A. Feely, D. Lockwood, D. M. Karl, and M. J. Church (2012), Biological production in the NE Pacific and its influence on air-sea $\mathrm{CO}_{2}$ flux: Evidence from dissolved oxygen isotopes and $\mathrm{O}_{2} / \mathrm{Ar}, J$. Geophys. Res. Ocean., 117(C5), doi:10.1029/2011JC007450.

Juranek, L. W., R. C. Hamme, J. Kaiser, R. Wanninkhof, and P. D. Quay (2010), Evidence of O 2 consumption in underway seawater lines: Implications for air-sea $\mathrm{O}_{2}$ and $\mathrm{CO}_{2}$ fluxes, Geophys. Res. Lett., 37(1), n/a-n/a, doi:10.1029/2009GL040423.

Kadko, D., and W. Johns (2011), Inferring upwelling rates in the equatorial Atlantic using 7Be measurements in the upper ocean, Deep Sea Res. Part I Oceanogr. Res. Pap., 58(6), 647-657, doi:10.1016/j.dsr.2011.03.004.

Kaiser, J. (2011), Technical note: Consistent calculation of aquatic gross production from oxygen triple isotope measurements, Biogeosciences, 8(7), 1793-1811, doi:10.5194/bg-8-1793-2011. 
Kaiser, J., and O. Abe (2012), Reply to Nicholson's comment on \&quot;Consistent calculation of aquatic gross production from oxygen triple isotope measurements\&quot; by Kaiser (2011), Biogeosciences, 9(8), 2921-2933, doi:10.5194/BG-9-2921-2012.

Kaiser, J., M. K. Reuer, B. Barnett, and M. L. Bender (2005), Marine productivity estimates from continuous $\mathrm{O}_{2} /$ Ar ratio measurements by membrane inlet mass spectrometry, Geophys. Res. Lett., 32(19), doi:10.1029/2005GL023459.

Kalnay, E. et al. (1996), The NCEP/NCAR 40-Year Reanalysis Project, Bull. Am. Meteorol. Soc., 77(3), 437-471, doi:10.1175/1520-0477(1996)077<0437:TNYRP>2.0.CO;2.

Karl, D. M., M. J. Church, J. E. Dore, R. M. Letelier, and C. Mahaffey (2012), Predictable and efficient carbon sequestration in the North Pacific Ocean supported by symbiotic nitrogen fixation, Proc. Natl. Acad. Sci., 109(6), 1842-1849, doi:10.1073/pnas.1120312109.

Karl, D. ., R. . Bidigare, and R. . Letelier (2001), Long-term changes in plankton community structure and productivity in the North Pacific Subtropical Gyre: The domain shift hypothesis, Deep Sea Res. Part II Top. Stud. Oceanogr., 48(8), 1449-1470, doi:10.1016/S0967-0645(00)00149-1.

Kavanaugh, M. T., S. R. Emerson, B. Hales, D. M. Lockwood, P. D. Quay, and R. M. Letelier (2014), Physicochemical and biological controls on primary and net community production across northeast Pacific seascapes, Limnol. Oceanogr., 59(6), 2013-2027, doi:10.4319/lo.2014.59.6.2013.

Kavanaugh, M. T., B. Hales, M. Saraceno, Y. H. Spitz, A. E. White, and R. M. Letelier (2014), Hierarchical and dynamic seascapes: A quantitative framework for scaling pelagic biogeochemistry and ecology, Prog. Oceanogr., 120, 291-304, doi:10.1016/j.pocean.2013.10.013.

Kemp, A. E. S., R. B. Pearce, I. Grigorov, J. Rance, C. B. Lange, P. Quilty, and I. Salter (2006), Production of giant marine diatoms and their export at oceanic frontal zones: Implications for $\mathrm{Si}$ and C flux from stratified oceans, Global Biogeochem. Cycles, 20(4), doi:10.1029/2006GB002698.

Kirk, J. T. O. (1994), Light and photosynthesis in aquatic ecosystems, Cambridge University Press, Cambridge.

Klein, P., and G. Lapeyre (2009), The Oceanic Vertical Pump Induced by Mesoscale and Submesoscale Turbulence, Ann. Rev. Mar. Sci., 1(1), 351-375, doi:10.1146/annurev.marine.010908.163704.

Knap, A., A. Michaels, A. Close, H. Ducklow, and A. Dickson (Eds.) (1996), Protocols for the Joint Global Ocean Flux Study (JGOFS) Core Measurements, Reprint, UNESCO 1994 IOC Manuals and Guides 29, UNESCO.

Knapp, G. P., M. C. Stalcup, and R. J. Stanley (1989), Dissolved oxygen measurments in sea water at the Woods Hole Oceanographic Institution, Woods Hole Oceanographic Institution, Woods Hole, MA.

Kruskal, W. H., and W. A. Wallis (1952), Use of Ranks in One-Criterion Variance Analysis, J. Am. Stat. Assoc., 47(260), 583, doi:10.2307/2280779. 
Kujawinski, E., K. Longnecker, and S. Rauch (2015), Project: Dissolved Organic Matter Composition in the Deep Atlantic Ocean, Biol. Chem. Oceanogr. Data Manag. Off. Available from: http://www.bcodmo.org/project $/ 2204$

Law, C. S., E. R. Abraham, A. J. Watson, and M. I. Liddicoat (2003), Vertical eddy diffusion and nutrient supply to the surface mixed layer of the Antarctic Circumpolar Current, J. Geophys. Res., 108(C8), doi:10.1029/2002JC001604.

Laws, E. A. (1991), Photosynthetic quotients, new production and net community production in the open ocean, Deep Sea Res. Part A. Oceanogr. Res. Pap., 38(1), 143-167.

Laws, E. A., P. G. Falkowski, W. O. Smith, H. Ducklow, and J. J. McCarthy (2000), Temperature effects on export production in the open ocean, Global Biogeochem. Cycles, 14(4), 1231-1246, doi:10.1029/1999GB001229.

Lê, S., J. Josse, and F. Husson (2008), FactoMineR : An $R$ Package for Multivariate Analysis, J. Stat. Softw., 25(1), doi:10.18637/jss.v025.i01.

Lee, Y. J. et al. (2015), An assessment of phytoplankton primary productivity in the Arctic Ocean from satellite ocean color/in situ chlorophyll- $a$ based models, J. Geophys. Res. Ocean., 120(9), 65086541, doi:10.1002/2015JC011018.

Levitus, S. E. (1982), Climatological atlas of the world ocean, NOAA Professional Paper 13, US Government Printing Office, Washington DC.

Lévy, M., R. Ferrari, P. J. S. Franks, A. P. Martin, and P. Rivière (2012), Bringing physics to life at the submesoscale, Geophys. Res. Lett., 39(14), doi:10.1029/2012GL052756.

Lévy, M., O. Jahn, S. Dutkiewicz, and M. J. Follows (2014), Phytoplankton diversity and community structure affected by oceanic dispersal and mesoscale turbulence, Limnol. Oceanogr. Fluids Environ., 4(1), 67-84, doi:10.1215/21573689-2768549.

Lévy, M. et al. (2015), The dynamical landscape of marine phytoplankton diversity., J. R. Soc. Interface, 12(111), doi:10.1098/rsif.2015.0481.

Lockwood, D., P. D. Quay, M. T. Kavanaugh, L. W. Juranek, and R. A. Feely (2012), High-resolution estimates of net community production and air-sea $\mathrm{CO} 2$ flux in the northeast Pacific, Global Biogeochem. Cycles, 26(4), n/a-n/a, doi:10.1029/2012GB004380.

Lomas, M. W., and N. R. Bates (2004), Potential controls on interannual partitioning of organic carbon during the winter/spring phytoplankton bloom at the Bermuda Atlantic time-series study (BATS) site, Deep Sea Res. Part I Oceanogr. Res. Pap., 51(11), 1619-1636, doi:10.1016/j.dsr.2004.06.007.

Longhurst, A. (1995), Seasonal cycles of pelagic production and consumption, Prog. Oceanogr., 36(2), 77-167, doi:10.1016/0079-6611(95)00015-1.

Lumpkin, R., and S. L. Garzoli (2005), Near-surface circulation in the Tropical Atlantic Ocean, Deep Sea Res. Part I Oceanogr. Res. Pap., 52(3), 495-518, doi:10.1016/j.dsr.2004.09.001. 
Mahadevan, A. (2016), The Impact of Submesoscale Physics on Primary Productivity of Plankton, Ann. Rev. Mar. Sci., 8(1), 161-184, doi:10.1146/annurev-marine-010814-015912.

Maranon, E., M. J. Behrenfeld, N. Gonzalez, B. Mourino, and M. V. Zubkov (2003), High variability of primary production in oligotrophic waters of the Atlantic Ocean: uncoupling from phytoplankton biomass and size structure, Mar. Ecol. Prog. Ser., 257, 1-11, doi:10.3354/meps257001.

Marra, J. (2009), Net and gross productivity: weighing in with 14C, Aquat. Microb. Ecol., 56(2-3), 123131, doi:10.3354/ame01306.

Marra, J. (n.d.), Approaches to the Measurement of Plankton Production, in Phytoplankton Productivity, pp. 78-108, Blackwell Science Ltd, Oxford, UK.

Martin, J. H., G. A. Knauer, D. M. Karl, and W. W. Broenkow (1987), VERTEX: carbon cycling in the northeast Pacific, Deep Sea Res. Part A. Oceanogr. Res. Pap., 34(2), 267-285, doi:10.1016/01980149(87)90086-0.

Martiny, A. C., S. Kathuria, and P. M. Berube (2009), Widespread metabolic potential for nitrite and nitrate assimilation among Prochlorococcus ecotypes, Proc. Natl. Acad. Sci., 106(26), 10787-10792, doi:10.1073/pnas.0902532106.

Martz, T. R., K. S. Johnson, and S. C. Riser (2008), Ocean metabolism observed with oxygen sensors on profiling floats in the South Pacific, Limnol. Oceanogr., 53(5-2), 2094-2111, doi:10.4319/1o.2008.53.5_part_2.2094.

McClain, C. R. (2009), A Decade of Satellite Ocean Color Observations*, Ann. Rev. Mar. Sci., 1(1), 1942, doi:10.1146/annurev.marine.010908.163650.

McGillicuddy, D. J. (2016), Mechanisms of Physical-Biological-Biogeochemical Interaction at the Oceanic Mesoscale, Ann. Rev. Mar. Sci., 8(1), 125-159, doi:10.1146/annurev-marine-010814015606.

McGillicuddy, D. J. et al. (2007), Eddy/wind interactions stimulate extraordinary mid-ocean plankton blooms., Science (80-. )., 316(5827), 1021-6, doi:10.1126/science.1136256.

Mémery, L., M. Arhan, X. . Alvarez-Salgado, M.-J. Messias, H. Mercier, C. . Castro, and A. . Rios (2000), The water masses along the western boundary of the south and equatorial Atlantic, Prog. Oceanogr., 47(1), 69-98, doi:10.1016/S0079-6611(00)00032-X.

Meyer, J. P., and M. A. Seaman (2013), A Comparison of the Exact Kruskal-Wallis Distribution to Asymptotic Approximations for All Sample Sizes up to 105, J. Exp. Educ., 81(2), 139-156, doi:10.1080/00220973.2012.699904.

Michaels, A. F., and M. W. Silver (1988), Primary production, sinking fluxes and the microbial food web, Deep Sea Res. Part I Oceanogr. Res. Pap., 35(4), 473-490, doi:10.1016/0198-0149(88)90126-4. 
Moore, L. R., A. F. Post, G. Rocap, and S. W. Chisholm (2002), Utilization of different nitrogen sources by the marine cyanobacteria Prochlorococcus and Synechococcus, Limnol. Oceanogr., 47(4), 989996, doi:10.4319/1o.2002.47.4.0989.

Moreno-Ostos, E., A. Fernández, M. Huete-Ortega, B. Mouriño-Carballido, A. Calvo-Díaz, X. Anxelu, G. Morán, and E. Marañón (2011), Size-fractionated phytoplankton biomass and production in the tropical Atlantic, Sci. Mar., 75(752), 379-389, doi:10.3989/scimar.2011.75n2379.

Mouriño-Carballido, B., R. Graña, A. Fernàndez, A. Bode, M. Varela, J. F. Domínguez, J. Escànez, D. de Armas, and E. Marañón (2011), Importance of $\mathrm{N}_{2}$ fixation vs. nitrate eddy diffusion along a latitudinal transect in the Atlantic Ocean, Limnol. Oceanogr., 56(3), 999-1007, doi:10.4319/1o.2011.56.3.0999.

Murray, J. W., R. T. Barber, M. R. Roman, M. P. Bacon, and R. A. Feely (1994), Physical and biological controls on carbon cycling in the equatorial pacific., Science (80-. )., 266(5182), 58-65, doi:10.1126/science.266.5182.58.

Murray, J. W., J. Young, J. Newton, J. Dunne, T. Chapin, B. Paul, and J. J. McCarthy (1996), Export flux of particulate organic carbon from the central equatorial Pacific determined using a combined drifting trap-234Th approach, Deep Sea Res. Part II Top. Stud. Oceanogr., 43(4-6), 1095-1132, doi:10.1016/0967-0645(96)00036-7.

Napoléon, C., L. Fiant, V. Raimbault, P. Riou, and P. Claquin (2014), Dynamics of phytoplankton diversity structure and primary productivity in the English Channel, Mar. Ecol. Prog. Ser., 505, 4964, doi:10.3354/meps 10772 .

Nicholson, D. P. (2011), Comment on: \&quot; Technical note: Consistent calculation of aquatic gross production from oxygen triple isotope measurements \&quot; by Kaiser (2011), Biogeosciences, 8, 2993-2997, doi:10.5194/bg-8-2993-2011.

Nicholson, D. P., S. R. Emerson, S. Khatiwala, and R. Hamme (2011), An inverse approach to estimate bubble-mediated air-sea gas flux from inert gas measurements, in Proceedings on the 6th International Symposium on Gas Transfer at Water Surfaces, Kyoto University Press.

Nicholson, D. P., S. T. Wilson, S. C. Doney, and D. M. Karl (2015), Quantifying subtropical North Pacific gyre mixed layer primary productivity from Seaglider observations of diel oxygen cycles, Geophys. Res. Lett., 42(10), 4032-4039, doi:10.1002/2015GL063065.

Nicholson, D., S. Emerson, and C. C. Eriksen (2008), Net community production in the deep euphotic zone of the subtropical North Pacific gyre from glider surveys, Limnol. Oceanogr., 53(5_part_2), 2226-2236, doi:10.4319/1o.2008.53.5_part_2.2226.

Nicholson, D., R. H. R. Stanley, and S. C. Doney (2014), The triple oxygen isotope tracer of primary productivity in a dynamic ocean model, Global Biogeochem. Cycles, 28(5), 538-552, doi:10.1002/2013GB004704.

Oksanen, J. et al. (2016), vegan: Community Ecology Package for R software. 
Ostle, C., M. Johnson, P. Landschützer, U. Schuster, S. Hartman, T. Hull, and C. Robinson (2015), Net community production in the North Atlantic Ocean derived from Volunteer Observing Ship data, Global Biogeochem. Cycles, 29(1), 80-95, doi:10.1002/2014GB004868.

Owens, S. A., S. Pike, and K. O. Buesseler (2015), Thorium-234 as a tracer of particle dynamics and upper ocean export in the Atlantic Ocean, Deep Sea Res. Part II Top. Stud. Oceanogr., 116, 42-59, doi:10.1016/j.dsr2.2014.11.010.

Palevsky, H. I., F. Ribalet, J. E. Swalwell, C. E. Cosca, E. D. Cokelet, R. A. Feely, E. V. Armbrust, and P. D. Quay (2013), The influence of net community production and phytoplankton community structure on $\mathrm{CO}_{2}$ uptake in the Gulf of Alaska, Global Biogeochem. Cycles, 27(3), 664-676, doi:10.1002/gbc.20058.

Palter, J. B., I. Marinov, J. L. Sarmiento, and N. Gruber (2013), Large-Scale, Persistent Nutrient Fronts of the World Ocean: Impacts on Biogeochemistry, in Chemical Oceanography of Frontal Zones, edited by I. Belkin, p. 38, Springer Berlin Heidelberg, Berlin.

Peterson, R. G., and L. Stramma (1991), Upper-level circulation in the South Atlantic Ocean, Prog. Oceanogr., 26(1), 1-73, doi:10.1016/0079-6611(91)90006-8.

Plattner, G.-K., N. Gruber, H. Frenzel, and J. C. McWilliams (2005), Decoupling marine export production from new production, Geophys. Res. Lett., 32(11), doi:10.1029/2005GL022660.

Pollard, R. T. et al. (2009), Southern Ocean deep-water carbon export enhanced by natural iron fertilization, Nature, 457(7229), 577-580, doi:10.1038/nature07716.

Prokopenko, M. G., O. M. Pauluis, J. Granger, and L. Y. Yeung (2011), Exact evaluation of gross photosynthetic production from the oxygen triple-isotope composition of $\mathrm{O}_{2}$ : Implications for the net-to-gross primary production ratios, Geophys. Res. Lett., 38(14), doi:10.1029/2011GL047652.

Quay, P., J. Stutsman, and T. Steinhoff (2012), Primary production and carbon export rates across the subpolar N. Atlantic Ocean basin based on triple oxygen isotope and dissolved $\mathrm{O}_{2}$ and $\mathrm{Ar}$ gas measurements, Global Biogeochem. Cycles, 26(2), doi:10.1029/2010GB004003.

Reuer, M. K., B. A. Barnett, M. L. Bender, P. G. Falkowski, and M. B. Hendricks (2007), New estimates of Southern Ocean biological production rates from O2/Ar ratios and the triple isotope composition of O2, Deep Sea Res. Part I Oceanogr. Res. Pap., 54(6), 951-974, doi:10.1016/j.dsr.2007.02.007.

Reygondeau, G., A. Longhurst, E. Martinez, G. Beaugrand, D. Antoine, and O. Maury (2013), Dynamic biogeochemical provinces in the global ocean, Global Biogeochem. Cycles, 27(4), 1046-1058, doi:10.1002/gbc.20089.

Rhein, M., M. Dengler, J. Sültenfuß, R. Hummels, S. Hüttl-Kabus, and B. Bourles (2010), Upwelling and associated heat flux in the equatorial Atlantic inferred from helium isotope disequilibrium, $J$. Geophys. Res., 115(C8), doi:10.1029/2009JC005772. 
Ribalet, F., A. Marchetti, K. A. Hubbard, K. Brown, C. A. Durkin, R. Morales, M. Robert, J. E. Swalwell, P. D. Tortell, and E. V. Armbrust (2010), Unveiling a phytoplankton hotspot at a narrow boundary between coastal and offshore waters, Proc. Natl. Acad. Sci., 107(38), 16571-16576, doi:10.1073/pnas.1005638107.

Richardson, T. L., and G. A. Jackson (2007), Small phytoplankton and carbon export from the surface ocean., Science (80-. )., 315(5813), 838-40, doi:10.1126/science.1133471.

Robinson, C., P. Holligan, T. Jickells, and S. Lavender (2009), The Atlantic Meridional Transect Programme (1995-2012), Deep Sea Res. Part II Top. Stud. Oceanogr., 56(15), 895-898, doi:10.1016/j.dsr2.2008.10.005.

Robinson, C. et al. (2006), The Atlantic Meridional Transect (AMT) Programme: A contextual view 1995-2005, Deep Sea Res. Part II Top. Stud. Oceanogr., 53(14-16), 1485-1515, doi:10.1016/j.dsr2.2006.05.015.

Romera-Castillo, C., R. T. Letscher, and D. A. Hansell (in press), New Nutrients Exert Fundamental Control on DOC Accumulation in the Surface Atlantic Ocean, Proc. Natl. Acad. Sci.

Sarma, V. V. S. S., O. Abe, A. Hinuma, and T. Saino (2006), Short-term variation of triple oxygen isotopes and gross oxygen production in the Sagami Bay, central Japan, Limnol. Oceanogr., 51(3), 1432-1442, doi:10.4319/1o.2006.51.3.1432.

Schlitzer, R. (2015), Ocean Data View,

Schmidtko, S., G. C. Johnson, and J. M. Lyman (2013), MIMOC: A global monthly isopycnal upperocean climatology with mixed layers, J. Geophys. Res. Ocean., 118(4), 1658-1672, doi:10.1002/jgrc.20122.

Serret, P., C. Robinson, M. Aranguren-Gassis, E. E. García-Martín, N. Gist, V. Kitidis, J. Lozano, J. Stephens, C. Harris, and R. Thomas (2015), Both respiration and photosynthesis determine the scaling of plankton metabolism in the oligotrophic ocean, Nat. Commun., 6 , doi:10.1038/ncomms7961.

Šidák, Z. (1967), Rectangular Confidence Regions for the Means of Multivariate Normal Distributions, $J$. Am. Stat. Assoc., 62(318), 626-633, doi:10.1080/01621459.1967.10482935.

Siegel, D. A., K. O. Buesseler, S. C. Doney, S. F. Sailley, M. J. Behrenfeld, and P. W. Boyd (2014), Global assessment of ocean carbon export by combining satellite observations and food-web models, Global Biogeochem. Cycles, 28(3), 181-196, doi:10.1002/2013GB004743.

Spitzer, W. S., and W. J. Jenkins (1989), Rates of vertical mixing, gas exchange and new production: Estimates from seasonal gas cycles in the upper ocean near Bermuda, J. Mar. Res., 47(1), 169-196, doi:10.1357/002224089785076370.

Stanley, R. H. R., and E. Howard (2013), Quantifying photosynthetic rates of microphytobenthos using the triple isotope composition of dissolved oxygen, Limnol. Oceanogr. Methods, 11, 360-373. 
Stanley, R. H. R., W. J. Jenkins, D. E. Lott, and S. C. Doney (2009), Noble gas constraints on air-sea gas exchange and bubble fluxes, J. Geophys. Res., 114(C11), doi:10.1029/2009JC005396.

Stanley, R. H. R., J. B. Kirkpatrick, N. Cassar, B. A. Barnett, and M. L. Bender (2010), Net community production and gross primary production rates in the western equatorial Pacific, Global Biogeochem. Cycles, 24(4), doi:10.1029/2009GB003651.

Steinberg, D. K., C. A. Carlson, N. R. Bates, S. A. Goldthwait, L. P. Madin, and A. F. Michaels (2000), Zooplankton vertical migration and the active transport of dissolved organic and inorganic carbon in the Sargasso Sea, Deep Sea Res. Part I Oceanogr. Res. Pap., 47(1), 137-158, doi:10.1016/S09670637(99)00052-7.

Steinberg, D. K., C. A. Carlson, N. R. Bates, R. J. Johnson, A. F. Michaels, and A. H. Knap (2001), Overview of the US JGOFS Bermuda Atlantic Time-series Study (BATS): a decade-scale look at ocean biology and biogeochemistry, Deep Sea Res. Part II Top. Stud. Oceanogr., 48(8), 1405-1447, doi:10.1016/S0967-0645(00)00148-X.

Stramma, L., and F. Schott (1999), The mean flow field of the tropical Atlantic Ocean, Deep Sea Res. Part II Top. Stud. Oceanogr., 46(1-2), 279-303, doi:10.1016/S0967-0645(98)00109-X.

Stukel, M. R., M. Kahru, C. R. Benitez-Nelson, M. Décima, R. Goericke, M. R. Landry, and M. D. Ohman (2015), Using Lagrangian-based process studies to test satellite algorithms of vertical carbon flux in the eastern North Pacific Ocean, J. Geophys. Res. Ocean., 120(11), 7208-7222, doi:10.1002/2015JC011264.

Stukel, M. R., and M. R. Landry (2010), Contribution of picophytoplankton to carbon export in the equatorial Pacific: A reassessment of food web flux inferences from inverse models, Limnol. Oceanogr., 55(6), 2669-2685, doi:10.4319/LO.2010.55.6.2669.

Swalwell, J. E., F. Ribalet, and E. V. Armbrust (2011), SeaFlow: A novel underway flow-cytometer for continuous observations of phytoplankton in the ocean, Limnol. Oceanogr. Methods, 9(10), 466-477, doi:10.4319/1om.2011.9.466.

Sweeney, E. N., D. J. McGillicuddy, and K. O. Buesseler (2003), Biogeochemical impacts due to mesoscale eddy activity in the Sargasso Sea as measured at the Bermuda Atlantic Time-series Study (BATS), Deep Sea Res. Part II Top. Stud. Oceanogr., 50(22), 3017-3039, doi:10.1016/j.dsr2.2003.07.008.

Świrgoń, M., and M. Stramska (2015), Comparison of in situ and satellite ocean color determinations of particulate organic carbon concentration in the global ocean, Oceanologia, 57(1), 25-31, doi:10.1016/j.oceano.2014.09.002.

Takahashi, T. et al. (2009), Climatological mean and decadal change in surface ocean pCO2, and net seaair CO2 flux over the global oceans, Deep Sea Res. Part II Top. Stud. Oceanogr., 56(8), 554-577, doi:10.1016/j.dsr2.2008.12.009. 
Thomalla, S., R. Turnewitsch, M. Lucas, and A. Poulton (2006), Particulate organic carbon export from the North and South Atlantic gyres: The 234Th/238U disequilibrium approach, Deep Sea Res. Part II Top. Stud. Oceanogr., 53(14), 1629-1648, doi:10.1016/j.dsr2.2006.05.018.

Tilstone, G., T. Smyth, A. Poulton, and R. Hutson (2009), Measured and remotely sensed estimates of primary production in the Atlantic Ocean from 1998 to 2005, Deep Sea Res. Part II Top. Stud. Oceanogr., 56(15), 918-930, doi:10.1016/j.dsr2.2008.10.034.

Trujillo-Ortiz, A., R. Hernandez-Walls, K. Barba-Rojo, and A. Castro-Perez (2007), ANDARTEST Anderson-Darling test for assessing normality of a sample data.,

Tsuchiya, M., L. D. Talley, and M. S. McCartney (1994), Water-mass distributions in the western South Atlantic; A section from South Georgia Island (54S) northward across the equator, J. Mar. Res., 52(1), 55-81, doi:10.1357/0022240943076759.

Uitz, J., H. Claustre, B. Gentili, and D. Stramski (2010), Phytoplankton class-specific primary production in the world's oceans: Seasonal and interannual variability from satellite observations, Global Biogeochem. Cycles, 24(3), n/a-n/a, doi:10.1029/2009GB003680.

Vaillancourt, R. D., J. Marra, M. P. Seki, M. L. Parsons, and R. R. Bidigare (2003), Impact of a cyclonic eddy on phytoplankton community structure and photosynthetic competency in the subtropical North Pacific Ocean, Deep Sea Res. Part I Oceanogr. Res. Pap., 50(7), 829-847, doi:10.1016/S09670637(03)00059-1.

Vallina, S. M., M. J. Follows, S. Dutkiewicz, J. M. Montoya, P. Cermeno, and M. Loreau (2014), Global relationship between phytoplankton diversity and productivity in the ocean, Nat. Commun., 5, doi:10.1038/ncomms5299.

Volk, T., and M. I. Hoffert (1985), The Carbon Cycle and Atmospheric CO2: Natural Variations Archean to Present, Geophysical Monograph Series, edited by E. T. Sundquist and W. S. Broecker, American Geophysical Union, Washington, D. C.

Wanninkhof, R. (2014), Relationship between wind speed and gas exchange over the ocean revisited, Limnol. Oceanogr. Methods, 12(6), 351-362, doi:10.4319/lom.2014.12.351.

Westberry, T., M. J. Behrenfeld, D. A. Siegel, and E. Boss (2008), Carbon-based primary productivity modeling with vertically resolved photoacclimation, Global Biogeochem. Cycles, 22(2), n/a-n/a, doi:10.1029/2007GB003078.

Williams, P. J. L. (1993), On the definition of plankton production terms, ICES Mar. Sci. Symp., 197, 919.

Williams, P. J. 1. B., and D. Lefèvre (1996), Algal 14 C and total carbon metabolisms. 1. Models to account for the physiological processes of respiration and recycling, J. Plankton Res., 18(10), 19411959, doi:10.1093/plankt/18.10.1941. 
Wilson, C., T. A. Villareal, N. Maximenko, S. J. Bograd, J. P. Montoya, and C. A. Schoenbaechler (2008), Biological and physical forcings of late summer chlorophyll blooms at $30^{\circ} \mathrm{N}$ in the oligotrophic Pacific, J. Mar. Syst., 69(3), 164-176, doi:10.1016/j.jmarsys.2005.09.018.

Wunsch, C., and R. Ferrari (2004), VERTICAL MIXING, ENERGY, AND THE GENERAL CIRCULATION OF THE OCEANS, Annu. Rev. Fluid Mech., 36(1), 281-314, doi:10.1146/annurev.fluid.36.050802.122121.

Xing, X., A. Morel, H. Claustre, D. Antoine, F. D'Ortenzio, A. Poteau, and A. Mignot (2011), Combined processing and mutual interpretation of radiometry and fluorimetry from autonomous profiling BioArgo floats: Chlorophyll a retrieval, J. Geophys. Res., 116(C6), C06020, doi:10.1029/2010JC006899.

Yeung, L. Y. et al. (2012), Impact of diatom-diazotroph associations on carbon export in the Amazon River plume, Geophys. Res. Lett., 39(18), doi:10.1029/2012GL053356.

Yoder, J. A., S. G. Ackleson, R. T. Barber, P. Flament, and W. M. Balch (1994), A line in the sea, Nature, 371(6499), 689-692, doi:10.1038/371689a0.

Zehr, J. P., E. J. Carpenter, and T. A. Villareal (2000), New perspectives on nitrogen-fixing microorganisms in tropical and subtropical oceans, Trends Microbiol., 8(2), 68-73, doi:10.1016/S0966-842X(99)01670-4.

Zhang, X., and H. G. Dam (1997), Downward export of carbon by diel migrant mesozooplankton in the central equatorial Pacific, Deep Sea Res. Part II Top. Stud. Oceanogr., 44(9-10), 2191-2202, doi:10.1016/S0967-0645(97)00060-X.

Anon (n.d.), Ssalto/Duacs satellite altimetry data, Available from: http://www.aviso.altimetry.fr/duacs/

\section{Chapter 3}

Abe, O., and N. Yoshida. 2003. Partial pressure dependency of 17O/16O and 18O/16O of molecular oxygen in the mass spectrometer. Rap. Comm. Mass Spec. 17: doi:10.1002/rcm.923

Abril, G., M.V. Commarieu, A. Sottolichio, P. Bretel, and F. Guerin. 2009. Turbidity limits gas exchange in a large macrotidal estuary. Est. Coast. Shelf Sci. 83: doi:10.1016/j.ecss.2009.03.006

Angert, A., S. Rachmilevitch, E. Barkan, and B. Luz. 2003. Effects of photorespiration, the cytochrome pathway, and the alternative pathway on the triple isotopic composition of atmospheric $\mathrm{O} 2$. Global Biogeochem. Cycles 17, doi:10.1029/2002GB001933

Ashworth, J., S. Coesel, A. Lee, E.V. Armbrust, M.V. Orellana, and N.S. Baliqa. 2013. Genome-wide diel growth state transitions in the diatom Thalassiosira pseudomona. Proc. Natl. Acad. Sci. 110(18): 7518-7523.

Attard, K.M., R.N. Glud, D.F. McGinnis, and S. Rysgaard. 2014. Seasonal rates of benthic primary production in a Greenland fjord measured by aquatic eddy correlation. Limnol. Oceanogr. 59(5): 1555-1569. 
Barkan, E., and B. Luz. 2003. High-precision measurements of $17 \mathrm{O} / 16 \mathrm{O}$ and $18 \mathrm{O} / 16 \mathrm{O}$ of O2 and O2/Ar ratio in air. Rap. Comm. Mass Spec. 17: doi:10.1002/rcm.1267

Barkan, E., and B. Luz. 2011. The relationships among the three stable isotopes of oxygen in air, seawater and marine photosynthesis. Rapid Commun. Mass Spectrom. 25: 2367-9.

Baschek, B., D.M. Farmer, and C. Garrett. 2006. Tidal fronts and their role in air-sea gas exchange. J. Mar. Res. 64: 483-515.

Berg, P., H. Røy, F. Janssen, V. Meyer, B. Jørgensen, M. Huettel, and D. de Beer. 2003. Oxygen uptake by aquatic sediments measured with a novel non-invasive eddy-correlation technique. Mar. Ecol. Prog. Ser. 261: $75-83$.

Blanchard, G.F., and P.A. Montagna. 1992. Photosynthetic response of natural assemblages of marine benthic microalgae to short- and long-term variations of incident irradiance in Baffin Bay, Texas. J. Phycol. 28: 7-14.

Borges, A.V., B. Delille, and L-S. Schiettecatte. 2004a. Gas exchange velocities of CO2 in three European estuaries (Randers Fjord, Scheldt, and Thames).Limnol. Oceanogr. 49: 1630-1641.

Borges, A.V., J-P. Vanderborght, L-S. Schiettecatte, F. Gazeau, S. Ferrón-Smith, B. Delille, and M. Frankignouille. 2004b. Variability of the gas transfer velocity of $\mathrm{CO} 2$ in a macrotidal estuary (the Scheldt). Estuaries 27: 593-603.

Bowen, J., B. Crump, L. Deegan, and J. Hobbiec. 2009. Increased supply of ambient nitrogen has minimal effect on salt marsh bacterial production. Limnol. Oceanogr. 54: 713-722.

Bowen, J., B.B. Ward, H.G. Morrison, J.E. Hobbie, I. Valiela, L.A. Deegan, and M.L. Sogin. 2011. Microbial community composition in sediments resists perturbation by nutrient enrichment. The ISME J. 5: 1540-1548.

Brewer, P.G., A.F. Hofmann, E.T. Peltzer, and W. Ussler III. 2014. Evaluating microbial chemical choices: The ocean chemistry basis for the competition between use of $\mathrm{O} 2$ or NO3- as an electron ecceptor. Deep Sea Res. I 87: 35-42.

Bromberg Gedan, K., B.R. Silliman, and M. D. Bertness. 2009. Centuries of Human-Driven Change in Salt Marsh Ecosystems. Ann. Rev. Mar. Sci. 1: 117-141.

Brotas, V., C R. Mendes, and P. Cartaxana. 2007. Microphytobenthic biomass assessment by pigment analysis: comparison of spectrophotometry and High Performance Liquid Chromatography methods. Hydrobiologia 587: 19-24.

Caffrey, J.M. 2003. Production, respiration, and net ecosystem metabolism in U.S. estuaries. Environ. Monitor. Assess. 81: 207-219.

Caffrey, J M. 2004. Factors controlling net ecosystem metabolism in US estuaries. Estuaries 27: p.90-101

Cahoon, L.B. 1999. The role of benthic microalgae in neritic ecosystems, p. 47-86. In A. D. Ansell, R. N. Gibson, and M. Barnes [eds.], Oceanography and marine biology, an annual review, v. 37. Taylor and Francis.

Cai, W.J. 2011. Estuarine and coastal ocean carbon paradox: CO2 sinks or sites of terrestrial carbon incineration? Ann. Rev. Mar. Sci. 3: 123-145. 
Cebrian, J., C. D. Foster, R. Plutchak, K. L. Sheehan, M.-E. C. Miller, A. Anton, K. Major, K. L. Heck, and S. P. Powers. 2007. The impact of Hurricane Ivan on the primary productivity and metabolism of marsh tidal creeks in the NorthCentral Gulf of Mexico. Aquat. Ecol. 42: 391-404.

Chanton, J.P., C.S. Martens, and C.A. Kelley. 1989. Gas transport from methane-saturated, tidal freshwater and wetland sediments. Limnol. Oceanogr. 34: 807-819.

Chanton, J.P., and G.J. Whiting. 1995. Trace gas exchange in freshwater and coastal marine environments: ebullition and transport by plants, p. 98-125. In P.A. Matson and R.C. Harriss [eds.], Biogenic Trace Gases: Measuring Emissions from Soil and Water, Blackwell Science, Oxford.

Charette, M. A. 2007. Hydrologic forcing of submarine groundwater discharge: Insight from a seasonal study of radium isotopes in a groundwater-dominated salt marsh estuary. Limnol. Oceanogr. 52: 230 239. doi:10.4319/10.2007.52.1.0230

Cole, J.J., D.L. Bade, D. Bastviken, M.L. Pace, and M. Van de Bogert. 2010. Multiple approaches to estimating air-water gas exchange in small lakes. Limnol. Oceanogr. Methods 8: 285-293.

Consalvey, M., D.M. Paterson, and G.J.C. Underwood. 2004. The ups and downs of life in a benthic biofilm: migration of benthic diatoms. Diatom Res. 19(2): 181-202.

Cook, P., B. Veuger, S. Böer, and J. J. Middelburg. 2007. Effect of nutrient availability on carbon and nitrogen incorporation and flows through benthic algae and bacteria in near-shore sandy sediment. Aquat. Microb. Ecol. 49: 165-180.

Cosper, E. 1982. Influence of light intensity on diel variations in rates of growth, respiration, and organic release of a marine diatom: comparison of diurnally constant and fluctuating light. J. Plankton Res. 4: 705-724.

Craig, H., and T. Hayward. 1987. Oxygen supersaturation in the ocean: biological versus physical contributions. Science 235(4785): 199-202.

Crosswell, J.R. 2015. Bubble clouds in coastal waters and their role in air-water gas exchange of CO2. J. Mar. Sci. Eng. 3: 866-890.

Davey, E., C. Wigand, R. Johnson, K. Sundberg, J. Morris, and C. T. Roman. 2011. Use of computed tomography imaging for quantifying coarse roots, rhizomes, peat, and particle densities in marsh soils. Ecol. Appl. 21: 2156-2171.

Deegan, L. A. 2002. Lessons learned: The effects of nutrient enrichment on the support of nekton by seagrass and salt marsh ecosystems. Estuaries 25: 727-742.

Deegan, L. A., J. L. Bowen, D. Drake, J. W. Fleeger, C. T. Friedrichs, K. A. Galván, J. E. Hobbie, C. Hopkinson, D. S. Johnson, J. M. Johnson, L. E. LeMay, E. Miller, B. J. Peterson, C. Picard, S. Sheldon, M. Sutherland, J. Vallino, and R. S. Warren. 2007. Susceptibility of salt marshes to nutrient enrichment and predator removal. Ecol. Appl. 17: S42-S63.

Deegan, L. A., D. S. Johnson, R. S. Warren, B. J. Peterson, J. W. Fleeger, S. Fagherazzi, and W. M. Wollheim. 2012. Coastal eutrophication as a driver of salt marsh loss. Nature 490: 388-92.

Dring, M. J., and D. H. Jewson. 1982. What Does 14C Uptake by Phytoplankton Really Measure? A Theoretical Modelling Approach. Proc. R. Soc. B Biol. Sci. 214: 351-368.

Dromgoole, F.I. 1978. The effects of oxygen on dark respiration and apparent photosynthesis of marine macro-algae. Aquatic Bot. 4: 281-297. 
Duarte, B., J. Freitas, J. Valentim, J.P. Medeiros, J.L. Costa, H. Silva, J.M. Dias, M.J. Costa, J.C. Marques, and I. Cacador. 2014. Abiotic control modelling of salt marsh sediments respiratory CO2 fluxes: application to increasing temperature scenarios. Ecological Indicators 46: 100-118.

Duarte, C. M., I. J. Losada, I. E. Hendriks, I. Mazarrasa, and N. Marbà. 2013. The role of coastal plant communities for climate change mitigation and adaptation. Nat. Clim. Chang. 3: 961-968.

Duarte, C. M., J. J. Middelburg, and N. Caraco. 2005. Major role of marine vegetation on the oceanic carbon cycle. Biogeosciences 2, doi:10.5194/bg-2-1-2005

Dutton, A., B.H. Wilkinson, J.M. Welker, G.J. Bowen, and K.C. Lohmann. 2005. Spatial distribution and seasonal variation in $180 / 160$ of modern precipitation and rover water across the conterminous USA. Hydrol. Process. 19(20): 4121-4146.

Eisenstadt, S., E. Barkan, and A. Kaplan. 2010. Enrichment of oxygen heavy isotopes during photosynthesis in phytoplankton. Photosynth. Res. 103(2): 97-103.

Engelsen, A., S. Hulth, L. Pihl, and K. Sundbäck. 2008. Benthic trophic status and nutrient fluxes in shallow-water sediments. Estuar. Coast. Shelf Sci. 78: 783-795.

Fagherazzi, S., P.L. Wiberg, S. Temmerman, E. Struyf, Y. Zhao, and P. A. Raymond. 2013. Fluxes of water, sediments, and biogeochemical compounds in salt marshes. Ecol. Proc. 2(3): 1-16.

Fenchel, T., and R. N. Glud. 2000. Benthic primary production and O2-CO2 dynamics in a shallow-water sediment: Spatial and temporal heterogeneity. Ophelia 53: 159-171.

Fisher, N.L., and K.H. Halsey. 2016. Mechanisms that increase the growth efficiency of diatoms in low light. Photosynt. Res. 129(2): 183-197.

Galván, K., J. W. Fleeger, and B. Fry. 2008. Stable isotope addition reveals dietary importance of phytoplankton and microphytobenthos to saltmarsh infauna. Marine 359: 37-49.

Galván, K., J. W. Fleeger, B. Peterson, D. Drake, L. A. Deegan, and D. S. Johnson. 2011. Natural abundance stable isotopes and dual isotope tracer additions help to resolve resources supporting a saltmarsh food web. J. Exp. Mar. Bio. Ecol. 410: 1-11.

Ganju, N.K., M. Hayn, S-N. Chen, R.W. Howarth, P.J. Dickhudt, A.L. Aretxabaleta, and R. Marino. 2012. Tidal and groundwater fluxes to a shallow, microtidal estuary: Constraining inputs through fieldd observations and hydrodynamic modeling. Estuaries and Coasts 35: doi:10.1007/s12237-0129515-X

Ganju, N.K., D.H. Schoellhamer, and B.A. Bergamaschi. 2005. Suspended sediment fluxes in a tidal wetland: Measurement, controlling factos, and error analysis. Estuaries 28: 812-822.

Gardner, L.R. 2005. Role of geomorphic and hydraulic parameters in governing pore water seepage from salt marsh sediments. Wat. Resour. Res. 41(7): doi:10.1029/2004WR003671

Gardner, L.R., and E.F. Gaines. 2008. A method for estimating pore water drainage from marsh soils using rainfall and well records. Estuar. Coast. Shelf Sci. 79: 51-58.

Gattuso, J.P., M. Frankignoulle, and R. Wollast. 1998. Carbon and carbonate metabolism in coastal aquatic ecosystems. Annu. Rev. Ecol. Syst. 29: 405-434.

Gattuso, J.P., B. Gentili, C.M. Duarte, H.A. Kleypas, J.J. Middelburg, and D. Antoinne. 2006. Light availability in the coastal ocean: impact on the distribution of benthic photosynthetic organisms and their contribution to primary production. Biogeosci. 3: 489-513. 
Gazeau, F. P. H., J. J. Middelburg, M. Loijens, J. P. Vanderborght, M.-D. Pizay, and J. P. Gattuso. 2007. Planktonic primary production in estuaries: a comparison of the $14 \mathrm{C}, \mathrm{O} 2$ and $18 \mathrm{O}$ methods. Aquat. Microb. Ecol. 46: 96-106.

Glazer, B.T., A.G. Marsh, K. Stierhoff, and G.W. Luther, III. The dynamic response of optical oxygen sensors and voltammetric electrodes to temporal changes in dissolved oxygen concentrations. 2004. Analytica Chimica Acta 518: 93-100.

Glud, R.N., M. Kuhl, F. Wenzhofer, and S. Rysgaard. 2002. Benthic diatoms of a high Arctic fjord (Young Sound, NE Greenland): importance for ecosystem primary production. Mar. Ecol. Prog. Ser. 238: $15-29$.

Halsey, K.H., A.J. Milligan, and M.J. Behrenfeld. 2010. Physiological optimization underlies growth rateindependent chlorophyll-specific gross and net primary production. Photosynth. Res. 103(2): 125137.

Halsey, K.H., A.J. Milligan, and M.J. Behrenfeld. 2014. Contrasting strategies of photosynthetic energy utilization drive lifestyle strategies in ecologically important eukaryotes. Metabolites 4(2): 260-280.

Halsey, K.H., R.T. O’Malley, J.R. Graff, A.J. Milligan, and M.J. Behrenfeld. 2013. A common partitioning strategy for photosynthetic products in evolutionarily distinct phytoplankton species. New Phytologist 198(4): 1030-1038.

Hamme, R.C., and S. R. Emerson. 2004. The solubility of neon, nitrogen, and argon in distilled water and seawater. Deep-Sea Res. I 51: 1517-1528.

Hamme, R.C., and S.R. Emerson. 2006. Constraining bubble dynamics and mixing with dissolved gases: Implications for productivity measurements by oxygen mass balance. J. Mar. Res. 64: doi:10.1357/002224006776412322

Happey-Wood, C.M., and P. Jones. Rhytms of vertical migration and motility in intertidal benthic diatoms with particular reference to Pleurosigma angulatum. Diatom Res. 3(1): 83-93.

Harrison, E.L., F. Veron, D.T. Ho, M.C. Reid, P.M. Orton, W.R. McGillis. 2012. Nonlinear interaction between rain and wind induced air-water gas exchange. J. Geophys. Res. 117: doi:10.1029/2011JC007693

Harvey, J.W., P.G. Germann, and W.E. Odum. 1987. Geomorphological control of subsurface hydrology in the creekbank zone fo tidal marshes. Estuar. Coast. Shelf Sci. 25: 677-691.

Heaton, T.H.E., and J.C. Vogel. 1981. "Excess air” in groundwater. J. Hydrol. 50: 201-216.

Helman, Y., E. Barkan, D. Eisenstadt, and A. Kaplan. 2005. Fractionation of the three stable oxygen isotopes by oxygen-producing and oxygen-consuming reactions in photosynthetic organisms. Plant Physiology 138(4): 2292-2298.

Ho, D.T., P. Schlosser, and P.M. Orton. 2011. On factors controlling air-water gas exchange in a large tidal river. Est. Coast. 34: doi:10.1007/s12237-011-9396-4

Hopkinson, C. S., W.-J. Cai, and X. Hu. 2012. Carbon sequestration in wetland dominated coastal systems - a global sink of rapidly diminishing magnitude. Curr. Opin. Environ. Sustain. 4: 186-194.

Hopkinson, C. S., A. E. Giblin, J. Tucker, and R. H. Garritt. 1999. Benthic Metabolism and Nutrient Cycling along an Estuarine Salinity Gradient. Estuaries 22: 863. 
Howarth, R. W. 1984. The ecological significance of sulfur in the energy dynamics of salt marsh and coastal marine sediments. Biogeochemistry 1: 5-27.

Jahnke, R. A., J. R. Nelson, R. L. Marinelli, and J. E. Eckman. 2000. Benthic flux of biogenic elements on the Southeastern US continental shelf: influence of pore water advective transport and benthic microalgae. Cont. Shelf Res. 20: 109-127.

Juranek, L. W., and P. D. Quay. 2013. Using triple isotopes of dissolved oxygen to evaluate global marine productivity. Ann. Rev. Mar. Sci. 5: 503-24.

Kaiser, J. 2011. Technical note: Consistent calculation of aquatic gross production from oxygen triple isotope measurements. Biogeosci. 8: doi:10.5194/bg-8-1793-2011

Kearns, P.J, J.H. Angell, E.M. Howard, L.A. Deegan, R.H.R. Stanley, and J.L. Bowen. In press. Nutrient enrichment induces dormancy and decreases diversity of active bacteria. Nature Comm.

King, G.M., and W.J. Wiebe. 1978. Methane release from soils of a Georgia salt marsh. Geochim. Cosmochim. Acta 42: 343-348.

Klump, S., Y. Tomonaga, P. Kienzler, W. Kinzelbach, T. Baumann, D.M. Imboden, R. Kipfer. 2007. Field experiments yeild new insights into gas exchange and excess air formation in natural porous media. Geochim. Cosmochim. Acta 2007: 1385-1397.

Koop-Jakobsen, K., and A. Giblin. 2009. Anammox in tidal marsh sediments: the role of salinity, nitrogen loading, and marsh vegetation. Estuaries and Coasts 32: 238-245.

Koop-Jakobsen, K., and A. Giblin. 2010. The effect of increased nitrate loading on nitrate reduction via denitrification and DNRA in salt marsh sediments. Limnol. Ocean. 55: 789-802.

Koretsky, C.M, C. Meile, and P.V. Cappellen. 2002. Quantifying bioirigation using ecological parameters: a stochastic approach. Geochem. Trans. 3: 17-30, doi: 10.1186/1467-4866-3-17.

Kremer, J.N., A. Reischauer, and C. D'Avanzo. 2003. Estuary-specific variation in the air-water gas exchange coefficient for oxygen. Estuaries 26: 829-836.

Kromkamp, J. C., E. P. Morris, R. M. Forster, C. Honeywill, S. Hagerthey, and D. M. Paterson. 2006. Relationship of intertidal surface sediment chlorophyll concentration to hyperspectral reflectance and chlorophyll fluorescence. Estuaries and Coasts 29: 183-196.

Laws, E.A. 1991. Photosynthetic quotients, new production and net community production in the open ocean. Deep-Sea Res. 38(1): 143-167.

Lellis-Dibble, K. A., K. E. McGlynn, and T. E. Bigford. 1998. Estuarine Fish and Shellfish Species in U.S. Commercial and Recreational Fisheries: Economic Value as an Incentive to Protect and Restore Estuarine Habitat. USDC NOAA Tech. Mem. NMFS-F/SPO-90

Li, S., N.E. Levin, and L.A. Chesson. 2015. Continental scale variation in 17O-excess of meteoric waters in the United States. Geochim. Cosmochim. Acta 164: 110-126.

Longmuir, I.S. 1954. Respiration rate of bacteria as a function of oxygen concentration. Biochem. J. 57(1): 81-87.

Luciano, K.E. 2007. Investifation into the relationship between morphology and tidal flux in ditched and non-ditched salt marsh creek systems, Rowley, Massachusetts. BS thesis, Virginia Institute of Marine Science. 
Luz, B., and E. Barkan. 2000. Assessment of Oceanic Productivity with the Triple-Isotope Composition of Dissolved Oxygen. Science (80-. ). 288: 2028-2031.

Luz, B., and E. Barkan. 2005. The isotopic ratios $17 \mathrm{O} / 16 \mathrm{O}$ and $18 \mathrm{O} / 16 \mathrm{O}$ in molecular oxygen and their significance in biogeochemistry. Geochim. Cosmochim. Acta 69: 1099-1110.

Luz, B., and E. Barkan. 2009. Net and gross oxygen production from O2/Ar, 17O/16O and 18O/16O ratios. Aquat. Microb. Ecol. 56: 133-145.

Luz, B., and E. Barkan. 2010. Variations of $17 \mathrm{O} / 16 \mathrm{O}$ and $18 \mathrm{O} / 16 \mathrm{O}$ in meteoric waters. Geochim. Cosmochim. Acta 74(22): 6276-6286.

Luz, B., and E. Barkan. 2011. Proper estimation of marine gross O2 production with $17 \mathrm{O} / 16 \mathrm{O}$ and 18O/16O ratios of dissolved oxygen. Geophys. Res. Lett. 38(19): doi:10.1029/2011GL049138.

MacIntyre, H. L., R. J. Geider, and D. C. Miller. 1996. Microphytobenthos: The Ecological Role of the "Secret Garden" of Unvegetated, Shallow-Water Marine Habitats. I. Distribution, Abundance and Primary Production. Estuaries 19: 186.

McGlathery, K., K. Sundbäck, and I. Anderson. 2004. The importance of primary producers for the benthic nitrogen and phosphorus cycling., p. 231-261. In S.L. Nielsen, G.T. Banta, and M.F. Pedersen [eds.], The influence of Primary Producers on Estuarine Nutrient Cycling. Aquatic Ecological Progress Series.

Mcleod, E., G. L. Chmura, S. Bouillon, R. Salm, M. Björk, C. M. Duarte, C. E. Lovelock, W. H. Schlesinger, and B. R. Silliman. 2011. A blueprint for blue carbon: toward an improved understanding of the role of vegetated coastal habitats in sequestering CO 2. Front. Ecol. Environ. 9: $552-560$.

Middelburg, J. J., C.M. Duarte, and J.P. Gattuso. 2004. Respiration in coastal benthic communities. In P.A. del Giorgio, and P.J. le B. Williams [eds.], Respiration in aquatic ecosystems, Oxford University Press.

Millero, F.J., R. Feistel, D.G. Wright, and T.J. McDougall. 2008. The composition of standard seawater and the definition of the reference-composition salinity scale. Deep-Sea Res. I 55: 50-72.

Morlock, S.E., H.T. Nguyen, and J.H. Ross. 2002. Feasibility of acoustic doppler veloccity meters for the production of discharge records from U.S. Geological Survey streamflow-gaging stations. USGS water-res. invest. rep. 01-4157

Morris, J. T., K. Sundberg, and C. S. Hopkinson. 2013. Salt marsh primary production and its responses to relative sea level and nutrients in estuaries at Plum Island, Massachusetts, and North Inlet, South Carolina, USA. Oceanography 26: 78-84.

Munro, D.R., P.D. Quay, L.W. Juranek, and R. Goericke. 2013. Biological production rates off the Southern California coast estimated from triple $\mathrm{O} 2$ isotopes and $\mathrm{O} 2$ :Ar gas ratios. Limnol. Oceanogr. 58(44): 1312-1328.

Nepf, H.M. 2012. Flow and transport in regions with aquatic vegetation. Annu. Rev. Fluid Mech. 44: $123-142$.

Nicholson, D. 2011b. Comment on: Technical note: Consistent calculation of aquatic gross production from oxygen triple isotope measurements by Kaiser (2011). Biogeosci. 8: 2993-2997. 
Nicholson, D., S. Emerson, S. Khatiwala, and R. C. Hamme. 2011a. An inverse approach to estimate bubble-mediated air-sea gas flux from inert gas measurements. Proceedings on the 6th Intl. Symp. on Gas Transfer at Water Surfaces. Kyoto University Press.

Nicholson, D., R.H.R. Stanley, and S.C. Doney. 2014. The triple oxygen isotope tracer of primary productivity in a dynamic ocean model. Glob. Biogeochem. Cycles 28(5): 538-552.

Nidzieko, N.J., J.A. Needoba, S.G. Monismith, K.S. Johnson. 2014. Fortnightly tidal modulations affect net community production in a mesotidal estuary. Estuaries and Coasts 37(suppl 1): S91-S110.

Ní Longphuirt, S., J. Clavier, J. Grall, L. Chauvaud, F. Le Loc'h, I. Le Berre, J. Flye-Sainte-Marie, J. Richard, and A. Leynaert. 2007. Primary production and spatial distribution of subtidal microphytobenthos in a temperate coastal system, the Bay of Brest, France. Est. Coast. Shelf Sci. 74(3): 367-380.

Nuttle, W.K. 1988. The extent of lateral water movement in the sediments of a New England salt marsh. Wat. Resources Res. 24: 2077-2085.

Nuttle, W.K., and H.F. Hemond. 1988. Salt marsh hydrology: Implications for biogeochemical fluxes to the atmosphere and estuaries. Glob. Biogeochem. Cycles 2: 91-114.

Odum, H. T. 1956. Primary production in flowing waters. Limnol. Oceanogr. 1: 102-11.

Pascal, P.-Y., J. W. Fleeger, H. T. S. Boschker, H. M. Mitwally, and D. S. Johnson. 2013. Response of the benthic food web to short- and long-term nutrient enrichment in saltmarsh mudflats. Mar. Ecol. Prog. Ser. 474: 27-41.

Peckol, P., and J.S. Rivers. 1996. Contribution by macroalgal mats to primary production of a shallow embayment under high and low nitrogen-loading rates. Estuar. Coastal Shelf Sci. 43: 311-325.

Pinckney, J., and R.G. Zingmark. 1993. Photophysiological responses of intertidal benthic microalgal communities to in situ light environments: Methodological considerations. Limnol. Oceanogr. 38(7): 1373-1383.

Pringault, O., V. Tassas, and E. Rochelle-Newall. 2007. Consequences of respiration in the light on the determination of production in pelagic systems. Biogeosciences 4: 105-114.

Prokopenko, M.G., O.M. Pailuis, J. Granger, and L.Y. Yeung. 2011. Exact evaluation of gross photosynthetic porduction from the oxygen triple-isotope composition of O2: Implications for the netto-gross primary production ratios. Geophys. Res. Lett. 38(14): doi:10.1029/2011GL047652

Rao, A.M.F., and M.A. Charette. 2011. Benthic nitrogen fixation in an eutrophic estuary affected by groundwater discharge. J. Coast. Res. 28(2): 477-485.

Raupach, M.R. 1994. Simplified expressions for vegetations roughness length and zero-plane displacement as function of canopy height and area index. Boundary-layer Meteor. 71: 211-216.

Raymond, P.A., and J. J. Cole. 2001. Gas exchange in rivers and estuaries: Choosing a gas transfer velocity. Estuaries 24: doi:10.2307/1352954

Revsbech, N. P., and B. B. Jørgensen. 1983. Photosynthesis of benthic microflora measured with high spatial resolution by the oxygen microprofile method: Capabilities and limitations of the method. Limnol. Oceanogr. 28: 749-756. 
Round, F.E., and J.D. Palmer. 1966. Persistant, vertical-migration rythms in benthic microflora: ::. Field and laboratory studies on diatoms from the banks of the River Avon. J. Mar. Biol. Assoc. U.K. 46(1): 191-214.

Sand-Jensen, K., and S. Nielsen. 2004. Estuarine primary producers, p. 17-57. In S.L. Nielsen, G.T. Banta, and M.F. Pedersen [eds.], Estuarine nutrient cycling: the influence of primary producers: the fate of nutrients and biomass. Aquatic Ecological Progress Series.

Sarma, V.V.S.S., O. Abe, and T. Saino. 2008. Spatial variations in time-integrated plankton metabolic rates in Sagami Bay using triple oxygen isotopes and O2:Ar ratios. Limnol. Oceanogr. 53(5): 17761783.

Shepard, C. C., C. M. Crain, and M. W. Beck. 2011. The protective role of coastal marshes: a systematic review and meta-analysis. PLoS One 6: e27374.

Spitzer, W S, and W J Jenkins. 1989. Rates of vertical mixing, gas-exchange and new production: Estimates from seasonal gas cycles in the upper ocean near Bermuda, J. Mar. Res., 47: doi: $10.1357 / 002224089785076370$

Spivak, A., and J. Ossolinski. 2016. Limited effects of nutrient enrichment on bacterial carbon sources in salt marsh tidal creek sediments. Mar. Ecol. Prog. Ser. 544: 107-130. doi:10.3354/meps11587

Stanley, R.H.R., B. Baschek, D.E. Lott III, W.J. Jenkins. 2009b. A new automated method for measuring noble gases and their isotopic ratios in water samples. Geochem. Geophys. Geosys. 10: doi:10.1029/2009GC002429

Stanley, R.H.R., and E.M. Howard. 2013. Quantifying photosynthetic rates of microphytobenthos using the triple isotope composition of dissolved oxygen. Limnol. Oceanogr. Methods 11: 360-373.

Stanley, R.H.R., W.J. Jenkins, D.E. Lott III, and S.C. Doney. 2009a. Noble gas constraints on air-sea gas exchange and bubble fluxes. J. Geophys. Res. 114: doi:10.1029/2009JC005396

Stevens, M.R. P. Diaz, and D.E. Smits. 2007. Evaluation of the acoustic doppler velocity meter for computation of discharge records of the three sites in Colorado, 2004-2005. USGS sci. invest. rep. 2007-5236

Sundbäck, K., E. Lindehoff, and E. Granéli. 2011. Dissolved organic nitrogen: an important source of nitrogen for the microphytobenthos in sandy sediment. Aquat. Microb. Ecol. 63: 89-100.

Teal, J. M. 1962. Energy Flow in the Salt Marsh Ecosystem of Georgia. Ecology 43: 614.

Tengberg, A., P.O.J. Hall, U. Andersson, B. Linden, O. Styrenius, G. Boland, F. de Bovee, B. Carlsson, S. Ceradini, A. Devol, G. Duineveld, J.U. Friemann, R.N. Glud, A. Khripounoff, J. Leather, P. Linke, L. Lund-Hansen, G. Rowe, P. Santschi, P. de Wilde, U. Witte. 2005. Intercalibration of benthic flux chamebrs: II. Hydrodynamic characterization and flux comparisons of 14 different designs. Mar. Chem. 94(1-4): 147-173.

Thamdrup, B., J.W. Hansen, B.B. Jorgensen. 1998. Temperature dependence of aerobic respiration in a coastal sediment. FEMS Microbio. Ecol. 25: 189-200.

Thiemens, M. H., S. Chakraborty, and G. Dominguez. 2012. The physical chemistry of mass-independent isotope effects and their observation in nature. Annu. Rev. Phys. Chem. 63: 155-77.

Thiemens, M. H., and J. E. Heidenreich. 1983. The mass-independent fractionation of oxygen: a novel isotope effect and its possible cosmochemical implications. Science 219: 1073-5. 
Tobias, C., A.E. Giblin, J. McClelland, J. Tucker, B. Peterson. 2003. Sediment DIN fluxes and preferential recycling of benthic microalgal nitrogen in a shallow macrotidal estuary. Mar. Ecol. 257: 25-36.

Tobias, C., and S. C. Neubauer. 2009. Salt marsh biogeochemistry - an overview, p. 445-492. In G. Perillo, E. Wolanski, D.R. Cahoon, and M.M. Brinson [eds.], Coastal wetlands: an integrated ecosystem approach. Elsevier.

Turner, R. E. 2010. Beneath the Salt Marsh Canopy: Loss of Soil Strength with Increasing Nutrient Loads. Estuaries and Coasts 34: 1084-1093.

Turner, R. E., B. L. Howes, J. M. Teal, C. S. Milan, E. M. Swenson, and D. D. Goehringer-Toner. 2009. Salt marshes and eutrophication: An unsustainable outcome. Limnol. Oceanogr. 54: 1634-1642.

Tyler, A., K. McGlathery, and I. Anderson. 2003. Benthic algae control sediment-water column fluxes of organic and inorganic nitrogen compounds in a temperate lagoon. Limnol. Ocean. 48: 2125-2137.

Valiela, I. 1995. Marine Ecological Processes, Springer.

Valiela, I., and J. M. Teal. 1979. The nitrogen budget of a salt marsh ecosystem. Nature 280: 652-656.

Vallino, J. J., C. S. Hopkinson, and R. H. Garritt. 2005. Estimating estuarine gross production, community respiration and net ecosystem production: a nonlinear inverse technique. Ecol. Modell. 187: 281-296.

Van Raalte, C., I. Valiela, J. M. Teal, and D. Van Raalte. 1976. Production of epibenthic salt marsh algae : Light and nutrient limitation. Limnol. Oceanogr. 21: 862-872.

Vieillard, A.M., R.W. Fulweiler, Z.J. Hughes, and J.C. Carey. 2011. The ebb and flood of silica: Quantifying dissolved and biogenic silica fluxes from a temperate salt marsh. Est. Coast. Shelf Sci. 4: 415-423.

Voulgaris, G., and S.T. Meyers. 2004. Temporal variability of hydrodynamics, sediment concentration and sediment settling velocity in a tidal creek. Cont. Sheld Res. 24: 1659-1683.

Wanninkhof, R. 1992. Relationship between wind speed and gas exchange over the ocean. J. Geophys. Res. 97: doi: 10.1029/92JC00188

Weiss, R F. 1971. Solubility of Helium and Neon in Water and Seawater. J. Chem. Eng. Data 16: 235241.

Weiss, R F, and T K Kyser. 1978. Solubility of krypton in water and sea water. J. Chem. Eng. Data 23: 69-72.

Whitney, D.E., and W.M. Darley. 1983. Effect of light intensity upon salt marsh benthic microalgal photosynthesis. Mar. Bio. 75: 249-252.

Williams, P. J. 1. B., and D. Lefèvre. 1996. Algal 14 C and total carbon metabolisms. 1. Models to account for the physiological processes of respiration and recycling. J. Plankton Res. 18: 1941-1959.

Wilson, A.M., and L.R. Gardner. 2006. Tidally driven groundwater flow and solute exchange in a marsh: Numerical simulations. Wat. Resources Res. 42: doi:10.1029/2005WR004302

Wilson, A.M., and J.T. Morris. 2012. The influence of tidal forcing on groundwater flow and nutrient exchange in a salt marsh-dominated estuary. Biogeochem. 108: 27-38. 
Wilson, C.A., Z.J. Hughes, D.M. Fitzgerald, C.S. Hopkinson, V. Valentine, A.S. Kolker. 2014. Saltmarhs and tidal creek morphodynamics: Dynamic equilibroum of northern latitude salt marshes?

Geomorphology 213: 99-115.

Wood, D, and R Caputi. 1966. Solubilities of $\mathrm{Kr}$ and Xe in fresh and sea water. U.S. Naval Radiological Defense Laboratory, Tech. Rep.USNRDL-TR-988

Yacobi, Y. Z., N. Perel, E. Barkan, and B. Lus. Unexpected underestimation of primary productivity by $18 \mathrm{O}$ and $14 \mathrm{C}$ methods in a lake: Implications for slow diffusion of isotope tracers in and out of cells. Limnol. Oceanogr. 52: 329-337.

Yeung, L.Y., W.M. Berelson, E.D. Young, M.G. Prokopenko, N. Rollins, V.J. Coles, J.P. Montoya, E.D. Carpenter, D.K. Steinberg, R.A. Foster, D.G. Capone, P.L. Yager. 2012. Impact of diatom-diazotroph associations on carbon export in the Amazon River plume, Geophys. Res. Lett. 39(18): doi:10.1029/2012GL053356

Zappa, C.J., W.R. McGillis, P.A. Raymond, J.B. Edson, E.J. Hintsa, H.J. Zemmelink, J.W.H. Dacey, and D.T. Ho. 2007. Environmental turbulent mixing controls air-water gas exchange in marine and aquatic systems.

\section{Chapter 4}

Bieri, R. H. 1971. Dissolved noble gases in marine waters. Earth Planet. Sci. Lett. 10: 329-333. doi:10.1016/0012-821X(71)90038-0

Borges, A. V., B. Delille, L.-S. Schiettecatte, F. Gazeau, G. Abril, and M. Frankignoulle. 2004. Gas transfer velocities of $\mathrm{CO}_{2}$ in three European estuaries (Randers Fjord, Scheldt, and Thames). Limnol. Oceanogr. 49: 1630-1641. doi:10.4319/lo.2004.49.5.1630

Caffrey, J. M. 2004. Factors controlling net ecosystem metabolism in U.S. estuaries. Estuaries 27: 90101. doi:10.1007/BF02803563

Cheng, C. H., M. Huettel, and R. A. Wildman. 2014. Ebullition-enhanced solute transport in coarsegrained sediments. Limnol. Oceanogr. 59: 1733-1748. doi:10.4319/1o.2014.59.5.1733

Cole, J. J., and N. F. Caraco. 1998. Atmospheric exchange of carbon dioxide in a low-wind oligotrophic lake measured by the addition of SF 6 . Limnol. Oceanogr. 43: 647-656. doi:10.4319/1o.1998.43.4.0647

Cole, J. J., D. L. Bade, D. Bastviken, M. L. Pace, and M. Van de Bogert. 2010. Multiple approaches to estimating air-water gas exchange in small lakes. Limnol. Oceanogr. Methods 8: 285-293. doi:10.4319/1om.2010.8.285

Crosswell, J. R. 2015. Bubble Clouds in Coastal Waters and Their Role in Air-Water Gas Exchange of CO2. J. Mar. Sci. Eng. 3: 866-890. doi:10.3390/jmse3030866

Crusius, J., and R. Wanninkhof. 2003. Gas transfer velocities measured at low wind speed over a lake. Limnol. Oceanogr. 48: 1010-1017. doi:10.4319/lo.2003.48.3.1010

Hamme, R. C., and S. R. Emerson. 2004. The solubility of neon, nitrogen and argon in distilled water and seawater. Deep Sea Res. Part I Oceanogr. Res. Pap. 51: 1517-1528. doi:10.1016/j.dsr.2004.06.009 
Holocher, J., F. Peeters, W. Aeschbach-Hertig, W. Kinzelbach, and R. Kipfer. 2003. Kinetic Model of Gas Bubble Dissolution in Groundwater and Its Implications for the Dissolved Gas Composition. Environ. Sci. Technol. 37: 1337-1343. doi:10.1021/es025712z

Jenkins, W. J., D. E. Lott, M. W. Davis, S. P. Birdwhistell, and M. O. Matthewson. 1991. Measuring Helium Isotopes and Tritium in Seawater Samples.

Kremer, J. N., A. Reischauer, and C. D'Avanzo. 2003. Estuary-specific variation in the air-water gas exchange coefficient for oxygen. Estuaries 26: 829-836. doi:10.1007/BF02803341

Linacre, E. T. 1993. Data-sparse estimation of lake evaporation, using a simplified Penman equation. Agric. For. Meteorol. 64: 237-256. doi:10.1016/0168-1923(93)90031-C

MacIntyre, S., A. Jonsson, M. Jansson, J. Aberg, D. E. Turney, and S. D. Miller. 2010. Buoyancy flux, turbulence, and the gas transfer coefficient in a stratified lake. Geophys. Res. Lett. 37. doi:10.1029/2010GL044164

Maeck, A., H. Hofmann, and A. Lorke. 2014. Pumping methane out of aquatic sediments \&amp;ndash; ebullition forcing mechanisms in an impounded river. Biogeosciences 11: 2925-2938. doi:10.5194/bg-11-2925-2014

Manning, C. C., R. H. R. Stanley, D. P. Nicholson, and others. 2016. Quantifying air-sea gas exchange using noble gases in a coastal upwelling zone. IOP Conf. Ser. Earth Environ. Sci. 35: 12017. doi:10.1088/1755-1315/35/1/012017

Manning, C. C., R. H. R. Stanley, and D. E. Lott. 2016. Continuous Measurements of Dissolved Ne, Ar, $\mathrm{Kr}$, and Xe Ratios with a Field-Deployable Gas Equilibration Mass Spectrometer. Anal. Chem. 88: 3040-3048. doi:10.1021/acs.analchem.5b03102

Nuttle, W. K., and H. F. Hemand. 1988. Salt marsh hydrology: Implications for biogeochemical fluxes to the atmosphere and estuaries. Global Biogeochem. Cycles 2: 91-114. doi:10.1029/GB002i002p00091

Staehr, P. A., D. Bade, M. C. Van de Bogert, G. R. Koch, C. Williamson, P. Hanson, J. J. Cole, and T. Kratz. 2010. Lake metabolism and the diel oxygen technique: State of the science. Limnol. Oceanogr. Methods 8: 628-644. doi:10.4319/lom.2010.8.0628

Stanley, R. H. R., B. Baschek, D. E. Lott, and W. J. Jenkins. 2009. A new automated method for measuring noble gases and their isotopic ratios in water samples. Geochemistry, Geophys. Geosystems 10: n/a-n/a. doi:10.1029/2009GC002429

Stanley, R. H. R., and E. M. Howard. 2013. Quantifying photosynthetic rates of microphytobenthos using the triple isotope composition of dissolved oxygen. Limnol. Oceanogr. Methods 11: 360-373. doi:10.4319/lom.2013.11.360

Stanley, R. H. R., W. J. Jenkins, D. E. Lott, and S. C. Doney. 2009. Noble gas constraints on air-sea gas exchange and bubble fluxes. J. Geophys. Res. 114: C11020. doi:10.1029/2009JC005396

Tsai, J.-L., and B.-J. Tsuang. 2005. Aerodynamic roughness over an urban area and over two farmlands in a populated area as determined by wind profiles and surface energy flux measurements. Agric. For. Meteorol. 132: 154-170. doi:10.1016/j.agrformet.2005.07.008

Vachon, D., and Y. T. Prairie. 2013. The ecosystem size and shape dependence of gas transfer velocity versus wind speed relationships in lakes. Can. J. Fish. Aquat. Sci. 70: 1757-1764. doi:10.1139/cjfas2013-0241 
Walmsley, J. L. 1988. On theoretical wind speed and temperature profiles over the sea with applications to data from sable island, Nova Scotia. Atmosphere-Ocean 26: 203-233. doi:10.1080/07055900.1988.9649300

Weiss, R. F., and T. K. Kyser. 1978. Solubility of krypton in water and sea water. J. Chem. Eng. Data 23: 69-72. doi:10.1021/je60076a014

Wilson, C. A., Z. J. Hughes, D. M. FitzGerald, C. S. Hopkinson, V. Valentine, and A. S. Kolker. 2014. Saltmarsh pool and tidal creek morphodynamics: Dynamic equilibrium of northern latitude saltmarshes? Geomorphology 213: 99-115. doi:10.1016/j.geomorph.2014.01.002

Winslow, L., J. Zwart, R. Batt, H. Dugan, R. Woolway, J. Corman, P. Hanson, and J. Read. LakeMetabolizer: An R package for estimating lake metabolism from free- water oxygen using diverse statistical models. Inl. Waters In press.

Wood, D., and R. Caputi. 1966. Solubilities of $\mathrm{Kr}$ and Xe in fresh and sea water. U.S. Naval Radiological Defense Laboratory, San Francisco, CA.

\section{Chapter 5}

Angert, A., S. Rachmilevitch, E. Barkan, and B. Luz. 2003. Effects of photorespiration, the cytochrome pathway, and the alternative pathway on the triple isotopic composition of atmospheric $\mathrm{O}_{2}$. Global Biogeochem. Cycles 17. doi:10.1029/2002GB001933

Argow, B. A., Z. J. Hughes, and D. M. FitzGerald. 2011. Ice raft formation, sediment load, and theoretical potential for ice-rafted sediment influx on northern coastal wetlands. Cont. Shelf Res. 31: 1294-1305. doi:10.1016/j.csr.2011.05.004

Bender, M., K. Grande, K. Johnson, and others. 1987. A comparison of four methods for determining planktonic community production. Limnol. Oceanogr. 1085-1098.

Berman-Frank, I., P. Lundgren, Y.-B. Chen, H. Küpper, Z. Kolber, B. Bergman, and P. Falkowski. 2001. Segregation of Nitrogen Fixation and Oxygenic Photosynthesis in the Marine Cyanobacterium Trichodesmium. Science (80-. ). 294: 15434-1537. doi:DOI: 10.1126/science.1064082

Birmingham, B. C., J. R. Coleman, and B. Colman. 1982. Measurement of photorespiration in algae. Plant Physiol. 69: 259-62. doi:10.1104/PP.69.1.259

Buapet, P., L. M. Rasmusson, M. Gullström, and others. 2013. Photorespiration and Carbon Limitation Determine Productivity in Temperate Seagrasses J.F. Valentine [ed.]. PLoS One 8: e83804. doi:10.1371/journal.pone.0083804

Cebrian, J., C. D. Foster, R. Plutchak, K. L. Sheehan, M.-E. C. Miller, A. Anton, K. Major, K. L. Heck, and S. P. Powers. 2007. The impact of Hurricane Ivan on the primary productivity and metabolism of marsh tidal creeks in the NorthCentral Gulf of Mexico. Aquat. Ecol. 42: 391-404.

Coleman, M. L., R. Raiswell, A. Brown, and others. 1993. Microbial Mineralization of Organic Matter: Mechanisms of Self-Organization and Inferred Rates of Precipitation of Diagenetic Minerals [and Discussion]. Philos. Trans. R. Soc. London A Math. Phys. Eng. Sci. 344. doi:DOI: 10.1098/rsta.1993.0076 
Deegan, L. A., D. S. Johnson, R. S. Warren, B. J. Peterson, J. W. Fleeger, S. Fagherazzi, and W. M. Wollheim. 2012. Coastal eutrophication as a driver of salt marsh loss. Nature 490: 388-92. doi:10.1038/nature11533

Dickson, A. G., C. L. Sabine, and J. R. Christian. 2007. Guide to Best Practices for Ocean CO2 Measurements.

Dupraz, C., R. P. Reid, O. Braissant, A. W. Decho, R. S. Norman, and P. T. Visscher. 2009. Processes of carbonate precipitation in modern microbial mats. Earth-Science Rev. 96: 141-162. doi:10.1016/j.earscirev.2008.10.005

Eisenstadt, D., E. Barkan, B. Luz, and A. Kaplan. 2010. Enrichment of oxygen heavy isotopes during photosynthesis in phytoplankton. Photosynth. Res. 103: 97-103. doi:10.1007/s11120-009-9518-Z

Fenchel, T., and R. N. Glud. 2000. Benthic primary production and O2-CO2 dynamics in a shallow-water sediment: Spatial and temporal heterogeneity. Ophelia 53: 159-171. doi:10.1080/00785236.2000.10409446

Forbrich, I., and A. E. Giblin. 2015. Marsh-atmosphere $\mathrm{CO}_{2}$ exchange in a New England salt marsh. J. Geophys. Res. Biogeosciences 120: 1825-1838. doi:10.1002/2015JG003044

Garcia, H. E., and L. I. Gordon. 1992. Oxygen solubility in seawater: Better fitting equations. Limnol. Oceanogr. 37: 1307-1312. doi:10.4319/lo.1992.37.6.1307

Garcia, H. E., and L. I. Gordon. 1993. Erratum: Oxygen solubility in seawater: better fitting equations. Limnol. Oceanogr. 38: 656.

Gedan, K., A. Altieri, and M. Bertness. 2011. Uncertain future of New England salt marshes. Mar. Ecol. Prog. Ser. 434: 229-237. doi:10.3354/meps09084

Giblin, A. E., and R. W. Howarth. 1984. Porewater evidence for a dynamic sedimentary iron cycle in salt marshes1. Limnol. Oceanogr. 29: 47-63. doi:10.4319/lo.1984.29.1.0047

Halsey, K. H., and B. M. Jones. 2015. Phytoplankton Strategies for Photosynthetic Energy Allocation. Ann. Rev. Mar. Sci. 7: 265-297. doi:10.1146/annurev-marine-010814-015813

Halsey, K. H., A. J. Milligan, and M. J. Behrenfeld. 2010. Physiological optimization underlies growth rate-independent chlorophyll-specific gross and net primary production. Photosynth. Res. 103: 125137. doi:10.1007/s11120-009-9526-z

Hancke, K., and R. Glud. 2004. Temperature effects on respiration and photosynthesis in three diatomdominated benthic communities. Aquat. Microb. Ecol. 37: 265-281. doi:10.3354/ame037265

Hartig, E. K., V. Gornitz, A. Kolker, F. Mushacke, and D. Fallon. 2002. Anthropogenic and climatechange impacts on salt marshes of Jamaica Bay, New York City. Wetlands 22: 71-89. doi:10.1672/0277-5212(2002)022[0071:AACCIO]2.0.CO;2

Hedges, J. I., J. A. Baldock, Y. Gélinas, C. Lee, M. L. Peterson, and S. G. Wakeham. 2002. The biochemical and elemental compositions of marine plankton: A NMR perspective. Mar. Chem. 78: 47-63.

Helman, Y., E. Barkan, D. Eisenstadt, B. Luz, and A. Kaplan. 2005. Fractionation of the three stable oxygen isotopes by oxygen-producing and oxygen-consuming reactions in photosynthetic organisms. Plant Physiol. 138: 2292-8. doi:10.1104/pp.105.063768 
Hopkinson, C. 2000. Transects of the Parker River Plum Island Sound Estuary conducted at dawn and dusk, containing dissolved oxygen, conductivity, temperature, percent saturation, $\mathrm{pH}, \mathrm{DIC}$ and $\mathrm{pCO} 2$ data.doi:doi:10.6073/pasta/30017c0f0d8f92226d243baabc8ef15f

Hopkinson, C. S., A. E. Giblin, J. Tucker, and R. H. Garritt. 1999. Benthic Metabolism and Nutrient Cycling along an Estuarine Salinity Gradient. Estuaries 22: 863. doi:10.2307/1353067

Hopkinson, C. S., W.-J. Cai, and X. Hu. 2012. Carbon sequestration in wetland dominated coastal systems - a global sink of rapidly diminishing magnitude. Curr. Opin. Environ. Sustain. 4: 186-194.

Howard, E. M., R. H. R. Stanley, I. Forbrich, A. E. Giblin, D. E. Lott, and K. L. Cahill. Using noble gases in a shallow aquatic envrionment to compare common gas exchange parameterizations and to constrain efflux of oxygen by ebullition. Submitted.

Jahnke, R. A., J. R. Nelson, R. L. Marinelli, and J. E. Eckman. 2000. Benthic flux of biogenic elements on the Southeastern US continental shelf: influence of pore water advective transport and benthic microalgae. Cont. Shelf Res. 20: 109-127.

Johnston, M. E. 2003. Importance of metabolism in the development of salt marsh ponds. Biol. Bull. 205: 248-249.

Jovanovic, Z., M. Pedersen, M. Larsen, E. Kristensen, and R. Glud. 2015. Rhizosphere O2 dynamics in young Zostera marina and Ruppia maritima. Mar. Ecol. Prog. Ser. 518: 95-105. doi:10.3354/meps11041

Kaiser, J. 2011. Technical note: Consistent calculation of aquatic gross production from oxygen triple isotope measurements. Biogeosciences 8: 1793-1811. doi:10.5194/bg-8-1793-2011

Karsten, U., L. A. Franklin, K. Lüning, and C. Wiencke. 1998. Natural ultraviolet radiation and photosynthetically active radiation induce formation of mycosporine-like amino acids in the marine macroalga Chondrus crispus (Rhodophyta). Planta 205: 257-262. doi:10.1007/s004250050319

Kinney, E., and C. Roman. 1998. Response of primary producers to nutrient enrichment in a shallow estuary. Mar. Ecol. Prog. Ser. 163: 89-98. doi:10.3354/meps163089

Koch, E., and G. Gust. 1999. Water flow in tide- and wave-dominated beds of the seagrass Thalassia testudinum. Mar. Ecol. Prog. Ser. 184: 63-72. doi:10.3354/meps184063

Koretsky, C. M., C. Meile, and P. Van Cappellen. 2002. Quantifying bioirrigation using ecological parameters: a stochastic approach $\uparrow$. Geochem. Trans. 3: 17. doi:10.1186/1467-4866-3-17

Larsen, S., L. P. Nielsen, and A. Schramm. 2015. Cable bacteria associated with long-distance electron transport in New England salt marsh sediment. Environ. Microbiol. Rep. 7: 175-179. doi:10.1111/1758-2229.12216

Laws, E. A. 1991. Photosynthetic quotients, new production and net community production in the open ocean. Deep Sea Res. Part A. Oceanogr. Res. Pap. 38: 143-167.

Lewis, E., and D. Wallace. 1998. Program developed for CO2 system calculations. 21.

Li, S., N. E. Levin, and L. A. Chesson. 2015. Continental scale variation in 17O-excess of meteoric waters in the United States. Geochim. Cosmochim. Acta 164: 110-126. doi:10.1016/j.gca.2015.04.047

Limentani, G. B., M. C. Ringo, F. Ye, M. L. Bergquist, and E. O. MCSorley. 2005. Beyond the t-Test: Statistical Equivalence Testing. Anal. Chem. 77: 221-226. doi:10.1021/ac053390m 
Llewellyn, C. A., and R. L. Airs. 2010. Distribution and abundance of MAAs in 33 species of microalgae across 13 classes. Mar. Drugs 8: 1273-91. doi:10.3390/md8041273

Ludwig, R., F. A. Al-Horani, D. de Beer, and H. M. Jonkers. 2005. Photosynthesis-controlled calcification in a hypersaline microbial mat. Limnol. Oceanogr. 50: 1836-1843.

doi:10.4319/1o.2005.50.6.1836

Luther, G. W., A. Giblin, R. W. Howarth, and R. A. Ryans. 1982. Pyrite and oxidized iron mineral phases formed from pyrite oxidation in salt marsh and estuarine sediments. Geochim. Cosmochim. Acta 46: 2665-2669. doi:10.1016/0016-7037(82)90385-4

Luz, B., and E. Barkan. 2010. Variations of $17 \mathrm{O} / 16 \mathrm{O}$ and $18 \mathrm{O} / 16 \mathrm{O}$ in meteoric waters. Geochim. Cosmochim. Acta 74: 6276-6286. doi:10.1016/j.gca.2010.08.016

Luz, B., and E. Barkan. 2011. Proper estimation of marine gross $\mathrm{O}_{2}$ production with ${ }^{17} \mathrm{O} /{ }^{16} \mathrm{O}$ and ${ }^{18} \mathrm{O} /$ ${ }^{16} \mathrm{O}$ ratios of dissolved $\mathrm{O}_{2}$. Geophys. Res. Lett. 38. doi:10.1029/2011GL049138

MacIntyre, H. L., R. J. Geider, and D. C. Miller. 1996. Microphytobenthos: The Ecological Role of the "Secret Garden" of Unvegetated, Shallow-Water Marine Habitats. I. Distribution, Abundance and Primary Production. Estuaries 19: 186. doi:10.2307/1352224

MacIntyre, H. L., T. M. Kana, T. Anning, and R. J. Geider. 2002. PHOTOACCLIMATION OF PHOTOSYNTHESIS IRRADIANCE RESPONSE CURVES AND PHOTOSYNTHETIC PIGMENTS IN MICROALGAE AND CYANOBACTERIA1. J. Phycol. 38: 17-38. doi:10.1046/j.1529-8817.2002.00094.x

Marra, J., and R. T. Barber. 2004. Phytoplankton and heterotrophic respiration in the surface layer of the ocean. Geophys. Res. Lett. 31. doi:10.1029/2004GL019664

Massé, A., O. Pringault, and R. De Wit. 2002. Experimental study of interactions between purple and green sulfur bacteria in sandy sediments exposed to illumination deprived of near-infrared wavelengths. Appl. Environ. Microbiol. 68: 2972-81. doi:10.1128/aem.68.6.2972-2981.2002

Millette, T. L., B. A. Argow, E. Marcano, C. Hayward, C. S. Hopkinson, and V. Valentine. 2010. Salt Marsh Geomorphological Analyses via Integration of Multitemporal Multispectral Remote Sensing with LIDAR and GIS. J. Coast. Res. 265: 809-816. doi:10.2112/JCOASTRES-D-09-00101.1

Murray, L., and R. L. Wetzel. 1987. Oxygen production and consumption associated with the major autotrophic components in two temperate seagrass communities. Mar. Ecol. Prog. Ser. 13: 231-239.

Nepf, H., and M. Ghisalberti. 2008. Flow and transport in channels with submerged vegetation. Acta Geophys. 56: 753-777. doi:10.2478/s11600-008-0017-y

Nicholson, D. P., R. H. R. Stanley, E. Barkan, D. M. Karl, B. Luz, P. D. Quay, and S. C. Doney. 2012. Evaluating triple oxygen isotope estimates of gross primary production at the Hawaii Ocean Timeseries and Bermuda Atlantic Time-series Study sites. J. Geophys. Res. 117: C05012. doi:10.1029/2010JC006856

Nicholson, J. A. M., J. F. Stolz, and B. K. Pierson. 1987. Structure of a microbiol mat at Great Sippewissett Marsh, Cape Cod, Massachusetts. FEMS Microbiol. Lett. 45: 343-364. doi:10.1111/j.1574-6968.1987.tb02411.x

Nidzieko, N. J., J. A. Needoba, S. G. Monismith, and K. S. Johnson. 2014. Fortnightly Tidal Modulations Affect Net Community Production in a Mesotidal Estuary. Estuaries and Coasts 37: 91-110. doi:10.1007/s12237-013-9765-2 
Niel, C. B. 1932. On the morphology and physiology of the purple and green sulphur bacteria. Arch. Mikrobiol. 3: 1-112. doi:10.1007/BF00454965

PEDERSEN, O., T. BINZER, and J. BORUM. 2004. Sulphide intrusion in eelgrass (Zostera marina L.). Plant, Cell Environ. 27: 595-602. doi:10.1111/j.1365-3040.2004.01173.x

Pinckney, J. L., and H. W. Paerl. 1997. Anoxygenic photosynthesis and nitrogen fixation by a microbial mat community in a Bahamian hypersaline lagoon. Appl. Environ. Microbiol. 63: 420-6.

Pinckney, J., and R. Zingmark. 1993. Biomass and production of benthic microalgal communities in estuarine habitats. Estuaries 16: 887-897. doi:10.2307/1352447

Pringault, O., V. Tassas, and E. Rochelle-Newall. 2007. Consequences of respiration in the light on the determination of production in pelagic systems. Biogeosciences 4: 105-114. doi:10.5194/bg-4-1052007

Prokopenko, M. G., O. M. Pauluis, J. Granger, and L. Y. Yeung. 2011. Exact evaluation of gross photosynthetic production from the oxygen triple-isotope composition of O 2 : Implications for the net-to-gross primary production ratios. Geophys. Res. Lett. 38: n/a-n/a. doi:10.1029/2011GL047652

Rao, A. M. F., S. Y. Malkin, S. Hidalgo-Martinez, and F. J. R. Meysman. 2016. The impact of electrogenic sulfide oxidation on elemental cycling and solute fluxes in coastal sediment. Geochim. Cosmochim. Acta 172: 265-286. doi:10.1016/j.gca.2015.09.014

Rao, A., N. Risgaard-Petersen, and U. Neumeier. 2016. Electrogenic sulfur oxidation in a northern saltmarsh (St. Lawrence Estuary, Canada). Can. J. Microbiol. 62: 530-537. doi:10.1139/cjm-20150748

Romano, C., J. Widdows, M. Brinsley, and F. Staff. 2003. Impact of Enteromorpha intestinalis mats on near-bed currents and sediment dynamics: flume studies. Mar. Ecol. Prog. Ser. 256: 63-74. doi:10.3354/meps 256063

Rosenberg, G., D. S. Littler, M. M. Littler, and E. C. Oliveira. 1995. Primary Production and Photosynthetic Quotients of Seaweeds from São Paulo State, Brazil. Bot. Mar. 38: 369-378. doi:10.1515/botm.1995.38.1-6.369

Spivak, A. C., K. Gosselin, E. M. Howard, G. Mariotti, I. Forbrich, and R. H. R. Stanley. Small ponds are semi-isolated biogeochemical hotspots in salt marsh ecosystems. Sumbitted.

Staehr, P. A., D. Bade, M. C. Van de Bogert, G. R. Koch, C. Williamson, P. Hanson, J. J. Cole, and T. Kratz. 2010. Lake metabolism and the diel oxygen technique: State of the science. Limnol. Oceanogr. Methods 8: 628-644. doi:10.4319/lom.2010.8.0628

Stephens, E. A., O. Braissant, and P. T. Visscher. 2008. Spirochetes and salt marsh microbial mat geochemistry: Implications for the fossil record. Carnets Geol. 9.

Swider, K. T., and J. E. Mackin. 1989. Transformations of sulfur compounds in marsh-flat sediments. Geochim. Cosmochim. Acta 53: 2311-2323. doi:10.1016/0016-7037(89)90353-0 
Trüper, H. G. 1964. Sulphur metabolism in Thiorhodaceae. II. stoichiometric relationship of CO2 fixation to oxidation of hydrogen sulphide and intracellular sulphur inChromatium okenii. Antonie Van Leeuwenhoek 30: 385-394. doi:10.1007/BF02046751

Vachon, D., and Y. T. Prairie. 2013. The ecosystem size and shape dependence of gas transfer velocity versus wind speed relationships in lakes. Can. J. Fish. Aquat. Sci. 70: 1757-1764. doi:10.1139/cjfas2013-0241

Vallino, J. J., C. S. Hopkinson, and R. H. Garritt. 2005. Estimating estuarine gross production, community respiration and net ecosystem production: a nonlinear inverse technique. Ecol. Modell. 187: 281-296.

Vasquez-Cardenas, D., J. van de Vossenberg, L. Polerecky, and others. 2015. Microbial carbon metabolism associated with electrogenic sulphur oxidation in coastal sediments. ISME J. 9: 19661978. doi:10.1038/ismej.2015.10

Veiderpass, V., and A. Stigebrandt. 2012. The impact of waves on the distribution of submerged macrophytes in Kalvöfjord, Sweden - A study of "comfort zones"; Vatten 68: 255-264.

Whitney, D. E., and W. M. Darley. 1983. Effect of light intensity upon salt marsh benthic microalgal photosynthesis. Mar. Biol. 75: 249-252. doi:10.1007/BF00406009

Williams, P. J. I., and J. E. Robertson. 1991. Overall planktonic oxygen and carbon dioxide metabolisms: the problem of reconciling observations and calculations of photosynthetic quotients. J. Plankton Res. 13: $153-169$.

Wilson, C. A., Z. J. Hughes, D. M. FitzGerald, C. S. Hopkinson, V. Valentine, and A. S. Kolker. 2014. Saltmarsh pool and tidal creek morphodynamics: Dynamic equilibrium of northern latitude saltmarshes? Geomorphology 213: 99-115. doi:10.1016/j.geomorph.2014.01.002

Winslow, L. A., J. A. Zwart, R. D. Batt, H. Dugan, R. I. Woolway, J. Corman, P. C. Hanson, and J. S. Read. 2016. LakeMetabolizer: An R package for estimating lake metabolism from free-water oxygen using diverse statistical models. Inl. Waters 6: 622-636.

\section{Chapter 6}

Manning, C. C., R. H. R. Stanley, and D. E. Lott. 2016. Continuous Measurements of Dissolved Ne, Ar, $\mathrm{Kr}$, and Xe Ratios with a Field-Deployable Gas Equilibration Mass Spectrometer. Anal. Chem. 88: 3040-3048. doi:10.1021/acs.analchem.5b03102

Steig, E. J., V. Gkinis, A. J. Schauer, S. W. Schoenemann, K. Samek, J. Hoffnagle, K. J. Dennis, and S. M. Tan. 2014. Calibrated high-precision 17O-excess measurements using cavity ring-down spectroscopy with laser-current-tuned cavity resonance. Atmos. Meas. Tech. 7: 2421-2435. doi:10.5194/amt-7-2421-2014 
216 